\title{
Plant Associations and Related Botanical Inventory of the Beaverhead Mountains Section, Montana
}

\section{Prepared for:}

United States Department of the Interior

Bureau of Land Management

P.O. Box 36800

Billings, Montana 59107-6800

Submitted by:

Stephen V. Cooper, Catherine Jean, and Bonnie L. Heidel

December, 1999

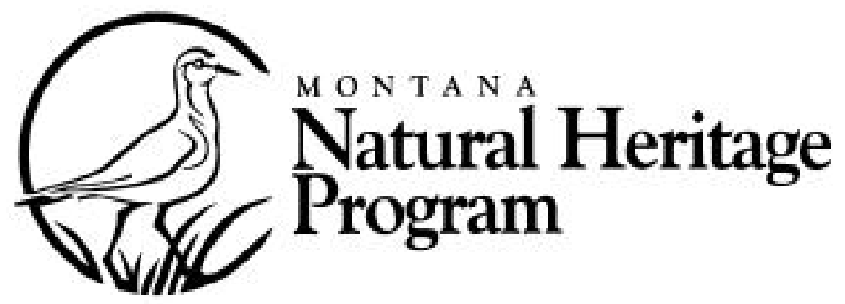




\section{Plant Associations and Related Botanical Inventory of the Beaverhead Mountains Section, Montana}

(C) 1999 Montana Natural Heritage Program

State Library Building • P.O. Box 201800 • 1515 East Sixth Avenue • Helena, MT 59620-1800 • 406-444-3009

BLM Agreement Number 1422E930A960015

Task Order Number 16

This document should be cited as follows:

Cooper, S. V., Jean, C. and B. L. Heidel. 1999. Plant associations and related botanical inventory of the Beaverhead Mountains Section, Montana. Unpublished report to the Bureau of Land Management. Montana Natural Heritage Program, Helena. 235 pp. 


\section{Table of Contents}

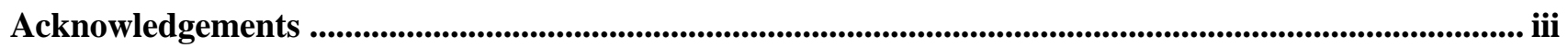

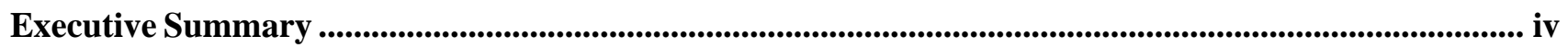

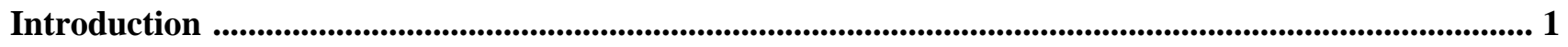

Study Area

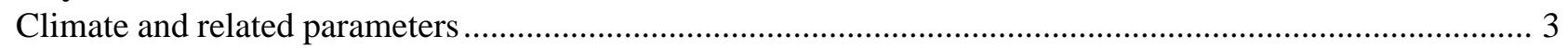

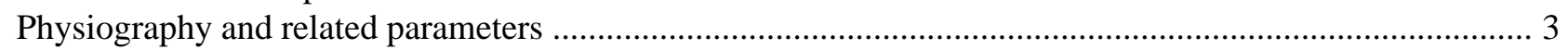

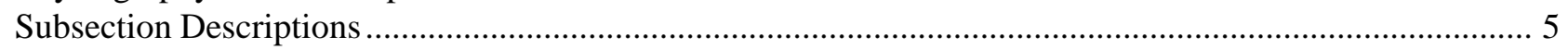

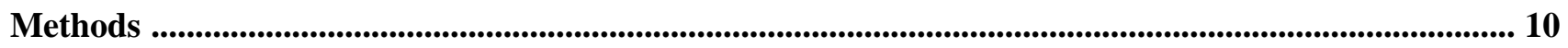

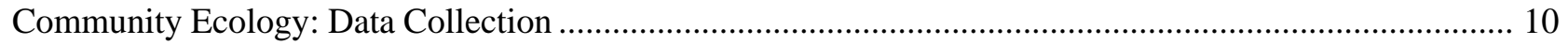

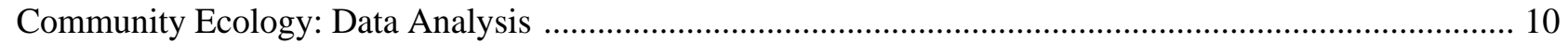

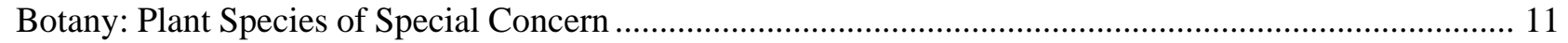

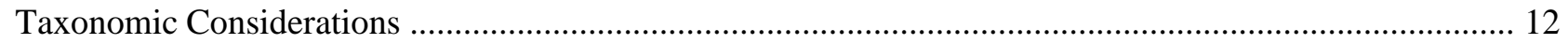

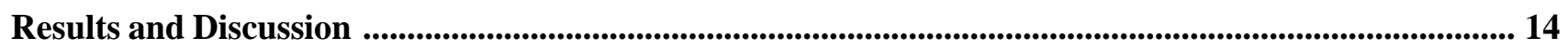

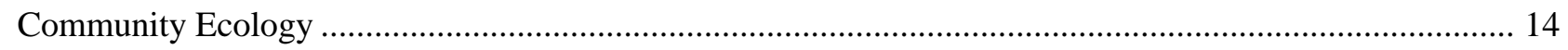

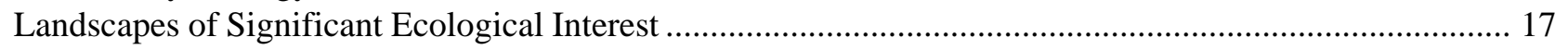

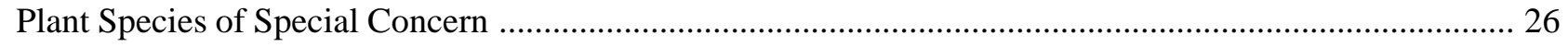

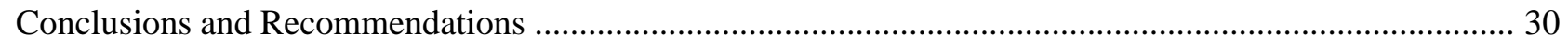

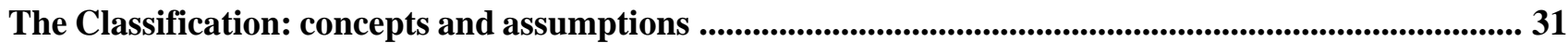

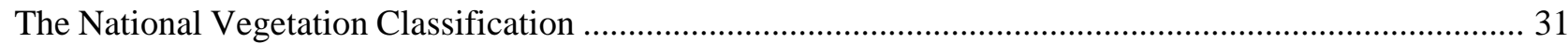

Plant Associations of the Beaverhead Mountains Section: Vegetation key and descriptions ...................... 33

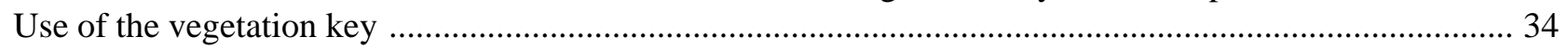

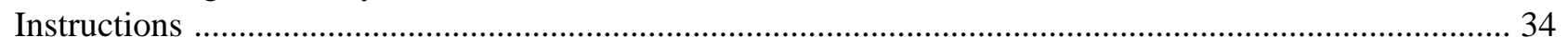

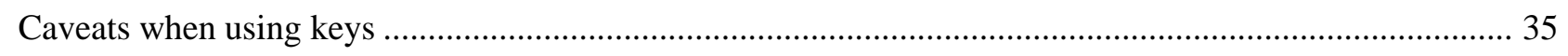

Key to Physiognomic Types (Lifeform Categories) ........................................................................................... 37

Vegetation Classication: Descriptions of Types ...........................................................................................76

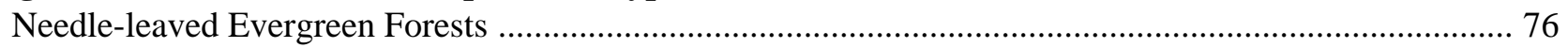

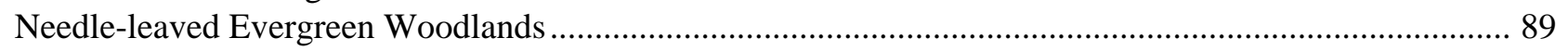

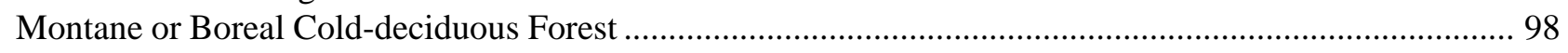

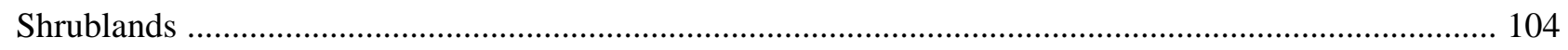

Dwarf-Shrublands including Dwarf-Shrub Herbaceous ................................................................ 126

Herbaceous Vegetation Lacking a Significant Shrub/Tree Component ................................................ 142

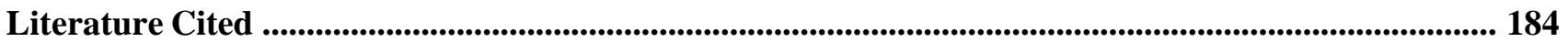

\section{Appendices}

Appendix A: Flora of the Beaverhead Mountains Section ................................................................... 194

Appendix B: Natural Heritage Global Rank Definitions and Their Modifiers ......................................... 220

Appendix C: Plant Associations of the Beaverhead Mountains Section ................................................. 221

Appendix D: Plant Associations: Principal and Substantiating References ............................................. 230 


\section{Figures}

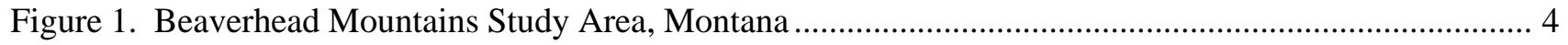

Figure 2. Beaverhead Mountains Section and Subsection Boundaries, Montana ........................................ 6

Figure 3. Locations of Select Globally Rare and Exemplary Plant Associations, Beaverhead Mountains

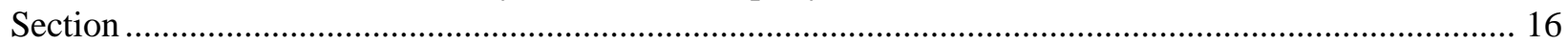

Figure 4. Locations of Plant Species of Special Concern, Beaverhead Mountains Section .......................... 27

\section{Tables}

Table 1. Synonymy of dominant and indicator plant species for the Beaverhead Mountains Section ............ 13

Table 2. Distribution of community types by lifeform and global rank .................................................. 15

Table 3. State and regional endemic plant species in the Beaverhead Mountains Section ........................... 19

Table 4. Globally rare plant species in the Beaverhead Mountains Section ............................................... 26

Table 5. State or globally rare plants known only from the Beaverhead Mountains Section ......................... 29 


\section{Acknowledgements}

The authors gratefully acknowledge the financial assistance provided by the USDI Bureau of Land Management (BLM): we especially appreciate the efforts of Brian Hockett, BLM range conservationist, Dillon, MT, in helping us locate and access field sites and for providing background information on such sites. Brian also shared his extensive knowledge of rangeland ecosystems of the ecoregion; should this document contain any misinterpretations of these systems the blame rests squarely with the authors. The USDA Forest Service, particularly Annie Greene of the Beaverhead National Forest and John Caratti of the R-1 Regional Office, were generous in supplying plot information in appropriate databases.

Preceding ecologists/botanists at the Montana Natural Heritage Program who laid the groundwork for this publication in terms of plot sampling, data reduction and analysis include Peter Achuff, Alistair McAlpine, Lisa Roe, Jim Vanderhorst, and Bonnie Heidel; Rob DeVelice was instrumental in securing funding for these early efforts and served both as the primary field ecologist and data analyst. Bonnie Heidel, Peter Achuff, Ken Scow, Jim Vanderhorst and Lisa Roe provided valuable assistance in voucher specimen identification. Margaret Beer, John Hinshaw, Cedron Jones, Scott LeeChadde, Debbie Dover, Kathy Jurist and Diana Schwab are appreciated for their conscientious efforts in data entry, quality control, slide scanning and the myriad support activities that contributed to this document. 


\section{Executive
Summary}

This project represents a comprehensive effort to identify and document all plant community types present in the Montana portion of the Beaverhead Mountains Ecoregional Section. The study area is an ecoregion defined by R.G. Bailey that extends from the Centennial Mountains south of Red Rock Lakes National Wildlife Refuge in southwestern Montana, west to the Continental Divide along the Beaverhead Mountains and includes the headwaters of the Beaverhead, Madison and Clarks Fork River.

Our goal for this project was to compile ecology plot data, validate and refine existing plant association types and combine them into a new classification following the National Vegetation Classification Standard. We tapped a number of previous and ongoing studies, and conducted extensive plant community surveys during 1998 field season. The result is a classification documenting 273 plant communities in the Beaverhead Mountains Section of southwest Montana.

This classification has three major components. The first is a complete list of 273 plant associations with state and global Heritage ranks that reflect their conservation status. The second component is a dichotomous plant association key organized by physiognomic type. The key is arranged according to the National Vegetation Classification, using the concept of forest, woodland, shrubland and herbaceous vegetation, and includes a number of newly identified vegetation types. The third component consists of standardized descriptions for 130 plant associations.

The results of this study underscore the high diversity of plants and vegetation types in this region of Montana. Over half of the 480 plant associations identified for the state of Montana (57\%) were documented to occur within the Beaverhead Mountains Section. This is especially impressive, given that the region comprises less than one-tenth of the state's total land area.

Of these 480 plant associations, just over $25 \%$ were ranked G3 or higher (some tentatively), indicating rangewide/global rarity or imperilment. The rarest community documented (ranked G1) was Artemisia cana/Leymus cinereus shrubland, found in a single location at Big Muddy Creek. Some of the communities ranked as imperiled (G2) occur in bottomlands or riparian areas, and are at greatest risks from certain grazing practices, weeds, and in some cases conversion to agriculture. Others occur as small patches in alpine or high subalpine environments, and are not particularly threatened. Yet another community, Elymus lanceolatus / Phacelia hastata, occurs in the Centennial Sandhills and relies on a natural disturbance regime maintained by animal- or fire-induced blowouts. Over time, some communities will decrease in rank, as additional stands are discovered while others may become imperiled and increase in rank due to loss of habitat or the increase of noxious weeds.

In addition to plant communities, this study also identified 130 plant species of global or state special concern that occur in the Beaverhead Mountains Section. This represents $30 \%$ of all special concern vascular plant species tracked in Montana, and includes $41 \%$ of all those that are globally significant (G1-G3). Most of these occur on at one or more locations on BLM administered public lands. The Beaverhead Section also supports 18 plant species that are endemic to Montana (occur nowhere else); this represents $31 \%$ of Montana's endemic plant species. The regions geological diversity, vertical relief, and the unique intersection Pacific and Gulf (of Mexico) storm tracks, as well as the overlap of several floristic elements explain the diversity of plants and vegetation communities.

Finally, six landscapes of exceptional ecological significance were identified, based upon the diversity and conditions of vegetation communities represented, and are described in this report. They include Bannock, Blacktail \& Robb-Ledford, Centennial Mountains \& Valley, Centennial Sandhills, Johnson Gulch-Deer Canyon Creek, and Morrison Lake Wetlands and Baldy Mountain. Each includes BLM lands, as well as other types of public ownership.

The Beaverhead Mountains Section is the first large ecoregional unit in Montana with a comprehensive Vegetation Classification compiled in a single document. This report reflects our current knowledge, and is by no means exhaustive. Additional survey work is needed to better document the globally imperiled plant communities and to provide better information to assist in their conservation and management. There is also a need for additional inventory and documentation of good quality, representative communities, and significant landscapes. These areas, and the limited resources they support, are of considerable ecological value and are worth managing to maintain their ecological value character. 


\section{Introduction}

The Beaverhead Mountains Section in southwestern Montana is a complex physical environment supporting a diverse landscape that is reflected in the number and physiognomy of natural plant communities. A classification of these communities gives land managers, researchers and interested citizens a statewide perspective of community distribution and variation. This perspective is crucial when identifying important areas for conservation and for making sound management prescriptions or predictions regarding future desired conditions of Montana's natural heritage. This project publication represents one milestone in the course of developing a comprehensive classification of Montana's plant communities.

This project endeavors to provide a compilation and description of plant communities within the Beaverhead Mountains Section (BVHDMS), in southwest Montana. It constitutes both an update of previous studies and a terminal report for recent plant community and botanical survey work conducted by the Montana Natural Heritage Program (MTNHP). The Beaverhead Mountains Section is an ecoregion defined by R. G. Bailey (1995, McNab and Avers 1994) within the Middle Rocky Mountain SteppeConiferous Forest-Alpine Meadow Province. The Montana portion of this section is an area of some 7,050,000 acres. We choose this ecoregion for a pilot effort in developing a comprehensive community classification because 1) an ecoregion is expected to be a meaningful ecological unit for the classification of plant communities, 2) BVHDMS has been intensively sampled for community classification purposes and 3) this area possesses a wealth of plant community diversity and is pivotal to our understanding of the states natural resources.

Several preceding MTNHP efforts have concentrated on the inventory of BLM lands within the Dillon Resource Area (RA), to a lesser extent the Headwaters RA and peripherally the Garnet RA. These efforts have emphasized locating, sampling and describing 1) what appeared to be previously undescribed communities and 2) common communities in good to excellent condition (relatively free of disturbance) 3) habitats expected to harbor sensitive, endangered or threatened plant species. We have also attempted to seek out and include data not generated by the MTNHP, but rather produced by other reliable sources such as the U. S. Forest Service's ECODATA plots (from Region 1 and the Beaverhead National Forest) and plots from classifications already in use, such as those of U. S. Forest Service research personnel, e.g. R. D. Pfister (Pfister et al. 1977) and W. F. Mueggler (Mueggler and Stewart 1980) and the Montana Riparian / Wetland Association (Hansen et al. 1995). In many cases the vegetation types (habitat types or climax plant associations) defined by the Forest Service and other researchers constitute initial attempts at classification; however, these classifications and their imbedded vegetation types have withstood the test of time and been shown to effectively partition the landscape into units of comparable biologic potential.

More recently there has been an impetus to create a national vegetation classification (Loucks 1996), a classification focused more on existing vegetation than on potential natural vegetation (as has been the case in Montana).

The initial effort in this regard has been "International classification of ecological communities: Terrestrial vegetation of the United States Volume I. The National Vegetation Classification System: development, status, and applications" Grossman et al. (1998) and Volume 2 "The National Vegetation Classification System: List of types" Anderson et al. 1998. Both these volumes are products of The Nature Conservancy and their stated purpose is to serve as useful implements for making on-theground conservation and resource management decisions. The Federal Geographic Data Committee-Vegetation Subcommittee (on which all federal agencies are represented) has produced a document "National Vegetation 
Classification Standard, June 1997" which, among other things, subscribes to the TNC National Vegetation Classification, at least accepting all the hierarchical levels that treat physiognomy (structure and appearance of vegetation) and reserving judgement only on the floristically defined levels, alliance and plant association, pending analysis and a report from the Ecological Society of America.

To make a classification useful, a means of somewhat unequivocally and objectively identifying taxonomic units must be provided in the form of a key. In this report we have adopted the National Vegetation Classification hierarchy and its keys, at least down to the Formation level. At the floristic levels (alliance and plant association) we have both adopted, as originally constructed, and modified the keys of preceding ecologists/ researchers. Dichotomous keys are not classifications, but they are the window to the classification and sincere attention has been paid to capturing the classificatory ideals of our predecessors, while simultaneously synthesizing and incorporating insights gained in subsequent sampling. This resulting classification with its accompanying vegetation key and descriptions of plant associations will be useful for the identification of plant community types (referred to more formally by their taxonomic status as plant associations), especially sensitive ones and natural areas where management prescriptions need adjusting to maintain habitat values. An important function of the classification is to serve as a surrogate for the categorization of environmental variation; it also serves as a reference system for baseline monitoring of environmental impacts and vegetation recovery. 


\section{Study Area}

This report is generated from natural plant communities sampled or in some manner documented to occur within the Montana portion (Figure 1) of the Beaverhead Mountains Section (BVHDMS) and referenced as M332E in Bailey et al. (1994). The Montana portion alone comprises just over 7,000,000 acres and together with an almost equally large expanse in east-central Idaho constitute the largest Section within the Middle Rocky Mountain Steppe - Coniferous Forest- Alpine Meadow Province (M332, Bailey et al. 1994). The Montana portion of BVHDMS encompasses virtually all of Beaverhead and Silver Bow Counties, slightly more than the western half portion of Madison County, the southern half of Powell County, and all but the northernmost portion of Deer Lodge County (Figure 1).

\section{Climate and related parameters}

All of the study area experiences, with local variations and permutations, a continental climate with cold, relatively dry winters with the months of greatest precipitation being May and June. It is a cool, dry country where sagebrush-grass communities cover the valleys and forests are restricted to higher elevations. Most of this ecoregion lies in the rainshadow of Oregon's Blue Mountains and central Idaho's high mountain mass, meaning it receives lesser amounts of precipitation from Pacific storm systems than other Montana ecoregions to the north. Average annual precipitation varies from over 50 inches at the Pioneer Mountain's crest to between 8 and 16 inches throughout the bulk of the study area (see sheets 3 and 4 in Ross and Hunter 1976). Summers are relatively dry with orographically generated precipitation events (moisture source often being northward flowing Gulfsteam air masses) having their greatest frequency and impact on anywhere in Montana, in these southwestern mountains. The average length of the freeze-free season varies from less than
30 days to more than 70 days, at highest and lowest elevations, respectively (see Figure 13 in Montagne et al. 1982).

The severity of climate is attested to the relatively narrow forested zone, lower timberline being at 6,000 to 7,500 feet and the upper at 9,300 to 9,500 feet. In fact, the driest of ranges south of Dillon have forest restricted to north-facing slopes and ravines and sagebrushgrass communities extend up through what would conventionally be the forested subalpine zone. The fact that throughout this ecoregion Pinus ponderosa (Ponderosa pine) is replaced by Pseudotsuga menziesii (Douglas fir) and/or Pinus flexilis (limber pine) at lower treeline also attests to a cold climate. The presence of Larix lyallii (subalpine larch) at upper treeline in the Anaconda-Pintlar Ranges is quite possibly testimony to the high snow load experienced by these northernmost-ranges within this ecoregion. Other observations could be made with regard to species distribution ostensibly correlated with climatic parameters but the above cited are the most salient in distinguishing this ecoregion.

\section{Physiography and related parameters}

The study area encompasses approximately $7,050,000$ million acres and ranges in elevation from 4,500 ft. on the Beaverhead River at Silver Star to $11,154 \mathrm{ft}$. at the summit of Tweedy Mtn. in the East Pioneer Range, encompassing no less than nine distinct mountain ranges. This area's diverse topography, high relief and climatic variability give rise, when overlain on a broad spectrum of parent material types ranging from intrusive and extrusive volcanics, calcareous and non calcareous sedimentary, coarse-grained metamorphic, all textures of valley fill sediment, to an abundance of unique environments. The East Pioneer, Tobacco Root, Pintlar, Beaverhead, Gravelly, Snowcrest, Tendoy and Centennial Ranges have all experienced Pleistocene alpine glaciation (Montagne 1972) and today support extensive alpine communities. Beyond the above observations there is 
Figure 1. Beaverhead M ountains Section Study Area, M ontana

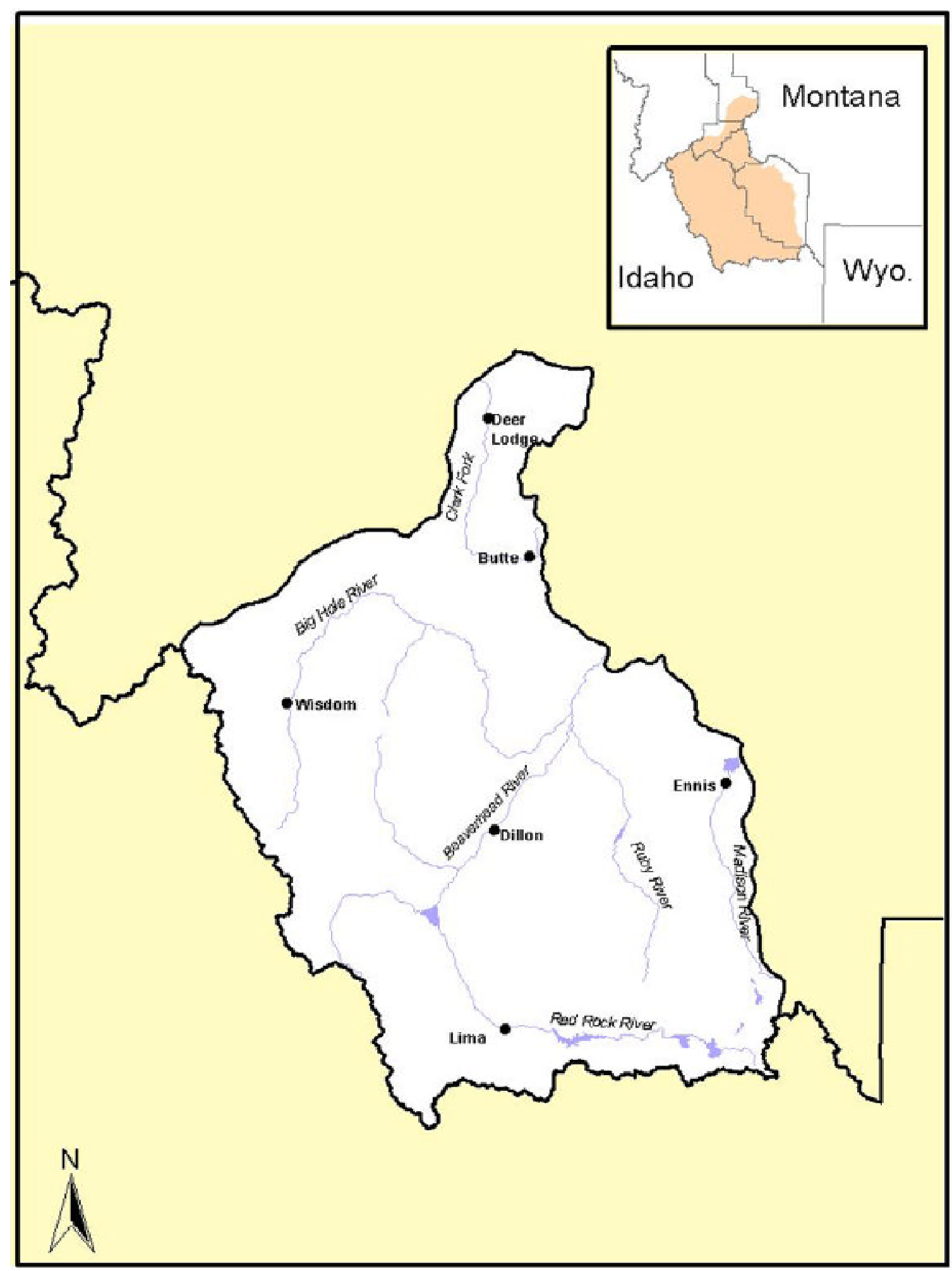


little that can be said that applies to and distinguishes the whole BVHDMS ecoregion. The incredible diversity of the physical landscape is reflected by the fact that the section contains eleven subsections (Figure 2), more than any other section within the state; the differences among subsections are based largely on geological or geomorphologic criteria, that is, they are comprised of mountain ranges or aggregations thereof and the intervening valleys. This diversity is best addressed by considering the eleven subsections presented below in alphabetical order according to their subsection designations (small case letter trailing the capital E). These brief descriptions have largely been abstracted from Nesser et al. (1997).

This setting also supports high vascular plant species diversity in terms of total numbers, endemism, and biogeographic elements. County floristic information is not available for Beaverhead and adjoining counties, but it is considered likely that the majority of the over 2,200 species and taxa below the species level documented in southwestern Montana occur in the study area. This is based on state floristic information initially compiled by Dorn (1984), augmented below the species level and crossreferenced to state sector by the U.S. Forest Service (Pierce 1997). The list of plants in southwestern counties represents roughly $80 \%$ of the vascular plant species in the state.

The diverse flora of the Beaverhead Mountains ecoregion is made up of species that have their centers of distribution in the Rocky Mountain, Great Basin or Great Plains; with fewer species present that are centered in the Snake River Plains, Columbia Plateau, Vancouverian, Boreal, and Arctic regions (Heidel et al. 1993). This does not include the widespread native species with no apparent centers of distribution, and the non-native species. It follows that the high floristic diversity is conducive to producing the notable community diversity found for the ecoregion.

In addition to these widely-distributed species, there are 18 Montana plant species of concern present in this region that are state or regional endemics, i.e., restricted in their worldwide distribution to an area that is half the size of Montana or less. This tally of endemic species is among the highest in the state. It was previously reported that southwestern Montana has the highest levels of endemism in Montana (Lesica1995). While information on the state flora and the Beaverhead Mountains ecoregion are too preliminary for quantitative analysis, the ecoregion has state significance in sheer numbers of species and levels of endemism.

\section{Subsection D escriptions}

\section{332E a C ontinental D ivide U plands}

This subsection is composed of block faulted mountains (the Beaverhead Mountains of the Bitterroot Range) comprised of a variety of igneous sedimentary and metasedimentary rocks, including those of the Boulder Batholith. Elevations range from 5,300 to 10,200 feet whereas precipitation evidences about a three-fold range, from 10 to 30 inches. The potential vegetation ranges from foothill prairie at lowest elevations, to Pseudotsuga menziesii (Douglas fir) forest (currently mostly Pinus contorta-dominated), to alpine meadows and barrens. The primary natural disturbances are fire and mass wasting. Land use is varied, divided among livestock grazing, mining, timber harvest, recreational use and development.

\section{332E b E ast Pioneer M ountains}

Mostly calcareous substrates (largely limestone) comprise the block-faulted mountains at the southern end whereas at northern end intrusive volcanics, mostly granitics, predominate. This subsection encompasses the most vertical relief (from 6,000 to 11,150 feet), the highest elevations and the most area within the alpine zone (elevations above approximately 9,400 feet). Mean annual precipitation varies from 10 to $40+$ inches. Potential vegetation is dominated by Douglas fir and Western sprucefir forests with abundant alpine meadows and barrens at the highest elevations; fragments of foothill prairie extend upslope as park-like inclusions in a mostly forested landscape. Fire 
Figure 2. Beaverhead M ountains Section and Subsection Boundaries, M ontana

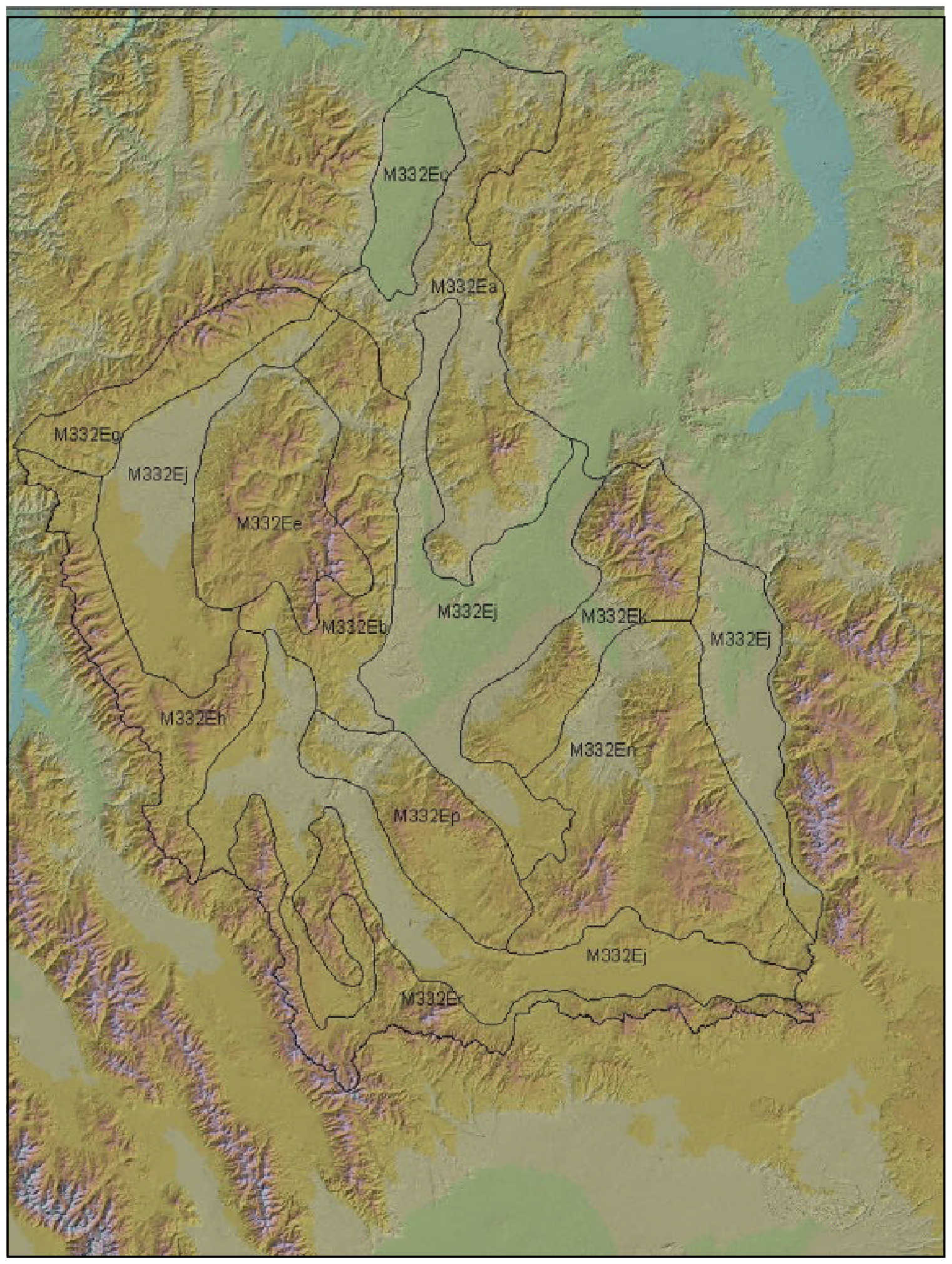


is the primary natural disturbance and the current dominant land use is livestock grazing, followed by timber harvest and recreation.

\section{332E c D eerlodge Valley}

This intermontane valley has formed in Tertiary sedimentary rocks and been subsequently filled with both Tertiary and Quaternary deposits. Elevations range from basins at approximately 4,400 feet, where salt-affected vegetation types are notable, to 6,000 feet where potential vegetation is sagebrush steppe (predominantly mountain big sagebrushdominated) and foothills prairie (including remnant rough fescue [Festuca campestris] prairie). Mean annual precipitation ranges from 11 to 16 inches. Agriculture and livestock grazing are the predominant land uses with urban/suburban development growing in importance.

\section{332E e West Pioneer M ountains}

This mountain mass has considerably less vertical relief (5,500 to 9,500 feet) and appears as a dissected planar surface rather than jagged peaks when compared to M332Eb. It is notable for its lithological diversity, including volcanics (granitics), metamorphic (gneiss and phyllite), sedimentary and metasedimentary parent material. Potential vegetation ranges from mountain big sagebrush-dominated steppe on the lower slopes to Douglas-fir at montane elevations to Western spruce-fir forest at the highest elevations; elevations within this subsection are insufficient to support alpine vegetation.

\section{332E g Anaconda M ountains}

This subsection is characterized by block faulted mountains composed primarily of granular plutonic rock, mostly monzonite and granodiorite and altered by alpine glaciation, which has created numerous classic U-shaped valleys mantled with glacial till and drift. Elevations range from 6,000 to 10,900 feet, with the highest portions of the range receiving in excess of 40 inches annual precipitation. Potential vegetation is primarily Douglas-fir in the lower montane and Western spruce-fire forest in the upper montane and subalpine zones with diverse alpine communities repre- sented. Past wildfires have left most of the range cloaked with seral lodgepole pine (Pinus contorta) forests. Among BVHDMS subsections this one is notable for both extensive heath-dominated alpine communities, (reflecting the subsection's acidic parent materials and high precipitation) and numerous stands of Larix lyallii (subalpine larch) at the highest forested elevations, generally on rocky, exposed substrates.

\section{332E h Beaverhead M ountains}

This subsection, which takes it name from the spine of mountains that traverse its length, is to a greater degree than other sections comprised of metasedimentary rocks (mostly argillite) that have been block faulted and subsequently modified by alpine glaciation. Elevations range from 6,800 to 10,600 . As with the other sections, potential vegetation ranges from Douglas-fir forests in the montane to Western spruce-fir in the montane to subalpine zone to alpine meadows and barrens above approximately 9,5000 feet. It differs from other subsections in having greater annual precipitation (20 to 40 plus inches) and consequently a preponderance of the landscape is forested. The primary natural disturbance is fire. Timber harvest and, to a lesser extent, livestock grazing and mining are the primary land uses.

\section{332E $h$ Southwest M ontana Intermontane Basins and Valleys}

This landscape is characterized by intermontane basins and broad valleys that have formed in alluvium, glacial deposits and Tertiary volcanic materials. Elevations range from 4,700 to 7,600 feet and the drainage density is low. Mean annual precipitation ranges from 9 to 21 inches, about $10 \%$ of which falls as snow. Much of the Red Rocks Basin and Big Hole Valley have cryic temperature regimes because of their high base elevations and cold air ponding; this is in distinction to the remainder of the section where the temperature regime is simply frigid. The predominant potential vegetation is sagebrush steppe (any of the three subspecies of Artemisia tridentata as well as $A$. tripartita, A. nova, A. arbuscula and A. cana) and steppe (dominated by Festuca idahoensis [Idaho fescue] and Pseudoroegneria spicata 
[bluebunch wheatgrass]). The primary natural disturbances are flooding and fire. Anthropogenic influences are livestock grazing, agriculture and urban/suburban development with hay production probably constituting the single largest replacement of native vegetation with introduced species and greatest alteration of the landscape.

\section{332E k R uby / T obacco R oot M ountains}

Both ranges comprising this landscape are fault block mountains, however the lower (to 9,400 feet), unglaciated Ruby Range is primarily limestone with some quartzite whereas the higher (to 10,600) glacially sculpted and more dissected Tobacco Root Range has a broader spectrum of rock exposed, including gneiss, granite, sandstone, quartzite, and limestone. For the subsection as a whole annual precipitation ranges from 13 to 35 inches. Judging by vegetation patterns alone, the Ruby Range is considerably drier than the Tobacco Root Range with steppe vegetation extending higher up itsflanks, having Artemisia nova (black sagebrush) as a significant shrub steppe component and having a greater extent of subalpine parks and virtually no subalpine fir (Abies lasiocarpa) represented. The Tobacco Root Range has abundant alpine meadow and barrens above 9,500 feet as well as extensive Western spruce-fir subalpine forests and at lower elevations Douglas-fir forests. The primary natural disturbance is fire. Past land use centered on mining and timber harvest but today livestock grazing and recreation are preeminent.

\section{332E n G ravelly / Snowcrest M ountains}

Both ranges are characterized by block faulted mountains composed of a variety of deposited materials, including shale, siltstone and sandstone; extrusive volcanics are very localized. Both ranges have also been significantly modified by glaciation and mass wasting. The terrain at the crest of the Gravelly Range is very gentle for a mountainous setting and supports the most extensive and accessible alpine terrain within the Section. Elevations range from 5,800 to 10,600 feet. Mean annual precipitation ranges from 14 to 30 plus inches. Both ranges lie in rainshadows of mountains to the west so that lower timberline is relatively elevated and mountain grasslands and shrublands are well represented on warmer aspects that would be forested in wetter climatic regimes. Potential forest vegetation is Douglas-fir at lower timberline which gives way to Western spruce-fir forest at higher elevations. Nearly pure stands of whitebark pine (Pinus albicaulis) are not uncommon on warmer-drier exposures at upper treeline. The primary natural disturbances are fire and mass wasting (slump landforms are abundantly evident in the sedimentary portion of the Gravelly Range). Livestock grazing and mining are the predominant land uses.

\section{332E p B lacktail M ountains}

This block faulted range is comprised of a variety of igneous, sedimentary and metamorphic rocks; alpine glaciation has impacted only the range's highest points. Elevations range from 6,900 to 9,500 feet. This is a comparatively dry subsection attributable to its rainshadow position in the lee of higher mountains to the west and southwest; mean annual precipitation ranges from 14 to 20 inches, about $50 \%$ falling as snow. The xeric nature of this subsection is borne out in the vegetation, which is predominantly grassland and shrub steppe on all but north-facing slopes of higher elevations. Potential forest vegetation is Douglas-fir or limber pine, except at the highest elevations on cool exposures where Western spruce-fir forests are found. Alpine turf and cushion plant communities are very limited, found on the highest ridgelines. Land use is predominantly livestock grazing with timber harvest and mining considered ancillary.

\section{332E r Southern Beaverhead M ountains}

Sedimentary, metamorphic (especially quartzite) and igneous rocks that have been block faulted characterize this subsection comprised of the Centennial, Tendoy and Southern Beaverhead Mountains and Lima Peaks (considered part of the Tendoy Range by some). Alpine glaciation and extensive mass wasting have significantly altered the land- 
scape (slumps and earth flows are especially evident in sedimentary materials on the flanks of the Centennial Range). Elevations range from 7,000 feet at the upper fringes of valleys within subsection M332Ej to almost 11,200 feet at Eighteen Mile Peak in the Beaverhead Mountains. Much of the landscape is characterized by mountain grasslands (Festuca idahoensis-dominated, Idaho fescue) and shrublands (Artemisia tridentata ssp. vaseyanadominated, mountain big sagebrush) with elevations above approximately 9,400 feet characterized by alpine grasslands and turf, cushion plant communities and extensive fellfields and barrens. The extensive limestone substrates are droughty, tending not to support forest. Douglas-fir and limber pine (Pinus flexilis) constitute the potential forest series at lower elevations and on drier sites. Western spruce-fir forest predominate at higher elevations and Engelmann spruce (Picea engelmannii) seems to be favored over Abies lasiocarpa on the extensive calcareous substrates. This subsection, primarily on aeolian deposits and other substrates on the flanks of the Centennial Mountains, boasts the most extensive development of aspen (Populus tremuloides). The primary natural disturbances are fire and mass wasting. Livestock grazing and recreation constitute the major land uses. 


\section{M ethods}

\section{Community, E cology: D ata Collection}

Original field sampling by the Montana Natural Heritage Program was conducted in three phases; an initial phase was centered on ten areas with acreage varying from 50 to 2,400 acres, selected by personnel of the Bureau of Land Management and sampled by R. DeVelice (1992). General descriptions of each of these ten sites included assessment of their biodiversity significance, current and past land use and suggested conservation management needs. A second phase of field sampling was conducted by S. Cooper and P. Lesica ( intensive in 1994, very limited in 1995 and 1996) based on reconnaissance information collected by Lesica, $\mathrm{J}$.

Vanderhorst and B. Heidel in the course of inventorying for threatened and endangered species in the Centennial Valley and the Tendoy Range and surrounding valley lands. A third field season was undertaken in 1998 to explore those remaining sites or landscapes that has not received coverage in the preceding years and to increase the sampling of under documented plant associations.

In each of these areas sampling centered on putative rare or previously undescribed communities and on common communities in good to excellent condition (based on standard range criteria). To minimize the confounding influence of disturbance (man-induced) on vegetation patterns, areas intensively grazed, herbicide treated, mechanically disturbed, artificially seeded or irrigated were not sampled. Plots were established within portions of stands that appeared homogeneous, both in vegetation composition and site factors. Within a given area an attempt was made to sample the vegetation characteristic of different landscape positions or at least capture the communities distinctly different in composition occurring within a circumscribed topography. Plot selection focused on existing stands of vegetation with the objective of describing the composition of seral, as well as mature vegetation; however, bias toward more mature stages is implicit through the selection of undisturbed sites.

Within each $375 \mathrm{~m}^{2}$ (used through 1992) or 1/ 10 acre plot (radii respectively of $35.8 \mathrm{ft}$ and $37.3 \mathrm{ft}$.) all vascular species were recorded and their height and canopy cover (Daubenmire 1959) estimated using a 12 class scale. Abiotic site information, including elevation, aspect, slope, parent material, landform, slope position and erosion characteristics, was also recorded for all plots. Only the initial 52 plot sample of DeVelice (1992) systematically collected information necessary to determine soil subgroup and water holding capacity from a 20-inch deep reconnaissance soil pit.

All plot information was collected on Community Survey Forms and entered into E.C.A.D.S (Ecological Classification And Description System), the evolutionary descendent of ECODATA (USDA 1987), a USDA Forest Service community sampling package. In the course of the 1998 field season, 108 new plots were added to the ECADS database.

\section{Community E cology: D ata Analysis}

Plot data was acquired from US Forest Service Region 1 and combined with the MTNHP plot data. Species composition and cover data for each plot was then compared to existing classification schemes to determine the community type assignment. A number of plots did not fit any existing plant association description within Montana or elsewhere, in which case they were set aside for consideration as representing new or unique communities. Plot data were not subjected to rigorous objective (multivariate) analysis. Though this is not a scientifically rigorous process, for the limited number of plots that did not fit, this constituted an efficient approach. The various iterations in the software and database structure (variations of ECODATA and ECADS) as well as the changing plant 
synonymy and its bearing on the database presented a logistic impasse at this time; we were unable to marshall all the plot data and produce synthesis and constancy/cover tables for all the plant associations with good plot data. Instead, we have produced these tables for the plot data collected in field season 1998 . The following section describes the process we have followed in the past for analyzing our plot database.

Analysis constituted a combination of ordination, to describe general patterns of communities in relation to environmental gradient, and classification to ascertain and describe community types.

Objective classification was accomplished using two-way indicator species analysis (TWINSPAN, Hill 1979b) whereas STRATA (USDA Forest Service) was employed to subjectively allocate plots to community types based on vegetation similarity values as well as subjectively perceived similarities and differences in site factors and treatments. Ordination was performed using detrended correspondence analysis (DECORANA, Hill 1979a). Species cover values for each plot constituted the input data. Both TWINSPAN and DECORANA are based on the mathematical strategy (i.e., reciprocal averaging; Hill 1979a, b) and thus afford direct comparison between the results of the two techniques. No data transformations were employed and all default options in TWINSPAN algorithm were used, except that pseudospecies cut levels were set at 0,5 and 20 percent cover. All default options were employed when running the ordination algorithms. To reduce variation (beta diversity) when running ordinations datasets were stratified by lifeform with 10 percent combined cover of trees or shrubs sufficient to place plots within these lifeform groups. Forbdominated plots were not separated from graminoid-dominated. Further reduction in beta diversity and resulting clarity of vegetation patterns was achieved by separating the obviously moist to wet-site plots from those of uplands.

Given the existence of numerous accepted classifications and not wanting to create unnecessary confusion, we were conservative in recognizing new community types.

TWINSPAN was particularly effective in pointing to particular plots that were placed in TWINSPAN classes that differed significantly from those categories they would be placed in existing classifications or following existing keys. TWINSPAN is also efficiently employed in constructing keys, i.e. looking for diagnostic species and defining appropriate cover values at which to make the breaks between community types. Ordination, in addition to helping refine the classification, assisted in describing and interpreting general patterns of vegetation communities and environment. For example, DECORANA extracts the dominant compositional gradient from the species data matrix. The environmental controls of these compositional gradients are then interpreted based on comparisons with the abiotic site data.

\section{Botany: Plant Species of Special Concern}

\section{Locations of Plants of Special Concern} previously documented within Beaverhead Mountains Section were queried from the Heritage Program Biological Conservation Database (BCD) and their biological diversity significance summarized. Key elements of plant species biodiversity significance were identified in three categories:

1. Globally imperiled or vulnerable vascular plant species, including state and regional endemics and species otherwise vulnerable on a rangewide basis. This corresponds with global ranks G1-G3 used by The Nature Conservancy and network of natural heritage programs.

2. Vascular plant elements that occur in this region of Montana to the exclusion of all other regions in the state. These are species for which the Beaverhead Mountains Section ecoregion represents their only conservation opportunities in Montana. 
3. Montana plant species of special concern occurring in aggregates within the region regardless of their global rank or state distribution, a third level of conservation priority. These are species that occur in patterns of overlapping distribution, collectively reflecting conservation priorities.

We did not conduct new fieldwork but drew heavily from secondary sources to synthesize available botanical literature, species documentation, and many years of study into a cohesive landscape picture. Areas within the Beaverhead Mountains Section have been the center of many plant surveys since the start of the Montana Natural Heritage Program and the start of the botany program in the Bureau of Land Management - Montana Office. Results from these studies have already been processed as element occurrence data, and synthesized as species status and biology information in BCD, as well as cross-referenced in a supporting bibliographic database.

Each species is ranked by a standardized set of criteria. Each place where a Montana plant species of special concern has been documented is referred to as an element occurrence record, and many of these are ranked in considering such factors as population numbers and threats. The point is that all of this information provides a basis for weighing the species and occurrences together, between agencies, and with other ecoregions.

\section{T axonomic C onsiderations}

Plant scientific names used in this report generally correspond to those found in manuals of the Montana flora (Booth 1950, Booth and Wright, Dorn 1984) or the regional manual of high repute "Flora of the Pacific Northwest" (Hitchcock and Cronquist 1976). However, where there is a discrepancy between any of these references and Kartesz (1994) we have incorporated the Kartesz taxonomic treatment to maintain consistency with other states and current taxonomic research. This has resulted in incorporating unfamiliar-sounding names for some dominant and indicator species, particularly the grasses of the Triticeae (Agropyron and Elymus in the traditional sense). Thus, western wheatgrass, listed as Agropyron smithii in Hitchcock and Cronquist (1976) and as Elymus smithii in Dorn (1984), has become Pascopyrum smithii (Kartesz 1994). Use of common names may be preferable for such controversial or mercurial groups, especially for ease of communication in informal situations. Table 1 presents the synonymy for common dominant and indicator species encountered in the Beaverhead Mountains Section; we have also tried to consistently list synonyms in the vegetation keys but have removed them from the headings of the plant association descriptions. Synonymy is also provided in the Beaverhead Mountains Section floristic list (Appendix A). 
Table 1. Synonymy of dominant and indicator plant species for the Beaverhead Mountains. Section Arranged by lifeform (tree, shrub, graminoid and forbs) and alphabetically by scientific name in column 2 .

\begin{tabular}{|c|c|c|c|}
\hline Common Name & Hitchcock \& Cronquist (1976) & Dorn (1984) & Kartesz (1994) \\
\hline black cottonwood & Populus trichocarpa & P. balsamifera & P. balsamifera ssp. trichocarpa \\
\hline Sitka alder & Alnus sinuata & Alnus viridis & A. viridis ssp. sinuata \\
\hline low sagebrush & Artemisia arbuscula v. arbuscula & A. arbuscula & A. arbuscula ssp. arbuscula \\
\hline early low sagebrush & Artemisia arbuscula v. arbuscula & A. longiloba & A. arbuscula ssp. longiloba \\
\hline black sagebrush & Artemisia arbuscula v. nova & A. nova & A. nova \\
\hline Gardner's saltsage & Atriplex nuttallii & Atriplex gardneri & A. gardneri \\
\hline bog birch & Betula glandulosa & B. glandulosa & Betula nana \\
\hline red-osier dogwood & Cornus stolonifera & C. stolonifera & Cornus sericea \\
\hline shrubby cinquefoil & Potentilla fruticosa & Pentaphylloides floribunda & P. floribunda \\
\hline Booth willow (billberry willow) & Salix myrtillifolia & Salix boothii & S. boothii \\
\hline bearded wheatgrass & Agropyron caninum & Elymus trachycaulus & E. trachycaulus \\
\hline thick-spiked wheatgrass & Agropyron dasystachyum & Elymus lanceolatus & E. lanceolatus \\
\hline western wheatgrass & Agropyron smithii & Elymus smithii & Pascopyrum smithii \\
\hline bluebunch wheatgrass & Agropyron spicatum & Elymus spicatus & Pseudoroegneria spicata \\
\hline long-stolon or sun sedge & Carex pensylvanica & C. pensylvanica & Carex inops ssp. heliophila \\
\hline beaked sedge & Carex rostrata & C. rostrata & Carex utriculata \\
\hline inland saltgrass & Distichlis stricta & D. stricta & Distichlis spicata \\
\hline few-flowered spike-rush & Eleocharis pauciflora & E. pauciflora & Eleocharis quinqueflora \\
\hline giant or basin wildrye & Elymus cinereus & E. cinereus & Leymus cinereus \\
\hline rough fescue & Festuca scabrella & F. scabrella & Festuca campestris \\
\hline spike fescue & Leucopoa kingii & Hesperochloa kingii & Festuca kingii \\
\hline smooth woodrush & Luzula hitchcockii & Luzula glabrata & L. glabrata v. hitchcockii \\
\hline Sandberg's bluegrass & Poa sandbergii & Poa secunda & P. secunda \\
\hline alkali bluegrass & Poa juncifolia & P. juncifolia & Poa secunda \\
\hline slender-flowered scurf-pea & Psoralea tenuiflora & P. tenuiflora & Psoralidium tenuiflorum \\
\hline softstem bulrush & Scirpus validus & S. validus & Scirpus tabernaemontani \\
\hline bottlebrush squirreltail & Sitanion hystrix & Elymus elymoides & E. elymoides \\
\hline false spikenard & Smilacina racemosa & S. racemosa & Maianthemит racemosum \\
\hline starry Solomon-plume & Smilacina stellata & S. stellata & Maianthemum stellatum \\
\hline green needlegrass & Stipa viridula & S. viridula & Nasella viridula \\
\hline
\end{tabular}




\section{Results and D iscussion}

\section{Community E cology}

Of the 480 plant associations identified for the whole of Montana, over half (57\%) occur within the Beaverhead Mountains Section (see Appendix A). Given that this Section comprises less than one tenth of the state's total land area, this concentration of community diversity is very high. There are two major reasons for this. First, the Beaverhead Mountains Section has received greater attention in terms of field sampling, of both plants and vegetation, than any other section in Montana; when more environments are explored, more community types are documented. Secondly, as noted in the description of the study area, this region exhibits the greatest geological diversity in the state, the most vertical relief, and the unique intersection of Pacific and Gulf (of Mexico) storm tracks, as well as the overlap of several floristic elements. The further effects of glaciation and periglacial climates have resulted in strong environmental gradients that drive species sorting and produce a high diversity of communities. This is exemplified by upland shrubland (or dwarf-shrubland) types. Shrublands types are more diverse here than elsewhere in Montana because of the many sagebrush taxa present as community dominants or indicators (all subspecies of Artemisia tridentata as well as A. tripartita, A. arbuscula, A. arbuscula ssp. longiloba, $A$. nova, A. cana, $A$. pedatifida, A. frigida, and A. scopulorum).

The Montana Natural Heritage Program tracks the diversity of plants, animals and natural communities (plant associations) at both state and global scales. Species and communities are given status ranks that reflect their conservation status based on rarity/extent, condition, trends and threats. Appendix B gives an explanation of the Natural Heritage Global Ranks. Table 2 illustrates the distribution of global ranks in the Beaverhead section by lifeform group.

Just over $25 \%$ of the plant associations in the
Beaverhead Section are ranked G3 and higher, indicating rangewide vulnerability or imperilment. Many of these rankings are considered uncertain (denoted "?") because they are based on limited information. As more surveys are conducted, some will likely prove to be less rare than current data indicate. Other types will likely prove more imperiled, as we come to better understand stresses and threats to them.

The single plant association ranked G1 is the Artemisia cana / Leymus cinereus shrubland. This association was documented from a single location at Big Muddy Creek in the BVHDMS on an elevated landform above a seep-spring area at $6620 \mathrm{ft}$ elevation. Elymus trachycaulus and Poa nevadensis are also found in the shrub understory.

Seven percent (19) of the 277 associations are ranked G2 (including G2? and G2G3) or higher. A number of $\mathrm{G} 2$ plant associations occur as small or large patch communities. This rank is justified, not so much on number of occurrences, but on threats. Communities particularly under threat from grazing practices include, among others, Artemisia cana / Leymus cinereus, A. tridentata ssp. vaseyana / Leymus cinereus, A. tridentata spp. tridentata / L. cinereus, Artemisia tripartita / Pseudoroegneria spicata, Artemisia arbuscula ssp. longiloba / Elymus lanceolatus and Bromus carinatus $-B$. anomalus. These same communities also bear lesser threats from cultivation (type conversion), noxious weeds, and roads.

For example, livestock and associated agricultural practices have demonstrably reduced the acreage of some riparian shrublands such as Artemisia tridentata ssp. tridentata / Leymus cinereus. The deepsoiled riparian terraces and valley bottom landforms that support this plant association have received enormous past livestock pressure. Much of the area has been converted to agricultural crops. These same productive bottomlands are prime habitat for a host of alien and native increasers; the most aggressive among which are Poa pratensis 
Table 2. Distribution of community types by lifeform group and global rank.

\begin{tabular}{|c|c|c|c|c|c|c|c|c|c|c|}
\hline $\begin{array}{l}\text { Lifeform Group } \\
\text { (Subclass level of the }\end{array}$ & $\begin{array}{l}\text { Glo } \\
\text { (hig }\end{array}$ & $\begin{array}{ll}\text { I Ran } \\
\text { lumer }\end{array}$ & $\begin{array}{l}\text { from rar } \\
\text { l value). }\end{array}$ & $\begin{array}{l}\text { (low } \\
\text { anks a }\end{array}$ & $\begin{array}{l}\text { nerical } v \\
\text { defined } \mathrm{i}\end{array}$ & $\begin{array}{l}\text { e) to c } \\
\text { Appenc }\end{array}$ & $\begin{array}{l}\text { Amon an } \\
\text { B. }\end{array}$ & demon & rabl & ecure \\
\hline & G 1 & $\begin{array}{l}\text { G2 } \\
\text { G2 ? } \\
\text { G 2 Q }\end{array}$ & $\begin{array}{l}\mathrm{G} 2 \mathrm{G} 3 \\
\mathrm{G} 2 \mathrm{G} 3 ? \\
\mathrm{G} 2 \mathrm{G} 4\end{array}$ & $\begin{array}{l}\text { G3 } \\
\text { G3? } \\
\text { G3 Q }\end{array}$ & $\begin{array}{l}\text { G3G4 } \\
\text { G } 3 \text { G } 4 \text { ? } \\
\text { G } 3 \text { G } 5\end{array}$ & $\begin{array}{l}\text { G4 } \\
\text { G4? } \\
\text { G4Q }\end{array}$ & G4G5 & $\begin{array}{l}\text { G 5 } \\
\text { G5 ? }\end{array}$ & G? & $\begin{array}{l}\text { Total by } \\
\text { lifeform }\end{array}$ \\
\hline $\begin{array}{l}\text { Deciduous Forest and } \\
\text { Woodland }\end{array}$ & & & & 1 & & 4 & & 2 & 5 & 12 \\
\hline Evergreen Forest & & 3 & & 5 & 2 & 20 & 1 & 24 & & 55 \\
\hline Evergreen Woodland & & 1 & 1 & 6 & 1 & 11 & 1 & 9 & & 30 \\
\hline Shrubland & 1 & 2 & 2 & 20 & 4 & 24 & 1 & 8 & 13 & 75 \\
\hline Dwarf-shrublands & & 3 & 2 & 5 & 1 & 1 & 1 & 2 & 1 & 16 \\
\hline Herbaceous & & 4 & 1 & 22 & 1 & 27 & & 12 & 17 & 85 \\
\hline Total by G-rank & 1 & 13 & 6 & 59 & 9 & 87 & 4 & 57 & 36 & 273 \\
\hline
\end{tabular}

(IIKentucky bluegrass), Poa palustris (fowl bluegrass), Phleum pratense (timothy), Bromus inermis (smooth brome) and Juncus balticus (Baltic rush).

While weeds are considered perhaps the greatest threat to biodiversity within this ecoregion (and throughout the western U. S.), none of these species are considered weeds, although it is clear that they have significantly and irreversibly reduced biodiversity within this ecosystem. Poa pratensis is perhaps the most serious threat to native communities, as it possesses broad ecological amplitude, is very aggressive and tolerates intensive grazing. It even threatens some of the most broadly distributed and seemingly secure communities, particularly those of mesic environments where, for instance, Deschampsia cespitosa (tufted hairgrass) is currently a dominant or indicator species.

Another group of communities considered imperiled (G2 and greater) those of alpine and high subalpine environments, which occur only in restricted environments. Examples include Salix arctica / Polygonum bistortoides, Juncus parryi / Erigeron ursinus, Festuca idahoensis Festuca kingii and Cassiope mertensiana / Carex paysonis (Figure 3). These communi- ties occur as small patches in habitats that are highly restricted, at least in Montana. Given the difficulty of access, inventory of these types is still limited. These communities are not especially threatened, though livestock grazing, particularly sheep with their preference for forbs could adversely impact community composition.

One globally imperiled (G2) community that is known from relatively thorough surveys is Elymus lanceolatus / Phacelia hastata. This is a seral community that occurs only in the Centennial Sandhills (Figure 4), where it is sustained by animal- and/or fire-induced blowouts at regular intervals. Without this disturbance regime, the community would succeed to Artemisia tridentata spp. tridentata /Stipa comata or A. tridentata ssp. vaseyana / Festuca idahoensis, and its complement of sensitive species could be lost from the ecosystem (Lesica and Cooper 1998).

Many other community types in the

Beaverhead Section are ranked "G?" indicating that no rank has yet been assigned. This group is made up of several shrubland types that we propose elevating to a trinomial name (Artemisia tridentata ssp. vaseyana / Festuca idahoensis - Geranium viscosissimum 
Figure 3. Locations of Select globally rare and exemplary plant associations, B eaverhead M ountains Section

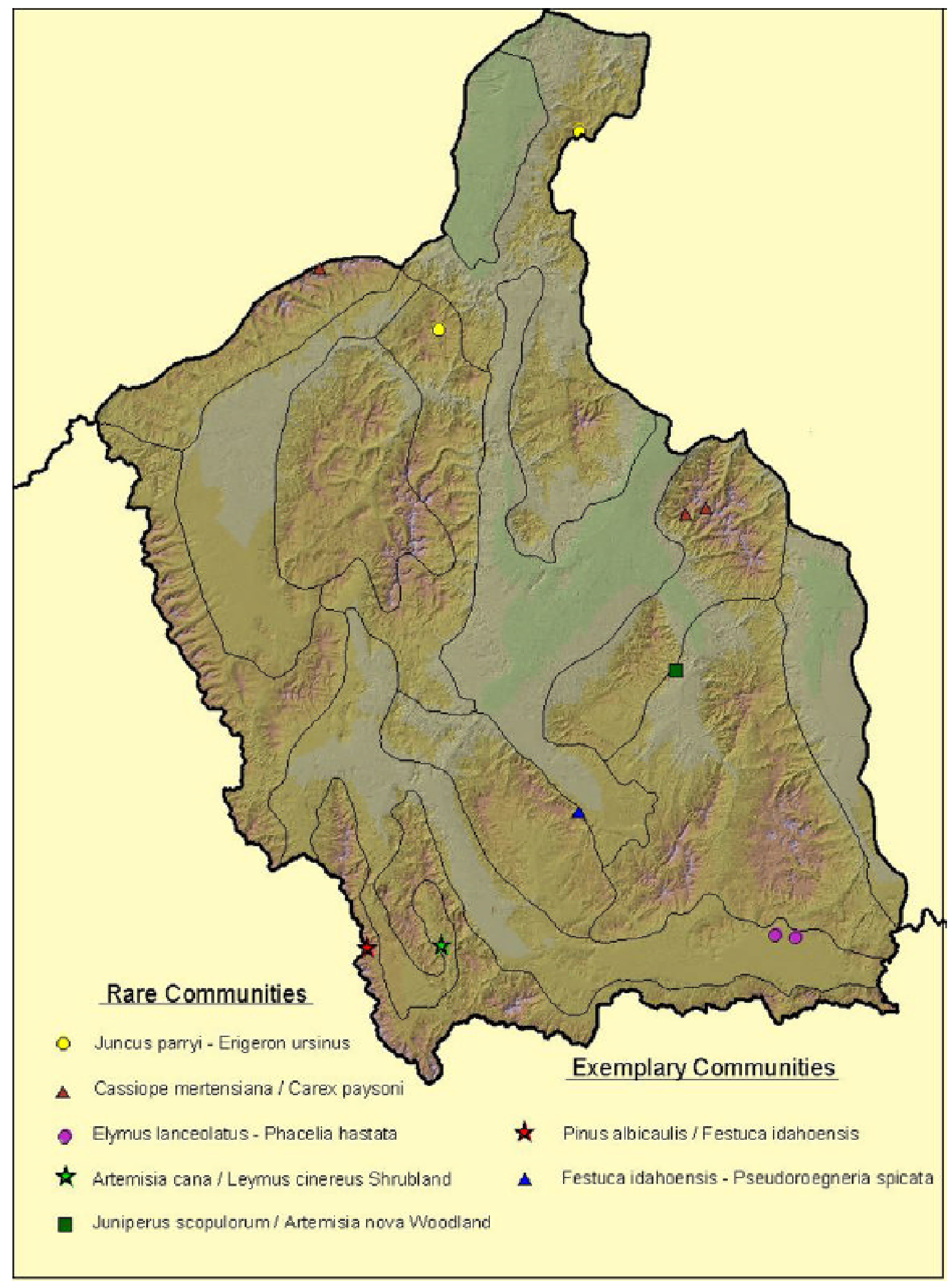


Shrubland), as well as a few newly defined community types (Bromus carinatus - Bromus anomalus Herbaceous Vegetation) and a couple of anthroprogenic communities ( $\mathrm{Poa}$ pratensis Herbaceous Vegetation).

The high global ranks assigned to some communities are probably outdated and need revision. Examples include Alnus viridis ssp. sinuata (Sitka alder), Salix arctica / Polygonum bistortoides and Artemisia tripartita / Festuca idahoensis, all of which are broadly distributed (across several states) and are under no particular threat. For instance, surveys have shown A. viridis ssp. sinuata $\mathrm{p}$. a. to be a very conspicuous, long-persisting mostly seral community type in northwestern Montana, occurring in the subalpine zone where logging operations have caused soil compaction. Although it is considerably less common type in the BVHDMS, it is not imperiled on a state or global basis.

The Montana Natural Heritage Program also assigns state ranks to community types, applying the factors in Appendix B. at a state level. Appendix A includes a complete list of current state ranks for communities in the Beaverhead section. The following rank qualifiers are used with S-ranks only: A- Accidental or casual in the state; infrequent and outside usual range, EExotic established in the state and community named for said exotic, P-Potential that element (plant community) occurs in the state but no extant or historic occurrences reported, $\mathbf{R}$ Reported to occur in the state but without a basis for accepting or rejecting the report.

In our analysis of plot records for this project, we identified three plant communities thought to be in Montana, but which had not previously been documented. These are Artemisia tridentata spp. tridentata / Festuca idahoensis, Artemisia tridentata spp.tridentata /

Pseudoroegneria spicata and Purshia tridentata / Pseudoroegneria spicata. These types can now be given state ranks.

Six globally significant community types (G1G3 ranks) have a state rank of $S$ ? These include Puccinellia nuttalliana, Festuca idahoensis - Danthonia intermedia,
Pseudoroegneria spicata - Oryzopsis

hymenoides, Salix brachycarpa / Carex

aquatilis, Artemisia tripartita/

Pseudoroegneria spicata, and Artemisia cana /

Leymus cinereus. For these types our in-state

knowledge of the status and distribution is fragmentary and we recommend additional efforts to locate occurrences, both to better document their distribution and status, and to assist in their conservation.

For community types that are considered rare in Montana (S1-S3) but widespread and secure globally (G4-G5), the Heritage Program seeks to document locations where they exist in exemplary, high quality condition. Some of these locations, as well as those supporting excellent stands or assemblages of globally significant types, are discussed below.

\section{L andscapes of Significant E cological I nterest}

The identification of landscapes harboring concentrations of plants, animals and communities of high conservation value facilitates conservation planning by bringing attention to areas of representative or unique characteristics. The Nature Conservancy is currently pursuing "ecoregional planning" for the Middle Rockies-Blue Mountain Ecoregion that includes the Beaverhead Mountains Section. This planning effort relies on computer models and actual biological data to parsimoniously select a portfolio of potential conservation sites. The goal of this planning is to protect biological diversity through conservation planning. The portfolio of sites will be screened by outside experts (agencies, academia, etc.) to detect errors of omission and commission. When the TNC efforts are complete a number of potential conservation sites within the Beaverhead Mountains Section will have been identified and shared with agencies to facilitate future consideration of these sites.

Observations and field studies by The Montana Natural Heritage Program have elevated the importance of natural landscapes in the BVHDMS. This brief sketch of landscapes 
and their biodiversity significance is an initial effort calling attention to priority landscapes in the BVHDMS. These landscapes serve as a focus for conducting further site studies and may provide the impetus for future conservation easements, or special agency designations such as Area of Critical Environmental Concern (ACEC) or Botanical Interest Area. These landscape areas, some of which are already managed for special purposes e.g. State Game Management Area, provide a broadened perspective to managers and land owners as to the biological treasures they have under their aegis and may promote appreciation and appropriate stewardship.

\section{Bannack L andscape}

Location: The landscape encompasses the undeveloped ridge systems and Grasshopper Creek valley in and adjoining Bannack State Park, the historic capital of Montana Territory, located about 20 miles southwest of Dillon. It is made up of Bureau of Land Management (BLM) lands, and state lands that are managed by BLM.

Environment: Bannack lies at the heart of one of the major limestone/dolomite formations, or Madison group formations, in southern Montana. The contact zones between this sedimentary group of formations and igneous intrusions are often rich in precious minerals, and the historical Bannack settlement on Grasshopper Creek marked Montana's first major gold discovery. The valley was the hub of past placer mining, and is an area of concentrated pit mining activity today. Most of the surrounding uplands are made up of Tertiary basin sediments.

Bannack is surrounded by a major north-south ridge valley system at the southern end of the Pioneer Mountains, nestled in the steeply dissected Grasshopper Creek valley. There is over 1,000 foot relief within the 1,000 acre Park, and the surrounding uplands rise hundreds of feet higher along the ridge to the north and south. They are all part of extensive foothills made up of jagged outcrops, rugged hills and rolling plains.
Vegetation/Other Biological Features:

Outstanding examples of the widespread Cercocarpus ledifolius/Pseudoroegneria spicata shrubland (mountain mahogany/ / bluebunch wheatgrass) cover the valley slopes. Limber pines are scattered along the ridge outcrops and Douglas fir woodland covers the highest spine of the ridges. Sagebrush steppe prevails throughout most of the area, dominated by Artemisia tridentata var. vaseyana / Pseudoroegneria spicata shrubland (mountain big-sage / bluebunch wheatgrass). At the Park entrance, coarse alluvial deposits are dominated by Stipa comata (needle and thread grass) and Pseudoroegneria spicata (bluebunch wheatgrass).

This rugged landscape provides habitat for one of highest concentration of state and regional endemic plant species in southwestern Montana, including some of the largest known populations for four of them (Table 3). None of these globally rare plants have sites that are protected in Montana, and their low-elevation populations as found in the Bannack area are vulnerable. We note that some of them are no longer tracked but have been moved to the watch list - they are relatively secure in the state thanks to places like Bannack. The majority of Bannack's endemic species are located in the mountain mahogany shrubland, at the valley's edge.

There is also a historic record of Penstemon lemhiensis (Lemhi Penstemon) from the Grasshopper Valley in the Bannack area, but it is possibly extirpated. Two more species that are peripheral Great Basin species that were once considered rare in Montana are present in the Bannack area: Arenaria kingii and Pediocactus simpsonii.

The full scope of biological significance hinges on boundary delimitation. The Bannack area is assumed to include at least one mile to the north and northeast of the Park. The Cold Spring Creek to the east and Henneberry Ridge to the southeast represent two of the larger occurrences of Astragalus scaphoides (Bitterroot Milkvetch) in Montana. The Microwave Tower to the north represents the 
Table 3. State and regional endemic plant species in the Bannack Landscape

\begin{tabular}{|l|l|l|l|}
\hline Species & Common name & $\begin{array}{l}\text { Species Status } \\
\text { Comments }\end{array}$ & $\begin{array}{l}\text { Distribution and abundance } \\
\text { comments }\end{array}$ \\
\hline $\begin{array}{l}\text { Astragalus } \\
\text { scaphoides }\end{array}$ & Bitterroot Milkvetch & Regional endemic & $\begin{array}{l}\text { Center of species' dis tribution in } \\
\text { Montana; large populations east of } \\
\text { Bannack }\end{array}$ \\
\hline $\begin{array}{l}\text { Astragalus } \\
\text { terminalis }\end{array}$ & Railhead Milkvetch & Regional endemic & East of Bannack \\
\hline $\begin{array}{l}\text { Lesquerella } \\
\text { pulchella }\end{array}$ & Beautiful Bladderpod & State endemic & $\begin{array}{l}\text { One of largest low elevation } \\
\text { populations }\end{array}$ \\
\hline $\begin{array}{l}\text { Lomatium } \\
\text { attenuatum }\end{array}$ & $\begin{array}{l}\text { Taper-tip Desert- } \\
\text { parsley }\end{array}$ & Regional endemic & $\begin{array}{l}\text { One of largest low elevation } \\
\text { populations }\end{array}$ \\
\hline $\begin{array}{l}\text { Phacelia incana } \\
\text { Hoary Phacelia }\end{array}$ & Regional endemic & $\begin{array}{l}\text { One of largest low elevation } \\
\text { populations }\end{array}$ \\
\hline $\begin{array}{l}\text { Sphaeromeria } \\
\text { argentea }\end{array}$ & Chicken Sage & Regional endemic & One of largest populations \\
\hline $\begin{array}{l}\text { Delphinium bicolor } \\
\text { ssp. Calcicola }\end{array}$ & Limestone Larkspur & $\begin{array}{l}\text { State endemic - no } \\
\text { longer tracked }\end{array}$ & One of largest populations \\
\hline Mimulus suksdorfii & $\begin{array}{l}\text { Suksdorfs } \\
\text { Monkeyflower }\end{array}$ & $\begin{array}{l}\text { Possibly a regional } \\
\text { endemic - no longer } \\
\text { tracked }\end{array}$ & Present at Bannack \\
\hline $\begin{array}{l}\text { Oryzopsis contracta } \\
\text { no longer tracked }\end{array}$ & Contracted Ricegrass & $\begin{array}{l}\text { Regional endemic - } \\
\text { Montana }\end{array}$ \\
\hline
\end{tabular}

only sensitive plant conservation site on the Dillon Area of BLM, with an exclosure around part of a Penstemon lemhiensis (Lemhi Penstemon) population representing another regional endemic.

All of the species found in the Bannack area occur elsewhere on the landscape, but the Bannack area represents the highest concentration, and significant large populations for four of the tracked endemic species.

Condition/Conservation Value: Despite its history as an early hub for exploration and settlement in Montana, the ridges outside of the townsite and mining sites are surprisingly intact. The proximity of high biodiversity significance neighboring a historic settlement, ghost town, and state park; with all of its contemporary recreation and interpretive values, offers special education opportunities on top of the conservation value.

The Grasshopper Valley is among the few places where spotted knapweed (Centaurea maculosa), a species that could spread throughout the landscape, is present in Beaverhead County. Diligent weed control efforts have been effective to date. Noxious weed invasion from the valley and from spread by vehicles or stock poses a major potential threat to all of the globally rare plant species in this area.

The majority of inventory work has been concentrated on plant species in Bannack State Park based on a study for Montana Department of Fish, Wildlife and Parks (Vanderhorst 1995). Status survey work for the BLM conducted on some of the regional endemic species has entailed visits to this and adjoining areas. We note that plant information is incomplete, and that information on animals and on plant communities still needs to be addressed to assess conservation values.

\section{Blacktail \& R obb-Ledford L andscape}

Location: Alkali Creek and the East Fork of Blacktail Creek watersheds imprecisely define this landscape boundary. It covers the benchlands and foothills on the west flank of the Snowcrest Range to include all of the Blacktail State Wildlife Management Areas (SWMA) and approximately the southern half of Robb-Ledford (SWMA); hence the place name.

Environment: This landscape is comprised of rolling benchlands, mostly mantled with 
ancient alluvium and underlain by sedimentary formations, with abrupt ridges and hogbacks more prominent approaching the Snowcrest Range. There are also outcrops, configured into prominent spires in places, of erosion resistant, unique sedimentary materials. Proper functioning first, second and third order steams, along with numerous seeps and springs, are also found in this landscape.

\section{Vegetation/Other Biological Features: This} landscape came to our attention through simple reconnaissance; driving through we noted localized areas that appeared at a distance to be weedy monocultures or planted introduced grasses. Upon closer inspection we found exceedingly productive grasslands dominated by highly palatable native graminoids such as Stipa richardsonii (Richardson's needlegrass), Stipa occidentalis (western needlegrass), Bromus carinatus (mountain brome), $B$. anomalus (nodding brome), and Elymus trachycaulus (bearded wheatgrass). These grasslands repeat in landscape positions where accumulation, either moisture (snow or groundwater), aeolian materials or both are deposited along toeslopes in lees of hills and north- and east facing slopes of ravines and draws. Soils are deep and nearly rock-free. The composition of these rich grasslands is unique in a landscape where grasslands are nearly always dominated by Festuca idahoensis (Idaho fescue), Pseudoroegneria spicata (bluebunch wheatgrass) and Pascopyrum smithii (western wheatgrass) or Elymus lanceolatus, (thickspike wheatgrass). It appeared that livestock grazing was of low intensity in the vicinity of these grasslands, though livestock grazing is by no means totally absent on these SWMA lands.

Besides those grasslands previously mentioned, this landscape includes a wide diversity of types, the majority of which appear to be in high ecological condition. Other grasslands range from Pascopyrum smithii (western wheatgrass) and Elymus trachycaulus (bearded wheatgrass) found on heavy soils at lower slope positions to Stipa comata,

Pseudoroegneria spicata and Calamagrostis montanensis (plains reedgrass) found on sandy, thin-soil, or rocky and warm aspects.
Shrublands within this landscape are less diverse but include high quality occurrences of Artemisia tridentata ssp. vaseyana dominated communities as well as some uncommon state communities including Artemisia tridentata ssp. vaseyana - Symphoricarpos oreophilus / Bromus carinatus and A. tridentata ssp. vaseyana - S. oreophilus / Festuca idahoensis and $A$. tridentata ssp. vaseyana - S. oreophilus / Elymus trachycaulus. These three shrublands are not rare, but are rarely found north of ID or NV. Scattered Populus tremuloides (trembling aspen) groves are also found, but grazing had significantly impacted the few examined. The quality and composition of the riparian stringers has not been examined but willows appear healthy and not overly browsed.

Condition/Conservation Values: This landscape is notable for its representation of relatively intact grassland-shrubland ecosystems that are highly characteristic for this ecoregion and predominantly in good to excellent ecological condition. The area has a few road and agricultural impacts, such as the creation of scattered introduced-grass hay meadows. Although our reconnaissance was minimal, riparian vegetation seemed little impacted by livestock. Habitats found in this landscape are replicated in numerous places across the ecoregion, yet this landscape has both unique communities, not found elsewhere in Montana and communities that have reached their northernmost extension. We suspect that past management is the unique aspect of this landscape and that these communities owe their presence to managing for game rather than strictly livestock.

\section{Centennial M ountains/ Valley D iversity G radient}

Location: This landscape is described as a 1-2 mile-wide swath that extends from the south shore of Upper Red Rock Lake southward and upslope to the Continental Divide at the crest of the Centennial Range. It includes lands managed as wilderness by the Red Rock Lakes National Wildlife Refuge and an 85-acre Research Natural Area (RNA) also under USFWS management. A portion of the area 
managed by the Bureau of Land Management is designated as the Centennial Mountains Wilderness Study Area. Experimental grazing units are administered by the Agricultural Research Service.

Environment: This landscape spans an enormous environmental gradient from aquatic beds on the south shore of Upper Red Rock Lake to alpine tundra on sedimentary substrates in the vicinity of Taylor and Sheep Mountains. This south face of the Centennial Range is largely composed of calcareous substrates (predominantly Madison limestone) with some outcrops of quartzite and extrusive volcanics as well. Annual precipitation of 20.1 inches at a nearby valley location (Lakeview at 6,700 feet) would make this the wettest of BVHDMS valleys. Near the crest of the range annual precipitation is expected to exceed 50 inches.

Natural fire, in the form of underburns in the Populus tremuloides-dominated stands and stand-replacing in the upslope forested landscape, is the major natural disturbance with avalanches running a close second on this steep and snowy terrain. Windthrow is possibly a factor in the lower-elevation wetland forests around the lake's margin.

\section{Vegetation/Other Biological Features: The} diversity of communities encompassed by this gradient would be among the greatest in the state and most of the inventoried portion is known to be in good condition. At the lowest elevations along the south shore of Upper Red Rocks Lake, there are unexplored aquatic beds in the shallow waters (personal communication John Pierce) followed by emergent Carex utriculata (beaked sedge) and C. aquatilis (water sedge). Sites lacking prolonged inundation are dominated by Calamagrostis canadensis (bluejoint reedgrass) and C. stricta (narrow-spiked reedgrass) which quickly give way to Salix (willow)-dominated communities and Populus tremuloides (trembling aspen) groves. The undergrowth of $P$. tremuloidesdominated stands range from wetland-associated graminoids (including all the above-cited) to mesic and wetland associated shrubs (Cornus sericea [red-osier dogwood], Salix boothii [Booth willow], S. geyeriana [Geyer willow]. Even species characteristic of uplands, Calamagrostis rubescens (pine grass), Carex geyeri (elk sedge) and Symphoricarpos albus (common snowberry) can be found in the undergrowth of $P$. tremuloides-dominated stands. Well-developed stands of subirrigated Picea engelmannii (Engelmann spruce) are also found just inland from the lake's margin. The elevation gradient then ascends through sagebrush-dominated communities, principally Artemisia tridentata ssp. vaseyana / Festuca idahoensis (mountain big sagebrush / Idaho fescue) found just below lower treeline.

At lower elevations, late seral to old-growth forests are dominated by Pseudotsuga menziesii (Douglas-fir) with Pinus flexilis (limber pine) and Picea engelmannii as primary associates. The putative climax tree species on all but the very lowest elevations is Abies lasiocarpa (subalpine fir). Given the current intensive moose browsing on this tree species and the fire return interval, it will never become an important component of these stands. Particularly notable for their extreme age (>300 years) and characteristic open structure are old-growth stands of Pinus flexilis on warm exposures. On highly exposed sites with thin soils, good condition Pseudoroegneria spicata (bluebunch wheatgrass) stands dominate the grassland habitat. The very highest points in the gradient have received only cursory examination but are reputed to harbor good examples of alpine turf with Carex rupestris (curly sedge) and Carex elynoides (Kobresia-like sedge) communities. The alpine also includes fellfields of Silene acaulis [moss campion] and Phlox pulvinata [cushion phlox] and snowbed communities where Sibbaldia procumbens [creeping sibbaldia] and Carex nigricans [black alpine sedge] are important components.

The only known extant occurrence of Whipple's beardtongue (Penstemon whippleanus) has been documented from avalanche chute runout areas in this area (Culver 1993). This area's abundant avalanche chutes and talus slopes are potential habitat for dwarf goldenweed (Haplopappus nanus), 
which is known only from an historical collection on slopes south of Red Rock Lakes.

Condition/Conservation Value: Past timber cutting has been sporadic, largely constituting high grading of large Douglas-fir for building. Grazing has occurred at varying intensities at the lower elevations and may have caused some compositional modification to Artemisiadominated stands. The condition of lands under ARS management is unknown, but it might be assumed that an excellent record of use could be obtained.

The extremely broad gradient of mostly highcondition communities represented by this landscape gradient constitutes a superlative example of southwestern Montana environments and the communities they support. There is no comparable gradient now under protection of any kind.

\section{Centennial Sandhills}

Location: The Centennial Sandhills occur in the northeast corner of the Centennial Valley, a broad trough at the head of the Red Rock River. The valley floor lies at 6,500 to 6,600 feet with the Centennial Mountains abruptly rising to ca. 10,000 feet on the south and grading more gently into the Gravelly and Snowcrest Ranges on the north. The Centennial Sandhills are found in a band 1-2 miles wide and ca. 9 miles long on the north side of the Upper and Lower Red Rocks Lakes. The ownership is divided among private, Bureau of Land Management and US Fish and Wildlife Service with the first two named holding most of the land. In terms of a conservable landscape it might be prudent to include the adjacent wetlands (to the edge of lake) on the Red Rock Lakes National Wildlife Refuge. This land is both fragile and probably constitutes primary or alternative habitat for some species and supports unique wetland communities as well.

Environment: The sandhills are small, generally stabilized dunes that formed when sands deposited in the basins of Red Rocks Lakes, probably in the late Pleistocene (ca.10,000-12,000 BP). At this time with the waning of cold and pluvial climates the lakes retreated, exposing shorelines. Prevailing winds from the southwest have blown finer materials far to the east and drifted the lessmobile sand to the northeast where it has formed primary dunes with low ridges running southwest-northeast, paralleling the prevailing winds. Superimposed on these older ridges are blowout or parabolic dunes that are ellipsoidal to elongate U-shaped and 30-60 feet high. Leeward slopes are steep (30-60\% slope), while windward slopes are more gently sloping and often bowl-, crater-, or trough-shaped. Shapes of individual dunes are modified from parabolic shapes by strong cross winds and differential growth of stabilizing vegetation.

Vegetation/Other Biological Features: The Centennial Sandhills are unique for having a concentration of both sensitive plant species and plant communities. The highly ranked community types include those that comprise the long-term stable or climax vegetation such as Artemisia tridentata ssp. tridentata / Stipa comata [basin big sagebrush / needle-andthread], A. tridentata ssp. tridentata / Festuca idahoensis [basin big sagebrush / Idaho fescue], Artemisia tripartita / F. idahoensis [threetip sagebrush / Idaho fescue] and A. tripartita / Stipa comata [three-tip sagebrush / needle-andthread]. The Sandhills also include highly distinctive seral communities, including Chrysothamnus viscidiflorus / Stipa comata [green rabbitbrush / needle-and-thread] and Elymus lanceolatus / Phacelia hastata [western wheatgrass / silverleaf phacelia] and Leymus flavescens (yellow wildrye) herbaceous vegetation. The dominance of $A$. tridentata ssp.

tridentata (along with variable amounts of $A$. tridentata ssp. vaseyana) on upland settings, such as the stabilized dunes is a unique situation not seen elsewhere in Montana. A.

tridentata ssp. tridentata in Montana and most of the Intermountain West is almost invariably confined to drainageways and alluvial flats.

Four plant species considered rare in Montana occur in the Centennial Sandhills (Lesica and Shelly 1991). Astragalus ceramicus var. apus (painted milk-vetch, G4T3/S1) and Oenothera 
pallida var. idahoensis (Idaho pale eveningprimrose, G5T4Q/S1) occur only in the Centennial Sandhills and the sandhills of southeastern Idaho. Cryptantha fendleri (Fendler's cryptantha, G4/S1) is sparsely distributed across much of western North America, but is known from only two Montana locations. Leymus flavescens (yellow wildrye, G4/S1) occurs in sandy soil in general across the northwestern U. S., but in Montana occurs only in the Centennial Sandhills.

Zoological rarities found only here in Montana or recorded as new to the Centennial Valley, but occurring more commonly to the south include the Great Basin pocket mouse, blacktailed jackrabbit and Preble's shrew.

Condition/Conservation Values: This is a unique site by virtue of the combination of geology and highly ranked biological features. Though this is the second largest sand dune complex in the state, after the Medicine Lake Sandhills, in terms of its generally good to excellent range condition, abundance of elements and lack of threats the Centennial Sandhills landscape has a significantly higher ranking as a conservation site. Noxious weed threats are at this time virtually nil and the natural processes (fire and grazing of both large and diminutive herbivores) that maintained the seral conditions necessary for the perpetuation of various species and communities are present and capable of being manipulated for conservation purposes. The most significant threat to this landscape would be landscape fragmentation and the cessation of fire or mechanical disturbance that would allow successional processes to proceed to their endpoint and eliminate the sensitive seral species and communities.

\section{Johnson G ulch-D eer C anyon C reek, Prairie to Peak G radient (Tendoy Mountains)}

Location: The boundaries of this landscape are currently ill-defined but encompasses a watershed-based area that stretches from near the roadhead in T11S, R12W, Section 13 to the crest of the Tendoy Mountains in the vicinity T12S, R10W, Section 7. It would include

Bureau of Land Management as well as private lands at lowest elevations, with state parcels at mid elevations and US Forest Service holdings on the higher ridges and crests.

Environment: This elevation transect rises from 6,200 feet at the confluence of an ephemeral stream draining lower Johnson Gulch with Medicine Lodge Creek to approximately 9,390 at the crest of the Tendoy Mountains. This transect spans predominantly calcareous parent materials (limestone), including calcareous alluvial and colluvial fill materials. Situated in the rainshadow of the Beaverhead Mountains to the west, this country is very dry, receiving between 8 and 20 (25) inches of precipitation per year, but is very typical in terms of climate, as well as geological substrate and geomorphology, of much of the BVHDMS.

\section{Vegetation/Other Biological Features: The} vegetation elements encompassed by this landscape are primarily upland range communities (grassland and shrubland) and woodland with forested types (Pseudotsuga menziesiidominated) restricted to moderate to steep north-facing slopes. The rangeland communities are the elements of focus; they are predominantly in good or better ecological condition. At lowest elevations there are high quality examples of Artemisia tridentata ssp. vaseyana- and A. tripartita dominated communities on the gentle to steep sideslopes. A. tridentata ssp. tridentata is found in drainageways and other alluvial positions. In some locations Leymus cinereus has been exhibiting a trend of increasing coverage, both in association with basin big sagebrush and as a grassland co-dominant or indicator species (trend ostensibly due to deferred grazing when it is most vulnerable [personal communication, Brian Hockett]). A very incomplete reconnaissance has not as yet revealed A. nova communities, but they are expected. At mid to the highest reaches of the subalpine was found a nearly complete array of the community types involving Festuca idahoensis and A. tridentata ssp. vaseyana as dominants/diagnostic species; the more mesic, collecting positions supported 
excellent examples of $F$. idahoensis / Elymus trachycaulus, A. tridentata ssp. vaseyana / F. idahoensis / Geranium viscosissimum, A. tridentata ssp. vaseyana / F. idahoensis / Elymus trachycaulus and F. idahoensis / Carex scirpoidea. Excellent examples of alpine turf, mostly Calamagrostis purpurascens - Carex rupestris and Carex elynoides associations, cap the crest of the range. High quality examples of Cercocarpus ledifolius-dominated communities are present, including the somewhat rare (G3) C. ledifolius / F. idahoensis. An old-growth (>300 years) Pinus flexilis (limber pine) stand (predominately P. flexilis / Juniperus communis) occupies the west-facing slopes in Section 35 and on the north-facing slopes within this same Section are excellent examples of late seral stands potentially dominated by Picea engelmannii (Engelmann spruce).

Condition/Conservation Values: This is a prime landscape for representing a wide diversity of high-quality rangeland communities, including grasslands, shrublands, and woodlands. The elevational range represented encompasses all but the most xeric of southwestern Montana plant communities and includes a significant amount of subalpine grasslands and alpine turf (bighorn sheep populations are noted to frequent these highelevation grasslands). Grazing intensity reduction or change in timing of use on some of the encompassed allotments has ostensibly resulted in recovery in some community types such as the resurgence of Leymus cinereus (basin wild rye) in some drainageway locations.

\section{M orrison Lake Wetlands to Baldy M ountain Transect (B eaverhead M ountains)}

Location: This landscape encompasses major portions of Sections 21, 22, 23 and 24 of Range 12 West and Township 14 South and could incorporate portions of adjoining sections if watershed boundaries are used to define the site. It stretches from the Morrison Lake Wetlands, embedded in the flats east of the Beaverhead Range, to the crest of the range at
Baldy Mountain. Ownership is primarily by the U. S. Forest Service with basal plain acreage under Bureau of Land Management control. By including private lands within Section 19 a greater diversity of shrublands could be encompassed.

Environment: This transect represents, with the exception of the Morrison Lake Wetlands, a landscape in relatively good condition and highly typical of the BVHDMS ecoregion. The Morrison Lake Wetlands are somewhat atypical because southwestern Montana has relatively few wetlands (only those of Centennial Valley/Red Rock Lakes could be considered extensive). From the calcareous colluvial and alluvial fill of the gently rolling flats at 8,200 feet the transect rises abruptly to 10,773 at Baldy Mountain on the Continental Divide. This whole transect is set in calcareous parent material, predominantly limestone and lying to the east of the Continental Divide it is in somewhat of a rainshadow of the Beaverhead Mountains. Combining a substrate that weathers to shallow, well-drained soils with a dry climatic regime fosters an extremely dry environment as denoted by a strongly compressed or truncated forested zone. The Morrison Lake Wetlands are comprised of a group of alkaline fens, marshes, meadows and temporary ponds interspersed in a matrix of shrub-steppe. Many of the wetlands are less than an acre. Morrison Lake supports no emergent vegetation, only aquatics, however, just east of the lake is a spring-fed mire with a floating-mat peatland.

Vegetation/Other Biological Values: This landscape transect has been inventoried in piecemeal fashion, the Morrison Lake Wetlands having been documented by Lesica (1990) and the alpine communities having received a one-day sampling by S. V. Cooper and P. Lesica (Cooper and Lesica 1997). Cooper and Lesica also documented some riparian and upland vegetation in the vicinity of Indian Creek as it passes through Section 24. The above-cited botanical/ecological explorations support this landscape as a prime representation of the western portion of BVHDMS. The alpine-subalpine plant associations include 
excellent examples of Carex elynoides (Kobresia-like sedge), Festuca idahoensis Carex scirpoidea (Idaho fescue / Canadian single-spike sedge), Dryas octopetala / Carex rupestris (white dryas / curly sedge) dwarfshrub herbaceous, D. octopetala / Polygonum viviparum (white dryas / alpine bistort) dwarfshrubland herbaceous, Salix arctica / Polygonum bistortoides (Arctic willow / American bistort) dwarf-shrubland and Salix glauca (glaucous willow) dwarf-shrubland.

The limited portion of upslope landscape with trees dominant appeared to be old-growth woodland stands with Pseudotsuga menziesii (Douglas-fir) dominant and Pinus flexilis (limber pine) well represented as a seral species. The higher elevation sites were only cursorily noted and appeared to be quite open with Picea engelmannii (Engelmann spruce) dominant and scattered Pinus albicaulis (whitebark pine). A complete reconnaissance of the forested portion is necessary and would be expected to produce old-growth stands of $P$. albicaulis (given that old-growth $P$. menziesii exists at lower elevations). Pseudotsugadominated old-growth forest vegetation on the flats to the west of Morrison Lake is classified as Picea engelmannii / Senecio streptanthifolius (Engelmann spruce / Rocky Mountain butterweed), a common forest type of calcareous substrates only found in southwestern Montana.

Lesica (1990) coined new community names, largely based on dominance by layer, for wetland/aquatic community types found in the Morrison Lakes Wetland. These communities, their names and/or composition, have not been subsequently crosswalked to the NVCS or Hansen et al. (1995). Lesica described the following communities: Carex utriculataDeschampsia cespitosa (beaked sedge - tufted hairgrass), C. utriculata / Potamogeton pectinatus (beaked sedge / fennel-leafed pondweed), Pentaphylloides floribunda / Juncus balticus (shrubby cinquefoil / Baltic rush), Salix brachycarpa / Carex scirpoidea (short-fruited willow / Canadian single-spike sedge), Eleocharis palustris - Carex athrostachya (common spikesedge - slender- beaked sedge), Eleocharis palustris /

Potamogeton gramineus (common spikesedge / grass-leaved pondweed), Alopecurus aequalis / Hippuris vulgaris (short-awn foxtail / common mare's tail), and Myriophyllum spicatum Ranunculus aquatilis (spiked water-milfoil white water-buttercup, an aquatic type).

Several of the above-named communities have not been documented to occur elsewhere in the ecoregion and are quite possibly constitute unique community elements.

The shrub-steppe uplands are moderately to heavily grazed and characterized by Artemisia tridentata ssp. vaseyana-dominated types, predominantly A. tridentata ssp. vaseyana / Festuca idahoensis (mountain big sagebrush / Idaho fescue) with minor inclusions of Artemisia arbuscula ssp. longiloba / F. idahoensis (early low sagebrush / Idaho fescue) and quite possibly other shrub steppe types. In the riparian environment Leymus cinereus (basin wildrye) appeared to be making a comeback in A. tridentata ssp. tridentata stands where protected from grazing. Willow communities were not classified to type but appeared to have received past hard use by both domestic stock and wild ungulates.

Within this landscape only the Morrison Lake Wetlands were explicitly inventoried for species ranked as sensitive by MTNHP and the following were found; Aquilegia formosa, Astragalus argophyllus, Astragalus leptaleus, Carex parryana ssp. idahoa, Gentiana aquatica, Senecio debilis, and Thalictrum alpinum.

Condition/Conservation Values: The lower elevations of this transect have been livestock grazed for decades causing most damage to the wetlands where drawdown zones have been trampled and compacted and weedy species introduced, including Poa pratensis (Kentucky bluegrass), Taraxacum officinale (dandelion) and the noxious weed Cirsium canadense (Canada thistle). Grazing intensities were very high at the time of wetland site visitation and continued abuse could result in lowered species diversity and the introduction and facilitation of exotics. Grazing could have a direct delete- 
rious impact on the rare $C$. parryana ssp. idahoa because it is both highly palatable and susceptible to grazing. We have not checked the grazing records from the U. S. Forest Service for insights as to how the mountain grasslands may have been impacted; from cursory examination they appeared to not to have been adversely impacted. The considerable diversity of vegetation types and sensitive species present and under threat from grazing gives impetus for considering this an important conservation site. While there might possibly be more appropriate landscapes for conservation, this one was forwarded at this time because it has relatively more existing information.

\section{Plant Species of Special Concern}

The Beaverhead Mountains Section harbors $41 \%$ (19) of all the globally rare Montana vascular plant species that are tracked by the Montana Natural Heritage Program. These species are listed in Table 4 (below) and their distributions shown in Figure 4. This concen- tration of globally rare plants is among the highest in the state and includes several state or regional endemics. The Beaverhead Mountains Section has among the highest levels of plant endemism in the state; 18 of the 57 taxa (31\% percent) are of conservation concern.

In addition to the Montana plant species of special concern that are listed below, there are several more regional endemics (e.g., Sphaeromeria capitata, Stanleya tomentosa, and Townsendia nuttallii) on the state "watch" list. These are found primarily or only in this area of Montana and are of limited distribution but not officially tracked by the Montana Natural Heritage Program.

What is the conservation status of these globally rare species? Almost all are on agency sensitive species lists. Four species are restricted to alpine or subalpine habitats without identifiable threats (Chrysothamnus parryi ssp. montanus, Draba globosa, Draba ventosa, and Saxifraga tempestiva). Two species are known only from historic records (Primula alcalina, Thelypodium paniculatum),

Table 4. Globally rare plant species in the Beaverhead Mountains section

\begin{tabular}{|c|c|c|c|}
\hline SCIENTIFIC NAME $^{\text {T }}$ & COMMON NAME & GRANK & SRANK \\
\hline Arabis fecunda* & Sapphire Rockcress & $\mathrm{G} 2$ & S2 \\
\hline Astragalus ceramicus var. apus* & Painted Milkvetch & G4T3 & $\mathrm{S} 1$ \\
\hline Astragalus scaphoides* & Bitterroot Milkvetch & G3 & S2 \\
\hline Astragalus terminalis* & Railhead Milkvetch & G3 & S2 \\
\hline Carex parryana ssp. idahoa* & Idaho Sedge & G4T2 & S2 \\
\hline Carex stenoptila & Small-winged Sedge & G3? & $\mathrm{S} 2$ \\
\hline Chrysothamnus parryi ssp montanus & Centennial Rabbitbrush & G5T1 & $\mathrm{S} 1$ \\
\hline Draba globosa & Round-fruited Draba & G3 & $\mathrm{S} 1$ \\
\hline Draba ventosa & Wind River Draba & G3 & S1 \\
\hline Lesquerella pulchella* & Beautiful Bladderpod & G2 & S2 \\
\hline Lomatium attenuatum* & Taper-tip Desert-parsley & G3 & $\mathrm{S} 2$ \\
\hline Penstemon lemhiensis* & Lemhi Beardtongue & G3 & $\mathrm{S} 2$ \\
\hline Phacelia incana* & Hoary Phacelia & G3 & S2 \\
\hline Primula alcalina & Idaho Primrose & G1 & SX \\
\hline Saxifraga tempestiva & Storm Saxifrage & G2 & $\mathrm{S} 2$ \\
\hline Sphaeromeria argentea* & Chicken Sage & G3? & $\mathrm{S} 2$ \\
\hline Spiranthes diluvialis & Ute Ladies' Tresses & $\mathrm{G} 2$ & S2 \\
\hline Thelypodium paniculatum & Northwestern Thelypody & G2G3 & SH \\
\hline Thlaspi parviflorum & Small-flowered Pennycress & G3 & $\mathrm{S} 2$ \\
\hline
\end{tabular}

\footnotetext{
${ }^{1}$ Species that are bold-faced are known from extant populations at elevations below alpine and subalpine and are relatively vulnerable. Species that are asterisked have a significant number (over 33\%) of total occurrence numbers on BLM-administered lands.
} 
Figure 4. L ocations of Plant Species of Special C oncern, Beaverhead M ountains Section

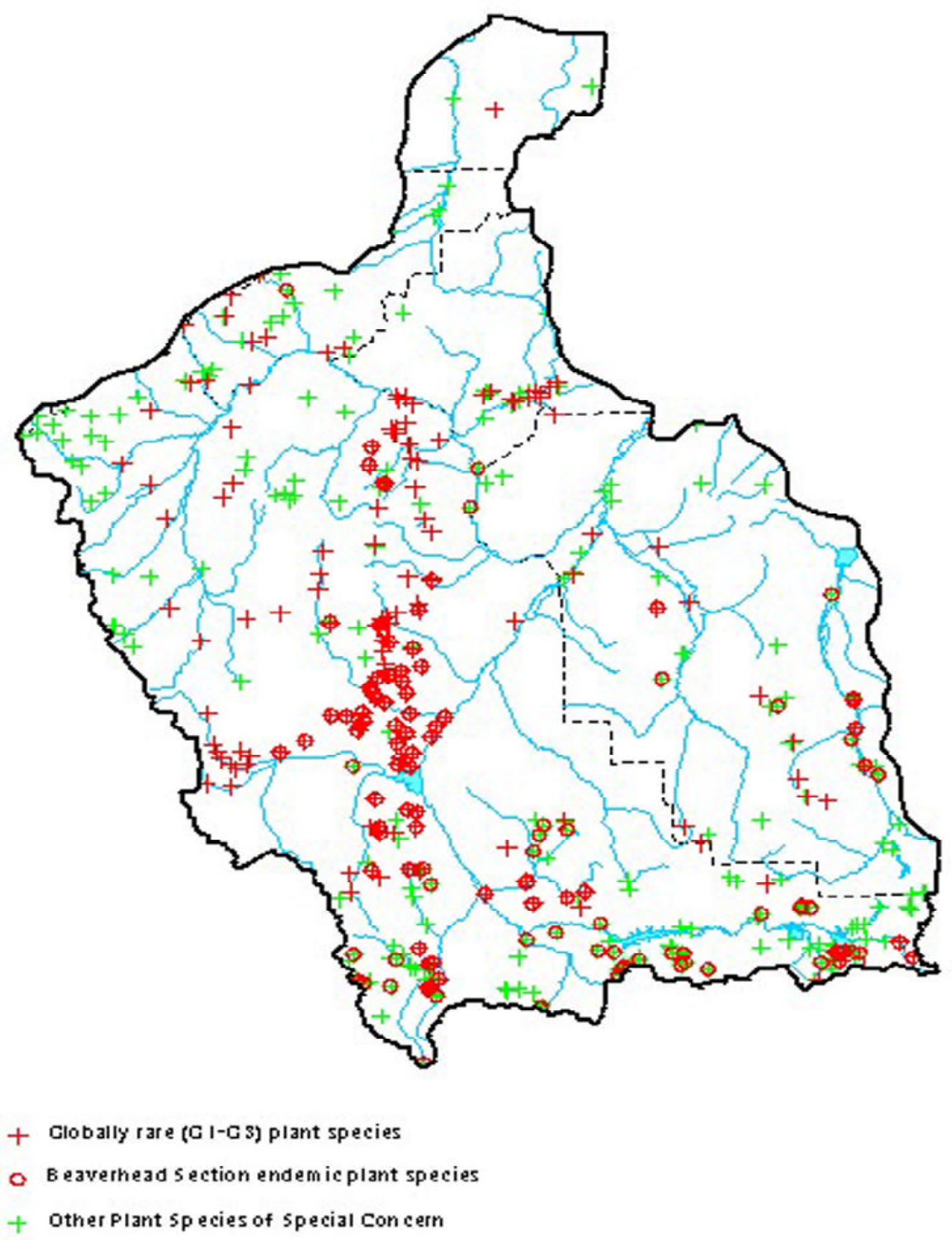

MTNHF Decemb a 15, 188 
and focused surveys are recommended in the areas from which they were originally known (Monida area, Alaska Basin area). The remaining 13 globally rare species (bold-faced in Table 4) stand as the most important conservation priorities for this Section. Ten of these species have at least $1 / 3$ of all their Montana records on BLM holdings (asterisked in Table $4)$.

Twenty-eight species that are globally rare occur on BLM-administered lands in this area (bold-faced in Tables 4 and 5). All of the globally rare species on the watch list are also on BLM lands. Fortunately, some or many are not under immediate threats. This is not to say that no conservation is needed. Continued vigilance for noxious weeds is an immediate and far-reaching need for maintaining plant diversity in general.

Many of the globally rare species we are reporting here for the Beaverhead Section represent newly documented additions to the state flora, some of them discovered as recently as last year. New species discoveries are added to the Montana plant species of special concern list if they are a native species known from fewer than 5 locations, in natural habitat, without evidence of adventive behavior indicated by increasing under unnatural conditions. Many of the new records are from lower elevations, so the remoteness of the species' habitat is inadequate explanation for their not having been discovered earlier. The relatively large number of new species reflects the floristic diversity as well as the incompleteness of basic floristic survey.

There are also a high number of State-rare plants that are restricted to this area of Montana. Ten percent (33) of all Montana vascular plant species of special concern occur exclusively in this region and no other parts of the state (Table 5, Figure 4). This includes 10 of the globally rare species noted above.

What is the conservation status of the state-rare species restricted to this area? Again, this question can be answered only indirectly. Many are on agency sensitive species lists.
Only three of the species are restricted to alpine or subalpine habitat (Braya humilis, Erigeron

leiomerus, and Oxytropis parryi) where threats are generally low. Three are known only from historic records (Calochortus bruneaunis,

Haplopappus nanus, Phacelia scopulina), and therefore cannot be protected. Focused surveys for these three species are recommended in conjunction with conservation and management activities taking place in the three areas from which they were originally known (Red Rock Valley south of Lima, Centennial Mountains, and Big Hole Valley near Melrose). The remaining 22 species (bold-faced in Table 5) represent the secondary conservation priorities for this region. Eighteen of these 22 species have at least one-third of their Montana records on BLM-administered lands.

Altogether, a total of about 130 Montana plant species of special concern occur in the Beaverhead Mountains. This includes globally rare species, state-rare species that are restricted to this portion of Montana, and staterare species that are more broadly distributed in the state. Their presence may represent welldeveloped habitat, habitat extremes, rare habitat, or undocumented habitat. In some measure, these species act as a surrogate for identifying unusual plant associations and exceptionally high quality conditions.

The 680 known occurrences of these species are not randomly distributed across the landscape but are very restricted (Figure 4). The patterns of aggregation fall into general categories by elevation range and habitat structure: valley wetland, foothills-montane wetland, sand dunes, and foothills-montane grasslands and shrublands. These "aggregates" of rare species, and the specific habitat conditions that represent, suggest a third set of conservation priorities for this region.

By analyzing the distribution of all the rare species describe for this section, it would be possible to identify places on BLM lands where their co-occurrence would provide opportunities to protect many species in aggregate. This analysis could emphasize those groups of higher conservation priority, and 
would focus on occurrences known to be of high quality (represented by an A or B quality rank). It could also include lands managed by other agencies, to yield a regional map of priority sites for rare plant conservation.

Finally, while there has been extensive botany work over large areas of the Beaverhead Mountains ecoregion, it is not exhaustive and provides no more than an initial data set and preliminary information for management and conservation of this extremely diverse landscape. This region, because of its high contribution to plant diversity at both the global and state scales, merits significant effort toward better understanding both the distribution and conservation needs of the rare flora.

Table 5. State or globally rare plants known ONLY from the Beaverhead Mountains Section

\begin{tabular}{|c|c|c|c|}
\hline SCIENTIFIC NAME & COMMON NAME & GRANK & SRANK \\
\hline Agastache cusickii $*$ & Cusick's Horse-mint & G3G4 & $\mathrm{S} 1$ \\
\hline Aquilegia formosa & Sitka Columbine & G5 & S1 \\
\hline Astragalus ceramicus var. apus* & Painted Milkvetch & G4T3 & S1 \\
\hline Astragalus scaphoides* & Bitterroot Milkvetch & G3 & S2 \\
\hline Astragalus terminalis* & Railhead Milkvetch & G3 & $\mathrm{S} 2$ \\
\hline Balsamorhiza hookeri & Hooker's Balsam-root & G5 & S1 \\
\hline Braya humilis & Low Braya & G4 & S1 \\
\hline Calochortus bruneaunis & Bruneau Mariposa Lily & G5 & SH \\
\hline Chrysothamnus parryi ssp montanus & Parry's Mountain & G5T1 & S1 \\
\hline Elymus flavescens* & Sand Wildrye & G4 & S1 \\
\hline Erigeron leiomerus & Smooth Fleabane & G4 & S1 \\
\hline Erigeron tener & Slender Fleabane & G4 & S1 \\
\hline Eriogonum caespitosum* & Mat Buckwheat & G5 & S1 \\
\hline Haplopappus nanus & Dwarf Goldenweed & G5 & S1 \\
\hline Ipomopsis congesta ssp crebrifolia* & Ballhead Gilia & G5T4? & $\mathrm{S} 2$ \\
\hline Kochia americana* & Red Sage & G5 & S1 \\
\hline Lesquerella pulchella* & Beautiful Bladderpod & $\mathrm{G} 2$ & $\mathrm{~S} 2$ \\
\hline Lomatium attenuatum* & Taper-tip Desert-parsley & G3 & $\mathrm{S} 2$ \\
\hline Lomatogonium rotatum* & Felwort & G5 & S1 \\
\hline Oenothera pallida var. idahoensis* & Pale Evening-primrose & G5T4Q & S1 \\
\hline Oxytropis parryi & Parry's Crazyweed & G5 & S1 \\
\hline Phacelia scopulina & Dwarf Phacelia & G4 & $\mathrm{SH}$ \\
\hline Phacelia incana* & Hoary Phacelia & G3 & $\mathrm{S} 2$ \\
\hline Primula alcalina & Mealy primrose & G4G5 & S2 \\
\hline Puccinellia lemmonii* & Lemmon's Alkaligrass & G4 & S1 \\
\hline Ranunculus hyperboreus & High-arctic Buttercup & G5 & S1 \\
\hline Ribes velutinum & Desert Gooseberry & G5 & S1 \\
\hline Sphaeralcea munroana* & White-stemmed Globe- & G4 & S1 \\
\hline Sphaeromeria argentea* & Chicken Sage & G3? & $\mathrm{S} 2$ \\
\hline Stellaria jamesiana & James Stitchwort & G5 & S1 \\
\hline Stephanomeria spinosa* & Spiny Skeletonweed & G4 & S1 \\
\hline Thelypodium paniculatum & Northwestern Thelypody & G2G3 & SH \\
\hline Townsendia florifer* & Showy Townsendia & G5 & S1 \\
\hline
\end{tabular}




\section{Conclusions and Recommendations}

This is the first ecoregion section in Montana to have a comprehensive Vegetation Classification assembled into a single document. This project provides a snapshot of plant communities in the Beaverhead Mountains Section and their status. It reflects our current knowledge and is by no means exhaustive. While there is potential to utilize the dataset for more advanced and comprehensive analyses of the vegetation community, we now have a satisfactory vegetation community classification for management, monitoring and conservation objectives.

The project goal was to compile ecology plot data, validate and refine existing plant association types and combine into a new classification following the National Vegetation Classification Standard. The classification has three major components: 1) a list of Plant Associations for Beaverhead Mountains Section in Montana, 2) the Key to Plant Associations, and, 3) Plant Association descriptions.

The MTNHP acquired all known ecology plot data sets, except for that of the Montana Wetland and Riparian Research Program, and utilized the material for this analysis. All plots that could be substantiated by a legal location descriptor were assigned to a plant association type and mapped into Geographic Information System. Other plant associations were added to the Beaverhead list based on distribution descriptions completed by other authors, primarily Hansen et al. (1995).

The Key to Plant Associations is a dichotomous key that identifies physiognomic vegetation types using the new National Vegetation Classification. The key includes every major vascular plant group or lifeform encountered in the BVHDMS. The key in combination with the plant association descriptions provides a very powerful tool for the identification of plant communities.
The MTNHP prepared 130 plant association descriptions and included in this classification. This project provided the raw material to develop dozens of new community descriptions (Characterization Abstracts) and to standardize over 100 of our older descriptions, all of which will be maintained as part of the Montana Natural Heritage Program data system. These community descriptions provide the basis for crosswalking with other classification efforts, such as rangeland sites currently employed by the BLM and NRCS. The process of examining plot data, reviewing, editing and preparing new plant community descriptions has provided the framework for our future revision of community ranks.

In the future we plan to develop a Web-based field guide that can produce current plant association distribution maps along with community descriptions and their conservation values. Such products should be valuable in conservation planning when one needs a spatial representation of rare or high quality community elements. Such map products could well serve land managers as they deal with landscape diversity and pinpoint opportunities to manage for, or promote, community diversity, as well as protecting existing landscapes with concentrations of valuable (rare) elements, including communities, plant and animal species. The prospect for producing online locations of sample plots on US Geological Survey 1:24,000 scale maps (or meaningful aggregations, such as 1:250,000 quadrangles) is in sight.

Our next phase of community ecology in Beaverhead Mountains Section will benefit from this analysis. Additional survey to better locate and identify conservation opportunities for the globally imperiled plant communities is needed. There is additional need to inventory for good quality, representative communities, and significant landscapes. Exemplary communities and landscapes of significant ecological interest are important heritage for Montana and worth managing to retain their natural character. 


\section{The assification: concepts and assumptions}

\section{The $N$ ational Vegetation Classification}

The list of plant associations for Beaverhead Mountains Section, their descriptions and the accompanied vegetation key is a product of the Montana Natural Heritage Program. The Heritage program network has worked cooperatively with The Nature Conservancy (TNC) by first developing a National Vegetation Classification Standard (reworked UNESCO 1973 and Driscoll et al. 1984) that was accepted as the framework to be used by all federal agencies (FGDC 1997). The National Vegetation Classification Standard (NVCS) is hierarchical and combines floristics at the lowest levels (associations and alliances) and physiognomy and broad ecological modifiers at the highest levels of the hierarchy. Physiognomy pertains to the physical features of the vegetation i.e. forest vs. herbaceous vegetation. This approach allows the characteristics of vegetation patterns to be referenced at multiple spatial scales. The upper levels of the hierarchy were adopted as a national standard by Secretary of the Interior, Bruce Babbit in 1997. The floristic levels of the Vegetation Classification in the United States is currently in a review draft phase.

The physiognomic/structural levels of the NVCS have proven useful in mapping the natural and semi-natural vegetation of the U. S. in order to assess the conservation status of species and their habitats as part of the USGS Gap Analysis Program (GAP). The floristically based units, alliances and plant associations, have proven much more difficult to define, both conceptually and individually, for example, what constitutes the defining parameters of the Abies lasiocarpa /Vaccinium scoparium forest plant association? There are several concepts and assumptions worth mentioning here. Although we have set out to conform to the conventions of the NVCS and its approach to classification there is one very significant point in the approach which has not been incorporated to any extent in our work or that of our predecessors; that is to describe existing vegetation, rather than potential vegetation. Most of the vegetation types treated within our work and those of our predecessors were considered potential natural vegetation types (habitat types sensu Daubenmire 1969) and named for the climax plant association. This remains a distinct drawback or handicap to the MTNHP.

To illustrate: A very common plant association (climax) in western Montana is Abies lasiocarpa / Clintonia uniflora; seldom is the forest canopy dominated by A. lasiocarpa. The most common seral overstory dominant (often monospecific) for this type is Pinus contorta. At this point in time there has never been a $P$. contorta / C. uniflora plant association recognized. Rather, foresters/ecologists have treated this condition as a $P$. contorta cover type of the A. lasiocarpa / C. uniflora habitat type (recognizing the sites potential to support A. lasiocarpa, this being the diagnostic species for the series level) and applied a structural descriptor (say pole or mature size class). They have also recognized the site's potential to support a variety of undergrowth dominants, using the presence of $C$. uniflora as an indicator of a particular environmental regime. The NVCS would create a new plant association based on existing Pinus contortadominated stands; in this case one would have to envision an association named $P$. contorta / C. uniflora.

There are many significant points to be addressed in classifying seral forest vegetation if we are to adhere to describe existing vegetation only: 1) do we consider all stages of a sere and assign each a plant association name, or describe seral communities with structural and compositional labels, such as seedling/sapling stage with Acer glabrum dominant in the tallest layer of a potential 
Abies lasiocarpa / Vaccinium scoparium p. a., 2) how does one discriminate among canopy species (for purposes of assigning type name) when there is no clear expressed dominance? is the choice based on dominance or the specie's indicator value?, and 4) given that succession is a complex function of many interacting variables that determine what "pathway" a disturbed stand will take, and that for a given environment, vegetation will more or less converge to a dynamic stable endpoint, do we continue to track what this endpoint might be when we collect information on seral plots?

Also worth noting is that the NVCS separates shrubland from shrub herbaceous types by distinguishing a shrub type as having shrub cover greater than $25 \%$. While this is an artificial distinction, it creates some confusion because our local shrubland classifications, principally Mueggler and Stewart (1980), recognize shrubland types as having cover sufficient to convey a "shrubby aspect", which they conceived of as being $5 \%$ or greater canopy cover. In this report we have treated most of the Artemisia tridentata-dominated types as shrublands (as was done by Mueggler and Stewart), but in fact almost none of the sampled stands meet the NVCS shrubland criteria.

Another classification quandary was what to do with vegetation units, termed "phases" by various researchers (Pfister et al. 1977, Mueggler and Stewart 1980, Hansen et al. 1995, Cooper et al. 1987) and "not tracked by the Heritage Programs in the NVC units." Considerable information concerning environment-vegetation relationships could be forfeited by not recognizing phases of plant associations. Thus, within this manuscript, we have elevated phases to plant associations where information is sufficient to do so. (In the vegetation keys that follow some phases have been designated and their characteristics are described within the plant association of which they are a component. A prime example (from northwestern Montana) of the information that would be obscured by not tracking phases is to consider selected phases of the
Abies lasiocarpa / Clintonia uniflora plant association. The Menziesia ferruginea and Xerophyllum tenax phases are environmentally distinct, the $M$. ferruginea phase occurring on cool aspects at higher elevations and the $X$. tenax phase occurring on southerly aspects and extending from the lowest to highest elevations at which $C$. uniflora co-occurs. The Aralia nudicaulis phase occurs on very distinct positions in the landscape, bottomlands and toe-slope seepage areas. These different phases not only have distinct flora, but differ significantly in their productivity (based on tree site index), the tree species that are expected to be major seral dominants, fuel models and fire regime. Thus we would propose that the three phases named above be treated as three distinct plant associations; A. lasiocarpa / C. unifloraAralia nudicaulis, A. lasiocarpa / $M$.

ferruginea / C. uniflora, and A. lasiocarpa / C. uniflora - X. tenax.

A natural question is why, if phases represent distinct floristic compositions and environments, they were not treated as plant associations in the first place. These classifications were initially promoted as a boon to management and the researchers intuited that managers would not want a lot of complexity (too many habitat types or plant associations recognized) and phases were, at that time, a way of tracking the complexity of vegetation/environment relationships.

Occasionally phases have been created to recognize regional floristic permutations of a common type. It should be noted that the environmental range of one of the phases of $A$. lasiocarpa / C. uniflora may be just as great as that encompassed by a particular plant association with no phases, say A. lasiocarpa / Galium triflorum or A. lasiocarpa / Vaccinium cespitosum. It is exceedingly difficult to measure all the physical parameters of a vegetation type's environment, which is precisely why habitat types or plant associations have been embraced as surrogate indicators of environment. 


\section{Plant Associations of the Beaverhead M ountains Section: Vegetation key and descriptions}

We have developed a dichotamous key to the vegetation types and prepared written descriptions for approximately $50 \%$ of the plant associations (total of 273) occurring in the Beaverhead Head Mountains Section. The approach of the Montana Natural Heritage Program has been to incorporate the classifications or type descriptions of other researchers into our more encompassing classification; this is because the work of our predecessors has been peer reviewed and found to be scientifically rigorous as well as eminently applicable by managers. Usually, but not always, these classifications of regional and/or physiognomic types (e.g. Pfister et al. 1977 of forested types on national forest holdings) have been crosswalked with those of neighboring states or provinces so that a westwide and eventually national classification can be constructed. Though a purported national classification of plant associations has been published, "International classification of ecological communities: Terrestrial vegetation of the United States" Anderson et al. (1998, Volume II), it is important to note that this is more a listing of types at the plant association level and not a formal taxonomy. Without a formal taxonomy (key to plant associations) there is no consistent way one can identify a piece of vegetation on the ground. One can go to all the disparate original sources for a type's description but often even these sources do not always have a "working key" that can be conveniently and consistently employed to differentiate one vegetation type from another.

One of the critical points of a community's description is how it differs from those communities to which it is most closely related (either floristically or environmentally). Vegetation keys at the alliance and plant association levels are shorthand means of discriminating among vegetation types. Though the TNC claims to have produced a national vegetation classification (Anderson et al. 1998) in fact at the alliance and plant association levels it is nothing more than a laundry list with no convenient way of identifying the vegetation with which one is dealing and for this reason cannot be effectively employed. Thus, we deemed it imperative that vegetation keys be developed for this report so that users of the classification would not feel stymied in trying to put names on and understand vegetation types. However, these keys are perhaps a weak link in this report because they have not enjoyed a critical analysis but rather have been pieced together, incorporating first those keys from original sources. These keys have also not been tested, except with existing plot data in an office setting. It should be appreciated that some fraction of the vegetation extant on the landscape will not key to a particular plant community type. This is because the type may be unique, tied to a particular substrate type for example, and was not sampled in the inventory process. For efficiency, sampling is stratified by major environmental parameters leaving literally hundreds, if not thousands, of square miles of this ecoregion unvisited.

Alternatively, leads in the key could have inadvertently left gaps through which certain types have fallen.

The key to the various physiognomic types was designed to be in accord with the National Vegetation Classification (NVC) and its concepts of forest, woodland, shrubland, etc. In other words, above the floristically based Alliance and Plant Association levels, we have largely subscribed to the NVC treatment of structure and cover to define units from the highest levels (Order) all the way down to Formation.

The keys within various physiognomic types, say woodlands or shrublands, are based on floristic composition. The floristic composition is weighted by canopy cover as defined by Daubenmire (1959), regardless of the life form being tallied. Daubenmire's canopy cover is conceived to be the vertical projection of a imaginary polygon about the outermost perimeter of a plant's canopy and expressed as a fraction of the total plot area sampled; this concept of cover has largely been 
accepted in the western US, particularly in Montana.

We have tried to capture the insights and good science of our predecessors who have constructed keys to various components (e. g. just forest or alpine types) of the vegetation of southwestern Montana, basing our keys on theirs to the extent possible. A significant number of plant associations have been newly described or identified since these preceding keys were written. We have intercalated these plant associations not treated by previous works into our keys based on the principles that guided our predecessor's key construction (at least as we interpreted their efforts).

In general, keys written for Montana vegetation, regardless of the dominant lifeform, have been structured to identify first the wettest or most environmentally unique sites (as reflected in their unique flora), or the most shade tolerant (in the case of forested alliances). The order progresses to associations successively drier, or less tied to specific site conditions or supporting less shade tolerant tree species.

The vegetation key is a tool that functions best when combined with a vegetation description. A formalized and highly reviewed description of a plant association in a global context is referred to as a Community Characterization Abstract (CCA). The production of carefully edited CCAs is critical in turn to producing useful floristic level classifications. Very few of these abstracts have been produced to date and we have provided the few CCAs that are applicable to the BVHDMS. The plant association descriptions within this report constitute an important source of information for the future synthesis of CCAs.

\section{U se of the vegetation key}

\section{Instructions}

1) Seek a homogenous site. Homogeneity (environment and history of use) and the vegetation cover are primary considerations in plot location selection. The plot being inventoried and classified should be representative of the stand as a whole, if not, then relocate the plot and re-estimate plant cover (thoroughly reconnoiter a stand before taking a plot sample).

a) Note that environmental gradients are often steep and that the size of homogeneous vegetation types may be extremely restricted $\left(<10 \mathrm{~m}^{2}\right)$, espe cially in alpine environments.

b) Homogeneity is most easily appraised as an area supporting a particular suite of dominants and subordinate indicator species.

2) Accurately identify and estimate canopy cover for all indicator species used in the key. In the following key and main body of text the canopy coverage (c.c.) concept employed is that of Daubenmire (1959); a vertical projection about the outermost perimeter of a plant's canopy expressed as a fraction of the area sampled. The following terms are applied when referring to species in the leads of the vegetation keys.

Present: trace to $100 \%$ canopy coverage (c.c.) versus Absent: 0\% c.c.

Common: species with $1 \%$ or more c.c. versus Scarce: having less than $1 \%$ c.c.

Well represented: species with at least $5 \%$ c.c. versus Poorly represented: less than 5\% c.c.

Abundant: species having $25 \%$ or greater c.c. versus Not Abundant: species having less than $25 \%$ c.c.

Reproducing Successfully: Generally at least 10 seedlings or saplings per acre and not confined to microsites. 
3) On sites where the vegetation is obviously depauperate (unusually sparse) due to heavy grazing, browsing, or closed canopy stage of forest vegetation succession adjust the key downward to reflect the reduced canopy cover (e.g." well represented" would become "common").

4) If severely disturbed or early seral conditions are encountered, the p. a. (at least as regards potential vegetation, especially forested conditions) is best determined by extrapolating from the nearest relatively undisturbed mature stand with similar site conditions (slope, aspect, elevation, and soils).

5) The following key is dichotomous; all criteria for one of the two choices of each couplet must be satisfied to obtain a correct determination. (NOTE: There are many couplets where one on the choices leads to an "undefined" or "undescribed" vegetation type. There are several reasons for this, the most obvious one being it that not all the landscape has been inventoried and there exist many permutations of site and vegetation composition that have not been described.)

6) Identify the lifeform group using "KeY To Physiognomic Types (Lifeform CategoRIES)."

7) Within the correct lifeform follow the key literally to identify a) in the case of forest and woodland vegetation the series (or alliance) level and then progress to the plant association (p. a.) level; b) in the case of other lifeforms (shrublands, grasslands, etc.) the key will identify plant associations directly.

8) Consult the table of contents to obtain the page number for the physiognomic types. Plant associations are ordered alphabetically within lifeform (e.g. forest shrubland, dwarf shrubland, etc.).

\section{C aveats when using keys}

1) This key is generally structured to identify, within lifeform types, the wettest sites first and progresses to successively drier sites.

2) In applying the key to actual field conditions the cover definitions cited above might need adjusting to the next lower coverage class, e.g. "well represented" becomes "common." This may be necessary when the closed canopy stage of forest succession obtains, or when grazing pressure has altered community composition.

3) In the case of early successional stages, particularly with regard to potentially forested sites, the current stand composition may not "key out" to a described plant association; this is because the keys are intended for use with relatively mature vegetation. It is the intention of the National Vegetation Classification Standard (NVC) to treat all existing vegetation types, including seral stages, as discrete plant associations, but this intention has not been realized for any appreciable area in the western US. See Arno et al. (1985, 1986), Steele (1988) or Hansen et al. (1995) for approaches dealing with classification and description of seral vegetation; Hansen et al. (1995, pages 2427 and Figure 3 ) is particularly helpful in explaining terminology such as plant association versus community type and habitat type.

4) To discriminate shrublands (including dwarf-shrublands) from "non-shrublands" we have included the NVC approach in normal typeface but in boldface type we have included an alternative set of leads which should be followed at this time. The boldface leads are necessary because of the significant discrepancy between the NVC criteria for a shrubland (at least $25 \%$ canopy cover of shrubs) versus the criteria employed by Mueggler and Stewart (1980), the most authoritative guide to rangeland types in Montana and the source whose key the Montana Natural Heritage Program has adhered to in the past. Mueggler and Stewart (1980) distinguish a "shrubland aspect" which they define as having woody plants common, with at least $5 \%$ canopy cover. It should be 
noted that, based on average canopy cover of the shrub component, none of the shrubland types classified by Mueggler and Stewart would qualify as shrublands by NVC criteria. In other words, virtually all the upland shrublands would be classified by the NVC as shrub herbaceous (i. e. a temperate or subpolar grassland with a sparse shrub layer). We have insufficient resources at this time to follow the NVC model which would distinguish between, for example, an Artemisia tridentata / Festuca idahoensis Shrubland and an A. tridentata / F. idahoensis Shrub Herbaceous type; the only difference between these types might be the canopy cover of $A$. tridentata. We indicate in the written description for the plant association whether it is considered a shrubland or shrub herbaceous type by the NVC.

5) The keys and plant association type descriptions are arranged by decreasing size of the dominant lifeform and within lifeform the upland sites are placed first followed by wetland/riparian types. The order of presentation of plant association descriptions is alphabetical within dominant lifeforms.

6) Once a stand has been keyed to plant association, the description of said associa- tion may not exist in this document, but references to the association, including the best sources for its description, will be found in Appendix D.

7) Some plant associations will key out in both upland and wetland keys because a) their habitat conditions are known to span that range, b) field personnel may be unfamiliar with the fine points of ascertaining wetland criteria so that by including some essentially wetland associations (that to the uninitiated appear as upland types) in the upland key, these types will be correctly identified to plant association.

8) The dichotomous key is only a convenience for identifying plant associations / community types; it is not the classification! Validate your determinations by comparing vegetation/site characteristics with parameters of plant association descriptions; be especially aware that vegetation composition and environmental conditions described come from sampled sites and personal observation and almost certainly do not include all the sites (the total range of environments) over which the type is found. 


\section{Key to Physiognomic Types (Lifeform Categories)}

The leads below are slightly altered or reworded from the National Vegetation Classification (NVC) with the following exception: To discriminate shrublands (including dwarf-shrublands) from "nonshrublands" we have included the NVC approach in normal typeface but in boldface type we have included an alternative set of leads which should be followed at this time (see \#4 under "Caveats when using keys" for a complete explanation).

The ultimate NVC classification unit designated in the physiognomic key below is subclass; once physiognomic type is determined continue with the Series or Alliance Level Keys for forests and woodlands and for the shrublands, etc. go directly to the plant association keys which are arranged in the following order; shrublands, dwarf-shrublands, grasslands, perennial forb vegetation, nonvascular vegetation)

1. Trees (deciduous or evergreen) dominant with at least $25 \%$ canopy cover, or rarely less than $25 \%$, if the cover of other life forms (shrub, dwarf-shrub, herb, nonvascular) is less than $25 \%$ and tree cover exceeds the combined cover of these other lifeforms.

1. Trees not dominant, their cover less than $25 \%$ and if less than $25 \%$ then the combined cover of other lifeforms is greater than that of the canopy trees

2. Tree crowns at least partially overlapping, forming 60 to $100 \%$ canopy cover FOREST

a. Evergreen trees (non-deciduous conifers) present and reproducing successfully (10 trees per acre, minimum) and not confined to microsites (note that at closed canopy stage there may be no evidence of reproduction by any conifer species).

EVERGREEN FOREST

b. Evergreen trees absent or, if present, then restricted to microsites and not reproducing successfully (unthrifty specimens, generally $<10$ trees / acre).....

DECIDUOUS FOREST

2. Tree crowns usually not touching, forming 25 to $60 \%$ canopy cover WOODLAND (see a \& b below)

a. Evergreen trees (non-deciduous conifers) present and reproducing successfully (10 trees per acre, minimum) and not confined to microsites (note that at "closed canopy" stage there may be no evidence of reproduction by any conifer species) EVERGREEN WOODLAND b. Evergreen trees absent or, if present, then restricted to microsites and not .reproducing successfully (unthrifty specimens, generally $<10$ trees / acre)....

DECIDUOUS WOODLAND

3. Shrubs generally greater than $0.5 \mathrm{~m}$ tall with individuals not touching to overlapping and canopy cover greater than $25 \%$; shrub canopy cover (uncommonly) may be less than $25 \%$ if the canopy cover of other life forms (dwarf-shrub, herbs, nonvasculars) is less than $25 \%$ and exceeded by that of the shrubs [N.B., 1) by following this lead only shrub communities with a size potential greater than that of dwarf-shrubs will be found, 2) some types considered shrublands prior to NVC will now be found in shrub herbaceous vegetation; a list of dwarf-shrub species used to define plant associations will be found in the following lead, \#4] SHRUBLAND (NVC DEFINITION)

$3^{*}$. Shrubs generally greater than $0.5 \mathrm{~m}$ tall and presenting, in the aggregate, a shrubland aspect with at least $5 \%$ canopy cover SHRUBLAND (Definition currently used by MTNHP)

3. Not as above, i. e. shrubs either less than $0.5 \mathrm{~m}$ tall or their combined cover less than $25 \%$ 4

$3^{\star}$. Not as above $\left(3^{\star}\right)$; shrubs either less than $0.5 \mathrm{~m}$ tall or in the aggregate not presenting a shrubland aspect, their canopy cover less than $5 \%$

4. Dwarf-shrubs (as individuals or clumps with a potential height less than $0.5 \mathrm{~m}$ ) generally forming $25 \%$ or greater canopy cover [taxa include Artemisia arbuscula (low sagebrush), A. arbuscula ssp. longiloba (early low sagebrush), A. nova (black sagebrush), A. pedatifida (birdfoot sagebrush), A. tridentata ssp. wyomingensis (Wyoming big sagebrush), A. tripartita (three-tip sagebrush), Atriplex gardneri (Gardner's saltbush), Cassiope mertensiana (Mertens' mountain heather), Dryas octopetala (white dryas), Kalmia 
microphylla (small-leaved laurel), Phyllodoce empetriformis (red mountain-heath), P. glanduliflora (yellow mountain-heath), Pentaphylloides floribunda (shrubby cinquefoil), Salix arctica (alpine willow), S. candida (hoary willow), S. barratiana (Barratt willow), S. brachycarpa (short-fruited willow), S. glauca (glaucous willow), $S$. planifolia var. monica (dwarf planeleaf willow), S. reticulata (snow willow)]; though an uncommon condition, dwarf-shrub cover may be less than $25 \%$ but exceeding the combined cover of the other lifeforms present (shrubs, herbs, nonvasculars) which is less than $25 \%$.

DWARF-SHRUBLAND (NVC definition)

$4^{\star}$. Dwarf-shrubs (as individuals or clumps with a potential height less than $0.5 \mathrm{~m}$ ) forming a shrubland aspect with at least $5 \%$ canopy cover (see list of dwarf-shrub species in lead 4 above) DWARF-SHRUBLAND (Definition currently used by MTNHP)

4. Not as above; dwarf-shrub cover less than 25\%; graminoids, forbs or nonvasculars constitute the dominant canopy cover, though trees and shrubs may be present and have to $25 \%$ canopy cover ...... 5

$4^{\star}$. Not as above $\left(4^{\star}\right)$; dwarf-shrub cover insufficient to present a shrubland aspect, canopy cover less than $5 \%$

5. Herbs (graminoids, forbs, ferns) dominant, usually constituting at least $25 \%$ canopy cover; in the unusual case where herb canopy cover is less than $25 \%$ it still exceeds the cover of each of the other lifeforms present

5. Herbs not dominant, in the aggregate constituting less than $25 \%$ canopy cover......

6. Perennial graminoid vegetation constitutes at least $50 \%$ of the total herbaceous canopy cover......

6. Of the total herbaceous cover perennial graminoids constitute less than $50 \%$ and conversely perennial forbs (including biennials and ferns) constitute more than 50\% canopy cover

Perennial Forb Vegetation

7. Nonvascular vegetation (bryophytes, lichens, algae) generally comprise at least $25 \%$ canopy cover; in unusual instances nonvascular canopy cover may be less than $25 \%$ where it exceeds the cover of each of the other lifeforms present; areas dominated by crustose lichens should be placed in the Sparsely Vegetated Class NONVASCULAR Vegetation

7. Not as above.

8. Vegetation is scattered or nearly absent; total vegetation cover (excluding crustose lichens the cover of which often far exceeds $10 \%$ ) is generally $1-10 \%$.

8. Vegetation cover is less than $1 \%$ 


\section{KeY to EVERGREen ForestS AND WOOdLANDS [INCLUDES UPLAND AND WETLAND TYPES]}

\section{Key to Series Level}

[series are arranged alphabetically following this key; key based largely on Pfister et al. (1977), Steele et al. (1983), Hansen et al. (1995)]; series comparable to NVC Alliance Level if only potential vegetation were addressed]

1. Abies lasiocarpa (subalpine fire) present and reproducing successfully; not confined to microsites........

....................................................................................................... Abies lasiocarpa Series

1. A. lasiocarpa absent or not reproducing successfully and if present, confined to microsites ................ 2

2. Picea (spruce) spp. [mostly $P$. engelmannii (Engelmann spruce), but including hybrid swarms of $P$. engelmannii x $P$. glauca (white spruce)] present and reproducing successfully..... Picea spp. Series

2. Picea spp. absent or not reproducing successfully or confined to microsites

3. Pinus albicaulis (whitebark pine) present and reproducing successfully; $A$. lasiocarpa (subalpine fir)and Picea (spruce) spp. may be present with more than 10 seedlings/saplings but they are stunted, unthrifty specimens

Pinus albicaulis Series

3. P. albicaulis absent or not reproducing successfully....

4. Pinus flexilis (limber pine) present and reproducing successfully (though episodically ) and often sharing this status with Pseudotsuga menziesii (Douglas-fir)

Pinus flexilis Series

4. P. flexilis absent or not reproducing successfully

5. Pseudotsuga menziesii (Douglas-fir) present and reproducing successfully

5. P. menziesii absent or not reproducing successfully....

Pseudotsuga menziesii Series

6. Pinus contorta (lodgepole pine) occurring in virtually pure stands, not necessarily reproducing, lacking evidence as to climax potential. Pinus contorta Series

6. P. contorta absent or not reproducing successfully 7

7. Pinus ponderosa (ponderosa pine) present, reproducing successfully (though mainly episodically), not accidental or confined to microsites

Pinus ponderosa Series

7. P. ponderosa absent or accidental ....

8. Juniperus scopulorum (Rocky Mountain juniper) the indicated site dominant, having canopy cover greater than or equal to that of Cercocarpus ledifolius (curl-leaf mountain mahogany)

8. J. scopulorum not the indicated site dominant Juniperus scopulorum Series

9. Cercocarpus ledifolius* (curl-leaf mountain mahogany) the indicated site dominant

9. C. ledifolius not the indicated site dominant

Cercocarpus ledifolius Series Forest or woodland Series (Alliance) as yet unrecognized for the Beaverhead Mountains Section

${ }^{*}$ Cercocarpus ledifolius is considered a shrub in most floras, but those with experience in the Great Basin may consider it a tree; we have included it in both the woodland and forest key and the shrubland key. 


\section{Abies lasiocarpa (subalpine fir) Series Key to Plant Associations}

1. Sites at or above the cold limits of Pseudotsuga menziesii (Douglas-fir) and also meeting one of following criteria;

a. Pinus albicaulis (whitebark pine) well represented as either seral or climax component;

b. Luzula hitchcockii (smooth woodrush) present, not related to microsites;

c. Ribes montigenum (mountain gooseberry) present;

d. Stands at upper timberline, growth stunted, tree height not much exceeding $50 \mathrm{ft}$;

UPPER SUBALPINE \& TIMBERLINE HABITATS lead \#15

1. Not as above....

2. Ledum glandulosum (Labrador tea) well represented (at least $5 \%$ canopy cover),

Abies lasiocarpa / Ledum glandulosum p. a.

a. One, or any combination of, the following species well represented: Calamagrostis canadensis (bluejoint reedgrass), C. stricta (narrow-spiked reedgrass), Senecio triangularis (arrowleaf groundsel) ........................................................................ Calamagrostis canadensis phase

b. Not as above; $C$. canadensis, $C$. stricta, $S$. triangularis poorly represented; undergrowth may be variously dominated by Vaccinium scoparium (grouse whortleberry), $V$. cespitosum (dwarf huckleberry), Linnaea borealis (twinflower), Cornus canadensis (bunchberry), Calamagrostis rubescens (pinegrass), or Carex geyeri (elk sedge) .... Ledum glandulosum phase

2. L. glandulosum poorly represented 3

3. Calamagrostis canadensis (bluejoint reedgrass) or C. stricta (narrow-spike reedgrass), alone or in combination, well represented....

a. Vaccinium cespitosum (dwarf huckleberry) common

Abies lasiocarpa / Calamagrostis canadensis p. a.

b. V. cespitosum scarce. Vaccinium cespitosum phase

3. C. canadensis and C. stricta, singly or their combined cover poorly represented 4

4. Streptopus amplexifolius (twisted stalk), Senecio triangularis (arrowleaf groundsel), Mitella pentandra (five-stamened mitrewort) common, either individually or their combined cover (and not confined to microsites).

Abies lasiocarpa / Streptopus amplexifolius p. a.

a. Menziesia ferruginea (fool's huckleberry) well represented Menziesia ferruginea phase

b. M. ferruginea poorly represented. Streptopus amplexifolius phase

4. The above-listed forbs, alone or their combined cover, having less than $1 \%$ cover .... 5

5. Galium triflorum (sweetscented bedstraw) and Actaea rubra (baneberry) either singly or their combined canopy cover at least $1 \%$....

Abies lasiocarpa / Galium triflorum p. a.

5. G. triflorum and A. rubra, singly or combined cover, less than $1 \%$

6. Menziesia ferruginea (fool's huckleberry) well represented

6. M. ferruginea poorly represented.

Abies lasiocarpa / Menziesia ferruginea p.a.

7. Linnaea borealis (twinflower) common Abies lasiocarpa / Linnaea borealis p.a.

7. L. borealis scarce. 8

8. Vaccinium cespitosum (dwarf huckleberry) common .. Abies lasiocarpa / Vaccinium cespitosum p.a.

8. V. cespitosum scarce...... 9

9. Alnus viridis ssp. sinuata (Sitka alder) well represented

9. A. viridis ssp. sinuata poorly represented

Abies lasiocarpa / Alnus viridis ssp. sinuata p.a. 
10. Xerophyllum tenax (beargrass) common.

Abies lasiocarpa / Xerophyllum tenax p.a.

10. X. tenax scarce

11. Vaccinium membranaceum [big huckleberry, formerly $V$. globulare (globe huckleberry) in western Montana and northern Idaho] well represented. Abies lasiocarpa / Vaccinium globulare p.a.

11. V. membranaceum poorly represented

12. Vaccinium scoparium [grouse whortleberry, including V. myrtillus (dwarf bilberry)] well represented......

12. V. scoparium (and V. myrtillus) poorly represented Abies lasiocarpa / Vaccinium scoparium p.a.

13. Thalictrum occidentale (western meadowrue) or T. fendleri (Fendler's meadowrue) well represented.

13. T. occidentale poorly represented Abies lasiocarpa / Thalictrum occidentale p.a.

14. Clematis columbiana var. columbiana (formerly C. pseudoalpina, Rocky Mountain clematis) or C. columbiana var. tenuiloba (formerly $C$. tenuiloba, matted purple virgin's-bower) present or Pinus flexilis (limber pine) common (sites invariably with calcareous substrates)

Abies lasiocarpa / Clematis columbiana var. columbiana p.a.

14. C. columbiana var. columbiana and C. columbiana var. tenuiloba absent and $P$. flexilis scarce.......... 15

15. Calamagrostis rubescens (pine grass) well represented

15. C. rubescens poorly represented

Abies lasiocarpa / Calamagrostis rubescens p.a.

16. Carex geyeri (elk sedge) well represented

16. C. geyeri poorly represented Abies lasiocarpa / Carex geyeri p.a.

17. Arnica cordifolia (heartleaf arnica) undergrowth dominant.....Abies lasiocarpa / Arnica cordifolia p.a.

17. A. cordifolia not the undergrowth dominant Undefined plant associations within Abies lasiocarpa Series

18. Abies lasiocarpa (subalpine fir) and Picea (spruce) spp. scarce or, if common, then unthrifty specimens; Pinus albicaulis (whitebark pine) the indicated dominant tree at projected longterm stable state Pinus albicaulis Series

18. Not as above. 19

19. Timberline habitats; Abies lasiocarpa (subalpine fir) and Picea (spruce) spp. stunted, Pinus contorta (lodgepole pine) scarce, Menziesia ferruginea (fool's huckleberry) absent ....

Pinus albicaulis - Abies lasiocarpa p.a.

19. Not as above, forest environments with $A$. lasiocarpa at least $50 \mathrm{ft}$. at maturity. 20

20. Luzula glabrata var. hitchcockii (smooth woodrush, formerly L. hitchcocki) common

20. L. glabrata var. hitchcockii scarce, confined to snow deposition microsites

21. Vaccinium scoparium (grouse whortleberry, including V. myrtillus, dwarf bilberry) well represented; Pinus albicaulis (whitebark pine) persisting, even reproducing, well into longterm stable state due to open canopy nature of stand structure....

21. Not as above..... Abies lasiocarpa - Pinus albicaulis / Vaccinium scoparium p.a.

22. Ribes montigenum (mountain gooseberry) present and not relegated to microsites

Abies lasiocarpa / Ribes montigenum p.a.

22. R. montigenum absent. Undefined plant associations within Abies lasiocarpa Series 


\section{Cercocarpus ledifolius (curl-leaf mountain mahogany) Series Key to Plant Associations}

1. Festuca idahoensis (Idaho fescue) well represented or the dominant graminoid

1. F. idahoensis poorly represented and not the dominant graminoid Cercocarpus ledifolius / Festuca idahoensis p. a.

2. Pseudoroegneria spicata (bluebunch wheatgrass) or Oryzopsis hymenoides (Indian ricegrass), their individual or combined cover, constitute the dominant graminoids

Cercocarpus ledifolius / Pseudoroegneria spicata p. a.

2. P. spicata and O. hymenoides, individually or their combined cover, not the dominant graminoids Undefined Cercocarpus ledifolius-dominated plant association / community type

\section{Juniperus scopulorum (Rocky Mountain Juniper) Series Key to Plant Associations}

1. Cottonwood (Populus spp.) species well represented or Cornus sericea (red-osier dogwood), Poa pratensis (Kentucky bluegrass) or Agrostis stolonifera (redtop, also A. alba) singly or combined cover, common; riparian sites ............................... Juniperus scopulorum / Cornus stolonifera p. a.

1. Cottonwood (Populus spp.) species poorly represented and $C$. stolonifera, $P$. pratensis, and $A$. stolonifera , singly or their combined cover, scarce........................................................................ 2

2. Cercocarpus ledifolius (curl-leaf mountain mahogany) well represented

2. C. ledifolius poorly represented Juniperus scopulorum / Cercocarpus ledifolius p. a.

3. Artemisia nova (black sagebrush) well represented...... Juniperus scopulorum / Artemisia nova p. a.

3. A. nova poorly represented.

4. Artemisia tridentata (big sagebrush, usually subspecies vaseyana or tridentata) well represented

4. A. tridentata poorly represented Juniperus scopulorum / Artemisia tridentata p.a.

5. Pseudoroegneria spicata (formerly Agropyron spicatum, bluebunch wheatgrass) well represented or the dominant graminoid Juniperus scopulorum / Pseudoroegneria spicata p. a.

5. P. spicata poorly represented or not the dominant graminoid....

Undefined plant association(s) within the Juniperus scopulorum Series 


\section{Picea (spruce) spp. [predominantly $P$. engelmanni (Engelmann spruce) and $P$. engelmannii x $\boldsymbol{P}$. glauca (white spruce) hybrids] Series Key to plant associations}

1. Equisetum arvense (field horsetail) abundant

Picea spp. / Equisetum arvense p. a.

1. E. arvense not abundant.

2. Carex disperma (soft-leaved sedge) well represented

2. C. disperma poorly represented

Picea spp. / Carex disperma p. a.

3. Calamagrostis canadensis (bluejoint reedgrass), C. stricta (narrow-spiked reedgrass), or Senecio triangularis (arrowleaf groundsel) common, either alone or in combination

3. C. canadensis, C. stricta, and S. triangularis scarce.

Picea spp. / Calamagrostis canadensis p. a.

4. Cornus sericea (formerly C. stolonifera, red-osier dogwood) or Alnus incana (mountain alder) common.....

Picea spp. / Cornus sericea p. a.

4. C. sericea scarce

5. Two of the following moist-site forbs present or one or more common; Streptopus amplexifolius, (clasping-leaved twisted stalk), Galium triflorum (sweetscented bedstraw), Actaea rubra (baneberry) . . Picea spp. / Galium triflorum p. a.

5. Not as above.....

N.B. Sites above this level in the key are usually wetlands and/or associated with riparian features

4. Linnaea borealis (twinflower) common

Picea spp. / Linnaea borealis p. a.

4. L. borealis scarce.

5. Physocarpus malvaceus (mallow ninebark) well represented.

5. P. malvaceus poorly represented

Picea spp. / Physocarpus malvaceus p. a.

6. Maianthemum stellatum (formerly Smilacina stellata, starry Solomon's seal) or Thalictrum ccidentale (western meadowrue) common

Picea spp. / Maianthemum stellatum p.a.

6. M. stellatum and T. occidentale scarce

7. Senecio streptanthifolius (Rocky Mountain butterweed) present; undergrowth generally depauperate.

a. Pseudotsuga menziesii (Douglas-fir) common

Picea spp. / Senecio streptanthifolius p.a.

b. P. menziesii scarce, sites above its elevational limits

Pseudotsuga menziesii phase

7. S. streptanthifolius absent, undergrowth various

Picea spp. phase

Undefined p.a.'s. within Picea spp. Series 


\section{Pinus albicaulis (whitebark pine) Series Key to Plant Associations}

1. Vaccinium scoparium (grouse whortleberry) well represented (not necessarily dominant undergrowth species in the presence of high coverage's of various herbs, usually Arnica latifolia (broadleaf arnica) or A. cordifolia (heart-leaf arnica)

Pinus albicaulis / Vaccinium scoparium p. a.

1. V. scoparium poorly represented

2. Carex geyeri (elk sedge) well represented

2. C. geyeri poorly represented

Pinus albicaulis / Carex geyeri p.a.

3. Juniperus communis (common juniper), Astragalus miser (weedy milkvetch), or Shepherdia canadensis (Canada buffaloberry) well represented or dominant, either singly or collectively.....

3. Not as above.

Pinus albicaulis / Juniperus communis p.a.

4. Festuca idahoensis (Idaho fescue) common

Pinus albicaulis / Festuca idahoensis p.a.

4. F. idahoensis scarce Undefined p.a.'s./c.t.'s. within the Pinus albicaulis Series

\section{Pinus contorta (lodgepole pine) Series Key to Plant Associations}

1. Calamagrostis canadensis (bluejoint reedgrass) or C. stricta (narrow-spike reedgrass), singly or their combined cover, well represented

Pinus contorta / Calamagrostis canadensis c. t. (see a. below)

a. Vaccinium cespitosum (dwarf huckleberry) present having at least $1 \%$ canopy cover Vaccinium cespitosum phase

a. V. cespitosum scarce, having less than $1 \%$ Calamagrostis canadensis phase

1. C. canadensis and C. stricta, poorly represented, either individually or their combined cover 2

2. Vaccinium cespitosum (dwarf huckleberry) common ..... Pinus contorta / Vaccinium cespitosum c. t.

2. V. cespitosum scarce. 3

3. Linnaea borealis (twinflower) common Pinus contorta / Linnaea borealis p.a.

3. L. borealis scarce 4

4. Xerophyllum tenax (beargrass) well represented Pinus contorta / Xerophyllum tenax c. t.

4. X. tenax poorly represented.

5. Vacuum scoparium (grouse whortleberry) well represented

5. V. scoparium poorly represented.

Pinus contorta / Vaccinium scoparium c. t.

6 Thalictrum occidentale (western meadowrue) or T. fendleri (Fendler's meadow rue) well represented...

6. T. occidentale and T. fendleri poorly represented

Pinus contorta / Thalictrum occidentale c. t.

7. Calamagrostis rubescens (pine grass) well represented

7. C. rubescens poorly represented. Pinus contorta / Calamagrostis rubescens c. t.

8. Carex geyeri (elk sedge) well represented

8. C. geyeri poorly represented Pinus contorta / Carex geyeri p.a.

9. Purshia tridentata (antelope bitterbrush) common Pinus contorta / Purshia tridentata p. a. 
9. P. tridentata scarce

10.

10. Carex rossii (Ross sedge) well represented or the undergrowth dominant

10. C. rossii poorly represented or not the undergrowth dominant

Pinus contorta / Carex rossii p. a.

11. Arnica cordifolia (heartleaf arnica) or Astragalus miser (weedy milkvetch) well represented, either singly or their combined cover

Pinus contorta / Arnica cordifolia c. t.

11. A. cordifolia and A. miser poorly represented, alone or their combined cover

Undefined Pinus contorta-dominated type

\section{Pinus flexilis (limber pine) Series Key to Plant Associations}

1. Cercocarpus ledifolius (curl-leaf mountain mahogany) well represented

1. C. ledifolius poorly represented

Pinus flexilis / Cercocarpus ledifolius p.a.

2. Juniperus communis (common juniper) or J. horizontalis (creeping juniper) well represented

2. J. communis and J. horizontalis poorly represented

Pinus flexilis /Juniperus communis p.a.

3. Festuca campestris (formerly Festuca scabrella, rough fescue) common

3. F. campestris scarce Pinus flexilis / Festuca scabrella p. a.

4. Festuca idahoensis (Idaho fescue) well represented Pinus flexilis / Festuca idahoensis p.a.

4. F. idahoensis poorly represented 5

5. Pseudoroegneria spicata (formerly Agropyron spicatum, bluebunch wheatgrass) well represented Pinus flexilis / Agropyron spicatum p.a.

5. P. spicata poorly represented Undefined p.a.'s. within Pinus flexilis Series 


\section{Pseudotsuga menziesii (Douglas-fir) Series Key to plant associations}

1. Habitats on steep slopes (generally $>40 \%$ ) composed of unstable substrates, from gravel to coarse rock, and lacking soil development; undergrowth canopy cover sparse and spatially quite variable.

Pseudotsuga menziesii / Scree p. a.

1. Not as above; sites with some soil development due to stability of substrate; undergrowth cover more uniform reflecting lack of microsites.

2. Cornus sericea (red-osier dogwood), Salix spp. (willows, excepting S. scouleriana, Scouler willow), Actaea rubra (baneberry), Poa pratensis (Kentucky bluegrass) or Equisetum arvense (field horsetail) common, individually or their combined cover...............Pseudotsuga menziesii / Cornus sericea p.a.

2. Above listed species considered individually or their combined cover, scarce

3. Sites with at least one of the following wetland attributes present: hydrophytic vegetation, wetland hydrology, hydric soils.

Unclassified Wetland or Riparian Sites

3. Sites lacking any one of the above-listed wetland attributes

$4 \quad$ Vaccinium cespitosum (dwarf huckleberry) common

4. V. cespitosum scarce.

Pseudotsuga menziesii / Vaccinium cespitosum p.a.

5. Linnaea borealis (twinflower) common

Pseudotsuga menziesii / Linnaea borealis p.a.

5. L. borealis scarce

6. Physocarpus malvaceus (mallow ninebark) well represented.

6. P. malvaceus poorly represented

Pseudotsuga menziesii / Physocarpus malvaceus p.a.

7. Vaccinium membranaceum (big huckleberry, formerly V. globulare) well represented

7. V. membranaceum poorly represented

Pseudotsuga menziesii / Vaccinium membranaceum p.a.

8. Symphoricarpos albus (common snowberry) well represented.

8. S. albus poorly represented.....

Pseudotsuga menziesii / Symphoricarpos albus p.a.

9. Calamagrostis rubescens (pine grass) well represented

9. C. rubescens poorly represented.

Pseudotsuga menziesii / Calamagrostis rubescens p.a.

10. Carex geyeri (elk sedge) well represented

10. C. geyeri poorly represented

Pseudotsuga menziesii / Carex geyeri p.a.

11. Spiraea betulifolia (shiny-leaf spiraea) well represented

11. S. betulifolia poorly represented

Pseudotsuga menziesii / Spiraea betulifolia p.a.

12. Arctostaphylos uva-ursi (kinnikinnick) well represented

12. A. uva-ursi poorly represented.

Pseudotsuga menziesii / Arctostaphylos uva-ursi p.a.

13. Juniperus communis (common juniper) or Juniperus horizontalis (creeping juniper) the undergrowth dominant......

Pseudotsuga menziesii / Juniperus communis p.a. 
13. J. communis and J. horizontalis not the undergrowth dominants

14. Symphoricarpos oreophilus (mountain snowberry) well represented.

14. S. oreophilus poorly represented.

Pseudotsuga menziesii / Symphoricarpos oreophilus p.a.

15. Arnica cordifolia (heartleaf arnica) or Antennaria racemosa (raceme pussy-toes) the dominant undergrowth species.

Pseudotsuga menziesii / Arnica cordifolia p.a.

15. Neither $A$. cordifolia nor $A$. racemosa the dominant undergrowth species.

16. Cercocarpus ledifolius (curl-leaf mountain mahogany) or Juniperus scopulorum (Rocky Mountain juniper) or their combined cover well represented.....

16. C. ledifolius and J. scopulorum poorly represented.....

17. Cercocarpus ledifolius (curl-leaf mountain mahogany) having greater canopy cover than Juniperus scopulorum (Rocky Mountain juniper). .. Pseudotsuga menziesii / Cercocarpus ledifolius p.a.

17. C. ledifolius having less canopy cover than J. scopulorum

Pseudotsuga menziesii / Juniperus scopulorum p.a.

18. Festuca campestris (formerly Festuca scabrella, rough fescue) common

18. F. campestris scarce

Pseudotsuga menziesii / Festuca scabrella p.a.

19. Festuca idahoensis (Idaho fescue) common Pseudotsuga menziesii / Festuca idahoensis p.a.

19. F. idahoensis scarce

20. Pseudoroegneria spicata (formerly Agropyron spicatum, bluebunch wheatgrass) well represented . Pseudotsuga menziesii / Pseudoroegneria spicata p.a.

20. P. spicata poorly represented Undefined p.a. of the Pseudotsuga menziesii Series 


\title{
KEY TO DECIDUOUS FORESTS AND WOODLANDS INCLUDING UPLAND AND WETLAND TYPES
}

\author{
[based largely on Hansen et al. (1995) \& Mueggler (1988)]
}

1. Populus angustifolia (narrowleaf cottonwood) and $P$. balsamifera ssp. trichocarpa (formerly $P$. trichocarpa, black cottonwood) alone or their combined canopy cover, at least $25 \%$ or the dominant lifeform

1. P. angustifolia and $P$. balsamifera ssp. trichocarpa with $<25 \%$ canopy cover and not the dominant species, ............. P. tremuloides (trembling aspen) dominant and having at least $25 \%$ cover, singly or in combination with $P$. angustifolia or $P$. balsamifera

2. Populus balsamifera ssp. trichocarpa (black cottonwood) with greater canopy cover than $P$. angustifolia (narrowleaf cottonwood)

2. $P$. balsamifera ssp. trichocarpa having less canopy cover than $P$. angustifolia.

3. Site recently deposited alluvial bar or overflow reach with seedling and sapling size classes (dbh $<5.0$ in.) dominating .... Populus balsamifera ssp. trichocarpa / Recent Alluvial Bar p.a.

3. P. trichocarpa represented by pole or mature size classes....

4. Shrub species having at least $15 \%$ canopy cover or Cornus sericea (red-osier dogwood, formerly $C$. stolonifera) common

4. Shrub species having less than $15 \%$ canopy cover and Cornus sericea scarce.....

5. Cornus sericea (red-osier dogwood) common

P. balsamifera ssp. trichocarpa / Cornus sericea p. a.

5. C. sericea scarce; undergrowth may be dominated by any combination of Symphoricarpos albus (western snowberry), S. occidentalis (common snowberry), Rosa woodsii (woods rose), or Rosa acicularis (prickly rose)

P. balsamifera ssp. trichocarpa / Symphoricarpos occidentalis p. a.

6. Poa pratensis (Kentucky bluegrass), Poa palustris (fowl bluegrass), or Agrostis alba (redtop) abundant, or dominating the undergrowth........P. balsamifera ssp. trichocarpa / Poa pratensis p. a.

6. Not as above. Undefined p. a. within $P$. balsamifera ssp. trichocarpa Series

7. Populus angustifolia (narrowleaf cottonwood) seedling or sapling size classes (dbh $<5.0$ in.) dominate recently deposited alluvial bars. Populus angustifolia / Recent Alluvial Bar p.a.

7. $P$. angustifolia pole or mature size classes dominate the site, regardless of substrate 8

8. Shrub species having a single or combined canopy cover of $25 \%$ or Cornus sericea (red-osier dogwood) at least common.

8. Shrub species having less than $15 \%$ canopy cover

9. Cornus sericea (red-osier dogwood) common

Populus angustifolia / Cornus stolonifera p. a.

9. C. sericea scarce; undergrowth may be dominated by any combination of Symphoricarpos albus (western snowberry), S. occidentalis (common snowberry), Rosa woodsii (woods rose), or Rosa acicularis (prickly rose)

Populus angustifolia / Symphoricarpos occidentalis p. a.

10. Poa pratensis (Kentucky bluegrass), Poa palustris (fowl bluegrass), or Agrostis alba (redtop), singly or combined cover, abundant, or dominant

10. Not as above.

Populus angustifolia / Poa pratensis p. a.

11. Calamagrostis canadensis (bluejoint reedgrass) or C. stricta (narrow-spike reedgrass), singly or in combination, well represented ..................... Populus tremuloides / Calamagrostis canadensis p. a

11. C. canadensis or C. stricta, either singly or their combined canopy cover, poorly represented............ 12 
12. Salix (willow) spp., Cornus sericea (red-osier dogwood), Alnus incana (mountain alder) or Prunus virginiana (common chokecherry), individually or combined canopy cover, well represented......

Populus tremuloides / Cornus sericea p.a.

12. Salix spp., C. sericea, A. incana, and P. virginiana, individually or combined cover, poorly represented

13. Any two of the following species common (not confined to microsites) or any single one well represented; Angelica arguta (sharptooth angelica), Osmorhiza occidentalis (western sweet-cicely), Heracleum lanatum (cow parsnip), Actaea rubra (baneberry), or Galium triflorum (sweetscented bedstraw) Populus tremuloides / Osmorhiza occidentalis p. a.

13. Not as above; considered individually all of the above species poorly represented and as pairs not more than scarce.....

14. One or more members of the tall forb group prominent, their single or combined canopy cover at least 10\%; Agastache urticifolia (stinging nettle), Aster engelmannii (Engelmann's aster), Delphinium occidentale (western larkspur), Hackelia floribunda (showy stickseed), Heracleum lanatum (cow parsnip), Mertensia ciliata (mountain bluebell), Osmorhiza occidentalis (western sweet-cicely), Senecio serra (tall butterweed), Valeriana occidentalis (western valerian)

Populus tremuloides / tall forb p.a.

14. No single one of the above species or any combination thereof constituting $10 \%$ canopy cover... 15

15. Considered singly or in any combination, any of the following species having at least $10 \%$ canopy cover; Thalictrum fendleri (Fendler's meadowrue) T. occidentale (western meadowrue), Osmorhiza chilensis (western sweet-cicely), O. depauperata (blunt-fruit sweet-cicely), Geranium viscosissimum (sticky geranium), Populus tremuloides / Thalictrum fendleri p.a.

15. None of the above-listed species, singly or in any combination, totaling $10 \%$ cover 16

16. Calamagrostis rubescens (pine grass) or Carex geyeri dominant, their individual or combined cover, at least $10 \%$ Populus tremuloides / Calamagrostis rubescens p.a.

16. Neither C. rubescens nor C. geyeri nor their combined cover exceeding $10 \%$

17. Bromus carinatus (mountain brome), B. anomalus (nodding brome), Elymus glaucus (blue wildrye), Elymus trachycaulus (bearded wheatgrass), Stipa occidentalis (western needlegrass), alone or in any combination, comprising at least $10 \%$ canopy cover.....

Populus tremuloides / Bromus carinatus p.a.

17. Not as above; singly or in combination, the above species comprise less than $10 \%$ cover 18

18. Poa pratensis (Kentucky bluegrass) dominates the undergrowth

18. P. pratensis not the undergrowth dominant. Undefined p. a. within Populus tremuloides Series 


\section{Key to Shrubland Plant Associations INCLUDING UPLAND AND WETLAND TYPES}

[based largely on Hansen et al. 1995, Mueggler and Stewart 1980]

1. Sites with at least one of the following wetland attributes: hydrophytic vegetation, hydric soils, wetland hydrology .......... see "KeY to WeTLAND / RIPARIAN ShrubLAND CommunitieS" following lead 49

1. Sites lacking any of the wetland attributes listed above

2. Pentaphylloides floribunda (shrubby cinquefoil, formerly Potentilla fruticosa) well represented (often partially obscured by lush herbaceous vegetation; other shrubby taxa may be well represented as well)

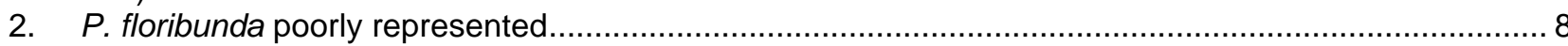

3. Carex utriculata (beaked sedge), C. atherodes (awned sedge), C. vesicaria (inflated sedge), $C$. aquatilis (water sedge), singly or combined cover at least $10 \%$

Pentaphylloides floribunda / Carex utriculata p. a.

3. C. utriculata, $C$. atherodes, $C$. vesicaria, and $C$. aquatilis poorly represented, singly or combined........ 4

4. Deschampsia cespitosa (tufted hairgrass) common, or only present as scattered individuals under intensive grazing regimes

Pentaphylloides floribunda / Deschampsia cespitosa p. a.

4. D. cespitosa scarce. 5

5. Festuca campestris (rough fescue, formerly F. scabrella) common, or only present as scattered individuals with intensive grazing......

Pentaphylloides floribunda / Festuca campestris p. a.

5. F. campestris scarce

6. Festuca idahoensis (Idaho fescue) well represented (only common with intensive grazing).....

Pentaphylloides floribunda / Festuca idahoensis p. a.

6. F. idahoensis poorly represented (or scarce under intensive grazing regime)

7. Potentilla ovina (sheep cinquefoil) common, the dominant of a depauperate undergrowth; substrates may be water-scoured and eroded

Pentaphylloides floribunda / Potentilla ovina p. a.

6. P. ovina scarce and not the undergrowth dominant

Undefined Pentaphylloides floribunda-dominated vegetation

8. Artemisia cana (silver sagebrush) having at least $10 \%$ canopy cover (other Artemisia taxa may be present)

8. A. cana having less than $10 \%$ cover. 14

9. Leymus cinereus (formerly Elymus cinereus, giant or basin wildrye) well represented (or common under intensive spring grazing regime)

Artemisia cana / Leymus cinereus p. a.

9. L. cinereus poorly represented 10

10. Poa secunda (Sandberg's bluegrass, only that portion of the taxon formerly known as Poa nevadensis [Nevada bluegrass] or $P$. juncifolia [alkali bluegrass]), or Carex praegracilis (clustered field sedge) common, singly or combined cover .... Artemisia cana / Poa secunda p.a.

10. P. nevadensis, $P$. juncifolia, and $C$. praegracilis scarce, individually or any combination. 11

11. Pascopyrum smithii (formerly Agropyron smithii, western wheatgrass) or Elymus lanceolatus (formerly A. dasystachyum, thick-spike wheatgrass) well represented....

Artemisia cana / Pascopyrum smithii p. a.

11. P. smithii and E. lanceolatus poorly represented 12 
12. Either Festuca idahoensis (Idaho fescue) or Elymus trachycaulus (bearded wheatgrass) well represented (only common under grazing).

a. F. idahoensis well represented see $a$ \& b below

b. F. idahoensis poorly represented and E. trachycaulus well represented.

. Elymus trachycaulus p. a.

12. F. idahoensis and E. trachycaulus poorly represented.......

13. Either Stipa comata (needle-and-thread) or Poa pratensis (Kentucky bluegrass) well represented

a. Stipa comata well represented and P. pratensis poorly represented

See a \& b below

....................................................................................... Artemisia cana / Stipa comata p.a.

b. S. comata poorly represented and $P$. pratensis well represented

Artemisia cana / Poa pratensis p.a.

13. S. comata and P. pratensis both poorly represented

Undefined p.a. within Artemisia cana alliance

14. Artemisia tridentata ssp. tridentata (Basin big sagebrush) either singly, or its combined cover with $A$. tridentata ssp. vaseyana (Vasey or mountain big sagebrush), well represented (other Artemisia taxa may be present) and Sarcobatus vermiculatus (black greasewood) scarce (should S. vermiculatus be common then lead \#72)

14. A. tridentata v. tridentata or its combined cover with $A$. tridentata ssp. vaseyana poorly represented and $S$. vermiculatus scarce.

15. Rhizomatous wheatgrasses, Elymus lanceolatus (thick-spike wheatgrass, formerly Agropyron dasystachyum) or Pascopyrum smithii (western wheatgrass, formerly A. smithii), common, individually or their combined cover....... Artemisia tridentata v. tridentata / Pascopyrum smithii p.a.

15. E. lanceolatus and Pascopyrum smithii or other wheatgrasses scarce

16. Festuca idahoensis (Idaho fescue) well represented (or only common if intensive grazing a factor)......

16. F. idahoensis poorly represented Artemisia tridentata v. tridentata / Festuca idahoensis p.a.

17. Stipa comata (needle-and-thread) or $S$. spartea (porcupine-grass) well represented

17. S. comata and S. spartea poorly represented Artemisia tridentata v. tridentata / Stipa comata p.a. Undefined p.a. within Artemisia tridentata v. tridentata Alliance

18. Artemisia tridentata ssp. vaseyana (mountain big sagebrush) well represented ................................ 19

18. A. tridentata ssp. vaseyana poorly represented ..................................................................... 27

19. Leymus cinereus (formerly Elymus cinereus, basin or giant wildrye) well represented (only common under intensive grazing regime)

19. L. cinereus poorly represented Artemisia tridentata ssp. vaseyana / Leymus cinereus p.a.

20. Festuca campestris (formerly Festuca scabrella, rough fescue) common (only as scattered plants with intensive grazing)

20. F. campestris scarce Artemisia tridentata ssp. vaseyana / Festuca scabrella p.a.

21. Symphoricarpos oreophilus (mountain snowberry) well represented.

21. S. oreophilus poorly represented

22. Bromus carinatus (mountain brome), B. anomalus (nodding brome) or $B$. inermis var. pumpellianus (pumpelly brome) well represented, individually or combined coverage.

Artemisia tridentata ssp. vaseyana - Symphoricarpos oreophilus / Bromus carinatus p. a.

22. B. carinatus, B. anomalus and B. inermis var. pumpellianus poorly represented, individually or their combined cover. 
23. Festuca idahoensis (Idaho fescue) well represented (only common under intensive grazing)

Artemisia tridentata ssp. vaseyana - Symphoricarpos oreophilus / Festuca idahoensis p. a.

23. F. idahoensis poorly represented

24. Pascopyrum smithii (western wheatgrass) or Elymus lanceolatus (thick-spike wheatgrass) well represented, individually or their combined coverage; Festuca idahoensis (Idaho fescue) poorly represented...................................... Artemisia tridentata ssp. vaseyana / Pascopyrum smithii p. a.

24. P. smithii and E. lanceolatus poorly represented, individually or combined........................................ 25

25. Festuca idahoensis (Idaho fescue) well represented (only common under intensive grazing regime)..... Artemisia tridentata ssp. vaseyana / Festuca idahoensis p.a. (see a-e below for proposed modifications of this associations, elevating phases to the p. a. level and defining new p. as.

25. F. idahoensis poorly represented . 26

a. Pascopyrum smithii (western wheatgrass) or Elymus lanceolatus (thick-spike wheatgrass) well represented ....................... A. tridentata ssp. vaseyana / F. idahoensis - Pascopyrum smithii p. a. b. Not as above; Stipa occidentalis (western needlegrass) or Stipa richardsonii (Richardson's needlegrass) common ........... A. tridentata ssp. vaseyana / F. idahoensis - Stipa occidentalis p. a. c. Not as above; Elymus trachycaulus (bearded wheatgrass), Bromus carinatus (mountain brome) or B. anomalus (nodding brome) common, individually or in any combination; or Geranium viscosissimum (sticky geranium), Potentilla glandulosa (sticky cinquefoil), or Potentilla gracilis (slender cinquefoil) present

A. tridentata ssp. vaseyana / F. idahoensis - Geranium viscosissimum p. a. d. Not as above, Pseudoroegneria spicata (bluebunch wheatgrass) well represented (reduce to common under intensive grazing regimes).

A. tridentata ssp. vaseyana / F. idahoensis - Pseudoroegneria spicata p. a. e. Not as above; $P$. spicata poorly represented...... undefined $\boldsymbol{A}$. tridentata ssp. vaseyana vegetation type

26. Pseudoroegneria spicata (formerly Agropyron spicatum, bluebunch wheatgrass) well represented (reduce to common under intensive grazing regimes)

Artemisia tridentata ssp. vaseyana / Pseudoroegneria spicata p.a.

26. P. spicata scarce Undefined/unrecorded Artemisia tridentata ssp. vaseyana vegetation type

27. Artemisia nova (black sagebrush) well represented (other Artemisia taxa may be present, even well represented).

27. A. nova poorly represented. 30

28. Festuca idahoensis (Idaho fescue) well represented Artemisia nova / Festuca idahoensis p. a.

28. F. idahoensis poorly represented 29

29. Pseudoroegneria spicata (bluebunch wheatgrass) or Oryzopsis hymenoides (Indian ricegrass) common, individually or their combined cover

Artemisia nova / Pseudoroegneria spicata p.a.

29. A. spicatum and $O$. hymenoides scarce..... Undefined p. a. within Artemisia nova Alliance

30. Cercocarpus ledifolius (curl-leaf mountain mahogany) well represented

30. C. ledifolius poorly represented (and by population structure not likely to increase)

31. Festuca idahoensis (Idaho fescue) well represented (only common if intensively grazed)

31. F. idahoensis poorly represented (or scarce with grazing). Cercocarpus ledifolius / Festuca idahoensis p.a.

32. Pseudoroegneria spicata (bluebunch wheatgrass) or Oryzopsis hymenoides (Indian ricegrass), individual or combined cover, the dominant graminoids

Cercocarpus ledifolius / Pseudoroegneria spicata p.a.

32. P. spicata and $O$. hymenoides, singly or combined cover, not the dominant graminoids Undefined p. a. within Cercocarpus ledifolius Alliance 
33. Artemisia tridentata ssp. wyomingensis (Wyoming big sagebrush) well represented

33. A. tridentata ssp. wyomingensis poorly represented.

34. Elymus lanceolatus (thick-spike wheatgrass) or Pascopyrum smithii (western wheatgrass), individually or their combined cover, common....

34. E. lanceolatus and P. smithii scarce Artemisia tridentata ssp. wyomingensis / Elymus lanceolatus p.a.

35. Pseudoroegneria spicata (bluebunch wheatgrass) well represented (only common with intensive grazing)

35. P. spicata (poorly represented) Artemisia tridentata ssp. wyomingensis / Pseudoroegneria spicata p.a. ............................................... Undefined p.a. within Artemisia tridentata ssp. wyomingensis Alliance

36. Artemisia tripartita (three-tip sagebrush) the dominant shrub spp. (Artemisia spp). 37

36. A. tripartita not the dominant shrub spp.

37. Festuca idahoensis (Idaho fescue) well represented (only common under intensive grazing)

37. F. idahoensis poorly represented Artemisia tripartita / Festuca idahoensis p. a.

38. Elymus lanceolatus (thick-spike wheatgrass, formerly Agropyron dasystachyum) or Pascopyrum smithii (western wheatgrass, formerly Agropyron smithii), individually or in combination, common and the dominant graminoids Artemisia tripartita / Elymus lanceolatus p. a.

38. E. lanceolatus and $P$. smithii, individually or their combined cover, poorly represented and neither being the dominant graminoids.

39. Pseudoroegneria spicata (bluebunch wheatgrass, formerly Agropyron spicatum) well represented (only common under intensive grazing) and, excepting Poa secunda (Sandberg's bluegrass), constitutes the dominant graminoid

Artemisia tripartita / Pseudoroegneria spicata p. a.

39. P. spicata poorly represented, not the dominant graminoid

Undefined p. a. within Artemisia tripartita Alliance

40. Rhus trilobata (skunk-bush sumac, formerly $R$. aromatica) well represented, as scattered patches, or the dominant shrub

40. R. trilobata poorly represented, not the dominant shrub

41. Festuca idahoensis (Idaho fescue) well represented or dominant graminoid

41. F. idahoensis poorly represented, not dominant graminoid

Rhus trilobata / Festuca idahoensis p.a.

42. Pseudoroegneria spicata (bluebunch wheatgrass) common or dominant graminoid

42. P. spicata scarce or not the dominant graminoid

Rhus trilobata / Pseudoroegneria spicata p.a.

Undefined p. a. within Rhus aromatica Alliance

43. Chrysothamnus viscidiflorus (green rabbitbrush) well represented or the dominant shrub

43. C. viscidiflorus poorly represented or not the dominant shrub

44. Stipa comata (needle-and-thread) common or the dominant herbaceous species

44. S. comata scarce, not the dominant herbaceous species

Chrysothamnus viscidiflorus / Stipa comata p. a. Undefined Chrysothamnus viscidiflorus-dominated vegetation type

45. Sarcobatus vermiculatus (black greasewood) well represented (valley locations with saline or alkaline soils) 46

45. S. vermiculatus poorly represented 49 
46. Distichlis spicata (formerly D. stricta, inland saltgrass) well represented (usually alkali flat conditions prevail, for example salt efflorescence) ................ Sarcobatus vermiculatus / Distichlis spicata p. a.

46. D. spicata poorly represented, habitat conditions various .......................................................... 47

47. Leymus cinereus (formerly Elymus cinereus, basin or giant wildrye) common

47. L. cinereus scarce, not the dominant graminoid. Sarcobatus vermiculatus / Leymus cinereus p.a.

48. Pascopyrum smithii (western wheatgrass) or Elymus lanceolatus (thick-spike wheatgrass), individually or their combined cover, the dominant component of herbaceous vegetation

Sarcobatus vermiculatus / Pascopyrum smithii p. a.

48. P. smithii not the dominant herb Undefined/unrecorded p.a./c.t. of the Sarcobatus vermiculatus alliance

49. Undefined/unreported shrubland type(s) or see "Key to DwarF-Shrubland PLant Associations, Including Upland and WetLand Types" or go to next lead "Key to "WetLand / Riparian Shrubland Communities"

\section{Key to WetLand / Riparian Shrubland Communities}

(see Alpine Key for high subalpine or alpine habitats where dwarf or prostrate shrubs [for example Salix spp.] are well represented )

1. Salix spp. (willow species) with at least $10 \%$ canopy cover 2

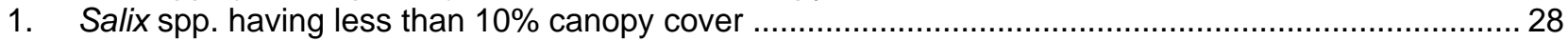

2. Salix geyeriana (Geyer willow), S. boothii (Booth willow), S. drummondiana (Drummond willow) or $S$. lutea (yellow willow) with at least $10 \%$ canopy cover, individually or combined

2. Above listed willow species, individually or combined, less than $10 \%$ canopy cover

3. Salix lutea (yellow willow) with greater canopy cover than $S$. drummondiana (Drummond willow) or the combined cover of $S$. geyeriana (Geyer willow) and S. boothii (Booth willow)

3. S. lutea having less canopy cover than $S$. drummondiana or combined cover of $S$. geyeri and S. boothii

4. Carex utriculata (beaked sedge), C. vesicaria (inflated sedge), $C$. atherodes (awned sedge), $C$. aquatilis (water sedge) or $C$. lenticularis (lentil-fruit sedge), individually or in any combination, well represented (5\% or greater canopy cover)....

Salix lutea / Carex utriculata p. a.

4. The above listed Carex spp., either individually or in any combination, having less than $5 \%$ cover ...... 5

5. Calamagrostis canadensis (bluejoint reedgrass), C. stricta (narrow-spiked reedgrass) or Deschampsia cespitosa (tufted hairgrass), individually or in combination, having at least $5 \%$ canopy cover Salix lutea / Calamagrostis canadensis p.a.

5. C. canadensis, C. stricta and D. cespitosa individually or their combined cover less than $10 \%$. Salix lutea Plant Association (a default community)

6. Salix drummondiana (Drummond willow) with a greater canopy cover than the individual or combined cover of S. boothii and S. geyeriana.

6. S. drummondiana with less cover than the combined or individual cover of $S$. boothii and S. geyeriana

7. Carex utriculata (beaked sedge), C. vesicaria (inflated sedge), C. atherodes (awned sedge), C. aquatilis (water sedge) or $C$. lenticularis (lentil-fruit sedge), individually or in any combination, well represented (5\% canopy cover)..................................... Salix drummondiana / Carex utriculata p. a.

7. The above listed Carex spp., either individually or in any combination, having less than $5 \%$ cover ...... 8

8. Calamagrostis canadensis (bluejoint reedgrass), C. stricta (narrow-spiked reedgrass) or Deschampsia cespitosa (tufted hairgrass), individually or in combination, having at least $5 \%$ canopy cover

Salix drummondiana / Calamagrostis canadensis p.a. 
8. Not as above; the above listed species, either singly or their combined canopy cover less than $5 \%$....... Salix drummondiana Plant Association (a default community)

9. Salix boothii (Booth willow) having at least as much canopy cover as S. geyeriana (Geyer willow) .... 10

9. S. boothii having less canopy cover than S. geyeriana .................................................................. 12

10. Carex utriculata (beaked sedge), C. vesicaria (inflated sedge), C. atherodes (awned sedge), C. aquatilis (water sedge) or $C$. lenticularis (lentil-fruit sedge), individually or in any combination, well represented ( $5 \%$ canopy cover)

Salix boothii / Carex utriculata p. a.

10. None of the above listed Carex spp., either individually or in any combination, exceeding $5 \%$ cover.. 11

11. Calamagrostis canadensis (bluejoint reedgrass), C. stricta (narrow-spiked reedgrass) or Deschampsia cespitosa (tufted hairgrass), individually or in combination, having at least $5 \%$ canopy cover

Salix boothii / Calamagrostis canadensis p.a.

11. Not as above; the above listed species, either singly or their combined canopy cover less than $5 \%$.......

Undefined Salix boothii-dominated p.a.

12. Carex utriculata (beaked sedge), C. vesicaria (inflated sedge), C. atherodes (awned sedge), C. aquatilis (water sedge) or $C$. lenticularis (lentil-fruit sedge), individually or in any combination, well represented (5\% or greater canopy cover)

Salix geyeriana / Carex utriculata p. a.

12. All of the above-listed Carex spp. with individual or combined cover less than $5 \%$ 13.

13. Calamagrostis canadensis (bluejoint reedgrass) or $C$. stricta (narrow-spiked reedgrass) well represented ( $5 \%$ or greater canopy cover), either individually or in combination

Salix geyeriana / Calamagrostis canadensis p.

13. Not as above; the above listed species, either singly or in combination, poorly represented (canopy cover less than $5 \%$ )

14. Deschampsia cespitosa (tufted hairgrass) well represented ( $>5 \%$ canopy cover); if site grazed intensively $D$. cespitosa need only be common (greater than $1 \%$ )

14. D. cespitosa poorly represented (<5\% canopy cover) Salix geyeriana / Deschampsia cespitosa p. a.

15. Considered singly or in any combination, the following primarily disturbance associated species, dominate the herbaceous layer; Poa pratensis (Kentucky bluegrass), P. palustris (fowl bluegrass), Phleum pratense (timothy), Agrostis stolonifera (redtop), or Juncus balticus (Baltic rush)

Salix geyeriana / Mesic graminoids p. a.

15. None of the above-listed species, singly or in any combination dominate the herbaceous layer Undefined Salix geyeriana-dominated p. a.

16. Canopy cover of Salix planifolia (planeleaf willow) or S. commutata (undergreen willow), or their combined cover, is at least $10 \%$.....

16. S. planifolia or S. commutata, their individual or combined cover, is less than $10 \%$.

17. Carex aquatilis (water sedge) or C. utriculata (beaked sedge) well represented, individually or in combination Salix planifolia / Carex aquatilis p.a.

17. C. aquatilis and C. utriculata poorly represented

18. Carex nebrascensis (Nebraska sedge) or C. simulata (short-beaked sedge) well represented, either individually or in combination. Salix planifolia / Carex nebrascensis p.a.

18. C. nebrascensis and C. simulata poorly represented, individually or in combination Undefined p. a. within Salix planifolia or S. commutata alliances

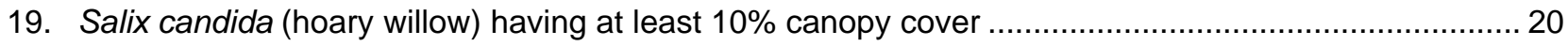

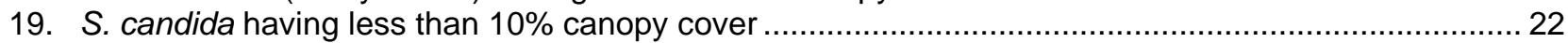


20. Carex utriculata (beaked sedge) or $C$. aquatilis (water sedge), or any combination of the two, well represented

20. C. rostrata and C. aquatilis poorly represented, individually or in combination.

21. Carex lasiocarpa (slender sedge), C. lanuginosa (woolly sedge), or C. buxbaumii (Buxbaum's sedge) well represented, individually or in any combination .... Salix candida / Carex lasiocarpa p. a.

21. Not as above; above listed sedges poorly represented, individually or in any combination Undefined p. as. within Salix candida alliance

22. Salix wolfii (Wolf's willow) having at least $10 \%$ canopy cover 23

22. S. wolfii with less than $10 \%$ canopy cover..... 25

23. Carex utriculata (beaked sedge), C. vesicaria (inflated sedge), C. atherodes (awned sedge), C. aquatilis (water sedge) or $C$. lenticularis (lentil-fruit sedge), considered singly or in any combination, well represented. Salix wolfii / Carex utriculata p.a.

23. Any of the above-listed species, considered singly or in any combination, poorly represented ........... 24

24. Deschampsia cespitosa (tufted hairgrass) or Juncus balticus (Baltic rush) or their combined cover well represented; accept $D$. cespitosa as common (1\% or greater canopy cover) where grazing is intensive

Salix wolfii / Deschampsia cespitosa p.a.

24. D. cespitosa or J. balticus or their combined cover poorly represented.

Undefined p. a. within Salix wolfii alliance

25. Individual non-Salix (non-willow) species having a greater canopy cover than any individual Salix species

25. Individual Salix species with greater canopy than any individual non-Salix species

26. Salix bebbiana (Bebb willow) with greater canopy cover than any other Salix species

26 S. bebbiana having less canopy cover than any other Salix species.

Salix bebbiana p.a.

27. Salix exigua (sandbar willow) having greater canopy cover than any other Salix species

27. S. exigua having less canopy cover than any other individual Salix species

Salix exigua p. a. Undefined Salix spp.-dominated riparian-wetland site type

28. Betula nana (formerly B. glandulosa, bog birch) having at least $10 \%$ canopy cover 29

28. B. nana having less than $10 \%$ canopy cover.

29. Carex utriculata (beaked sedge), C. vesicaria (inflated sedge), C. atherodes (awned sedge), C. aquatilis (water sedge) or $C$. lenticularis (lentil-fruit sedge), individually or in any combination, well represented ( $5 \%$ canopy cover).

Betula nana / Carex utriculata p.a.

29. All of the above-listed Carex spp. with individual or combined canopy covers less than $5 \%$ Undefined p. a. within $B$. nana alliance

30. Kalmia microphylla (small-leaved laurel) having at least $10 \%$ canopy cover 31

30. K. microphylla having less than $10 \%$ canopy cover 32

31. Carex scopulorum (Holm's Rocky Mountain Sedge) or C. nigricans (black alpine sedge) or their combined cover well represented

Kalmia microphylla/Carex scopulorum p.a.

31. C. scopulorum or C. nigricans or their combined cover poorly represented

Undefined $K$. microphylla-dominated p. a.

32. Betula occidentalis (water or river birch) having at least $10 \%$ canopy cover and with the greatest cover of the tallest vegetation layer. Betula occidentalis p. a.

32. B. occidentalis having less than $10 \%$ canopy cover. 33 
33. Alnus incana (mountain alder) having at least $10 \%$ canopy cover and with greatest canopy cover in tallest layer Alnus incana p. a.

33. A. incana having less than $10 \%$ canopy cover or not having the greatest canopy cover of the tallest layer

34. Pentaphylloides floribunda (shrubby cinquefoil, formerly Potentilla fruticosa) having at least $10 \%$ canopy cover....

34. P. floribunda having less than $10 \%$ canopy cover

35. Carex utriculata (beaked sedge), C. atherodes (awned sedge), C. vesicaria (inflated sedge), $C$. aquatilis (water sedge), individually or in any combination, having at least $10 \%$ canopy cover

Pentaphylloides floribunda / Carex utriculata p. a.

35. C. utriculata, C. atherodes, C. vesicaria, and C. aquatilis poorly represented, singly or combined...... 36

36. Deschampsia cespitosa (tufted hairgrass) or Juncus balticus (Baltic rush) or their combined cover well represented; under intensive grazing $D$. cespitosa may be only common

Pentaphylloides floribunda / Deschampsia cespitosa p. a.

36. D. cespitosa and $J$. balticus poorly represented...

Undefined p. a. within Pentaphylloides floribunda alliance

37. Artemisia cana (silver sagebrush) well represented 38

37. A. cana poorly represented. 42

38. Leymus cinereus (formerly Elymus cinereus, giant or basin wildrye) well represented (or merely common under intensive spring grazing regime).

Artemisia cana / Leymus cinereus p. a.

38. L. cinereus poorly represented

39. Poa secunda (Sandberg's bluegrass, considering only that portion of the taxon formerly known as Poa nevadensis [Nevada bluegrass] or $P$. juncifolia [alkali bluegrass]), or Carex praegracilis (clustered field sedge) common, singly or their combined cover ... Artemisia cana / Poa secunda p.a.

39. P. secunda and C. praegracilis scarce, individually or any combination.

40. Pascopyrum smithii (formerly Agropyron smithii, western wheatgrass) or Elymus lanceolatus (formerly $A$. dasystachyum, thick-spike wheatgrass) well represented

40. P. smithii and E. lanceolatus poorly represented Artemisia cana / Pascopyrum smithii p. a.

41. Either Festuca idahoensis (Idaho fescue) or Elymus trachycaulus (bearded wheatgrass) well represented (reduce to only common under intensive grazing) see a \& b below a. F. idahoensis well represented Artemisia cana / Festuca idahoensis p.a.

b. F. idahoensis poorly represented and E. trachycaulus well represented.... Artemisia cana / Elymus trachycaulus p. a.

41. F. idahoensis and E. trachycaulus poorly represented Undefined p. a. within Artemisia cana alliance

42. Sarcobatus vermiculatus (black greasewood) well represented or if vegetation depauperate then the shrub layer dominant; $S$. vermiculatus often not shrub layer dominant (expect Artemisia or Chrysothamnus spp.).

42. S. vermiculatus poorly represented or, if depauperate conditions obtain, then not the shrub layer dominant

43. Distichlis spicata (inland saltgrass, formerly Distichlis stricta) well represented (alkali flats conditions prevail) Sarcobatus vermiculatus / Distichlis stricta p.a.

43. D. stricta poorly represented. 44 
44. Leymus cinereus (formerly Elymus cinereus, basin wildrye) well represented (reduced to only common under intensive grazing) Sarcobatus vermiculatus / Elymus cinereus p.a.

44. L. cinereus poorly represented

45. Pascopyrum smithii (syn. Agropyron smithii, western wheatgrass) or Elymus lanceolatus (syn. A. dasystachyum, thickspike wheatgrass) the dominant component of herbaceous layer

Sarcobatus vermiculatus / Pascopyrum smithii p.a.

45. P. smithii and E. lanceolatus not the dominant undergrowth component

Undescribed Sarcobatus vermiculatus p.a.

46. Betula occidentalis (water birch) having at least $15 \%$ canopy cover and constituting the greatest canopy cover in the tallest layer

Betula occidentalis p. a.

46. B. occidentalis having less than $15 \%$ canopy cover or not having the greatest canopy cover within the tallest stratum

47. Alnus incana (mountain alder) having at least $15 \%$ canopy cover and having the greatest cover of the tallest layer present

Alnus incana p. a.

47. A. incana having less than $15 \%$ canopy cover or without the greatest canopy cover in the tallest stratum

48. Shepherdia argentea (thorny buffaloberry) having at least $15 \%$ canopy cover and comprising the greatest canopy cover of the tallest stratum Shepherdia argentea p. a.

48. S. argentea having less than $15 \%$ cover or not exhibiting the greatest canopy cover among species of the uppermost stratum

49. Rosa woodsii (woods rose) or R. acicularis (prickly rose) or any combination of the two having at least $15 \%$ canopy cover and they comprise greatest cover in the tallest vegetation layer.....

Rosa woodsii p. a.

49. R. woodsii and R. acicularis, or any combination of the two having less than $15 \%$ cover or not comprising the greatest canopy cover in the uppermost vegetation layer

50. Symphoricarpos occidentalis (western snowberry) or $S$. albus (common snowberry), their individual or combined cover greater than $15 \%$ and having the greatest canopy cover in the tallest layer......

Symphoricarpos occidentalis p.a.

50. S. occidentalis and S. albus or their combined cover less than $15 \%$ or not comprising the greatest canopy cover in the tallest vegetation layer.

51. Site having at least one of the following wetland criteria present: hydrophytic vegetation, hydric soils, wetland hydrology..... Undefined riparian-wetland p.a.

51. Site not exhibiting any of the above-listed wetland criteria. Upland Site 


\section{Key to Dwarf-Shrubland Plant Associations INCLUDING UPLAND AND WETLAND TYPES}

[Dwarf-shrubs (as individuals or clumps with a potential height less than $0.5 \mathrm{~m}$ ) generally forming $25 \%$ or greater canopy cover (taxa include Artemisia arbuscula (low sagebrush), A. arbuscula ssp. longiloba (early low sagebrush), A. nova (black sagebrush), A. pedatifida (birdfoot sagebrush), A. tridentata ssp. wyomingensis (Wyoming big sagebrush), A. tripartita (three-tip sagebrush), Atriplex gardneri (Gardner's saltbush), Cassiope mertensiana (Mertens' mountain heather), Dryas octopetala (white dryas), Kalmia microphylla (small-leaved laurel), Phyllodoce empetriformis (red mountain-heath), P. glanduliflora (yellow mountain-heath), Pentaphylloides floribunda (shrubby cinquefoil), Salix arctica (alpine willow), S. candida (hoary willow), S. barratiana (Barratt willow), S. brachycarpa (short-fruited willow), S. glauca (glaucous willow), S. planifolia var. monica (dwarf planeleaf willow), S. reticulata (snow willow)); though an uncommon condition, dwarf-shrub cover may be less than $25 \%$ but exceeding the combined cover of the other lifeforms present (shrubs, herbs, nonvasculars) which is less than $25 \%$ ]

1. Salix spp. (willow species) with at least $10 \%$ canopy cover ........................................................ 2

1. Salix spp. having less than $10 \%$ canopy cover ........................................................................ 14

2. Salix planifolia var. monica (dwarf planeleaf willow) the dominant Salix spp..................................... 3

2. S. planifolia var. monica not the dominant Salix spp................................................................. 4

3. Carex scopulorum (Holm's Rocky Mountain sedge) well represented and dominating the herbaceous layer (though Deschampsia cespitosa (tufted hairgrass) attains this status as well) .....

Salix planifolia var. monica / Carex scopulorum p. a.

3. C. scopulorum poorly represented and not the herbaceous layer dominant.

Undefined Salix planifolia var. monica-dominated vegetation type

4. Salix candida (hoary willow) the dominant Salix spp................................................................ 5

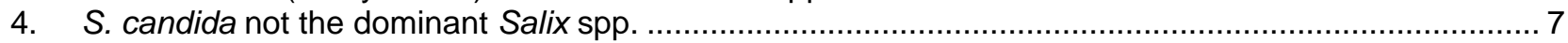

5. Carex utriculata (beaked sedge), C. atherodes (awned sedge), C. vesicaria (inflated sedge), $C$. aquatilis (water sedge) well represented (cover $\geq 5 \%$ )............... Salix candida / Carex utriculata p. a.

5. None of the above-listed species well represented, individually or in combination............................... 7

6. Carex lasiocarpa (slender sedge), C. buxbaumii (Buxbaum's sedge), or C. lanuginosa (wooly sedge) well represented (cover $\geq 5 \%$ ), singly or combined

Salix candida / Carex lasiocarpa p. a.

6. C. lasiocarpa, C. buxbaumii, C. lanuginosa poorly represented, singly or combined cover Undefined Salix candida-dominated vegetation type

7. Salix brachycarpa (short-fruited willow) the dominant Salix spp. ................................................. 8

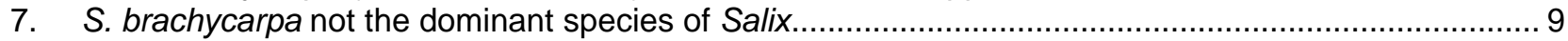

8. Carex utriculata (beaked sedge) or $C$. aquatilis (water sedge) well represented, singly or combined cover ................................................ Salix brachycarpa / Carex utriculata - Carex aquatilis p. a.

8. S. brachycarpa not the dominant Salix spp........ Undefined Salix brachycarpa-dominated vegetation type

9. Salix reticulata (snow willow) the dominant Salix spp; Dryas octopetala (white dryas) not abundant .. 10

9. S. reticulata not the dominant Salix spp; D. octopetala may be abundant ...................................... 11

10. Caltha leptosepala (elkslip marsh-marigold) present (often abundant), but not necessarily dominating a diverse forb layer Salix reticulata / Caltha leptosepala p. a.

10. C. leptosepala not present .................................... Undefined Salix reticulata-dominated vegetation type

11. Salix arctica (Arctic willow) the dominant Salix spp..................................................................... 12

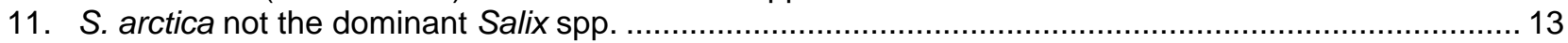


12. Polygonum bistortoides (American bistort) or Polygonum viviparum (alpine bistort), singly or combined cover, well represented, though somewhat ephemeral and diminutive often contributing the most forb cover

Salix arctica / Polygonum bistortoides p. a.

12. P. bistortoides and $P$. viviparum poorly represented

Undefined S. arctica-dominated vegetation type

13. Salix glauca (glaucous willow) the dominant Salix spp., undergrowth variable Salix glauca p. a.

13. S. glauca not dominating the shrub layer Undefined Salix-dominated vegetation type

14. Kalmia microphylla (small-leaved laurel) having at least $10 \%$ canopy cover 15

14. K. microphylla having less than $10 \%$ canopy cover 16

15. Carex scopulorum (Holm's Rocky Mountain sedge) or Carex nigricans (black alpine sedge) well represented, singly or in combination Kalmia microphylla / Carex scopulorum p. a.

15. C. scopulorum and $C$. nigricans poorly represented, singly or in combination Undefined Kalmia microphylla vegetation type

16. Phyllodoce empetriformis (red mountain-heath) or $P$. glanduliflora (yellow mountain-heath) dominating the shrub layer, singly or in concert, their canopy cover generally abundant 17

16. P. empetriformis and $P$. glanduliflora not the shrub-layer dominants, not abundant.

17. Antennaria lanata (woolly pussy-toes) common.. Phyllodoce empetriformis / Antennaria lanata p. a.

17. A. lanata scarce Undefined Phyllodoce empetriformis-dominated vegetation type

18. Cassiope mertensiana (Mertens' mountain heather) dominating the shrub layer..... 19

18. C. mertensiana not the shrub-layer dominant

19. Carex paysonis (Payson sedge) common Cassiope mertensiana / Carex paysonis p. a.

19. C. paysonis scarce. Undefined Cassiope mertensiana vegetation type

20. Dryas octopetala (white dryas) as the shrub-layer dominant, generally abundant 21

20. D. octopetala not the shrub-layer dominant.

21. Relatively mesic sites of protected slopes giving the impression of nearly total vegetation coverage (turf sites); Polygonum viviparum (alpine bistort), P. bistortoides (American bistort), Zigadenus elegans (glaucous death-camas) and Oxytropis viscida (sticky crazyweed) common, either singly or in any combination..... Dryas octopetala / Polygonum viviparum p. a.

21. Not as above, $P$. viviparum, $P$. bistortoides, $Z$. elegans, and $O$. viscida scarce; vegetation coverage various but not sparse.

22. Sparsely vegetated sites of exposed positions (ridgetops, shoulders, saddles, etc.) with Dryas octopetala occurring in distinct clumps of highly variable coverage, usually surrounded by bare ground or gravel (rock pavement); commonly occurring forb-layer components include Phlox pulvinata (cushion phlox), Oxytropis campestris (slender crazyweed), Minuartia obtusiloba (Arctic sandwort), Douglasia montana (Rocky Mountain Douglasia); Carex rupestris (curly sedge) and C. elynoides (Kobresia-like sedge) dominate the sparse graminoid layer.....

Dryas octopetala / Carex rupestris p. a.

22. Not as above. Undefined Dryas octopetala-dominated vegetation type

23. Any of the following species well represented or constituting the dwarf-shrub layer dominant, Atriplex gardneri (Gardner's saltsage, formerly A. nuttallii), Artemisia arbuscula (low sagebrush), A. arbuscula ssp. longiloba (early low sagebrush), A. nova (black sagebrush), A. pedatifida (birdfoot sagebrush), A. tridentata ssp. wyomingensis (Wyoming big sagebrush), A. tripartita (three-tip sagebrush)

23. Not as above; none of the named species well represented or constituting dwarf-shrub layer dominant 
24. Pentaphylloides floribunda (shrubby cinquefoil, formerly Potentilla fruticosa) well represented (often partially obscured by lush herbaceous vegetation; other shrubby taxa may be well represented as well)......

24. P. floribunda poorly represented

Undefined dwarf-shrub vegetation type

25. Carex utriculata (beaked sedge), C. atherodes (awned sedge), C. vesicaria (inflated sedge), $C$. aquatilis (water sedge), singly or combined cover at least $10 \%$

Pentaphylloides floribunda / Carex utriculata p. a.

25. C. utriculata, C. atherodes, C. vesicaria, and C. aquatilis poorly represented, singly or combined...... 26

26. Deschampsia cespitosa (tufted hairgrass) common, or only present as scattered individuals under intensive grazing regimes

Pentaphylloides floribunda / Deschampsia cespitosa p. a.

26. D. cespitosa scarce

27. Juncus balticus (Baltic rush) well represented and dominating the herbaceous layer

Pentaphylloides floribunda / Juncus balticus p. a.

27. J. balticus poorly represented and not the herbaceous layer dominant

28. Festuca campestris (rough fescue, formerly F. scabrella) common, or only present as scattered individuals with intensive grazing...................Pentaphylloides floribunda / Festuca campestris p. a.

28. F. campestris scarce 29

29. Festuca idahoensis (Idaho fescue) well represented (only common with intensive grazing)..... ........................................................... Pentaphylloides floribunda / Festuca idahoensis p. a.

29. F. idahoensis poorly represented (or scarce under intensive grazing regime) 30

30. Potentilla ovina (sheep cinquefoil) common, the undergrowth dominant of a depauperate undergrowth; substrates may be water-scoured and eroded

30. P. ovina scarce and not the undergrowth dominant

Pentaphylloides floribunda / Potentilla ovina p. a. Undefined Pentaphylloides floribunda-dominated vegetation

31. Atriplex gardneri (Gardner's saltsage, formerly A. nuttallii) well represented or the dwarf-shrub layer dominant.....

31. A. gardneri poorly represented and not the dwarf-shrub layer dominant

32. Oryzopsis hymenoides (Indian ricegrass) common or the herbaceous layer dominant

Atriplex gardneri / Oryzopsis hymenoides p. a.

32. O. hymenoides scarce and not the herbaceous layer dominant Undefined Atriplex gardneri vegetation type

33. Artemisia pedatifida (birdsfoot sagebrush) well represented or the dwarf-shrub layer dominant ......... 34

33. A. pedatifida poorly represented and not the dwarf-shrub layer dominant. 35

34. Festuca idahoensis (Idaho fescue) common

34. F. idahoensis scarce

Artemisia pedatifida / Festuca idahoensis p. a. Undefined Artemisia pedatifida vegetation type

35. Artemisia arbuscula (low sagebrush) or A. arbuscula ssp. longiloba (early low sagebrush) well represented or the dwarf-shrub layer dominants

35. A. arbuscula and A. arbuscula ssp. longiloba poorly represented or not the dwarf-shrub layer dominants

36. A. arbuscula ssp. longiloba (early low sagebrush) well represented and dominates the dwarf-shrub layer

36. A. arbuscula ssp. longiloba poorly represented and not the dwarf-shrub layer dominant......... 39 
37. Festuca idahoensis (Idaho fescue) well represented (only common, if grazing intensity is high). Artemisia arbuscula ssp. longiloba / Festuca idahoensis p. a.

37. F. idahoensis poorly represented (or scarce in the presence of intensive grazing 38

38. Elymus lanceolatus (thick-spike wheatgrass, formerly Agropyron dasystachyum) or Pascopyrum smithii (western wheatgrass, formerly Agropyron smithii), individually or in combination, well represented and the dominant graminoids

Artemisia arbuscula ssp. longiloba / Elymus lanceolatus p. a.

38. E. lanceolatus and $P$. smithii, individually or their combined cover, poorly represented and not the dominant graminoids. undefined Artemisia arbuscula ssp. longiloba vegetation type

39. Festuca idahoensis (Idaho fescue) well represented (may be only common with intensive grazing).......

39. F. idahoensis poorly represented Artemisia arbuscula / Festuca idahoensis p. a.

40. Pseudoroegneria spicata (bluebunch wheatgrass, formerly Agropyron spicatum) well represented ........ Artemisia arbuscula / Pseudoroegneria spicata p. a.

40. P. spicata poorly represented Undefined Artemisia arbuscula vegetation type

41. Artemisia tridentata ssp. wyomingensis (Wyoming big sagebrush) well represented and the dwarf-

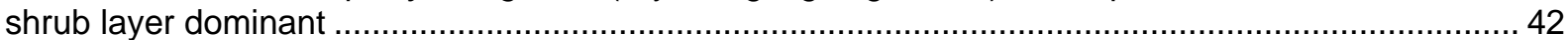

41. A. tridentata ssp. wyomingensis poorly represented or not the dwarf-shrub layer dominant............... 44

42. Elymus lanceolatus (thick-spike wheatgrass, formerly Agropyron dasystachyum) or Pascopyrum smithii (western wheatgrass, formerly Agropyron smithii), individually or in combination, common and the dominant graminoids ......... Artemisia tridentata ssp. wyomingensis / Pascopyrum smithii p. a.

42. E. lanceolatus and $P$. smithii, individually or their combined cover, poorly represented and not the dominant graminoids

43. Pseudoroegneria spicata (bluebunch wheatgrass, formerly Agropyron spicatum) well represented (only common under intensive grazing) and, excepting Poa secunda (Sandberg's bluegrass) the dominant graminoid ..........Artemisia tridentata ssp. wyomingensis / Pseudoroegneria spicata p. a.

43. P. spicata poorly represented, not the dominant graminoid

Undefined Artemisia tridentata ssp. wyomingensis vegetation type

44. Artemisia tripartita (three-tip sagebrush) the dominant dwarf-shrub ............................................. 45

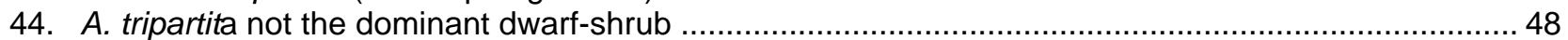

45. Festuca idahoensis (Idaho fescue) well represented (only common under intensive grazing)

45. F. idahoensis poorly represented Artemisia tripartita / Festuca idahoensis p. a.

46. Elymus lanceolatus (thick-spike wheatgrass, formerly Agropyron dasystachyum) or Pascopyrum smithii (western wheatgrass, formerly Agropyron smithil), individually or in combination, common and the dominant graminoids Artemisia tripartita / Elymus lanceolatus p. a.

46. E. lanceolatus and $P$. smithii, individually or their combined cover, poorly represented and not the dominant graminoids

47. Pseudoroegneria spicata (bluebunch wheatgrass, formerly Agropyron spicatum) well represented (only common under intensive grazing) and, excepting Poa secunda (Sandberg's bluegrass), constitutes the dominant graminoid Artemisia tripartita / Pseudoroegneria spicata p. a.

47. P. spicata poorly represented, not the dominant graminoid Undefined Artemisia tripartita-dominated vegetation type

48. Artemisia nova (black sagebrush) well represented and the dominant dwarf-shrub. 49 
48. A. nova poorly represented and not the dominant dwarf-shrub

49. Pseudoroegneria spicata (bluebunch wheatgrass, formerly Agropyron spicatum) or Oryzopsis hymenoides (Indian ricegrass) singly or in combination, common or the dominant graminoids, excepting Poa secunda (Sandberg's bluegrass) ....... Artemisia nova / Pseudoroegneria spicata p. a.

49. $P$. spicata and $O$. hymenoides, singly or in combination, scarce and not the dominant graminoids

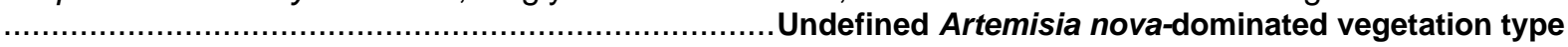

50. Re-enter dwarf-shrub key at beginning lead and re-evaluate the effects of grazing on diagnostic species; see next lead if stand remains unclassifiable on second pass.

50. Undefined dwarf-shrub types should be accumulated here! 


\section{Key to Grassland Plant Associations INCLUDING UPLAND AND WETLAND TYPES}

In addition to vegetation types dominated by members of the Poaceae (grasses) we include here communities dominated by members of the families Juncaceae (rush family, including Juncus spp.[rush], Luzula spp. [woodrush]), Cyperaceae (sedge family, including Carex spp. [sedge]. Cyperus spp. [galingale], Dulichium arundinaceum [dulichium], Eleocharis spp. [spike rush], Eriophorum spp. [cotton grass], Kobresia spp. [kobresia], Scirpus spp. [bulrush], Typhaceae (cattail family, Typha spp. [cattails], Juncaginaceae (arrow grass family, including Lilaea scilloides (flowering quillwort) and Triglochin spp. [arrow grass]). Not included as "graminoid" types are vegetation types named for members of the ferns and "fern allies" including Equisetaceae (horsetail family), Lycopodiaceae (club moss family), Marsileaceae (pepperwort family), Ophioglossaceae (grape fern family), Polypodiaceae (fern family), Selaginellaceae (spike moss family); these herbaceous, non-graminoid groups are found under forbs

1. Sites ranging from temporarily flooded to permanently saturated, includes all wetlands (and some near-wetlands) wherein a graminoid is the first designated diagnostic species; sites dominated by or supporting as a diagnostic taxon any of the following species

Agrostis stolonifera (redtop)

Bromus inermis (smooth brome)

Calamagrostis canadensis (bluejoint reedgrass), C. stricta (narrow-spiked reedgrass)

Carex aperta (Columbia sedge), C. aquatilis (water sedge), C. atherodes (awned sedge), C. buxbaumii (Buxbaum's sedge), C. lanuginosus (woolly sedge), C. lasiocarpa (slender sedge), C. lenticularis (lentil-fruit sedge), C. limosa (mud sedge), C. nebrascensis (Nebraska sedge), C. praegracilis (clustered field sedge), C. scopulorum (Holm's Rocky Mountain sedge), C. simulata (short-beaked sedge), C. utriculata (beaked sedge), C. vesicaria (inflated sedge)

Deschampsia cespitosa (tufted hairgrass)

Distichlis spicata (inland saltgrass)

Eleocharis acicularis (needle spike-rush), E. palustris (common spikesedge), E. quinqueflora (formerly $E$.

pauciflora, few-flowered spike-rush), E. rostellata (beaked spike-rush)

Glyceria borealis (northern mannagrass)

Hordeum jubatum (foxtail barley)

Juncus balticus (Baltic rush)

Leymus cinereus (formerly Elymus cinereus, basin wildrye)

Pascopyrum smithii (western wheatgrass)

Phalaris arundinacea (reed canarygrass)

Poa pratensis (Kentucky bluegrass), P. palustris (fowl bluegrass)

Scirpus acutus (hardstem bulrush), S. tabernaemontani (formerly S. validus, softstem bulrush)

Sporobolus airoides (alkali sacaton)

Typha angustifolia (narrow-leaved cattail), Typha latifolia (common cattail)

1. Sites drier than described above or occurring at the highest elevations of the subalpine zone (above the distribution of Pseudotsuga menziesii [Douglas-fir] as a climax species and at the upper distribution limits of Artemisia tridentata ssp. vaseyana (mountain big sagebrush) and, with the exception of Deschampsia cespitosa (tufted hairgrass) in the sub-alpine/alpine, not supporting any of the above-listed species as dominants or diagnostic spp.

see following section with subheading GraminoId-Dominated CommunitIES: UPLANDS, High-SUBALPINE, AND ALPINE

Note: Leads 2 through 10 key to wetland plant associations with Carex spp. (sedges) as diagnostic species

2. Carex spp. (sedge species) listed in \#1, individually or their combined canopy cover at least $25 \%$ or these taxa dominant

2. Carex spp. having less than $25 \%$ single or combined cover and not dominant .............................. 11

3. Carex utriculata (beaked sedge), C. vesicaria (inflated sedge), C. atherodes (awned sedge), individually or their combined cover, at least $25 \%$

Carex utriculata p. a. (see a, b, c) a. Carex aquatilis (water sedge), C. lenticularis (lentil-fruit sedge) and grass species, individually or in combination having less than $5 \%$ cover. Carex utriculata phase b. Carex aquatilis and $C$. lenticularis, singly or combined, at least well represented 
c. C. aquatilis and C. lenticularis, singly or combined, poorly represented; grass species or Juncus balticus (Baltic rush) well represented, either as single species or their combined cover.....

Deschampsia cespitosa phase

3. C. utriculata, C. vesicaria, and C. atherodes, individually or their combined cover, less than $25 \% \ldots . . . .4$

4. Carex aquatilis (water sedge), C. aperta (Columbia sedge) and C. lenticularis (lentil-fruited sedge), as individual species or combined, having at least $25 \%$ canopy cover..Carex aquatilis p. a.(see a \& b) a. Juncus balticus (Baltic rush) and grass species, as lone species or in combination, having less than $5 \%$ canopy cover Carex aquatilis phase b. J. balticus or grass species having at least 5\% canopy cover ........... Deschampsia cespitosa phase

4. C. aquatilis, $C$. aperta, and $C$. lenticularis, individually or combined, having less than $25 \%$ cover ........ 5

5. Carex limosa (mud sedge) having at least $25 \%$ canopy cover Carex limosa p. a.

4. C. limosa having less than $25 \%$ canopy cover....

6. Carex lasiocarpa (slender sedge), C. lanuginosa (woolly sedge), and C. buxbaumii (Buxbaum's sedge), individually or in any combination, having at least $25 \%$ canopy cover ... Carex lasiocarpa p. a.

6. C. lasiocarpa, C. lanuginosa, and C. buxbaumii, individually or in combination, having less than $25 \%$ canopy cover 7.

7. Carex simulata (short-beaked sedge) having at least $25 \%$ canopy cover Carex simulata p. a.

7. C. simulata having less than $25 \%$ canopy.....

8. Carex scopulorum (Holm's Rocky Mountain sedge) having $25 \%$ or greater canopy cover

8. C. scopulorum having less than $25 \%$ canopy cover. Carex scopulorum p. a.

9. Carex nebrascensis (Nebraska sedge) with a greater canopy cover than any other individual sedge species..... Carex nebrascensis p. a.

9. C. nebrascensis having less canopy cover than other sedge species present. 10

10. Site with at least one of the following wetland attributes present; hydrophytic vegetation, hydric soils, wetland hydrology..... Unclassified wetland or riparian site

10. Site lacking any of the above listed wetland attributes Upland site (see second item of lead \#1)

Note: Leads 11 through 35 key to wetland (or near-wetland) plant associations wherein Carex spp. are not the diagnostic species

11. Typha latifolia (common cattail) or $T$. angustifolia (narrow-leaved cattail), individually or their combined canopy cover, at least $25 \%$ or constituting the dominant vegetation ....... Typha latifolia p. a.

11. T. latifolia and $T$. angustifolia having less than $25 \%$ canopy cover and not constituting the dominant vegetation

12. Scirpus acutus (hardstem bulrush) or S. tabernaemontani (formerly $S$. validus, softstem bulrush), individually or in combination, constitute the dominant vegetation. Scirpus acutus p. a.

12. S. acutus and S. tabernaemontani, individually or in combination, not the dominant species..... 13

13. Phalaris arundinacea (reed canarygrass) having $25 \%$ canopy cover or is the dominant species

Phalaris arundinacea p. a.

13. $P$. arundinacea having less than $25 \%$ canopy cover and not the dominant species 14.

14. Equisetum fluviatile (water horsetail) having at least $25 \%$ canopy or is the dominant species.

14. E. fluviatile having less than $25 \%$ canopy cover and not the dominant species Equisetum fluviatile p. a.

15. Eleocharis palustris (common spikesedge) or E. acicularis (needle spike-rush), individually or in combination, having $25 \%$ canopy cover or constitute the dominant species .. Eleocharis palustris p. a. 
15. E. palustris and E. acicularis, individually or in concert, with less than $25 \%$ canopy cover and not dominant.....

16. Eleocharis quinqueflora (formerly Eleocharis pauciflora, few-flowered spike-rush) and E. rostellata (beaked spike-rush), individually or in combination, having 25\% canopy cover or constituting the dominant species....

Eleocharis quinqueflora p.a.

16. E. pauciflora and E. rostellata having less than $25 \%$ canopy cover and not the dominant species ..... 17

17. Glyceria borealis (northern mannagrass) with at least $25 \%$ canopy cover or the dominant graminoid ....

17. G. borealis having less than $25 \%$ canopy cover and not the dominant graminoid. Glyceria borealis p. a.

18. Calamagrostis canadensis (bluejoint reedgrass) or C. stricta (narrow-spiked reedgrass), individually or in concert, having at least $25 \%$ canopy cover or constituting the dominant graminoids

Calamagrostis canadensis p. a.

18. C. canadensis and C. stricta having less than $25 \%$ cover, individually or combined, and not the dominant graminoids.

19. Deschampsia cespitosa (tufted hairgrass) having at least $15 \%$ canopy cover and constituting the dominant graminoid

Deschampsia cespitosa p. a.

19. D. cespitosa having less than $15 \%$ cover and is not the dominant graminoid 20.

20. Juncus balticus (Baltic rush) the dominant graminoid

20. J. balticus not the dominant graminoid

21. Carex praegracilis (clustered field sedge) or Muhlenbergia richardsonis (mat muhly), individually or combined canopy cover, well represented Juncus balticus - Carex praegracilis $\mathrm{p}$. a.

21. C. praegracilis and $M$. richardsonis, their individual or combined coverage, poorly represented .......... 22

22. Juncus balticus (Baltic rush) virtually a monospecific dominant, other species characteristically having less than $5 \%$ cover; generally occurring on continuously flooded or saturated soils, often drawdown zones of limnic environments.

Juncus balticus p. a.

22. J. balticus not a monospecific dominant; other graminoids and forbs occupying the site, though they may not be as dominant as $J$. balticus. Undefined Juncus balticus vegetation type

23. Distichlis spicata (inland saltgrass, formerly D. stricta) having at least $15 \%$ canopy cover or dominating the graminoid layer.

Distichlis spicata p. a.

23. D. spicata having less than $15 \%$ cover and not the graminoid layer dominant...... 24.

24. Leymus cinereus (giant wildrye, formerly Elymus cinereus) well represented (characteristically on temporarily or seasonally flooded sites......

24. L. cinereus poorly represented

25. Puccinellia nuttalliana (Nuttall's alkaligrass) or $P$. distans (weeping alkaligrass) or Poa secunda (Sandberg's bluegrass, formerly Poa juncifolia, alkali bluegrass), their individual or combined coverage well represented or dominant

Leymus cinereus - Puccinellia nuttalliana p. a.

25. Not as above, $P$. nuttalliana, $P$. distans and $P$. secunda poorly represented and not dominant..... Undefined Leymus cinereus vegetation type

26. Puccinellia nuttalliana (Nuttall's alkaligrass) well represented or dominant; alkaline seeps or flats

26. P. nuttalliana poorly represented and not dominant

Puccinellia nuttalliana p. a.

26. P. nuttallianapoorly represented and not dominant....

27. Sporobolus airoides (alkali sacaton) well represented or the dominant graminoid; typically on seasonally flooded alkaline seeps or alluvial flats

Sporobolus airoides p. a. 
27. S. airoides poorly represented and not the dominant graminoid....

28. Pascopyrum smithii (western wheatgrass, formerly Agropyron smithii) well represented or dominant graminoid; characteristic of basins or swales or alluvial flats (usually with heavy-textured soils)......

Pascopyrum smithii / alluvial flat p. a.

28. P. smithii poorly represented and not the dominant graminoid

29. Poa secunda (Sandberg's bluegrass, formerly Poa juncifolia or P. secunda ssp. juncifolia, alkali bluegrass) well represented and dominant of graminoid layer; community characteristic of seasonally or temporarily flooded alluvial flats

Poa secunda p. a.

29. P. secunda poorly represented and not dominant graminoid 30

Note: At lead 30 begins a section of wetland (or near wetland) vegetation types characterized by introduced graminoids.

30. Poa palustris (fowl bluegrass) having a greater canopy cover than other individual herbaceous species.

Poa palustris c. t.

30. P. palustris canopy cover less than that of other herbaceous species. 31

31. Agrostis stolonifera (redtop) having a greater canopy cover than that of other individual herbaceous species. Agrostis stolonifera c. t.

31. A. stolonifera canopy cover less than that of other herbaceous species 32

32. Hordeum jubatum (foxtail barley) having a greater canopy cover than that of other individual species ... Hordeum jubatum c. t.

32. H. jubatum canopy cover less than that of other herbaceous species 33

33. Bromus inermis (smooth brome) having a greater canopy cover than that of other individual species

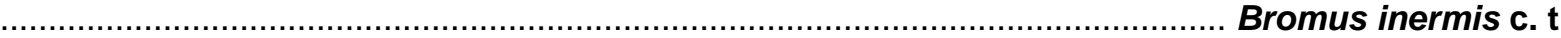

33. B. inermis canopy cover less than that of other herbaceous species 34

34. Poa pratensis (Kentucky bluegrass) ) having a greater canopy cover than other individual herbaceous species. Poa pratensis c. t.

34. P. pratensis canopy cover less than that of other herbaceous species 35

35. Sites possessing at a minimum one of the following wetland criteria: hydrophytic vegetation, wetland hydrology, hydric soils. Unclassified or unreported wetland or riparian site (see also "Riparian Dominance Types of Montana" [Hansen et al. 1988] to identify and describe the site)

35. Sites lacking all of the above-cited wetland criteria..... Upland site

\section{Graminoid-dominated Communities; UpLANDS, INCLUDING VARIOUS High-sUbaLPINE AND ALPINE ENVIRONMENTS}

1. Sites ranging from moist uplands to seasonally saturated flats and seeps (including jurisdictional wetlands); dominated by any one of the following species, Juncus balticus (Baltic rush), Deschampsia cespitosa (tufted hairgrass), Carex praegracilis (clustered field sedge), Muhlenbergia richardsonis (mat muhly), Hordeum jubatum (foxtail barley), Leymus cinereus (giant or basin wildrye), Pascopyrum smithii (western wheatgrass), Puccinellia nuttalliana (Nuttall's alkaligrass), $P$. distans (weeping alkaligrass).

1. Sites drier than described above or occurring at highest elevations of subalpine zone (above distribution of Pseudotsuga menziesii (Douglas-fir) as climax species, upper distribution of Artemisia 
tridentata ssp. vaseyana [mountain big sagebrush]) upward into alpine and, with the exception of $D$. cespitosa in the sub-alpine/alpine, not supporting any of the above listed

species as dominants

2. Deschampsia cespitosa (tufted hairgrass) well represented (only common if grazing intensive) ........... 3

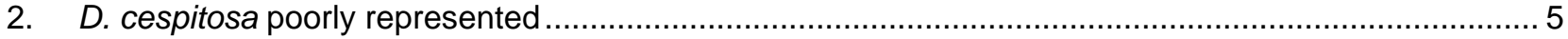

3. Potentilla diversifolia (diverse-leaved cinquefoil) common or Phleum alpinum (alpine timothy) and Trisetum spicatum (spike trisetum) present; Pseudoroegneria spicata (bluebunch wheatgrass) not present; high subalpine to alpine meadows ...... Deschampsia cespitosa / Potentilla diversifolia p.a.

3. $\quad P$. diversifolia scarce, $P$. alpinum and $T$. spicatum absent; habitats various. 4

4. Carex spp. (sedge species, e.g) well represented or the dominant graminoids along with $D$. cespitosa

Deschampsia cespitosa - Carex spp.

4. Not as above, Carex spp. not the dominant graminoids. see a \& b below

a. Festuca idahoensis (Idaho fescue) well represented (or only common under intensive grazing)

Festuca idahoensis - Deschampsia cespitosa p. a.

b. F. idahoensis poorly represented (the following type represents largely lower elevation sites too wet to support $F$. idahoensis, yet not formal wetlands)

Deschampsia cespitosa p. a. (as described by Hansen et al. [1995])

5. Juncus balticus (Baltic rush) is well represented

5. J. balticus poorly represented

6. Carex praegracilis (clustered field sedge) or Muhlenbergia richardsonis (mat muhly), individually or combined, well represented Juncus balticus-Carex praegracilis p.a.

6. C. praegracilis and $M$. richardsonis, individually or combined cover, well represented 7

7. Juncus balticus (Baltic rush) virtually a monospecific dominant, among the few species present $(<5)$; characteristically of drawdown zones of lemnic environments (includes just the most depauperate stands incorporated in Hansen et al. [1995])

Juncus balticus p.a.

7 J. balticus not a monospecific dominant; other graminoids and forbs occupying the site, though their canopy cover may be less than that of $\mathrm{J}$. balticus (includes most of the $\mathrm{J}$. balticus community type as conceived of by Hansen et al. [1995]) Juncus balticus p. a.

8. Distichlis spicata (inland saltgrass, formerly D. stricta) having at least $15 \%$ canopy cover or dominating the graminoid layer..... Distichlis spicata p. a.

8. D. spicata having less than $15 \%$ canopy cover and not the dominant graminoid

9. Leymus cinereus (giant wildrye, formerly Elymus cinereus) well represented (characteristically on temporarily or seasonally flooded sites.

9. L. cinereus poorly represented

10. Puccinellia nuttalliana (Nuttall's alkaligrass) or $P$. distans (weeping alkaligrass) or Poa secunda (Sandberg's bluegrass, formerly Poa juncifolia, alkali bluegrass), their individual or combined coverage well represented or dominant....

Leymus cinereus - Puccinellia nuttalliana p. a.

10. Not as above, $P$. nuttalliana, $P$. distans and $P$. secunda poorly represented and not dominant. Undefined Leymus cinereus vegetation type

11. Puccinellia nuttalliana (Nuttall's alkaligrass) well represented or dominant; alkaline seeps or flats Puccinellia nuttalliana p. a.

11. P. nuttalliana poorly represented and not dominant 12

12. Sporobolus airoides (alkali sacaton) well represented or the dominant graminoid; typically on seasonally flooded alkaline seeps or alluvial flats Sporobolus airoides p. a.

12. S. airoides poorly represented and not the dominant graminoid. 13 
13. Pascopyrum smithii (western wheatgrass, formerly Agropyron smithii) well represented or dominant graminoid; characteristic of basins or swales or alluvial flats (usually with heavy-textured soils).....

Pascopyrum smithii / alluvial flat p. a.

13. P. smithii poorly represented and not the dominant graminoid 14

14. Poa secunda (Sandberg's bluegrass, formerly Poa juncifolia or $P$. secunda ssp. juncifolia, alkali bluegrass) well represented and dominant of graminoid layer; community characteristic of seasonally or temporarily flooded alluvial flats

Poa secunda p. a.

14. P. secunda poorly represented and not dominant graminoid

...See lead \#30 in the initial portion of "KEY TO GRASSLAND TYPES, UPLAND AND WETLAND" that treats wetlands

15. Communities of high subalpine to alpine sites, generally above $8,500 \mathrm{ft}$., or if occurring lower then associated with windswept shoulders and ridges

15. Not as above, communities of elevations generally considered to lie below the upper subalpine, roughly less than $8,500 \mathrm{ft}$. at this latitude and in this climatic regime.

16. Bromus carinatus (mountain brome) or $B$. anomalus (nodding brome) well represented, individually or collectively (other graminoids may be dominant) ........Bromus carinatus - Bromus anomalus p. a.

16. B. carinatus or $B$. anomalus poorly represented, individually or collectively. 17

17. Stipa richardsonii (Richardson's needlegrass) abundant (only well represented if grazing intensive) . 18

17. S. richardsonii not abundant (and poorly represented in the presence of grazing) 19

18. Festuca idahoensis (Idaho fescue) well represented (or common if grazing intensive): Note, the following p.a. largely replaces $F$. idahoensis $-S$. richardsonii p. a.

Stipa richardsonii - Festuca idahoensis p. a.

18. F. idahoensis poorly represented Undefined Stipa richardsonii vegetation type

19. Festuca campestris (formerly F. scabrella, rough fescue) well represented (only common under intensive grazing)

19. F. campestris poorly represented (or scarce under grazing) 22

20. Festuca idahoensis (Idaho fescue) well represented, Pseudoroegneria spicata (formerly Agropyron spicata, bluebunch wheatgrass), if present, with less cover than $F$. idahoensis

Festuca campestris - Festuca idahoensis p.a.

20. F. idahoensis (Idaho fescue) poorly represented, $P$. spicata (bluebunch wheatgrass) common or at least equal in cover to $F$. idahoensis....

21. Pseudoroegneria spicata (formerly Agropyron spicatum, bluebunch wheatgrass) common ( reduced to scattered individuals with intensive grazing) .. Festuca campestris - Pseudoroegneria spicata p.a.

21. P. spicatum scarce.................... Undefined/unreported p.a./c.t. within Festuca campestris alliance

22. Festuca idahoensis (Idaho fescue) well represented (or only common under intensive grazing) ......... 23

22. F. idahoensis poorly represented (or scarce under intensive grazing); $D$. cespitosa absent............... 27

23. Potentilla diversifolia (diverse-leaved cinquefoil), Carex scirpoidea (Canadian single-spike sedge), C. filifolia (thread-leaved sedge) or Phleum alpinum (alpine timothy), their single or combined cover, common; sites at higher reaches of subalpine extending to alpine, extending lower on cold, exposed sites (includes two Mueggler \& Stewart (1980) types, F. idahoensis - Carex scirpoidea p.a. (hypothesized) and $F$. idahoensis-Carex filifolia p.a. (verified))

Festuca idahoensis / Potentilla diversifolia p a.

23. P. diversifolia, C. scirpoidea, C. filifolia, P. alpinum scarce, either individual or combined cover; sites not of alpine to high subalpine, or not exposed or colder than prevailing climate.

24. Geranium viscosissimum (sticky geranium), Potentilla gracilis (slender cinquefoil) present and/or Bromus carinatus (mountain brome), B. anomalus (nodding brome) or Elymus trachycaulus (formerly Agropyron caninum, bearded wheatgrass) common, individually or combined 
24. Not as above. Festuca idahoensis - Elymus trachycaulus p.a.

25. Pascopyrum smithii (western wheatgrass) or Elymus lanceolatus (formerly Agropyron dasystachyum, thick-spike wheatgrass), their individual or combined cover, common

Festuca idahoensis / Pascopyrum smithii p.a.

25. P. smithii and A. dasystachyum, their individual or combined cover, scarce .................................... 26

26. Pseudoroegneria spicata (formerly Agropyron spicatum, bluebunch wheatgrass) common (accept merely present under intensive grazing regime) Festuca idahoensis - Pseudoroegneria spicata p.a.

26. P. spicata scarce. Undefined/unreported Festuca idahoensis vegetation type

27. Pascopyrum smithii (western wheatgrass) or Elymus lanceolatus (formerly Agropyron dasystachyum, thick-spike wheatgrass), their individual or combined cover, common ............................................ 28

27. P. smithii and E. lanceolatus scarce except in stands where $P$. spicata well represented .................. 30

28. Pascopyrum smithii (western wheatgrass) a nearly monospecific dominant of gentle flats and bottomlands with fine-textured (heavy) soils

Pascopyrum smithii / alluvial flats p.a.

28. P. smithii not virtually the only species of importance, sites including more than fine-textured bottomlands

29. Elymus lanceolatus (thick-spike wheatgrass) common and usually dominant component of early seral stages on sandy substrates Elymus lanceolatus / Phacelia hastata p.a.

29. Elymus lanceolatus scarce and/or not dominant.... Undefined $P$. smithii and/or E. lanceolatus vegetation types

30. Pseudoroegneria spicata (bluebunch wheatgrass) well represented (only common under intensive grazing, or where badland conditions or a high cover of exposed substrate, usually gravel, exists).... 31

30. A. spicatum poorly represented (or scarce with grazing)

31. Rhizomatous wheatgrasses, principally Elymus lanceolatus (thick-spike wheatgrass) and Pascopyrum smithii (western wheatgrass) common, Carex stenophylla usually present.

Pseudoroegneria spicata - Pascopyrum smithii p.a.

31. Rhizomatous wheatgrasses scarce; $C$. stenophylla usually absent. 32

32. Bouteloua gracilis (blue grama) well represented Pseudoroegneria spicata - Bouteloua gracilis p.a.

32. B. gracilis poorly represented

33. Oryzopsis hymenoides (Indian ricegrass) present; sites either badlands with naturally (mostly) eroded conditions or excessively drained sandy soils.

Pseudoroegneria spicata - Oryzopsis hymenoides p.a.

33. O. hymenoides absent, sites not as above.

34. Ridge shoulders or convexities exposed to prevailing winds (usually with soil deflation and much, $60 \%+$, exposed substrate); at or below the upper subalpine zone; cushion plants (e.g Eritrichium nanum, Douglasia montana, Townsendia spp. ) or compact forbs (e.g. Oxytropis campestris, Stenotus acaulis [formerly Haplopappus acaulis]) dominate the undergrowth; graminoid component far reduced relative to forbs

34. Not as above

Pseudoroegneria spicata / Cushion Plants p.a.

35. Poa secunda (Sandberg's bluegrass) present, usually graminoid 2nd in importance to Pseudoroegneria spicata (bluebunch wheatgrass); Stipa comata (needle-and-thread) or S. spartea (porcupine-grass) may be conspicuous components on lower alluvial slopes and benches and valley bottoms ....

Pseudoroegneria spicata - Poa secunda p.a.

35. P. secunda absent, not as above ......... Undefined/unreported Pseudoroegneria spicata vegetation type 
36. Stipa comata (needle-and-thread) or S. spartea (porcupine grass) and/or Bouteloua gracilis (blue

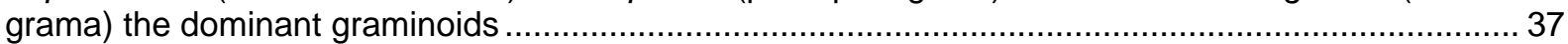

36. S. comata, S. spartea and B. gracilis not the dominant graminoids ................................................ 39

37. Psoralidium tenuiflorum (formerly Psoralea tenuiflora, slender-flowered scurf-pea) present, usually the dominant forb in depauperate vegetation; substrate sandy

37. P. tenuiflorum absent

Stipa comata / Psoralidium tenuiflorum p. a.

38. Bouteloua gracilis (blue grama) common

Stipa comata - Bouteloua gracilis p.a.

38. B. gracilis scarce Undefined / unreported Stipa comata vegetation type

39. Distichlis spicata (formerly $D$. stricta, inland saltgrass) common, usually the dominant graminoid in salt-or alkali-affected sites

Distichlis spicata p.a.

39. D. spicata poorly represented, may not be the dominant graminoid

Undefined / unreported herbaceous p.a./c.t. for southwestern MT

Note: from lead 40 to 66 the vegetation types treated are of subalpine to alpine environments, both wetlands and uplands

40. Stands dominated by graminoids (grasses, sedges, rushes etc.).

40. Stands not graminoid-dominated, but rather by forbs.

see "Key to Perennial Forb Vegetation Including UPLAND and WetLand Types"

41. Wetland sites with a floristic composition and/or soils/hydrology meeting wetland criteria 42

41. Sites not wetlands, not meeting wetland criteria

42. Wetland sites dominated by Carex scopulorum (Holm's Rocky Mountain sedge) C. aquatilis (water sedge), C. lenticularis (lentil-fruit sedge) or C. aperta (Columbia sedge), singly or in combination ..... 43

42. Site not dominated by any combination of the above-listed species

Undefined Graminoid-dominated Wetland

43. Site dominated by Carex scopulorum (Holm's Rocky Mountain sedge)

43. Site not dominated by $C$. scopulorum.

44. Caltha leptosepala (elkslip marsh-marigold) or Senecio cymbalarioides (few-leaved groundsel), singly or combined, well represented and diagnostic forbs Carex scopulorum / Caltha leptosepala p. a.

44. C. leptosepala and S. cymbalarioides poorly represented.... Undefined Carex scopulorum vegetation type

45. Sites dominated by or having greater canopy cover of Carex simulata (short-beaked sedge) than any other single graminoid. Carex simulata p. a.

45. Sites dominated by graminoids other than C. simulata 46

46. Carex aquatilis (water sedge), C. lenticularis (lentil-fruit sedge) or C. aperta (Columbia sedge), singly or in combination, dominant or well represented.

Carex aquatilis p. a. (see a \& b below)

a. Grass species or Juncus balticus (Baltic rush), alone or in combination, poorly represented....

b. Grass species and J. balticus, alone or in combination, poorly represented

Carex aquatilis Phase

Deschampsia cespitosa Phase

46. C. aquatilis, C. lenticularis, and $C$. aperta, singly or in combination, poorly represented and not dominant

47. Snowbed communities (sites with greater accumulations of snow than other landscape positions and/or later meltoff of snow) 
47. Not snowbed sites, not characterized by above average snow deposition or late meltoff.

48. Sites where snow is very long-persisting (longer than any other vegetated position) and Carex nigricans (black alpine sedge) is dominant and usually abundant

48. C. nigricans scarce, not the canopy dominant. Carex nigricans p. a.

49. Snowbed sites often with depauperate herb cover, with Juncus drummondii (Drummond's rush), Antennaria lanata (woolly pussy-toes) and/or Sibbaldia procumbens (creeping sibbaldia) present and dominant ............................................................. Juncus drummondiana / Antennaria lanata p. a.

49. Snowbed sites but not as above, lacking J. drummondii, A. lanata and S. procumbens as dominants ...

50. Juncus parryi (Parry's rush) the dominant graminoid with Erigeron ursinus (Bear River fleabane) common and consistently the dominant forb, though cover seldom exceeds $5 \%$.

50. J. parryi not the dominant graminoid and $E$. ursinus not dominant forb

Juncus parryi / Erigeron ursinus p. a. Undefined Snowbed Sites

51. Stands dominated by one, or combinations, of the following six graminoids Deschampsia cespitosa (tufted hairgrass), Festuca idahoensis (Idaho fescue), Festuca kingii (spike-fescue, formerly Hesperochloa kingii or Leucopoa kingii) Bromus inermis var. pumpellianus (formerly $B$. pumpellianus, pumpelly brome), Juncus balticus (Baltic rush) or Carex obtusata (blunt sedge)......... 52

51. Stands not dominated by any one, or combination, of the above listed graminoid species..... 59

52. Deschampsia cespitosa (tufted hairgrass) well represented, the dominant graminoid of moist to wet meadows.

52. D. cespitosa poorly represented and not the dominant graminoid

53. Caltha leptosepala (elkslip marsh-marigold) and/or Senecio cymbalarioides (few-flowered groundsel), singly or in combination, well represented or dominating the forb layer of wet meadows .....

Deschampsia cespitosa / Caltha leptosepala p. a.

53. C. leptosepala and S. cymbalarioides, alone or combined cover not dominant and poorly represented .

54. Potentilla diversifolia (diverse-leaved cinquefoil) common.

54. P. diversifolia scarce

Deschampsia cespitosa / Potentilla diversifolia p. a. ..Undefined Deschampsia cespitosa vegetation type

55. Festuca idahoensis (Idaho fescue), Carex obtusata (blunt sedge), or Bromus inermis var. pumpellianus (pumpelly brome, formerly B. pumpellianus) or any combination of the three well represented or comprising the dominant graminoids

55. Neither F. idahoensis, C. obtusata nor B. inermis var. pumpellianus or any combination of the three dominating the graminoid component.

56. Potentilla diversifolia (diverse-leaved cinquefoil) common

56. P. diversifolia scarce

Festuca idahoensis / Potentilla diversifolia p. a.

57. Festuca kingii (spike fescue, formerly Hesperochloa kingii or Leucopoa kingii) the graminoid with greatest coverage in rather depauperate communities

57. F. kingii not the indicated dominant

Undefined Graminoid-dominated vegetation type

58. Oxytropis campestris (slender crazyweed) present and characteristic forb

58. O. campestris not present.

Festuca kingii / Oxytropis campestris p. a. ..Undefined Festuca kingii vegetation type 
59. Turf communities (commonly characterized by an abundance of dwarf, fibrous-rooted graminoids, usually Carex spp. [sedge species], but forbs may dominate some stands); dominant graminoids with individual or combined cover exceeding 15\% including Carex elynoides (Kobresia-like sedge), $C$. rupestris (curly sedge), C. scirpoidea (Canadian single-spike sedge), C. phaeocephala (dunhead sedge), C. albonigra (black-and-white-scaled sedge), C. atrata (blackened sedge), Festuca ovina (sheep fescue), and Calamagrostis purpurascens (purple reedgrass) ....

59. Not graminoid-dominated or having reduced total $(<50 \%)$ canopy coverage from turf communities as described above.

60. Moist turf sites with one or any combination of the following Carex spp. (sedge species) dominant; $C$. scirpoidea (Canadian single-spike sedge), C. phaeocephala (dunhead sedge), C. albonigra (blackand-white-scales sedge), C. atrata (blackened sedge)

60. Dry turf sites (grading to cushion plant environments with much more open structure) with one or any combination of the following species dominant; Carex rupestris (curly sedge), C. elynoides (Kobresia-like sedge), C. obtusata (blunt sedge), Festuca ovina (sheep fescue), Calamagrostis purpurascens (purple reedgrass)......

61. Geum rossii (Ross's avens) common (usually well represented and the dominant forb); substrates non-calcareous Carex scirpoidea / Geum rossii p. a.

61. Geum rossii scarce, other forbs dominant. 62

62. Potentilla diversifolia (diverse-leaved cinquefoil) and/or Phlox pulvinata (cushion phlox) dominant and/or common Carex scirpoidea / Potentilla diversifolia p. a.

62. Not as above, $P$. diversifolia and P. pulvinata scarce and not dominant. Undefined moist alpine turf vegetation types

63. Turf nearly continuous, Carex rupestris (curly sedge), C. obtusata (blunt sedge) and Calamagrostis purpurascens (purple reedgrass) not dominant or codominant graminoids; forbs a minor component....

Carex elynoides p. a.

63. Turf patchy, C. rupestris, C. obtusata or Calamagrostis purpurascens (purple reedgrass) dominant graminoids with openings forb dominated and much exposed gravel/rock substrate existing.....

64. Calamagrostis purpurascens (purple reedgrass) well represented or dominant graminoid. Calamagrostis purpurascens - Carex rupestris p. a.

64. C. purpurascens poorly represented and not the dominant graminoid 65

65. Carex rupestris (curly sedge) or C. obtusata (blunt sedge) the dominant graminoid, well represented; Potentilla ovina (sheep cinquefoil) or Phlox pulvinata (cushion phlox) common, individually or combined, usually the dominant forbs

Carex rupestris / Potentilla ovina p. a.

65. P. ovina and $P$. pulvinata, singly or combined, scarce and not the dominant forbs Undefined / unrecorded Carex rupestris vegetation types

66. Forb dominated turf communities ranging from dense generally continuous plant cover to somewhat open plant cover characterized by well-distributed clumps of erect forbs, though cushion plants may be dominant ..........................see "Key to Perennial Forb Vegetation TyPeS, UPLAND AND WeTLAND"

66. More like cushion plant than turf communities, with erect forbs sparse and cushion plants dominant and/or there exists much exposed substrate 


\section{Key to Perennial Forb Vegetation INCLUDING UPLAND AND WETLAND TYPES}

We include here as "forb-dominated" types vegetation types named for members of the ferns and "fern allies" including Equisetaceae (horsetail family), Lycopodiaceae (club moss family), Marsileaceae (pepperwort family), Ophioglossaceae (grape fern family), Polypodiaceae (fern family), Selaginellaceae (spike moss family) in addition to myriad other families, e.g. Asteraceae, Polymoniaceae, etc., the component species of which are traditionally thought of as forbs. Not included are members of the Poaceae (grasses) or members of the families Juncaceae (rush family, including Juncus spp.[rush], Luzula spp. [woodrush]), Cyperaceae (sedge family, including Carex spp. [sedge]. Cyperus spp. [galingale], Dulichium arundinaceum [dulichium], Eleocharis spp. [spike rush], Eriophorum spp. [cotton grass], Kobresia spp. [kobresia], Scirpus spp. [bulrush], Typhaceae (cattail family, Typha spp. [cattails], Juncaginaceae (arrow grass family, including Lilaea scilloides (flowering quillwort) and Triglochin spp. [arrow grass]);

1. Sites ranging from moist uplands to temporarily flooded to permanently flooded or saturated; dominated by having one of the following diagnostic species present, Angelica arguta (sharptooth angelica) or Angelica dawsonii (Dawson's angelica), Equisetum fluviatile (water horsetail), Parnassia fimbriata (fringed grass-of-parnassas), Senecio triangularis (arrowleaf groundsel), Salicornia rubra (red glasswort)

1. Not as above; sites drier than described above or occurring at highest elevations of the subalpine zone (above distribution of Pseudotsuga menziesii [Douglas-fir] as climax species, at upper distribution of Artemisia tridentata ssp. vaseyana [mountain big sagebrush]) upward into the alpine zone

2. Equisetum fluviatile (water horsetail) abundant (having at least $25 \%$ canopy cover

2. E. fluviatile not abundant, canopy less than $25 \%$.

3. Senecio triangularis (arrowleaf butterweed or groundsel) well represented or any two of the following species common $S$. triangularis, Parnassia fimbriata (fringed grass-of-Parnassas), Angelica arguta (sharptooth angelica), A. dawsonii (Dawson's angelica)

Senecio triangularis $\mathrm{p}$. a.

3. Not as above; $S$. triangularis poorly represented and all combinations of two of the above-listed species are scarce, less than $5 \%$ canopy cover.

4. Salicornia rubra (red glasswort) having a greater canopy cover than any other individual herbaceous species Salicornia rubra p. a.

4. S. rubra having a lesser canopy cover than any other individual herbaceous species....

Undefined or undescribed perennial forb-dominated vegetation type

5. Geum rossii (Ross's avens) as erect forb, dominant or co-dominant.............................................. 6

5. G. rossii present, not dominant, cespitose and reduced in size ................................................. 9

6. Erect forb Trifolium parryi (Parry's clover) dominant or co-dominant in forb layer

6. T. parryi absent or not dominant/co-dominant...... Geum rossii-Trifolium parryi p. a. ${ }^{1}$

7. Cushion plant Trifolium nanum (dwarf clover) dominant/co-dominant in forb layer

7. T. nanum not dominant/co-dominant Geum rossii - Trifolium nanum p. a. ${ }^{1}$

8. Cushion plant Trifolium dasyphyllum (whip-root clover) dominant/co-dominant

8. T. dasyphyllum not dominant/co-dominant Trifolium dasyphyllum - Geum rossii p. a. ${ }^{1}$ Undescribed / undefined turf community types 
9. Fellfield (high degree of exposed rock) or cushion plant environments (exposed, wind-blasted positions, usually ridge crests, slope shoulders and saddles); cushion plants range from a dominant aspect of community to slightly subordinate to erect forbs.

9. Sites not fellfields nor cushion plant communities, rather they include steep, either dry or wet slopes with a high degree of exposed substrate $(>70 \%)$

10. Cushion plant communities wherein Geum rossii (Ross's avens)and/or Minuartia obtusiloba (formerly Arenaria obtusiloba, arctic sandwort) are common or dominants of the forb layer (which is often depauperate with scattered plants).

Geum rossii - Arenaria obtusiloba p. a.

10. Neither G. rossii nor M. obtusiloba common, nor forb layer dominants

11. Moderately dense, single tier plant cover of prostrate and cespitose forbs, graminoid component depauperate, with Phlox pulvinata (cushion phlox) and Trifolium dasyphyllum (whip-root clover) providing more c.c. than other forbs ......................... Phlox pulvinata - Trifolium dasyphyllum p. a.

11. Not as above, neither P. pulvinata nor T. dasyphyllum the most important cushion plants..... 12

12. Scattered mixture of erect and cushion plants and graminoids with Antennaria microphylla (rosy pussy-toes) and/or Artemisia scopulorum (Rocky Mountain sagewort), alone or in combination, having the greatest forb cover ....................... Antennaria microphylla - Artemisia scopulorum p. a. ${ }^{1}$

12. Cushion plant communities with neither A. microphylla nor $A$. scopulorum nor combinations of the two dominating the forb layer.

13. Cushion plants dominant aspect of communities with varying combinations of the three species Phlox multiflora (many-flowered phlox), Trifolium nanum (dwarf clover) and Eritrichium nanum (pale alpine forget-me-not) providing the majority of canopy cover

Phlox multiflora-Triflorum nanum p. a. ${ }^{1}$

13. None of the above three species, alone or in combination, providing the dominant aspect of vegetation cover.

Undefined Cushion Plant Vegetation Types

14. Sites with predominantly northerly exposures, moderate to steep slopes with a high degree (at least $75 \%$ ) of exposed substrate of which more than $80 \%$ is soil or gravel; soils moist to saturated throughout growing season: vegetative canopy cover is much reduced, not exceeding $40 \%$ but with no characteristic species assemblage

Alpine Fellfield / Moist

14. Sites not as above 15

15. Sites with uniformly steep ( $>40 \%$ ), often unstable slopes of southeast through west-facing exposures; of the large amount of exposed substrate ( $>55 \%$ ) about $50 \%$ is gravel; Elymus scribneri (formerly Agropyron scribneri, spreading wheatgrass) is usually the one species with higher coverage and constancy in this "type" than other p.a.'s....

Alpine Fellfield / Dry

15. Sites not as described above. Undefined community types of Subalpine and Alpine Environments

${ }^{1}$ Thelenius and Smith (1985) 


\section{Vegetation \\ Classication: \\ Descriptions \\ of Types}

\section{N eedle-leaved E vergreen Forests}

Abies lasiocarpa / Arnica cordifolia Forest

(ABILAS / ARNCOR) subalpine fir / heartleaf arnica

\section{Natural Heritage Conservation}

Rank- G5 / S5

Environment- This is a major community type in the drier mountain ranges of Montana east of the Continental Divide, especially on calcareous substrates, ranging into northwestern Wyoming. It is a common, distributed on sites drier than those supporting Abies lasiocarpa / Thalictrum occidentale and occurring at generally higher elevations, usually transitional to the moister/colder Abies lasiocarpa / Vaccinium scoparium forest. Though more prevalent on northerly exposures, it is found on all aspects and degrees of slope. The observed elevation range was 7,300 to $8,900 \mathrm{ft}$., but the actual range is no doubt broader.

Vegetation- Limited data and reconnaissance observations indicate Pinus contorta and Pseudotsuga menziesii and all admixtures dominate seral stages and persist to near climax conditions. Abies lasiocarpa and Picea engelmannii are generally slow to reestablish following disturbance. Waist high 50-year-old Abies specimens are not unusual. Pinus albicaulis is scattered to abundant in the highest elevation examples of this type.

Undergrowth is generally somewhat depauperate depending on degree of canopy closure, but even young stands may have less than 5\% cover. Arnica cordifolia is the diagnostic forb (by default); others commonly occurring are Pyrola secunda, Aster conspicuus, and Osmorhiza chilensis or $O$. depauperata. In early seral stands Shepherdia canadensis, Juniperus communis or Symphoricarpos oreophilus are often well represented but decline to scattered individuals as the tree canopy closes.

Other Studies- This is a common type east of the Continental Divide in Montana and its areal extent increases with the increase in calcareous substrates. In central Idaho (Steele et al. 1981) it is a common type on quartzite and mixed volcanics which is also the case in northwestern Wyoming (Steele et al. 1983). Abies lasiocarpa / Arnica cordifolia is purported to occur as far south as Colorado.

Element Code- CEGL000298

Edition / Author- 99-11-16 / S.V. Cooper, MTNHP

Abies lasiocarpa / Calamagrostis canadensis Forest

(ABILAS / CALCAN) subalpine fir / bluejoint reedgrass

\section{Natural Heritage Conservation Rank- G5 / S5}

\section{Environment- Abies lasiocarpa /}

Calamagrostis canadensis forest association is broadly distributed near and east of the Continental Divide and is usually confined to small restricted areas that poorly drained or sub-irrigated sites that have surface water during late spring and early summer. These sites often border on streams and wet to moist, Juncus- or Carex-dominated species meadows. Where impermeable layers direct water to the surface this type can occur on mountain slopes. In general the stand size of Abies lasiocarpa / Calamagrostis canadensis are smaller than can be delineated on 1:24,000 scale maps, except as inclusions or a component of a complex.

Vegetation- The cool, wet conditions restrict the tree flora to predominantly three species. Picea (spruce) is usually the dominant species 
in mature to old-growth stands and persists as a minor climax or co-climax species with Abies lasiocarpa. Pinus contorta (lodgepole pine) often is the major seral species in younger stands. Pinus albicaulis (whitebark pine) is a minor component associated with drier hummocks in higher elevation stands.

Calamagrostis canadensis (bluejoint reedgrass) or C. stricta, indicator species for the type is the dominant graminoid, generally occurring with greater than 5\% canopy cover and averaging around $20 \%$. Senecio triangularis (alternative indicator species), Veratrum viride, Trollius laxus, Streptopus amplexifolius, and Dodecatheon jeffreyi constitute the high-constancy forb component that is associated with these wet sites. Thalictrum occidentale and Arnica latifolia, generally associated with merely moist sites, also have high constancy and may be well represented. In the Red Rocks Lake NWR expression of this type, shrubs are a minor component except where Ribes lacustre and Vaccinium scoparium which may attain abundance on hummocks and raised areas surrounding tree bases.

Only the Calamagrostis canadensis and Galium triflorum phases have been identified in the Centennial Range and Valley in general. The G. triflorum phase represents an equally moist but slightly warmer microclimate as denoted by the presence of $G$. triflorum and Actaea rubra.

Element Code- CEGL000300

Edition / Author- 99-11-16 / S.V. Cooper, MTNHP

Abies lasiocarpa / Calamagrostis rubescens Forest

(ABILAS / CALRUB) subalpine fir / pine grass

\section{Natural Heritage Conservation \\ Rank- G4G5 / S3}

Environment- Abies lasiocarpa / Calamagrostis rubescens forest is common on the south-facing flank of the Centennial Range but on the north-facing Montana portion it is a less prevalent type from 6,600 to at least $8,800 \mathrm{ft}$. on warm exposures. It usually grades to Abies lasiocarpa / Arnica cordifolia or Abies lasiocarpa / Thalictrum occidentale on cooler exposures and exists in complex mosaics with Abies lasiocarpa / Carex geyeri that occasion the question as to what factors may differentiate these types.

Vegetation- Description of this type comes from mid-seral stands that have a mixed canopy dominance split between Pseudotsuga menziesii and Pinus contorta. Abies lasiocarpa and Picea engelmannii are slow to reestablish on these dry sites. Pinus albicaulis occurs scattered at the highest elevations of this type.

The undergrowth is typically dominated in younger open stands by a near sward of Calamagrostis rubescens, usually accompanied by Carex geyeri, and the forbs, Thalictrum occidentalis, Fragaria virginiana, Aster conspicuus and Arnica cordifolia are characteristically present, though poorly represented, (excepting A. cordifolia). In older stands or those in the "closed canopy" stage $C$. rubescens may be reduced to scattered patches or even poorly represented. In open seral conditions Shepherdia canadensis and Juniperus communis may be well represented.

Soils- Very limited data preclude saying much about this type other than to observe that it occurred on rhyolite substrates in the Centennial Range north flank but also is known from sedimentary substrates on the south flank.

Other Studies- Abies lasiocarpa / Calamagrostis rubescens is a common type from central Montana (Pfister et al. 1977), where it occurs on calcareous substrates, to northern Utah (Mauk \& Henderson 1984) and central Colorado (Karmokova et al. 1988). It occurs as far west as the eastern flank of the Cascades in Washington and Oregon (Williams and Smith 1990, Williams and Lillybridge 1985) and is prevalent in Oregon's Blue Mountains (Johnson \& Simon 1987). 
Element Code- CEGL000301

Edition / Author- 99-11-16 / S.V. Cooper, MTNHP

Abies lasiocarpa / Carex geyeri Forest

(ABILAS / CARGEY) subalpine fir / elk sedge

\section{Natural Heritage Conservation \\ Rank- G5 / S3}

Environment- The Abies lasiocarpa / Carex geyeri Forest is a minor type throughout the Centennial Range but gains greater prominence in the Greater Yellowstone, encompassing some of the driest sites within the Abies lasiocarpa series. Sampled sites ranged from 7,600 to $8,600 \mathrm{ft}$., but the lower portion of this range could be extended considerably based on reconnaissance observations. Slopes of all degrees of inclination are included and aspects range from primarily east- through south- to west facing. This type often grades to Pseudotsuga menziesii / Carex geyeri or Calamagrostis rubescens on yet drier (lower elevation) sites and to Abies lasiocarpa / Thalictrum occidentale or Abies lasiocarpa / Arnica cordifolia forest on moister exposures.

Vegetation- Sampled stands were both late mature and early seral and thus encompassed much variability in structure and composition. The lower elevation examples of this type are dominated throughout their existence by Pseudotsuga menziesii with Abies lasiocarpa and Picea engelmannii only slowly establishing. Long-lived Pinus contorta may also form nearly pure stands on this type, but the usual condition is to occur mixed with Pseudotsuga. Stands from higher elevations often have A. lasiocarpa establishing immediately following disturbance but $P$. menziesii is still a major component. Pinus albicaulis (or P. flexilis) can also be a major long-lived seral component.

Because we recognize forb-rich conditions as conveying a higher moisture status and belonging to preferentially recognized plant associations, the undergrowth conditions in
Abies lasiocarpa / Carex geyeri Forest are mostly typified by the dominance of Carex geyeri with only scattered forbs. In open stands $C$. geyeri may approach a sword-like coverage. Forbs commonly present in minor amounts include Thalictrum occidentale, Astragalus miser, Fragaria virginiana, Aster foliaceus and Arnica cordifolia. In early- to mid-seral conditions Shepherdia canadensis and Juniperus communis are usually well represented.

Soils- Very limited information for this type indicates it occurs preferentially on noncalcareous substrates, which agrees with type descriptions from other areas. We found it exclusively on rhyolite or various forms of extrusive igneous in the Centennial Range and others (Steele et al. 1983, Steele et al. 1981, and Alexander et al. 1986) also report it from volcanic substrates that weather to other than fine-textured substrates. Only in central MT has it been reported from calcareous substrates (Pfister et al. 1977).

Other Studies- Abies lasiocarpa / Carex geyeri Forest is recorded as a minor type in central Montana ranges (Pfister et al. 1977), as a major type within the granitics of central Idaho's Batholith and occurs as a minor type as far south as northwestern Colorado (Hoffman and Alexander 1980) and Utah. Across its range it appears associated with volcanics of various kinds, both extrusive and intrusive.

Element Code- CEGL000304

Edition / Author- 99-11-16 / S.V. Cooper, MTNHP

Abies lasiocarpa / Ribes montigenum Forest

(ABILAS / RIBMON) subalpine fir / mountain gooseberry

\section{Natural Heritage Conservation \\ Rank- G5 / S4}

Environment- Abies lasiocarpa / Ribes montigenum Forest is found within the upper subalpine zone, generally beyond the limits of Pseudotsuga, and well within an environment 
wherein Pinus albicaulis is an important seral component. We noted this type as low as 7,500 $\mathrm{ft}$ on steep, north-facing slopes and extending to upper forest line (circa 8,600 ft.) on the Centennial Range's Big Table Mountain; Pfister et al (1977) noted this type extending to $9,000 \mathrm{ft}$ in the higher portion of the Centennials. At upper elevations of its distribution Abies lasiocarpa / Ribes montigenum Forest occurred on all aspects but seemed to be associated with slopes having late snow release. This type generally grades to Abies lasiocarpa / Arnica cordifolia and Abies lasiocarpa / Carex geyeri at lower elevations and to Abies lasiocarpa / Vaccinium scoparium on more moist sites. At highest elevations groves of Abies lasiocarpa / Ribes montigenum (on north slopes or depressions) form a mosaic with Artemisia tridentata var. vaseyana -dominated shrublands or Festuca idahoensis-dominated grasslands.

Vegetation- Only older, late-mature to decadent stands were sampled and from their structure we infer that the early seral tree species are Pinus albicaulis and Abies lasiocarpa and to a much lesser degree Picea engelmannii and even Pseudotsuga menziesii (though ideally these sites are above the cold limits of Pseudotsuga). With stand break-up (heavy mortality in $P$. albicaulis and $A$. lasiocarpa at 150 to 200 years, or older) a second flush of $A$. lasiocarpa and $P$. engelmannii is established. As remarked by Pfister et al. (1977) these sites are apparently unfavorable for Pinus contorta.

Stands with closed canopies are quite depauperate, however with canopy openings Ribes montigenum is common to well represented, usually in openings that appear to receive greater snow deposition than the majority of the stand. Forbs with a consistent presence include Pyrola secunda, Aquilegia flavescens, and Arnica cordifolia. The higher elevations of BLM Centennial holdings are used as sheep range which may account for the greater than expected cover of Poa pratensis and lesser amounts of forbs.

Soils- All sampled stands occurred on Tertiary volcanics which weather into nutrient-poor, acidic medium-textured soils. The ground surface had less than $10 \%$ exposed rock or soil and more than $80 \%$ litter.

Other Studies- This type was originally described from Utah by Pfister (1972) and documented for south-central and southwestern Montana by Pfister et al. (1977) and adjacent Challis and Open Northern Rockies Sections by Steele et al. (1981). It is sporadically distributed across western Wyoming and here evidences some departure from the type, e.g. the importance of seral $P$. contorta, as opposed to the lack of this species described above for southwestern MT. The eastern extremity of distribution is Colorado (Langenheim 1962) and to the south, southern Utah (Youngblood and Mauk 1985).

\section{Element Code- CEGL000331 \\ Edition / Author- 99-11-16 / S.V. Cooper, MTNHP}

\section{Abies lasiocarpa / Thalictrum occidentale Forest \\ (ABILAS / THAOCC) subalpine fir / western meadowrue}

\section{Natural Heritage Conservation \\ Rank- G4 / S3}

Environment- Abies lasiocarpa / Thalictrum occidentale a common plant association in northwestern Wyoming and extends at least as far as the north-facing flank of the Centennial Range in Montana; it has not been documented from areas farther north. It occurs on cool, moist slopes of all degrees of inclination and primarily on north- to east-facing aspects. The sampled elevation range was 7,400 to $8,200 \mathrm{ft}$., but in reconnaissance was noted to occur as low as 7,100 and as high as 8,400 ft. Abies lasiocarpa / Thalictrum occidentale grades to Abies lasiocarpa / Arnica cordifolia or Calamagrostis rubescens on warmer, drier exposures.

Vegetation- The sampled stands in the Centennial Range were primarily of late-seral to old-growth conditions with long-lived (300+ 
years) Pseudotsuga menziesii, or occasionally Pinus contorta, dominating the upper canopy and A. lasiocarpa and Picea engelmannii at least common, usually abundant as understory pole and sapling-sized components. At the highest elevations of this type Pinus albicaulis can be an important seral component; the importance of $P$. contorta also appears to increase with increasing elevation. Based on age of breast-height cores it appears extensive stand-replacing fires occurred 182+ and 248+ years ago. Fire-scarred individuals were not found within stands, only on ridgelines where stem density is less and fuel loading is light.

Undergrowth cover is strongly dependent upon tree canopy cover; open stands have a nearly continuous forb cover and closed, generally younger stands have scattered forbs totaling less than 5\% cover. Thalictrum occidentale, Osmorhiza chilensis (?) or Arnica cordifolia are dominant, but the two foregoing spp. must be at least well-represented (common in closed canopy stands) to be diagnostic for the type. Other forbs with relatively high constancy are Aquilegia flavescens, Aster engelmannii and Erythronium grandiflorum. Calamagrostis rubescens, Poa pratensis and Carex geyeri are the only graminoids with even moderate constancy and virtually never attain the swardlike cover they typically do in other associations. Total shrub cover seldom exceeds 5\% but Lonicera utahensis, Symphoricarpos oreophilus and Shepherdia canadensis are present with at least $50 \%$ constancy.

Soils- Tertiary extrusive volcanics, predominantly rhyolite, dominate much of the north flank of the Centennial Range and all of the sample plots were located on this substrate which has weathered to deep, well-drained substrates. This type has also been noted on sedimentary substrates, primarily sandstone. We suspect calcareous substrates are effectively drier and support primarily Abies lasiocarpa / Arnica cordifolia or Abies lasiocarpa / Juniperus communis.

Other Studies- This type was first described for northwestern Wyoming and eastern Idaho by Steele et al. (1983) and a portion of Pfister et al. (1977) plots may also better fit the description of this type than that of Abies lasiocarpa/Arnica cordifolia, where they were grouped. Cole (1982) has described a very similar type for the Blue Mountains of northwestern Oregon.

Comments- Cattle grazing in this type has led, where canopy cover is reduced, to the introduction of locally dense patches of Poa pratensis and trampling of native vegetation; where surfaces remain moist into mid-to latesummer, soil compaction and hummocking are evident.

\section{Element Code- CEGL000338}

Edition / Author- 99-11-16 / S.V. Cooper, MTNHP

\section{Picea (engelmannii x glauca, engelmannii) / Equisetum arvense Forest \\ (PICEA / EQUARV) spruce / common horsetail}

\section{Natural Heritage Conservation Rank- G4 / S4}

Environment- Picea/ Equisetum arvense is a major wetland type within the Picea series, being present throughout the Intermountain West, though little appropriately wet habitat is present in southwestern Montana. Patch size usually does not exceed a few tenths of an acre. Picea/ Equisetum arvense generally occurs on flat sites such as sub-irrigated terraces, seeps, fen margins and toeslopes that receive sufficient subsurface flow to be continuously saturated, or nearly so. Standing water may be present well into summer.

Vegetation- Stands are characteristically dominated by mature, not infrequently old growth size Picea with a low density of often severely suppressed specimens in the understory. Scattered Abies lasiocarpa and Pinus contorta are often present on raised microsites (windthrow hummocks) and may be generally distributed on drier sites within the type, but these species never create a dominant aspect. 
The wettest or youngest examples of this type have Salix spp. (often S. bebbiana) present but more typically Cornus sericea, Ribes lacustre and Symphoricarpos albus are the only shrubs present. Equisetum arvense is diagnostic for this type, frequently with minor coverage, especially on sites with a thick carpet of bryophytes. A tally of other obligate or facultative hydrophytes, e.g. Streptopus amplexifolius, Parnassia fimbriata, Senecio triangularis, Geum macrophyllum and Calamagrostis canadensis (or C. neglecta) should confirm the hydrophytic nature of these sites.

Soils- The only site sampled for soils was developed on fine-textured alluvium and given the landscape position of other sites noted in reconnaissance various textures of alluvium would be expected. Generally a thick mat of mor humus is expected (up to $18 \mathrm{~cm}$ thick): for the sampled site humus was only $8 \mathrm{~cm}$ thick, but the upper soil horizons are also rich with organic material.

Other Studies- This association, or the very similar P. engelmannii / Equisetum arvense, has been documented from the east slope of the Cascades in Washington (Williams \& Lillybridge 1983, Williams and Lillybridge 1990) and Oregon (Kovalchik 1987), east to Montana (Pfister et al. 1977, Hansen et al. 1995) and south through Wyoming (Steele et al. 1983), to Idaho (Steele et al. 1981), Utah (Padgett et al. 1989) and Colorado (Cooper and Cottrell 1990).

Comments- This association is particularly susceptible to livestock/wildlife trampling throughout the growing season; resulting damages include exposed substrate and consequent erosion, loss of vegetation and introduction of weeds.

\section{Element Code- CEGL000408}

Edition / Author- 99-11-16 / S.V. Cooper, MTNHP

\section{Picea engelmannii / Calamagrostis canadensis Forest}

(PICENG / CALCAN) Spruce/Bluejoint reedgrass

\section{Natural Heritage Conservation Rank- G4 / S3}

Environment- The Picea engelmannii / Calamagrostis canadensis community occurs at low to high elevations in the mountains throughout Montana. It is generally restricted to flat to gently sloping sites with poor drainage, such as fen and lake margins, toeslopes, and low stream and river terraces. Soil texture varies from silt to sandy loam with some redox concentrations present. This community is usually temporarily flooded in the spring, and stands have a high water table year round. Stands are characterized by a large amount of micro-topography stemming from windthrown spruce. Adjacent wetter communities include Salix drummondiana or Betula glandulosa shrublands, or Carex sp. dominated flats. Adjacent drier communities are usually upland conifer forests dominated by Abies lasiocarpa or Pseudotsuga menziesii (Hansen et al. 1995).

Range- This community is present in Montana, Idaho, Utah, and Wyoming.

Vegetation- The overstory is dominated by spruce, and Abies lasiocarpa may also be present. There is low coverage of shrubs, although the diversity of shrub species present is fairly high. Calamagrostis canadensis and/ or Calamagrostis stricta dominate the ground layer. Other associated species include Carex utriculata, Solidago canadensis, and Equisetum arvense (Hansen et al. 1995).

Similar Communities- Youngblood et al. (1985) described a similar community for western Wyoming and Jankovsky-Jones (1997) for southeastern Idaho. Some Utah stands in Padgett et al. (1989) which are classified as Conifer/Calamagrostis canadensis have a Picea engelmannii - dominated overstory. 
the Picea/Calamagrostis canadensis community is a late seral phase of the Abies lasiocarpa/Calamagrostis canadensis habitat type described by Pfister et al. (1977). Gaps in the forest that are created by disturbances such as windthrow are dominated by shrubs such as Alnus incana and Salix drummondiana.

Management- Timber productivity in this type is moderate to high. Because of high water tables, windthrow following harvest can be a problem, as can soil damage resulting from harvesting and site preparation; timing of activities is important to avoid damage.

Palatability of Calamagrostis canadensis is moderate to high, and foliage is most palatable when young. However, wet conditions during this time period make soil susceptible to damage from livestock. If levels of utilization of Calamagrostis canadensis stay high for long periods, production of Calamagrostis canadensis can decline (Hansen et al. 1995).

Other Studies- In Montana and Idaho, Picea glauca and Picea engelmannii hybrids are common, thus, lumping both species together is practical for classification purposes (Hall and Hansen 1997; Hansen et al. 1995). The type here represents stands dominated by $P$. engelmannii or Picea hybrids as described by Pfister et al. (1977), Steele et al. (1981) and Mauk and Henderson (1984).

\section{Element Code- CEGL000356}

Edition / Author- 99-10-18 / J. Greenlee, MTNHP

Picea (engelmannii x glauca, engelmannii) / Galium triflorum Forest.

(PICEA/GALTRI) Engelmann spruce / sweetscented bedstraw

\section{Natural Heritage Conservation Rank- G4 / S4}

Environment- Picea / Galium triflorum is usually associated with benches and terraces bordering riparian reaches, seep areas and moist toeslopes, from the lowest forested elevations to the mid subalpine zone. In southwestern MT the observed range was from 7,400 to $8,400 \mathrm{ft}$., considerably extending the range cited (2,800-7,050 ft.) by the Montana Riparian/Wetland Association (Hansen et al. 1995). In the vicinity of the drier Tendoy Range, Picea / Galium triflorum has a wide elevational range, but in the Centennial Range it occurs only at lower treeline. Upslope and at higher elevations with comparable environments, it gives way to Abies lasiocarpa / Actaea rubra. In other words, at least in southwestern Montana, this type is present only beyond the geographical or ecological limits of Abies lasiocarpa. In our study area Picea / Galium triflorum has very limited acreage as it was noted in reconnaissance to occur only as a narrow stringer in toeslope positions or on very narrow streamside terraces. These sites are considered to receive greater than average moisture due to subsurface flow, but most sites probably would not be considered jurisdictional wetlands.

Vegetation - We have followed the naming convention of Pfister et al. (1977) and perpetuated by Hansen et al. (1995) by referring to Picea at the genus level recognizing that most of the Montana populations are hybrid swarms of $P$. glauca $\times$ P. engelmannii. Cursory observations of cone size, scale morphology and length of the free scale indicated that $P$. engelmannii characters were predominant. In the portions of this p.a. with higher moisture status Picea generally dominates a closed mature canopy and other age classes are poorly represented. Pseudotsuga menziesii and Pinus contorta are important seral species on the drier portion of this habitat.

Undergrowth coverage is highly variable but generally the diagnostic species Galium triflorum, Actaea rubra and Streptopus amplexifolius are only present, seldom even common and only approach well represented. Other moist-site species with moderate constancy are Heracleum lanatum, Smilacina stellata, Mertensia ciliata, and Geum macrophyllum and on disturbed sites (by cattle) Urtica dioica. Thalictrum occidentale, Osmorhiza chilensis and Taraxacum officinale 
(on disturbed sites) often are well represented. Ribes lacustre, Symphoricarpos albus and Rubus parviflorus are the only shrubs consistently present, though only $S$. albus is ever well represented. Some sites support a nearly continuous layer of bryophytes, but others have only a solid layer of litter.

Soils- Alluvial and colluvial deposits are the predominant substrates, they are derived from the country rock and generally silty in texture.

Other Studies- Picea engelmannii / Galium triflorum was first described by Pfister et al. (1977) as occurring from central MT southward, reaching its greatest abundance in the Gallatin Nat. Forest vicinity. It is also common in northwestern Wyoming (Steele et al. 1983) and central Idaho (Steele et al. 1981) recognized here by the same suite of indicator species, though apparently pure $P$. engelmannii populations dominate these extra-MT sites.

Comments- This type is especially susceptible to livestock trampling damage early in the growing season when soils are saturated; damages include vegetation destruction and severe hummocking and weed introduction.

\section{Element Code- CEGL000409}

Edition / Author- 99-11-16 / S.V. Cooper, MTNHP

\section{Picea (engelmannii x glauca, engelmannii) / Senecio streptanthifolius Forest (PICEA / SENSTR) spruce / Rocky Mountain butterweed}

\section{Natural Heritage Conservation Rank-}

\section{G4 / S4}

Environment- This is the driest of Picea series types identified for Montana. Within the southwestern Montana study area, as is true regarding its overall distribution, it occurs only on calcareous substrates; thus it is areally extensive only in the Tendoy and Ruby ranges, where it was sampled and noted in reconnaissance. Both ranges are also in a rainshadow further exacerbating site severity. Its elevation range was from 7,600 to $8,800 \mathrm{ft}$ with generally cool aspects and steeper slopes predominating. Sites often have considerable exposed soil, gravel and rock with litter not much exceeding 40\%; alternatively, some sites have bryophyte coverage exceeding $40 \%$.

Adjacent sites with yet drier regimes are within the Pseudotsuga menziesii or Pinus flexilis series or occasionally an open Festuca idahoensis- or Artemisia tridentata $v$. tridentata-dominated complex; moister sites occupy restricted environments within this mountain range.

Vegetation- Seral tree dominants on this association are Pseudotsuga menziesii and Pinus flexilis and here they are slow growing. Picea is slow to establish on these droughty sites and is equally slow growing. Severity of site is also reflected in canopy structure, which usually does not exceed $80 \%$ and often is around 40-50\%, especially where natural mortality has removed canopy dominants.

The undergrowth is generally low in coverage, not exceeding $30 \%$, even when canopy structure is quite open. Composition can be quite diverse with many of the consistently present species drawn from the adjacent grasslands, such as Potentilla diversifolia, Townsendia montana, Astragalus miser, Synthyris pinnatifida, Antennaria microphylla, Solidago multiradiata and Frasera speciosa. None of the undergrowth species assert dominance in terms of cover, even the diagnostic Senecio streptanthifolius. Only Arnica cordifolia is well represented. On steep north-facing slopes bryophyte cover may be substantial, exceeding $20 \%$.

Soils- As noted above all examples of this type occurred on calcareous substrates, usually limestone. Limestone weathers to a silty texture but these soils have much exposed gravel and rock, in combination usually exceeding $20 \%$.

Other Studies- This type was first identified by Pfister et al. (1977) for Montana. It has not been recognized elsewhere, though the portion of Picea engelmannii / Arnica cordifolia, 
which occurs on calcareous substrates in the Absaroka and Owl Creek Ranges of northwestern Wyoming and supports $S$. streptanthifolius, is virtually identical.

\section{Element Code- CEGL000414}

Edition / Author- 99-11-16 / S.V. Cooper, MTNHP

Pinus contorta / Carex rossii Forest (PINCON / CARROI) lodgepole pine / Ross sedge

\section{Natural Heritage Conservation Rank- G5 / SP}

Environment- Within the Dillon R.A. this community is apparently restricted to peculiar parent materials, possibly hydrothermally altered volcanics. Pinus contorta / Carex rossii was noted as very small stands (mostly $<1$ acre) at scattered, low gradient valley locations surrounded by a mosaic of Artemisia tridentata- and Festuca idahoensis-dominated shrublands/grasslands. The soils are lightcolored, highly reflective, virtually without structure but well drained and have very little incorporated organic matter. We suspect these substrates, as is typical for hydrothermally altered substrates, are more acidic than those of the surrounding rangelands; the actual composition for these parent materials have not been confirmed by persons with geological expertise.

Vegetation- Tree canopies are open (40-70\% cover) and dominated by Pinus contorta and Pinus flexilis. These trees were the only species noted to be reproducing. Although these sites are well within range of other conifers, seed dispersal, most particularly Pseudotsuga menziesii, none have been able to colonize these sites. This may be due to the unusual nature of the substrate and the fact that this environment is outside the climatic window of species associated with subalpine conditions.

The undergrowth is very sparse (combined cover not exceeding 5\%) with extremely low species diversity, generally fewer than five species per 1/10 acre. Carex rossii is the most abundant species and, with Carex geyeri, the only graminoids encountered. Penstemon aridus was the only forb consistently present.

Soils- Except for 5-20\% litter, the surface is exposed soil with little incorporated organic matter resulting in a notably light-colored and reflective surface. There is virtually no profile development and soils are well drained. Expected low $\mathrm{pH}$ values and perhaps peculiar micronutrient and nutrient levels strongly condition the vegetation response.

Other studies- Pinus contorta / Carex rossii is described as an uncommon community type from the Yellowstone region southward to the Wind River Range (Steele et al. 1983); in the Yellowstone region it supports only scattered Pinus flexilis (or P. albicaulis) and is associated with extrusive volcanics (mostly rhyolite) that weather to a coarse-textured, excessively-drained substrate, much like those that it occupies within our study area.

This plant association may require a special recognition, e.g. Pinus contorta / Carex rossiihydrothermal sites, as the substrate is so unusual as to constitute a unique environment.

\section{Element Code- CEGL000144}

Edition / Author- 99-11-16 / S.V. Cooper, MTNHP

\section{Pseudotsuga menziesii / Arnica cordifolia Forest. \\ (PSEMEN / ARNCOR) Douglas-fir / heartleaf arnica}

\section{Natural Heritage Conservation Rank-} G4 / S4

Environment- Pseudotsuga menziesii / Arnica cordifolia occupies the coolest-driest exposures supporting the Pseudotsuga series in southwestern Montana, on moderate to steep slopes with north- through east-facing aspects. The sampled range was 6,200 to $8,050 \mathrm{ft}$. but the upper elevational limits are expected to be somewhat greater. Pseudotsuga menziesii / 
Arnica cordifolia is often noted to extend continuously from slope bottoms, just above toeslopes, to near ridge shoulders or to grade to Pseudotsuga menziesii / Symphoricarpos oreophilus on upslope positions. This type is generally set in a landscape where drier exposures are Artemisia tridentata or Festuca idahoensis-dominated rangelands, though there may be a narrow bordering fringe of open Pseudotsuga- or Pinus flexilis-dominated forest with bunchgrass undergrowth.

Vegetation- Forest canopy cover is generally greater than $60-70 \%$, dominated by Pseudotsuga menziesii with minor amounts, usually less than $10 \%$ canopy cover, of Pinus flexilis and Juniperus scopulorum. Scattered, stunted individuals (termed "accidentals") of Abies lasiocarpa or Picea indicate a transition to the moister-cooler environments characterized by these species in late successional stages.

Undergrowth is normally depauperate, especially in stands approaching late seral to old-growth status. The shrub component is very sparse with only Ribes viscosissimum and Symphoricarpos oreophilus consistently represented. Artemisia tridentata skeletons are present in younger stands indicating these sites may pass through a seral stage dominated by this species and other rangeland components. Poa nervosa appears to be the most constant of graminoids but is not well represented. Forb diversity is moderately diverse with the diagnostic Arnica cordifolia generally well represented to abundant and Antennaria racemosa, Geranium viscosissimum, Astragalus miser, Potentilla gracilis having high constancy. Bryophyte and lichen combined cover generally exceeds $30 \%$ and runs as high as $90 \%$ plus.

Soils- All sampled stands occurred on calcareous (limestone) substrates, but this type was noted on volcanics in the course of reconnaissance. These materials weather to loams to silt loams and have little $(<10 \%)$ exposed rock, gravel or soil.

Comments- Sampled stands had all been entered either for clearcutting or salvage; pressure on these stands to provide timber and fuel for the mining industry was very intense in the latter decades of 1800's and opening decades of 1900's.

Other Studies- Pseudotsuga menziesii / Arnica cordifolia has been identified on a variety of parent materials for central and southwestern Montana (Pfister et al. 1977) where it is considered too dry to support Calamagrostis rubescens or Carex geyeri (and their comparably named Pseudotsuga menziesii series plant associations). This type extends to northwestern Wyoming (Steele et al. 1983) and east central Idaho (Steele et al. 1981) where two phases are recognized.

Element Code- CEGL000427

Edition / Author- 99-11-16 / S.V. Cooper, MTNHP

Pseudotsuga menziesii / Calamagrostis rubescens Forest

(PSEMEN / CALRUB) Douglas-fir / pine grass

\section{Natural Heritage Conservation Rank-} G5 / S5

Environment- Pseudotsuga menziesii / Calamagrostis rubescens is an uncommon type on BLM holdings, documented only from the Madison Co. vicinity on relatively warm, moist slopes at 5,900 ft upward to ridge crests. This type was not found in the Tendoy Range and noted only as fragments in the Centennial Range and vicinity, but reasons for its absence were not apparent.

Vegetation- The tree canopy is usually closed and dominated by Pseudotsuga menziesii; Pinus flexilis and Juniperus scopulorum may be well represented, but usually they occur just as scattered individuals. These sites are accessible and generally support only secondgrowth stands.

Depending on canopy density and other factors the undergrowth can range from a luxuriant 
sward of Calamagrostis rubescens and Carex geyeri with associated forbs to depauperate conditions where $C$. rubescens occurs in thin patches and forbs are scarce. Forbs with high frequency and that are occasionally well represented include Arnica cordifolia, Antennaria racemosa, Aster conspicuus, and Galium boreale.

Soils- This type has been documented from both granitic and calcareous substrates with sandy and silty loams. There is little exposed soil or gravel/rock; litter and bryophytes contribute most ground cover.

\section{Other Studies- Pseudotsuga menziesii /} Calamagrostis rubescens is such a common and broadly distributed plant association in Montana that four phases have been recognized reflecting different geographical influences and ecological conditions. Likewise for Idaho (Steele et al. 1981), Wyoming (Steele et al. 1983), Washington (Daubenmire and Daubenmire 1968) on into southern Utah (Mauk and Henderson 1984) geographical and ecological distinctions have been recognized at the phase level of the association.

\section{Element Code- CEGL000429}

Edition / Author- 99-11-16 / S.V. Cooper, MTNHP

\section{Pseudotsuga menziesii / Juniperus communis Forest \\ (PSEMEN / JUNCOM) Douglas-fir / common juniper}

\section{Natural Heritage Conservation Rank- G4 / S4}

Environment- This is a very minor type of small stand size on the Red Rocks Lake National Wildlife Refuge (RRLNWR). In both the Beaverhead Mountains Section and in central Montana, where is has been previously inventoried, it is one of the warmest and driest plant associations in the Pseudotsuga series. On the RRLNWR, this type occurred at the lower forest margin, on southerly or westerly exposures on both calcareous and volcanic substrates. RRLNWLR expressions of the Pseudotsuga menziesii / Juniperus communis type quite clearly differ from the type at large by lacking seral Pinus ponderosa. It is unknown how Pseudotsuga menziesii / Juniperus communis of the RRLNWLR differs from other comparably dry Pseudotsuga series types, such as Pseudotsuga menziesii / Symphoricarpos albus or Pseudotsuga menziesii/ Arnica cordifolia. All these types are considered too dry to support Carex geyeri or Calamagrostis rubescens (or Pinus contorta, except when acid-weathering substrates are involved)

The tree canopy is relatively open, grading to that of woodland ( $<60 \%$ tree canopy cover). Short-statured Pseudotsuga menziesii dominates all developmental stages and Pinus flexilis is a minor associate, especially on calcareous substrates; the Refuge, Centennial Valley and much of southwestern MT are too cold to support Pinus ponderosa as a component species.

Vegetation- Arctostaphylos uva-ursi and Juniperus communis dominate the undergrowth, where Spiraea betulifolia is often well represented. Both the graminoid and forb layers are depauperate with the only even moderately constant species being Festuca idahoensis, Carex rossii, Galium boreale, Smilacina racemosa and Astragalus miser.

\section{Element Code- CEGL000439}

Edition / Author- 99-11-16 / S.V. Cooper, MTNHP

Pseudotsuga menziesii / Physocarpus malvaceus Forest

(PSEMEN / PHYMAL) Douglas-fir / ninebark

\section{Natural Heritage Conservation Rank- G5 / S5}

Environment- Pseudotsuga menziesii /

Physocarpus malvaceus is a common forest type of the Garnet Resource Area but is a minor type within the Dillon R.A. It is found 
primarily on steep, northwest, through north, to east-facing slopes. Slopes often have a bryophyte / lichen encrusted rocky limestone substrate that verges on unstable. This type is occasionally found to extend continuously from toe-slopes or drainage bottoms up to slope shoulders. Most of this type is found below $6,000 \mathrm{ft}$., the documented occurrences being below $4,600 \mathrm{ft}$. It was noted to grade to Pseudotsuga menziesii /Scree of steeper, rockier positions and Pseudotsuga menziesii / Symphoricarpos oreophilus (or S. albus) or Pseudotsuga menziesii /Calamagrostis rubescens on warmer exposures.

Vegetation- Pseudotsuga menziesii dominates the mostly closed canopy from seral through old-growth stages with Pinus ponderosa and Juniperus scopulorum consistently represented, their cover generally not exceeding $20 \%$. $P$. ponderosa persists into the old-growth stage reproducing where canopy gaps are sufficiently large.

The undergrowth is dominated by shrubs, with Physocarpus malvaceus and Acer glabrum usually being abundant and Symphoricarpos albus, Spiraea betulifolia, Clematis occidentalis and Berberis repens consistently common to well represented. Calamagrostis rubescens is the only graminoid with high constancy, but in southwestern MT lacks the high coverage that distinguishes a phase of this association. Smilacina stellata and Aster conspicuus have high constancy and are often abundant; Heuchera parviflora and Disporum trachycarpum are consistently present but poorly represented.

Soils- All examples of Pseudotsuga menziesii / Physocarpus malvaceus in southwestern Montana were noted for calcareous substrates (this is not true throughout its much broader geographic range). In degree of development, rock and gravel content and depth soils are highly variable; some steep north-facing sites verge on being scree sites but an intact, nearly continuous bryophyte layer indicates little movement (such habitat conditions also obtain in eastern Washington and northern Idaho).
Other Studies- Pseudotsuga menziesii / Physocarpus malvaceus is one of the plant associations recognized in the Daubenmire's (1968) pioneering work of forest classification in eastern Washington and northern Idaho. Now this plant association (or regional permutations thereof) is recognized from as far west as interior British Columbia (Braumandl and Curran 1992) and the Okanogan of Washington State (Williams and Lillybridge 1983) to as far south as northern Utah (Mauk and Henderson 1984).

Comments- Being broadly distributed, there is much floristic diversity within this type. It also manifests the phenomena of factor compensation in a remarkable fashion; in the moist climatic regime of northern Idaho its found only on warmer exposures, south-facing slopes and in the much drier regimes of central and southwestern Montana on steep northerly exposures.

\section{Element Code- CEGL000447}

Edition / Author- 99-11-16 / S.V. Cooper, MTNHP

\section{Pseudotsuga menziesii / Spiraea betulifolia Forest \\ (PSEMEN / SPIBET) Douglas-fir / white spiraea}

\section{Natural Heritage Conservation Rank- G5 / S4}

Environment- Pseudotsuga menziesii / Spiraea betulifolia is cited as a minor type in Montana (Pfister et al. 1977) and northwestern Wyoming (Steele et al. 1983) but on the northern flank of the Centennial Range it is an important type. It is found along with Pseudotsuga menziesii / Calamagrostis rubescens, at lower timberline and generally on other than north-facing slopes. If one uses the approach of Steele (et al. 1983) who recognizes the indicator importance of S. betulifolia (wellrepresented) to be greater than that of Calamagrostis rubescens, then many of the warmer, calcareous exposures at lower elevations $(<7,000 \mathrm{ft}$.) would be identified as 
either the Calamagrostis rubescens or Spiraea betulifolia phases of Pseudotsuga menziesii / Spiraea betulifolia. (We have followed the Montana approach that treats stands with $C$. rubescens or Carex geyeri dominance as members of the Calamagrostis rubescens type.) Often Pseudotsuga menziesii / Spiraea betulifolia borders on valley locations or parks of Festuca idahoensis- or Artemisia tridentata ssp. vaseyana-dominated grasslands/ shrublands. North-facing slopes adjoining the valley are sometimes sufficiently moist to support the Picea engelmannii or Abies lasiocarpa series.

Vegetation- The tree stratum is usually dominated by $P$. menziesii with a minor component of Pinus flexilis, especially on calcareous substrates; or Populus tremuloides at the lower fringes of the type; or P. menziesii mixed with Pinus contorta on volcanic substrates. Spiraea betulifolia is a major component as a low shrub layer that sometimes includes high coverage of Pachistima myrsinites and Berberis repens and the presence of Amelanchier alnifolia (heavily browsed) and Symphoricarpos oreophilus. Arnica cordifolia, Aster conspicuus, Aster engelmannii, Antennaria racemosa, Fragaria virginiana and Osmorhiza chilensis comprise the forbs that are most predictably represented. In the modal expression of this type the graminoid component is minimal with only $C$. rubescens, C. geyeri, and Poa nervosa consistently present.

\section{Element Code- CEGL000457}

Edition / Author- 99-11-16 / S.V. Cooper, MTNHP

\section{Pseudotsuga menziesii / Symphoricarpos oreophilus Forest \\ (PSEMEN / SYMORE) Douglas-fir / mountain snowberry}

Natural Heritage Conservation Rank- G5 / S3

Environment- Pseudotsuga menziesii / Symphoricarpos oreophilus occupies cooler exposures, moderate to steep slopes with northwest- through southeast-facing aspects. The sampled range was 6,200 to $8,050 \mathrm{ft}$. but the upper elevation limits are expected to be somewhat greater; it often was noted to extend continuously from midslope to near ridge shoulders. This type is generally set in a landscape where drier exposures are Artemisia tridentata or Festuca idahoensis-dominated rangelands though there may be a narrow bordering fringe of open Pseudotsuga- or Pinus flexilis-dominated forest with bunchgrass undergrowth.

Vegetation- Pseudotsuga menziesii dominates a closed tree stratum wherein Pinus flexilis and Juniperus scopulorum are consistently present, usually not exceeding $20 \%$ cover. All the stands we sampled had been entered at some time in the past for clearcutting or salvage logging. Abundant skeletons of Artemisia tridentata in younger stands indicate a seral stage probably dominated by this and other rangeland species.

Undergrowth tends to be depauperate in number of species and coverage with no set pattern of dominance; we noted shrubs or graminoids or forbs could be dominant. Symphoricarpos is $100 \%$ constant but seldom even well represented; other highly constant shrubs include Ribes cereum and Juniperus communis. Festuca occidentalis and Poa pratensis and $P$. nervosa are consistently present, sometimes well represented. Arnica cordifolia, Solidago multiradiata, Astragalus miser and Phlox multiflora have a high constancy and are occasionally well represented. Bryophyte and lichen cover varies widely from $30 \%$ plus to virtually none, the variability ostensibly based on time since the last disturbance.

Soils- Pseudotsuga menziesii / Symphoricarpos oreophilus was found only on calcareous substrates. Almost invariably these substrates weather to silt loams or loams. Surfaces have less than 5\% exposed gravel, rock and soil (combined); they are covered with litter or bryophytes/lichens. 
Other Studies- Pseudotsuga menziesii / Symphoricarpos oreophilus is very broadly distributed, ranging from east of the Cascades to northeastern Washington (Williams and Lillybridge 1983, 1985) and south to Blue Mountains of Oregon (Johnson and Simon 1987). It experiences a large distribution gap in northern Idaho and western Montana but then in the expansive calcareous substrates of southwestern Montana it resurges in importance. This type is known from central Idaho (Steele et al. 1981), western Wyoming (Steele et al. 1983) and Colorado (Johnson 1987) to southern Utah (Youngblood and Mauk 1985) and northern New Mexico (Muldavin 1994). It is found on other than calcareous substrates in regions outside southwestern Montana.

\section{Element Code- CEGL000462}

Edition / Author- 99-11-16 / S.V. Cooper.

\section{N eedle-leaved E vergreen Woodlands}

\section{Abies lasiocarpa / Juniperus communis Woodland \\ (ABILAS / JUNCOM) subalpine fir / common juniper}

\section{Natural Heritage Conservation Rank- G4G5 / S3}

Environment- This is a very common plant association, identified from the drier mountain ranges of eastern Oregon and Washington, eastward into Montana and Wyoming and south as far as New Mexico and Arizona. Within the context of the relatively mesic, generally north-facing flank of the Centennial Range, Abies lasiocarpa / Juniperus communis represents habitats experiencing greater moisture stress than other common A. lasiocarpa types having either western meadowrue (Thalictrum occidentale), pinegrass (Calamagrostis rubescens) or shiny-leaf spiraea (Spiraea betulifolia) as the diagnostic species. On Sheep Mountain RNA, Abies lasiocarpa /
Juniperus communis was encountered on warmer exposures (those with a westerly component), and above 7,800 ft., though it is capable of occurring at much lower elevations. It generally grades to Abies lasiocarpa / Ribes montigenum (mountain gooseberry), or to Abies lasiocarpa / Thalictrum occidentale and Abies lasiocarpa / Arnica cordifolia of moister environments.

Being a woodland, tree canopy cover is generally below $60 \%$ and tree form approaches "stunted" with heights barely exceeding 40 feet at more than 350 years of age. Picea engelmannii (Engelmann spruce) and Abies lasiocarpa are present mostly in the sapling/ seedling layer, though scattered mature and old-growth Picea engelmannii are typically present as well. This association is at the dry extreme of Abies lasiocarpa distribution and this species probably will never achieve canopy dominance. The canopy dominant in most of these stands is Pseudotsuga menziesii (Douglas-fir), though Pinus flexilis (limber pine) is a major component in patches.

Vegetation- Juniperus communis (common juniper) dominates the undergrowth, its cover generally exceeding $10 \%$. The graminoid element is especially depauperate with only traces of Poa reflexa (nodding bluegrass) and Carex rossii (Ross sedge). Aster conspicuus (showy aster) is the only forb consistently occurring well represented; $A$. conspicuus, Potentilla gracilis (slender cinquefoil), and Sedum lanceolatum (lanceleaved stonecrop) were noted as the prevalent forbs consistently present throughout these drier woodland environments.

Element Code- CEGL000919

Edition / Author- 99-11-16 / S.V. Cooper.

Cercocarpus ledifolius / Festuca idahoensis Woodland

(CERLED / FESIDA) curl-leaf mountain mahogany / Idaho fescue

Natural Heritage Conservation RankG3 / S2 
Environment- The only documented occurrence for southwestern Montana is on steep residual mountain slopes at elevations around 6000 feet. The total cover of soil, gravel, and rock usually exceeds $40 \%$ and the soil surface is stable with no evidence of accelerated erosion. Parent materials are granitic and soils are Ustorthents and are moderately deep and very cobbly. Textures vary from loamy sands to sandy loams. The soils are non-calcareous and their available water holding capacities are low due to the coarse soil textures and abundant coarse fragments. This association can be expected on other, especially calcareous, parent materials.

Adjacent Communities- The Cercocarpus ledifolius / Festuca idahoensis and C. ledifolius I Pseudoroegneria spicata types are ecologically similar and intergrade. Of the two types, C. ledifolius / Pseudoroegneria spicata occurs in slightly more xeric situations. The Pseudotsuga menziesii / F. idahoensis type may be found on adjacent more mesic sites that are less rocky and have deeper soils.

Vegetation- Cercocarpus ledifolius and Pseudoroegneria spicata are abundant. Festuca idahoensis cover generally exceeds $5 \%$. Other species that are generally present with cover exceeding $1 \%$ include Artemisia frigida, A. tridentata, Opuntia polyacantha, and Poa sandbergii. Bromus tectorum may be abundant where surface disturbance has occurred through grazing.

Other Studies- This type has been previously described from northeastern Oregon (Johnson and Simon 1987) and central Idaho (Schlatterer 1972).

Element Code- CEGL000962

Edition / Author- 99-11-16 / S.V. Cooper.

Juniperus scopulorum / Artemisia tridentata Woodland

(JUNSCO / ARTTRI) Rocky Mountain juniper / big sagebrush

Natural Heritage Conservation Rank-

\section{G2Q / S2?}

Environment- The type occurs on plateaus and residual mountain slopes at elevations between 5500 and 6000 feet. The total covers of soil, gravel, and rock averages $70 \%$. The soil surface is sometimes unstable because of a lack of adequate vegetation cover.

Soils are generally shallow or very shallow (i.e., lithic) and are derived from igneous or sedimentary parent materials and feature strong to violent effervescence. Textures vary from sandy loams to silt loams and are gravelly to very gravelly. Available water holding capacity varies from low to medium.

Vegetation- Juniperus scopulorum is the only tree species present. Species exceeding 5\% cover in the undergrowth include Artemisia tridentata, Opuntia polyacantha, Pseudoroegneria spicata, Aristida longiseta, Bouteloua gracilis, Oryzopsis hymenoides, and Stipa comata.

Adjacent Communities- The Artemisia tridentata / Pseudoroegneria spicata community type often occurs on adjacent less rocky sites with deeper soils. More xeric (or at least rockier) sites with similar soils feature the Juniperus scopulorum / Cercocarpus ledifolius community type.

Other Studies- This type has not been previously described but has been reported as a possible type in Colorado and Wyoming (Bourgeron and Engelking 1994).

Element Code- CEGL000743

Edition / Author- 99-11-16 / S.V. Cooper

Juniperus scopulorum / Cercocarpus ledifolius Woodland

(JUNSCO / CERLED) Rocky Mountain juniper / mountain mahogany

\section{Natural Heritage Conservation Rank-} G3? / S3?

Environment- This type occurs predominantly on steep dip slopes and residual mountain 
slopes at elevations between 5500 and 6500 feet. The total cover of soil, gravel, and rock usually exceeds $50 \%$ and the soil surface is often unstable because of a lack of adequate vegetation cover

Vegetation- Juniperus scopulorum is the only tree species present. Abundant Cercocarpus ledifolius characterizes the undergrowth. Other species that are generally present with cover exceeding $1 \%$ include Artemisia frigida, Opuntia polyacantha, Pseudoroegneria spicata, and Oryzopsis hymenoides.

Soils- Parent materials are predominantly quartzite or limestone and soils are generally Orthents and are shallow (lithic) and gravelly to very stony. Textures vary from loamy sands to loams. Most of the soils exhibit strong and/ or violent effervescence. Available water holding capacity is mostly low due to the coarse soil textures, abundant coarse fragments, and shallow depth.

Adjacent Communities- The Juniperus scopulorum / Cercocarpus ledifolius and C. ledifolius / Pseudoroegneria spicata types are ecologically similar and intergrade. Of the two types, J. scopulorum /C. ledifolius occurs in slightly more mesic situations. The $J$. scopulorum / Artemisia tridentata and A. tridentata / Pseudoroegneria spicata types adjoin J. scopulorum / Cercocarpus ledifolius on drier sites. The Pseudoroegneria spicata / Bouteloua gracilis type is often found on adjacent less rocky and less steep sites.

Other Studies- This type has been described only for Montana (Chaffee 1981).

\section{Element Code- CEGL000744}

Edition / Author- 99-11-16 / S.V. Cooper, MTNHP

$$
\begin{gathered}
\text { Juniperus scopulorum / Pseudoroegneria } \\
\text { spicata Woodland } \\
\text { (JUNSCO / AGRSPI) rocky mountain } \\
\text { juniper / bluebunch wheatgrass }
\end{gathered}
$$

Natural Heritage Conservation Rank-
G4 / S4

Environment: In eastern Montana this community type is commonly found in low to moderate relief rolling uplands as well as in badland arroyos/draws, often occurring adjacent to Juniperus scopulorum / Oryzopsis hymenoides but on warmer exposures (not strictly north-facing) with the same moderate to steep slopes. In eastern MT three of the four sampled stands were on calcareous substrates, though this h.t. is not confined to these substrates in this region. It has also been recorded for the BVHDMS occurring on rocky, primarily volcanic substrates with thin soils; substrate conditions here appear to be the determining factors in its occurrence. It is primarily associated with lower treeline positions and slope and aspect are only weak determining factors. In eastern MT., Juniperus scopulorum / Pseudoroegneria spicata also has more exposed soil and rock (often exceeding $50 \%$ ) than the associated Juniperus scopulorum / Oryzopsis hymenoides, . The same situation often obtains in western MT where bare soil and exposed rock usually constitute $60 \%$ plus of the substrate and litter is never more than $20 \%$ and adjacent shrubland types often have more developed, deeper soils.

Vegetation: As a result of the ubiquitous past cutting for fencing, stands of Juniperus scopulorum / Pseudoroegneria spicata in eastern MT are rather open, with coverage of 8 to $12 \mathrm{ft}$ tall Juniperus scopulorum not exceeding 50\%; in BVHDMS the tree canopy are also quite open (at most approaching 30\% canopy cover). We speculate tree coverage does not much exceed these figures due to limitations of site factors. The higher coverage of shrubs (up to $20 \%$ for Artemisia tridentata and A. frigida combined) that are reported in northeastern MT as opposed to southeastern Montana (Hansen and Hoffman 1988) is also attributable to seral conditions. In BVHDMS A. tridentata ssp. vaseyana and ssp. wyomingensis, A. frigida, Chrysothamnus nauseosus, and $C$. viscidiflorus have the highest constancy but comprise less than 5\% cover in the aggregate. The undergrowth is dominated by graminoids, chief among which and diagnostic of the type is Pseudoroegneria 
spicata, always well represented (40\% ave. cover in eastern MT only $10 \%$ on the BVHDMS). Carex filifolia and Koeleria cristata have high constancy and Bouteloua curtipendula is consistently present in the easternmost occurrences of this type. Forb diversity is moderately high and higher in western than eastern MT with not much overlap in high constancy species between regions; regardless of location, forb coverage's are generally low, not exceeding $10 \%$ except in the most open stands.

Other Studies: In a study centered on southeastern Montana, Hansen and Hoffman (1988) have best documented this type and Brown (1971) has also described it for badland landforms in the Ashland District, Custer National Forest. This association has been described as relatively common in Wyoming and extends as far south as Colorado.

\section{Element Code- CEGL000748}

Edition / Author- 99-12-03 / S.V. Cooper, MTNHP

Pinus albicaulis - Abies lasiocarpa Woodland [Provisional] (PINALB - ABILAS) whitebark pine subalpine fir

\section{Natural Heritage Conservation Rank-}

\section{G5? / S5}

Environment- The type occurs on glaciated mountain ridges and upper slopes at elevations above 8900 feet. The total cover of soil, gravel, and rock exceeds $50 \%$. The soil surface is often unstable because of a lack of adequate vegetation cover.

Vegetation- Pinus albicaulis, Abies lasiocarpa, Picea engelmannii, and occasionally Pseudotsuga menziesii occur in varying amounts in the often open, stunted, and wind-deformed tree layer. The undergrowth is highly variable in composition and few individual species ever exceed 5\% cover.

Adjacent Communities- This type occurs at, or near, upper timberline. It is often bordered below by the Abies lasiocarpa - Pinus albicaulis / Vaccinium scoparium type and above by alpine scrub. Unvegetated talus slopes sometimes interfinger with this type.

Soils- Soils are typically gravelly to very gravelly, feature a cryic temperature regime, and may be strongly to violently effervescent. Textures are sandy loams to loams. Soil depth is shallow to moderately deep. Available water holding capacity varies from low to medium.

Other Studies- This type has been described only in Montana, where it is quite common (Pfister et al. 1977).

Comments- Pinus albicaulis - Abies lasiocarpa, is actually a syntaxonomic unit above the plant association level because considerable undergrowth variation is accommodated within it as originally described. Pfister et al (1977) did not choose to decompose this unit on the basis of various undergrowth assemblages because it encompasses environments with very low production and similar management implications.

\section{Element Code- CEGL000128}

Edition / Author- 99-11-16 / S.V. Cooper, MTNHP

Pinus albicaulis / Festuca idahoensis Woodland

(PINALB / FESIDA) whitebark pine / Idaho fescue

\section{Natural Heritage Conservation Rank-}

\section{G4 / S4}

Environment- The Pinus albicaulis / Festuca idahoensis woodland association is a minor type recorded from the highest forested elevations, generally above 8,600 ft., of the Centennial and Tendoy Ranges on wind-swept ridgelines and west and southwest-facing slopes; this association is expected in other dry southwestern Montana Ranges. Ostensibly drier sites are Festuca idahoensis- or Artemisia 
tridentata var. vaseyana-dominated; on moister or less stressful exposures are found Abies lasiocarpa / Ribes montigenum, Abies lasiocarpa / Arnica cordifolia and Picea / Senecio streptanthifolius.

Vegetation- Pinus albicaulis dominates an open canopy generally not exceeding $60 \%$ coverage. Scattered Pinus contorta and stunted Picea or Abies lasiocarpa may be present; ring counts or these species taken in the most open portion of these stands indicated ages of 40 to 105 years for trees not exceeding $6 \mathrm{ft}$, thus indicating lack of potential to establish dominance from stand opening treatments. Undergrowth in undisturbed stands of this type is dominated by Festuca idahoensis, but in the Centennials and Tendoys sheep grazing has led to reduction of the forb component and the introduction of Poa pratensis, which has become well represented in some stands. Trisetum spicatum and Poa nervosa are consistently present though coverages are seldom even common. Forbs consistently present include Antennaria microphylla, Solidago multiradiata and Astragalus miser.

Soil- Pinus albicaulis / Festuca idahoensis was noted to occur on both calcareous and extrusive volcanics which weather to silt loam and loam textures. Exposed substrate is commonly less than $10 \%$ and litter is usually $80 \%$ plus and relatively deep (1 inch plus).

Other Studies- This is a minor to incidental type from southern Montana and northwestern Wyoming (Steele et al. 1983) and east central Idaho (Steele et al. 1981) and is not reported elsewhere.

\section{Element Code- CEGL000755}

Edition / Author- 99-11-16 / S.V. Cooper, MTNHP

Pinus flexilis / Cercocarpus ledifolius Woodland

(PINFLE / CERLED) limber pine / curlleaf mountain mahogany

Natural Heritage Conservation Rank-

\section{G3G4 / S3}

Environment- Pinus flexilis / Cercocarpus ledifolius has been sampled only in the Limestone Hills vicinity, in the proximity of the Elkhorn Range but has been noted in reconnaissance in the Tendoy and Greenhorn ranges. In both areas Pinus flexilis / Cercocarpus ledifolius is associated with moderate to steep slopes with west- to southfacing aspects and calcareous substrates. In the Tendoys it occurs as a narrow fringe, rather ecotonal, between the drier (or at least less rocky) Artemisia tridentata and Festuca idahoensis-dominated rangelands and pure Cercocarpus ledifolius stand and on moister sites open to closed forests of the and Pseudotsuga menziesii series.

Vegetation- Tree canopies are very open with scattered Pinus flexilis and Pseudotsuga menziesii and, in younger stands, well represented Juniperus scopulorum. The shrub stratum is dominated by well represented to abundant Cercocarpus ledifolius; Artemisia frigida and Gutierrezia sarothrae are consistently present but poorly represented. Pseudoroegneria spicata and Oryzopsis hymenoides (or O. micrantha) and, only in extreme southwestern Montana, Festuca kingii are the dominant graminoids; their cover varies widely but never was noted to exceed $10-15 \%$. Forbs diversity may be appreciable but cover seldom exceeds trace amounts, with the exception of Cymopterus bipinnatus and Petrophytum caespitosum.

\section{Soils- Pinus flexilis / Cercocarpus ledifolius} has been documented only from calcareous substrates in Montana, sites with shallow soils and rock and gravel exposure usually exceeding 30\%. Soils are silt loams but the gravel content severely diminishes their water holding capacity but may facilitate its deep percolation to leves tapped by deep rooted shrubs and trees.

Other Studies- This minor type was first recorded for Montana in a preliminary report (DeVelice 1992) regarding southwestern counties and it is very similar to Pseudotsuga menziesii / Cercocarpus ledifolius described 
for the Pryor Mountains (DeVelice and Lesica 1993). This association extends as an incidental type to northwestern Wyoming (Steele et al. 1983) and eastern central Idaho (Steele et al. 1981) but further to the south in northern Utah it is more extensive (Mauk and Henderson 1984).

\section{Element Code- CEGL000804}

Edition / Author- 99-11-16 / S.V.Cooper, MTNHP

Pinus flexilis / Festuca idahoensis Woodland

(PINFLE / FESIDA) limber pine / Idaho fescue

\section{Natural Heritage Conservation Rank- G5 / S5}

\section{Environment- Pinus flexilis / Festuca}

idahoensis is commonly found on west- to south-facing aspects of moderate to steep slopes often associated with upper slope, windexposed conditions. The observed elevation range was 7,500 to $8,800 \mathrm{ft}$., though in fact it is probably greater. Adjacent drier sites support Festuca idahoensis and Artemisia tridentata var. vaseyana-dominated rangeland communities; it grades to Pseudotsuga menziesii series sites of moister habitats at lower elevations and to Picea series sites at upper elevations.

Vegetation- Widely spaced Pinus flexilis dominate the upper statum in these open, savanna-like all-aged appearing stands (at least stands not readily accessible to tree harvesting) wherein total canopy cover usually does not exceed $60 \%$. Pseudotsuga menziesii may be well represented or even co-dominant, but size-class distributions indicate $P$. flexilis will continue to be at least a co-dominant component. Juniperus scopulorum may be well represented as a long-persisting seral species. Scattered, old (>250 yrs.) fire-scarred individuals (both P. menziesii and P. flexilis) with multiple fire-scars may be present indicating a past history of ground-fires or fires creating a mosaic of age classes.
Undergrowth is dominated by bunchgrasses with Festuca idahoensis common indicating habitats more moist than those supporting just Pseudoroegneria spicata or Hesperochloa kingii as dominants. In extreme southwestern Montana $H$. kingii is a conspicuous, occasionally dominant, component. Forb diversity is often high with Phlox hoodii, Machaeranthera canescens, Astragalus miser, Senecio canus and Linum perenne being consistently represented. Shrubs commonly found scattered in canopy openings are Juniperus communis, Artemisia tridentata var. vaseyana, Ribes cereum and Chrysothamnus nauseosus.

Soils- All stands sampled and noted in reconnaissance were developed on calcareous substrates having much, usually $20 \%$ plus, exposed soil and gravel/rock; these substrates have weathered to silt loam textures.

\section{Other Studies- Pinus flexilis / Festuca} idahoensis is a major plant association of calcareous substrates along the Rocky Mountain Front and ranges of central Montana (Pfister et al. 1977) and continues as a notable component to south-central and southwest Montana and extends as a minor type to eastcentral Idaho (Steele et al. 1981) and northwestern Wyoming in the rainshadow of the Absaroka Range and the very dry Owl Creek Range (Steele et al. 1983).

Comments- Intensive cattle grazing in this type can virtually eliminate the bunchgrass component, or at least reduce the cover of $F$. idahoensis and Pseudoroegneria spicata to the point where site potential is not discernable; the fact that grass cover potentially is considerably less than that of open rangelands does not seem to lessen the degree of impact.

\section{Element Code- CEGL000805}

Edition / Author- 99-11-16 / S.V.Cooper, MTNHP 
Pinus flexilis / Juniperus communis Woodland

(PINFLE / JUNCOM) limber pine / common juniper

\section{Natural Heritage Conservation Rank- G5 / S4}

Environment- Within the study area, this minor type was sampled at $5,800 \mathrm{ft}$. on calcareous substrates with moderate exposures. It was not encountered in reconnaissance further south than the Dewey, MT vicinity (it is common along the Rocky Mountain Front to the north). Drier sites are occupied by Cercocarpus ledifolius/Pseudoroegneria spicata and moister ones by the Pseudotsuga menziesii series.

Vegetation- The one plot is typical for the type at large as described by Pfister et al. (1977) with Pinus flexilis dominating the canopy and Pseudotsuga menziesii and Juniperus scopulorum as subordinate species, but with population structure indicating Pseudotsuga would not outcompete $P$. flexilis in the long term.

The undergrowth is dominated by the trailing shrub Arctostaphylos uva-ursi and Juniperus communis. Under appreciable grazing pressure the highly palatable bunchgrass $P$ seudoroegneria spicata was present as mostly consumed scattered clumps. The lack of Festuca idahoensis may be attributable to grazing pressure as it is common in the general vicinity. Astragalus miser is the dominant forb as it is in most occurrences of this association.

Soils- Sampled substrates were calcareous sandstones and weathered to silty sands with approximately $20 \%$ exposed gravel and rock. With high shrub cover the litter cover was comparably high.

Other Studies- Pinus flexilis / Juniperus communis is found as far west as the Blue Mountains of Oregon (Cole 1982) and Lost River and Lemhi Ranges of Idaho (Steele et al. 1981) where it is an incidental type and increases in importance to the east where it's extensive along the Rocky Mountain Front of Montana (Pfister et al. 1977) and Wyoming (Steele et al. 1983). In southeastern Wyoming (Alexander et al. 1986) and northwestern Colorado (Johnson 1987) it's extent is again reduced relative to its representation in Montana.

\section{Element Code- CEGL000807}

Edition / Author- 99-11-16 / S.V.Cooper, MTNHP

Pinus flexilis / Pseudoroegneria spicata Woodland

(PINFLE / PSESPI) limber pine / bluebunch wheatgrass

\section{Natural Heritage Conservation Rank-}

G4 / S4

Environment- Pinus flexilis / Pseudoroegneria spicata is found on the driest exposures capable of supporting trees, steep southeastthrough southwest-facing slopes. The noted study area elevation range was from 5,500 to $7,600 \mathrm{ft}$. but this association could occur at higher elevations. This type is usually found in a mosaic of grassland/shrubland on deeper soils with adjacent forest types being Pseudotsuga menziesii / Cercocarpus ledifolius, Pseudotsuga menziesii / Juniperus communis and Pinus flexilis / Juniperus communis on rockier sites.

Vegetation- Widely spaced Pinus flexilis usually dominates the canopy, often in conjunction with Juniperus scopulorum and Pseudotsuga menziesii; in early seral stands $J$. scopulorum can have greater cover than other tree species, but in the aggregate the visual expression is that of a very open forest or savanna.

Undergrowth cover is intrinsically low and with cattle grazing may look especially depauperate. Pseudoroegneria spicata well represented is diagnostic for the type; usually it is the dominant herb but in the southwestern extreme Festuca kingii may be a co-dominant 
or even dominant with cattle grazing preferentially removing Pseudoroegneria spicata. Contribution of herbs from contiguous grasslands, e.g. Oryzopsis hymenoides, Koeleria cristata, Phlox hoodii, Senecio canus, Liatris punctata, Draba spp., Oxytropis spp. and Linum perenne, often produce a species-rich undergrowth. Combined shrub cover seldom exceeds 5\% with Artemisia frigida, A. tridentata, Gutierrezia sarothrae, Ribes cereum and Symphoricarpos oreophilus being the most commonly represented.

Soils- This type was noted only on calcareous substrates, both sandstone and limestone, with exposed surfaces of soil, gravel and rock often exceeding $60 \%$; from the descriptions of others there is reason to expect this type on other than calcareous parent materials.

Other Studies- This type is found all along the Rocky Mountain Front and scattered ranges of central (Pfister et al. 1977) as well as southwestern Montana (DeVelice 1992). This type is not identified for contiguous states to the south because the cline of increasing Festuca kingii toward the south causes these Pinus flexilis -dominated stands with Pseudoroegneria spicata to be termed Pinus flexilis / Festuca kingii (see Steele et al. 1981, 1983); Pinus flexilis / Festuca kingii extends to the Medicine Bow Range of southeastern Wyoming (Alexander et al. 1986).

\section{Element Code- CEGL000813}

Edition / Author- 99-11-16 / S.V.Cooper.

Pseudotsuga menziesii / Cercocarpus ledifolius Woodland

(PSEMEN / CERLED) Douglas-fir / curlleaf mountain mahogany

\section{Natural Heritage Conservation Rank-} G4? / S?

\section{Environment- Pseudotsuga menziesii / Cercocarpus ledifolius is restricted to dry, often steep (>40\%) upper slopes and ridges with south- to west-facing aspects. Sites}

mostly have shallow soils and much (>30\%) exposed substrate that often approaches scree in lack of stability. Sampled elevation range was 5,800 to $6,600 \mathrm{ft}$, though its noted range included higher elevations. Pseudotsuga menziesii / Symphoricarpos oreophilus occurs on relatively more mesic, or less rocky sites and Cercocarpus ledifolius / Pseudoroegneria spicata is also part of what is often a fairly fine-scaled mosaic of forest/tall shrub communities.

Vegetation- Canopy structure is usually quite open, savanna-like with total combined cover of Pseudotsuga menziesii and Juniperus scopulorum, the only tree species present, not exceeding 50\% and often as little as 5\%. Pinus flexilis was not recorded where substrates are crystalline (Silverbow Co.) but was noted in reconnaissance of calcareous substrates (Beaverhead Co.).

Shrub cover, the two principal ones of which are Cercocarpus ledifolius and Artemisia tridentata, is at least as variable as the tree cover but seldom exceeds 50\%. Artemisia frigida and Chrysothamnus nauseosus have high constancy and scarce coverage. The herb component is poorly represented and only Pseudoroegneria spicata, Poa sandbergii and Lappula redowskii have greater than 50\% constancy. Combined bryophyte and lichen cover ranged as high as $40 \%$, but usually they are merely traces.

Soils- All sampled stands occurred on coarsetextured soils derived from granitic parent materials and had as much as $90 \%$ exposed gravel and rock; deep percolation of precipitation subsequently tappable by trees and shrubs is the factor assumed to favor these lifeforms on these sites.

Other Studies- DeVelice and Lesica (1993) have described Pseudotsuga menziesii / Cercocarpus ledifolius for the Pryor Mountains and vicinity. It extends from the study area into east-central Idaho (Steele et al.1981) and southeastern Idaho and southwestern Wyoming (Steele et al. 1983), where it is found on a variety of substrates, and 
is documented as far south as southern Utah (Youngblood and Mauk 1985).

Element Code- CEGL000897

Edition / Author- 99-11-16 / S.V.Cooper, MTNHP

Pseudotsuga menziesii / Festuca idahoensis Woodland

(PSEMEN / FESIDA) Douglas-fir / Idaho fescue

\section{Natural Heritage Conservation Rank- G4 / S4}

\section{Environment- Pseudotsuga menziesii /}

Festuca idahoensis is a dry site type but was found on all aspects depending on local precipitation patterns; in rainshadow positions it is found on north-facing aspects whereas in main mountain masses it is associated with steep, west- to south-facing slopes. The observed elevation range was 6,600 to 7,800 $\mathrm{ft}$., but there is no reason it could not be found beyond both of these extremes. It grades to Pseudotsuga menziesii / Pseudoroegneria spicata or Artemisia tridentata var. vaseyanaor Festuca idahoensis-dominated rangelands on drier aspects or more densely forested Pseudotsuga-dominated community types of moister exposures.

Vegetation- Pseudotsuga menziesii dominates the canopy and all size classes including reproduction, whereas Pinus flexilis and Juniperus scopulorum are reduced to minor components (cover $<10 \%$ ) of the canopy and reproduce only in larger canopy gaps. Stands with warmer exposures tend to be more open ( $<60 \%$ cover) than those of cooler northerly slopes (approaching 90\% canopy cover).

Artemisia tridentata var. vaseyana and Chrysothamnus viscidiflorus are present as scattered individuals and many skeletons of $A$. tridentata may be present in younger stands indicating this type passes through an $A$. tridentata-dominated seral stage. Herbaceous components are those of the adjacent grasslands, but with reduced cover. Festuca idahoensis is the dominant graminoid except were grazing pressure is intensive, then $P o a$ sandbergii, Koeleria cristata or Festuca kingii (only in the southwestern extreme) become dominant. Astragalus miser and Antennaria microphylla are common, occasionally abundant, forbs. Lichen and bryophyte cover varies widely, but apparently is related to stand age (or history) being higher in older, more dense ones..

Soils- Parent materials include both crystalline and calcareous substrates and generally there is less than $20 \%$ exposed substrate, most of which is soil. The relatively high content of woody debris is mostly from sagebrush skeletons or toppled-over old wolf trees.

Other Studies- The Pseudotsuga menziesii / Festuca idahoensis p.a. has a broad geographic distribution, from east of the Cascades in Washington (Williams and Lillybridge 1983) and British Columbia (McLean 1970) to northwestern Colorado (Bourgeron and Engelking 1994), but has its greatest areal extent along the Rocky Mountain Front and isolated ranges of central Montana (Pfister et al. 1977).

\section{Element Code- CEGL000900}

Edition / Author- 99-11-16 / S.V.Cooper, MTNHP

Pseudotsuga menziesii / Scree Woodland (PSEMEN / SCREE) Douglas-fir / scree

\section{Natural Heritage Conservation Rank- G5 / S4}

Environment- Pseudotsuga menziesii / Scree is a widespread plant association in Montana previously recognized by the simple designation of "Scree" without dominant overstory identified. This association is distinguished by its unique substrate, unstable, generally steep slopes; potentially this type can be found on slopes of any aspect or angle of repose providing they are unstable, but as sampled and noted it occurs on those with any aspect, but greater than 50\% slope. All sampled 
conditions and the most extensive stands (on quartzite) were in Granite Co. but this type was noted for the Tendoy and Centennial Ranges as limited linear patches on substrates of various origin. The sampled elevation range was 4,400 to $5,400 \mathrm{ft}$. but it has been noted over a much wider range; potentially it is coextensive with the distribution of Pseudotsuga.

Vegetation- Due to existing severe site conditions trees are usually widely spaced and slow growth with a wolfy form; combined canopy cover ranged from 20 to $70 \%$, dominated by Pseudotsuga menziesii but with Juniperus scopulorum and Pinus ponderosa well represented on lower elevation sites. The combination of unstable substrates, low undergrowth productivity and widely spaced trees has resulted in a high proportion of these stands developing into old-growth, with Pseudotsuga 2-3 ft. in diameter.

The undergrowth is depauperate, in both diversity and cover, in the graminoid and forb components with only Pseudoroegneria spicata, Heuchera parviflora and Phacelia hastata having high constancy. Shrubs take advantage of their deep rooting habit to become by far, the undergrowth component with the greatest coverage (and highest diversity); Acer glabrum consistently dominated this layer with Ribes cereum, Amelanchier alnifolia, and Symphoricarpos oreophilus (S. albus to the northwest of area) consistently present, occasionally well represented.

Soils- The unstable nature of these substrates is their distinguishing character; parent material includes calcareous and non-calcareous sedimentary as well as crystalline materials. The combined exposure of soil, gravel and rock usually exceeds $50 \%$ and runs as high as $90 \%$ with litter the only other important component. Usually even the litter and bryophyte components are underlain by coarse fragments. Soil development is minimal with no horizonation evident.

Other Studies- For Montana and Idaho this type was first described under the broader designation "Scree" (Pfister et al. 1977, Cooper et al. 1987) but this syntaxon included enormous variation as it included all tree series. Pseudotsuga menziesii/scree has been formally described only for southern Colorado and northern New Mexico (DeVelice et al. 1986, Fitzhugh et al. 1987) largely because other regional forest classifications (e.g. Steele et al. 1983, Youngblood and Mauk 1985) have been biased toward commercial forests, which Pseudotsuga menziesii / scree is clearly not. Using a key based on vegetation alone these sites would key to Pseudotsuga menziesii / Acer glabrum (Steele et al. 1983) which is a very different, highly productive association of central and northern Idaho.

\section{Element Code- CEGL000911}

Edition / Author- 99-11-16 / S.V.Cooper, MTNHP

\section{M ontane or B oreal C old- deciduous F orest}

Populus balsamifera ssp. trichocarpa / Poa pratensis Forest

(POPBST / POAPRA) black cottonwood / Kentucky bluegrass

\section{Natural Heritage Conservation Rank- $\mathrm{G}$ ? / S?}

Environment- Populus balsamifera ssp. trichocarpa / Poa pratensis is cited by Hansen et al. (1995) to be a major forested riparian type of western Montana; it is uncommon within the study area being found on alluvial terraces of major steams and rivers as well as bordering ponds and lakes. The type as defined by Hansen et al. is a default class within the Populus balsamifera ssp. trichocarpa series and thus very inclusive of both environmental and vegetation variation. This type incorporates sites subjected to periodic disturbances such as flooding and erosion/deposition. Intensive grazing further adds to site heterogeneity and can also result in solid swards of introduced, rhizomatous grasses (eventually to weed-choked terraces). Upslope these sites usually abruptly give way 
to Artemisia tridentata var. vaseyana or tridentata dominated shrub steppe.

Vegetation- This type has a simple two layer structure of mostly open Populus balsamifera ssp.trichocarpa over a sward of introduced rhizomatous grasses, Poa pratensis and Phleum pratense and the rhizomatousappearing Poa palustris. Other commonly occurring grasses include Bromus inermis, Carex praegracilis, C. utriculata and Phalaris arundinacea. Solidago gigantea, Smilacina stellata, Thermopsis montana and Taraxacum officinale are consistently present and capable of occurring well represented.

Soils- There was a conspicuous lack of genetic horizonation in these alluvium-derived soils of loam to silty loam to coarse sand that had a notable gravel component at greater depths. There is notable spatial heterogeneity due to active and past fluvial processes.

Other Studies- The Populus balsamifera ssp. trichocarpa / Poa pratensis zootic disclimax has been described for only Montana; it has been documented by Hansen et al. (1995) to be a major riparian type of low- to mid-elevations throughout the foothills and mountains of Montana.

\section{Element Code- CEGLMTNH31}

Edition / Author- 99-11-16 / S.V.Cooper, MTNHP

Populus tremuloides / Bromus carinatus Forest

(POPTRE / BROCAR) trembling aspen / mountain brome

\section{Natural Heritage Conservation Rank- G5 / S?}

Environment- This is a broadly distributed aspen-dominated type documented from the mountain ranges of the Great Basin and western Utah; it has not previously been described from a location as far north as Red Rock Lakes NWR. On the Refuge Populus tremuloides / Bromus carinatus occurs on the lower slopes and toeslope positions of the
Centennial escarpment; generally these sites

have deep and relatively rock-free soils with an upper horizon having a strong blocky structure and very low chroma (dark, humus-rich soils).

Vegetation- Populus tremuloides is the dominant tree and forms a nearly continuous canopy as well as having seedling and sapling size classes present in some stands. Conifer "invasion" appears to be very slow on these sites; seedlings and very scattered saplings of Picea engelmannii and Abies lasiocarpa were noted, though the predominant seed-rain would have been from Pseudotsuga menziesii.

In addition to $P$. tremuloides dominating the tree canopy, this association is identified when the undergrowth component is dominated by one of the following tall grasses Bromus carinatus, Elymus glaucus, or Elymus trachycaulus (syn. Agropyron caninum) and the tall forb component is poorly represented. On the Refuge the first two listed grasses were almost always the dominants. Other frequently abundant graminoids, include Poa pratensis, Carex hoodii, C. petasata, Stipa occidentalis, and Poa pratensis. Forbs consistently present include, include Achillea millefolium, Thalictrum occidentale (or T. fendleri), Osmorhiza chilensis, Geranium viscosissimum, and Potentilla gracilis.

Element Code- CEGL000573

Edition / Author- 99-11-16 / S.V.Cooper, MTNHP

Populus tremuloides / Calamagrostis canadensis Forest

(POPTRE / CALCAN) trembling aspen / bluejoint reedgrass

\section{Natural Heritage Conservation Rank-}

G3 / S2

Environment- The Populus tremuloides /

Calamagrostis canadensis association is described as an incidental type within Montana (Hanson et al. 1995) and not listed at all for the Intermountain Region (Mueggler 1988) therefore the occurrences on the Red Rock 
Lakes NWL Refuge can be considered somewhat unique, pending further inventory. Hanson et al. (1995) probably never sampled Populus tremuloides stands in the vicinity of the Refuge and the higher elevations of southwestern Montana because the stands on the Refuge occur at considerably higher elevations than cited for the Populus tremuloides / Calamagrostis canadensis type by Hansen et al. (1995). Populus tremuloides / Calamagrostis canadensis occurs on flats and depressions along the Centennnial Mtns. escarpment as small stands ( $<1$ acre) or as fringes on herb- or shrub-dominated wetlands and generally is associated with seasonally saturated soils.

Vegetation- The observed stand structure was rather open, verging on woodland $(<60 \%$ canopy cover) and having $P$. tremuloides as the sole dominant of the upper canopy; seedlings and saplings of Picea engelmannii and Abies lasiocarpa are widely scattered. These stands are putatively seral to Picea engelmannii I Calamagrostis canadensis or Abies lasiocarpa / Calamagrostis canadensis but completion of the sere would require hundreds of years disturbance-free.

The undergrowth dominant, Calamagrostis canadensis, usually occurs in a sward with high cover values (> 40\%). Other graminoids include Elymus glaucus, Bromus ciliatus, and Elymus trachycaulus and where disturbance has occurred Phleum pratense and Poa pratensis. Forbs usually constitute a minor presence, individual species generally not exceeding 5\% cover; commonly present in Refuge stands are Smilacina stellata, Geranium richardsonii, Galium triflorum, Osmorhiza occidentalis and Geum macrophyllum. Shrubs are a minor component with Rosa woodsii, Symphoricarpos albus, and Salix bebbiana being most typical.

\section{Element Code- CEGL000574}

Edition / Author- 99-11-16 / S.V.Cooper, MTNHP

\section{Populus tremuloides / Calamagrostis rubescens Forest}

(POPTRE / CALRUB) trembling aspen / pine grass

\section{Natural Heritage Conservation Rank- G5? / S4}

Environment- Mueggler (1988) noted Populus tremuloides / Calamagrostis rubescens to be an especially common type in the Centennial Mtns. of Idaho and Gros Ventre Range of western Wyoming; southwestern Montana reflects this pattern as well with this community type the prevalent Populus tremuloidesdominated vegetation type. Populus tremuloides / Calamagrostis rubescens occupies gentle to moderate slopes and benches irrespective of aspect or slope configuration. It is generally associated with landscape positions that could be inferred to be collecting positions (e.g. in the lee of a knoll or ridge) where in the past aeolian material would have been deposited or currently where snow deposition would be greater. Though extensive soil measurements were not taken this type does seem to occur with deeper and relatively rock free profiles (at least for upper horizons).

Vegetation- The successional status of this association is still debated (Mueggler 1988); it is usually dominated in all size strata by $P$. tremuloides (inferring existence of a climax plant association) with only the occasional presence of conifer regeneration to suggest that some stands may be slowly successional to conifer dominance. On the Refuge the conifer species most likely to replace $P$. tremuloides are, in order of probability, Abies lasiocarpa, Pseudotsuga menziesii and Pinus contorta. Very often P. tremuloides forms a nearly continuous upper canopy.

Shrubs such as Symphoricarpos albus, $S$. oreophilus, Berberis repens, Rosa woodsii and Arctostaphylos uva-ursi occur scattered, not as a discrete layer. The type is characterized by the dominance/codominance of Calamagrostis rubescens and/or Carex geyeri, which often 
occur in such density as to create a sward-like aspect. Other graminoids consistently present, but with low cover, include Elymus glaucus,

Bromus carinatus, Elymus trachycaulus, Stipa occidentalis and where disturbance has favored their intrusion Poa pratensis and Phleum pratense. Total forb cover is generally less than $10 \%$ and includes Geranium viscosissimum, Lupinus agents, Thalictrum occidentale (or T. fendleri), Achillea millifolium, Fragaria vesca (or $F$. virginiana). High coverages of $A$. millefolium, Taraxacum officinale, Hackelia floribunda and Astragalus miser are indicative of past or continuing grazing (usually intensive).

\section{Element Code- CEGL000575}

Edition / Author- 99-11-16 / S.V.Cooper, MTNHP

Populus tremuloides / Poa pratensis Forest (POPTRE / POAPRA) trembling aspen / Kentucky bluegrass

\section{Natural Heritage Conservation Rank-} G? / S?

Environment- Populus tremuloides / Poa pratensis is a common grazing-induced community type found along the foothills to the Centennial Range, often associated with slumps and pockets of loess soils. This type generally occupies benches or gentle north- or east-facing slopes. The classification manual of Montana's riparian/wetland vegetation (Hansen et al. 1995) recognizes a portion of this type to occupy wetland sites (including requisite soil and hydrological properties). However, the Populus tremuloides / Poa pratensis sites we noted were candidate wetlands; at most they could be considered mesic sites in water receiving positions but lacking even facultative hydrophytes. Adjacent communities are the putatively less grazingimpacted Populus tremuloides /Thalictrum fendleri c.t. and Pseudotsuga menziesii / Calamagrostis rubescens or on drier transitions Artemisia tridentata ssp vaseyana /Festuca idahoensis.
Vegetation- The overstory is generally a closed canopy of Populus tremuloides; the understory may be dominated (but cover not exceeding $10-20 \%$ ) by a younger age-class of $P$.

tremuloides but more often a scattering (cover $<10 \%$ ) of Pseudotsuga menziesii, Abies lasiocarpa or Picea engelmannii is found. These "climax" species are slow to establish and equally slow to exert dominance. Two 80100 year old $P$. tremuloides-dominated stands had no more than $5 \%$ of the aforenamed climax tree species and they had all established within the last 30 years. Wild ungulate browsing on the Abies was evident but the other two species fared no better in their growth rates.

Like the conifers, the shrubs, Symphoricarpos oreophilus, Berberis repens, Prunus virginiana (heavily browsed) and Rosa woodsii, are an insignificant component of the vegetation complex. This type generally supports a lush sward of Poa pratensis in which Bromus carinatus, B. ciliatus Elymus trachycaulus and Elymus glaucus have been reduced to minor components from what are assumed to be their pre-disturbance levels. Phleum pretense well represented was assumed to denote an even further degraded condition. Forb composition may be diverse and even include indicators of relatively mesic conditions (Thalictrum occidentale, T. fendleri, Osmorhiza chilensis, Geranium viscosissimum and Valeriana dioica) but coverage's of mesic indicator, even combined, do not exceed $5 \%$ whereas weedy species (Taraxacum officinale, Trifolium repens) may be abundant.

Soils- The only excavated pit showed deep $(>80 \mathrm{~cm})$ silt loam with no rock or gravel and a mollic epipedon at least $20 \mathrm{~cm}$ thick. Other exploratory excavations of this c.t. found the upper $10-20 \mathrm{~cm}$ to be mollic with virtually no rock or gravel. In extremely impacted areas $P$. pratensis may be reduced to poorly represented and $T$. officinale is dominant.

Other Studies- Mueggler's monograph (1988) of aspen in the Intermountain region describes Populus tremuloides / Poa pratensis as a minor type, a grazing-induced deflection of the Populus tremuloides alliance with Thalictrum 
fendleri, Symphoricarpos oreophilus, or Calamagrostis rubescens community types from Utah, Nevada, northward to the BridgerTeton N.F. of Wyoming and Targhee of Idaho. In Colorado it is speculated to be a disturbanceinduced version of Populus tremuloides / Calamagrostis rubescens. In Montana Hansen et al. (1995) recognize Populus tremuloides / Poa pratensis as a major type, part of the riparian complex, though not always a jurisdictional wetland. Hansen et al's concept of this type is probably broader than those of the Intermountain region as they cite a different suite of browsed-out shrubs e.g. Cornus sericea, Prunus virginiana, Alnus incana and Salix species.

\section{Element Code- CEGLMTHP27}

Edition / Author- 99-11-16 / S.V.Cooper, MTNHP

Populus tremuloides / Tall Forbs Forest (POPTRE / Tall Forbs) Trembling aspen / Tall Forb

\section{Natural Heritage Conservation Rank-}

\section{G5 / S3}

Environment- Though Populus tremuloides / Tall Forb is the most commonly encountered $P$. tremuloides-dominated plant association in the Intermountain Region it is relatively uncommon on the RRLNWLR. Generally it occurs on concave to undulating landform configurations. Soils are relatively deep, rock-free and developed from a number of parent material types including limestone, sandstone, quartzite and andesite. Environmental parameters have not been definitively identified that would make this type differentiable from Populus tremuloides / Thalictrum fendleri, (or Populus tremuloides / Calamagrostis rubescens) and it does grade to these types in a landscape context. We speculate that this type occurs on slightly more mesic landscape positions relative to the other types. Stands on the RRLNWLR are small $(<1 \mathrm{ac})$ and have received significant past disturbance by livestock. Refuge examples of this type appear to be near-climax aspen communities with little indication that conifers will ever achieve dominance.

Vegetation- The unifying distinctions for the type is the presence and usual prominence of one or more members of what Mueggler (1988) has termed the "tall forb group", lack of a distinct shrub layer, and inconsequential amounts of conifers in the tree stratum. The tall forb guild is comprised of Valeriana occidentalis, Osmorhiza occidentalis, Hackelia floribunda, Senecio serra, Agastache urticifolia, Mertensia paniculata, Delphinium occidentale, Aster engelmannii and Heracleum lanatum (syn. H. sphondylium). On Refuge stands $O$. occidentale, A. engelmannii, and $H$. lanatum are most likely to be layer dominants in undisturbed conditions, whereas $A$. urticifolia and $H$. floribunda more typically exploit disturbance conditions.

The tree stratum characteristically is composed of only P. tremuloides. Abies lasiocarpa and Picea engelmannii may be present as scattered seedlings and saplings; Abies is very heavily browsed by moose and has little chance of escaping sapling status.

Shrub are present, usually Symphoricarpos oreophilus, Berberis repens and Ribes spp., but their combined cover does not generally exceed 5\%. Low forbs with high constancy in this type include Geranium richardsonii, $G$. viscosissimum, Osmorhiza chilensis (or $O$. depauperata), Thalictrum occidentale (or T. fendleri) and Potentilla glandulosa. The common graminoid component, which can have appreciable cover though obscured by the tall forbs, includes Bromus carinatus, Elymus glaucus, and Elymus trachycaulus.

\section{Element Code- CEGL000618}

Edition / Author- 99-11-16 / S.V.Cooper, MTNHP

Populus tremuloides / Thalictrum fendleri Forest

(POPTRE / THAFEN) trembling aspen / Fendler's meadowrue 
Natural Heritage Conservation RankG5 / S?

Environment- Populus tremuloides /

Thalictrum fendleri, is a common community type found along the foothills to the Centennial Range, often associated with slumps and pockets of loessal soils. This type generally occupies benches or gentle north- or eastfacing slopes. This type was not found above $7,400 \mathrm{ft}$ elevation. Adjacent communities of drier (?) environments are Populus tremuloides /Calamagrostis rubescens, Pseudotsuga menziesii / Calamagrostis rubescens or Artemisia tridentata ssp vaseyana/Festuca idahoensis; it grades to Populus tremuloides / Cornus sericea or most often Populus tremuloides /Osmorhiza occidentalis of moister environments (recognized by $5 \%$ coverage of Viola canadensis, Heracleum lanatum, Actaea rubra, O. occidentalis or Galium triflorum).

Vegetation- The overstory is generally a closed canopy of Populus tremuloides; the understory may be dominated (but cover not exceeding $10-20 \%$ ) by a younger age-class of $P$. tremuloides but more often a scattering (cover < 10) of Pseudotsuga menziesii, Abies lasiocarpa or Picea engelmannii is found. These "climax" species are slow to establish and equally slow to exert dominance. A $P$. tremuloides-dominated stand in excess of 100 years old had no more than $10 \%$ of the aforenamed climax tree species and they had all established within the last 40 years. Wild ungulate browsing on the Abies lasiocarpa was evident but the other two species fared no better in their growth rates.

Shrubs, including the highly constant Symphoricarpos oreophilus, Rosa woodsii, and Berberis repens, are poorly represented; Shepherdia canadensis can be well represented in small patches and may represent fragments of the Populus tremuloides / Shepherdia canadensis c.t. but does not appear to be associated with a particular environmental feature. The dominant aspect of this c.t. in good condition is that of a low-forb layer with highly variable coverage of introduced rhizomatous (Poa pratensis, Phleum pratense) and native grasses (Bromus ciliatus, $B$. vulgaris, B. carinatus, Elymus glaucus and Agropyron caninum) The coverage of introduced grasses may be abundant on grazing-impacted sites. In its modal form the diagnostic forbs Thalictrum fendleri (or T. occidentale), Osmorhiza chilensis (or $O$. depauperata), and Geranium viscosissimum have at least $10 \%$ cover, singly or in the aggregate. Other species noted to be consistently present and associated with even more mesic conditions than those listed above include Ligusticum filicinum, Geranium richardsonii, Geum macrophyllum, Smilacina stellata, and Mertensia ciliata. Taraxacum officinale, Descurainia pinnata and Myosotis arvensis are increaser forbs with grazing.

Soils- The only excavated pit showed deep $(>100 \mathrm{~cm})$ silt loam with no rock or gravel and a mollic epipedon at least $30 \mathrm{~cm}$ thick. Other exploratory excavations of this c.t. found the upper profile to be mollic and totally lacking rock or gravel. The litter layer is continuous and very thin, as is the humus layer as is typical for productive Populus tremuloidesdominated stands. In extremely overgrazed areas $P$. pratensis may be reduced to poorly represented and $T$. officinale and Achillea millifolium are dominant.

Other Studies- For the Intermountain region Mueggler (1988) has defined as areally extensive a Populus tremuloides /Thalictrum fendleri c.t. that ranges from northwestern Wyoming to eastern Idaho (as /Geranium viscosissimum c.t. Mueggler and Campbell 1982) and Utah (Mueggler and Campbell 1986) and southeastern Wyoming (Alexander et al. 1986). Populus tremuloides /Thalictrum fendleri is apparently very abundant in western Colorado (Johnson 1987).

Comments- Mueggler (1988) remarks that this c.t. appears to slowly succeed to the Abies lasiocarpa / Osmorhiza chilensis habitat type; population structure of stands in our study area seem to substantiate this observation.

Element Code- CEGL000619 
Edition / Author- 99-11-16 / S.V.Cooper, MTNHP

\section{Shrublands}

Artemisia cana / Pacopyrum smithii
Shrubland
(ARTCAN / PASSMI) silver sagebrush /
western wheatgrass

\section{Natural Heritage Conservation Rank- G4 / S4}

Environment- (The following description characterizes the type as found in northeastern MT; it is a minor type in southwestern MT, noted only in passing). The ARTCAN / Pascopyrum smithii association is found on level to gently sloping, narrow to extremely broad alluvial (floodplain) terraces and coalescing alluvial fans and upslope may occur in swales and gentle depressions. These sites are moister than contiguous upslope vegetation and in some cases may constitute wetland sites (none of our sampled stands were, this can only be determined by hydrological monitoring or examination of soil characteristics). Substrates are generally moderately fine to fine textured, being derived from sediments deposited in low energy environments (or in the case of basins and swales from slopewash), have a high water holding capacity and are well- to imperfectly drained. As speculated in other studies (Jorgenson 1979, Hansen et al 1991) perched or high water tables may influence the rooting zone for a portion of the year. A variety of community types are found to occur adjacent on upland sites, most commonly Stipa comata Bouteloua gracilis and Pascopyrum smithii Stipa comata, whereas moister positions are frequently dominated by the Symphoricarpos occidentalis and Rosa woodsii dominated associations or Sarcobatus vermiculatusdominated types when the setting is in highly erosive to badlands topography.

Vegetation- Artemisia cana having at least $5 \%$ canopy cover is diagostic of this type, but its cover usually exceeds $30 \%$. None of the sites supported the robust 4-5 ft tall specimens cited by Hansen and Hoffman (1988) or Mueggler and Stewart (1980) for favorable site conditions. Artemisia frigida was consistently present in low amounts (greater than 10\% where cattle grazing intensive) and other shrub species were only sporadic. Graminoids dominate the herbaceous layer with Pacopyrum smithii usually dominant, but in our samples Stipa viridula, $S$. comata and Bouteloua gracilis were all dominant or co-dominant in at least one stand (they also had greater than $75 \%$ constancy). This variability is speculated to reflect differing grazing pressure as stands were not chosen for pristine condition (stand with $B$. gracilis dominant had A. smithii and $S$. viridula confined to canopies of $A$. cana). The forb component is insignificant; none had even $50 \%$ constancy.

Other Studies- This, or closely related, types have been documented in other areas of MT; southeastern (Hansen and Hoffman 1988), southwestern (Mueggler and Stewart 1980) and central (Jorgensen 1979). The most comprehensive sampling (43 stands) of this type is that performed by the Montana Riparian Association (Hansen et al. 1995) for the entire state. This type has been described only for Montana, North and South Dakota (Hansen et al. 1984).

\section{Element Code- CEGL001072}

Edition / Author- 99-12-03 / S.V.Cooper, MTNHP

Artemisia tridentata ssp. tridentata / Festuca idahoensis Shrubland (ARTTST / FESIDA) basin big sagebrush / Idaho fescue

\section{Natural Heritage Conservation Rank- G4? / SP}

Environment- This type is found on moist lower slopes or short slopes at 6,600-7,200 ft in the Tendoy Mountains and repeats in the Centennial Sandhills as the late seral stage of stabilized dune sands. Adjacent less mesic sites are dominated by Artemisia tridentata vaseyana and Festuca idahoensis. Moister sites with finer textured soils support Artemisia 
tridentata ssp.tridentata with an understory dominated by Pascopyrum smithii and/or Leymus cinereus.

Vegetation- Artemisia tridentata ssp. tridentata is the dominant shrub with $10-20 \%$ canopy cover. Chrysothamnus spp. and Tetradymia canescens are present in many stands. Grass canopy cover is $50-70 \%$. Festuca idahoensis dominates, and Pseudoroegneria spicata is also common. Forb cover is generally ca. $10 \%$ with moderate diversity. Astragalus adsurgens, Penstemon aridus and Senecio canus are often present. Mosses and lichens may be common.

Soils- Soils are deep and silty or loamy in texture; the Centennial Sandhills composed of aeolian sand to loamy sands constitute a rather unique substrate. From 10 to $20 \%$ of the surface is bare, and surface gravel is usually present, with the exception of the Centennial Sandhills representatives of the type.

Other Studies- Mueggler and Stewart (1980) do not mention that Artemisia tridentata ssp. tridentata may occur with Festuca idahoensis in Montana. Similar vegetation is reported for Idaho, Oregon and Washington (Bourgeron and Engelking 1994).

\section{Element Code- CEGL001014}

Edition / Author- 99-11-16 / S.V.Cooper, MTNHP

Artemisia tridentata ssp. tridentata / Pascopyrum smithii Shrubland (ARTTST / PASSMI) basin big sagebrush / western wheatgrass

\section{Natural Heritage Conservation Rank-} $\mathrm{G} 3$ ? / S3

Environment- This type is common on gently slope to nearly level stream terraces at 5,9007,200 ft in the Tendoy Mountains; very limited areas of Artemisia tridentata ssp. tridentata / Pascopyrum smithii were noted in reconnaissance of the Centennial Valley and other locations within the northern portion of
Dillon Resource Area. Sites are often associated with dry stream channels and thus have a low gradient. The landscape location and vegetation might be considered riparian but the type is not a jurisdictional wetland using soils and hydrological criteria. Parent material is alluvium derived from limestone and quartzite. Adjacent upslope sites support sagebrush steppe dominated by Artemisia tridentata ssp. tridentata or vaseyana and Festuca idahoensis; in the Yellowstone Ecosystem this type was noted to grade to Artemisia cana / Pascopyrum smithii of moister regimes. Cool slopes are dominated by Pseudotsuga menziesii or Pinus flexilis forests, and very rocky slopes support Cercocarpus ledifolius woodlands.

Vegetation- Artemisia tridentata ssp. tridentata is the dominant shrub with canopy cover ranging from 10 to $50 \%$.

Chrysothamnus nauseosus and $C$. viscidiflorus occur, poorly represented, in many stands. The subshrub, Artemisia frigida, may be common in some stands. Grass cover is high to nearly continuous in most stands. Pascopyrum smithii and/or Elymus lanceolatus (in our concept of the type) are the dominant and diagnostic grass species. Poa pratensis and Leymus cinereus may be abundant. Pseudoroegneria spicata and small bluegrass's such as Poa secunda, $P$. juncifolia, or $P$. cusickii are often present. Forb cover is usually $10 \%$ or less, and diversity is low to moderate. Achillea millefolium, Antennaria microphylla, Erigeron compositus, Senecio canus and Taraxacum officinale are species with high constancy. Mosses and lichens are often present and may be common.

Soils- Soils are very deep with a silty to loamy texture. Some may be slightly saline. The amount of bare soil surface varies between $10 \%$ and $70 \%$, but there is no surface gravel.

Other Studies- Mueggler and Stewart (1980) describe associations dominated by Sarcobatus vermiculatus and either Pascopyrum smithii or Leymus cinereus for western Montana. These types have a similar herbaceous layer to Artemisia tridentata ssp. tridentata /

Pascopyrum smithii but the dominant shrub is 
S. vermiculatus. An Artemisia tridentata ssp. tridentata / Pascopyrum smithii c.t. is reported for Colorado and Nevada, and an Artemisia tridentata ssp. tridentata /Leymus cinereus type is reported for Colorado, Idaho and Oregon (Bourgeron and Engelking 1994).

Comments- Most sites that are mesic enough to support Artemisia tridentata ssp. tridentata will probably also support a robust grass such as Leymus cinereus. Heavy spring and early summer grazing of these bottomland sites would favor the shorter, rhizomatous Pascopyrum smithii over the more robust, bunch-forming E. cinereus. We hypothesize that heavy grazing in the early part of the century removed most $E$. cinereus from these sites. With light grazing it is conceivable that E. cinereus could eventually regain dominance. This type might best be considered a disclimax of the Artemisia tridentata ssp. tridentata / Leymus cinereus plant association but only exclosures established before the cattle industry was initiated could pose an answer.

\section{Element Code- CEGL001017}

Edition / Author- 99-11-16 / S.V. Cooper, MTNHP

Artemisia tridentata / Stipa comata Shrubland

(ARTTRI / STICOM) basin big sagebrush / needle-and-thread

\section{Natural Heritage Conservation Rank- G4Q / S4}

Environment- Artemisia tridentata (ssp. tridentata) / Stipa comata is endemic to stabilized sand dunes in the Centennial Valley at 6,600-6,700 ft. Adjacent blowouts support Stipa comata / Psoralea tenuiflora as a seral community type.

Vegetation- Artemisia tridentata ssp. tridentata is the dominant shrub with $10-30 \%$ canopy cover. Chrysothamnus spp. are present in many stands. Grass canopy cover is ca. 40$70 \%$ with Stipa comata, Festuca idahoensis, or Elymus lanceolatus sharing dominance, depending on the stage of succession. Forbs have $10-30 \%$ cover and moderate to high diversity. Opuntia fragilis, Lupinus argenteus, Comandra umbellata and Artemisia dracunculus may be common. Mosses and lichens are absent.

Soils- Soils are deep and sandy. Gravel and rock are absent. $40-60 \%$ of the soil surface is bare.

Other Studies- Vegetation dominated by Artemisia tridentata ssp. tridentata and Stipa comata is reported for Idaho, Washington and Oregon (Bourgeron and Engelking 1994). This type occurs at low elevations in Washington and common forbs are very different than those of the Montana type (Daubenmire 1970).

Comments- This type might be considered a seral stage of Artemisia tridentata ssp. tridentata /Festuca idahoensis or an edaphic phase of this type. However, it appears to be stable over long time periods and has different soil, forb and bryophyte components than typical Artemisia tridentata ssp. tridentata / Festuca idahoensis.

The relationship between this type and the closely related Stipa comata phase of Artemisia tripartita / Festuca idahoensis is unclear. Both are found in apparently similar habitats. Fire and/or grazing may play a role in maintaining these different associations in the Centennial Sandhills landscape.

\section{Element Code- CEGL001010}

Edition / Author- 99-11-16 / S.V. Cooper, MTNHP

Artemisia tridentata ssp. vaseyana / Leymus cinereus Shrubland

(ARTTSV / LEYCIN) mountain big sagebrush / basin wildrye

\section{Natural Heritage Conservation Rank-} G4? / S?

Environment- Gentle to moderate slopes and terraces with warm aspects, deep soils and 
mesic moisture regimes may support examples of Artemisia tridentata ssp vaseyana / Leymus cinereus. Stands occur at 6,900-7,200 ft or higher in the Red Rock River drainage.

Slightly drier sites support Artemisia tridentata ssp vaseyana / Festuca idahoensis, while cooler sites support Pseudotsuga menziesii or Pinus flexilis forests. Adjacent rocky slopes support Cercocarpus ledifolius woodlands.

\section{Vegetation- Artemisia tridentata vaseyana is} the dominant shrub with 20-30\% canopy cover. Artemisia tripartita, Tetradymia canescens and Chrysothamnus spp. are often present but not common. Grass cover is $70-90 \%$. Dominant grasses are Leymus cinereus and Festuca idahoensis. Agropyron caninum, A. dasystachyum and Bromus carinatus may be common in some stands. Poa pratensis occurs in stands that have been heavily grazed. Forbs have $10-20 \%$ cover, but diversity is only low to moderate. Achillea millefolium, Cirsium spp., Erysimum inconspicuum and Potentilla gracilis are present in many stands. Mosses may be common in some stands.

Soils- Soils are deep with a loamy or silty in texture and are often derived from alluvium. Some sites may be slightly saline. Bare ground and surface gravel are uncommon.

Other Studies- This type has been described for Idaho (Bourgeron and Engelking 1994).

Comments- This type might also be considered a mesic phase of Artemisia tridentata ssp vaseyana/Festuca idahoensis. At this time the successional relationships of Festuca idahoensis and Leymus cinereus are not clear. Heavy spring or early summer grazing probably results in a decrease of $E$. cinereus, while light summer grazing may favor this species. Although this type is now confined to very mesic sites, it may not have been so restricted prior to introduction of livestock.

\section{Element Code- CEGL001027}

Edition / Author- 99-11-16 / S.V. Cooper, MTNHP
Artemisia tridentata ssp. vaseyana / Pseudoroegneria spicata Shrubland (ARTTSV / PSESPI) mountain big sagebrush / bluebunch wheatgrass

\section{Natural Heritage Conservation Rank- G5 / S?}

Environment- This type is common on steep slopes or occasionally ridges or terraces at 5,000-6,000 $\mathrm{ft}$ on the east slopes of the Pioneer Mountains and the Block Mountain area; it was noted in reconnaissance in the Tendoy Range on south-facing slopes. Common associated parent materials are basalt, quartzite or mixed sedimentary. Adjacent shallower, rocky soils are dominated by Cercocarpus ledifolius. Artemisia tridentata ssp. tridentata and Pascopyrum smithii dominate adjacent terraces.

Vegetation- Artemisia tridentata ssp. vaseyana is the dominant shrub with $10-40 \%$ canopy cover. Scattered Chrysothamnus nauseosus plants are usually present. The subshrubs, Artemisia frigida and Gutierrezia sarothrae are common in most stands. Grass canopy cover is generally 40-70\%. Pseudoroegneria spicata is the dominant species; Stipa comata, Bouteloua gracilis and Poa secunda are other common species. Forb canopy cover is $10-30 \%$, and diversity is low to moderate. Opuntia polyacantha, Phlox muscoides and Erigeron compositus are common species. Mosses and lichens are often common.

Soils- Soils have a loamy or sandy clay texture. The surface may be as much as $20 \%$ bare soil, but rock and gravel account for up to $70 \%$ cover.

Other Studies- Mueggler and Stewart (1980) describe this type for western Montana. Similar communities have been reported for most of the Northern Rocky Mountains and intermountain states (Bourgeron and Engelking 1994). Mueggler and Stewart (1980) discuss how the types described from different areas 
differ.

Comments- Most of the country south of Horse Prairie Creek may be too high to support extensive stands of this type.

Element Code- CEGL001030

Edition / Author- 99-11-16 / S.V. Cooper, MTNHP

\begin{tabular}{c} 
Artemisia tridentata ssp. wyomingensis / \\
Elymus lanceolatus Shrubland \\
(ARTTSW / ELYLAN) Wyoming big \\
sagebrush / thick-spike wheatgrass \\
\hline
\end{tabular}

\section{Natural Heritage Conservation Rank-} G4 / S?

Environment- This type occurs on gently sloping alluvial fans and terraces. Our single example was from $6,650 \mathrm{ft}$, but Artemisia tridentata ssp. wyomingensis/Elymus lanceolatus might be expected to occur at 5,000-7,500 ft. Artemisia arbuscula ssp. longiloba and Elymus lanceolatus or Leymus cinereus and Poa juncifolia dominate adjacent stands on heavier soils with poorer drainage. Adjacent slopes with deeper soils may support stands dominated by Artemisia tridentata vaseyana and Festuca idahoensis or Pseudoroegneria spicata.

Vegetation- Artemisia tridentata ssp. wyomingensis is the dominant shrub. Chrysothamnus spp. may also be present. The subshrub, Artemisia frigida, is usually common. Grass canopy cover is moderate to high and dominated by the midgrass species, Elymus lanceolatus and Stipa viridula. Poa cusickii and $P$. secunda may also be present. Forb cover is low, and diversity is low to moderate. Phlox hoodii and Astragalus adsurgens are common species. Mosses and lichens are uncommon.

Soils- Soils are fine-textured. Much of the soil surface is bare, and surface gravel is uncommon.

Other Studies- Jorgensen (1979) describes vegetation dominated by Artemisia tridentata wyomingensis and Elymus lanceolatus in central Montana. This type, or a very similar analogue, is reported for Wyoming and Colorado as Artemisia tridentata var. wyomingensis /Elymus lanceolatus var. albicans (Bourgeron and Engelking 1994).

Comments- Elymus lanceolatus and Pascopyrum smithii have overlapping habitat requirements throughout much of Montana (see Coupland 1961 and Jorgensen 1979). In much of Montana the two types, Artemisia tridentata ssp. wyomingensis / Pascopyrum smithii and Artemisia tridentata ssp. wyomingensis / Elymus lanceolatus may be indistinguishable except for the different dominant rhizomatous wheatgrasses.

\section{Element Code- CEGL001044}

Edition / Author- 99-11-16 / S.V. Cooper, MTNHP

Betula glandulosa / Carex utriculata Herbaceous Vegetation

(BETGLA / CARUTR) bog birch/beaked sedge

\section{Natural Heritage Conservation Rank- G4? / S4}

Environment- This community type occurs adjacent to beaver ponds, lakes, or marshes, and on seeps, swales and wet alluvial terraces adjacent to low gradient meandering streams (Hansen et al. 1995). This community occurs on fairly wet sites with peat accumulation, indicating a predominance of anaerobic processes. In contrast, some willow stands, like Salix drummondiana stands, commonly occur on soils that are better aerated, and hence are not usually found in peatlands. Soils are commonly flooded until mid summer, and are saturated year round on wetter sites. Redox concentrations are present in some mineral soils; redox depletions (gleyed soil) occur rarely. Organic matter accumulations may form floating, quaking mats as this type encroaches onto open water. Drier extremes have shallow organic horizons overlying 
deeper mineral soil (Hansen et al. 1995).

Range- Betula glandulosa / Carex utriculata is a minor type at mid elevations in western Montana (Hansen et al. 1995), and throughout Idaho (Moseley et al. 1991, Bursik and Moseley 1995).

Adjacent Communities- Salix drummondiana, S. geyeriana, Carex utriculata or C. lasiocarpa may dominate adjacent wetter sites. Drier wetland communities support Poa pratensis, Populus trichocarpa, and Potentilla fruticosa. At higher elevations, adjacent wetland forests are often dominated by Picea engelmannii or Abies lasiocarpa. Adjacent uplands support habitat types from the Abies lasiocarpa, Pseudotsuga menziesii, and Pinus ponderosa series, depending on elevation and aspect (Hansen et al. 1995).

Vegetation- Betula glandulosa contributes an average of $35 \%$ to the overstory. Minor amounts of Potentilla fruticosa and Salix species are usually present. The canopy cover provided by the various shrubs is sparse to moderate, but the herbaceous layer cover is high. Associated shrubs include Rhamnus alnifolia and various willows. Understory species composition is dependent on water levels. The wettest sites support Carex utriculata and C. aquatilis. Geum macrophyllum and the graminoides Poa pratensis and Agrostis stolonifera are often present in drier micro-sites and/or disturbed sites (Hansen et al. 1995).

Similar Communities- The Betula glandulosa / Carex utriculata habitat type is equivalent to Betula glandulosa / Carex rostrata (Hansen et al. 1995), which had been previously described in an unpublished study by Pierce (1986). Carex utriculata was erroneously referred to as Carex rostrata in earlier taxonomic and ecological studies (Griffiths 1989). Pierce (1986) described a similar community with an understory dominated by Deschampsia cespitosa. Other communities with a Betula glandulosa overstory and Carex lasiocarpa understory occur in northern Idaho and northwest Montana (Jankovsky-Jones 1997, Chadde et al. 1998, and Greenlee 1999).
Betula glandulosa / Carex cusickii plant associations also exist in northwest Montana (Greenlee 1999).

Succession - The Betula glandulosa / Carex utriculata community type represents a fairly stable type. Grazing my decrease the vigor of bog birch and increase the presence of species tolerant of grazing including Agrostis stolonifera, Poa pratensis, Poa palustris, and Juncus balticus.

Management - Saturated soils are highly susceptible to soil compaction and streambank sloughing when used by livestock and heavy machinery. Overuse may result in reduced vigor or eventual elimination of shrubs from the site. Burning of this type can temporarily increase productivity of Carex species. However, care should be taken when burning along streambanks because of the excellent erosion protection provided by Betula glandulosa / Carex utriculata habitat type (Hansen et al. 1995).

Wildlife Values- Betula glandulosa is a valuable browse species for elk (Kufeld 1973). Communities dominated by Betula glandulosa may function to stabilize channel banks (frequently creating overhanging banks) and provide shade creating quality fish habitat.

Element Code- CEGL001079

Edition / Author- 95-09-05/L. Williams

Cercocarpus ledifolius / Pseudoroegneria spicata Shrubland

(CERLED / AGRSP); curl-leaf mountain mahogany / bluebunch wheatgrass

\section{Natural Heritage Conservation Rank- G4Q / S4}

Environment- Mountain mahogany woodlands often dominate on steep, rockygravelly slopes, usually with warm, southeastthrough west-facing aspects at 5,400-7,900 ft throughout the study area; these conditions are usually found on upper and shoulder positions of ridge and hill slopes. Adjacent cool slopes 
may support Pseudotsuga menziesii or Pinus

flexilis forests. Adjacent warm slopes with deeper soils/less rock are dominated by the grasses Festuca idahoensis and

Pseudoroegneria spicata with or without Artemisia tridentata vaseyana or A. tripartita. Many stands grade into barren rock outcrops or talus slopes.

Vegetation- Stands are dominated by Cercocarpus ledifolius with canopy cover of $10-60 \%$ with a mean of $30 \%$. Artemisia tridentata vaseyana and Chrysothamnus viscidiflorus are often common. Juniperus scopulorum occurs in about half of the stands with a mean cover of $6 \%$ but its presence cannot be convincingly linked to particular habitat conditions or stand history. The subshrub, Artemisia frigida, is present in most stands. Grass cover is sparse.

Pseudoroegneria spicata and Oryzopsis hymenoides are the herbs with the highest constancy; the presence of either as common is diagnostic for the type. Poa secunda and Stipa comata are present in many stands. Forb cover is low but diversity is often moderate to high. No species occurred in even half of the sampled stands. Achillea millefolium, Opuntia polyacantha and Taraxacum officinale were the only species with constancy higher than $25 \%$. Mosses are present in most stands, and lichens are occasionally found.

Soils- Textures are sandy to sandy loam. An average of greater than $65 \%$ of the soil surface is bare or covered with rock or gravel. As Mueggler and Stewart (1980) suspected this type is not confined to calcareous substrates but is often found on these substrates because they weather to the appropriate rocky-gravelly substrate; this type was also found on granitics, extrusive volcanics and quartzite.

Other Studies- Mueggler and Stewart (1980) describe this type for western Montana; however, their sampling was limited to calcareous parent materials. Similar vegetation has been described for Idaho (Schlatterer 1972, Lewis 1975, Scheldt and Tisdale 1970). Vegetation with the same dominants has been reported for Colorado, Utah, Nevada and
Wyoming (Bourgeron and Engelking 1994).

Comments- Stands with Juniperus scopulorum have sometimes been segregated as a separate type (Bourgeron and Engelking 1994); however, we could find no consistent habitat or floristic differences between these two putative types in our study area, and indirect gradient analysis did not separate them. There is a tendency for J. scopulorum to be present only non-calcareous sites.

The presence of large quantities of pellets and strongly hedged $C$. ledifolius indicates that this vegetation type is very important for deer, particularly as winter range.

\section{Element Code- CEGL000967}

Edition / Author- 99-11-16 / S.V. Cooper, MTNHP

Chrysothamnus viscidiflorus / Stipa comata Shrubland

(CHRVIS / STICOM) green rabbitbrush / needle-and-thread

\section{Natural Heritage Conservation Rank-}

\section{Environment- The Chrysothamnus}

viscidiflorus / Stipa comata c.t. has been found only on the Centennial Sandhills as a putative seral stage to the Artemisia tridentata ssp vaseyana / Festuca idahoensis or Artemisia tridentata ssp. tridentata / Stipa comata community types. Chrysothamnus viscidiflorus / Stipa comata apparently represents a relatively early stage in the colonization of sandy substrates that have blownout (lost their original vegetation cover leaving a raw substrate). It can occur in any position in this dune system but most often was noted in drier exposures of upslope positions because these are the locations most often subject to disturbance.

Vegetation- Total vegetative cover on these sites is generally low, not exceeding $30 \%$, with Chrysothamnus viscidiflorus dominant and other shrubs, Tetradymia canescens,

Chrysothamnus nauseosus and Leptodactylon 
pungens, scattered. Stipa comata or Elymus lanceolatus, Opuntia polyacantha, Psoralea tenuiflora, and Phacelia hastata dominate the forb layer, but none are even well represented, the combined forb cover being less than $15 \%$.

Soils. The substrate is fine-textured sand with more than $90 \%$ exposed and only a trace of litter. There is no evidence of soil development with the exception of a trace of organic matter darkening the surface.

Other Studies- There is no comparable community type listed for the western United States and only one type (in Utah) wherein Chrysothamnus viscidiflorus is the diagnostic species (Chrysothamnus viscidiflorus / Leymus salinus ssp. salinus; Bourgeron and Engelking 1994).

\section{Element Code- CEGLMTNH12}

Edition / Author- 99-11-16 / S.V. Cooper, MTNHP

Cornus sericea Shrubland
(CORSER) red-osier dogwood

\section{Natural Heritage Conservation Rank-} G4 / S3

Environment- This type is typically adjacent to stream and river channels, but it can occupy a diversity of landforms. It may appear as dense linear bands on alluvial benches in narrow canyons or broad thickets on islands and floodplains of major streams and rivers. It may also occur on well-watered sites below beaver dams. Most occurrences have evidence of annual or near-annual flooding (Manning and Padgett 1995; Hall and Hansen 1997). Soils of this community are classified as Inceptisols, Entisols, or Mollisols. Where sites are located outside of the active floodplain, a litter/duff layer 2 inches or more thick may accumulate. Surface horizons are comprised of a wide range of alluvial materials with textures ranging from silty clay to sandy loam. These layers may be relatively shallow or as deep as 5 feet. Underlying layers are typically coarse sands, gravels, and cobbles that facilitate the movement of aerated groundwater through the subsurface layers which may be important for the longevity of stands. Water availability ranges from high, where this type occupies floodplains immediately adjacent to active channels and mottled and gleyed soils may occur (Manning and Padgett 1995; Hall and Hansen 1997; Crowe and Clausnitzer 1997), to low on upper, remote floodplain sites.

Range- This is a widespread type known from Washington, Oregon, Idaho, Nevada, and Montana.

Adjacent Communities- Because of the wide geographic range for this type, communities of adjacent uplands can be coniferous forest, aspen, sagebrush-steppe, and pinyon-juniper types.

Vegetation- Cornus sericea forms a dense, closed canopy, often excluding understory shrub and herbaceous species. Cornus sericea is usually the only species with high cover values. Associated species vary with geographic location and elevation, but commonly associated shrubs include Rosa woodsii, Ribes hudsonianum, Acer glabrum, Salix exigua, S. lutea, and Clematis ligusticifolia. Because of its wide range, a great diversity of herbaceous species are associated with this community, usually in low cover (Manning and Padgett 1995; Hansen et al. 1995; Hall and Hansen 1997; Crowe and Clausnitzer 1997).

Similar Communities- Cornus sericea is a community dominant in several associations. This community, however, lacks the structural diversity of the other types, for example the Alnus incana / Cornus sericea and Cornus sericea-Salix sp. types from Nevada (Manning and Padgett 1995). The relationship of this community with the Cornus sericea / Heracleum lanatum and C. sericea / Galium triflorum types from Utah and eastern Idaho (Youngblood et al. 1985, Padgett et al. 1989) is unclear.

Succession- This is considered an early seral community, typically colonizing sites adjacent 
to streams. The herbaceous cover is often sparse, probably due to the dense overstory canopy and regular flooding, scouring, and deposition. The latter factor is probably responsible for maintaining this as a persistent community type on the landscape. The presence of tall shrubs or trees in some stands may represent succession toward Alnus incana, Populus trichocarpa, $P$. tremuloides, $P$. angustifolia, Picea engelmannii, Pseudotsuga menziesii, or other communities.

Management- The herbaceous biomass varies widely and is largely dependent on the density of the dogwood canopy (Crowe and Clausnitzer 1997). Ratings for red-osier dogwood palatability for livestock range from low (Manning and Padgett 1995; Crowe and Clausnitzer 1997) to "ice cream" (Hansen et al. 1995; Hall and Hansen 1997), but the stands are often so dense that they limit grazing in many cases. This community functions in a variety of ways to promote stream health. Redosier dogwood forms dense root networks that stabilize streambanks against lateral cutting and erosion, provides cover in the form of overhanging branches and banks, and shades channels, effectively moderating extreme summer temperature fluctuations (Hall and Hansen 1997). Dogwood sprouts vigorously after a fire and germination of it's seed-bank is stimulated by fire (Crowe and Clausnitzer 1997).

Wildlife Values- Red-osier dogwood provides food and cover for mule deer, moose, elk, cottontail rabbits, snowshoe hares, and many birds. The fruits are an important back bear food and are also eaten by songbirds, grouse, quail, partridge, cutthroat trout, ducks, crows, mice, and other mammals. Deer mice, meadow voles, and other small rodents eat the young stems and bark. Red-osier dogwood often grows in dense thickets because of its layering ability. These thickets provide good mule deer fawning and rearing areas and nesting habitat for many songbirds (Hansen et al. 1995, Crowe and Clausnitzer 1997).

Element Code- CEGL001165
Edition / Author- 98-01-02/ B. Moseley

\section{Pentaphylloides floribunda / Deschampsia cespitosa Shrubland \\ (POTFRU / DESCES) shrubby cinquefoil / tufted hairgrass}

\section{Natural Heritage Conservation Rank-} G4 / S4

Environment- These alkaline meadows occur on sub-irrigated flood plains, stream terraces and gentle lower slopes, often in association with springs or seeps; though facultative hydrophytes are often abundant these do not consistently possess hydrologic regimes or soil characteristics indicating jurisdictional wetlands. This c.t. was found at 5,000-7,500 ft throughout the study area, most often in areas of calcareous parent material. Most examples of this type have hummock-hollow microtopography. Salix spp., Carex utriculata or Carex simulata fen or Juncus balticus and Deschampsia cespitosa often dominate wetter sites. Drier sites may be dominated by Leymus cinereus, Carex praegracilis and Puccinellia distans. Artemisia tridentata, A. tripartita or A. longiloba and Festuca idahoensis dominates adjacent upland communities.

Vegetation- Pentaphylloides floribunda is the only common shrub with $10-30 \%$ cover.

Dominant graminoids, usually well represented, are Juncus balticus and Deschampsia cespitosa. Other common species include Carex praegracilis and Muhlenbergia richardsonis. Forbs are diverse and may be abundant, up to $35 \%$ cover. Common species include Aster occidentalis, Senecio debilis, Allium schoenoprasum and Valeriana edule. Taraxacum officinale, Poa pratensis and Iris missouriensis may be common in stands that have been heavily impacted by livestock grazing. Mosses are common in some stands.

Soils- Soils have a silty texture and an alkaline reaction; some may be slightly saline as well. Soils are moist to wet for at least the first half of the growing season. There is very little bare 
ground and no surface gravel.

Other Studies- Hansen et al. (1995) and Lesica (1990) describe a Pentaphylloides floribunda / Deschampsia cespitosa habitat type for western Montana. Similar vegetation has been described for Idaho, Utah and western Wyoming (Youngblood et al. 1985, Padgett et al. 1989, and Chadde et al. 1988).

Comments- Hansen et al. (1995) believes that the abundance of Juncus balticus is positively correlated with overgrazing. However, we believe that other factors, such as hydrologic regime, salinity and soil reaction may also affects the relative dominance of $J$. balticus and Deschampsia cespitosa (see comments under Juncus balticus - Carex praegracilis

The Pentaphylloides floribunda / Deschampsia cespitosa c.t. is similar in composition to the Deschampsia cespitosa c.t. and the Juncus balticus - Carex praegracilis c.t. The presence of Pentaphylloides floribunda is undoubtedly related to the degree of soil aeration, with Pentaphylloides floribunda increasing soils become drier. However, this relationship is also influenced by the degree of hummocking. Hummocks provide a better-aerated environment and allow $P$. fruticosa to occur in areas where it otherwise would not. It is not known how hummocks are formed, but frost action may play a role. Trampling by livestock enhances the hummocks and probably allows shrubs to become larger and more frequent. It is also possible that trampling by livestock and other large animals is the primary cause of the hummocks. Deschampsia cespitosa and Juncus balticus - Carex praegracilis types can probably be converted to Pentaphylloides floribunda / Deschampsia cespitosa by the formation of hummocks.

This vegetation is productive and often remains green throughout the growing season, making it attractive to livestock, especially late in the year. The moist to wet soils and generally small size of stands make this type very prone to degradation. Trampling by livestock, especially during the spring and early summer results in the formation or enhancement of hummocks. The presence of substantial hummocking may cause an increase of the shrub, Pentaphylloides floribunda.

Element Code- CEGL001107

Edition / Author- 99-11-16 / S.V. Cooper, MTNHP

Pentaphylloides floribunda / Potentilla
ovina Shrubland
(PENFLO / POTOVI) shrubby cinquefoil /
sheep cinquefoil

Natural Heritage Conservation RankG? / S?

Environment- This uncommon type occurs in broad swales of gently sloping alluvial fans in areas of calcareous parent material. These sites may be sub-irrigated during part of the year; they have the appearance of being salt-affected and possibly experience soil loss through sheet erosion on an annual basis. Pentaphylloides floribunda / Potentilla ovina is found at 7,000$7,400 \mathrm{ft}$ towards the headwaters of Big Sheep Creek in the Beaverhead and Tendoy mountains. Artemisia tridentata ssp. wyomingensis or A. longiloba and Festuca idahoensis dominates adjacent upland vegetation.

Vegetation- The only shrub is Pentaphylloides floribunda with canopy cover of ca. 30\%. Ground layer vegetation is sparse. Graminoid canopy cover is usually less than $20 \%$; species with high constancy and at least common coverage are Muhlenbergia richardsonis, Pascopyrum smithii, Carex scirpoidea and Poa juncifolia. Forbs frequently present include Potentilla ovina, Gentiana affinis, Viola nephrophylla, Aster occidentalis and Hedysarum sulphurescens. Mosses and lichens are absent, perhaps stripped by a flooding regime.

Soils- Soils have a silty texture and a near neutral to alkaline reaction. They appear to be shallow and stony with ca. one third of the surface covered with gravel or rock. Over $50 \%$ 
of the soil surface is bare.

Other Studies- Similar vegetation has not been previously described.

Comments- Soil appears to have been removed from these sites by water, although this seems implausible given the nearly level slope. Perhaps wind erosion following severe overgrazing caused the shallow soils. This type bears some similarity to Pentaphylloides floribunda / Deschampsia cespitosa; however, it has very different soils and herbaceous species that indicate drier conditions.

Trampling by livestock during the spring when these sites are wet results in soil compaction and a decrease in productivity.

\section{Element Code- CEGLMTHP17}

Edition / Author- 99-11-16 / S.V. Cooper, MTNHP

Salix boothii / Calamagrostis canadensis Shrubland

(SALBOO / CALCAN) Booth's willow / Bluejoint reedgrass

\section{Natural Heritage Conservation Rank- G3G4Q / SR}

Environment- The Salix boothii /

Calamagrostis canadensis association can be found in montane habitats in western Montana (from valley bottoms to mid-elevations in the mountains) and in the mountains of central and eastern Montana. It is frequently found on alluvial terraces where beaver activity has created a series of dams that raise the local water table, along streams, and near seeps or springs. Soils are usually deep silt or sand overlying more sand, gravel, or cobbles. This community usually floods during spring, with the water level within $1 \mathrm{~m}$ of the surface the rest of the year. Nearby wetter communities could include Carex utriculata, Salix geyeriana / Carex utriculata, Typha latifolia, or open water, and nearby drier communities could include Populus balsamifera ssp. trichocarpa / Cornus sericea, Calamagrostis canadensis,
Deschampsia cespitosa, or Juncus balticus. A variety of adjacent uplands could occur nearby ranging from conifer dominated communities to dry shrublands such as Artemisia tridentata associations (Hansen et al. 1988, Hansen et al. 1995).

\section{Range- The Salix boothii / Calamagrostis} canadensis association is found in Montana, Utah, Idaho, and western Wyoming.

Vegetation: The Salix boothii / Calamagrostis canadensis association typically has a dense overstory dominated by Salix boothii, and an understory dominated by Calamagrostis canadensis. In contrast to Salix geyerianadominated communities, these stands are more often closed and less easily accessible by large ungulates, while Salix geyeriana stands have a more open corridor aspect (Padgett et al. 1989). Other willows commonly found in this community are Salix geyeriana and Salix drummondiana; however, Salix boothii is clearly the dominant willow. Common associated understory species are Carex utriculata, Geum macrophyllum, Mentha arvensis, Solidago canadensis, and Equisetum arvense (Hansen et al. 1995).

Similar Communities- This community has also been documented in Utah (Padgett et al. 1989), Idaho and western Wyoming (Youngblood et al. 1985). Other studies (Hansen et al. 1995, Hall and Hansen 1997) include Salix boothii-dominated stands within a Salix geyeriana habitat type for management purposes, since Salix boothii and Salix geyeriana are often codominant within a stand. Other authors (e.g. Padgett et al. 1989) separate Salix boothii-dominated stands as a separate plant association due to structural differences between Salix boothii and Salix geyeriana stands, although based on the descriptions in Padgett et al. (1989), there is some degree of overlap between the two plant associations.

\section{Succession- Salix boothii / Calamagrostis} canadensis stands are fairly stable if the hydrologic regime remains unchanged. Kittel et al. (1998) suggest that flooding events in Salix boothii/Carex utriculata communities can 
result in sediment deposition, which raises the floodplain surface higher above the water table. As the floodplain aggrades, the site could become less saturated, which could cause the graminoid understory to change towards Calamagrostis canadensis. Similarly, Kittel (1994) states that distance from the stream channel can change the degree of soil saturation, and thereby influence the understory composition. Removal of beaver from a Salix boothii/Carex utriculata stand could also cause compositional changes. Unmaintained beaver dams could break, and cause a lowering of the water table, which could cause a shift in the dominant understory graminoid towards Calamagrostis canadensis (Hansen et al. 1995).

\section{Management- Calamagrostis canadensis is} moderately to highly palatable and with high grazing pressure, the vigor, reproductive success, and competitive ability of this grass will decrease. Exotic pasture grasses such as Poa pratensis or Agrostis stolonifera may then increase. Livestock grazing in this association should be avoided when the soils are wet to avoid churning of the soil surface. Salix boothii / Calamagrostis canadensis stands exposed to heavy browsing pressure usually show reduced vigor of the willow species, such as highlining, clubbing, or dead clumps, with eventual decrease in willow coverage (Hansen et al. 1995).

\section{Element Code- CEGL001175}

Edition / Author- 99-10-18 / J. Greenlee, MTNHP

Salix candida / Carex utriculata Shrubland (SALCAN / CARUTR) Hoary willow Beaked sedge

\section{Natural Heritage Conservation Rank-}

\section{G3 / S3}

Environment- This community is found in montane to lower subalpine habitats in western, southwestern, and central Montana. It occurs on peat deposits that have developed around the margins of lakes and ponds, and around springs/seeps. These sites are seasonally flooded and have water tables at or near the surface throughout the growing season. Adjacent wetter communities include Carex lasiocarpa floating mats, rooted aquatic vegetation like Nuphar sp., or open water. Adjacent drier communities include those dominated by Pentaphylloides floribundal Deschampsia cespitosa. Uplands are usually dominated by coniferous forest (Hansen et al. 1995).

Vegetation- Salix candida grows as a low shrub and dominates the shrub layer, although canopy coverage tends to be low (10\%-40\%). There may be a few scattered shrubs with greater stature than Salix candida, like Betula glandulosa. A dense cover of Carex utriculata, and Carex aquatilis, Carex simulata dominates the understory, and Triglochin maritimum can also compose significant amounts of the understory coverage. Forb coverage is usually low (Hansen et al. 1995).

Similar Communities- Chadde et al. (1998) mention a Salix candida / Carex lasiocarpa peatland community for western Montana which is similar to Salix candida / Carex utriculata, except that the understory is dominated by Carex lasiocarpa; this type is also supported by unpublished plot data on file at MTNHP. Lesica (1986) also notes Salix candida as an important shrub component in dwarf carr vegetation at the Pine Butte Fen on the Rocky Mountain Front.

Succession- The successional dynamics of this community are poorly understood. It is most likely a fairly stable community, as the peat deposits upon which this community is found require a stable hydrologic regime.

Management- The organic soils of this type are easily damaged by livestock use, especially when wet. However, due to the wetness of this type, it most likely does not receive much livestock use in any case. The response of Salix candida to fire has not been documented.

Element Code- CEGL001188 
Edition / Author- 99-10-18 / J.Greenlee, MTNHP

\section{Salix exigua Temporarily Flooded Shrubland \\ (SALEXI) sandbar willow}

\section{Natural Heritage Conservation Rank- G5 / S5}

Environment- This type occurs on gravelly alluvial parent materials on flat flood plains and terraces in wide valley bottoms.

Elevations are between 5000 and 5500 feet. Litter plus wood cover equals or exceeds $60 \%$ of the ground surface and soil cover is typically around $20 \%$. The soil surface is unstable because of a lack of adequate vegetation cover and the active fluvial disturbance regime present on S. exigua sites.

Vegetation- Salix exigua dominates the overstory (often with a total canopy cover of $100 \%$ ). The undergrowth is typically very depauperate due to the intense competition with S. exigua and seldom exceeds 5\% total cover. Species that are often present in this sparse undergrowth include Cirsium arvense, Mentha arvensis, and Phalaris arundinacea.

Soils- Soils are often Aquic Udifluvents, are very deep with sandy textures, have a low available water holding capacity, and are noncalcareous. Coarse fragment contents vary from gravelly to very cobbly.

Adjacent Communities- Adjacent sites are often open water. Adjacent drier riparian communities often feature the Populus balsamifera ssp. trichocarpa / Cornus sericea type.

Other Studies- This type has been described as ubiquitous and abundant in Montana by Hansen et al. (1991); it is reported for the northwest, where it is comparatively rare, by Kovalchik (1987) and as far to the southeast as Colorado (Kittel and Lederer 1993).

Element Code- CEGL001197
Edition / Author- 99-11-16 / S.V. Cooper, MTNHP

Salix drummondiana / Calamagrostis canadensis Shrubland

(SALDRU / CALCAN) drummond's willow / bluejoint reedgrass

\section{Natural Heritage Conservation Rank- G3 / SP}

Environment- Elevation ranges from 2320 to 8200 feet throughout the range of the community. Type occurs on low gradient slopes adjacent to beaver ponds, lakes, marshes, rivers and streams, or on toeslopes below upland sites. Soils are coarse to fragmented loams or grass peat over deep, erosive, moderately fine textured alluvium (Kovalchik 1993, Tuhy and Jensen 1982). Hansen et al. (1995) notes soil textures range from silt to clay loam; mottling and gleyed soils are common. Type is relatively dry compared to other willow plant association (Kovalchik 1993). Water levels range from at the surface to $100 \mathrm{~cm}$ below the surface during the growing season.

Range- This community is a minor type in Colorado, Utah, Idaho, Washington, and Montana.

Adjacent Communities- Adjacent wetter sites may support Salix drummondiana / Carex utriculata, Carex utriculata, C. aquatilis, or $C$. scirpoidea var. pseudoscirpoidea types, or open water. Drier sites may support Salix dominated types with a Poa pratensis or Juncus balticus understory, or Potentilla fruticosa, Alnus incana or conifer dominated types (Hansen et al. 1995, Kovalchik 1993).

Vegetation- Salix drummondiana dominates the tall shrub layer (25-60\% cover). Salix geyeriana, Salix boothii and Salix monticola are sometimes present in lesser amounts than the dominant shrub. Lonicera involucrata, Ribes spp., Alnus incana, and Pentaphylloides floribunda are usually present with up to $15 \%$ cover individually. Calamagrostis canadensis contributes at least $5 \%$ and up to $60 \%$ cover to 
the understory. Other species with high constancy include Carex microptera, $C$. utriculata, C. aquatilis, Deschampsia cespitosa, Aster foliaceus, and Fragaria virginiana.

Similar Communities- Similar communities include Tuhy's (1981) Salix drummondiana / Ribes lacustre /Thalictrum occidentale, Mutz and Queiroz's (1983) Salix drummondianaSalix boothii / Calamagrostis canadensis, Baker's (1989) Salix drummondiana-Salix monticola/Calamagrostis canadensis-Carex rostrata, and Kittel et al.'s (1998) Salix drummondiana / mesic forb types.

Grazing pressure will cause a decrease in Calamagrostis canadensis and Deschampsia cespitosa, with a corresponding increase in either introduced or less desirable species such as Ribes setosum, Urtica dioica, and Equisetum arvense. Abundance of Calamagrostis canadensis suggests that communities may be seral stages of Abies lasiocarpa/Calamagrostis canadensis habitat type. The development of a conifer overstory tends to reduce and eventually eliminate the shade intolerant Salix species without affecting the herbaceous layer (Tuhy and Jensen 1982, Hansen et al. 1995).

Management- The vigor of Salix spp. in these communities appears directly related to streambank stability and rate of sedimentation into stream systems (Tuhy et al. 1982). Sustained grazing decreases the vigor, reproductive success, and competitive ability of Calamagrostis canadensis and Deschampsia cespitosa. To maintain vigor and prevent damage to soils and vegetation, grazing should be deferred until soils dry. Proper levels of grazing should range from light to moderate. Overuse by livestock will result in reduced vigor of willow species present, illustrated by uneven stem age distribution, highlining, and clubbing or dead clumps. With continued overuse, willows may be eventually eliminated from the site (Hansen et al. 1995).

Wildlife Values- Abundant food, cover, and proximity to water provide habitat for numerous wildlife species and songbirds.
Moose and beaver tend to heavily utilize most species of willow.

Element Code- CEGL002667

Edition / Author- 1996-06-13/L. Williams

Salix drummondiana / Carex utriculata Shrubland

(SALDRU / CARUTR) drummond's willow / beaked sedge

\section{Natural Heritage Conservation Rank- G3 / S5}

Environment- The community is found in narrow to wide valleys on alluvial terraces adjacent to streams of low or moderate gradients (Mutz and Queiroz 1983; Hansen et al. 1995; Hall and Hansen 1997). These streams are often moderately entrenched, Rosgen C types (Kovalchik 1993). It is equally common adjacent to poorly drained or impounded areas such as beaver ponds, peatlands, lakes, marshes, seeps, springs, and road crossings (Kovalchik 1993; Moseley et al. 1994; Hansen et al. 1995). Though on mostly flat ground, channels and hummocks (Mutz and Queiroz 1983) characterize the microtopography. As with landform settings, soils vary from Entisols and Histosols to Mollisols. Soils adjacent to moderate gradient streams are often poorly developed, coarse textured, and sandy with high gravel and cobble content. These soils allow the water necessary to support Carex utriculata to easily pass through (Hansen et al. 1995). In wider valleys, clay and silt-loam or organic soils are more common. Gleying and mottling are often present, typical of a spring/summer surface water table followed by the water table dropping to $100 \mathrm{~cm}$ below the surface by late summer (Kovalchik 1993). Organic loam and sedge peat soils, with high available water content, are up to $1 \mathrm{~m}$ deep and classified as Cumulic Cryaquolls and Terric, Hemic, Sapric, and Fibric Histosols (Mutz and Queiroz 1983; Kovalchik 1993). A 5-cm surface litter/duff layer may be present. The soils of this community are held together by sod mats formed by Carex species and willow cover 
which effectively stabilize stream banks (Hansen et al. 1995).

Range- The Salix drummondiana / Carex utriculata community type is known from Montana, Idaho, Washington, and probably western Wyoming.

Adjacent Communities- Communities adjacent to Salix drummondiana / Carex utriculata include other Salix drummondiana types with slightly drier moisture regimes. Examples are Salix drummondiana / Calamagrostis canadensis, Salix drummondiana / Carex scopulorum var. prionophylla, and Salix drummondiana / Poa pratensis (Mutz and Queiroz 1983, Hansen et al. 1988, Kovalchik 1993, Hansen et al. 1995). Other adjacent communities with similar moisture levels are Salix geyeriana / Carex rostrata, Salix boothii / Carex rostrata, Salix farriae / Carex scopulorum var. prionophylla, and Salix wolfii communities (Mutz and Queiroz 1983, Kovalchik 1993, Hall and Hansen 1997, Walford et al. 1997). Slightly drier adjacent communities include Alnus incana / Calamagrostis canadensis, Alnus incana / Carex utriculata, Potentilla fruticosa / Deschampsia cespitosa, and Deschampsia cespitosa communities. Wetter adjacent communities are herbaceous types (Carex utriculata, Carex aquatilis, or Carex lasiocarpa dominated) and Salix farriae / Carex utriculata (Kovalchik 1993, Hansen et al. 1995). Adjacent uplands are Abies lasiocarpa, Pseudotsuga menziesii, Picea engelmannii, or Pinus ponderosa habitat types (Hansen et al. 1988, Hansen et al. 1995).

Vegetation- The Salix drummondiana / Carex utriculata community type is variable, often having mixed Salix and Carex species present. Salix drummondiana is usually dominant with 30 to $55 \%$ cover and 70 to $100 \%$ constancy (Kovalchik 1993, Hansen et al. 1995, Jankovsky-Jones 1997). Other tall willow species, such as Salix geyeriana, S. boothii, $S$. sitchensis, S. lasiandra, S. bebbiana, and $S$. pseudomonticola, usually have less than $40 \%$ cover and less than $30 \%$ constancy. While these species form a tall shrub canopy (to $4 \mathrm{~m}$ ), shorter species, such as Salix farriae or Salix planifolia, can be prominent in the understory (Mutz and Queiroz 1983, Kovalchik 1993, Hansen et al. 1995). Where Salix species have been reduced by beaver or overgrazing, Betula glandulosa (10 to $15 \%$ cover), Spiraea douglasii, or Ribes species may be important (Hansen et al. 1995). Picea engelmannii, Abies lasiocarpa, and Alnus incana are also occasionally present. The herbaceous layer is dominated by Carex utriculata (10 to $39 \%$ cover, about $80 \%$ constancy) and Carex aquatilis (less than $34 \%$ cover, less than $80 \%$ constancy) with Carex vesicaria also common. Other associated Carex, having low cover and constancy, include Carex lanuginosa, $C$. lasiocarpa, C. lenticularis, and $C$. nebrascensis. Other common graminoid species, with low constancy but occasionally moderate cover (less than 40\%), are Calamagrostis canadensis, Phalaris arundinacea, Scirpus microcarpus, Glyceria species, and Juncus species (Mutz and Queiroz 1983; Kovalchik 1993; Hansen et al. 1995; Jankovsky-Jones 1996; Jankovsky-Jones 1997). Due to the dense Salix and Carex species cover, overall forb cover is low and mainly around shrub bases. Widespread species are Epilobium ciliatum, Geum macrophyllum, and Equisetum arvense. Less common species (but occasionally with higher cover) include Saxifraga arguta, Galium species, Petasites sagittatus, and Aster modestus (Mutz and Queiroz 1983, Kovalchik 1993, Hansen et al. 1995, Jankovsky-Jones 1996, Jankovsky-Jones 1997). Moss cover is often high.

Similar Communities- Earlier studies lumped this community within broader Salix / Carex rostrata [often misidentified, actually Carex utriculata], Salix drummondiana-Salix boothii/ Carex rostrata-Carex aquatilis, and Salix/Carex rostrata-Carex aquatilis communities (Tuhy and Jensen 1982, Mutz and Queiroz 1983, Walford et al. 1997). Likewise, in eastern Idaho, western Wyoming, and Utah, it may have been kept within the Salix boothii / Carex rostrata or Salix geyeriana / Carex rostrata community types (Youngblood et al. 1985, Padgett et al. 1989). These communities often 
have high cover and constancy of Salix drummondiana (to the level of co-dominance) making lumping of types seems logical (Hansen et al. 1995, Hall and Hansen 1997). Salix drummondiana communities, with their mixed Salix species composition, may be transitional to other community types (Kovalchik 1993). In addition, Salix sitchensis is easily confused with Salix drummondiana (with which it may hybridize). Salix sitchensis sometimes co-dominates stands making community identification difficult (JankovskyJones 1997).

The edaphic and hydrologic situations which allow Carex utriculata dominance also promote many different Salix species. However, dominance by any one Salix species can be the result of many factors such as elevation or grazing (Hall and Hansen 1997). Tall willow communities similar to Salix drummondiana / Carex utriculata (often with high cover and constancy of Salix drummondiana) include: Salix drummondianaSalix boothii / Carex rostrata-Carex aquatilis, Salix boothii / Carex rostrata, Salix geyeriana / Carex rostrata, Salix lutea / Carex rostrata, and Salix drummondiana / Carex aquatilis (Mutz and Queiroz 1983, Youngblood et al. 1985, Padgett et al. 1989, Hansen et al. 1995, Hall and Hansen 1997, Walford et al. 1997, Kittel et al. 1998). Short willow species may dominate at higher elevations. Salix drummondiana is sometimes present in short willow communities such as: Salix candida/ Carex utriculata; Salix farriae/Carex utriculata; and Salix wolfii/Carex rostrata (Youngblood et al. 1985; Padgett et al. 1989; Kovalchik 1993; Hansen et al. 1995; Walford et al. 1997). Other Carex species may be more common than Carex utriculata in similar communities due to variations in seral status or other factors. These include Salix boothii / Carex aquatilis, Salix geyeriana / Carex aquatilis, and Salix drummondiana / Carex scopulorum var. prionophylla (Youngblood et al. 1985; Padgett et al. 1989; Kovalchik 1993; Hansen et al. 1995; Hall and Hansen 1997).

Succession- The successional origin of Salix drummondiana / Carex utriculata is not well known. Both Salix drummondiana and Carex utriculata can be colonizers of fresh, mineral alluvium (Hansen et al. 1995, Walford et al. 1997). Thus, when alluvium is exposed, such as post-flood silt deposits around willow roots or after a beaver dam breaks, these species may invade. Alternately, Carex utriculata might invade on silt deposited in open beaver ponds, then allowing later Salix invasion as the site dries (Mutz and Queiroz 1983). Another hypothesis, taken from the similar Salix boothii / Carex utriculata type, is that a Salix community existed before the beaver dam. The beaver dam was built, flooding the Salix but not eliminating it, subsequent siltation allowed Carex utriculata to invade, and Salix rejuvenated later (Youngblood et al. 1985, Padgett et al. 1989). Whatever the origin, stability of the Salix drummondiana / Carex utriculata community is indicated by a thick accumulation of organic matter (Kovalchik 1993). Disturbance by livestock or beaver will reduce Salix drummondiana cover and allow graminoids, especially introduced species, to increase (Mutz and Queiroz 1983). If willows are reduced too much, beaver will leave in search of food and fail to maintain dams washed out by storms. The water table will then lower as the stream downcuts and the community will change toward a drier Salix drummondiana / Calamagrostis canadensis or Abies lasiocarpa type (Hansen et al. 1988; Hansen et al. 1995).

Management- Salix drummondiana / Carex utriculata can be a productive community but will decrease if soils are damaged or hydrologic conditions change. For example, recreation trails, road building, agriculture (including draining with ditches), and livestock grazing easily damage organic soils through compaction and reduction of water holding capacity (Mutz and Queiroz 1983; Moseley et al. 1994; Hansen et al. 1995). These activities may also cause streambank sloughing as well as premature soil drying, the loss of vegetative protection, and eventual loss of the community. Beavers are also important in maintaining necessary hydrologic conditions. Thick shrub cover and excessive wetness often limit activities in this community. Livestock forage 
value varies with season and historic use, but both Salix drummondiana and Carex utriculata are fair to good forage in the spring (Hansen et al. 1988, Hansen et al. 1995). Overgrazing of willows decreases their vigor and can eliminate them from the site allowing graminoid cover to increase. This may occur with a late summer and fall grazing regime, which reduces willow regrowth and allows sedges, with their underground root reserves, to later proliferate. Thus, long rest periods are needed to maintain the community (Hansen et al. 1995).

Prescribed fire effectively rejuvenates dead clumps because Salix drummondiana sprouts vigorously after fire (quick, hot fires are preferred over slow, cool burns). Fires also increase Carex rostrata but only if ungrazed before and after the fire (Hansen et al. 1995). Both Salix drummondiana and Carex rostrata (and Carex aquatilis and C. vesicaria) are excellent for re-vegetation over the long-term and provide good erosion control (Hansen et al. 1995).

Wildlife Values- In the winter, Salix drummondiana shoots are heavily browsed by moose. Throughout the year Salix drummondiana is utilized by beaver and provides fair forage for elk and deer. Songbirds also utilize Salix species habitat for feeding and nesting. In addition to Salix root masses, the dense Carex rostrata and Carex aquatilis sod overhangs undercut banks creating prime fish habitat (Hansen et al. 1988; Hansen et al. 1995; Hall and Hansen 1997; Walford et al. 1997).

\section{Element Code- CEGL002631}

Edition / Author- 1998-11-25 / Chris Murphy

Salix glauca Shrubland (SALGLA) Glaucus willow

\section{Natural Heritage Conservation Rank-}

G3? / S2

Environment- The single stand of Salix glauca occurred on a moderate to steep upper, northfacing slope, just in the lee of a divide ridge at
9,900 ft in the Snowcrest Range. We observed but did not sample other examples of this type in the Gravelly Range. This site was a definite snow catchment area. Adjacent associations were Carex elynoides turf and moist slopes.

Vegetation- Salix glauca had canopy cover of 60\%; no other shrubs were present. Trace amounts of Poa alpina and Elymus lanceolatus were present, but total graminoid cover was only $1 \%$. Forb cover was $60 \%$. Common species included Aster alpigenus, Hedysarum sulphurescens, Senecio crassulus and Synthyris pinnatifida. Lichens and mosses covered $7 \%$ of the ground surface.

Soils- Parent material was calcareous sandstone. Exposed ground and gravel constituted $6 \%$ of the surface. Depths of litter and duff were 1.0 in and 0.5 in respectively. The high surface organic matter probably reflects low rates of decomposition due to low insolation and late snowmelt. Percent coarse fragments were very different for different microsites but averaged $30 \%$ for the stand. The texture of the fine fraction was sandy loam. Soil $\mathrm{pH}$ was 7.6. Organic matter content was $14 \%$, mean total nitrogen was $0.31 \%$, and C: $\mathrm{N}$ ratio was $28: 1$.

Productivity- Our estimates are based on only three plots in one stand and should be considered only rough approximations. Salix glauca produced $759 \mathrm{lbs} /$ acre. Graminoid productivity was $12 \mathrm{lbs} / \mathrm{acre}$, and forb productivity was $759 \mathrm{lbs} / \mathrm{acre}$. Total productivity was $1,530 \mathrm{lbs} / \mathrm{acre}$.

Other Studies- Achuff and Corns (1982) describe three community types dominated by Salix glauca from the Canadian Rockies.

These associations contain other shrubs (i.e., Salix, Betula, and Potentilla) and better developed graminoid cover. Salix glauca associations were observed near treeline in the Front Range of Montana, south of Glacier National Park (Cooper and Lesica, pers. obs.). Associations dominated by Salix glauca occur on gentle lee slopes on the east end of the Beartooth Range (Lesica 1991). Common understory species in these communities were 
Carex paysonis, Deschampsia cespitosa, Geum rossii and Lupinus argenteus. In the Beartooth Range of Montana and the Rocky Mountains of Colorado, Salix planifolia and S. glauca dominated associations found on cool moist slopes having late snow release (Johnson and Billings 1962, Komarkova and Webber 1978).

\section{Element Code- CEGL001136}

Edition / Author- 97 -10 / S.V. Cooper et al.

\section{Salix geyeriana / Calamagrostis} canadensis Shrubland

(SALGEY / CALCAN) Geyer's willow / Bluejoint reedgrass

\section{Natural Heritage Conservation Rank- G5 / S4}

Environment- The Salix geyerianal Calamagrostis canadensis association can be found in montane habitats in western Montana (from valley bottoms to mid-elevations in the mountains) and in the mountains of central and eastern Montana. It is frequently found on alluvial terraces where beaver activity has created a series of dams that raise the local water table, along streams, and near seeps or springs. Soils are usually deep silt or sand overlying more sand, gravel, or cobbles. This community usually floods during spring, with the water level within $1 \mathrm{~m}$ of the surface the rest of the year. Nearby wetter communities could include Carex utriculata, Salix geyerianal Carex utriculata, Typha latifolia, or open water, and nearby drier communities could include Populus balsamifera ssp. trichocarpa / Cornus sericea, Calamagrostis canadensis, Deschampsia cespitosa, or Juncus balticus. A variety of adjacent uplands could occur nearby, ranging from conifer dominated communities to dry shrublands such as Artemisia tridentata associations (Hansen et al. 1988, Hansen et al. 1995).

Range- This community or one very similar to it occurs in Montana, Utah, Nevada, and Idaho.

Vegetation- The Salix geyerianal Calamagrostis canadensis association typically has an overstory dominated by Salix geyeriana, which occurs as large clumps, and an understory dominated by Calamagrostis canadensis. These stands have an open corridor aspect, while Salix boothii communities are more often closed and less easily accessible by large ungulates (Padgett et al. 1989). Other willows commonly found in this community are Salix boothii and Salix drummondiana; however, Salix geyeriana is clearly the dominant willow. Common associated understory species are Carex utriculata, Geum macrophyllum, Mentha arvensis, Solidago canadensis, and Equisetum arvense (Hansen et al. 1995).

Similar Communities- This community has also been documented in Utah (Padgett et al. 1989) and Idaho (Hall and Hansen 1997, Youngblood et al. 1985). Several studies (Hansen et al. 1995, Hall and Hansen 1997) include in this association stands dominated by Salix boothii, a willow that is frequently a codominant with Salix geyeriana. Other authors (e.g. Padgett et al. 1989) separate Salix boothii-dominated stands as a separate plant association, based on structural differences between Salix boothii and Salix geyeriana stands. Kittel et al. (1998) describe a Salix geyeriana-Salix monticola/Calamagrostis canadensis community which has an understory that is similar to Salix geyerianal Calamagrostis canadensis but which has Salix monticola in the overstory as a codominant instead of Salix boothii. In Nevada, Manning and Padgett (1995) describe a Salix geyerianal Mesic graminoid association which is similar to Salix geyeriana/Calamagrostis canadensis, although the understory of the former is apparently more diverse.

Succession- Salix geyeriana/Calamagrostis canadensis stands are fairly stable if the hydrologic regime remains unchanged. Kittel et al. (1998) suggest that flooding events in Salix geyeriana/Carex utriculata communities can result in sediment deposition, which raises the floodplain surface higher above the water table. As the floodplain aggrades, the site could become less saturated, which could cause the graminoid understory to change towards 
Calamagrostis canadensis. Similarly, Kittel (1994) states that distance from the stream channel can change the degree of soil saturation, and thereby influence the understory composition. Removal of beaver from a Salix geyeriana/Carex utriculata stand could also cause compositional changes. Unmaintained beaver dams could break, and cause a lowering of the water table, which could cause a shift in the dominant understory graminoid towards Calamagrostis canadensis (Hansen et al. 1995).

Management- Calamagrostis canadensis is moderately to highly palatable and with high grazing pressure, the vigor, reproductive success, and competitive ability of this grass will decrease. Exotic pasture grasses such as Poa pratensis or Agrostis stolonifera may then increase. Livestock grazing in this association should be avoided when the soils are wet to avoid churning of the soil surface. Salix geyerianal Calamagrostis canadensis stands exposed to heavy browsing pressure usually show reduced vigor of the willow species, such as highlining, clubbing, or dead clumps, with eventual decrease in willow coverage (Hansen et al. 1995).

\section{Element Code- CEGL001205}

Edition / Author- 99-10-15 / J.Greenlee, MTNHP

Salix geyeriana / Carex utriculata Shrubland

(SALGEY / CARUTR) Geyer's willow / Beaked Sedge

\section{Natural Heritage Conservation Rank- G5 / S5}

Environment- The Salix geyeriana / Carex utriculata association can be found in montane habitats in western Montana (from valley bottoms to mid-elevations in the mountains) and in the mountains of central and eastern Montana. It is frequently found on alluvial terraces where beaver activity has created a series of dams that raise the local water table, along streams, and near seeps or springs. Soils are usually fine textured mineral soils that accumulate during periodic flooding events, and they may have a surface organic horizon. Soil reaction is neutral to moderately alkaline (pH 7.0 to 7.5), and this community is usually flooded during spring and early summer, with the water level near the surface the rest of the year. Nearby wetter communities could include Carex utriculata, Typha latifolia, or open water, and nearby drier communities could include Salix geyeriana/Calamagrostis canadensis, Calamagrostis canadensis, Deschampsia cespitosa, or Juncus balticus. A variety of adjacent uplands could occur nearby, ranging from conifer dominated communities to dry shrublands such as Artemisia tridentata associations (Hansen et al. 1988, Hansen et al. 1995).

Range- This community is found in Montana, Idaho, eastern Oregon, Utah, Nevada, Colorado, and Wyoming.

Vegetation- The Salix geyeriana / Carex utriculata association typically has an overstory dominated by Salix geyeriana which occurs as large clumps and an understory dominated by Carex utriculata. Other willows commonly found in this community are Salix boothii and Salix drummondiana. Common associated understory species are Carex aquatilis, Geum macrophyllum, and Epilobium ciliatum. This community can occur as a part of a diverse mosaic of wetland types depending on degree and frequency of flooding, scouring, channel changes, and beaver activity (Hansen et al. 1995).

Similar Communities- This community has been documented by a number of studies in other western states: eastern Oregon (Kovalchik 1987), Utah (Padgett et al. 1989), Nevada (Manning and Padgett 1995), Idaho (Hall and Hansen 1997), Colorado (Kittel et al. 1998), and Wyoming (Chadde et al. 1988). Several studies (Hansen et al. 1995, Hall and Hansen 1997) include in this association stands dominated by Salix boothii, a willow that is frequently a codominant with Salix geyeriana. Other authors (e.g. Padgett et al. 1989) separate Salix boothii-dominated stands as a separate 
plant association, based on structural differences between Salix boothii and Salix geyeriana stands.

Succession- Salix geyeriana / Carex utriculata stands are fairly stable if the hydrologic regime remains unchanged. However, flooding events can result in sediment deposition, which raises the floodplain surface higher above the water table. As the floodplain aggrades, the site could become less saturated, which could cause the graminoid understory to change. Similarly, Kittel (1994) states that distance from the stream channel can change the degree of soil saturation, and thereby influence the understory composition. Removal of beaver from a Salix geyeriana/Carex utriculata stand could also cause compositional changes. Unmaintained beaver dams could break, and cause a lowering of the water table, which could cause a shift in the dominant understory graminoid (Hansen et al. 1995).

\section{Management- Salix geyeriana / Carex} utriculata stands exposed to heavy browsing pressure usually show reduced vigor of the willow species, such as highlining, clubbing, or dead clumps, with eventual decrease in willow coverage. Livestock disturbance in drier Salix geyeriana / Carex utriculata stands can result in increases in cover of exotic pasture grasses like Poa pratensis or Agrostis stolonifera

\section{Element Code- CEGL001207}

Edition / Author- 1999-10-15 / J. Greenlee, MTNHP

\begin{tabular}{c} 
Salix planifolia / Carex nebrascensis \\
Shrubland \\
(SALPAL / CARNEB) planeleaf willow / \\
Nebraska sedge \\
\hline
\end{tabular}

\section{Natural Heritage Conservation Rank-} $\mathrm{G}$ ? / S?

\section{Environment- Salix planifolia / Carex} nebrascensis is a minor community type, generally occurring as small ( $<1$ acre) patches in the Centennial Valley vicinity on flat or gently sloping meadows and on lower slopes with a variety of aspects. The high water tables found in Salix planifolia / Carex nebrascensis result from lateral subirrigation though in several instances this type was noted to be associated with fluvial conditions as narrow stringer of small, first order streams. This community is usually is the moistest on the local moisture gradient and grades to Deschampsia cespitosa-, Poa pratensis- or Juncus balticus- dominated c.t.'s. of drier positions or more usually abruptly gives way to the Artemisia tridentata ssp. vaseyana or Artemisia tripartita / Festuca idahoensis c.t.

Vegetation- Shrub dominance on sampled occurrences varied between Salix brachycarpa and Salix. planifolia (ssp. unknown due to inopportune sampling time) and both species and Pentaphylloides floribunda were noted to be present in most stands, but coverages never exceeded $20 \%$ (see comments). The dominant aspect of these stands is a solid sward of midheight graminoids, Carex nebrascensis, Carex praegracilis, Carex. simulata, Calamagrostis stricta, Deschampsia cespitosa and Juncus balticus; only the first thee named are abundant. Well represented Pedicularis groenlandica is the dominant forb and Senecio sphaerocephalus, Aster occidentalis and Sisyrinchium angustifolium are frequently present, often well represented. Basal area of graminoids and about $10 \%$ litter account for the $20 \%$ of the ground that is not a carpet of bryophytes.

Soils- The upper 10-20 $\mathrm{cm}$ of profiles have at least $50 \%$ fibrous to histic organic matter and the remainder is a silty or clayey mineral material; in one case the mineral material was a $3 \mathrm{~cm}$. thick layer intercalated between two histic organic layers. Soils were saturated to the surface in late July.

Other Studies- This description should be considered preliminary as this exact community type has not been described elsewhere; in fact, a misidentification of Salix spp. on our part could place it with any number of Salix-dominated community types with $C$. nebrascensis as a dominant undergrowth component. Youngblood et al. (1985) describe a Salix planifolia c.t. of "low 
elevations" in the Centennial Range and Yellowstone Plateau and note that Carex utriculata and Juncus balticus dominate the undergrowth therefore having a high resemblance to this type.

Comments- Judging by the intensive hedging of the Salix species (mature specimens not over $2 \mathrm{dm}$ when potential is $1 \mathrm{~m}$ plus) in these and adjacent Salix-dominated stands we surmise the wild ungulate pressure to be intensive on these sites. Some hummocking is present but not enough to create difficult walking. Cattle use has not yet markedly altered composition (potential for creating Poa pratensis- and Juncus balticus-dominated stands).

\section{Element Code- CEGLMTHP20}

Edition / Author- 97 -10 / S.V. Cooper et al.

\begin{tabular}{c}
\hline Sarcobatus vermiculatus / Distichlis \\
spicata Shrubland \\
(SARVER / DISSPI) black greasewood / \\
inland saltgrass
\end{tabular}

Natural Heritage Conservation RankG4 / S2

Environment- This uncommon type is found on wide stream terraces and lower alluvial fans often in areas of calcareous parent material such as the Centennial Valley. Higher terraces with less saline soil may support stands dominated by Sarcobatus vermiculatus or Artemisia tridentata ssp. tridentata and Pascopyrum smithii or Leymus cinereus. Nearby slopes are dominated by Artemisia tridentata or A. tripartita and Pseudoroegneria spicata or Festuca idahoensis.

Vegetation-Sarcobatus vermiculatus is the dominant shrub although canopy cover is often less than $10 \%$. Artemisia tridentata ssp. tridentata and Chrysothamnus nauseosus are also often present. Halophytic grasses including Distichlis stricta, Puccinellia distans, and/or Poa juncifolia dominate the ground layer. Forbs are uncommon, and diversity is low. Lepidium densiflorum, Haplopappus uniflorus and Antennaria microphylla may be present. Mosses and lichens are absent.

Soils- Soils are silty and deep. The surface horizons are saline. Most of the soil surface is bare, and gravel is absent.

Other Studies- Mueggler and Stewart (1980, p. 76) state that they have reason to believe that this type occurs in western Montana, but they do not describe it. Similar vegetation has been described for eastern Washington (Daubenmire 1970), Colorado, Idaho and Oregon

(Bourgeron and Engelking 1994).

Comments- Sarcobatus vermiculatus /

Distichlis stricta and Sarcobatus vermiculatus / Pascopyrum smithii are similar types; this type might also be considered a saline phase of the Sarcobatus vermiculatus / Pascopyrum smithii c.t. (see Comments under Sarcobatus vermiculatus / Pascopyrum smithii).

\section{Element Code- CEGL001363}

Edition / Author- 99-11-16 / S.V. Cooper. MTNHP

Sarcobatus vermiculatus / Leymus cinereus
Shrubland
(SARVER / LEYCIN) black greasewood /
basin wildrye

\section{Natural Heritage Conservation Rank-} G3 / S3

Environment- This association characteristically occurs in low precipitation areas as comparatively narrow bands along river and stream flood plains. Sarcobatus vermiculatus is typically found on heavy textured, poorly drained saline or alkaline substrates. According to Mueggler and Stewart (1980) environmental distinctions separating this type from Sarcobatus vermiculatus / Pascopyrum smithii have not been identified but Sarcobatus vermiculatus / Leymus cinereus does appear to be associated with better drained, less alkaline soils, such as occur on concave toe slopes (and not on flats). This association is documented to occur throughout the west, from Washington south to California 
and east to Nevada and Wyoming.

Vegetation- Tussocks of the tall grass Leymus cinereus comprising at least $1 \%$ canopy cover distinguish this association from the related Sarcobatus vermiculatus / Pascopyrum smithii or Sarcobatus vermiculatus / Distichlis stricta associations. As in the Sarcobatus vermiculatus / Pascopyrum smithii type, $S$. vermiculatus is the shrub with the highest cover values, followed closely by Artemisia tridentata ssp. tridentata (or A. tridentata ssp. wyomingensis). The large shrub stature relative to that of the associated vegetation cause this type to be treated as a Shrubland (their combined cover usually not exceeding $15 \%$ ). The low shrubs Artemisia frigida and Chrysothamnus viscidiflorus are noted to increase on disturbed sites and may have in excess of $10 \%$ cover. Technically speaking, combined shrub canopy cover values are never sufficient $(>25 \%$ ) for this to be classified as other than a shrub herbaceous type. Pascopyrum smithii and/or Elymus lanceolatus are the only abundant graminoids but others with high constancy include Pseudoroegneria spicata, Koeleria macrantha, Carex filifolia and Poa juncifolia. Forb diversity and cover is higher here than in related $S$. vermiculatus-dominated types; Sphaeralcea coccinea, Aster falcatus, Aster. chilensis and Iva axillaris are consistently present in trace amounts.

Comments- Mueggler and Stewart (1980) observe that overgrazing causes the decline of Pascopyrum. smithii and Pseudoroegneria. pseudoroegneria and that persistent heavy use can cause the decrease of Koeleria cristata and Leymus. cinereus. Krall et al. (1970) found early spring grazing, prior to the "boot" developmental stage, to be very detri-mental to Leymus cinereus. We suspect that some proportion of this type has been convert-ed to the Sarcobatus vermiculatus / Pascopy-rum smithii association through overgrazing in the early spring because $P$. smithii, though it is more palatable, is less susceptible than Leymus. cinereus to the pressures of inappropriately timed grazing.

Element Code- CEGL001366
Edition / Author- 99-12-03 / S.V. Cooper

Shepherdia argentea Shrubland (SHEARG) thorny buffaloberry

\section{Natural Heritage Conservation Rank- G3G4/S3}

Environment: Shepherdia argentea is a minor type documented only from the wetlands between Twin Bridges and Dillon Valley where it occurs in subirrigated valley positions in an extensive complex mosaic with other riparian and wetland communities. In this landscape $S$. argentea forms patchily distributed copses with other shrubs, the intervening ground being dominated by Pascopyrum smithii, Sporobolus airoides and other mesic graminoids. The southwestern MT occurrences contrast with those of Phillips and Valley Counties where it occurs as small stands (mostly < 1/5 acre) on the most mesic positions, often $\mathrm{V}$-shaped ravines at drainage headwaters, in a rolling uplands or badlands landscape mosaic. This landscape position agrees with the observations of Hansen et al. [1991] who targeted riparian areas specifically and found the type along the Sun, Milk, Missouri and Yellowstone Rivers. Stands range from small to large patch and show much internal heterogeneity in both microtopography and vegetation, with clumpy distribution of $S$. argentea (and other shrubs). The smallest stands are not much more than individual clumps or narrow stringers of $S$. argentea.

Soils- Soils are developed from fine-textured alluvium whereas those of northeastern Montana are developed from glacial drift or shales. Several sites were noted to have gleyed and mottled soils; only one sampled stand in northeastern Montana at a drainage headland qualified as jurisdictional wetland with gleying and mottling within 6 in of surface. Being productive sites, the ground cover is primarily litter, though much bare soil is exposed where animal trails are concentrated.

Vegetation: Shepherdia argentea, a tall shrub 
(4 to $7 \mathrm{ft}$ tall in eastern MT sites and to 12 to $15 \mathrm{ft}$ tall in Beaverhead Mountains Section) is usually abundant, forming a patchy distribution. Symphoricarpos occidentalis, Rosa woodsii (or R. acicularis) or Juniperus horizontalis may have greater cover, but occur in the low shrub layer. In eastern MT., Ribes setosum is consistently present as a mid to tall shrub. The forb layers form two sampled stands were very different, apparently reflecting differences in soil moisture. The wet-site herb layer was dominated by Poa palustris (eastern MT) or Sporobolus airoides and Elymus trachycaulus (western MT) whereas the drier stands were dominated by Pascopyrum smithii in both regions.

Other Studies: For Montana, Shepherdia argentea was first described in the southeast by Hansen and Hoffman (1988) and subsequently documented to range from southwestern, through central, to eastern sections by Hansen et al. (1995). Other northern Great Plains occurrences are described from North Dakota (Nelson 1961 and Boldt et al. 1978) and cited from South Dakota (Faber-Langendoen 1993).

Comments- At least in eastern Montana, due to landscape position and structure, these sites are heavily used by wild ungulates for cover; domestic stock also use these sites preferentially. Either/both of these groups are probably implicated in the introduction of Euphorbia esula (leafy spurge) to these moist habitats that are so favorable to its propagation.

\section{Element Code- CEGL001128}

Edition / Author- 99-12-03 / S.V. Cooper, MTNHP

\section{D warf-Shrublands including D warf-Shrub $\mathrm{H}$ erbaceous}

\author{
Artemisia arbuscula ssp. longiloba / \\ Elymus lanceolatus Dwarf-shrub \\ Herbaceous Vegetation \\ (ARTASL / ELYLAN) early low \\ sagebrush / thick-spike wheatgrass
}

\section{Natural Heritage Conservation Rank- $\mathrm{G}$ ? / S?}

Environment- Artemisia arbuscula ssp. longiloba / Elymus lanceolatus is found on gently sloping terraces and lower slopes of alluvial fans as well as broad, low ridges at $6,600-7,200 \mathrm{ft}$, usually in areas of calcareous parent material. Adjacent communities on moist stream terraces may be dominated by Pentaphylloides floribunda, Juncus balticus, Carex praegracilis, Deschampsia cespitosa, Leymus cinereus or Poa juncifolia. Deeper or better-drained soils are dominated by Artemisia tridentata ssp. wyomingensis or A. tripartita and Elymus lanceolatus or Festuca idahoensis.

Vegetation- Artemisia arbuscula ssp. longiloba is the dominant shrub with 10-40\% canopy cover. Other common shrubs include Chrysothamnus nauseosus and C. viscidiflorus. Artemisia frigida is a common subshrub. Grass cover was 30-70\%. Elymus lanceolatus is the dominant tall grass with $10-50 \%$ cover. Other common grasses include Poa secunda, $P$. cusickii and P. juncifolia. Forb cover is only ca. $10 \%$, and diversity is low. Highly constant, usually poorly represented forb species include Phlox hoodii, Antennaria microphylla, A. parvifolia and Erigeron compositus. Mosses are absent, but lichens may be common.

Soils- Soils have a silty to clayey texture and are usually calcareous and perhaps saline as well. $30-70 \%$ of the soil surface is bare. There 
is little or no surface gravel.

Other Studies- Vegetation dominated by Artemisia arbuscula ssp. longiloba and Pascopyrum is reported for Colorado (Bourgeron and Engelking 1994).

Comments- This type is used by antelope and may be important deer and elk winter range. See comments under Artemisia nova / Pseudoroegneria spicata. This association occurs only in southern Beaverhead County in Montana.

\section{Element Code- CEGLMTHP01}

Edition / Author- 99-11-16 / S.V. Cooper, MTNHP

Artemisia arbuscula ssp. longiloba / Festuca idahoensis Dwarf-shrub Herbaceous Vegetation

(ARTASL / FESIDA) early low sagebrush / Idaho fescue

\section{Natural Heritage Conservation Rank- G3? / S2}

Environment- Gentle slopes of broad low ridges, alluvial fans and terraces at 6,900-7,200 $\mathrm{ft}$ may support vegetation dominated by Artemisia arbuscula ssp. longiloba and Festuca idahoensis. This type usually occurs in areas of calcareous parent material. Sheet erosion is evident in many stands. Adjacent deeper and/ or better-drained soils support Artemisia tridentata wyomingensis or vaseyana and Festuca idahoensis or Pseudoroegneria spicata. Elymus lanceolatus / Festuca idahoensis gradually merges into the Artemisia arbuscula ssp. longiloba /Elymus lanceolatus c.t. on lower and gentler slopes with heavier soils.

Vegetation- The dominant shrub is Artemisia arbuscula ssp. longiloba with 10-20\% canopy cover. Chrysothamnus viscidiflorus occurs in most stands. Artemisia tridentata and A. tripartita may occur when they occupy adjacent stands. Total grass canopy cover averages ca. 40\%. Festuca idahoensis is the dominant grass with $20-40 \%$ cover. Other common grasses include Elymus lanceolatus, Pseudoroegneria spicata Koeleria cristata and Poa secunda. Forb cover is rarely greater than 10\%. Common species include Antennaria microphylla, Geum triflorum, Linum lewisii, Phlox hoodii and Taraxacum officinale.

Mosses are rare, but lichens are common in some stands.

Soils- Soils have a silty to clayey texture and are usually calcareous and perhaps saline as well. $20-70 \%$ of the soil surface may be bare or covered with gravel.

Other Studies- Similar vegetation has been reported for Nevada (Zamora and Tueller 1973) and Idaho (Tisdale et al. 1965) and likely occurs in Wyoming and Oregon as well (Bourgeron and Engelking 1994).

Comments- See comments under Artemisia nova / Pseudoroegneria spicata. In Montana, this association is known only from southern Beaverhead County.

\section{Element Code- CEGL001522}

Edition / Author- 99-11-16 / S.V. Cooper, MTNHP

Artemisia nova / Pseudoroegneria spicata Dwarf-shrubland

(ARTNOV / PSESPI) black sagebrush / bluebunch wheatgrass

\section{Natural Heritage Conservation Rank- G4G5 / S3}

Environment- This type is common on gentle to steep slopes, alluvial fans and terraces in areas of calcareous parent material; generally it is present on slopes with south- or west-facing aspects. It is found in the Tendoy Mountains north to Badger Pass at 5,500-7,200 ft. Cercocarpus ledifolius or Pinus flexilis dominates adjacent rockier soils. Artemisia tridentata vaseyana or Artemesia tridentata tridentata and Festuca idahoensis, Pseudoroegneria spicata or Pascopyrum smithii dominates deeper soils. 
Vegetation- Artemisia nova is the dominant shrub with coverages up to $40 \%$. Chrysothamnus nauseosus is usually common. Common subshrubs include Artemisia frigida and Gutierrezia sarothrae. Pseudoroegneria spicata is well represented and usually the dominant grass with canopy cover of 10-40\%. Stipa comata and Oryzopsis hymenoides are highly constant but generally are not even well represented. Forb cover is sparse, but diversity is moderate. Opuntia polyacantha, Phlox hoodii, Stephanomeria runcinata, Erigeron tweedyi and Penstemon aridus are often present. Mosses are absent, and lichens are rare.

Soils- Soils have a silty or sandy texture and are usually stony, shallow and derived from calcareous parent material. Up to $80 \%$ of the ground may be bare or covered with gravel or rock.

Other Studies- Mueggler and Stewart (1980) first described this type for western Montana as Artemisia arbuscula/Pseudoroegneria spicata, lumping the nova and arbuscula subspecies of A. arbuscula. Various authors have subsequently recognized the distinct ecology, if not taxonomy, of these taxa. Similar vegetation has been reported for Oregon, Nevada, Idaho, Colorado and Wyoming (Bourgeron and Engelking 1994).

Comments- There has been a good deal of confusion surrounding the taxonomic nomenclature of the low sagebrush Artemisia nova, Artemisia arbuscula and Artemisia. longiloba in Montana. Hitchcock and Cronquist (1973) place all three taxa under Artemisia. arbuscula. Schulz (1984) recognizes Artemisia. nova as a distinct species, but places Artemisia. longiloba as a subspecies of Artemisia. arbuscula. Beetle (1982) recognizes all three as separate species. This variable nomenclature has caused confusion in the vegetation classification literature.

The closely related Artemisia nova / Festuca idahoensis c.t. also occurs in southwest Montana, but we failed to sample any stands.
Element Code- CEGL001424

Edition / Author- 99-11-16 / S.V. Cooper. P

Atriplex gardneri / Oryzopsis hymenoides Dwarf-shrubland

(ATRGAR / ORYHYM) Gardner's saltbush / indian ricegrass

\section{Natural Heritage Conservation Rank-}

G3 / S3

Environment- The type occurs on steep badland sites at elevations around 5000 feet. Bare soil cover totals at least $60 \%$ and most of the remaining ground cover is comprised of coarse fragments. The soil surface is unstable because of a lack of adequate vegetation cover and gully erosion is common.

Parent materials are sedimentary rocks and soils are very shallow and poorly developed. Soils range from not gravelly to very gravelly and are non-calcareous. Textures are generally very fine (e.g., silty clay loams) and available water holding capacity is low.

Vegetation- Total vegetation cover in this badlands type seldom exceeds $5 \%$.

Characteristic species include Atriplex nuttallii, Chrysothamnus nauseosus, and Oryzopsis hymenoides.

Adjacent Communities- Vegetation on adjacent more stable soils include the Artemisia tridentata / Pseudoroegneria spicata type on non-alkaline soils and the Sarcobatus vermiculatus / Pascopyrum smithii type on alkaline soils.

Other Studies- This type has not previously been reported in Montana but has been reported in Colorado and is listed as questionably present in Oregon and Wyoming (Bourgeron and Engelking 1994).

Element Code- CEGL001444

Edition / Author- 99-11-16 / S.V. Cooper, MTNHP 
Cassiope mertensiana / Carex paysonis Dwarf-shrubland

(CASMER / CARPAY) Merten's mossheather / Payson's sedge

\section{Natural Heritage Conservation Rank-}

G2? / S2

\section{Environment- Cassiope mertensiana / Carex} paysonis is uncommon in the study area, occurring at the base of gentle, north- or eastfacing slopes at 9,400-9,600 ft in the Anaconda and Tobacco Root ranges. This type probably also occurs in the Pioneer Range. These cool, protected sites have deep snow during the winter, and release comes somewhat late in the growing season. They often showed signs of frost-churning and solifluction, suggesting that they receive additional upslope moisture. Cassiope mertensiana / Carex paysonis exists in a mosaic with other snowbed associations such as Carex nigricans and Juncus drummondii / Antennaria lanata and often occurs adjacent to moist turf communities such as Salix arctica / Polygonum bistortoides and Carex scirpoidea / Geum rossii. Cassiope mertensiana / Carex paysonis probably experiences earlier snow release than the other mountain-heather community, Phyllodoce empetriformis / Antennaria lanata

Vegetation- Mean canopy cover of shrubs was $60 \%$. Cassiope mertensiana and Salix arctica were the dominant shrubs; we refer to this community as Cassiope mertensiana because of its greater stature and generally greater abundance compared to Salix. arctica. Phyllodoce glanduliflora was present in one stand. Mean graminoid cover was 23\%, with Carex paysonis the dominant one. Poa alpina and Carex scirpoidea were common; Poa fendleriana and Deschampsia cespitosa were well represented in one stand. Mean forb cover was $30 \%$. Geum rossii and Potentilla diversifolia were common species, and Erigeron simplex and Polygonum bistortoides were frequent. Antennaria lanata and Juncus drummondii were notable for their absence or low cover. Mean cover of lichens and mosses was less than $1 \%$.

Soils- Parent materials were quartzite, gneiss and granite. Bare ground and gravel covered $11 \%$ of the surface, while rock cover was $8 \%$. Mean depth of litter was 0.2 in, and mean depth of duff was 0.1 in. Percent coarse fragments varied from $9 \%$ to $35 \%$ with a mean of $23 \%$. Mean texture of the fine fraction was sandy clay. Soil $\mathrm{pH}$ ranged from 5.8 to 6.2 with a mean of 6.0. Mean organic matter content was $19 \%$, mean total nitrogen was $0.52 \%$, and C:N ratio was 19:1. Soils were moderately deep and moist to wet in late July.

Productivity- We measured productivity in only one stand, and Cassiope was difficult to clip accurately. Thus, our production estimates are only rough approximations. Shrub productivity was $237 \mathrm{lbs} / \mathrm{acre}$, graminoid productivity was $267 \mathrm{lbs} / \mathrm{acre}$, and forb productivity was $712 \mathrm{lbs} /$ acre. Total productivity was $1216 \mathrm{lbs} / \mathrm{acre}$.

Other Studies- Associations dominated by Cassiope mertensiana have been reported for the Canadian Rockies, the Cascade Range and northwest Montana. Phyllodoce spp. were often codominant. See discussion under Phyllodoce empetriformis / Antennaria lanata dwarf shrubland.

\section{Element Code- CEGL001396}

Edition / Author- 97 -10 / S.V. Cooper et al.

Dryas octopetala / Carex rupestris Dwarfshrub Herbaceous Vegetation

(DRYOCT / CARRUP) Mountain avens / curly sedge

\section{Natural Heritage Conservation Rank- G4 / S3}

Environment- This sparsely vegetated community type occurred on broad ridge tops, shoulders, saddles and upper slopes at 9,2009,700 ft in the Pioneer and Anaconda ranges. Distinct patterning was apparent, with Dryas 
octopetala forming mats surrounded by bare ground or rock pavement. Mats were either evenly spaced or aligned along the edge of stepped terraces or wind rows. Bamberg and Major (1968) report that Dryas mats in the Big Snowy Range of Montana demonstrated appreciable yearly downslope movement. However, wind rows at Siyeh Pass in Glacier Park were relatively stable. Dryas octopetala / Carex rupestris usually occurred in a matrix of dry or moist turf communities such as Carex elynoides or Carex scirpoidea / Potentilla diversifolia. This community type is closely related to Dryas octopetala / Polygonum viviparum and the two may intergrade. However, Dryas octopetala / Polygonum viviparum occurred on wetter sites, had higher total cover, and had more species, such as Salix reticulata, Polygonum spp., and Poa alpina, indicative of more mesic conditions.

Vegetation- Mean cover of dwarf shrubs was $38 \%$. Dryas octopetala was the only common shrub species; Cassiope mertensiana, Pentaphylloides floribunda and Salix arctica each occurred in one stand. Mean graminoid cover was $13 \%$. Common species included Carex rupestris and C. elynoides. Calamagrostis purpurascens, Festuca ovina and Poa secunda were locally distributed, individually seldom attaining more than 5\% cover. Mean forb cover was only $15 \%$, the second lowest value among all community types. Oxytropis campestris and Phlox pulvinata were common species found in most stands. Anemone drummondii, Minuartia obtusiloba, Douglasia montana, Geum rossii, Oxytropis viscida, Potentilla diversifolia, Zigadenus elegans and Selaginella densa were common in some stands. Cover of mosses and lichens was less than $1 \%$.

Soils- Parent materials were limestone, granite and quartzite, with limestone predominating. Bare ground and gravel covered $40 \%$ of the surface. Mean depths of litter and duff were both 0.1 in. Percent coarse fragments ranged from $33 \%$ to $54 \%$ with a mean of $42 \%$.

Texture of the fine fraction varied from sandy clay to sandy clay-loam, and mean texture was sandy clay. Soil $\mathrm{pH}$ ranged from 6.2 to 7.8 with a mean of 7.3. Mean organic matter content was $12 \%$, mean total nitrogen was $0.34 \%$, and C:N ratio was $36: 1$. Soils evidenced past erosion events and are often unstable. Sandy clay-loam soils, which are derived from calcareous parent materials, often show evidence of frost churning.

Productivity- Our estimates are based on only three stands, and two of these occurred on barren soils derived from partially metamorphosed limestone. These soils likely have unusual physio-chemical properties that deter plant establishment and growth.

Consequently, our production estimates for this type are probably low. Shrub productivity varied from 44 to $651 \mathrm{lbs} /$ acre with a mean of $157 \mathrm{lbs} / \mathrm{acre}$. Graminoid productivity ranged from 15 to $89 \mathrm{lbs} /$ acre with a mean of $33 \mathrm{lbs} /$ acre. Forb productivity varied from 8 to 148 lbs/acre with a mean of $43 \mathrm{lbs} / \mathrm{acre}$. Total productivity averaged $233 \mathrm{lbs} /$ acre.

Other Studies- Achuff and Corns (1982) describe an alpine type from the Canadian Rockies dominated by Dryas octopetala and Kobresia myosuroides, but this community has many mesic site indicators and is more similar to our Dryas octopetala/Polygonum viviparum. Douglas and Bliss (1977) describe Dryas fellfields from the North Cascades of Washington. Besides Dryas ctopetala, only a handful of other species, including Festuca ovina and Minuaritia obtusiloba, were common. Associations dominated by Dryas octopetala, Carex rupestris, Carex. elynoides and Carex. scirpoidea occur in the Big Snowy and Flint Creek ranges of Montana (Bamberg and Major 1968). Dryas communities in Glacier Park, Montana appear compositionally intermediate between those in the Flint Creek Range and those of the Canadian Rockies (Bamberg and Major 1962, Choate and Habeck 1967). Johnson and Billings (1962) state that Dryas octopetala colonizes wind-eroded sites and is very limited on the Beartooth Plateau of south-central Montana and adjacent Wyoming. Urbanczyk and Henderson (1994) described vegetation dominated by Dryas octopetala and Carex. rupestris on steep north slopes below snowbanks in Idaho's Lemhi Range. 
Communities dominated by Dryas octopetala and Carex rupestris from the Rocky Mountains of Colorado are associated with high levels of calcium according to Komarkova and Webber (1979) and Willard (1978), but Eddleman and Ward (1984) found no such relationship.

Festuca ovina, Geum rossii, Silene acaulis and Trifolium nanum are also common in the Colorado representation of this type.

Our two Dryas-dominated types Dryas octopetala / Carex rupestris and Dryas octopetala / Polygonum viviparum appear to be at the drier and wetter ends of a moisture gradient. The former type predominates in relatively dry mountain ranges of the Central and Northern Rockies and on the east side of the Cascades, while the latter type is more common in the Canadian Rockies and the wetter ranges of the Northern Rockies (see Other Studies under Dryas octopetala / Polygonum viviparum).

\section{Element Code- CEGL001892}

Edition / Author- 97 -10 / S.V.Cooper et al.

Dryas octopetala / Polygonum viviparum
Dwarf-shrub Herbaceous Vegetation
(DRYOCT / POLVIV) white dryas /
viviparous bistort

\section{Natural Heritage Conservation Rank- G3? / S2}

Environment- This minor type was found in both the wettest, (Anaconda and Madison) and driest (Tendoy) ranges. Small occurrences of this type were noted but not sampled in other mountain ranges. This vegetation was generally found on northerly-facing gentle to steep slopes. Evidence of disturbance, including solifluction, slumps and earthflows, were also common. Only trace amounts of rock were exposed but gravel ranged from 5 to $30 \%$.

Vegetation- Mats of Dryas octopetala, ranging in cover from 30 to $80 \%$, and Salix reticulata (5-20\% c.c) provided the dominant aspect of this c.t. Graminoid canopy cover was low, not exceeding 5\%, and composed of the common turf species Carex elynoides, $C$. rupestris, and Festuca ovina as well as Poa alpina. Average forb cover was also relatively low, $14 \%$, with dominance shared among the diagnostic species for the type, Polygonum viviparum, $P$. bistortoides, Zigadenus elegans and Oxytropis viscida. Other forbs with high constancy, low coverage and some degree of fidelity to this type were Lloydia serotina, Senecio crassulus, Smelowskia calycina, Oxytropis campestris and Pedicularis cystopteridifolia. Two plots had moss and lichen coverages in excess of $50 \%$ adding to the impression of a smooth blanket of vegetation.

Soils- Parent materials were limestone and quartzite. Average litter and duff depth were, respectively 0.4 and $0.5 \mathrm{in}$. Coarse fragment content ranged from 8 to $45 \%$ and averaged $30 \%$. Mean textural class of the fine fraction was sandy clay. Soil reaction for calcareous sites ranged from 7.4 to 7.6; the lone quartzite sample was more than one $\mathrm{pH}$ unit lower at 6.2. Mean organic matter content was $25 \%$, mean total nitrogen was $0.75 \%$, and $\mathrm{C}: \mathrm{N}$ ratio was 18:1. This type had the highest average organic matter and nitrogen content of any non-wetland community in our study.

Productivity- Of the two plots clipped, the one from the rocky site registered only $548 \mathrm{lbs} / \mathrm{acre}$ (46\% shrub) whereas the one with only trace amounts of exposed rock and soil produced $1229 \mathrm{lbs} /$ acre (97\% shrub).

Other Studies- Vegetation similar to Dryas octopetala / Polygonum viviparum is common in the Canadian Rockies (Achuff and Corns 1982, Hrapko and LaRoi 1978). Canadian types have high diversity of lichens and mosses and are considered successionally mature. Concentrating on calcareous substrates of several Montana ranges, Bamberg and Major (1968) sampled many stands of what they termed zonal alpine vegetation, but did not explicitly group stands into community types. On the basis of their stand tables it appears Dryas octopetala / Polygonum viviparum is a major c.t. in Glacier National Park and Big Snowy Mountains. A similar turf type occurs 
in the Flint Creek Range. In the Colorado Rockies, Willard (1979) described moist fellfield communities dominated by $D$. octopetala with significant $P$. viviparum cover and lichens and mosses, but lacking dwarf Salix spp.; she described dwarf willow communities as being confined to snowbed environments. McGraw (1985) found that $D$. octopetala consists of at least two distinct ecotypes in Alaska: one that occurs in cool, moist habitats and one that is found on dry, exposed sites. Similar ecotypic differentiation would explain the dominance of D. octopetala in the relatively cool, moist Dryas octopetala / Polygonum viviparum c.t. as well as the drier Dryas octopetala / Carex rupestris c.t.

\section{Element Code- CEGL001894}

Edition / Author- 97 -10 / S.V.Cooper et al.

Phyllodoce empetriformis / Antennaria lanata Dwarf-shrubland

(PHYEMP / ANTLAN) Mountain-heather / woolly pussytoes

\section{Natural Heritage Conservation Rank- \\ G3 / S3}

\section{Environment- Phyllodoce empetriformis /} Antennaria lanata was locally common on gentle to moderate, protected slopes between 9,200 and 10,100 ft in the Anaconda, Madison and Pioneer ranges, associated with crystalline parent materials in these wetter ranges of our study area. These sites are undoubtedly well covered by snow during the winter, and melt off probably occurs relatively late in the season, although not as late as in the Carex nigricans c.t. In addition to other snowbed associations such as Carex nigricans and Juncus drummondii / Antennaria lanata, Phyllodoce empetriformis /Antennaria lanata often grades to moist turf communities such as Salix arctica / Polygonum bistortoides and Carex scirpoidea / Geum rossii. A typical toposequence on a lee slope might be Carex scirpoidea / Geum rossii on the lower slope, Phyllodoce empetriformis /Antennaria lanata at the base of the slope, with Carex nigricans in the bottom.
Vegetation- Dwarf shrubs provide the dominant aspect with a mean cover of $55 \%$. Phyllodoce empetriformis and Vaccinium scoparium were common in all four stands, while $P$. glanduliflora and Cassiope mertensiana were common in two of the stands. Mean graminoid cover was low, $20 \%$. Carex paysonis, Juncus drummondii and Poa fendleriana were common graminoids occurring in all or most stands. Forb cover was 35\%; Antennaria lanata, Polygonum bistortoides and Sibbaldia procumbens were consistently present, though only A. lanata occurred with greater than $5 \%$ cover. Mean cover of mosses and lichens was less than $1 \%$.

Soils- Parent materials were granite and quartzite. Cover of bare ground and gravel was $15 \%$ with $8 \%$ cover of rock. Mean depths of litter and duff were both 0.1 in. Percent coarse fragments varied from $0 \%$ to $14 \%$ with a mean of $7 \%$. Mean texture of the fine fraction was sandy clay-loam. Soil reaction ranged from 6.0 to 6.4 with a mean $\mathrm{pH}$ of 6.1 . Mean organic matter content was $14 \%$, mean total nitrogen was $0.30 \%$, and C:N ratio was 23:1. Soils underlying \%. Phyllodoce empetriformis were relatively deep and generally still moist in late July.

Productivity- We measured productivity in only two stands, and the heather species proved difficult to clip accurately. Thus, our production estimates are only rough approximations. Mean shrub productivity was $166 \mathrm{lbs} /$ acre. Graminoid productivity had a mean of $133 \mathrm{lbs} / \mathrm{acre}$, and forb productivity had a mean of $104 \mathrm{lbs} /$ acre. Mean total productivity was $403 \mathrm{lbs} /$ acre.

Other Studies- Mountain-heather communities similar to Phyllodoce empetriformis / Antennaria lanata have been reported for Banff and Jasper national parks in the Canadian Rockies by Achuff and Corns (1982) and Hrapko and LaRoi (1978). The Canadian types had similar composition, but Phyllodoce glanduliflora and Cassiope mertensiana were the dominant heather species. Mountainheather communities from the North Cascades 
of Washington are more similar to our Phyllodoce empetriformis /Antennaria lanata (Douglas 1972, Douglas and Bliss 1977). Whereas Douglas (1972) combines all Phyllodoce- and Cassiope-dominated associations into one community type, Douglas and Bliss (1977) designate separate community type dominated by Phyllodoce empetriformis, Phyllodoce glanduliflora and Cassiope. mertensiana. In the North Cascades, where snowpack is much higher than most areas of the northern Rockies, these communities are not confined to areas of late snow release. Apart from these studies, Choate and Habeck's (1967) mention of a similar type at Logan Pass in Glacier National Park in northwest Montana is the only other reference to heatherdominated communities. Thus, mountainheather associations appear to be confined to the mountains north and west of our study area.

Element Code- CEGL001405

Edition / Author- 97 -10 / S.V.Cooper et al.

Salix reticulata / Caltha leptosepala Dwarf-shrubland

(SALRET / CALLEP) snow willow / marsh marigold

\section{Natural Heritage Conservation Rank- G3 / S2}

Environment- Sampled in only the Tendoy and Gravelly ranges, Salix reticulata / Caltha leptosepala appears to be a minor type, environmentally and floristically related to Salix arctica / Polygonum bistortoides. The relative paucity of this c.t. can be explained, at least in part, by lack of appropriate habitat (i.e., gentle to steep north-facing slopes). Slopes with this aspect and possessing a soil mantle are not common in the predominantly northsouth trending ranges of the study area. Slopes with northerly aspects did occur as spur ridges but often they were merely boulder fields. Both stands carpeted active solifluction slopes and were subirrigated from late-persisting snowfields lying above. Ostensibly these sites could be as wet as Carex scopulorum / Caltha leptosepala, differing by lacking stagnant water and possessing both unstable substrates and possibly long-persisting snowpacks.

Vegetation- The prevailing aspect of this c.t. was a lush green carpet of dwarf shrub species (average c.c. 70\%), among which Salix reticulata $(=S$. nivalis $)$ was dominant, but Salix rotundifolia (= Salix dodgeana) and Salix arctica also figure prominently. The graminoid component was sparse, not exceeding $20 \%$ c.c. with Carex haydenii, Carex nova, Carex scirpoidea, Deschampsia cespitosa, Luzula spicata and Poa alpina having at least $5 \%$ c.c. in one or more stands. Averaging $21 \%$ c.c., Caltha leptosepala was a diagnostic species (only forb with $100 \%$ constancy) and it, along with Silene acaulis, were the only forbs with more than $10 \%$ coverage.

Soils- Both stands were developed on limestone but were notably low in coarse fragment content $(<10 \%)$. Litter and duff depths were less than 0.5 in. Mean soil texture was clay. Despite the wet conditions, soil reaction was typical for calcareous substrates (7.5 average). Mean organic matter content was $15 \%$, mean total nitrogen was $0.40 \%$, and $\mathrm{C}: \mathrm{N}$ ratio was $24: 1$.

Productivity- The wide range in productivity, 517-1,670 lbs/acre, despite the similarity of site parameters, is partly explained by the fact that the low-productivity site had experienced snow release just prior to sampling. The higher figure would be more typical for the c.t. The shrub fraction of total production was $56 \%$ and $78 \%$.

Other Studies- Stands dominated by Caltha leptosepala and Trollius laxus with a significant contribution by Salix arctica and Salix reticulata are occasionally found in alpine seepage areas in the Canadian Rockies (Achuff and Corns 1982). Johnson and Billings (1962) describe for the Beartooth Range small areas of soil frost disturbance dominated by Salix arctica or S. reticulata and Trifolium parryi. They infer these sites to be in an early stage of recolonization and describe no more extensive communities with dwarf Salix 
spp. dominant. Our sites are like those of the Beartooth Range but disturbance (solifluction and congeliturbation) has apparently occurred on a much larger scale. Willard (1979) describes alpine marshes in Colorado Rockies dominated by Carex scopulorum and Caltha leptosepala with Salix arctica a common species (see discussion under Carex scopulorum / Caltha leptosepala). In her Colorado study area $S$. reticulata is apparently rare.

\section{Element Code- CEGL001435}

Edition / Author- 97 -10 / S.V.Cooper et al.

\section{H erbaceous Vegetation with a Sparse N eedle- leaved or microphyllus E vergreen Shrub Layer}

\section{Artemisia cana / Elymus trachycaulus Shrub Herbaceous Vegetation (ARTCAN / ELYTRA) silver sage / bearded wheatgrass}

\section{Natural Heritage Conservation Rank- $\mathrm{G}$ ? / S?}

Environment- This is a very minor vegetation component found on terraces, wide valley bottoms and fans. On the Red Rocks Lake National Wildlife Refuge we noted a number of very small areas where Elymus trachycaulus (syn. Agropyron caninum) constituted the dominant graminoid under a light canopy (10$20 \%$ cover) of Artemisia cana. Poa juncifolia and Poa cusickii are commonly associated with this community. The forb component is depauperate and composed of increaser species such as Achillea millifolium, Artemisia ludoviciana, Tragopogon dubius and Antennaria microphylla.

Vegetation- This type has not been described elsewhere and may represent a seral stage of Artemisia cana / Pascopyrum smithii (identified by the dominance of Pascopyrum smithii or Elymus lanceolatus). That Artemisia cana / Elymus trachycaulus would constitute a grazing "disclimax" is somewhat improbable given that Elymus trachycaulus is only slightly less palatable than the highly palatable Pascopyrum smithii; one would not expect one species to be so highly differentially grazed that it would be virtually eliminated and yet a companion species would remain in a vigorous condition. The Artemisia cana / Pascopyrum smithii type is common in southwestern Montana, but is often highly impacted by grazing and "converted" to Artemisia cana / Poa pratensis.

\section{Element Code- CEGLMTHP37}

Edition / Author- 99-11-16 / S.V. Cooper, MTNHP

Artemisia cana / Festuca idahoensis Shrub Herbaceous Vegetation

(ARTCAN / FESIDA) Silver sage / Idaho fescue

\section{Natural Heritage Conservation Rank- G3? / S3?}

Environment- This plant association was first named by Mueggler and Stewart (1980) and described as occurring in southwestern Montana (Yellowstone Park vicinity) as small patches on deep, loamy, alluvial soils along mountain streams above 6,000 feet elevation. It occurs as a very minor landscape component on the RRLNWLR associated with virtually identical environmental parameters as described by Mueggler and Stewart (1980) for the type at large.

Vegetation- Vegetation composition is that of the more moist portions of the Artemisia tridentata ssp. vaseyana / Festuca idahoensis habitat type, with high cover (> 30-40\%) for Festuca idahoensis, and variable cover of other mesic graminoids including Bromus carinatus, Bromus anomalus, Stipa occidentalis, and Elymus trachycaulus (syn. Agropyron caninum). The shrub component, which generally does not exceed 15-20\% canopy cover, is dominated by Artemisia cana with 
scattered A. tridentata ssp. tridentata or A. tridentata ssp. vaseyana. The forb component is rich with Geranium viscosissimum,

Potentilla gracilis, Geum triflorum, and Agoseris glauca contributing the greatest cover; the moistest sites support appreciable amounts of Polygonum bistortoides and Carex petasata. Taraxacum officinale, Achillea millifolium and Trifolium spp. have high cover where grazing has altered communities.

\section{Element Code- CEGL001552}

Edition / Author- 99-11-16 / S.V. Cooper, MTNHP

Artemisia cana / Poa pratensis Shrub Herbaceous Vegetation

(ARTCAN / POAPRA) silver sage / Kentucky bluegrass

Natural Heritage Conservation Rank$\mathrm{G}$ ? / S?

Environment- Artemisia cana / Poa pratensis is a grazing induced "disclimax" of any one of a number of plant associations including Artemisia cana / Festuca idahoensis, Artemisia cana / Elymus trachycaulus or most probably Artemisia cana / Pascopyrum smithii. Within greater southwestern Montana Artemisia cana I Pascopyrum smithii is the most abundant association dominated by Artemisia cana (though within this region the type is relatively scarce); presumably the Centennial Valley is not unique so that most Artemisia cana / Poa pratensis would be derived from overgrazing of Artemisia cana / Pascopyrum smithii. Usually occurring as stringers on the first (or second) terrace up from small mountain streams, these communities occupy mesic to wet sites, with deep alluvial soils.

Vegetation- In the more severe cases of "conversion" the scattered shrub canopy (combined cover $<20 \%$ ) of Artemisia cana and occasionally A. tridentata ssp. vaseyana or $A$. tridentata ssp. tridentata is imposed over a sward of Poa pratensis and Trifolium spp. In less impacted stands graminoid remnants of past vegetation, such as Carex petasata,
Pascopyrum smithii, Elymus lanceolatus, Bromus ciliatus, or Stipa occidentalis will be present. Cover for Achillea millifolium, Trifolium spp., Potentilla gracilis, and Agoseris glauca appears to increase under these conditions; where disturbance has been severe Taraxacum officinale can form a virtually continuous carpet (often with Trifolium spp.).

\section{Element Code- CEGLMTHP38}

Edition / Author- 99-11-16 / S.V. Cooper, MTNHP

Artemisia tridentata ssp. vaseyana /

Festuca idahoensis Shrub Herbaceous Vegetation

(ARTTSV / FESIDA) mountain big sagebrush / Idaho fescue

\section{Natural Heritage Conservation Rank-} G5 / S4

Environment- Artemisia tridentata ssp vaseyana/Festuca idahoensis is common on slopes and upper terraces at $6,000-8,500 \mathrm{ft}$ throughout the study area. Parent materials included limestone, quartzite, volcanic, igneous and alluvium. Adjacent cooler slopes support Pseudotsuga menziesii or Pinus flexilis forests. Sites with shallow soil are often dominated by Cercocarpus ledifolius. Nearly level sites of terraces with finer textured soils may be dominated by Artemisia tridentata ssp. tridentata.

Vegetation- Artemisia tridentata ssp. vaseyana is the dominant shrub with canopy cover of 10$70 \%$. Chrysothamnus viscidiflorus is the only other frequent and common shrub. Grass cover is generally high. Festuca idahoensis is the dominant grass with mean canopy cover of $48 \%$. Other common grasses are Pseudoroegneria spicata and Koeleria cristata. Forbs are generally abundant, and diversity is moderate to high. Common species include Achillea millefolium, Antennaria microphylla, Besseya wyomingensis, Eriogonum umbellatum, Geum triflorum and Taraxacum officinale. Mosses are uncommon in most stands. Particularly mesic sites at higher 
elevations have robust grasses such as Bromus carinatus and Elymus lanceolatus and higher coverage of mesic forbs such as Geranium viscosissimum, Galium boreale, Geum triflorum and Potentilla gracilis.

Soils- Soils have a loamy texture and are generally deep. Up to $30 \%$ of the ground may be bare. Gravel is uncommon or absent.

Other Studies- Mueggler and Stewart (1980) describe this type for western Montana. Similar vegetation has been reported for all states in the Northern Rocky Mountains and intermountain states (Bourgeron and Engelking 1994). Mueggler and Stewart (1980) discuss how these associations differ from those in Montana.

Comments- Mesic stands supporting an abundance of robust grasses and forbs is considered the Geranium viscosissimum phase of the type (Mueggler and Stewart 1980).

Elk and mule deer are common in these habitats. Pocket gophers are evident in some stands.

\section{Element Code- CEGL001533}

Edition / Author- 99-11-16 / S.V. Cooper, MTNHP

Artemisia tridentata ssp. wyomingensis / Pseudoroegneria spicata Shrub Herbaceous Vegetation (ARTTSW / PSESPI) Wyoming big sagebrush / bluebunch wheatgrass

\section{Natural Heritage Conservation Rank- G4 / S3}

Environment- Gently sloping alluvial fans and terraces may support stands of Artemisia tridentata ssp. wyomingensis / Pseudoroegneria spicata. The single sample stand was at 7,400 ft, but this type might be expected to occur at 5,000-7,500 ft. Adjacent stands with heavier soils and poor drainage are dominated by Artemisia arbuscula ssp. longiloba and Elymus lanceolatus or Festuca idahoensis or Leymus cinereus and Poa juncifolia. Adjacent slopes with deeper soils may support stands dominated by Artemisia tridentata vaseyana and Festuca idahoensis.

Vegetation- Artemisia tridentata ssp. wyomingensis is the dominant shrub with a cover of at least $10 \%$; in the course of reconnaissance cover was noted to vary from $5-10 \%$ (in the establishment phase) to $30 \%$ plus in stands heavily impacted by grazing. Chrysothamnus spp. may also be present. Grass cover may be as high as $60 \%$, and Pseudoroegneria spicata is the dominant species. Poa secunda and Koeleria cristata are other high constancy grasses; with light grazing they are poorly represented but increase somewhat in proportion to increasing grazing pressure. Common forbs include Phlox hoodii and Antennaria microphylla. Mosses and lichens are uncommon or absent.

Soils- Soils are silty in texture. Much of the surface is bare, and surface gravel is common.

Other Studies- Vegetation dominated by Artemisia tridentata wyomingensis and Pseudoroegneria spicata is described for western Montana by Mueggler and Stewart (1980) who do not differentiate between subspecies wyomingensis and vaseyana.

Jorgensen (1979) describes this type for central Montana. This type has also been reported for Washington, Oregon, Nevada, Idaho, Wyoming and Colorado (Bourgeron and Engelking 1994).

Comments- Subspecies vaseyana appears to be more common than subspecies wyomingensis in the high country of southwest Montana. We expect subspecies wyomingensis to be common only in the more windswept or otherwise arid sites where subspecies vaseyana is excluded.

\section{Element Code- CEGL001535}

Edition / Author- 99-11-16 / S.V. Cooper, MTNHP 
Artemisia tripartita / Elymus lanceolatus

Shrub Herbaceous Vegetation

(ARTTRP / ELYLAN) three-tip sagebrush / thickspike wheatgrass

\section{Natural Heritage Conservation Rank-}

$\mathrm{G}$ ? / S?

Environment- This type is found on nearly level slopes of stream terraces and alluvial fans at ca. 6,500-6,700 $\mathrm{ft}$ in the Centennial Valley. Vegetation of lower positions in the landscape tends to be wetlands dominated by Juncus balticus or Deschampsia cespitosa. Artemisia tripartita / Festuca idahoensis is the most common type on adjacent slopes or betterdrained positions with coarser-textured soils.

Vegetation- Artemisia tripartita is the dominant shrub with canopy cover of 10-20\%. Chrysothamnus nauseosus and Chrysothamnus viscidiflorus are common in most stands. Grass cover varies between 40 and $60 \%$. Elymus lanceolatus is usually the dominant grass. Small cespitose bluegrass's, such as Poa juncifolia, Poa secunda and Poa cusickii, are often common. Forb cover is ca. $10 \%$, and diversity is low. Phlox hoodii, Antennaria microphylla and Antennaria parvifolia are common species. Mosses and lichens are absent.

Soils- Soils are deep and silty in texture. Some sites may be slightly saline. $40-70 \%$ of the soil surface is bare, and gravel is uncommon.

Other Studies- This type has not been previously described.

Comments- Examples of this unusual vegetation have been heavily grazed by livestock in the past and probably obscures the actual potential vegetation of these sites. Vegetation dominated by Artemisia tripartita and Leymus cinereus is reported for Idaho (Bourgeron and Engelking 1994), and a type dominated by Artemisia tripartita and Stipa comata occurs in Washington (Daubenmire 1970). Artemisia tripartita / Elymus lanceolatus may be a seral stage of vegetation similar to one of these types. A possible scenario envisions these sites as seral to
Artemisia tridentata ssp. tridentata (or vaseyana), quite possibly the Artemisia. tridentata ssp. tridentata / Leymus cinereus or Artemisia tridentata ssp. tridentata /

Pascopyrum smithii c.t.s.

\section{Element Code- CEGLMTHP08}

Edition / Author- 99-11-16 / S.V. Cooper

\begin{tabular}{c} 
Artemisia tripartita / Festuca idahoensis \\
Shrub Herbaceous Vegetation \\
(ARTTRP / FESIDA) Three-tip sagebrush \\
/ Idaho fescue \\
\hline
\end{tabular}

\section{Natural Heritage Conservation Rank-} G3 / S3?

Environment- This type is common on gentle to moderate slopes and ridges at $6,300-7,500 \mathrm{ft}$ within the drainage of the Red Rock River. The Stipa comata phase is common on the stabilized sandhills at $6,600-6,700 \mathrm{ft}$ at the northeast end of the Centennial Valley. Soils are derived from many different parent materials. Finer textured soils of adjacent terraces support Artemisia tripartita / Elymus lanceolatus, while steeper, higher or cooler slopes are dominated by Artemisia tridentata vaseyana and Festuca idahoensis. The Stipa comata phase of Artemisia tripartita / Festuca idahoensis occupies long-term stabilized dunes in the Centennial Valley; areas that have been stabilized for shorter time support Stipa comata I Psoralea tenuiflora.

Vegetation- Artemisia tripartita is the dominant shrub with canopy cover of $10-30 \%$. Artemisia tridentata ssp. vaseyana, Chrysothamnus viscidiflorus and Tetradymia canescens are often present. Total grass cover is generally high, $60-80 \%$ in most stands.

Festuca idahoensis is usually the dominant species, but Elymus lanceolatus, Pseudoroegneria spicata and Koeleria cristata are also common. Stipa comata is common and its dominance defines the Stipa comata phase. Forb canopy cover and diversity are moderate. Common forbs are Achillea millefolium, Antennaria microphylla, Geum triflorum, Comandra umbellata, Lupinus 
sericeus, Phlox hoodii and Taraxacum

officinale. Mosses and lichens may be present but are uncommon.

Soils- Soils have a silty or loamy texture. Usually less than $30 \%$ of the surface is bare, and gravel is uncommon. The Stipa comata phase occurs on sandy soils with no surface gravel.

Other Studies- Daubenmire (1970) first described Artemisia tripartita / Festuca idahoensis as a zonal climax plant association for eastern Washington, but many of the common subordinate species are different from those of our study area. Mueggler and Stewart (1980) were the first to document this type for southwestern Montana. Similar vegetation has been described for Idaho (Hironaka 1977) and vegetation dominated by these two species is also reported for Colorado (Bourgeron and Engelking 1994).

Comments- The Stipa comata phase occurs on sandy rather than silty or loamy soils and is characterized by having Stipa comata rather than Elymus lanceolatus or Pseudoroegneria spicata as important subordinate grasses. See Comments under Artemisia tridentata ssp. tridentata /Stipa comata.

Mueggler and Stewart (1980) imply that stands dominated by Artemisia tripartita may occur on sites that are drier than optimum for $A$. tridentata vaseyana. This hypothesis is supported by our observation that $A$. tridentata vaseyana stands are often on cooler or higher elevation slopes. Capable of root sprouting as a reproductive tactic, Artemisia tripartita also resprouts after fire, while $A$. tridentata is killed, so fire history may play a role in determining the distribution of these plants across the landscape. Nonetheless, it is difficult to clearly distinguish between the ecological niches of these two sagebrush species, at least in the landscapes where they co-occur.

\section{Element Code- CEGL001536}

Edition / Author- 99-11-16 / S.V. Cooper, MTNHP
Artemisia tripartita / Pseudoroegneria spicata Shrub Herbaceous Vegetation (ARTTRP / PSESPI) three-tip sagebrush / bluebunch wheatgrass

\section{Natural Heritage Conservation Rank-} G2G3 / S?

\section{Environment- Artemisia tripartita /}

Pseudoroegneria spicata is a community of very limited acreage in the Beaverhead Mountains Section, noted only south of the Clark Fork Reservoir. It almost always occurs as small or linear patches on west- to southfacing ridges and convex slope shoulders; sites where solar insolation and prevailing winds exceed the tolerance of Festuca idahoensis. These sites usually have an abrupt transition to Artemisia tripartita / Festuca idahoensis or Artemisia tridentata ssp. vaseyana / Festuca idahoensis of less moisture-stressed positions.

Vegetation- These droughty sites have insufficient cover (10-20\%) of Artemisia tripartita to be considered shrub stands. Other shrubs that occur, generally with less than 5\% cover, include Chrysothamnus viscidiflorus, Artemisia frigida and Artemisia tridentata var. vaseyana. Pseudoroegneria spicata and Koeleria cristata dominate the herbaceous layer, though their coverage is commonly less than $30 \%$. Forbs often include Phlox hoodii, Achillea millifolium, Antennaria parviflora, A. microphylla and Erigeron compositus, none having more than $5 \%$ cover.

Soils- Generally there is more than $70 \%$ exposed substrate (including soil, gravel and rock) and litter is confined to the immediate vicinity of shrub and bunchgrass canopies. Parent materials include extrusive volcanics and alluvial outwash.

Other Studies- Artemisia tripartita /

Pseudoroegneria spicata was first described by Daubenmire (1970) for portions of the Columbia Basin of Washington State where it 
occurs in much the same manner it does within the Centennial Valley, as isolated patches typifying hot, dry exposures within what is Artemisia tripartita / Festuca idahoensis dominated landscape (considered a climatic climax only in the Columbia Basin). It apparently is most extensive southward in Idaho's Snake River Plain and also occurs as an uncommon type in Oregon (Bourgeron and Engelking 1994).

Comments- Within the Centennial Valley Artemisia tripartita / Pseudoroegneria spicata we speculate to be a successional community type, seral to Artemisia tridentata ssp. vaseyana / Pseudoroegneria spicata. We noted, but did not sample, several small stands with mixed $A$. tridentata ssp. vaseyana and $A$. tripartita and occurring in the same landscape positions as Artemisia tripartita /

Pseudoroegneria spicata. The severity of these sites may slow the rate at which $A$. tridentata ssp. vaseyana recolonizes and ultimately replaces Artemisia. tripartita (in the absence of fire).

\section{Element Code- CEGL001538}

Edition / Author- 99-11-16 / S.V. Cooper, MTNHP

Pentaphylloides floribunda / Festuca
campestris Shrub Herbaceous
(PENFLO / FESCAM) Shrubby cinquefoil
/ rough fescue

\section{Natural Heritage Conservation Rank-}

\section{G4 / S4}

Environment- Owning to the fact that the geographic range of Festuca campestris, as a community dominant, extends on the east side of the Continental Divide southward to approximately $46^{\circ}$ north latitude this association is found only north of this latitude in the Anaconda-Pintlar Range and on the upper slopes of the Deerlodge Valley Subsection. It is most prevalent on gently sloping or rolling topography but can occur on steep slopes within mountain parklands above the 4,500 to 6,000 feet elevation range quoted by Mueggler and Stewart (1980). This is a moderately mesic foothill to mountain type that likely falls in the 20 to 30 inch precipitation zone.

Soils- Parent materials on which it is noted to occur include sandstone, limestone and glacial drift; soils are moderately deep, 10 to 18 inches rooting depth. Considerable surface rock may be exposed, although little bare soil is minimal. In the more productive Danthonia intermedia Phase, litter and vegetation combine to cover $98 \%$ of the surface.

Vegetation- Like the Pentaphylloides floribunda / Festuca idahoensis association, but even more so, the shrub component, dominated by Pentaphylloides. floribunda (5$30 \%$ canopy cover), can be rendered quite obscure by the luxuriant graminoids, principally the tall tussock grass Festuca campestris; at least as the type occurs in Montana it would be best considered a dwarfshrub type because Pentaplylloides floribunda seldom is taller than 4-5 decimeters. Artemisia frigida and Gutierrezia sarothrae are subshrubs that along with Juniperus horizontalis (dwarf-shrub) attain a high constancy but seldom are well represented, even with overgrazing. Festuca idahoensis and Koeleria macrantha have high constancy in both phases but are distinctly secondary to Festuca campestris. Forb diversity is generally high, those of high constancy being Galium boreale, Gaillardia aristata, Achillea millefolium, Solidago missouriensis and Campanula rotundifolia.

The Danthonia intermedia phase differs significantly (floristics coupled with environmental parameters of mesic situation) and it should be considered a separate association (Pentaphylloides floribunda / Festuca campestris - Danthonia. intermedia); this phase is typified by Danthonia intermedia being well represented and the relative abundance of Elymus trachycaulus, Carex obtusata, Potentilla gracilis, Lupinus sericeus, Agoseris glauca, Geranium viscosissimum and Erigeron specious. 
Though not named by Mueggler and Stewart (1980), the drier phase should receive an association designation as well and be known as Pentaphylloides. floribunda / Festuca campestris - Pseudoroegneria spicata. It is typified by a relatively high constancy and coverage of Pseudoroegneria spicata and Carex scirpoidea and the consistent presence of a number of other graminoids that do not occur, or if they do, at much reduced levels in the mesic phase including Danthonia parryi, Muhlenbergia cuspidata, Muhlenbergia. richardsonis and Poa secunda. It lacks the mesic forbs of the more productive Pentaphylloides floribunda / Festuca campestris - Danthonia intermedia but dwarf shrubs are virtually exclusive to this phase along with the forbs Chrysopsis villosa, Senecio canus, Erigeron caespitosus and Oxytropis sericea.

Comments- This association should be partitioned into two, Pentaphylloides floribunda / Festuca campestris - Danthonia intermedia and Pentaphylloides floribunda / Festuca campestris - Pseudoroegneria spicata, based on floristic and environmental differences. This association is very similar to those in the Festuca campestris alliance. It is well suited to grazing by cattle and horses by virtue of having its forage comprised principally of tussock grasses. Pentaphylloides floribunda increases appreciably with overgrazing and complicates management because it is difficult to control, sprouting readily following burning or spraying $(2,4-\mathrm{D})$. Those forbs decreasing on sheep range include Lupinus sericeus, Gaillardia aristata, Galium boreale, Senecio canus and possibly Potentilla gracilis; conversely these same forbs are candidates to increase on cattle range.

\section{Element Code- CEGL001503}

Edition / Author- 98-12-03 / S.V. Cooper

Pentaphylloides floribunda / Festuca idahoensis Shrub Herbaceous (PENFLO / FESIDA) shrubby cinquefoil / Idaho fescue

\section{Natural Heritage Conservation Rank-} G3 / S3

\section{Environment- Pentaphylloides floribunda /}

Festuca idahoensis is an uncommon association in Montana, found only east of the Continental Divide, however it ranges southward into central Idaho, northern Nevada and western Wyoming. In Montana it has been found at elevations between 6,600 and 8,600 feet on gentle mountain slopes with moderately deep soils derived from granitics. Normally the soil surface is extensively covered with vegetation and litter. This association is considered moderately mesic, usually falling within the 20 to 30 inch precipitation zone.

Vegetation- Given the relatively short stature of Pentaphylloides floribunda combined with its low canopy cover (seldom exceeding 15\%) it is appropriate to classify this type as shrub herbaceous (most Montana stands in fact would qualify for dwarf-shrub status). Occasionally Artemisia tridentata ssp. vaseyana and A. cana are present in trace amounts. Festuca idahoensis and Danthonia intermedia are the dominant graminoids; at higher elevations in south central Montana Carex obtusata may also be well represented. The mesic and relatively cold nature of these sites is revealed in the low cover of Pseudoroegneria spicata and presence of several Stipa spp and Elymus trachycaulus. The most common forbs are Besseya wyomingensis, Campanula rotundifolia, Achillea millifolium, Arenaria congesta, and Potentilla gracilis; Geum triflorum is sometimes the dominant forb.

Other Studies- Schlatterer (1972) has described an equivalent vegetation type from the White Cloud, Boulder and Pioneer Mountains of central Idaho. Mueggler and Stewart (1980) cite the type from Yellowstone National Park.

Comments- Over $60 \%$ of the total vegetation of this type consists of palatable grasses and sedges, the most productive of which are Festuca idahoensis and Danthonia intermedia; the first of the forenamed species in addition to 
Elymus trachycaulus and Carex obtusata are the primary decreasers with overgrazing. Pentaphylloides floribunda, filling a niche akin to that of Artemisia tridentata ssp. vaseyana in Artemisia tridentata ssp. vaseyana / Festuca idahoensis, generally increase appreciably with grazing pressure, along with Geum triflorum and Potentilla gracilis.

\section{Element Code- CEGL001502}

Edition / Author- 98-12-03 / S.V. Cooper

Salix arctica / Polygonum bistortoides Dwarf Shrubland

(SALARC / POLBIS) Arctic willow / American bistort

\section{Natural Heritage Conservation Rank-} G2G3 / S2S3

Environment- Though our definition of this c.t. is based on only two plots, the fact that this type is recognized elsewhere in the Rocky Mountains allows us to compare and interpret our data. Sites were documented in the East Pioneer and Anaconda-Pintlar Ranges on lower to mid-slopes of gentle terrain (and noted in reconnaissance in the Beaverhead Mountains). We interpret these sites as wetland/turf hybrids in terms of both environment and vegetation. Both sites were potentially in water-receiving positions; one community was intercalated between snowbeds upslope and drier turf c.t's. downslope, while the other was developed on an ephemeral spring with spongy ground throughout. Landscape positions of Salix arctica / Polygonum bistortoides were much like those supporting Salix reticulata / Caltha leptosepala but with a higher probability of wind scouring. Vegetation composition also indicated a drier environment than that of Salix reticulata / Caltha leptosepala.

Vegetation- These stands were dominated by Salix arctica (50\% c.c.) with lesser amounts of Dryas octopetala. Moss coverage in excess of $50 \%$ adds to the visual impression of a blanket of vegetation. Graminoid cover averaged $15 \%$, contributed mostly by Poa alpina and moist- site Carex spp., C. albonigra, C. phaeocephala or C. nova. Forb cover averaged $30 \%$. The diagnostic forb, Polygonum bistortoides (10\% c.c.), was among several with relatively high coverages, including $P$. viviparum, Geum rossii, Potentilla diversifolia, Aster alpigenus and Claytonia lanceolata.

Soils- Parent material included quartzite and granite-limestone mix from a contact zone. Average litter and duff depths were 0.1 in and 0.4 in respectively. Coarse fragment content ranged from 19 to $33 \%$. Mean texture of the fine fraction was clay. Soil reactions were slightly acid, averaging 6.50. Mean organic matter content was $16 \%$, mean total nitrogen was $0.43 \%$, and $\mathrm{C}: \mathrm{N}$ ratio was $25: 1$

Productivity- Total productivity ranged from 798 to $1,095 \mathrm{lbs} /$ acre with shrub productivity constituting 32 to $81 \%$ of the total; graminoid and forb coverage were 148 and 295 lbs/acre, respectively.

Other Studies- Salix arctica dominates in some snowbed communities of the Canadian Rockies (Achuff and Corns 1982, Hrapko and LaRoi 1978). Potentilla diversifolia and Polygonum viviparum were common species in the above-cited communities; however, snowbed indicator species, such as Antennaria lanata, Phyllodoce glanduliflora and Cassiope mertensiana were also common. Johnson and Billings (1962) discuss small disturbance sites within moist Deschampsia cespitosa meadows with vegetation very similar to Salix arctica / Polygonum bistortoides (see Other Studies section Salix reticulata / Caltha leptosepala for expanded discussion). In the Colorado Rockies Willard (1979) described snowbed vegetation dominated by $S$. arctica; her community type had high coverages of Geum rossii, Polygonum spp., Festuca ovina, mosses and lichens and was more similar to that of our study area than those cited from the Canadian Rockies. Baker (1983) described late snowbank communities dominated by $S$. arctica and $S$. reticulata for the Sangre de Cristo Range of New Mexico.

Element Code- CEGL001431 
Edition / Author- 97 -10 / S.V. Cooper et al.

Sarcobatus vermiculatus / Pascopyrum smithii Shrub Herbaceous Vegetation (SARVER / PASSMI) black greasewood / western wheatgrass

Natural Heritage Conservation RankG4 / S4

Environment- This uncommon type is found on wide stream terraces, often in areas of calcareous parent material such as the Ruby and Tendoy Mountains. Higher terraces with less alkaline-saline soil may support stands dominated by Artemisia tridentata ssp. tridentata and Pascopyrum smithii or Leymus cinereus. Nearby slopes are dominated by Artemisia tridentata and Pseudoroegneria spicata or Festuca idahoensis.

Vegetation- Sarcobatus vermiculatus is the dominant shrub, averaging approximately $10 \%$ canopy cover; its cover may be less but its stature in comparison to the associated species still conveys a shrubland aspect. Artemisia tridentata ssp. tridentata and Chrysothamnus viscidiflorus can be conspicuous components, their cover approaching that of $S$. vermiculatus. The subshrubs, Artemisia frigida and Atriplex gardneri, may also be common. Pascopyrum smithii and/or Elymus lanceolatus are the dominant and diagnostic species, often forming a thin sword. Forbs are scarce. Opuntia polyacantha or Taraxacum officinale may be present. Soil lichens or algae may be present as well where livestock trampling has been minimal.

Soils- Soils are silty and deep. The surface horizons are usually alkaline-saline. Most of the ground surface is bare soil and gravel is rare or absent.

Other Studies- Mueggler and Stewart (1980) describe this type for western Montana, and Hansen et al. (1995) describe it mainly for eastern Montana. Jorgensen (1979) described a very similar Sarcobatus vermiculatus /
Elymus lanceolatus type from central Montana. Sarcobatus vermiculatus / Pascopyrum smithii is also reported from Wyoming (Bourgeron and Engelking 1994). A very similar type dominated by Sarcobatus vermiculatus and Pascopyrum smithii with the addition of Leymus cinereus has also been described for western Montana (Mueggler and Stewart 1980).

Comments- Although Mueggler and Stewart (1980) state that Poa juncifolia decreases with grazing, we believe that under late season grazing it may increase, while rhizomatous wheatgrasses decrease. Mueggler and Stewart (1980) indicate an inability to distinguish environment differences between Sarcobatus vermiculatus / Leymus cinereus and Sarcobatus vermiculatus / Pascopyrum smithii; the differences could be more attributable to grazing history than habitat. Most examples of Sarcobatus vermiculatus / Pascopyrum smithii are close to water and on nearly level terrain. Consequently most stands have been severely impacted by livestock grazing. Leymus cinereus may have been present in many of these stands prior to the heavy livestock grazing in the early part of the century (see Comments- under Leymus cinereus / Puccinellia nuttalliana).

\section{Element Code- CEGL001508}

Edition / Author- 99-11-16 / S.V. Cooper, MTNHP

\section{H erbaceous Vegetation L acking a Significant Shrub/ Tree Component}

\author{
Bromus carinatus - Bromus anomalus \\ Herbaceous Vegetation \\ (BROCAR - BROANO) mountain brome \\ - nodding brome
}

Natural Heritage Conservation RankG? / S?

Environment- This community is associated 
with sites that are both protected and receive more moisture than other positions in the landscape, either through snow deposition or subsurface input. Sites are usually found on gently inclined ( $<30 \%$ slope) lee slopes with northwest- to northeast-facing aspects in toeslope positions with concave to straight slope shapes. That these are lee slopes is partially corroborated by the soils. They are deep silt loams, often without rock (of any size class) in the upper $20 \mathrm{~cm}$, but not having more than $5 \%$ stone to a depth of $40 \mathrm{~cm}$ in any of the examined profiles; we speculate that these soils originate primarily from the deposition of aeolian material and in part from slopewash of fines. Stands are usually small, less than 2-3 acres and often only a fraction of an acre. Adjacent vegetation is almost invariably dominated by the more mesic plant associations within the Artemisia tridentata ssp. vaseyana and Festuca idahoensis alliances, usually those communities denoted by Elymus trachycaulus, Stipa richardsonii, Geranium viscosissimum, Potentilla gracilis and $P$. glandulosa.

Range- This association has only been found on the lower slopes of the Snowcrest and Blacktail Ranges and various hills and foothills between these ranges; it was particularly in evidence on the Blacktail State Wildlife Management Area and occurred to a lesser degree and in a more degraded condition on the Robb-Ledford State Wildlife Management Area. We speculate that it could occur to the southward in Idaho, Nevada and Utah if one envisions it as a seral community resulting from the burning (or otherwise destruction) of the shrub component of Artemisia tridentata ssp. vaseyana / B. carinatus and Symphoricarpos oreophilus / B. carinatus communities. Anecdotal information from the foothills of the Rocky Mountain Front suggests this type occurs as small meadow patches within a primarily forested landscape.

Vegetation- This community is distinguished by its dearth of shrubs; Artemisia tridentata ssp. vaseyana Chrysothamnus nauseosus and Symphoricarpos oreophilus have the highest constancy but coverages seldom exceed trace amounts. The modal to highest quality (least disturbed) expression of this type is that of dense grassland, the tussocks set so closely that from a distance one appears to be viewing a grain field or vegetation dominated by rhizomatous grasses. The non-rhizomatous (approaching bunchgrass form) grasses Bromus carinatus and B. anomalus usually dominate the community and true bunchgrasses such as Stipa richardsonii, S. occidentalis, Elymus trachycaulus, Festuca idahoensis, Poa juncifolia and Danthonia intermedia are important components as well, their cover occasionally exceeding that of the diagnostic species. These communities are notable both for their dense cover of graminoids, whose combined individual cover values approach or exceed $100 \%$, and for the variety of graminoids, usually exceeding 10 species per plot. In stands of ostensibly degraded condition Poa pratensis and occasionally

Phleum pratense are well represented to abundant and probably indicative of past grazing pressure, quite possibly by horse herds. The forb component is highly variable in coverage, ranging from nearly $100 \%$ in stands where the graminoid component is reduced, to $20-40 \%$ in stands with the highest coverage of graminoids. Any of the following forbs may be both well represented and exhibit high constancy and their presence is indicative of the mesic nature of this type; Potentilla gracilis, Potentilla glandulosa, Geranium viscosissimum, Aster chilensis, Geum triflorum, Agoseris glauca and Galium boreale.

Similar Communities- Though this community occurs as small patches in unique environments, it is rather surprising that it has gone undescribed until now and in fact it is notable that there are no herbaceous community types noted in the NVCS with native Bromus spp. as diagnostic species. As noted above, it is quite conceivable that the successional seres of several shrub-dominated rangeland types of Idaho, Nevada and Utah would pass through a compositional stage approximating the community type described here. The community type most similar in environment and composition is Stipa richardsonii-Festuca idahoensis. This 
community occurs in the same region and landscape positions closely comparable to those of $B$. carinatus $-B$. anomalus and could be seen as a variant of it, as it possess many of the same species, but the dominance of the Bromus spp. has been replaced by that of $S$. richardsonii.

\section{Element Code- CEGLMTHP09}

Edition / Author- 99-11-22 / S. V. Cooper, MTNHP

\section{Calamagrostis canadensis Herbaceous Vegetation \\ (CALCAN) Bluejoint reedgrass}

\section{Natural Heritage Conservation Rank-} G4Q / S4

Environment- The Calamagrostis canadensis association is found in montane to subalpine habitats in the mountains of Montana. It is typically found in a variety of settings: in depressional landforms as one of the outer bands (i.e. the drawdown zone) of vegetation, in wet meadows, adjacent to stream courses and on alluvial terraces, and in moist forest openings. Soils in basin settings are generally loamy mineral soils, while those along low gradient streams are usually coarse textured alluvium. Calamagrostis canadensis communities usually flood in the spring and dry down by mid-summer. Adjacent wetter communities could include Carex aquatilis or Carex utriculata communities, and adjacent drier vegetation is usually upland coniferous forest (Hansen et al. 1988, Hansen et al. 1995).

Vegetation: Calamagrostis canadensis is the dominant species in this community, with canopy coverage averaging 70\% (Hansen et al. 1995). Traces of conifers and of shrubs can be found in this association, and Deschampsia cespitosa is a frequently associated graminoid. A wide variety of forbs are associated with this community, usually at low coverage.

Similar Communities- Similar communities have been described by Mattson (1984) for Yellowstone National Park, Padgett et al.
(1989) for Utah and southeastern Idaho, Jankovsky-Jones (1997) for northern Idaho, and eastern Oregon (Kovalchik 1987). Similar communities dominated by Calamagrostis stricta have been observed in Montana, and Hansen et al. (1995) have placed these in the Calamagrostis canadensis association because of similarities in management concerns. Other studies have documented Calamagrostis canadensis as the dominant understory species growing with a variety of other overstory species, including Picea sp. (Hansen et al. 1995), Abies lasiocarpa (Pfister et al. 1977), Salix drummondiana, Salix geyeriana, and Salix lutea (Hansen et al. 1995).

Succession- Successional dynamics of this community are poorly understood. Padgett et al. (1989) describe expansion of Calamagrostis canadensis into the moist borders of Pinus contorta stands dying from bark beetles, and ascribe this to increases in the water table due to less transpiration by the conifers. Hansen et al. (1995) suggest that Picea sp. /

Calamagrostis canadensis communities are late seral stages of the Abies lasiocarpa I Calamagrostis canadensis community, with shrubs dominating where disturbance removes the tree overstory. However, it is not clear whether the Calamagrostis canadensis community should be considered an early seral community that is ultimately invaded by conifers and/or shrubs. Changes in the composition of the Calamagrostis canadensis community can take place when there are changes in the hydrologic regime.

Management- Palatability of Calamagrostis canadensis varies from moderate to high, and heavy grazing can reduce the vigor of this grass and lead to an increase of other graminoids such as Poa pratensis, Poa palustris, Agrostis stolonifera, or Juncus balticus. Heavily grazed wetter sites can be converted to dominance by Juncus balticus or Carex nebrascensis. Hansen et al. (1995) also state that moderate late-season grazing of Calamagrostis canadensis has only limited impact on stands, especially when soils are dry.

Element Code- CEGL001559 
Edition / Author- 99-10-12 / J. Greenlee, MTNHP

Calamagrostis purpurascens - Carex rupestris Herbaceous Vegetation

(CALPUR - CARRUP) purple reedgrass curly sedge

\section{Natural Heritage Conservation Rank- $\mathrm{G}$ ? / S?}

Environment- The Calamagrostis purpurascens -Carex rupestris $\mathrm{p}$. a. is primarily a high subalpine to alpine vegetation type, having been documented from $8,900 \mathrm{ft}$. to the highest elevations of the Blacktail, Greenhorn and Tendoy Ranges (above 9,400 feet); it occupies the most exposed sites, generally including shoulders of ridges, ridgelines, and upper west and south-facing slopes. These upper slopes are flat to moderately sloping and usually convex to straight in shape. This type can be expected to occur in similar positions in mountain ranges adjacent to the ones cited above and that exist in the rain shadow of the Beaverhead Range. All BVRHDMS sampled sites and noted occurrences were developed on limestone and possessed soils with silt loam textures and a high rock content. In the study area it may occur on a wider variety of substrates than indicated based on the observation that in Wyoming (Regan et al. 1997) it is known to occur on non-calcareous sedimentary substrates. The amount of exposed substrate varies greatly, depending on exposure, but generally between dead and live Selaginella densa, bryophytes, and litter more than $70 \%$ of the surface has an organic cover. This type usually is the most areally extensive community in a mosaic of $C$. rupestris / Potentilla ovina, Carex elynoides turf communities, Festuca kingii / Oxytropis campestris grasslands that ostensibly occupy equally, if not more, stressful sites and Carex scirpoidea / Potentilla diversifolia, Festuca idahoensis / Potentilla diversifolia or Deschampsia / P. diversifolia communities found on more mesic, less exposed, or lower elevation sites.
Vegetation- These sites are recognized by Calamagrostis purpurascens being well represented and Carex rupestris or C. obtusata also being dominant or co-dominant; total graminoid canopy cover is mostly 50 to $70 \%$. However, graminoid diversity is low on these sites, the only other ones occurring regularly were Koeleria macrantha and Poa rupicola. No shrubs or subshrubs occurred in this type. Forb diversity is generally high with at least 15 and up to 28 per plot, but overall forb cover seldom exceeds $20 \%$. Forbs with the highest cover and constancy are Bupleurum americanum, Phlox pulvinata, Oxytropis campestris, Erigeron compositus and Potentilla ovina. Senecio canus, S. streptanthifolius and Arenaria obtusiloba simply have high constancy within the type. The cover of live (or photosynthetically active) Selaginella densa generally was not more than $20 \%$ but dead foliage/stems created anywhere from a trace to $30 \%$ cover.

Similar Communities- From Wyoming's Snowy Range a community of the same name (same dominant species) and occupying the same environmental niche has been described. South into Colorado as well as in Idaho (Urbanczyk and Henderson 1994, Richardson and Henderson 1999) and Montana (Cooper et al. 1997) several communities are documented wherein Carex rupestris alone is the dominant or diagnostic graminoid and the environments bear a strong resemblance to the one described here. Moseley (1985) has identified, based on a very limited sample in the alpine of eastcentral Idaho, a Calamagrostis purpurascens type. Urbanczyk and Henderson (1994) document a Calamagrostis purpurascens Carex elynoides community wherein Carex rupestris is a major component; they indicate that this community occupies positions less e than Carex elynoides turf sites. In southwestern Montana it has not been established what environmental parameters consistently distinguish Carex rupestris / Oxytropis campestris from Carex purpurascens / Carex. rupestris, though it is likely the degree of exposure. 


\section{Element Code- CEGLMTHP11}

Edition / Author- 99-11-22 / S. V. Cooper, MTNHP

Carex aquatilis Herbaceous Vegetation (CARAQU) Water sedge

\section{Natural Heritage Conservation Rank-} G5 / S5

Environment- Carex aquatilis communities can be found at mid to high elevations throughout Montana. It is typically found in depressional landforms, old channels along streams, fens, and in silted in beaver ponds. This community occurs both on mineral and organic soils, though more commonly on the latter. Soil reactions are usually acidic, and water levels in Carex aquatilis communities usually remain high throughout the growing season, occasionally dropping below the rooting zone in dry years. Adjacent wetter communities include Carex utriculata and Carex lasiocarpa stands, while drier communities could include Juncus balticus, Calamagrostis canadensis, or meadows dominated by Deschampsia cespitosa (Hansen et al. 1988).

Vegetation-Carex aquatilis is clearly the dominant species in this plant association, although it can be found growing with significant coverage of other graminoids, particularly Carex utriculata or Deschampsia cespitosa. Low coverage of shrubs such as Salix sp. or Pentaphylloides floribunda may also be found growing in this association. Low coverage of a variety of forbs may be found in this community; these forbs may include Mentha arvensis, Galium triflorum, Aster occidentalis, and Epilobium spp (Hansen et al. 1995, Hansen et al. 1988).

Similar Communities- Similar plant communities have been documented by other studies in eastern Oregon (Kovalchik 1987), Idaho (Hall and Hansen 1997), Utah (Padgett et al. 1989), Nevada (Manning and Padgett 1995), Wyoming (Youngblood et al. 1985), and Colorado (Kittel et al. 1998). Two phases, the Carex aquatilis and Deschampsia cespitosa phase, have been described for Montana (Hansen et al. 1995). Carex aquatilis has also been described growing as the dominant understory vegetation with Salix planifolia and Salix wolfii (Hansen et al. 1995).

Succession- Carex aquatilis communities probably represent a fairly stable plant association, although the successional pathways for this community are poorly understood. It can colonize expanses of mineral soil, such as dried out beaver ponds (Hansen et al. 1995), but it can also occupy sites on organic soils, which typically experience more anaerobic conditions.

Management- Carex aquatilis is considered moderately palatable to livestock, and poor grazing management practices can impact this plant community by causing decreases in Deschampsia cespitosa and increases in Juncus balticus and through trampling damage to organic soils. However, due to the rhizomatous habit of this sedge, disturbed sites do stand a chance of improving rapidly once the disturbance level is reduced (Kovalchik 1987). This species' rhizomes can also strongly anchor and stabilize streambanks.

Element Code- CEGL001802

Edition / Author- 99-10-05 / J. Greenlee, MTNHP

Carex buxbaumii Herbaceous Vegetation (CARBUX) buxbaum's sedge

\section{Natural Heritage Conservation Rank-} G3 / S3

Environment- This community type occurs in moderately broad valley bottoms, in depressional wetlands like glacial potholes, in peatlands, and on lake plains. Saturated soil conditions persist in the surface peat from mid spring to mid summer. Water levels may then drop to the soil surface or, on drier sites, to several decimeters below the surface.

Range- Carex buxbaumii is a minor community type in the Uinta Mountains of 
Utah, western and south-central Montana, Yellowstone National Park, and 4 disjunct areas of Idaho.

Adjacent Communities- In Montana, adjacent wetter communities include Scirpus acutus, Carex lasiocarpa, and Carex utriculata, and adjacent drier sites include Deschampsia cespitosa and Juncus balticus communities (Pierce 1986).

Vegetation- Carex buxbaumii is always dominant in this community, with $25 \%$ or greater cover. Carex aquatilis and/or Carex saxatilis are sometimes present and occasionally are co-dominants. Other associates include Deschampsia cespitosa, Caltha leptosepala, Eleocharis pauciflora (syn. E. quinqueflora), Senecio cymbalarioides, Pedicularis groenlandica, Ligusticum tenuifolium, Carex lanuginosa, Carex utriculata, Carex lasiocarpa, Carex muricata, Carex livida, Carex nebrascensis, Carex praegracilis, and Carex simulata (Padgett et al 1989).

Similar Communities- Includes the Carex buxbaumii - Carex saxatilis (Tuhy 1981) c.t. and Carex buxbaumii - Carex aquatilis (Mattson 1984) h.t. and phases. Hansen et al. (1995) lump this community with Carex lasiocarpa and Carex lanuginosa for management purposes. Pierce (1986) and Padgett et al. (1989) also describe this community type.

Management- Herbage production varies from low to moderate. Saturated soils are a natural deterrent to livestock grazing. Alteration of hydrology and subsequent dewatering may result in communities dominated by Carex buxbaumii being accessible to cattle. Fencing of these relatively small communities is a practical management method for restoration when the hydrologic regime is intact

\section{Element Code- CEGL001806}

Edition / Author - 95-06-09/ L. Williams
Carex elynoides Herbaceous Vegetation (CARELY) blackroot sedge

Natural Heritage Conservation RankG4 / S4

Environment- Carex elynoides was the most frequently sampled alpine c.t. in the BVHDMS. It was found in all eight mountain ranges and is undoubtedly, one of, if not the most extensive alpine vegetation type in our study area. It was most extensive in the drier Tendoy, Beaverhead, and Snowcrest ranges. Carex elynoides spanned a considerable range in elevation, 9,360 to 10,360 ft. All sites, because of topographic position and orientation, were inferred to be highly windimpacted and blown free of winter snow. More than half the stands occurred on ridge crests or shoulders with less than 20\% slope. Most of the remainder was on moderate to steep southwest- to west-facing slopes. This type often graded to grassland c.t's. or Carex scirpoidea / Potentilla diversifolia c.t. on more protected moist sites and cushion plantdominated sites with yet greater wind impact. Mean exposed bare soil, gravel and rock (23\%) was slightly greater than for grassland types; however, sites grading to cushion plant communities had as much as $70 \%$ substrate exposure.

Vegetation- The Carex elynoides type is characterized by a short ( $<4$ in), usually dense ground cover of fibrous-rooted graminoids (ave. c.c. $46 \%$ ) and forbs. Carex elynoides was strongly dominant ( $100 \%$ constancy, $27 \%$ c.c.) followed in decreasing order by the turfformers Carex rupestris and Festuca ovina. Other common graminoids were Calamagrostis purpurascens, Poa glauca, and Festuca (syn. Hesperochloa) kingii. Average forb cover was $31 \%$, only slightly less than that of grassland types. The dominance of Phlox pulvinata and Selaginella densa and the presence and occasional dominance of cushion plants set this type apart from grasslands. Forbs with high (>50\%) constancy included Cymopterus 
bipinnatus, Besseya wyomingensis, Hymenoxys

grandiflora, Oxytropis campestris, Potentilla diversifolia, and $P$. ovina.

Soils- Parent materials were predominantly limestone and calcareous sandstone, but quartzite and gneiss were also represented. Mean litter and duff depths were, respectively, 0.4 in and 0.3 in. Percent coarse fragments ranged from 8 to $75 \%$ with a mean of $33 \%$, a figure intermediate between the grassland and cushion plant c.t's. Texture of the fine fraction ranged from fine clay to sandy clay-loam, and the mean textural class was sandy clay. Soil reactions were slightly more basic than those of grasslands with an average $\mathrm{pH}$ of 7.5 for calcareous materials and 6.4 for non-calcareous substrates. Mean organic matter content was $16 \%$, mean total nitrogen was $0.57 \%$, and C:N ratio was 15:1.

Productivity- A nearly ten-fold range in productivity was recorded for both graminoids (80-682 lbs/acre) and forbs (115-977 lbs/acre). Average productivity's for graminoids, forbs and community total were respectively, 398, 398, and 796 lbs/acre. A cline of decreasing productivity occurred from solid turf conditions to near cushion plant conditions.

Other Studies- Carex elynoides turf communities are reported from similar environments on a variety of parent materials in neighboring ranges in Idaho (Moseley 1985, Caicco 1983, Urbanczyk and Henderson 1994, Richardson and Henderson 1999), calcareous sites in Montana (Bamberg and Major 1968), the Beartooth Plateau of Wyoming/Montana (Johnson and Billings 1962), throughout Colorado (Eddleman and Ward 1984, Willard 1979, Komarkova and Webber 1978), the Uinta Range of Utah (Lewis 1970), the Great Basin of Nevada (Loope 1969) and as far south as New Mexico (Baker 1983) and. Johnson and Billings (1962) consider C. elynoides and $G$. rossii dominated turf to be the climax vegetation type of their study area. Similar vegetation is not reported for the cooler and moister ranges to the north and west of our study area.

\section{Element Code- CEGL001852}

Edition / Author- 97-10 / S.V. Cooper et al.

Carex lanuginosa Herbaceous Vegetation (CARLAN) woolly sedge

\section{Natural Heritage Conservation Rank-} G3? / SP

Range- Carex lanuginosa dominated communities have also been documented for Idaho (Hall and Hansen 1997, Jankovsky-Jones 1997) and eastern Oregon (Kovalchik 1987).

Environment- Carex lanuginosa communities can be found at low to mid elevations in western and central Montana. These marsh communities are usually found in depressions, older riverine sloughs, wet meadow areas along creeks, and in wetlands formed by springs and seeps. Stands primarily occur on mildly brackish mineral soils that are seasonally flooded but which usually dry down by late summer. Adjacent wetter communities include shallow marsh communities dominated by Carex utriculata or Scirpus maritimus, while adjacent drier, less frequently flooded communities include Juncus balticus, Distichlis stricta, or stands of exotic pasture grasses like Phleum pratense. Upland communities are often dominated by Artemisia cana or Artemisia tridentata.

Vegetation- Carex lanuginosa typically dominates these communities, and due to its rhizomatous habit often forms dense stands with heavy cover. Common associated species include Mentha arvensis, Hordeum jubatum, and Juncus balticus.

Similar Communities- Hansen et al. (1995) included the Carex lanuginosa plant association in the Carex lasiocarpa habitat type for management considerations; however, the two communities differ in that Carex lasiocarpa tends to occur on slightly acidic, organic soils that are permanently wet, while Carex lanuginosa communities are often found in mildly brackish marshes that dry down seasonally (Lesica 1994). Carex lanuginosa dominated communities have also been 
documented for Idaho (Hall and Hansen 1997, Jankovsky-Jones 1997) and eastern Oregon (Kovalchik 1987).

Succession- Carex lanuginosa communities probably represent a fairly stable plant association, given a relatively stable hydrologic regime. Moderate disturbance could cause increases in Juncus balticus or any pasture grasses present, like Poa pratensis or Phleum pratense (Hansen et al. 1995).

Management- Carex lanuginosa is highly palatable, and communities can be impacted by season-long grazing, particularly when grazing management practices cause increased downcutting of stream channels, which could alter the hydrology of Carex lanuginosa communities located in floodplain settings. However, due to the rhizomatous habit of this sedge, disturbed sites do stand a chance of improving rapidly once the disturbance is removed and if the disturbance level isn't too high (Kovalchik 1987).

\section{Element Code- CEGL001809}

Edition / Author- 99-10-05 / J. Greenlee, MTNHP

Carex lasiocarpa Herbaceous Vegetation (CARLAS) Slender Sedge

\section{Natural Heritage Conservation Rank- G5 / S5}

Environment- The Carex lasiocarpa plant association usually occupies former lake basins, long-abandoned beaver ponds, potholes, and lake and stream margins that favor the accumulation of peat. Occasionally this community occurs as floating or quaking mats on fluid peat subsoil. This association can often be found in intermediate to rich fens. The soils are usually organic, with accumulations of sedge peat. This type is typically an indicator of a stable hydrologic regime with yearlong saturated soil conditions in the root zone at minimum. This community can tolerate yearlong flooded conditions.

Range- The Carex lasiocarpa community type is distributed globally throughout the northern hemisphere; in the western United States it is a minor type in eastern Washington, the Uinta Mountains of Utah, southeastern Idaho, throughout much of Montana, and in central Yellowstone National Park.

Adjacent Communities- Adjacent wetter sites may be dominated by either Carex utriculata, Carex aquatilis, or Carex nebrascensis communities. Drier sites may be dominated by Deschampsia cespitosa, Artemisia cana / Festuca idahoensis, or Juncus balticus communities. Adjacent uplands can be dominated by Artemisia tridentata, or a variety of conifer communities (Hansen et al. 1995).

Vegetation- Carex lasiocarpa dominates the community with $30-80 \%$ cover. It often forms monocultures in sedge meadows in Montana. Carex utriculata and Carex lanuginosa are often the only other species with high constancy.

Similar Communities- Some classifications include stands dominated by Carex lanuginosa in the Carex lasiocarpa plant association (Pierce 1986, Hansen et al. 1995), due to similarities in structure and management concerns. Carex lanuginosa tends to occur on mineral soils, while Carex lasiocarpa is most often found on organic soils (Hansen et al. 1988, Lesica 1994). Carex buxbaumii stands are also included in the Carex lasiocarpa habitat type by some classifications due to similarities in management concerns (Kovalchik 1987, Hansen et al. 1995).

Succession- Moderate disturbance will increase Carex aquatilis, Juncus balticus and associated forbs. Severe disturbance (resulting in dewatering) may lower the water table and cause the site to be dominated by Poa pratensis, Poa palustris, Potentilla anserina, or Agrostis stolonifera.

Management- Drought years may make occurrences of this community accessible to both domestic and wild grazing animals which could cause rutted and hummocky soils on margins. These sites are generally so wet as to 
preclude most types of recreational uses except fishing. Heavy disturbance such as from ORV use should be avoided because the organic soils are slow to recover from mechanical damage. High water tables make burning difficult, but fire can be used on sites adjacent to floodplains. Dominant sedges of this h.t. are resistant to damage by fire except where hot fires penetrate the peat soil. It has often been the policy of land managers to trap and kill beaver because they can be a nuisance. However, because beavers produce such desirable habitat and provide many beneficial stream functions, their removal from a riparian system needs to be closely evaluated (Hansen et al. 1995).

\section{Element Code- CEGL001810}

Edition / Author- 95-07-11/ L. Williams

Carex nebrascensis Herbaceous Vegetation (CARNEB) Nebraska sedge

\section{Natural Heritage Conservation Rank- G4 / S4}

Environment- Hansen et al. (1995) identify Carex nebrascensis as a minor type at low to mid elevations throughout Montana; within southwest Montana and particularly on the Red Rock Lakes National Wildlife Refuge it is also a minor type occurring in small to large patch's. Carex nebrascensis in general is found on subirrigated, gently inclined lower slopes, basins and swales and spans a broad range in hydrologic regimes from semipermanently flooded to saturated (soils drying to over a meter in depth). On the Refuge it is found most frequently as small patches (not individually mapped) in the wetland complexes surrounding Upper and Lower Red Rock Lakes as well as a riparian stinger and around scattered swales that might be vernally flooded. Carex nebrascensis generally occupies habitats intermediate (in hydrologic regime) between the wetter associations typically dominated by Carex utriculata or Carex aquatilis and the marginally drier associations Deschampsia cespitosa, Calamagrostis canadensis or
Pentaphylloides floribunda / Deschampsia. cespitosa.

Vegetation- Because Hansen et al. (1995) consider Carex nebrascensis to be purely a species responding to grazing disturbance they recognize a Carex nebrascensis community type only after all other possible diagnostic Carex spp. have been discounted. Carex nebrascensis should also have greater cover than any other Carex spp. We do not necessarily subscribe to the grazing disclimax hypothesis of Hansen et al. (1995) regarding this type. It seems to occupy a somewhat unique niche and perhaps is associated with alkaline substrates; Hansen et al. (1995) do not indicate what associations are grazing impacted so significantly as to drive them to Carex nebrascensis dominance (and thus the Carex nebrascensis c.t.).

The only shrubs noted in this type are errant specimens of Pentaphylloides floribunda or Lonicera involucrata. In most southwestern Montana examples of this type the cover of Carex nebrascensis exceeds 60-70\%; other graminoids of moderate to high constancy include Deschampsia cespitosa, Juncus balticus, Calamagrostis stricta (or Carex canadensis), Carex praegracilis, Poa palustris and Eleocharis palustris; the first three named spp. can have significant cover $(>5 \%)$. The forb component of these sites is depauperate; those most consistently present and with the greatest cover (seldom in excess of 5\%) include Geum macrophyllum, Epilobium ciliatum, Aster occidentalis, Mentha arvensis and Triglochin maritimum. Grazing impacted sites tend to have higher cover for $M$. arvensis and Taraxacum officinale.

Element Code- CEGL001813

Edition / Author- 99-11-16 / S.V. Cooper, MTNHP

Carex nigricans Herbaceous Vegetation (CARNIG) Black alpine sedge

Natural Heritage Conservation RankG4 / S3 
Environment- The Carex nigricans

community type was found between 9,500 and $10,000 \mathrm{ft}$ in the Anaconda, Madison, East Pioneer and Tobacco Root ranges, the wettest ranges within the BVHDMS. This distinctive community occurs on nearly level sites at the base of slopes and in valley and swale bottoms where blowing snow is deposited and melt off does not occur until well into the growing season. Carex nigricans occupied sites with perennially moist or saturated soil and with the shortest snow-free season of any snowbed c.t. In 1991, many of our stands had just begun to green-up in late July. Carex nigricans usually occurs in a matrix of small patches of wetland or other snowbed communities. Deschampsia cespitosa / Caltha leptosepala, Carex scopulorum / Caltha leptosepala, Juncus drummondii /Antennaria lanata and Phyllodoce empetriformis / Antennaria lanata are the associations most frequently found as matrix components.

Vegetation- Mean graminoid cover was $83 \%$. Carex nigricans was the absolute dominant with mean cover of $76 \%$. Other frequently occurring, but less abundant graminoids were Juncus drummondii, Phleum alpinum and Carex paysonis. Forbs had a mean cover of $21 \%$. The most common herbaceous species were Caltha leptosepala, Antennaria lanata and Erigeron peregrinus. No forb species occurred in all four stands, and only one, $A$. lanata occurred in three out of four. The dwarf shrubs Phyllodoce empetriformis and $P$. glanduliflora were present in small amounts in two stands, and Salix arctica was common in one stand. Mean cover of mosses and lichens was less than $1 \%$.

Soils- Parent materials were limestone, granite and gneiss. Bare ground and gravel covered only $2 \%$ of the surface. Mean depths of litter and duff were 0.4 in and 0.2 in respectively. Percent coarse fragments were always less than $10 \%$ with a mean of $3 \%$. Mean texture of the fine fraction was sandy clay. $\mathrm{pH}$ was 6.5 at the limestone site and varied between 5.8 and 6.2 when parent materials were crystalline. Mean $\mathrm{pH}$ for the type was 6.2. Mean organic matter content was $15 \%$, mean total nitrogen was $0.36 \%$, and C:N ratio was 20:1. Soils in late July, 1992 were always wet and cold.

Productivity- Due to retarded phenology in 1991, we measured productivity in only two stands. Graminoid productivity had a mean of $375 \mathrm{lbs} / \mathrm{acre}$, and forb productivity had a mean of $275 \mathrm{lbs} /$ acre. Mean productivity of dwarf shrubs was $22 \mathrm{lbs} / \mathrm{acre}$. Mean total productivity was $650 \mathrm{lbs} / \mathrm{acre}$. Carex nigricans was not fully mature when we clipped plots in these stands; thus, our estimates of graminoid and total productivity are low.

Other Studies- Communities dominated by Carex nigricans have been described for Banff and Jasper national parks in Alberta, Canada (Achuff and Corns 1982, Hrapko and LaRoi 1978). Composition is similar to our Carex nigricans association, although Luzula wahlenbergii $(=$ L. piperi $)$ was a common component of the Canadian types (and not recorded in the BVHDMS). Carex nigricans communities are more common and widely distributed in the Canadian Rockies, often occurring on slopes as well as level areas. Communities similar to the Carex nigricans association described herein are also common in the North Cascades of Washington (Douglas 1972, Douglas and Bliss 1977). Luetkea pectinata and bryophytes were common components in the North Cascades type. Rottman and Hartman (1985) report an association dominated by $C$. nigricans occurring in the center of sorted stone polygons in the San Juan Mountains of Colorado; Sibbaldia procumbens, Artemisia scopulorum and Juncus drummondii were the commonly associated forbs. Carex nigricans snowbed communities appear to be most common to the north and west of our study area.

Element Code- CEGL001816

Edition / Author- 97 -10 / S.V. Cooper et al.

Carex rupestris / Potentilla ovina Herbaceous Vegetation

(CARRUP / POTOVI) curly sedge / sheep cinquefoil 


\section{Natural Heritage Conservation Rank-}

G3 / S3

Environment- Carex rupestris / Potentilla ovina is transitional between an alpine turf and a cushion plant type. It occurs on exposed, windswept upper slopes, saddles and ridgetops, apparently strongly associated with soils developed from calcareous parent materials found in the Beaverhead, Madison, Pioneer, Tendoy and Blacktail Ranges. Appropriate habitat occurs in other ranges of southwestern Montana, especially the Centennial Range. It was sampled at elevations ranging from 9,300 to $10,400 \mathrm{ft}$. In several of the ranges, Carex rupestris / Potentilla ovina generally graded into the Carex elynoides or Carex scirpoidea / Potentilla diversifolia turf communities of deeper soils on more protected slopes, whereas in the Blacktail and Tendoy Ranges it graded to Festuca idahoensis-dominated community types, particularly Festuca idahoensis - Elymus trachycaulus. It grades to fellfield or cushion plant communities that typify harsher, more windswept sites.

Vegetation- Mean graminoid cover was $11 \%$. Important graminoids were Carex rupestris, Calamagrostis purpurascens, and $P$ oa secunda, and at higher elevations Festuca ovina, and Festuca kingii. Carex elynoides was common in some stands. Mean forb cover was $29 \%$. Consistently present and often well represented forbs include Potentilla ovina, Minuartia obtusiloba, Oxytropis campestris and Phlox pulvinata. Occurring consistently but with low cover is Eritrichium nanum, Bupleurum americanum, Cymopterus bipinnatus, Erigeron compositus and Senecio canus. Trifolium haydenii, Selaginella densa and Silene acaulis were well represented in some stands. The shrub, Pentaphylloides floribunda, was present in one stand. Lichen and moss canopy cover averaged less than $2 \%$.

Soils- Parent material was quartzite in one stand and limestone in the remaining seven stands. Bare ground and gravel covered $67 \%$ of the surface. Mean depths of litter and duff were both 0.1 in. Percent coarse fragments ranged from $40 \%$ to $66 \%$ with a mean of $57 \%$.
Texture of the fine fraction varied from sandy clay to sandy clay-loam with a mean class of sandy clay-loam. Soil $\mathrm{pH}$ varied from 6.9 to 8.2 with a mean of $7.8 ; \mathrm{pH}$ from the seven plots on limestone varied from 7.5 to 8.2 with a mean of 7.9. $\mathrm{pH}$ of the single plot on quartzite was 6.9. Mean organic matter content was $12 \%$, mean total nitrogen was $0.34 \%$, and C:N ratio was 32:1.

Productivity- Graminoid productivity varied from 35 to $253 \mathrm{lbs} /$ acre with a mean of $112 \mathrm{lbs} /$ acre. Forb productivity ranged from 89 to 759 lbs/acre with a mean of $277 \mathrm{lbs} / \mathrm{acre}$. Mean total productivity was $389 \mathrm{lbs} / \mathrm{acre}$. Cushion plant productivity is difficult to measure; thus, the forb estimates are only rough approximations. However, this community type was among the least productive in our study area.

Comments- Some stands at lower elevations are dominated by Calamagrostis purpurascens which caused us to recognize it as an alternative indicator with $C$. rupestris. Pseudoroegneria spicata is also present in the warmest and most exposed of these stands that may represent transitions to the Pseudoroegneria spicata / Cushion Plant association. Some stands may be impacted by sheep grazing or pocket gophers. Carex rupestris / Potentilla ovina sites may provide important winter range for bighorn sheep. Vegetation of these dry, exposed sites is easily damaged by motorized vehicles and recovers slowly.

Other Studies- Carex rupestris commonly dominates windswept fellfields in the Rocky Mountains. Lewis (1970) describes cushion plant communities in the Uinta Mountains of Utah dominated by $C$. rupestris, Festuca ovina and cushion plants such as Silene acaulis and Trifolium nanum. Willard (1979) describes a dry turf association dominated by $C$. rupestris, Potentilla nivalis and Silene acaulis for Rocky Mountain National Park in Colorado, and Komarkova and Webber (1978) report a fellfield community dominated by $C$. rupestris and Kobresia myosuroides from Niwot Ridge. Baker (1983) describes a C. rupestris-cushion 
community for the Sangre de Cristo Range of New Mexico. Moseley (1985) describes limestone fellfields dominated by $C$. rupestris and Potentilla ovina from east-central Idaho, while Urbancyzk and Henderson (1994) report cushion plant communities dominated by $C$. rupestris in the Lemhi Range of Idaho, but $P$. ovina was uncommon (D. Henderson, pers. comm.).

In our study area Carex rupestris / Potentilla ovina is ostensibly confined to calcareous parent materials, and Potentilla ovina, one of the dominant forbs, is a calciphile on these high elevation sites. In the limestone mountains to the north, most $C$. rupestris associations support Dryas spp. as an important component (Bamberg and Major 1968, Achuff and Corns 1982). To the east and south of our study area, $C$. rupestris fellfield communities on crystalline parent material are often codominated by Geum rossii or Dryas octopetala (Bliss 1956, Johnson and Billings 1962, Willard 1979) and are more similar to our Geum rossii / Minuartia obtusiloba c.t. . The Carex rupestris / Potentilla ovina c.t. may be endemic to calcareous ranges of southwest Montana and adjacent east-central Idaho.

\section{Element Code- CEGL001862}

Edition / Author- 97 -10 / S.V. Cooper et al.

Carex scirpoidea / Geum rossii Herbaceous Vegetation

(CARSCI / GEUROS) northern singlespike sedge / Ross' avens

\section{Natural Heritage Conservation Rank- G3 / S3}

Environment- We regard Carex scirpoidea / Geum rossii as a geographic/substrate variant of Carex scirpoidea / Potentilla diversifolia. It was a common plant association in the BVHDMS's relatively moist mountain ranges dominated by granitic or metamorphosed intrusive volcanics, the East Pioneer and Tobacco Root Ranges. It was also found in the Madison Range, exclusively on gneiss. It spanned nearly the full range of alpine elevations available in these ranges, from 9,300 to $10,320 \mathrm{ft}$. Sample sites were about evenly divided between low gradient slopes and steeper slopes. All aspects were represented. Most characteristic was some degree of enhanced effective moisture through increased snowpack or delayed snowmelt. Often Carex scirpoidea / Geum rossii turf occurred as patches scattered among boulders which act as snow fences creating eddy currents and increasing snowpack. Carex scirpoidea / Geum rossii graded to drier turf types, usually Carex elynoides, of more exposed positions and to DRY SLOPE or MOIST SLOPE c.t's. of steeper, unstable slopes.

Vegetation- Graminoid canopy cover averaged only $37 \%$, of which $24 \%$ was Carex

scirpoidea. Carex phaeocephala, C. atrata and C. albonigra were also dominant in at least one stand. Common turf graminoids $C$. rupestris, C. elynoides and Festuca ovina had moderate coverages or high constancy but are much less important than in the Carex scirpoidea / Potentilla diversifolia c.t. Other graminoids with high constancy were Luzula spicata, Poa alpina, P. secunda and Trisetum spicatum. The moister sites supported Deschampsia cespitosa, but its cover did not exceed 5\%. Carex scirpoidea / Geum rossii forb coverage averaged $51 \%$, very similar to that of Carex scirpoidea / Potentilla diversifolia. With the exception of Geum rossii, which was $100 \%$ constant and averaged $37 \%$ c.c. in this type, the two C. scirpoidea-dominated turf types had many forb species of high constancy or coverage in common e.g., Minuartia obtusiloba, Potentilla diversifolia, Phlox pulvinata, Polygonum bistortoides, Erigeron simplex, Lloydia serotina and Lupinus argenteus. Nonetheless, these two mesic turf types had fewer herbs in common (55) than were found in only one of the two types (63).

Soils- All soils were developed on intrusive igneous or metamorphosed substrates thereof. Carex scirpoidea / Geum rossii had roughly seven times more exposed soil, gravel and rock than did Carex scirpoidea / Potentilla diversifolia. Both litter and duff depths were shallow (0.2 in). Coarse fragment content 
ranged from 6 to $39 \%$ and averaged $19 \%$, twice that of the Carex scirpoidea / Potentilla diversifolia c.t. Texture of the fine fraction ranged from sandy clay to sandy loam, while the mean textural class was sandy clay-loam. $\mathrm{pH}$ values were low, averaging 5.9 and ranging from 5.5 to 6.3. Mean organic matter content was $14 \%$, mean total nitrogen was $0.45 \%$, and $\mathrm{C}: \mathrm{N}$ ratio was 18:1. Soils were more coarsetextured, and organic matter and nitrogen contents were lower than those of other turf communities.

Productivity- The high degree of variability in productivity appears to reflect the variability in exposed substrate. Total productivity ranged from 236 to $2669 \mathrm{lbs} /$ acre and averaged 964 lbs/acre, 272 and $692 \mathrm{lbs} /$ acre for graminoids and forbs, respectively.

Other Studies- The Carex scirpoidea var. scirpoidea c.t. described by Douglas and Bliss (1977) for the eastern North Cascades has strong floristic similarity with our Carex scirpoidea/Geum rossii p. a., except their type lacks Geum rossii. However, their Carex scirpoidea c.t. represents drier portions of moisture/snowmelt gradients from a much wetter climatic regime. Conversely, Thilenius and Smith (1985) describe as the moistest of their Absaroka Range alpine sites a Geum rossii-Trifolium parryi c.t. whose environmental parameters resemble those of Carex scirpoidea / Geum rossii but whose vegetation differs by having $C$. scirpoidea replaced by $C$. ebenea. In analogous fashion, the Sange de Cristo Range of New Mexico supports a Geum rossii meadow type in which C. heteroneura $(=C$. atrata $)$ and Deschampsia cespitosa are conspicuous components denoting the mesic nature and a similarity to our Carex scirpoidea / Geum rossii c.t. (Baker 1983). Lewis (1970) described vegetation dominated by Carex scirpoidea, Geum rossii and Deschampsia cespitosa from Utah's Uinta Mountains. Well-drained sites were dominated by $C$. scirpoidea, while D. cespitosa dominated areas of impeded drainage. One can only speculate on the ecological or phytogeographic significance of the fact that this association has not been reported immediately to the south in the high ranges of east-central Idaho.

Element Code- CEGL001866

Edition / Author- 97 -10 / S.V. Cooper et al.

Carex scirpoidea / Potentilla diversifolia Herbaceous Vegetation

(CARSCI / POTDIV) northern single-spike sedge / diverse-leaved cinquefoil

\section{Natural Heritage Conservation Rank-}

G3 / S2

Environment- Carex scirpoidea / Potentilla diversifolia is a moist turf type found in BVHDMS ranges with higher precipitation (Gravelly, Snowcrest, Anaconda-Pintlar and Madison). It occurred from 9,300 to $10,320 \mathrm{ft}$ associated with gentle, not nearly so windimpacted slopes as those of the Carex elynoides c.t did. Most of the sites had evidence of frost-sorting or solifluction lobes. We hypothesize these sites are turf because they occur in windswept positions (little winter snow accumulation), but they are also moist, being in runoff collecting positions or, if on slopes, then ones with low solar insolation (north-facing). Carex scirpoidea / Potentilla diversifolia grades to the Carex elynoides association on upper slopes and to wet meadows or snowbed communities of yet wetter sites. With the exception of wet meadow c.t's., Carex scirpoidea / Potentilla diversifolia had less exposed soil and rock (4\%) than any other alpine site, graminoiddominated plant association within the BVHDMS.

Vegetation- Dominance of Carex scirpoidea, C. atrata, C. phaeocephala, C. obtusata or a combination of these sedges is diagnostic for this c.t. Graminoid canopy cover averaged $66 \%$, of which $35 \%$ was $C$. scirpoidea. Carex elynoides, C. rupestris, Festuca ovina and Calamagrostis purpurascens were strongly represented. Elymus trachycaulus, Luzula spicata and Poa alpina had high constancy and low coverage. Average forb coverage (47\%) was high, reflecting the favorable moisture status of these sites. Forbs with high constancy (> 50\%) included those typical of moist sites 
such as Lloydia serotina, Erigeron simplex, Polygonum bistortoides, $P$. viviparum and Zigadenus elegans. Other high-constancy forbs included Cerastium arvense, Hymenoxys grandiflora, Lupinus argenteus, Pedicularis parryi, Solidago multiradiata, and most characteristically Potentilla diversifolia. Forbs consistently present, but more typical of dry turf or cushion plant communities, included Minuartia obtusiloba, Oxytropis campestris and Phlox pulvinata.

Soils- Samples were about evenly divided between calcareous (limestone and conglomerate) and noncalcareous (basalt, granite and quartzite) substrates. Average litter and duff depths were, respectively 0.6 in and 0.4 in. Coarse fragment content ranged from 0 to $31 \%$ and averaged $9 \%$. Texture of the fine fraction ranged from fine clay to sandy clay, and mean texture was sandy clay. Soil reaction was strongly conditioned by substrate type, averaging 7.2 for calcareous and 5.8 for noncalcareous types; both values were distinctly lower than for the drier turf types. Mean organic matter content was 20\%, mean total nitrogen was $0.73 \%$, and $\mathrm{C}: \mathrm{N}$ ratio was 13:1.

Productivity- The range in total productivity was relatively narrow, 1,127 to $1,426 \mathrm{lbs} / \mathrm{acre}$ (1283 lbs/acre average), with graminoids averaging 743 and forbs $540 \mathrm{lbs} / \mathrm{acre}$. These high values relative to, say the Carex elynoides p. a. (ave. $796 \mathrm{lbs} / \mathrm{acre}$ ), further substantiate the less stressful, higher moisture status of the Carex scirpoidea / Potentilla diversifolia c.t.

Other Studies- Douglas and Bliss (1977) describe a Carex scirpoidea var. scirpoidea community type from the eastern North Cascades of Washington that is vegetationally and physiognomically very similar to Carex scirpoidea / Potentilla diversifolia. However, in the moister Cascadian climate, their Carex scirpoidea community represents the dry, early snow-free end of an alpine continuum, occurring on well-drained slopes of all aspects. Stand tables from Bamberg and Major (1968) show plots for the Big Snowy Mountains of Montana that conform to our conception of this c.t.

Element Code- CEGL001867

Edition / Author- 97 -10 / S.V. Cooper et al.

Carex scopulorum / Caltha leptosepala Herbaceous Vegetation

(CARSCO / CALLEP) Holm's Rocky Mountain sedge / elkslip marsh marigold

\section{Natural Heritage Conservation Rank-}

G4 / S3

Environment- This association was extensive in the Gravelly and Madison Ranges, sampled in the Tobacco Roots, noted in the East Pioneer and to be expected in the other ranges based on the broad distribution of the dominant species. Saturated soil, often with standing water throughout the growing season, was the dominant feature. These sites span the range from wet meadow to fen and occur in shallow undrained depressions, low-gradient subirrigated positions and also adjacent to first order streams or rivulets. From the high values for basal area ( $8 \%$ average) and abundant moss (60\% average) and litter (30\% average), it follows that there was seldom more than a trace amount of exposed soil/gravel or rock.

Vegetation- Only trace amounts of Salix spp. were present. Without exception, the graminoid component, dominated by the diagnostic species Carex scopulorum or C. lenticularis, was extremely dense ( $88 \%$ mean canopy cover), though they did not exceed 8-12 inches. Other graminoids with high constancy or coverage were $C$. haydenii, Deschampsia cespitosa, Juncus drummondii, J. mertensiana and Poa alpina. The forb component, notably lacking in diversity, was dominated by several wet-site species, most commonly Caltha leptosepala (35\% mean c.c.), Pedicularis groenlandica, Polygonum bistortoides, Senecio cymbalarioides, Trollius laxus and Veronica wormskjoldii.

Soils- All parent materials were characterized as alluvium, mostly volcanic-derived. Four of the five sites had fibrous peat at least 6 in deep. 
Litter depths averaged 0.7 in; we did not discriminate duff from peat. No coarse fragments were found in any of the profiles. Soil texture ranged from clay to sandy clayloam with a modal condition being clay loam. Soil reaction for the one calcareous site $(\mathrm{pH}=$ 5.9) was the lowest of any calcareous site sampled; however, the soils derived from volcanic alluvium showed no trend of lower $\mathrm{pH}$ values (6.0 average) than other wet/moist sites. The only slightly acid values indicate minerotrophic sites having more in common with fens than bogs (as this c.t. has been termed in the literature). Mean organic matter content was $25 \%$, mean total nitrogen content was $0.70 \%$, and $\mathrm{C}: \mathrm{N}$ ratio was $17: 1$. Organic matter and nitrogen content were higher than in other wetland types and were equaled only by the Dryas octopetala / Polygonum viviparum turf.

Productivity- Average productivity for the c.t., 2,277 lbs/acre, was higher, particularly in the graminoid component, 1,720 lbs/acre, than that of any other study area c.t. However, the range, 1,426 to 4,123 lbs/acre, overlaps with a number of moist/wet site types. We speculate that these values are underestimates, as sampling invariably occurred prior to phenological optima. A protected site at 10,230 $\mathrm{ft}$ in the Madison range had total productivity of 4,123 lbs/acre, much higher than for comparable c.t.'s in the Central Rockies (May and Webber 1982, Scott and Billings 1964, Briggs and MacMahon 1983).

Other Studies- Virtually identical alpine marsh communities and environmental parameters are described for the Beartooth Plateau of Montana/Wyoming (Johnson and Billings 1962), Medicine Bow (Scott and Billings 1964) and Teton Ranges (Spence and Shaw 1981) of Wyoming and Colorado's Front Range (Willard 1979 and May and Webber 1982). Caltha leptosepala is the dominant forb in many alpine marshes of Utah's Uinta Mountains, but the dominant graminoids are Carex aquatilis or $C$. saxatilis, rather than $C$. scopulorum (Briggs and MacMahon 1983, Lewis 1970). Hansen et al. (1995) describe a similar type from subalpine and alpine areas of
Montana, but subalpine stands have a different forb composition. Similar communities have not been described north and west of our study area.

\section{Element Code- CEGL001823}

Edition / Author- 97 -10 / S.V. Cooper et al.

Carex simulata Herbaceous Vegetation (CARSIM) short-beaked sedge

\section{Natural Heritage Conservation Rank-} G4 / S3

Environment- This minor, but distinctive, fen community type was found confined to saturated organic soils developed in association with springs/subirrigation on stream terraces at 6,600-7,000 ft. in the vicinity of the Tendoy and Centennial Ranges; the actual elevation range is probably considerably greater. This vegetation type occurs on some of the wettest sites sampled in southwestern Montana. Plants generally occur on hummocks or mats between areas of standing water. Slightly drier adjacent communities are dominated by Pentaphylloides floribunda / Juncus balticus or Poa juncifolia, Puccinellia distans, and Carex praegracilis, or Salix spp. and Carex utriculata. Upland vegetation is dominated by various combinations of Artemisia tridentata (ssp. vaseyana or tridentata), A. cana or A. longiloba usually with Festuca idahoensis and/or Leymus cinereus.

Vegetation- Stands generally are somewhat depauperate, having fewer than ten species. Carex simulata is dominant and abundant. Carex nebrascensis, Eleocharis pauciflora (syn. E. quinqueflora), and Deschampsia cespitosa may be well represented in some stands. Forbs are generally not abundant. Triglochin palustre and Epilobium palustre, and Ranunculus cymbalaria are the most common forb species. Mosses are common, usually with $20-30 \%$ cover, though they can form a nearly continuous sward, occurring even where standing water existed at the time of sampling. 
Soils- Soils are Histosols. Reducing conditions, as evidenced by gleying, are $1 \mathrm{~cm}$ to greater than $20 \mathrm{~cm}$ below the surface. Water covers $30-80 \%$ of the surface.

Other Studies- Hansen et al. (1995) describe a Carex simulata habitat type from western Montana. Lesica (1990) described similar communities from higher elevations in southwest Montana, but significant coverages of Carex aquatilis and Salix brachycarpa were present in some stands. More species rich vegetation dominated by Betula glandulosa, Pentaphylloides floribunda and Carex simulata was described for north-central Montana (Lesica 1986). Fen vegetation dominated by $C$. simulata has also been described for Idaho, Wyoming and Utah (Padgett et al. 1989, Youngblood et al. 1985).

Comments- Light to moderate livestock grazing does occur in the Carex simulata c. t. Although the effects of the associated herbivory are probably minimal, trampling may alter the habitat by creating hummocks that permit the invasion of shrubs and alien species.

\section{Element Code- CEGL001825}

Edition / Author- 99-11-16 / S.V. Cooper, MTNHP

Carex utriculata Herbaceous Vegetation (CARUTR) beaked sedge

\section{Natural Heritage Conservation Rank-} G5 / S5

Environment- This is perhaps the most common of mid to high elevation wetland types in Montana; within the Centennial Valley it is rather uncommon with the strong exception of the Red Rock Lakes National Wildlife Refuge where perhaps the state's largest expanse occurs (it also occupies more area than any other single vegetation type on the Refuge). It is recognized by any of three sedges, Carex utriculata, Carex vesicaria, or Carex atherodes having at least $25 \%$ canopy cover. On the Refuge and throughout southwestern Montana C. utriculata is by far the dominant and diagnostic sedge and C. vesicaria is sporadically present with low cover values. Carex utriculata is found as a narrow, linear feature, bordering steams or pond margins with continuously saturated soils but its most extensive development (at least in the BVHDMS) is in the wetlands surrounding Upper and Lower Red Rocks Lakes. In places it forms a broad band, scores of meters wide in places, around the majority of these lake's perimeters. Even in late summer soils of these sites are saturated to the surface and often are even inundated by standing water. Substrates have a high organic content, though not all of the Refuge sites investigated had histic epipedons (probably mostly Inceptisols and Entisols prevail though Aquic Mollisols and Histosols also occur). Soils are formed from alluvium of various sources and are generally moderately fine-textured, though lenses of silty-clays were also encountered. On the Refuge this type most often borders directly on open water but in places there are distinct patches dominated by Typha latifolia, Scirpus acutus and Hippuris vulgaris intercalated between Carex utriculata and open water. Toward shallower/drier habitats Carex utriculata grades to Juncus balticusdomination, mostly as Juncus balticus plant association (near monoculture of J. balticus) or the Juncus balticus - Carex praegracilis plant association. This type forms floating mats as well, when under the influence of wave action, pieces of the anchored mat are dislodged.

Vegetation- Carex utriculata verges on being a monospecific dominant in the portions of this type occupying sites that are nearly continuously inundated; Carex aquatilis shares dominance on a very minor portion of the type. Total graminoid cover often approaches $100 \%$. The fact these are aggressively rhizomatous graminoids may explain site preemption (local dominance of one of the two species) and the low species diversity exhibited by this type, though the environment itself is considerably restrictive. Other graminoids consistently present, often well represented include Calamagrostis stricta and Juncus balticus. Shrubs, Salix spp., Lonicera involucrata and Pentaphylloides floribunda, and forbs, 
principally Epilobium ciliatum, Ranunculus spp. and Potentilla palustris are present in trace amounts.

Comments- This is an extremely common and broadly distributed wetland type, ranging as far south as New Mexico and Arizona (Bourgeron and Engelking 1994). In Montana probably more sampling has been done to document the regional permutations of this type than has been conducted for any other wetland type. Thus three phases are recognized of which Carex aquatilis is the one sampled and commonly represented in the BVHDMS; the Deschampsia cespitosa phase (representative of less inundated and more livestock-accessible environments) may have been enormously reduced to extirpated by heavy grazing of these riparian/wetland habitats, most especially those of the Centennial Valley and Refuge lands.

\section{Element Code- CEGL001562}

Edition / Author- 99-11-16 / S.V. Cooper, MTNHP

\section{Deschampsia cespitosa Herbaceous Vegetation}

(DESCES) Tufted hairgrass

\section{Natural Heritage Conservation Rank- G4 / S4}

Environment- This common plant association occurs on moist stream terraces, extensive subirrigated benchlands, small valley seeps and just above the drawdown zone of lakes and ponds. Within the BVHDMS some of the best quality stands, albeit of limited extent, occur throughout the lowlands of the Red Rock Lakes National Wildlife Refuge. Soils are generally silty to silty clays in texture. Sites with the BVHDMS exhibited little bare ground and no surface gravel. Adjacent sites are most frequently dominated by Juncus balticus, Pentaphylloides floribunda, Carex utriculata, C. simulata or Salix spp. Artemisia tridentata, A. tripartita, and/or Festuca idahoensis dominate adjacent upland vegetation.

Vegetation- The dominant aspect of these sites is a continuous dense canopy of graminoids, the dominants of which, Deschampsia cespitosa and Juncus balticus, are usually abundantly represented; however the mere presence of $D$. cespitos $a$ under intense grazing is enough to indicate these moist to wet sites are potentially dominated by this species. Muhlenbergia richardsonis and Carex praegracilis are other common graminoids. The cover and diversity of forbs is low to moderate. Aster occidentalis, Potentilla anserina, $P$. gracilis and Crepis runcinata are common species. Mosses are common; occasionally they will form a nearly continuous layer.

Comments- (see comments under Juncus balticus - Carex praegracilis). This vegetation is productive and often remains green throughout the growing season, making it attractive to livestock, especially late in the year. The moist to wet soils and generally small size of stands make this type very prone to degradation. Hansen et al. (1995) describe the Deschampsia cespitosa habitat type as a major landscape component for Montana. Similar types have been described for the Blackfoot River Valley (Lesica 1994) and the Blackfeet Indian Reservation (Lesica 1989). Wet meadows dominated by D. cespitosa have also been described for the alpine zone of southwestern Montana mountains (Cooper and Lesica 1992). Youngblood et al. (1985) described this vegetation type for Wyoming and Idaho; in fact this is one on the most widespread wetland types of the Intermountain West (Padgett et al. (1989) and also extends to near-coastal environments (Bourgeron and Engelking 1994).

\section{Element Code- CEGL001599}

Edition / Author- 99-11-16 / S.V. Cooper, MTNHP

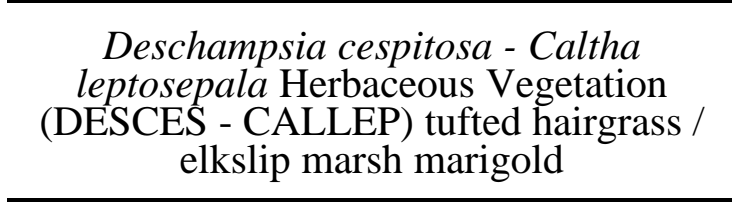

Natural Heritage Conservation Rank- 
G4 / S3

Environment- This c.t. was well represented in the Gravelly Range and also sampled in the Beaverhead and Madison Ranges; it was noted, but not sampled, in four of the other five major mountain ranges of the BVHDMS. It occurred at elevations as high as 10,100 feet, but was much more common in lower elevation collecting positions (either snow or percolating water) at the alpine/subalpine ecotone (approx. 9,200 to 9,500 feet). Sampled sites occupied flat to concave benches and slopes that did not exceed $15 \%$ slope and were north- through northeast-facing (and probably subirrigated). Small patches of this c.t. were noted on steeper slopes below persistent snowbanks and in swales. At time of sampling all soil profiles were saturated to the surface. Solifluction lobes were prominent even on the gentlest slopes.

Vegetation- The high coverage of moss (72\% mean) contributed dramatically to the lush appearance of this c.t. Only trace amounts of Salix spp. were found. Graminoid cover varied considerably, averaging 38\%. Deschampsia cespitosa was clearly the dominant graminoid; other moist site graminoids occurring with at least 5\% coverage were Carex atrata, $C$. nigricans, C. haydenii, Juncus drummondii and $J$. balticus. Though $D$. cespitosa clumps provided a recognition factor for this association, forb cover (68\% average) far outstripped that of the graminoids. Caltha leptosepala dominated ( $48 \%$ c.c.) the forb layer. Other forbs with high coverage but not necessarily high constancy were Aster foliaceus, Claytonia lanceolata, Erigeron peregrinus, Pedicularis groenlandica, Polygonum bistortoides, $P$. viviparum, Senecio cymbalarioides and Veronica wormskjoldii.

Soils- Parent materials included alluvium, limestone, sandstone, basalt and gneiss. Litter and duff depth averaged 0.6 in and 0.4 in, respectively. Coarse fragment content was consistently low, averaging only $3 \%$, with traces of gravel and rock found on the surface. Bare soil exposure was as high as $20 \%$, especially where pocket gopher (Thomomys talpoides) workings were extensive. Soil texture varied from clay to sandy clay with a mean of sandy clay. Soil reaction for calcareous substrates averaged 7.5 while that for noncalcareous was only 5.8. Mean organic matter content was $20 \%$, mean total nitrogen was $0.57 \%$, and $\mathrm{C}: \mathrm{N}$ ratio was $15: 1$.

Productivity- Total productivity ranged widely, from 621 to $3,197 \mathrm{lbs} /$ acre with a mean of $1,820 \mathrm{lbs} / \mathrm{acre}$. Graminoid productivity accounted for only $13 \%$ to $42 \%$ of the total. These productivity figures are likely underestimates as at least two sites were sampled prior to culmination of growth.

Other Studies- Mueggler and Stewart (1980) describe a Deschampsia cespitosa / Carex spp. c.t. for subalpine meadows of western Montana. It has high productivity but little contribution by forbs. Our Deschampsia cespitosa / Caltha leptosepala c.t. can be interpreted as a alpine extension of the Deschampsia series. Johnson and Billings (1962) have described a more broadly defined Deschampsia cespitosa vegetation type (with appreciable Carex scopulorum) for the Beartooth Range of Montana-Wyoming. They observe an increasing coverage of Caltha leptosepala indicates a transition to $C$. scopulorum-dominated bog conditions. Deschampsia cespitosa-dominated meadows extend southward to Colorado (Willard 1979, Bonham and Ward 1970, May and Webber 1982 and Eddleman and Ward 1984), Utah (Lewis 1970), and northern New Mexico (Baker 1983) but apparently lack the mesic to hydric forbs that characterize the Deschampsia cespitosa / Caltha leptosepala c.t. Their D. cespitosa-dominated types are apparently intermediate moisture status between our Deschampsia cespitosa / Potentilla diversifolia and Deschampsia cespitosa / Caltha leptosepala c.t's.

\section{Element Code- CEGL001882}

Edition / Author- 99-11-16 / S.V. Cooper, MTNHP 
Deschampsia cespitosa - Potentilla diversifolia Herbaceous Vegetation (DESCES - POTDIV) tufted hairgrass / divers-leaved cinquefoil

\section{Natural Heritage Conservation Rank-} G5 / S2

Environment- This community type occurred from treeline to over 10,000 ft in the Gravelly, Madison and Snowcrest ranges, but small examples can probably be found in all of the BVHDMS ranges. It was confined to cool slopes, valley bottoms and depressions where soils are deep and remain moist until at least mid-summer. This community type occupies the most mesic situations in the lower alpine zone. Snow cover during winter protects the plants, and although snow release comes moderately early in the season, the sites are often fed by meltwater from upslope snowfields. Deschampsia cespitosa/ Potentilla diversifolia was abundant on the old erosion terraces of the Gravelly Range and was often associated with slopes showing evidence of solifluction. This community type generally occurred in a matrix of drier grassland and moist or dry turf vegetation. It also graded into wetland communities, especially Deschampsia cespitosa / Caltha leptosepala. In the Gravelly Range it sometimes occurred immediately above shrublands dominated by Artemisia tridentata ssp. vaseyana or subalpine forests dominated by Picea engelmannii.

Vegetation- Graminoid cover in Deschampsia cespitosa / Potentilla diversifolia is high, averaging $78 \%$ and exceeded only by that of the Carex scopulorum / Caltha leptosepala marsh community. Deschampsia cespitosa is the dominant graminoid, often forming large tussocks. Carex atrata and Phleum alpinum are also important. Festuca idahoensis was common in stands at lower elevations, and Carex phaeocephala and Juncus balticus were locally common. The latter may have increased under the influence of livestock grazing (Hansen et al. 1995). Mean forb cover was 37\%. Potentilla diversifolia, Polygonum bistortoides and Senecio crassulus were the most abundant forbs. Cerastium arvense, Ranunculus eschscholtzii and Saxifraga oregana were also common. Mertensia ciliata was abundant in one stand. Mean cover of lichens and mosses was $3 \%$.

Soils- Parent materials for these stands were sandstone, limestone, quartzite and gneiss. Mean depths of litter and duff was 0.4 in and 0.8 in respectively. Generally soils supporting this community type were deep with dark, mollic-appearing epipedons and high moisture content throughout much of the growing season. Bare ground and gravel covered $6 \%$ of the surface. This type had the lowest coarse fragment content of all non-wetland types, ranging from $0-19 \%$ with a mean of $8 \%$. Texture of the fine fraction ranged from fine clay to loamy sand with a mean textural class of clay loam. Soil reaction varied from a low of 6.0 on soils derived from gneiss to 7.0 on soils derived from limestone and calcareous sandstone. Mean $\mathrm{pH}$ for the type was 6.5. Mean organic matter content was $18 \%$, mean total nitrogen was $0.65 \%$, and C:N ratio was $14: 1$

Productivity- Graminoid productivity varied between 850 and 2,350 lbs/acre with a mean of $938 \mathrm{lbs} /$ acre. Forb productivity ranged from 180 to $875 \mathrm{lbs} /$ acre with a mean of $729 \mathrm{lbs} /$ acre. Mean total productivity was $1,667 \mathrm{lbs} /$ acre and was highest on warmer aspects.

Other Studies- Deschampsia cespitosa / Potentilla diversifolia at lower elevations is very similar in environment and composition to Mueggler and Stewart's (1980) Festuca idahoensis / Deschampsia cespitosa habitat type. Though impossible to evaluate with stand tables, Mueggler and Stewart's Festuca idahoensis / Deschampsia cespitosa association probably also encompasses the moist turf community type, Carex scirpoidea / Potentilla diversifolia. These authors state that productivity of their type probably ranges between 1200 and $1500 \mathrm{lbs} / \mathrm{acre}$, somewhat lower than we measured. Johnson and Billings (1962) described wet meadows dominated by D. cespitosa and Carex scopulorum in the 
Beartooth Mountains of south-central Montana, and Lesica (1991) reported that drier communities dominated by $D$. cespitosa and Geum rossii also occur in this range. In North America, similar associations are best developed in the Rocky Mountains from southern Montana south to New Mexico where Baker (1983) reported communities similar in dominant vegetation and landscape position. Lewis (1970) described alpine meadow communities from the Uinta Mountains of Utah dominated by $D$. cespitosa, Polygonum bistortoides, and Geum rossii. These types differ by having $G$. rossii dominant instead of $P$. diversifolia and by the greater prominence of Trifolium spp. Bonham and Ward (1970) and Komarkova and Webber (1978) described similar D. cespitosa-dominated communities in Colorado with $G$. rossii and Trifolium parryi as diagnostic species. This community type in Rocky Mountain National Park, Colorado did not have an abundance of $T$. parryi, and Willard (1979) believes that this species has increased under the influence of livestock grazing in unprotected areas outside the park. Meadows dominated by $D$. cespitosa are reported for the Cascade Range of Washington (Hamann 1972 as cited in Willard 1979).

\section{Element Code- CEGL001889}

Edition / Author- 97 -10 / S.V. Cooper et al.

Distichlis spicata Herbaceous Vegetation (DISSPI) Inland saltgrass

\section{Natural Heritage Conservation Rank-} G5 / S4

Environment- Hansen et al. (1995) observe that this is a minor type in central and eastern Montana and only as an incidental type in southwestern Montana valleys; it occurs on the Red Rock Lakes National Wildlife Refuge as small patch communities incapable of being mapped, except as inclusions. Relatively high quality examples occur on the Refuge on saline or alkali basins and swales, seep areas and the drawdown zone of pond or lake margins.

Vegetation- Relatively pure patches of
Distichlis spicata with herbs typical of wetter or less saline (alkaline) environments occurring in not more than trace amounts identify this type. Scattered patches of the tussock grass, Puccinellia nuttalliana, are quite typical, as are lone specimens of Leymus cinereus. Distichlis spicata usually occurs in a matrix with Leymus cinereus - Puccinellia nuttalliana and

Sarcobatus vermiculatus-dominated communities representing slightly drier sites and usually abruptly transitioning to Juncus balticus- or Scirpus spp.-dominated communities occurring on wetter and/or less saline sites. Shrubs and forbs are very scattered if they occur at all. S. vermiculatus and Chrysothamnus nauseosus are the only shrubs noted in the type and Triglochin maritimum and Grindelia squarrosa are the only forbs consistently present on Refuge examples. Hansen et al. remark that Hordeum jubatum can increase markedly with grazing but only scattered $H$. jubatum was noted.

\section{Element Code- CEGL001770}

Edition / Author- 99-11-16 / S.V. Cooper, MTNHP

\section{Eleocharis palustris Herbaceous Vegetation \\ (ELEPAL) Common Spikerush}

\section{Natural Heritage Conservation Rank-}

\section{G5 / S5}

Environment- The Eleocharis palustris community type is found at low to moderate elevations, generally in wide, low gradient valleys of all shapes. Sites are wet basins, floodplains, meadows, gravel bars, and lake and pond margins. It typically occupies sites that are prone to yearly flooding or persistent surface water. Where streams are present, they are Rosgen's $\mathrm{C}$ and $\mathrm{E}$ stream types. Elevations range from 2,200 to at least 8,700 feet, depending on latitude (Hansen et al. 1995; Manning and Padgett 1995; Crowe and Clausnitzer 1997; Hall and Hansen 1997). Soils of this community type are classified as Mollisols, Entisols, Histosols, and Inceptisols. Textures are variable, ranging from sites that 
are very coarse-fragment rich to others that are deep and fine-textured. The surface is usually rich in organic matter and the litter accumulation may blend into rich, black organic muck soils. The fine-textured upper horizons often arise from alluvial deposition. Sand, gravel, and cobbles usually constitute the main body of deeper subsurface materials (Manning and Padgett 1995; Crowe and Clausnitzer 1997; Hall and Hansen 1997).

Range- Eleocharis palustris is a common type in California, Colorado, Idaho, Montana, Nevada, Oregon, Utah, Washington, Wyoming, and Saskatchewan. Essentially it has been documented from every western state except Arizona and New Mexico (Bourgeron and Engelking 1994; Anderson et al. 1998).

Adjacent Communities- Due to the wide geographic distribution of this type, adjacent upland communities are varied, including shrub-steppe, woodland, and coniferous forest types. Adjacent riparian communities may be dominated by an equally varied assortment of types including deciduous forest, tall shrub, low shrub, and herbaceous communities

Vegetation- Eleocharis palustris is an aggressive, rhizomatous species that nearly excludes all other species from establishing any significant cover. Common associates in high quality sites include Alopecurus aequalis, Mentha arvensis, Rumex crispus, Eleocharis acicularis, Carex utriculata, Glyceria spp., and Phalaris arundinacea. On some sites aquatic species, such as Hippuris vulgaris, Utriculata vulgaris, and Potamogeton natans, have high cover.

Similar Communities- In some cases, the Eleocharis palustris may be confused with E. rostellata, especially if the stolons of $E$. rostellata are not present or not obvious. Be sure of the plant's true identity. A misidentification will result in the wrong community type being identified and ecological confusion will result because the sites on which they occur are very different ecologically. It should be noted that Hansen et al. (1995) consider Eleocharis acicularis (needle spike- rush) to be an ecological analogue of $E$. palustris and use it as a diagnostic species for the E. palustris association.

Succession- Padgett at al. (1989) suggest that Eleocharis palustris can represent an early seral species on ponds and streambanks where water is at or above the ground surface. As siltation occurs over time, other communities, such as Carex utriculata, may replace it. However, due to the continual saturation and dense growth of Eleocharis palustris, once formed, stands appear difficult to displace and may persist as climax vegetation. If water levels rise, Scirpus spp. and Typha latifolia may be able to supplant E. palustris. Hansen et al. (1995) have observed that disturbance can drastically shift the vegetative composition of this type toward increaser or invader species such as Hordeum jubatum.

Management- Seasonally wet conditions and low palatability of Eleocharis palustris limit the grazing value of this type for livestock, even during drought years when upland forage dries early and dies back (Kovalchik 1987). Sites occupied by this type are typically inundated or at least saturated for much of the year so as to preclude most development. Trampling damage and soil churning occurs readily with livestock use and may result in a shift toward more disturbance tolerant species such as Hordeum jubatum, Carex nebrascensis, and Juncus balticus (Hall and Hansen 1997).

Wildlife Values- Broad zones of this type along streams, rivers, lakes, and reservoirs provide valuable feeding and nesting areas for waterfowl. Eleocharis palustris and associated plants are a valuable source of food and cover for waterfowl. Wild ungulates seldom browse this habitat type due to its low palatability (Hall and Hansen 1997).

\section{Element Code- CEGL001833}

Edition / Author- 98-12-08/ B. Moseley

Elymus lanceolatus - Phacelia hastata Herbaceous Vegetation (ELYLSL / PHAHAS) thickspike 
wheatgrass / silverleaf phacelia

\section{Natural Heritage Conservation Rank- G2 / S2}

Environment- This association has been observed only in the Centennial Valley in a landscape referred to as the Centennial Sandhills. It occurs on sand dune blow outs at elevations around 6,700 feet elevation. These sites may range from foredune apons, to moderately inclined dune faces and lee slope deposition areas. Bare soil (fine-textured sand) cover ranges from 60 to $90 \%$ and coarse fragments are absent. The soil surface is usually unstable because of a lack of adequate vegetation cover. Parent materials are eolian sand deposits. Soils are very deep Ustic Torripsamments lacking coarse fragments and are not calcareous. Available water holding capacity is low due to the coarse texture of the substrate.

Vegetation- Elymus lanceolatus is well represented to abundant within this community type; it and Stipa comata constitute the primary colonizing graminoids on these sites with shifting substrates. Other species that are generally present with cover ranging from 1 to $20 \%$ include Chrysothamnus nauseosus, Tetradymia canescens, Eriogonum ovalifolium v. celsum, Linum perenne, Phacelia hastata, Psoralea tenuifolia, Oryzopsis hymenoides, and S. comata.

Adjacent Communities- The Artemisia tripartita / Festuca idahoensis, A. tridentata ssp. tridentata / Festuca idahoensis and A. tridentata ssp. tridentata / Stipa comata plant associations are usually found on adjacent more stable soils and sites.

Other Studies- There are no other studies that describe similar communities.

\section{Element Code- CEGL001745}

Edition / Author- 99-11-16 / S.V. Cooper

\section{Equisetum fluviatile Herbaceous Vegetation (EQUFLU) Water horsetail}

\section{Natural Heritage Conservation Rank- G4 / S4}

Environment- Th Equisetum fluviatile plant association can be found at low to mid elevations in the mountains of central and southwestern Montana, and in the mountains and valleys of western Montana. It occurs in glacial potholes and lakes, old oxbows, and backwaters of rivers and streams. Soils are variable and they frequently include Mollisols, Entisols, and Histosols. The mineral soils usually have some degree of organic matter buildup. This community is usually flooded year-round. Adjacent wetter site can be dominated by aquatic communities such as Nuphar sp., Typha latifolia, or open water. A variety of drier communities can occur adjacent to Equisetum fluviatile communities, and these can include stands of Carex sp., Salix sp., or Phalaris arundinacea (Hansen et al. 1988, Hansen et al. 1995).

Range- This community occurs in Montana, Idaho, and Alberta.

Vegetation- This community is usually dominated by a dense, monotypic stand of Equisetum fluviatile. Scattered forbs may occur in the community, and these include Polygonum amphibium, Potamogeton gramineus, and Comarum palustre. Carex lasiocarpa and Carex utriculata can also occur in these stands in low amounts (Hansen et al. 1988, Hansen et al. 1995).

Similar Communities- This community has been documented in northern Idaho (Jankovsky-Jones 1997) and Alberta (Dirschl et al. 1974).

Succession- The successional dynamics of this community are poorly understood. If the hydrologic regime remains unchanged, it is likely that this community will be fairly stable. 
Management- This community is generally so wet that it receives very little livestock use.

\section{Element Code- CEGL001960}

Edition / Author- 99-10-18 / J. Greenlee, MTNHP

Festuca idahoensis - Deschampsia cespitosa Herbaceous Vegetation

(FESIDA - DESCES) Idaho fescue tufted hairgrass

\section{Natural Heritage Conservation Rank- G3 / S3}

Environment- Festuca idahoensis Deschampsia cespitosa is a minor subalpine meadow type of southwestern Montana. On the Red Rock Lakes National Wildlife Refuge (RRLNWLR) it is associated with especially mesic, or subirrigated sites at lower elevations $(<7,000$ feet) than previously cited for this type (8,000 feet, Mueggler and Stewart 1980). It generally occurs as small inclusions within more extensive Festuca idahoensis-dominated types. The loamy soils appeared to have a loess component. Very little soil surface is exposed because of the high productivity and consequent abundant litter generation. This type grades abruptly to and is often encompassed within Festuca idahoensis Elymus trachycaulus or other yet drier $F$. idahoensis-dominated type (i.e. the Festuca idahoensis - Deschampsia cespitosa type as found on the RRLNWLR and greater southwestern Montana constitutes moist pockets with favorable soils).

Vegetation- This association is recognized by the dominance of Festuca idahoensis and the presence of Deschampsia cespitosa and the absence of Pseudoroegneria spicata (these sites are too moist for this bunchgrass of xeric environments); Mueggler and Stewart (1980) do not make it clear whether the presence of Phleum alpinum or Trisetum spp. are also diagnostic. Festuca idahoensis is generally the dominant graminoid with $D$. cespitosa and Elymus trachycaulus having appreciable, though much reduced cover relative to $F$. idahoensis; other graminoids include Phleum alpinum, Luzula spicata, Danthonia intermedia, Carex scirpoidea (or C. obtusata) and Trisetum spp. Canopy cover for forbs usually exceeds 40\%; Polygonum bistortoides, Potentilla gracilis, P. diversifolia, Erigeron speciosus and Lupinus argenteus contribute the greatest cover and are consistently present.

Similar Communities- Deschampsia cespitosa I Potentilla diversifolia is a subalpine/alpine grassland type the sampled stands of which have appreciable amounts of $F$. idahoensis and would key to the F. idahoensis - D. cespitosa type in Mueggler and Stewart (1980). The $D$. cespitosa / $P$. diversifolia type was distinguished by Cooper and Lesica (1997) based on their impression that it constituted a type of higher elevations, colder environments. Given D. cespitosa's restricted environmental amplitude and proven affinity of for moist to wet sites it might improve the classification to name this type $D$. cespitosa $-F$. idahoensis, making certain that future keys to vegetation give priority to $D$. cespitosa over $F$. idahoensis.

\section{Element Code- CEGL001900}

Edition / Author- 99-11-16 / S.V. Cooper, MTNHP
Festuca idahoensis - Elymus trachycaulus Herbaceous Vegetation (FESIDA - ELYTRA) Idaho fescue - bearded wheatgrass

\section{Natural Heritage Conservation Rank- G4 / S3S4}

Environment- This type is common on upper slopes, rolling uplands and gentle ridgetops at 7,400-9,200 ft in the Tendoy and Centennial Mountains. These mesic, productive grasslands often occur in snow catchment areas such as lee slopes just below ridge lines. Vegetation dominated by Artemisia tridentata ssp. vaseyana often occurs below in slightly drier sites. Steep slopes on cool slopes support Pseudotsuga menziesii, Pinus flexilis, or $P$. albicaulis forests. Adjacent exposed ridge tops often support the cushion plant communities, 
Pseudoroegneria spicata-Cushion Plants, Calamagrostis purpurascens - Carex rupestris or Carex rupestris - Potentilla ovina.

Vegetation- Festuca idahoensis - Elymus trachycaulus is dominated by Festuca idahoensis and the robust grasses, Elymus trachycaulus and Bromus carinatus. Carex petasata and Koeleria cristata are other common graminoids. The introduced $\mathrm{Poa}$ pratensis may be common in sites that have experienced considerable grazing pressure. This association has high cover of tall and low forbs and high species diversity. Common forbs include Geum triflorum, Geranium viscosissimum, Arenaria congesta, Erigeron compositus, Achillea millefolium, Artemisia ludoviciana, Galium boreale, and Lupinus spp. Taraxacum officinale may be common in some lower stands with a history of grazing pressure. Moss and lichens are rare.

Soils- Soils are deep, often with a surface horizon of loess. Parent materials are limestone, mixed sedimentary or volcanic. Bare ground usually has less than $20 \%$ cover, and rock and gravel are minimal.

Other studies- Mueggler and Stewart (1980) describe this type for western Montana. They state that high abundance of forbs such as Geum triflorum and Achillea millefolium are the result of grazing pressure.

Comments- The mesic Geranium viscosissimum phase of this type described by Mueggler and Stewart (1980) occurs on loessal soils. Deer, elk and antelope frequently use this type. Disturbance due to pocket gophers (Thomomys talpoides) is abundant in this mesic phase. The G. viscosissimum phase we recommend to be elevated to association level creating $F$. idahoensis - E. trachycaulus / G. viscosissimum. The 1995 version of the southwestern classification (Cooper et al.) puzzled what to do with a few very mesic stands with low canopy cover of or lacking $F$. idahoensis, but rather are dominated by Stipa occidentalis, Danthonia intermedia, Elymus trachycaulus, Bromus carinatus and B. anomalus and Carex spp. To correct this gap in the classification several different grassland associations, most notably Bromus carinatus B. anomalus and Stipa occidentalis $-F$. idahoensis, have been proposed based on sampling conducted subsequent to the 1995 publication.

\section{Element Code- CEGL001614}

Edition / Author- 99-11-16 / S.V. Cooper, MTNHP

Festuca idahoensis - Potentilla diversifolia
Herbaceous Vegetation
(FESIDA / POTDIV) Idaho fescue /
diverse-leaved cinquefoil

Natural Heritage Conservation RankG3 / S3

Environment- Festuca idahoensis / Potentilla diversifolia is common below $9,900 \mathrm{ft}$ in the Beaverhead, Gravelly, Snowcrest and Tendoy ranges. This type was most common on warm slopes at the low limit of alpine vegetation (ca. $9,500 \mathrm{ft}$ ) where moderate to light snow cover melts early in the growing season. It abutted subalpine forest dominated by Picea engelmannii, Pinus albicaulis and Pseudotsuga menziesii, or graded into shrublands dominated by Artemisia tridentata ssp. vaseyana below treeline. Festuca idahoensis / Potentilla diversifolia merged with Deschampsia cespitosa / Potentilla diversifolia grassland on moister slopes and with Carex elynoides turf at higher elevations on warm, dry, wind-impacted slopes. Dominance of Bromus pumpellianus defines a phase that was locally abundant on cool slopes in the Snowcrest Range.

Vegetation- Mean graminoid cover of Festuca idahoensis / Potentilla diversifolia was 55\%. Festuca idahoensis was the dominant graminoid with Elymus trachycaulus ranking second in abundance. Carex obtusata, Poa secunda, C. scirpoidea and $P$. arctica were locally common, the former two on warm aspects and the latter two on cooler slopes or level areas with deeper soils. Mean forb cover was $34 \%$. Common forbs were Potentilla diversifolia, Phlox pulvinata and Polemonium 
viscosum. Polygonum bistortoides, Myosotis sylvatica and Cerastium arvense were frequent, and Geum triflorum and Trifolium haydenii were locally common. Mean cover of lichens and mosses was only $2 \%$.

Bromus pumpellianus phase- Two stands from cool slopes in the Snowcrest range were dominated by Bromus pumpellianus instead of Festuca idahoensis. Carex obtusata was abundant in one. These stands were otherwise compositionally similar to typical Festuca idahoensis/Potentilla diversifolia.

Soils- Parent material was generally sedimentary, with limestone and calcareous sandstone's predominating. Quartzite, calcareous conglomerate, and volcanic andesite were also represented. Mean litter depth was $0.6 \mathrm{in}$, and mean duff depth was 0.3 in. Bare ground and gravel covered $11 \%$ of the surface. Percent coarse fragments varied from 2-51\% with a mean of $20 \%$. Texture of the fine fraction ranged from fine clay to sandy clayloam; the mean textural class was sandy clay. Reaction of the soil was near-neutral $(\mathrm{pH}=6.7$ 7.5) with a mean $\mathrm{pH}$ of 7.2. Mean organic matter content was $19 \%$, mean total nitrogen was $0.66 \%$, and $\mathrm{C}: \mathrm{N}$ ratio was $14: 1$.

Productivity-Graminoid productivity varied between 180 and 1,130 lbs/acre with a mean of $726 \mathrm{lbs} / \mathrm{acre}$. Forb productivity varied between 160 and 1,270 lbs/acre with a mean of $778 \mathrm{lbs} /$ acre. Mean total productivity for Festuca idahoensis / Potentilla diversifolia was 1,504 lbs/acre. Productivity was highest on deep soils.

Other Studies- Festuca idahoensis / Potentilla diversifolia could be considered a highelevation phase of Mueggler and Stewart's (1980) Festuca idahoensis / Elymus trachycaulus habitat type. Although the dominant graminoids in the two types are similar, the important forbs are different, with $P$. diversifolia considered the diagnostic forb separating the two types. Potentilla gracilis, Geum triflorum and Achillea millefolium are the most abundant forbs in the lower-elevation type. Alpine grasslands similar to Festuca idahoensis / Potentilla diversifolia were described for east-central Idaho (Moseley 1985, Caicco 1983) where they were characterized by having the highest snowdeposition of all alpine communities (Moseley 1985). Alpine meadows dominated by Festuca thurberi, an ecological analogue of $F$. idahoensis, have been described for New Mexico (Baker 1983).

\section{Element Code- CEGL001623}

Edition / Author- 99-11-16 / S.V. Cooper, MTNHP

Festuca idahoensis - Pseudoroegneria spicata Herbaceous Vegetation

(FESIDA - PSESPI) Idaho fescue bluebunch wheatgrass

\section{Natural Heritage Conservation Rank- G4 / S4}

Environment- Mueggler and Stewart (1980) describe this association as the most frequently encountered grassland type in southwestern Montana. We have few samples of the type because it thought to be abundantly documented. We sampled it on moderate to steep, predominantly southerly-facing slopes in the Tendoy Mountains at 6,000-7,500 feet (the cited elevation range is 4,500 to 7,500 feet). On lower elevation sites it tends to occur on more moderate exposures and northerly aspects. Adjacent sites with deeper soils (or in some cases rockier soils) are dominated by Artemisia tridentata ssp. vaseyana. Rockier sites on warm slopes are often dominated by Cercocarpus ledifolius, while Pseudotsuga menziesii and Pinus flexilis forests occur on cooler slopes.

Vegetation- Stands are dominated by the diagnostic grasses Festuca idahoensis and Pseudoroegneria spicata; on lightly grazed sites their combined cover can exceed $70 \%$. Koeleria cristata is also common. The shrubs, Chrysothamnus nauseosus and Artemisia tridentata ssp. tridentata, may be present but are not common, their cover not exceeding $5 \%$. Forbs are diverse and abundant. Common 
species include Phlox hoodii, P. muscoides, Achillea millefolium, Antennaria microphylla and Draba oligosperma. Mosses and especially lichens may be common.

Soils- Soils are well-drained and silty in texture. Little of the surface is bare, but gravel is common.

Other Studies- This vegetation has been described for western Montana by Mueggler and Stewart (1980). The type has also been documented to extend from the Kamloops area of British Columbia southward through Washington, Idaho and Wyoming, (Bourgeron and Engelking 1994).

\section{Element Code- CEGL001624}

Edition / Author- 99-11-16 / S.V. Cooper, MTNHP

Festuca kingii - Oxytropis campestris Herbaceous Vegetation

(FESKIN / OXYCAM) Spike fescue/field crazyweed

\section{Natural Heritage Conservation Rank-}

G3? / S2

\section{Environment- Festuca kingii / Oxytropis}

campestris is a minor type documented as occurring from 9,500-9,800 feet on moderate to steep slopes, generally with warm aspects. Extensive stands of this type occurred only in the Beaverhead and Tendoy ranges, the westernmost and driest part of our study area. Although not particularly windswept, these areas receive little precipitation and snowmelt occurs early. Festuca kingii / Oxytropis campestris most often occurred in a mosaic of Festuca idahoensis / Potentilla diversifolia grassland and Carex elynoides turf communities. It occurred in stonier soils than other grassland types and at lower elevations than turf communities. Subalpine grasslands and shrublands dominated by Artemisia tridentata ssp. vaseyana generally occurred at lower elevations.

Vegetation- Mean graminoid cover was $37 \%$.
Festuca kingii was the dominant grass. Pseudoroegneria spicata, Poa fendleriana and $P$. secunda were present with less than 5\% cover in all three stands. Mean forb cover was 23\%. High constancy forbs included Oxytropis campestris, Phlox hoodii, Erigeron compositus and Cymopterus bipinnatus. The subshrub Artemisia frigida was a minor component of all three stands, and the shrubs, A. tridentata ssp. vaseyana and Chrysothamnus viscidiflorus were minor components in the lowest stand that bordered subalpine shrublands. Mean cover of lichens and mosses was less than $1 \%$.

Soils- Festuca kingii / Oxytropis campestris occurred only on soils derived from calcareous parent material, either limestone or Beaverhead conglomerate. Mean depths of litter and duff were 0.3 in and 0.2 in respectively. Bare ground and gravel covered $21 \%$ of the surface, and rock cover averaged $9 \%$. Percent coarse fragments ranged from $33-65 \%$ with a mean of $51 \%$. Mean texture of the fine fraction was sandy clay-loam. Soil reaction varied from 7.3-7.5, with a mean $\mathrm{pH}$ of 7.4. Mean organic matter content was $11 \%$, mean total nitrogen was $0.35 \%$, and $\mathrm{C}: \mathrm{N}$ ratio was $21: 1$. This community occurred on the shallowest, stoniest and sandiest soils of any grassland type, and organic matter and nitrogen levels are only half of that in the other two types.

Productivity- Graminoid productivity varied between 275 and $875 \mathrm{lbs} /$ acre with a mean of $613 \mathrm{lbs} /$ acre. Forb productivity ranged from 250 to $600 \mathrm{lbs} / \mathrm{acre}$ with a mean of $399 \mathrm{lbs} /$ acre. Shrub productivity in the lowest elevation stand was $253 \mathrm{lbs} / \mathrm{acre}$. Mean total productivity was $1096 \mathrm{lbs} / \mathrm{acre}$, appreciably less than that of the other two grassland types.

Other Studies- Although Festuca kingii occurs throughout much of the western U.S., similar alpine grassland associations have only been described for the calcareous ranges of eastcentral Idaho (Caicco 1983, Moseley 1985, Urbanczyk and Henderson 1994) and northwest Utah (Preece 1950, Ream 1964). In Idaho, where this association is more common, two types are recognized (Moseley 1985) based on differences in substrate stability. 


\section{Element Code- CEGL001912}

Edition / Author- 97 -10 / S.V. Cooper et al.

\section{Natural Heritage Conservation Rank-}

G3 / S3

\section{Environment- Geum rossii / Minuartia} obtusiloba is common on exposed, windswept upper slopes, saddles and ridgetops between 9,800 and 10,400 ft in the Pioneer and Tobacco Root ranges. This type occurred only on soils developed from crystalline parent material. This sparsely vegetated association usually graded into the Carex scirpoidea / Geum rossii turf community having deeper soils and protected exposures.

Vegetation- Mean graminoid cover was only 4\%. Festuca ovina was the only graminoid commonly present in appreciable amounts (canopy cover not exceeding 10\%). Luzula spicata and Carex elynoides had low coverage but were frequent, and Carex rupestris and Trisetum spicatum were locally common. Mean cover of forbs was $30 \%$. Geum rossii had the greatest constancy and cover of any forb; Minuartia obtusiloba (formerly Arenaria obtusiloba), Eritrichium nanum, Phlox pulvinata and Silene acaulis were common cushion plants. Selaginella densa and $S$. watsonii were locally abundant. Trace amounts of the shrubs, Ribes hendersonii and Dryas octopetala, occurred in one stand. Cover of mosses and lichens was less than $1 \%$.

Soils- Parent materials were granite and quartzite. Bare ground and gravel covered $47 \%$ of the surface. Mean depths of litter and duff were both less than 0.1 in. Percent coarse fragments varied from 355 to $70 \%$ with a mean of $49 \%$. Textural classes of the fine fraction ranged from sandy clay-loam to sand with a mean class of sandy loam. Soil $\mathrm{pH}$ ranged from 6.2 to 6.6 with a mean of 6.4. Mean

organic matter content was only $8 \%$, mean total nitrogen was $0.24 \%$, and $\mathrm{C}: \mathrm{N}$ ratio was 20:1. Soils had a sandier texture and lower levels of organic matter and nitrogen than most other community types sampled.

Productivity- Graminoid productivity ranged from 0 to $118 \mathrm{lbs} /$ acre with a mean of $41 \mathrm{lbs} /$ acre. Forb productivity varied from 192 to 651 lbs/acre with a mean of $453 \mathrm{lbs} / \mathrm{acre}$. Mean total productivity was $494 \mathrm{lbs} / \mathrm{acre}$. Cushion plant productivity is difficult to measure; thus, the forb estimates are only rough approximations. The low total productivity reflects the small graminoid contribution.

Other Studies- Fellfields and cushion plant communities similar to Geum rossii / Minuartia obtusiloba are common in the Rocky

Mountains of southern Montana south to Colorado. Bamberg and Major (1968) describe a fellfield community from the Flint Creek Range of Montana dominated by G. rossii, Carex elynoides, Lupinus argenteus and Potentilla concinna. Cushion plant communities in the Beartooth Range of Montana and Wyoming are dominated by $G$. rossii, Carex rupestris, Minuartia obtusiloba, Silene acaulis and Trifolium nanum (Johnson and Billings 1962, Lesica 1991). Bliss (1956) describes ridgetop vegetation in the Medicine Bow Mountains of Wyoming dominated by Carex rupestris and cushion plants such as Paronychia pulvinata, Selaginella densa, Minuartia obtusiloba, Phlox caespitosa and Trifolium dasyphyllum. Geum rossii was present but of secondary importance. Similar associations with varying amounts of Geum rossii have been described from Wyoming's Absaroka Range (Thilenius and Smith 1985) and the Uinta Range in Utah (Lewis 1970). In the Rocky Mountains of Colorado, exposed ridges and fellfields are dominated by cushion plants, such as Trifolium dasyphyllum, Paronychia pulvinata, Silene acaulis and Minuartia obtusiloba as well as Carex rupestris and Kobresia myosuroides (Komarkova and Webber 1978, Willard 1979). Geum rossii is dominant in turf communities but is of secondary importance in cushion plant associations in these areas. 


\section{Element Code- CEGL001965}

Edition / Author- 97 -10 / S.V. Cooper et al.

Hordeum jubatum Herbaceous Vegetation (HORJUB) Foxtail barley

\section{Natural Heritage Conservation Rank- G4 / S4}

Environment- This is an uncommon type west of eastern and central Montana; it is of very limited extent in BVHDMS, confined to drawdown zones of ponds or ephemeral ponds with moderately saline or alkali water. Early on in the growing season these sites are flooded but in the course of the summer the water table falls considerably below the surface leaving white crusts of salt efflorescence. Sites are recognized by the dominance of Hordeum jubatum (we noted minor amounts of $H$. brachyantherum in these stands; it is a close ecological analogue of $H$. jubatum and could be used as an alternative indicator for these environments). Some of the stands noted on the Red Rock Lakes National Wildlife Refuge may represent grazing disclimax of sites potentially dominated by Pascopyrum smithii, Distichlis spicata or Leymus cinereus.

Vegetation- A moderate coverage to dense sward of Hordeum jubatum, to the near exclusion of other species, characterizes these sites. Shrubs are not found in this type. Other graminoids present include those cited above as well as Poa pratensis and $H$. brachyantherum. Polygonum spp., Grindelia squarrosa and Rumex crispus are the only forbs consistently noted.

\section{Element Code- CEGL001798}

Edition / Author- 99-11-16 / S.V. Cooper, MTNHP

Juncus balticus Herbaceous Vegetation (JUNBAL) Baltic rush
G5 / S5

Environment- The Juncus balticus association is a major wetland association occurring from low to high elevations $(2,300$ to $9,000 \mathrm{ft}$. + ) throughout Montana; it is found throughout the BLM's Dillon R. A. and is especially abundant on the Red Rocks Lakes National Wildlife Refuge (RRLNWLR) where it characterizes some of the wetter sites, those continuously saturated and often having year-long standing water. The only wetter sites would be dominated by Carex utriculata, Carex aquatilis, Carex simulata, Scirpus spp. or Typha latifolia. What we have referred to as the Juncus balticus type is somewhat different from, though included within, the broadly described type of Hansen et al. (1995). The plant association we refer to as Juncus balticus is virtually a monoculture of Juncus balticus and occurs on sites that are largely beyond the foraging range of livestock (except in drier years) and hence could not be grazing-induced disclimax as Hansen et al. (1995) claim is the case for all $J$. balticus dominated sites. There is generally no appreciable amount of forage on these sites, save for Calamagrostis canadensis (C. stricta); sedge species present are coarse and unpalatable. We recognize a Juncus balticus - Carex praegracilis association that characterizes moist meadows to seasonally flooded wetlands and which could be a grazing disclimax, but this interpretation is open to question as well.

Vegetation- On the RRLNWLR the Juncus balticus type characterizes the sometimes broad vegetation zone occurring on slightly less wet sites (or with shallower standing water through growing season) than does the predominant wetland type, Carex utriculata Carex utriculata. The Juncus balticus type often was noted to grade to the Juncus balticus - Carex praegracilis or Deschampsia cespitosa associations of somewhat drier terrain. The form of J. balticus in this zone is especially robust (to $2.5-3 \mathrm{ft}$. tall). Other than the presence of scattered graminoids noted above the only other herbs regularly present are Menyanthes trifoliata, Triglochin maritimum, Polygonum amphibium, and Ranunculus spp.

Natural Heritage Conservation Rank- 
Element Code- CEGL001838

Edition / Author- 99-11-16 / S.V. Cooper, MTNHP

Juncus balticus - Carex praegracilis Herbaceous Vegetation

(JUNBAL - CARPRA) baltic rush clustered field sedge

\section{Natural Heritage Conservation Rank-}

$\mathrm{G}$ ? / S?

\section{Environment- Juncus balticus - Carex}

praegracilis is a wetland association common in subirrigated sites on stream terraces, pond and lake margins, and in swales of alluvial fans. It has been documented from 6,500$7,200 \mathrm{ft}$ in the major valleys of the Tendoy Mountains and in the Centennial Valley and almost certainly occurs broadly throughout the BVHDMS. Most examples of this type have developed hummock-hollow microtopography. Adjacent moister sites are often dominated by Pentaphylloides floribunda and Deschampsia cespitosa or Salix spp. and Carex spp.

Occasionally Juncus balticus/Carex praegracilis will adjoin Carex simulata- and Carex nebrascensis- dominated fens and wet meadows. Adjacent drier sites, though usually considered wetlands as well, are dominated by Poa juncifolia and Leymus cinereus. Festuca idahoensis and Artemisia tridentata, A.

tripartita or A. longiloba dominate adjacent uplands. This type appears to be most common in areas of predominantly calcareous parent material.

Vegetation- Graminoid cover is over $80 \%$ in most stands. Dominant species include Juncus balticus, Carex praegracilis and Muhlenbergia richardsonis. Other common graminoids are Deschampsia cespitosa and Poa juncifolia. Cover of forbs is variable, probably depending on grazing history and moisture regime. Forb diversity is low to moderate. Common species include Antennaria microphylla, Aster occidentalis, Crepis runcinata, Haplopappus uniflorus and Potentilla gracilis. Bryophyte coverage is highly variable, ranging from trace amounts to a nearly continuous blanket.
Soils- Soils have a silty to clayey texture and are moist throughout most of the growing season. They have a near-neutral or slightly alkaline reaction and may also be mildly saline in some cases. Bare soil is generally uncommon except for areas that are heavily trampled.

Other Studies- Hansen et al. (1995) describe the Juncus balticus "community type" for Montana and state that it is a grazing-induced disclimax of vegetation previously dominated by Deschampsia cespitosa or Calamagrostis canadensis. They report that Juncus balticus and Hordeum jubatum are the only common species, suggesting that their type is significantly different than Juncus balticus / Carex praegracilis of southwest Montana. For the Blackfeet Reservation of northwest Montana Lesica (1989) described a Deschampsia cespitosa / Juncus balticus association with composition similar to that of J. balticus / C. praegracilis. Similar vegetation has also been described for the Blackfoot River Valley of Montana (Lesica 1994) southward into Idaho and Wyoming (Youngblood et al. 1985) to as far south as southern Utah (Padgett et al. 1989).

Comments- If Juncus balticus plant associations are a grazing-induced disclimax of Deschampsia cespitosa associations (Hansen et al. 1995), D. cespitosa should be negatively correlated with abundance of J. balticus, forbs and exotics, and abundance of J. balticus should be positively correlated with abundance of forbs and exotics. This does not appear to be the case in our study area. A Pearson's correlation coefficient ( $r$ ) matrix is given below:

\begin{tabular}{lrrrr} 
& Junbal & Desces & Forbs & Exotics \\
\cline { 2 - 5 } Junbal & 1.000 & & & \\
Desces & 0.019 & 1.000 & & \\
Forbs & -0.297 & -0.272 & 1.000 & \\
Exotics & -0.122 & -0.020 & 0.110 & 1.000
\end{tabular}

There was almost no correlation between the cover of D. cespitosa and J. balticus. Both species decrease as the abundance of forbs and exotics increase, although the correlation is not strong. These results suggest that both species 
decrease with grazing disturbance and that the abundance of forbs is a better indicator of disclimax than the abundance of J. balticus, at least in the BVHDMS. Our ordination results suggest that Juncus balticus is dominant in slightly drier sites than those dominated by $D$. cespitosa.

Variety montanus is the common form of Juncus balticus in Montana. However one of our stands was a nearly complete monoculture of J. balticus var. vallicola, a more robust form of the species. This stand on a pond drawdown zone had the appearance of a bulrush monoculture. This vegetation may warrant recognition as a separate type if it occurs in other locations.

Trampling by livestock, especially during the spring and early summer results in the formation or enhancement of hummocks. The presence of substantial hummocking may allow the invasion of the shrub, Pentaphylloides floribunda, into habitats that are otherwise too wet.

\section{Element Code- CEGLMTHP14}

Edition / Author- 99-11-16 / S.V. Cooper, MTNHP

\section{Juncus drummondii - Antennaria lanata Herbaceous Vegetation (JUNDRU / ANTLAN) Drummond's rush / woolly pussytoes}

\section{Natural Heritage Conservation Rank- G3? / S2}

Environment- Small patches (tenths of an acre at most) of Juncus drummondii / Antennaria lanata are relatively common in swales and other snow-collection microtopography set within valleys and cirque basins between 9,600 and 10,000 ft in the Madison Range. This community is always associated with areas of late snowmelt; however, melt off probably occurs earlier than in communities dominated by Carex nigricans. Juncus drummondii / Antennaria lanata was often part of vegetation mosaic resulting from uneven snow deposition.
Commonly associated communities are moist turf, wetlands and other snowbed associations such as Carex scirpoidea / Geum rossii, Carex scopulorum / Caltha leptosepala, Carex nigricans and Phyllodoce empetriformis / Antennaria lanata .

Vegetation- Mean graminoid cover was $30 \%$. Dominant graminoids were Juncus drummondii, Poa fendleriana and Carex paysonis. Carex pyrenaica was locally common. Mean cover of forbs was $37 \%$ and common species included Antennaria lanata, Sibbaldia procumbens and Erigeron peregrinus. Arnica latifolia was common in one stand. The shrub, Vaccinium scoparium, was also common in this same stand. Mean cover of mosses and lichens was $2 \%$.

Soils- Parent materials were gneiss and granite. Bare ground and gravel covered $50 \%$ of the surface. Mean depths of litter and duff were both 0.1 in. Percent coarse fragments ranged from $6 \%$ to $17 \%$ with a mean of $13 \%$. Mean texture of the fine fraction was sandy clay. Although they occurred in topographically low positions, these relatively barren and unproductive communities were apparently underlain by shallow and perhaps excessively well-drained soils. Soil $\mathrm{pH}$ ranged from 6.0 to 6.1 with a mean of 6.1. Mean organic matter content was $12 \%$, mean total nitrogen was $0.21 \%$, and $\mathrm{C}: \mathrm{N}$ ratio was $24: 1$.

Productivity- Graminoid productivity varied between 200 and $270 \mathrm{lbs} /$ acre with a mean of $237 \mathrm{lbs} / \mathrm{acre}$. Forb productivity ranged from 150-860 lbs/acre with a mean of $460 \mathrm{lbs} / \mathrm{acre}$. Productivity of dwarf shrubs in one stand was $30 \mathrm{lbs} /$ acre. Mean total productivity was 726 lbs/acre. Highest productivity occurred on the deepest soils.

Other Studies- Antennaria lanata is a common component of snowbed communities in the North Cascades and Canadian Rockies, but the codominant species usually cited are Carex nigricans or dwarf shrubs such as Cassiope or Phyllodoce spp. rather than Juncus drummondii (Achuff and Corns 1982, Hrapko and LaRoi 1978, Douglas and Bliss 1977). 
Snowbed communities dominated by Juncus drummondii with Carex pyrenaica and Sibbaldia procumbens occur in the Rocky Mountains of Colorado (Komarkova and Webber 1978, Willard 1979); however, Antennaria lanata is not listed for the Colorado associations. Lesica (1991) reports communities very similar to Juncus drummondii / Antennaria lanata from the eastern edge of the Beartooth Mountains in south-central Montana. It appears that Juncus drummondii dominates snowbed communities in the central Rocky Mountains, while Antennaria lanata occupies a similar niche in the Canadian Rockies and the North Cascades. The two species codominate snowbed associations in the crystalline ranges of southern Montana.

\section{Element Code- CEGL001904}

Edition / Author- 97 -10 / S.V. Cooper et al.

Juncus parryi / Erigeron ursinus Herbaceous Vegetation

(JUNPAR / ERIURS) Parry's rush / bear fleabane

\section{Natural Heritage Conservation Rank-}

G2? / S2?

Environment- Examples of Juncus parryi / Erigeron ursinus were locally common near the base of gentle slopes with warm aspects. Both of our stands were between 9,500 and $9,800 \mathrm{ft}$ in the Gravelly Range. Although snow is expected to accumulate on these sites, this type of all the snowbed communities is perhaps least affected by late melt off. Festuca idahoensis / Potentilla diversifolia grassland was the common adjacent plant community. Juncus parryi / Erigeron ursinus is similar in physiognomy and habitat to Juncus drummondii / Antennaria lanata.

Vegetation- Mean graminoid cover was $35 \%$. Dominant graminoids were Juncus parryi and Festuca idahoensis, and Poa glauca was consistently present with low coverages. Mean cover of forbs was $25 \%$. Common species included Erigeron ursinus, E. peregrinus, E. simplex, Antennaria umbrinella and Lewisia pygmaea. Mean cover of mosses and lichens was $2 \%$.

Soils- Parent materials in the two stands were andesite and quartzite. Bare ground and gravel covered $47 \%$ of the surface, indicating that this was the most barren of our snowbed communities. Mean depths of litter and duff were 0.3 in and 0.1 in respectively. Mean percent coarse fragments was $25 \%$. Mean texture of the fine fraction was clay. Mean soil $\mathrm{pH}$ was 5.6. Mean organic matter content was $20 \%$, mean total nitrogen content was $0.64 \%$, and $\mathrm{C}: \mathrm{N}$ ratio was $15: 1$. This sparsely vegetated community type is similar to Juncus drummondii / Antennaria lanata, but the soils were even more stony and acidic.

Productivity- Mean graminoid productivity was $439 \mathrm{lbs} / \mathrm{acre}$, and mean forb productivity was $253 \mathrm{lbs} / \mathrm{acre}$. Mean total productivity was $692 \mathrm{lbs} /$ acre. Productivity is probably affected more by the shallow, poorly developed soils than late snow release.

Other Studies- Holway and Ward (1963) report snow accumulation areas in the Colorado Rocky Mountains dominated by Carex pyrenaica and Juncus parryi. Willard (1979) states that Juncus parryi is ecologically similar to J. drummondii but generally occurs at lower elevations. In our study area, Juncus parryi / Erigeron ursinus was associated with terrain supporting alpine grasslands, while Juncus drummondii / Antennaria lanata was associated with turf communities. Thus, it seems likely that these two community types occupy similar topographic positions, but Juncus drummondii / Antennaria lanata occupies cooler, moister habitats.

\section{Element Code- CEGL001906}

Edition / Author- 97 -10 / S.V. Cooper et al.

\begin{tabular}{c}
\hline Leymus cinereus - Puccinellia nuttalliana \\
Herbaceous Vegetation \\
(LEYCIN - PUCNUT) basin wildrye - \\
Nuttall's alkaligrass
\end{tabular}

Natural Heritage Conservation Rank- 
$\mathrm{G}$ ? / S?

Environment- Stream terraces and lower portions of alluvial valley floors at 6,500-7,000 feet within the BVHDMS support narrow corridors or extensive stands of Leymus cinereus - Puccinellia nuttalliana. This type is usually associated with areas dominated by limestone parent material. Adjacent moister vegetation types may be dominated by Juncus balticus, Pentaphylloides floribunda, Deschampsia cespitosa or Salix spp. with Carex spp. Adjacent upland vegetation supports stands dominated by Artemisia arbuscula ssp. longiloba, A. tripartita, or A. tridentata wyomingensis and Festuca idahoensis.

Vegetation- These species-poor associations are dominated by Poa juncifolia, Puccinellia nuttalliana (or P. distans) and Carex praegracilis. Leymus cinereus may be dominant, sometimes forming near monocultures, in stands that have not suffered intensive grazing pressure. The abundance and species composition of the forb component is variable and depends upon the moisture regime, soil texture, salinity, and degree of disturbance. Haplopappus uniflorus and Antennaria microphylla are the most common forb species. The shrub Chrysothamnus nauseosus is usually present and may be common. Mosses and lichens are absent.

Soils- Soils are usually saline with a silty or clayey texture. Reaction is neutral to alkaline. The surface is $60-90 \%$ bare with little or no gravel. Soils may be affected by natural or man-caused subirrigation.

Other studies- Similar vegetation dominated by Leymus cinereus, Pascopyrum smithii and Puccinellia distans in northwest Montana was briefly discussed by Mueggler and Stewart (1980, p. 75) and Lesica (1988); a general Leymus cinereus c.t. is found from Washington, Oregon, through Idaho to Colorado but has curious gaps (not cited for Wyoming or Utah) that may reflect a lack of inventory rather than an intrinsic absence (Bourgeron and Engelking 1994).
Comments- Leymus cinereus is highly palatable to livestock only early in the growing season; if grazed at this season it loses a significant portion of biomass that would have contributed to its potentially tall stature, thus making it very sensitive to intensive grazing. We believe that broad alluvial valleys of southwest Montana may have been dominated by $E$. cinereus before the advent of the livestock industry over 100 years ago. With the decline of this robust grass, smaller species adapted to saline soils, such as Poa juncifolia, Puccinellia distans and Carex praegracilis assumed dominance.

\section{Element Code- CEGLMTHP15}

Edition / Author- 99-11-16 / S.V. Cooper, MTNHP

$$
\begin{gathered}
\text { Pascopyrum smithii Herbaceous } \\
\text { Vegetation [Provisional] } \\
\text { (PASSMI) western wheatgrass }
\end{gathered}
$$

\section{Natural Heritage Conservation Rank- G3G5 / S4}

Environment- This is an uncommon type in southwestern MT, occurring on alluvial floodplains that experience regular overland flow flooding or heavy-textured soils. It is found on islands of the Big Hole River at ca. 5,000-5,200 ft. Slightly higher areas are dominated by Artemisia tridentata ssp. tridentata and Pseudoroegneria spicata. Adjacent sites can support Populus balsamifera ssp. trichocarpa forests. Hansen et al. (1995) consider at least a portion of this type to be wetland sites (jurisdictional wetlands).

Vegetation- Graminoid cover is high, but Pascopyrum smithii is the only dominant grass. Koeleria cristata, Poa pratensis and Carex stenophylla are other common species. Equisetum hyemale is the only common forb. A number of weedy forbs, such as Grindelia squarrosa, Cirsium arvense, Tragopogon spp. and Melilotus officinalis, may be present. Mosses and lichens are absent.

Soils- Soils are silt loams. There is no gravel 
on the surface.

Other Studies- Coupland (1950) reports a similar type from alluvial floodplains in Alberta. Hansen et al. (1995) cite this as an important type in central and eastern Montana and in varying degrees of importance it extends to Colorado and Utah (Bourgeron and Engelking 1994).

Comments- This vegetation is clearly seral to Populus balsamifera ssp. trichocarpa forest or Artemisia tridentata tridentata / Pascopyrum smithii. Flooding or ice scour apparently retards colonization by woody species. These sites are likely areas to be invaded by Euphorbia esula (leafy spurge).

\section{Element Code- CEGL001577}

Edition / Author- 99-11-16 / S.V. Cooper, MTNHP

Phalaris arundinacea Western Herbaceous Vegetation

(PHAARU) reed canarygrass

\section{Natural Heritage Conservation Rank- G5 / S4}

Vegetation- Phalaris arundinacea dominates (often with a total canopy cover of 100\%). The undergrowth is typically very depauperate due to the intense competition with $P$. arundinacea. Poa palustris may be well represented and Cirsium arvense and Mentha arvensis are often common. The total cover of the few additional species seldom exceeds $1 \%$. Adjacent wetter sites often feature open water while adjacent drier riparian communities include the Populus balsamifera ssp. trichocarpa / Cornus sericea type and a wide variety of other riparian types.

Environment- This type occurs on gravelly alluvial parent materials on flat flood plains in wide valley bottoms at elevations around 5000 feet. Litter and soil cover equals or exceeds $30 \%$ and $20 \%$ of the ground surface, respectively. The soil surface is generally stable with no evidence of accelerated erosion.

Soils- Soils are often Fluvaquents or Fluvents, are very deep with loamy sand to loam textures, medium available water holding capacity, and are non-calcareous. Coarse fragment contents vary from none to gravelly.

Other Studies- Within the western U. S. this type has been described only in Montana, where it is a common type from the western intermountain valleys to the eastern plains (Hansen et al. 1995). Some contend that Phalaris arundinacea is not a native species, hence this type should be recognized as a disturbance type.

\section{Element Code- CEGL001474}

Edition / Author- 99-11-16 / S.V. Cooper, MTNHP

Poa juncifolia Herbaceous Vegetation (POAJUN) alkali bluegrass

\section{Natural Heritage Conservation Rank-} G4? / S?

Environment- If were to slavishly follow the nomenclature of Kartez (1994) then this type would be known as the Poa secunda community. However, Poa juncifolia appears to us to be a distinct ecological entity, associated with alkali soils (as its common name indicates) and having a much more robust growth form with tussocks up to 2 to 3 times taller (and broader at the base) than those of $P$. secunda. These stands exist as small to large patches, commonly found on the western and central portions of the Refuge on finetextured alluvial flats that are seasonally saturated, but which dry deeply by late summer. Traces of salt efflorescence have been noted. These sites may constitute grazing disclimax of Pascopyrum smithii-, Elymus lanceolatus-, Leymus cinereus-, Hordeum jubatum- or even Puccinellia nuttallianadominated habitats; only appropriate exclosures could answer this question. The closest named type of the literature is described from southern Oregon alkali flats as $P o a$ secunda (P. juncifolia?) - Puccinellia lemmonii - Elymus elymoides (syn. Sitanion hystrix). 
Vegetation- The bunchgrass Poa juncifolia dominates a sparse herbaceous layer with canopy cover in the range of $15-30 \%$. Other graminoids consistently present with low cover values include Sitanion hystrix, Poa cusickii, Pascopyrum smithii (or Elymus lanceolatus), Hordeum jubatum, and Puccinellia nuttalliana. The only shrubs consistently present are scattered Chrysothamnus nauseosus or $C$. viscidiflorus. Forb diversity is low, mostly species associated with disturbance, e.g. Grindelia squarrosa, Descurainia pinnata, and various Erigeron spp., especially $E$. corymbosus.

\section{Element Code- CEGL001657}

Edition / Author- 99-11-16 / S.V. Cooper, MTNHP

Poa pratensis Herbaceous Vegetation [Provisional] (POAPRA) Kentucky bluegrass

\section{Natural Heritage Conservation Rank-} GW / SW

Environment- The Poa pratensis community type, recognized by a higher coverage of $\mathrm{Poa}$ pratensis than any other herb present, represents a widely distributed, grazing- or otherwise disturbance-induced disclimax of Refuge lands; it is probably the most extensive disclimax on both the Refuge and in the state in general. Poa pratensis, a rhizomatous exotic European meadow grass, has a very broad ecological amplitude and is found from true wetlands mixed with Juncus balticus,

Pascopyrum smithii and Deschampsia cespitosa types, to upland sites such as Festuca idahoensis - Pseudoroegneria spicata or $F$. idahoensis - Elymus trachycaulus; it develops its highest cover on grazing-impacted wet meadows and mesic grasslands characterized by medium-textured Mollisols, such as $F$. idahoensis - Stipa richardsonii or Bromus carinatus- and B. anomalus-dominated habitats. It also is found on sandy, as well as, dense clay soils, if the moisture status is favorable. As Hansen et al. (1995) note this is one of the driest of herb-dominated riparian or wetland community types.
Vegetation- Composition of these sites can vary from nearly pure swards of $P$. pratensis to mixtures of weedy species or increaser species, including Bromus inermis, Phleum pratense, Juncus balticus, Festuca arundinacea, Hordeum jubatum, Potentilla anserina, $P$. gracilis and Taraxacum officinale. Once a site has been captured by $P$. pratensis it is unlikely to revert to native cover due to the highly competitive nature of this species; it develops an extensive rhizome system that permits it to spread quickly and is extremely resistant to high levels of herbivory. We speculate that on the Refuge, as elsewhere, the native community types most often converted to $P$. pratensis dominance include Pascopyrum smithii and Deschampsia cespitosa.

\section{Element Code- CEGL003081}

Edition / Author- 99-11-16 / S.V. Cooper, MTNHP
Pseudoroegneria spicata - Cushion plant Herbaceous Vegetation
(PSESPI - CUSH) bluebunch wheatgrass - cushion plant

\section{Natural Heritage Conservation Rank- G3 / S3}

Environment- This uncommon type occurs on windswept, limestone ridge crests and upper slopes in the Tendoy and perhaps Centennial Mountains at 8,000-9,000 ft. Adjacent sites with deeper soils are often dominated by Artemisia tridentata vaseyana and Festuca idahoensis. Cool slopes below these ridges may support Pseudotsuga menziesii or Pinus flexilis forests.

Vegetation- The dominant graminoids are Pseudoroegneria spicata, Festuca kingii and Carex filifolia. Shrubs are completely absent. There is a rich assemblage of low forbs, including many cushion-forming species such as Cymopterus bipinnatus, Lesquerella alpina, Phlox hoodii, Eritrichium nanum, Oxytropis campestris and Townsendia spp. Forbs have significantly more basal cover than graminoids, 
especially in the most exposed sites. Mosses and especially lichens are common.

Soils- Soils are calcareous and shallow. Most of the surface is covered with gravel or the plant cushions.

Other Studies- DeVelice and Lesica (1993) described an extensive Pseudoroegneria spicata / Cushion plant type from windswept calcareous ridges at low to mid-elevations on the south side of the Pryor Mountains in southcentral Montana. Although the forbs in these two types have the same low, cushion-like physiognomy, there is little overlap in species composition. Lesica (1988) described a sparsely vegetated grassland dominated by Pseudoroegneria spicata and Eriogonum ovalifolium in northwest Montana; however, this type is developed on excessively drained gravel and exhibits a different suite of forbs, without the high diversity of Pseudoroegneria spicata / Cushion plant. Alpine cushion plant communities described by Cooper and Lesica (1992) also have a similar physiognomy and many forb species in common, but the common graminoids are Carex rupestris, C. elynoides and Festuca ovina.

Comments- This type may occur lower but only on the most extremely exposed and edaphically harsh sites. Extensive areas will occur only at higher elevations.

These sites may provide important winter range for bighorn sheep. Vegetation of these dry, exposed sites is easily damaged by motorized vehicles and recovers slowly.

\section{Element Code- CEGL001666}

Edition / Author- 99-11-16 / S.V. Cooper, MTNHP

Pseudoroegneria spicata - Oryzopsis hymenoides Herbaceous Vegetation (PSESPI / ORYHYM) bluebunch wheatgrass / Indian ricegrass

Natural Heritage Conservation RankG3 / S?
Environment- The type occurs on steep badland sites at elevations around 5500 feet. Bare soil cover totals 20 to $70 \%$ and most of the remaining ground cover is comprised of coarse fragments. The soil surface is unstable because of a lack of adequate vegetation cover and rill and sheet erosion is common. Parent materials are sedimentary rocks and soils are shallow and poorly developed. Soils range from not gravelly to very gravelly and are strongly to violently effervescent. Textures are sands to loams and available water holding capacity is low.

Vegetation- Total vegetation cover in this badlands type seldom exceeds $15 \%$. Characteristic species include Gutierrezia sarothrae, Pseudoroegneria spicata, Aristida longiseta, and Oryzopsis hymenoides.

Adjacent Communities- Vegetation on adjacent more stable soils include the Artemisia tridentata / Pseudoroegneria spicata and Pseudoroegneria spicata/Bouteloua gracilis types.

Other Studies- This type has not previously been reported in Montana but has been reported in Wyoming (Bourgeron and Engelking 1994).

\section{Element Code- CEGL001674}

Edition / Author- 99-11-16 / S.V. Cooper, MTNHP

Pseudoroegneria spicata - Pascopyrum smithii Herbaceous Vegetation (PSESPI - PASSMI) bluebunch wheatgrass - western wheatgrass

\section{Natural Heritage Conservation Rank-} G4 / S4

Environment- This uncommon type occurs on gently sloping alluvial fans at ca. 6,000-7,000 $\mathrm{ft}$. as well as moderate to steep eroding slopes at 6,600-7,500 $\mathrm{ft}$ in the Centennial Mountains. Vegetation dominated by Artemisia tridentata ssp. vaseyana and either Pseudoroegneria 
spicata or Festuca idahoensis occurs on adjacent deeper or less erodible soils.

Vegetation- Pseudoroegneria spicata and A. smithii/dasystachyum are common. Examples lower down on alluvial fans have abundant Calamagrostis montanensis and Stipa comata; both of these species probably increase with grazing at the expense of the wheatgrass and are also associated with coarser-textured soils. Gutierrezia sarothrae and Artemisia frigida are common subshrubs that also increase with grazing. Forbs are uncommon. Festuca kingii is common and occasionally well represented in examples of this type occurring on steep slopes of the Centennial Mountains. Subshrubs and other grasses are uncommon, but forbs are abundant and diverse. Common species include Ipomopsis congesta and Aster occidentalis. Mosses and lichens are rare.

Soils- This type appears to be most common on soils derived from calcareous parent materials, limestone or calcareous sandstone. Bare soil is abundant and surface gravel is common. Soils of slopes in the Centennial Mountains in the Peet Creek area are unstable and prone to slumping.

Other Studies- Mueggler and Stewart (1980) and Jorgensen (1979) describes this type for east and occasionally west of the Continental Divide in Montana. Their type is very similar to stands from southwest Montana that occur on alluvial fans. Pseudoroegneria spicataPascopyrum smithii is also reported from Wyoming ( Bourgeron and Engelking 1994).

Comments- Extensive areas of the Centennial Mountains between Sand Creek and Peet Creek are dominated by highly erodible soils derived from calcareous sandstone. The grasslands occurring on these soils have been included here, but they may constitute a distinct phase of the Pseudoroegneria spicata / Pascopyrum smithii c.t. or perhaps a distinct community type.

Heavy livestock grazing or motorized vehicle use of these sites is not desirable due to the erodible nature of the soil.

\section{Element Code- CEGL001675}

Edition / Author- 99-11-16 / S.V. Cooper, MTNHP

Pseudoroegneria spicata - Poa secunda
Herbaceous Vegetation
(PSESPI / POASEC) bluebunch
wheatgrass-Sandberg bluegrass

\section{Natural Heritage Conservation Rank-} G4? / S4

Environment- Pseudoroegneria spicata - Poa secunda is common on moderate to steep slopes and alluvial fans, usually with a warm aspect. Elevations range from 5,800-7,500 ft. Adjacent deeper soils often support vegetation dominated by Artemisia tridentata and Festuca idahoensis. Cooler aspects or higher elevations support woodlands dominated by Pinus flexilis or Pseudotsuga menziesii. Very rocky outcrops are dominated by Cercocarpus ledifolius. Most stands occur south of Dillon. Along the east front of the northern Tendoy Mountains, this vegetation often forms mosaics that include shallow drainage's and bald limestone outcrops.

Vegetation- Dominance is shared by Pseudoroegneria spicata Stipa comata and Oryzopsis hymenoides. Poa secunda and Koeleria cristata are present in small amounts in most stands. The subshrubs Artemisia frigida and Gutierrezia sarothrae are usually present. Forbs cover is low but diverse. Common species include Phlox hoodii, Haplopappus acaulis, Arenaria kingii, Penstemon aridus and Lesquerella alpina. Mosses are rare, but lichens may be common in some stands.

Soils- Parent materials are limestone or mixed sedimentary. $20-50 \%$ of the soils is exposed and gravel is abundant on the surface. Soils are generally well drained and often sandy in texture.

Other Studies- Pseudoroegneria spicata- $\mathrm{Poa}$ secunda (=AGRSP-POASEC) is described for 
western Montana by Mueggler and Stewart (1980). Daubenmire (1970) describes a similar type with lower forb diversity and without Stipa comata that was once extensive in eastern Washington. Pseudoroegneria spicata- Poa secunda apparently extends as far south as northern Colorado and Utah but is only a significant landscape component in Wyoming (Bourgeron and Engelking 1994).

Comments- All of our stands fit the description of the Stipa comata phase of the Pseudoroegneria spicata - Poa secunda c.t. (Mueggler and Stewart, 1980). Stipa comata, Poa sandbergii and the subshrubs Gutierrezia sarothrae and Artemisia frigida increase with increasing grazing pressure, but at higher intensities $S$. comata decreases.

\section{Element Code- CEGL001677}

Edition / Author- 99-11-16 / S.V. Cooper

Puccinellia nuttalliana Herbaceous Vegetation

(PUCNUT) Nuttall's alkaligrass

\section{Natural Heritage Conservation Rank- G3? / S?}

Environment- Puccinellia nuttalliana is an uncommon on the Refuge and globally ranked high for rarity as well. It is sparsely vegetated with Nuttall's alkaligrass (Puccinellia nuttallii) approaching monospecific dominance, occurring in small patches as a broken stringer along intermittent drainage's and on alluvial flats (mostly on the western fringe of Refuge). In some cases on the Refuge Puccinellia nuttalliana constitutes the vegetation band closest to the incised channel on a floodplain position with silty loam alluvial soils. Salt efflorescence was not observed but this community is known to occur on salt-affected soils that have a slightly wetter, temporarily inundated, moisture regime. The Puccinellia nuttalliana type has not been formally described from MT in the classification efforts of Hansen et al. (1995), but probably has been subsumed by these researchers within the Distichlis stricta or Pascopyrum smithii community types. Within the TNC tracking system this community type has been reported only from Colorado as G1? but Heidel and Cooper (1997) have documented it from western plains of Montana near the Rocky Mountain Front, noted it in field reconnaissance, and cited it from the Canadian literature (synonym: Puccinellia airoides, Dodd and Coupland 1966). We have recognized the type by $P$. nuttalliana having at least $10 \%$ cover and greater cover than any other forb indicative of yet drier moisture regimes. It is usually exists in a complex with Leymus cinereus - Puccinellia nuttalliana, Pascopyrum smithii / alluvial flats or Distichlis spicata- and Sarcobatus vermiculatusdominated types. In the vicinity of the Puccinellia nuttalliana sampling sites a comparable landscape position was occupied by what has been described as western wheatgrass - inland saltgrass grassland (Pascopyrum smithii-Distichlis spicata Herbaceous Vegetation; WY, ND).

Vegetation- The Refuge examples of this type have low diversity and are compositionally very similar to other observed Montana occurrences. Puccinellia nuttalliana dominants at around $40 \%$ canopy cover; inland saltgrass (Distichlis spicata), Hordeum jubatum (H. brachyantherum), Leymus cinereus, Grindelia squarrosa and Iva axillaris are the only other herbs exhibiting more than trace coverages or that are present with any consistency. Chrysothamnus nauseosus and Sarcobatus vermiculatus in trace amounts were the only shrubs noted within the type.

\section{Element Code- CEGL001799}

Edition / Author- 99-11-16 / S.V. Cooper, MTNHP

Scirpus acutus Herbaceous Vegetation (SCIACU) hardstem bulrush

\section{Natural Heritage Conservation Rank-} G5 / S5

Environment- This is a major wetland plant community throughout Montana noted as 
occurring from low to mid elevation (Hansen et al. 1995); the examples occur at the cited upper elevation limits of the type $(6,600$ feet $)$. On the refuge this type is found as a discontinuous fringe on the margins of ponds and is especially conspicuous on the margins of Upper and Lower Red Rock Lakes in water up to about six feet deep. It frequently grows with Typha latifolia or adjacent to T. latifoliadominated communities (where T. latifolia cover is greater than $25 \%$ the community is defined as a T. latifolia type). Scirpus acutus usually grades to the Carex utriculata - Carex utriculata type or Juncus balticus communities that characterize shallower water.

Vegetation- Though Hansen et al. (1995) include Scirpus validus (softstem bulrush) as an equivalent indicator species for this type we found only Scirpus acutus on the Refuge. Except for the situations where Typha latifolia occurs with significant cover, Scirpus. acutus generally forms dense near monocultures with Potamogeton amphibium, Potamogeton spp., Potentilla palustris, Eleocharis palustris and Triglochin maritimum being present in barely more than trace amounts.

\section{Element Code- CEGL001840}

Edition / Author- 99-11-16 / S.V. Cooper, MTNHP

Scirpus tabernaemontani Herbaceous Vegetation (SCITAB) Softstem bulrush

\section{Natural Heritage Conservation Rank- G4 / S3}

Environment- The Scirpus tabernaemontani association is typically found at low to mid elevations across Montana, but more frequently in the eastern part of the state. It is found in marshes, pond and lake margins, oxbow lakes, and backwater areas of rivers and streams. Soils are commonly Mollisols (Aquolls), Entisols (Aquents), or sometimes Histosols, and soil reaction ranges from neutral to moderately alkaline (pH 7.0 to 8.0). This community can occur in standing water up to
1-2 meters deep, and it is usually classified as semi-permanently flooded since the water levels can drop below the soil surface by the end of the growing season. This community occupies the same position in the landscape as Typha sp., with open water or aquatic communities occupying wetter spots and Salix sp., Carex sp., or Phalaris arundinacea communities occupying nearby drier sites (Hansen et al. 1988, Hansen et al. 1995).

Range- Communities of Scirpus tabernaemontani are widespread across the Great Plains states, and also occur in Montana and Idaho.

Vegetation- This plant association usually forms dense monocultures that can occupy large areas. Stems are usually 1-2 meters tall. The flooded conditions in which this species often grows precludes the establishment of other graminoids and forbs; if present, species like Potentilla palustris and Polygonum amphibium are often widely scattered. Its seeds require bare, moist soils for germination, so this species can rapidly colonize newly exposed mudflats and drawdown areas (Hansen et al. 1988, Hansen et al. 1995).

\section{Similar Communities- Scirpus}

tabernaemontani is synonymous with Scirpus validus. Hansen et al. (1995), Hall and Hansen (1997), and Kittel et al. (1998) have a Scirpus acutus habitat type in their classifications that includes all combinations of Scirpus acutus and S. tabernaemontani due to similarities in environmental conditions and management concerns. Scirpus tabernaemontani is often treated as a separate alliance in the Western Regional Vegetation Classification (Bourgeron and Engelking 1994). Other communities with this species growing as a dominant or codominant have been described: Scirpus tabernaemontani - Typha latifolia (e.g. Komarkova 1986), and Scirpus tabernaemontani - Typha spp. - (Sparganium spp., Juncus spp.) (Hoagland 1997). The Scirpus tabernaemontani type described here encompasses enough compositional and structural variation to include these other types. 
Succession- This species can colonize newly exposed mudflats and drawdown areas, and communities tend to be persistent if the hydrologic regime is stable.

Management- Softstem bulrush has low to moderate palatability to livestock, and because communities are usually flooded, access for livestock is usually difficult. When stands dry down, livestock may heavily utilize these communities if upland forage is sparse. Stands of softstem bulrush can buffer wave action on lakes and ponds (Hansen et al. 1995).

Element Code- CEGL002623

Edition / Author- 99-10-15 / J. Greenlee, MTNHP

Stipa comata - Bouteloua gracilis Herbaceous Vegetation (STICOM - BOUGRA) needle-and-thread / blue grama

\section{Natural Heritage Conservation Rank- G5 / S5}

Environment- Stipa comata-Bouteloua gracilis occurs from the floors and gently sloping coalesced alluvial fans of intermountain valleys upslope to where it intergrades with Festuca idahoensis- and Artemisia tridentata ssp. vaseyana-dominated rangelands. Lowest elevation occurrences are represented by valley bottoms (non-saline or alkaline sites) around 4,500 ft. and the type extends as high as $6,300 \mathrm{ft}$ as small patches with coarse-textured soils within a mosaic of more mesic vegetation. It is apparently not aspect or slope restricted within this elevation range but habitats within this landscape are generally low gradient ( $<25 \%$ slope). Mueggler and Stewart (1980) term Stipa comata-Bouteloua gracilis the driest of western Montana grassland habitat types; it is certainly the most extensive dry grassland type but is no drier than the more areally restricted Pseudoroegneria spicata - Oryzopsis hymenoides, Stipa comata / Psoralidium tenuiflorum or Pseudoroegneria spicata I Cushion Plant community types.
Vegetation- All sites sampled and noted in reconnaissance supported abundant Stipa comata, the diagnostic species, but cover was highly variable (20-80\%) depending on grazing history and intrinsic severity of site, ostensibly controlled by the amount of gravel and stone in substrate. Cover of Bouteloua gracilis, the other diagnostic species, is also highly variable but shows a clinal response of increasing cover west to east. It was only sporadically encountered in the westernmost valley between Tendoy and Beaverhead Ranges. Other graminoids frequently occurring, usually poorly represented, include Koeleria cristata, Carex stenophylla and Poa sandbergii.

Though ubiquitous on these sites it is here that the increase in cover of Artemisia frigida and Gutierrezia sarothrae with the increasing intensity of grazing is so apparent. Ceratoides lanata is also consistently present, in scare amounts. Forb cover and diversity is usually low, those species with the highest cover are often exotic weeds or escaped agricultural introductions (e.g. Melilotus officinalis, Salsola kali, Medicago sativa, Taraxacum officinale). Sphaeralcea coccinea, a increaser with grazing, is highly constant but scarce; no other forbs had even $50 \%$ constancy.

Soils- Given the position of these sites within the landscape and composition (dominance of Stipa comata) it follows that the soils are medium-textured to sandy, usually loamy Mollisols or Aridisols with free calcium carbonate at and near the surface. The amount of bare substrate (soil, gravel, rock) ranged from 20 to 50\%; most of the surface is covered with litter and cryptogamic crust (dominated by lichens), the relative proportions of which are highly variable.

Other Studies- Coupland (1961) in southern Saskatchewan described a Stipa comata Bouteloua gracilis faciation on sandy sites within the mixed-grass prairie. This major plant association extends southward along the western fringe of the Great Plains in eastern Montana, Wyoming and Colorado (Bourgeron and Engelking 1994). The study area constitutes the western extreme of its 
distribution. Daubenmire described a Stipa comata-Poa sandbergii habitat type in eastern Washington that is similar to Stipa comataBouteloua gracilis in landscape position, community type matrix and dominants (with the exception of Bouteloua gracilis absence in WA). He showed with detailed soil analyses that Stipa comata-dominated sites differed at 95\% confidence level from Agropyron- and Festuca-dominated sites in having both coarser (drier) and less fertile (lower exchange capacity and lower nutrient content) soils.

\section{Element Code- CEGL001699}

Edition / Author- 99-11-16 / S.V. Cooper, MTNHP

\section{Stipa comata - Psoralidium tenuiflorum Herbaceous Vegetation \\ (STICOM - PSOTEN) needle-and-thread - slender-leaved scurf-pea}

\section{Natural Heritage Conservation Rank- $\mathrm{G}$ ? / S?}

Environment- This seral type characterizes recently stabilized blowouts of sandhills at 6,600-6,900 ft on the northeast side of the Centennial Valley. Adjacent vegetation is dominated by various combinations of Artemisia tripartita or A. tridentata ssp. tridentata with Festuca idahoensis or Stipa comata or yet earlier, forb-dominated seral vegetation.

Vegetation- Grass canopy cover is generally less than 30\%. Dominant species are Stipa comata, Elymus lanceolatus and Oryzopsis hymenoides. Scattered Chrysothamnus nauseosus shrubs may be present. Forb cover is $10-30 \%$ with moderate diversity. Psoralidium tenuiflorum, Opuntia fragilis, and Phacelia hastata may be abundant. Other common forbs include Machaeranthera canescens, Allium textile and Comandra umbellata. Mosses and lichens are not present.

Soils- Soils are very sandy with little horizon development. They were probably stabilized relatively recently. $60-95 \%$ of the surface is bare.

Other Studies- Lesica (1987) described a similar association from the sandhills near Medicine Lake in Sheridan County, Montana. The Stipa comata / Bouteloua gracilis habitat type of Mueggler and Stewart (1980) is found at lower elevations and has Bouteloua gracilis and many different forbs than Stipa comata / Psoralidium tenuiflorum.

Comments- This vegetation is endemic to sandhills and other areas of very sandy soils. It is clearly a seral community associated with undeveloped soils and periodic disturbance from grazing, fire and/or wind.

\section{Element Code- CEGLMTHP23}

Edition / Author- 99-11-16 / S.V. Cooper, MTNHP

Stipa richardsonii - Festuca idahoensis Herbaceous Vegetation

(STIRIC - FESIDA) Richardson's needlegrass - Idaho fescue

\section{Natural Heritage Conservation Rank-} G1? / S?

Environment- Sites supporting this type are poorly documented in Montana. At least in southwestern Montana it occurs primarily in sheltered environments, such as toeslopes and leeward slopes in the foothills or lower slopes of mountain ranges, from 6,600 to $7,600 \mathrm{ft}$ (there is no reason to suspect that this community is restricted to this elevation range). These are locations one would expect to receive drifted snow or possibly be subirrigated or in some way have augmented moisture regimes. The fact that sites are in collecting positions is borne out by the soils, which are loams or silt loams in texture and have virtually no rock in the upper $20-30 \mathrm{~cm}$ of the profile. Given the soil characteristics and landscape position of the type we infer that soils are primarily aeolian in origin with some colluvial and slopewash fine materials added. Reflecting highly productive nature of these sites, the substrate surface is composed of 
copious litter and basal area and just a trace of exposed soil. This community is very circumscribed in area, usually less than 2-3 acres, because it is apparently confined to small lenses of aeolian materials. Adjacent vegetation is almost invariably dominated by the more mesic plant associations within the Artemisia tridentata ssp. vaseyana and Festuca idahoensis alliances, usually those communities (or phases) denoted by Elymus trachycaulus, Stipa richardsonii, Geranium viscosissimum, Potentilla gracilis and $P$. glandulosa.

Range- This community is currently known from the foothills and lower slopes of the Snowcrest, Gravelly, and Blacktail Mountain Ranges and the hills and ridge complexes between these ranges. It has been documented to occur in Wyoming as well within the Southern Rocky Mountain Steppe - Open Woodland - Coniferous Forest - Alpine Meadow Province.

Vegetation- The shrub component is present as scattered individuals of Artemisia tridentata ssp. vaseyana and Chrysothamnus nauseosus whose cover usually does not exceed trace amounts. Stands with plot data had a very strongly dominant tall graminoid layer that was so dense as to covey the look of an agricultural field when viewed from a distance (other stands, more open with less herbaceous cover, were noted in passing). Stipa richardsonii is conspicuously dominant with cover usually in excess of $70 \%$. Other graminoids of high constancy include Festuca idahoensis, Elymus trachycaulus, Stipa occidentalis, Danthonia intermedia, and Pascopyrum smithii, their individual cover seldom exceeds $10 \%$. The suite of mesic site forbs regularly present includes Gentiana affinis, Potentilla gracilis, Geum triflorum, Cirsium scariosum (or $C$. undulatum), Agoseris glauca, Campanula rotundifolia and Galium boreale; seldom does individual forb cover exceed 5\% and combined forb cover is usually in the range of $5-15 \%$.

Similar Communities- The most similar community in name and all other parameters is Festuca idahoensis - Stipa richardsonii defined by Mueggler and Stewart (1980) for southwestern Montana; their plots also demonstrate $S$. richardsonii to have high canopy cover, significantly greater than that of $F$. idahoensis. We recommend that their type be combined with the $S$. richardsonii $-F$. idahoensis association as this latter name more appropriately describes the community, which is $S$. richardsonii-dominated and similar in composition and abiotic features.

Element Code- CEGL001712

Edition / Author- 99-11-22 / S. V. Cooper, MTNHP

Typha latifolia Western Herbaceous Vegetation (TYPLAT) common cattail

\section{Natural Heritage Conservation Rank- G5 / S5}

Environment- The Typha latifolia community is a broadly distributed, extremely common emergent wetland type that occurs from low to mid elevations (Red Rock Lakes NWR examples occur 400 feet higher than type's cited range [Hansen et al. 1995]), characteristically on permanently to semipermanently flooded sites. Sites on the Refuge often are associated with water depths in excess of four feet and have a deep organic matter accumulated over silts and other fines. However, exposed mineral soil is required for germination and establishment Typha latifolia so that these sites presumably dry out in extreme years so as to facilitate these processes. The Typha latifolia type often occurs in small, individually unmapped patches, as part of a wetland mosaic with Scirpus acutus (in equally deep water) or as narrow stringers along channels removed from the main water bodies (in the case of Red Rocks Lake National Wildlife Refuge ) where Carex utriculata - Carex utriculata communities are found immediately adjacent on sites with shallower water depths. Hansen et al. (1995) indicate that Typha latifolia is not as tolerant of brackish water as is Scirpus acutus but this is not the factor separating these 
sites on RRLNWLR, rather it is probably a matter of priority of establishment and subsequent competition for substrate.

Vegetation- Typha latifolia (or T.

angustifolia), usually exceeding $75 \%$ canopy cover, dominate these sites with few other forbs or graminoids present due to the constantly wet conditions. Scattered individuals from adjacent slightly less wet communities often include Carex utriculata, Scirpus validus, Beckmannia syzigachne, Polygonum amphibium and Epilobium ciliatum.

Element Code- CEGL002010

Edition / Author- 99-11-16 / S.V. Cooper, MTNHP 


\section{Literature
Cited}

Achuff, P. L. and I. G. W. Corns. 1982. Vegetation. In W. D. Holland and G. M. Coen (eds.), Ecological (biophysical) land classification of Banff and Jasper National Parks Vol. 1. Alberta Institute of Pedology Publication No. SS-82-44.

Alexander, R. R., G. R. Hoffman and J. M. Wirsing. 1986. Forest vegetation of the Medicine Bow National Forest in southeastern Wyoming: A habitat type classification. USDA Forest Service Research Paper RM271. Rocky Mountain Forest and Range Experiment Station, Fort Collins, CO. 39 pp.

Anderson, M. et al. 1998. International Classification of Ecological Communities: Terrestrial Vegetation of the United States. Volume II. The National Vegetation Classification System: List of Types. The Nature Conservancy, Arlington, Virginia, USA.

Arno, S. F. and G. E. Gruell. 1983. Fire history of the forest-grassland ecotone in southwestern Montana. Journal of Range Management 36: 332-336.

Arno, S. F., D. G. Simmerman and R.E. Keane. 1985. Forest succession on four habitat types in western Montana. Gen. Tech. Rep. INT-177. Ogden, UT: USDA Forest Service, Intermountain Forest and Range Experiment Station. 74 pp.

Arno, S. F., D. G. Simmerman and R.E. Keane. 1986. Characterizing succession within a forest habitat type - - An approach designed for resource managers. Res. Note. INT-357. Ogden, UT: USDA Forest Service, Intermountain Research Station. 8 pp.
Bailey, R. G. 1995. Description of the ecoregions of the United States. $2^{\text {nd }}$ ed. rev. and expanded ( $1^{\text {st }}$ ed. 1980). Misc. Publ. No. 1391 (rev.), Washington, D. C: USDA Forest Service. 108 pp. with separate map at $1: 7,500,000$.

Baker, W. L. 1983. Alpine vegetation of Wheeler Peak, New Mexico, USA.: Gradient analysis, classification, and biogeography. Arctic and Alpine Research 15(2):223240.

Baker, W. L. 1989. Classification of the riparian vegetation of the montane and subalpine zones in western Colorado. Great Basin Naturalist 49(2):214-228

Bamberg, S. A., and J. Major. 1968. Ecology of the vegetation and soils associated with calcareous parent materials in three alpine regions of Montana. Ecological Monographs 38(2):127-167.

Beetle, A. A., and K. L. Johnson. 1982. Sagebrush in Wyoming. Wyoming Agricultural Experiment Station Bulletin 779. University of Wyoming, Laramie.

Billings, W. D. 1973. Arctic and alpine vegetations: similarities, differences and susceptibility to disturbance. Bioscience 23: 687-704.

Bliss, L.C. 1956. A comparison of plant development in microenvironments of arctic and alpine tundras. Ecological Monographs 26:303-337

Bonham, C. D. and R. T. Ward. 1970. Phytosociological relationships in alpine tufted hairgrass (Deschampsia caespitosa (L.) Beauv.) meadows. Arctic and Alpine Research 2: 267-275.

Booth, W. E. 1950. Flora of Montana, part 1, conifers and monocots. The Research Foundation at Montana State College, Bozeman, MT. 232 pp. 
Booth, W. E., and J. C. Wright. 1966. Flora of Montana, part 2, dicotyledons. Department of Botany and Microbiology, Montana State University, Bozeman, MT. 305 pp.

Bourgeron, P. S., and L. D. Engelking, eds. 1994. A preliminary vegetation classification of the western United States. Unpublished report by The Nature Conservancy, Western Heritage Heritage Task Force. Boulder, CO. 175 pp.

Briggs, G. M. and J. A. MacMahon. 1983. Alpine and subalpine wetland plant communities of the Uinta Mountains, Utah. Great Basin Naturalist 43: 523-530.

Caicco, S. L. 1983. Alpine vegetation of the Copper Basin area, south-central Idaho. Unpublished thesis, University of Idaho, Moscow. 99 pp.

Chadde, S. W., P. L. Hansen and R. D. Pfister. 1988. Wetland plant communities of the northern range, Yellowstone National Park, Montana Riparian Association, School of Forestry, University of Montana, Missoula.

Chaffee, G. B. 1981. Vegetation report, Limestone Hills quarry area. Report prepared for Continental Lime, Inc. by Econ, Inc., Helena, Montana. 41pp. + app.

Cole, D. N. 1982. Vegetation of two drainages in Eagle Cap Wilderness, Wallowa Mountains, Oregon. USDA Forest Service Research Paper INT-288.

Cooper, D. J. and T. R. Cottrell. 1990. Classification of riparian vegetation in the northern Colorado Front Range. Unpublished report prepared for The Nature Conservancy, Colorado Field Office, Boulder. 115 pp.

Cooper, S. V. and P. Lesica. 1992. Plant community classification for alpine vegetation on Beaverhead National Forest, Montana. Conservation Biology Research, Helena, MT. 80 pp.
Cooper, S. V. and P. Lesica. 1997. Plant community classification for alpine vegetation on the Beaverhead National Forest, Montana. Gen. Tech. Rep. INT-GTR-362. Ogden, UT: U. S. Department of Agriculture, Forest Service, Intermountain Research Station. $61 \mathrm{pp}$.

Cooper, S. V., P. Lesica, R. L. DeVelice, and T. McGarvey. 1995. Classification of southwestern Montana plant communities with emphasis on those of Dillon Resource Area, Bureau of Land Management. Montana Natural Heritage Program, Helena, MT. $154 \mathrm{pp}$.

Cooper, S. V., K. E. Neiman, R. Steele and D. W. Roberts. 1987. Forest Habitats of northern Idaho: A second approximation. USDA Forest Service, Intermountain Research Station. General Technical Report INT-236. Intermountain Research Station, Ogden, UT. 135 pp.

Cooper, S. V. and R. D. Pfister. 1981. Forest habitat types of the Blackfeet Indian Reservation. Review Draft, 5/21/81, for Bureau of Indian Affairs, Blackfeet Agency, Browning, MT.

Cooper, S. V. and R. D. Pfister. 1985. Forest habitat types of the Crow and Northern Cheyenne Indian Reservations. Termination report to Bureau of Indian Affairs, Billings Area Office (draft). USDA Forest Service, Intermountain Forest and Range Expriment Station, Ogden, UT.

Coupland, R. T. 1950. Ecology of mixed prairie in Canada. Ecological Monographs. 20(4): 271-315.

Coupland, R. T. 1961. A reconsideration of grassland classification in the Northern Great Plains of North America. Journal of Ecology 49: 135-167. 
Crowe, E. A. and R. R. Clausnitzer. 1997. Mid-Montane Wetland Plant Associations of the Malheur, Umatilla and WallowaWhitman National Forests. USDA Forest Service. Pacific Northwest Region. Technical Paper R6-NR-ECOL-TP-22-97

Daubenmire, R. 1959. A canopy-cover method of vegetational analysis. Northwest Science 33: 131-154.

Daubenmire, R. F. 1968. Plant communities: A textbook of plant synecology. Harper \& Row, New York. 300 pp.

DeVelice, R. L., J. A. Ludwig, W. H. Moir, and F. Ronco Jr. 1986. A classification of forest habitat types of northern New Mexico and southern Colorado. USDA Forest Service General Technical Report RM-131. Rocky Mountain Forest and Range Experiment Station, Ft. Collins, CO. 59 pp.

DeVelice, R. L. 1992. Classification of the plant communities of Beaverhead, Silver Bow and Madison Counties, Montana. Volume I (text). Montana Natural Heritage Program, Helena, MT. 35 pp.

DeVelice, R. L. 1992. Classification of the plant communities of Beaverhead, Silver Bow and Madison Counties, Montana. Volume II (appendices). Montana Natural Heritage Program, Helena, MT. 167 pp.

DeVelice, R. L. and P. Lesica. 1993. Plant community classification for vegetation on BLM lands, Pryor Mountains, Carbon County, Montana. Montana Natural Heritage Program, Helena, MT.

Daubenmire, R. 1959. A canopy-coverage method of vegetational analysis. Northwest Science 33(1):43-64.

Daubenmire, R. and J. B. Daubenmire. 1968. Forest vegetation of eastern Washington and northern Idaho. Washington Agricultural Experiment Station, College of Agriculture, Washington State University. 104 pp.
Daubenmire, R. 1970. Steppe vegetation of Washington. Washington Agricultural Experiment Station Technical Bulletin No. 62. Washington State University, Pullman.

Dorn, R. D. 1984. Vascular Plants of Montana. Mountain West Publishing, Cheyenne, WY. 276 pp.

Douglas, G. W. 1972. Subalpine plant communities of the western North Cascades, Washington. Arctic and Alpine Research. 4: 147-166.

Douglas, G. W. and L. C. Bliss. 1977. Alpine and high subalpine plant communities of the North Cascades Range, Washington and British Columbia. Ecological Monographs 47:113-150.

Driscoll, R.S., D.L. Merkel, D.L. Radloff, D.E. Snyder, and J.S. Hagihara. 1998. An Ecological Land Classification Framework for the United States. U.S. Department of Agriculture, Forest Service, Miscellaneous Publication 1439, Washington, D.C. USA.

Eddleman, L. E. and R. T. Ward. 1984. Phytoedaphic relationships in alpine tundra of north-central Colorado, USA. Arctic and Alpine Research 16: 343-359.

Fitzhugh, E. L., W. H. Moir, J. A. Ludwig and F. Ronco Jr. 1987. Forest habitat types in the Apache, Gila, and part of the Cibola National Forests. USDA Forest Service General Technical Report RM-145, Rocky Mountain Forest and Range Experiment Station, Fort Collins, CO. 116 pp.

FGDC. 1997. Federal Geographic Data Committee Vegetation Classification and Information Standards. FGDC Secretariat, U.S. Geological Survey, Reston, Virginia, USA. http://www.nbs.gov/fgdc.veg/. 
Grossman, D.H. et al. 1998. International Classification of Ecological Communities: Terrestrial Vegetation of the United States. Volume I. The national vegetation classification system: development, status, and applications. The Nature Conservancy, Arlington, Virginia. USA.

Habeck, J. R. and C. M. Choate. 1963. An analysis of krummholz communities at Logan Pass, Glacier National Park. Northwest Science 37:165-166.

Hansen, P. L. 1985. An ecological study of the vegetation of the Grand River/Cedar River, Sioux, and Ashland districts of the Custer National Forest. Unpublished dissertation, South Dakota State University, Brookings, SD.

Hansen, P. L., S. W. Chadde and R. D. Pfister. 1988. Riparian dominance types of Montana. University of Montana Miscellaneous Publication 49. Montana Forest and Conservation Experiment Station, Missoula. MT. $411 \mathrm{pp}$.

Hansen, P. L., and G. R. Hoffman. 1987. The vegetation of the Grand River/Cedar River, Sioux, and Ashland Districts of the Custer National Forest: A habitat type classification. USDA Forest Service, Rocky Mountain Forest and Range Experiment Station. General Techical Report RM-157. Rocky Mountain Forest and Range Experiment Station, Fort Collins, CO. 68 pp.

Hansen, P. L., R. D. Pfister, K. Boggs, B. J. Cook, J. Joy and D. K. Hinckley. 1995. Classification and management of Montana's riparian and wetland sites. Montana Forest and Conservation Experiment Station, School of Forestry, University of Montana. Misc. Publication number 54. $646 \mathrm{pp}$.

Harvey, S. J. 1982. Vegetation of Musselshell and Petroleum Counties, Montana. Unpublished report to the Soil Conservation Service, Roundup, MT.
Heidel, B. L. and S. V. Cooper. 1997. Botanical survey of the Alkali Lake area, Blackfeet Indian Reservation. Unpubished report to the Blackfeet Fish and Wildlife Department and the U. S. Fish and Wildlife Service. Montana Natural Heritage Program, Helena. 35pp. + app.

Hill, M. O. 1979a. DECORANA - A FORTRAN Program for Detrended Correspondence Analysis and Reciprocal Averaging. Ithaca, New York: Cornell University.

Hill, M. O. 1979b. TWINSPAN - A FORTRAN Program for Arranging Multivariate Data in an Ordered Two-Way Table by Classification of the Individuals and Attributes. Ithaca, New York: Cornell University.

Hironaka, M. 1977. Second year's report: habitat type classification for grasslands and shrublands of southern Idaho. College of Forestry, Wildlife and Range Science, University of Idaho, Moscow.

Hironaka, M., M. A. Fosberg and A. H. Winward. 1983. Sagebrush-grass habitat types of southern Idaho. Forestry, Wildlife, and Range Experiment Station Bulletin No. 15, University of Idaho, Moscow. 44 pp.

Hitchcock, C. L. and A. Cronquist. 1973. Flora of the Pacific Northwest. University of Washington Press, Seattle, WA. 730 pp.

Hoffman, G. R. and R. R. Alexander. 1980. Forest vegetation of the Routt National Forest in northwestern Colorado: a habitat type classification. USDA Forest Service Research Paper RM-221. Rocky Mountain Forest and Range Experiment Station, Fort Collins, CO. 41 pp.

Holway, J. G. and R. T. Ward. 1963. Snow and meltwater effects in an area of Colorado alpine. American Midland Naturalist 69(1):189-197 
Hrapko, J. O. and G. H. LaRoi. 1978. The alpine tundra vegetation of Signal Mountain, Jasper National Park. Canadian Journal of Botany 56: 309-332.

Johnston, B. C. 1987. Plant associations of Region Two. Edition 4. USDA Forest Service, Rocky Mountain Region. R2-Ecol87-2. 429 pp.

Johnson, C. and S. Simon. 1987. Plant associations of the Wallowa-Snake Province. USDA Forest Service, WallowaWhitman National Forest Region Six. R6ECOL-TP-255B-86. 373 pp.

Johnson, P. L., and W. D. Billings. 1962. The alpine vegetation of the Beartooth Plateau in relation to cryopedogenic processes and patterns. Ecological Monographs 32(2):105133.

Jorgensen, H. E. 1979. Vegetation of the Yellow Water Triangle. Montana Department of Fish and Game, Helena, MT.

Kartesz, J. 1994. Synonoymized checklist of the vascular flora of the United States, Canada and Greenland, Vols. I \& II. Timber Press, Arlington, VA.

Kittel, G. M. and N. D. Lederer. 1993. A preliminary classification of the riparian vegetation of the Yampa and San Miguel/ Dolores River Basins. Unpublished report prepared for Colorado Department of Health and the Environmental Protection Agency by The Nature Conservancy, Colorado Field Office, Boulder, CO.

Kittel, G., E. VanWie, and M. Damm. 1998. Salix drummondiana/Calamagrostis canadensis plant association. Characterization abstract. In: Osborn, R., G. Kittel, and M. Reid. 1998. Colorado Riparian Plant Associations and Western States Vegetation Classification. CDROM. U.S. Geological Survey, Mid-Continent Ecology Research Center, Fort Collins, CO.
Komarkova, V. K., R. R. Alexander and B. C. Johnston. 1988. Forest vegetation of the Gunnison and parts of the Uncompahgre National Forest: A preliminary habitat type classifiction. USDA Forest Service, Rocky Mountain Forest and Range Experiment Station. Research Paper RM-163. Rocky Mountain Forest and Range Experiment Station, Fort Collins, CO. 65 pp.

Komarkova, V. and P. J. Webber. 1978. An alpine vegetation map of Niwot Ridge, Colorado. Arctic and Alpine Research 10: 1-29.

Kovalchik, B. L. 1987. Riparian zone associations- Deschutes, Ochoco, Fremont and Winema National Forests. USDA Forest Service Technical Paper 279-97. Pacific Northwest Region, Portland, OR. 171 pp.

Kovalchik, B. L. 1993. Riparian plant associations on the National Forests of eastern Washington - Draft version 1. USDA Forest Service, Colville National Forest, Colville, WA. 203 pp.

Krall, J. L., J. R. Stroh, C. S. Cooper and S. R. Chapman. 1971. Effect of time and extent of harvesting basin wildrye. Journal of Range Management 24(6): 414-418.

Langenheim, J. H. 1962. Vegetation and environmental patterns I the Crested Butte area, Gunnison County, Colorado. Ecological Monographs 32:249-285.

Lesica, P. 1986. Vegetation and flora of Pine Butte Fen, Teton County, Montana. Great Basin Naturalist 46: 22-32.

Lesica, P. 1987. Medicine Lakes Sandhills Preserve Design. Prepared for The Nature Conservancy, Helena, MT.

Lesica, P. 1988. Giant Ripple Marks preserve design. Prepared for The Nature Conservancy, Helena, MT. 
Lesica, P. 1989. The vegetation and flora of glaciated prairie potholes on the Blackfeet Indian Reservation, Montana. Prepared for The Nature Conservancy, Helena, MT.

Lesica, P. 1990. Vegetation and sensitive vascular plants of Morrison Lake, Harkness Lakes and Nicholia Creek wetlands, Beaverhead County, Montana. Prepared for Beaverhead National Forest, Dillon, MT. 28 pp.

Lesica, P. 1991. The importance of the Line Creek Plateau for protecting biological diversity in the Greater Yellowstone Ecosystem. Report prepared for The Nature Conservancy, Helena, MT.

Lesica, P. 1994. The distribution of plant community diversity associated with glacial wetlands in the Ovando valley, Montana. Prepared for The Nature Conservancy, Helena, MT.

Lesica, P. and S. V. Cooper. 1998. Succession and disturbance in sandhills vegetation: Constructing models for managing biological diversity. Conservation Biology 13(2): 293-302.

Lesica, P. and S. J. Shelly. 1991. Sensitive, threatened, and endangered vascular plants of Montana. Montana Natural Heritage Program Occasional Publication No. 1. Helena, Montana.

Lewis, M. E. 1970. Alpine rangelands of the Uinta Mountains, Ashley and Wasatch national forests. USDA Forest Service, Intermountain Region, Ogden Utah.

Lewis, M. E. 1975. Plant communities of the Jarbridge Mountain complex, Humboldt National Forest. USDA Forest Service Intermountain Region, Ogden Utah.

Loope, L. L. 1969. Subalpine and alpine vegetation of northeastern Nevada. Unpublished thesis, Duke University, Durham, North Carolina.
Loucks, O. 1996. 100 years after Cowles: A national classification of vegetation. Bulletin of the Ecologiscal Society of America 77: 75-76.

May, D. E. and P. J. Webber. 1982. Spatial and temporal variation of the vegetation and its productivity, Niwot Ridge, Colorado. $\underline{\text { In }}$ J. C. Halfpenny (ed.), Ecological studies in the Colorado alpine. University of Colorado Institute of Arctic and Alpine Research Occasional Paper 37.

Manning, M. E. and W. G. Padgett. 1995. Riparian community type classification for Humboldt and Toiyabe National Forests, Nevada and eastern California. USDA, Forest Service, Intermountain Region. 306 pp.

Mattson, D. J. 1984. Classification and environmental relationships of wetland vegetation in central Yellowstone National Park. Unpublished thesis, University of Idaho, Moscow. 409 pp.

Mauk, R. L. and J. A. Henderson. 1984. Coniferous forest habitat types of northern Utah. USDA Forest Service, Intermountain Forest and Range Experiment Station. General Technical Report INT-170. Intermountain Forest and Range Experiment Station, Ogden, UT. 89 pp.

McGraw, J. B. 1985. Experimental ecology of Dryas octopetala ecotypes. III. Environmental factors and plant growth. Arctic and Alpine Research 17: 229-239.

McLean, A. 1970. Plant communities of the Smilkameen Valley, British Columbia and their relationship to soils. Ecological Monographs 40(4): 403-424.

McNab, W. H. and P. E. Avers. 1994. Ecological subregions of the United States: Section descriptions. WO-WSA-5 USDA Forest Service. Washington, D. C. 52 chapters plus append. 
Montagne, J. M. 1972. Quaternary systems, Wisconsin glaciation. In W. M. Mallory, et al. (eds.). Geologic atlas of the Rocky Mountain region. Rocky Mountain Association of Geologists. Hershfield Press, Denver, $\mathrm{CO}$.

Montagne, C., L. C. Munn, G. A. Nielsen, J. W. Rogers and H. E. Hunter. 1982. Soils of Montana. Bulletin 744. Montana Agricultural Experiment Station, Montana State University, Bozeman, MT.

Moseley, R. K. 1985. Synecological relationships of alpine spike-fescue grasslands in east-central Idaho. M.S. thesis, University of Idaho, Moscow.

Moseley, R.K., R.J. Bursik, M. Manusco. 1991. Floristic inventory of wetlands in Fremont and Teton counties, Idaho. Unpublished report on file IDCDC Dept Fish and Game, Boise, ID. 60 pp

Mueggler, W. F. 1988. Aspen community types of the Intermountain Region. USDA Forest Service General Tecnical Report INT-250. USDA Forest Service, Intermountain Research Station, Ogden, UT. $135 \mathrm{pp}$.

Mueggler, W. F. and R. B. Campbell Jr. 1982. Aspen community types on the Caribou and Targhee National Forests in southeastern Idaho. USDA Forest Service, Intermountain Forest and Range Experiment Station Research Paper INT-294. Ogden, Utah. 32 pp.

Mueggler, W. F. and R. B. Campbell Jr. 1986. Aspen community types of Utah. USDA Forest Service Research Paper INT-362, Intermountain Forest and Range Experiment Station, Ogden, UT.

Mueggler, W. F. and W. L. Stewart. 1980. Grassland and shrubland habitat types of western Montana. USDA Forest Service General Technical Report INT-66. Intermountain Forest and Range Experiment Station, Ogden, Utah. 154 pp.
Muldavin, E. 1994. Oregon Mountains sensitive species and plant community inventory. Unpublished report prepared by the New Mexico Natural Heritage Program, Albuquerque, NM.

Mutz, K. M. and J. Queiroz. 1983. Riparian community classification for the Centennial Mountains and South Fork Salmon River, Idaho. Unpublished report prepared for USDA Forest Service Intermountain Region under contract 53-84M8-2-0048 by Meiiji Resource Consultants, Layton, UT. 168 pp.

Nesser, J. A., G. L. Ford, C. L. Maynard, and D. Page-Dumroese. 1997. Ecological units of the Northern Region: Subsections. Gen. Tech. Rep. INT-GTR-369. Ogden, UT. USDA, Forest Service, Intermountain Research Station. 88 pp.

Noss, R. F. 1985. On characterizing presettlement vegetation: how and why. Natural Areas Journal 5: 5-19.

Padgett, W. G., A. P. Youngblood and A. H. Winward. 1989. Riparian community type classification of Utah and southeastern Idaho. USDA Forest Service Intermountain Region, R4- ECOL-89-01, Intermountain Research Station, Ogden, UT. 191 pp.

Pfister, R. D. 1972. Vegetation and soils in the subalpine forests of Utah. Unpublished dissertation, Washington State University, Pullman. 98 pp.

Pfister, R. D., B. L. Kovalchik, S. F. Arno and R. C. Presby. 1977. Forest habitat types of Montana. USDA Forest Service, Intermountain Forest and Range Experiment Station. General Technical Report INT-34. Intermountain Forest and Range Experiment Station, Ogden, UT. 174 pp.

Pierce, J. R. 1986. Wetland community types of west-central Montana. Unpublished draft report prepared for the USDA Forest Service, Region One, Missoula, MT. 57 pp. 
Preece, S. J. 1950. Floristic and ecological features of the Raft River Mountains of northwestern Utah. M.S. thesis, University of Utah, Salt Lake City.

Ream, R. R. 1964. The vegetation of the Wasatch Mountains, Utah and Idaho. Unpublished dissertation, University of Wisconsin, Madison. 190 pp.

Reed, P. B. Jr. 1988. National list of plant species that occur in wetlands: Northwest (Region 9). U. S. Fish and Wildlife Service Biological Report 88 (26.9). USDI Fish and Wildlife Service, Research and Development, Washington, DC. 89 pp.

Regan, C. M., R. C. Musselman, and J. D. Haines. 1997. Vegetation of the Glacier Lakes Ecosystem Experimental Site. Research Paper. RMRS-RP-1. Fort Collins, CO; U. S. Department of Agriculture, Forest Service, Rocky Mountain Research Station. 36pp.

Richardson, C. A. and D. M. Henderson. 1999. Classification and ordination of the alpine plant communities of Railroad Ridge, White Cloud Peaks, Idaho. Great Basin Naturalist 59(1): 63-78.

Roberts, D. W. 1980. Forest habitat types of the Bear's Paw Mountains and Little Rocky Mountains, Montana. Unpublished M. S. thesis, University of Montana, Missoula, MT.

Rollins, R. C. 1984. Studies in the Cruciferae of western North America II. Contributions to the Gray Herbarium 214: 1-18.

Rollins, R. C. and E. A. Shaw. 1973. The genus Lesquerella (Cruciferae) in North America. Harvard University Press, Cambridge, MA.

Ross, R. L. and H. E. Hunter. 1976. Climax vegetation of Montana based on soils and climate. USDA Soil Conservation Service, Bozeman, Montana. 64 pp.
Rottman, M. L. and E. L. Hartman. 1985. Tundra vegetation of three cirque basins in the northern San Juan Mountains, Colorado. Great Basin Naturalist 45: 87-93.

Schassberger, L. A. 1988. Effects of grazing on the habitat of Astragalus ceramicus var. apus in the Centennial Valley, Montana. M. A. thesis, University of Montana, Missoula.

Scheldt, R. S. and E. W. Tisdale. 1970. Ecology and utilization of curl-leaf mountain mahogany in Idaho. Station Note No. 15. Forest, Wildlife and Range Experiment Station, Univ. of Idaho, Moscow, ID. 2 pp.

Schlatterer, E. F. 1972. A preliminary description of plant communities found on the Sawtooth, White Cloud, Boulder, and Pioneer Mountains. USDA Forest Service Intermountain Region Mimeo Report.

$111 \mathrm{pp}$.

Schultz, L. M. 1984. Taxonomic and geographic limits of Artemisia subgenus Tridentatae (Beetle) McArthur (Asteraceae, Anthemideae). Page 20-45 in E. D. McArthur and B. L. Welch (eds.) Proceedings - Symposium on the biology of Artemisia and Chrysothamnus. USDA Forest Service, Intermountain Research Station, Ogden, UT.

Scott, D. and W. D. Billings. 1964. Effects of environmental factors on standing crop and productivity of an alpine tundra. Ecological Monographs 34: 243-270.

Spence, J. R. and R. J. Shaw. 1981. A checklist of the alpine and vascular flora of the Teton Range, Wyoming, with notes on biology and habitat preference. Great Basin Naturalist 41: 232-242.

Steele, R., R. D. Pfister, R. A. Ryker and J. A. Kittams. 1981. Forest habitat types of central Idaho. USDA Forest Service, Intermountain Forest and Range Experiment Station. General Technical Report INT-114. Ogden, UT. 138 pp. 
Steele, R., S. V. Cooper, D. M. Ondov, D. W. Roberts and R. D. Pfister. 1983. Forest habitat types of eastern Idaho-western Wyoming. USDA Forest Service, Intermountain Forest and Range Experiment Station. General Technical Report INT-144. Intermountain Forest and Range Experiment Station, Ogden, UT. 122 pp.

Tisdale, E. W., M. Hironaka, and M. A. Fosberg. 1965. An area of pristine vegetation in Craters of the Moon National Monument, Idaho. Ecology 46(3):349-352.

Thilenius, J. F. and D. R. Smith. 1985. Vegetation and soils of an alpine range in the Absaroka Mountains, Wyoming. USDA Forest Service General Technical Report RM-121. Rocky Mountain Forest and Range Experiment Station, Ft. Collins, CO. 18 pp.

Tuhy, J. S. 1981. Stream bottom community classification for the Sawtooth Valley, Idaho. Unpublished thesis, University of Idaho, Moscow. 230 pp.

Tuhy, J. S. and S. Jensen. 1982. Riparian classification for the Upper Salmon and Middle Fork Salmon River drainages, Idaho. Unpublished report prepared for the USDA Forest Service Intermountain Region by White Horse Associates, Smithfield, Utah. $183 \mathrm{pp}$.

UNESCO (United Nations Educational, Scientific, and Cultural Organization). 1973. International Classification and Mapping of Vegetation. Serices 6. Ecology and Conservation. United Nations, Paris, France.

Urbanczyk, S. M. and D. M. Henderson. 1994. Classification and ordination of alpine plant communities, Sheep Mountain, Lemhi County, Idaho. Madrono 41(3): 205-223.

U. S. Department of Agriculture, Forest Service. 1987. Ecosystem classification handbook. Forest Service Handbook 12/87 R-1 Supp.1. Missoula, MT: USDA, Forest Service, Northern Region.
U. S. Department of Agriculture, Forest Service, Region 1. 1997. Unpublished plant species databases. Includes: flora, autecology, fire effects, and establishment electronic databases originally in paradox.

Missoula, MT.

Vanderhorst, J. and P. Lesica. 1994. Sensitive plant survey in the Tendoy Mountains, Beaverhead County, Montana. Montana Natural Heritage Program, Helena.

Walford, G., G. Jones, W. Fertig, and K. Houston. 1997. Riparian and wetland plant community types of the Shoshone National Forest. Unpublished draft report prepared by Wyoming Natural Diversity Database, Laramie, WY, for The Nature Conservancy, and the USDA Forest Service. 227 pp.

Willard, B. E. 1979. Plant sociology of alpine tundra, Trail Ridge, Rocky Mountain National Park, Colorado. Colorado School of Mines Quarterly 74: 1-119.

Willard, B. E. and J. W. Marr. 1970. Effects of human activities on alpine tundra ecosystems in Rocky Mountain National Park, Colorado. Biological Conservation 2: 257265.

Williams, C. K. and T. R. Lillybridge. 1983. Forested plant associations of the Okanogan National Forest. USDA Forest Service Pacific Northwest Region. R6-Ecol-132b1983. $140 \mathrm{pp}$.

Williams, C. K. and T. R. Lillybridge. 1985. Forested plant associations of the Colville National Forest. Draft. Unpublished field guide prepared for USDA Forest Service.

Williams, C. K. and B. G. Smith. 1990.

Forested plant associations of the Wenatchee National Forest. Unpublished draft prepared for USDA Forest Service. $219 \mathrm{pp}$. 
Youngblood, A. P. and R. L. Mauk. 1985. Coniferous forest habitat types of central and southern Utah. USDA Forest Service, Intermountain Forest and Range Experiment Station. General Technical Report INT-187. Intermountain Research Station, Ogden, UT. $89 \mathrm{pp}$.

Youngblood, A. P., W. G. Padgett and A. H. Winward. 1985. Riparian community type classification of eastern Idaho - western Wyoming. USDA Forest Service Region 4 Ecology 85-01, Intermountain Research Station, Ogden, UT.

Zamora, B. and P. T. Tueller. 1973. Artemisia arbuscula, A. longiloba and A. nova habitat types in northern Nevada. Great Basin Naturalist 33: 225-242. 
Appendix A. Flora of the Beaverhead Mountains Section

Trees

\begin{tabular}{llll}
$\begin{array}{l}\text { USFS Reg. } \\
\mathbf{1} \text { Code }\end{array}$ & Synonymy & Species Name & Common Name \\
\hline ABILAS & ABILAS & Abies lasiocarpa & Subalpine Fir \\
JUNSCO & JUNSCO & Juniperus scopulorum & Rocky Mountain Juniper \\
LARLYA & LARLYA & Larix lyallii & Subalpine Larch \\
PICENG & PICENG & Picea engelmannii & Engelmann Spruce \\
PINALB & PINALB & Pinus albicaulis & Whitebark Pine \\
PINCON & PINCON & Pinus contorta & Lodgepole Pine \\
PINFLE & PINFLE & Pinus flexilis & Limber Pine \\
PINMON & PINMON & Pinus monticola & Western White Pine \\
PINPON & PINPON & Pinus ponderosa & Ponderosa Pine \\
POPANG & POPANG & Populus angustifolia & Narrowleaf Cottonwood \\
POPBST & POPBST & Populus balsamifera ssp. trichocarpa & Balsam Poplar \\
POPTRE & POPTRE & Populus tremuloides & Quaking Aspen \\
PSEMEN & PSEMEN & Pseudotsuga menziesii & Douglas-fir
\end{tabular}




\section{Appendix A. Flora of the Beaverhead Mountains Section Shrubs}

\begin{tabular}{|c|c|c|c|}
\hline $\begin{array}{l}\text { USFS Reg. } \\
1 \text { Code }\end{array}$ & Synonymy & Species Name & Common Name \\
\hline ACEGLA & ACEGLA & Acer glabrum & Rocky Mountain Maple \\
\hline ALNINC & ALNINC & Alnus incana & Mountain Alder \\
\hline ALNVSS & ALNVSS & Alnus viridis ssp. sinuata & \\
\hline AMEALN & AMEALN & Amelanchier alnifolia & Western Serviceberry \\
\hline AMEUTA & AMEUTA & Amelanchier utahensis & Utah Serviceberry \\
\hline ARCUVA & ARCUVA & Arctostaphylos uva-ursi & Kinnikinnick \\
\hline ARTARB & ARTARB & Artemisia arbuscula & Low Sagebrush \\
\hline ARTASL & ARTLOL & Artemisia arbuscula ssp. longiloba & Alkali Sagebrush \\
\hline ARTCAN & ARTCAN & Artemisia cana & Silver Sagebrush \\
\hline ARTFRI & ARTFRI & Artemisia frigida & Fringed Sagewort \\
\hline ARTNOV & ARTNOV & Artemisia nova & Black Sagebrush \\
\hline ARTTST & ARTTST & Artemisia tridentata ssp. tridentata & Basin Big Sagebrush \\
\hline ARTTSV & ARTTSV & Artemisia tridentata ssp. vaseyana & Mountain Big Sagebrush \\
\hline ARTTSW & ARTTSW & Artemisia tridentata ssp. wyomingensis & Wyoming Big Sagebrush \\
\hline ARTTRP & ARTTRP & Artemisia tripartita & Three-tip Sagebrush \\
\hline ATRGAR & ATRNUT & Atriplex gardneri & Gardner's Saltsage \\
\hline ATRNUT & ATRNUT & Atriplex nuttallii & Saltsage \\
\hline BETGLA & BETGLA & Betula glandulosa & Bog Birch \\
\hline BETOCC & BETOCC & Betula occidentalis & Water Birch \\
\hline CASMER & CASMER & Cassiope mertensiana & Mertens' Mountain Heather \\
\hline CASTET & CASTET & Cassiope tetragona & Four-angled Mountain Heather \\
\hline CEAVEL & CEAVEL & Ceanothus velutinus & Mountain Balm \\
\hline CERLED & CERLED & Cercocarpus ledifolius & Curl-leaf Mountain-mahogany \\
\hline CHIUMB & CHIUMB & Chimaphila umbellata & Common Prince's-pine \\
\hline CHRNAU & CHRNAU & Chrysothamnus nauseosus & Rubber Rabbitbrush \\
\hline CHRVIS & CHRVIS & Chrysothamnus viscidiflorus & Green Rabbitbrush \\
\hline CLECOU & CLECOU & Clematis columbiana & Matted Clematis \\
\hline CLELIG & CLELIG & Clematis ligusticifolia & Western Virgins-bower \\
\hline CLEOCC & CLEOCC & Clematis occidentalis & Western Blue Virginsbower \\
\hline CORSER & CORSTO & Cornus sericea & Red-osier Dogwood \\
\hline CRADOU & CRADOU & Crataegus douglasii & Black Hawthorn \\
\hline DRYOCT & DRYOCT & Dryas octopetala & White Dryas \\
\hline ELACOM & ELACOM & Elaeagnus commutata & Silverberry \\
\hline ERINAN & HAPNAN & Ericameria nana & Dwarf Goldenweed \\
\hline ERIMIC & ERIMIC & Eriogonum microthecum & Slenderbush Buckwheat \\
\hline GAUHUM & GAUHUM & Gaultheria humifusa & Alpine Wintergreen \\
\hline GUTSAR & GUTSAR & Gutierrezia sarothrae & Broom Snakeweed \\
\hline JUNCOM & JUNCOM & Juniperus communis & Common Juniper \\
\hline KALMIC & KALMIC & Kalmia microphylla & Small-leaved Laurel \\
\hline KRALAN & CERLAN & Krascheninnikovia lanata & Winterfat \\
\hline LEDGLA & LEDGLA & Ledum glandulosum & Labrador-tea \\
\hline LEPPUN & LEPPUN & Leptodactylon pungens & Prickly Phlox \\
\hline LINBOR & LINBOR & Linnaea borealis & Twinflower \\
\hline LONINV & LONINV & Lonicera involucrata & Twin-berry \\
\hline LONUTA & LONUTA & Lonicera utahensis & Utah Honeysuckle \\
\hline MAHREP & BERREP & Mahonia repens & Creeping Oregongrape \\
\hline MENFER & MENFER & Menziesia ferruginea & Menziesia \\
\hline PENFRU & PENFRU & Penstemon fruticosus & Bush Penstemon \\
\hline PENFLO & POTFRU & Pentaphylloides floribunda & Shrubby Cinquefoil \\
\hline PHILEW & PHILEW & Philadelphus lewisii & Mockorange; Syringa \\
\hline
\end{tabular}




\section{Appendix A. Flora of the Beaverhead Mountains Section Shrubs}

\begin{tabular}{|c|c|c|c|}
\hline $\begin{array}{l}\text { USFS Reg. } \\
1 \text { Code }\end{array}$ & Synonymy & Species Name & Common Name \\
\hline PHYEMP & PHYEMP & Phyllodoce empetriformis & Red Mountain-heath \\
\hline PHYGLA & PHYGLA & Phyllodoce glanduliflora & Yellow Mountain-heath \\
\hline PHYMAL & PHYMAL & Physocarpus malvaceus & Mallow Ninebark \\
\hline PRUVIR & PRUVIR & Prunus virginiana & Common Chokecherry \\
\hline PURTRI & PURTRI & Purshia tridentata & Antelope Bitter-brush \\
\hline RHAALN & RHAALN & Rhamnus alnifolia & Alder Buckthorn \\
\hline RHUTRI & RHUTRI & Rhus trilobata & Skunk-bush Sumac \\
\hline RIBAME & RIBAME & Ribes americanum & Black Currant \\
\hline RIBAUR & RIBAUR & Ribes aureum & Golden Currant \\
\hline RIBCER & RIBCER & Ribes cereum & Squaw Currant \\
\hline RIBHUD & RIBHUD & Ribes hudsonianum & Stinking Currant \\
\hline RIBINE & RIBINE & Ribes inerme & Whitestem Gooseberry \\
\hline RIBIRR & RIBOSI & Ribes irriguum & Idaho Gooseberry \\
\hline RIBLAC & RIBLAC & Ribes lacustre & Swamp Currant \\
\hline RIBMON & RIBMON & Ribes montigenum & Mountain Gooseberry \\
\hline RIBVIS & RIBVIS & Ribes viscosissimum & Sticky Currant \\
\hline ROSACI & ROSACI & Rosa acicularis & Prickly Rose \\
\hline ROSARK & ROSARK & Rosa arkansana & Arkansas Rose \\
\hline ROSWOO & ROSWOO & Rosa woodsii & Woods Rose \\
\hline RUBIDA & RUBIDA & Rubus idaeus & Red Raspberry \\
\hline RUBLAC & RUBLAC & Rubus laciniatus & Evergreen Blackberry \\
\hline RUBPAR & RUBPAR & Rubus parviflorus & Thimbleberry \\
\hline SALARC & SALARC & Salix arctica & Artic Willow \\
\hline SALBAR & SALBAR & Salix barclayi & Barclay's Willow \\
\hline SALBEB & SALBEB & Salix bebbiana & Bebb Willow \\
\hline SALBOO & SALBOO & Salix boothii & Booth Willow \\
\hline SALBRA & SALBRA & Salix brachycarpa & Short-fruited Willow \\
\hline SALCAN & SALCAN & Salix candida & Hoary Willow \\
\hline SALDIS & SALDIS & Salix discolor & Pussy Willow \\
\hline SALDRU & SALDRU & Salix drummondiana & Drummond Willow \\
\hline SALEXI & SALEXI & Salix exigua & Sandbar Willow \\
\hline SALFAR & SALFAR & Salix farriae & Farr's Willow \\
\hline SALGEY & SALGEY & Salix geyeriana & Geyer Willow \\
\hline SALGLA & SALGLA & Salix glauca & Glaucous Willow \\
\hline SALLEM & SALLEM & Salix lemmonii & Lemmon's Willow \\
\hline SALLUT & SALLUT & Salix lutea & Watson Willow \\
\hline SALPLA & SALPLA & Salix planifolia & Planeleaf Willow \\
\hline SALRIG & SALRIG & Salix rigida & Diamond Willow \\
\hline SALSCO & SALSCO & Salix scouleriana & Scouler Willow \\
\hline SALWOL & SALWOL & Salix wolfii & Wolf's Willow \\
\hline SAMCER & SAMCER & Sambucus cerulea & Blue Elderberry \\
\hline SAMRAC & SAMRAC & Sambucus racemosa & Red Elderberry \\
\hline SARVER & SARVER & Sarcobatus vermiculatus & Black Greasewood \\
\hline SHEARG & SHEARG & Shepherdia argentea & Thorny Buffaloberry \\
\hline SHECAN & SHECAN & Shepherdia canadensis & Canada Buffaloberry \\
\hline SORSCO & SORSCO & Sorbus scopulina & Cascade Mountain-ash \\
\hline SPIBET & SPIBET & Spiraea betulifolia & Shiny-leaf Spiraea \\
\hline SYMALB & SYMALB & Symphoricarpos albus & Common Snowberry \\
\hline SYMORE & SYMORE & Symphoricarpos oreophilus & Mountain Snowberry \\
\hline TETCAN & TETCAN & Tetradymia canescens & Gray Horse-brush \\
\hline
\end{tabular}


Appendix A. Flora of the Beaverhead Mountains Section

Shrubs

\begin{tabular}{llll}
$\begin{array}{l}\text { USFS Reg. } \\
\text { 1 Code }\end{array}$ & Synonymy & Species Name & Common Name \\
\hline VACCES & VACCES & Vaccinium cespitosum & Dwarf Huckleberry \\
VACMEM & VACMEM & Vaccinium membranaceum & Big Huckleberry \\
VACMYR & VACMYR & Vaccinium myrtillus & Dwarf Bilberry \\
VACSCO & VACSCO & Vaccinium scoparium & Whortleberry
\end{tabular}




\section{Appendix A. Flora of the Beaverhead Mountains Section Forbs}

\begin{tabular}{|c|c|c|c|}
\hline $\begin{array}{l}\text { USFS Reg. } \\
1 \text { Code }\end{array}$ & Synonymy & Species Name & Common Name \\
\hline ACHMIL & ACHMIL & Achillea millefolium & Common Yarrow \\
\hline ACOCOL & ACOCOL & Aconitum columbianum & Columbian Monkshood \\
\hline ACTRUB & ACTRUB & Actaea rubra & Baneberry \\
\hline AGACUS & AGACUS & Agastache cusickii & Cusick's Giant-hyssop \\
\hline AGAURT & AGAURT & Agastache urticifolia & Nettle-leaf Giant-hyssop \\
\hline AGOAUR & AGOAUR & Agoseris aurantiaca & Orange Agoseris \\
\hline AGOGLA & AGOGLA & Agoseris glauca & Pale Agoseris \\
\hline AGOHET & AGOHET & Agoseris heterophylla & Annual Agoseris \\
\hline AGOLAC & AGOLAC & Agoseris lackschewitzii & Pink Agoseris \\
\hline ALLBRA & ALLBRA & Allium brandegei & Brandegee Onion \\
\hline ALLBRE & ALLBRE & Allium brevistylum & Short-style Onion \\
\hline ALLCER & ALLCER & Allium cernuum & Nodding Onion \\
\hline ALLGEY & ALLGEY & Allium geyeri & Geyer's Onion \\
\hline ALLSCH & ALLSCH & Allium schoenoprasum & Chives \\
\hline ALLTEX & ALLTEX & Allium textile & Textile Onion \\
\hline ALLVIR & ALLVIR & Allotropa virgata & Candystick \\
\hline ALYALY & ALYALY & Alyssum alyssoides & Pale Alyssum \\
\hline ALYDES & ALYDES & Alyssum desertorum & Desert Alyssum \\
\hline ANAMAR & ANAMAR & Anaphalis margaritacea & Common Pearly-everlasting \\
\hline ANDFIL & ANDFIL & Androsace filiformis & Slender-stemmed Fairy-candelabra \\
\hline ANDOCC & ANDOCC & Androsace occidentalis & Western Fairy-candelabra \\
\hline ANDSEP & ANDSEP & Androsace septentrionalis & Northern Fairy-candelabra \\
\hline ANECYL & ANECYL & Anemone cylindrica & Candle Anemone \\
\hline ANEDRU & ANEDRU & Anemone drummondii & Drummond's Anemone \\
\hline ANEMUL & ANEMUL & Anemone multifida & Cliff Anemone \\
\hline ANENUT & ANEPAT & Anemone nuttalliana & Pasqueflower \\
\hline ANEPAR & ANEPAR & Anemone parviflora & Small-flowered Anemone \\
\hline ANETET & ANEMVT & Anemone tetonensis & Teton Anemone \\
\hline ANGARG & ANGARG & Angelica arguta & Sharptooth Angelica \\
\hline ANGROS & ANGROS & Angelica roseana & Rose's Angelica \\
\hline ANTALP & ANTALP & Antennaria alpina & Alpine Pussy-toes \\
\hline ANTANA & ANTANA & Antennaria anaphaloides & Tall Pussy-toes \\
\hline ANTARO & ANTARO & Antennaria aromatica & Aromatic Pussy-toes \\
\hline ANTCOR & ANTCOR & Antennaria corymbosa & Meadow Pussy-toes \\
\hline ANTDIM & ANTDIM & Antennaria dimorpha & Low Pussy-toes \\
\hline ANTLAN & ANTLAN & Antennaria lanata & Woolly Pussy-toes \\
\hline ANTLUZ & ANTLUZ & Antennaria luzuloides & Woodrush Pussy-toes \\
\hline ANTMIC & ANTMIC & Antennaria microphylla & Rosy Pussy-toes \\
\hline ANTNEG & ANTNEG & Antennaria neglecta & Field Pussy-toes \\
\hline ANTPAR & ANTPAR & Antennaria parvifolia & Nuttall's Pussy-toes \\
\hline ANTRAC & ANTRAC & Antennaria racemosa & Raceme Pussy-toes \\
\hline ANTROS & ANTMIC & Antennaria rosea & Rosy Pussy-toes \\
\hline ANTUMB & ANTUMB & Antennaria umbrinella & Umber Pussy-toes \\
\hline APOAND & APOAND & Apocynum androsaemifolium & Spreading Dogbane \\
\hline APOCAN & APOCAN & Apocynum cannabinum & Hemp Dogbane \\
\hline AQUCOE & AQUCOE & Aquilegia coerulea & Colorado Columbine \\
\hline AQUFLA & AQUFLA & Aquilegia flavescens & Yellow Columbine \\
\hline AQUFOR & AQUFOR & Aquilegia formosa & Sitka Columbine \\
\hline ARATHA & ARATHA & Arabidopsis thaliana & Mouse-ear Cress \\
\hline
\end{tabular}




\section{Appendix A. Flora of the Beaverhead Mountains Section Forbs}

\begin{tabular}{|c|c|c|c|}
\hline $\begin{array}{l}\text { USFS Reg. } \\
1 \text { Code } \\
\end{array}$ & Synonymy & Species Name & Common Name \\
\hline ARADRU & ARADRU & Arabis drummondii & Drummond's Rockcress \\
\hline ARAFEC & ARAFEC & Arabis fecunda & Sapphire Rockcress \\
\hline ARAGLA & ARAGLA & Arabis glabra & Towermustard \\
\hline ARAHIR & ARAHIR & Arabis hirsuta & Hairy Rockcress \\
\hline ARAHOL & ARAHOL & Arabis holboellii & Holboell's Rockcress \\
\hline ARALEM & ARALEM & Arabis lemmonii & Lemmon's Rockcress \\
\hline ARALYA & ARALYA & Arabis lyallii & Lyall's Rockcress \\
\hline ARAMIC & ARAMIC & Arabis microphylla & Littleleaf Rockcress \\
\hline ARANUT & ARANUT & Arabis nuttallii & Nuttall's Rockcress \\
\hline ARASPA & ARASPA & Arabis sparsiflora & Sicklepod Rockcress \\
\hline ARECAP & ARECAP & Arenaria capillaris & Thread-leaved Sandwort \\
\hline ARECON & ARECON & Arenaria congesta & Ballhead Sandwort \\
\hline AREKIN & AREKIN & Arenaria kingii & King's Sandwort \\
\hline ARGANS & POTANS & Argentina anserina & Silverweed Cinquefoil \\
\hline ARNALP & ARNALP & Arnica alpina & Alpine Arnica \\
\hline ARNCHA & ARNCHA & Arnica chamissonis & Meadow Arnica \\
\hline ARNCOR & ARNCOR & Arnica cordifolia & Heart-leaf Arnica \\
\hline ARNDIV & ARNDIV & Arnica diversifolia & Sticky Arnica \\
\hline ARNFUL & ARNFUL & Arnica fulgens & Orange Arnica \\
\hline ARNLAT & ARNLAT & Arnica latifolia & Broadleaf Arnica \\
\hline ARNLON & ARNLON & Arnica longifolia & Longleaf Arnica \\
\hline ARNMOL & ARNMOL & Arnica mollis & Hairy Arnica \\
\hline ARNPAR & ARNPAR & Arnica parryi & Nodding Arnica \\
\hline ARNRYD & ARNRYD & Arnica rydbergii & Rydberg's Arnica \\
\hline ARNSOR & ARNSOR & Arnica sororia & Twin Arnica \\
\hline ARTCAM & ARTCAM & Artemisia campestris & Pacific Sagewort \\
\hline ARTDRA & ARTDRA & Artemisia dracunculus & Tarragon \\
\hline ARTLUD & ARTLUD & Artemisia ludoviciana & Prairie Sagewort \\
\hline ARTMIC & ARTMIC & Artemisia michauxiana & Michaux Mugwort \\
\hline ARTSCO & ARTSCO & Artemisia scopulorum & Rocky Mountain Sagewort \\
\hline ASCSPE & ASCSPE & Asclepias speciosa & Showy Milkweed \\
\hline ASTALG & ASTALG & Aster alpigenus & Alpine Aster \\
\hline ASTASC & ASTCHI & Aster ascendens & Long-leaved Aster \\
\hline ASTCAM & ASTCAM & Aster campestris & Meadow Aster \\
\hline ASTCHI & ASTCHI & Aster chilensis & Long-leaved Aster \\
\hline ASTCOM & ASTFAL & Aster commutatus & White-prairie Aster \\
\hline ASTCON & ASTCON & Aster conspicuus & Showy Aster \\
\hline ASTEAT & ASTEAT & Aster eatonii & Eaton's Aster \\
\hline ASTENG & ASTENG & Aster engelmannii & Engelmann's Aster \\
\hline ASTFAL & ASTFAL & Aster falcatus & White-prairie Aster \\
\hline ASTFOL & ASTFOL & Aster foliaceus & Leafy Aster \\
\hline ASTHES & ASTHES & Aster hesperius & Marsh Aster \\
\hline ASTINT & ASTINT & Aster integrifolius & Thick-stemmed Aster \\
\hline ASTLAE & ASTLAE & Aster laevis & Smooth Aster \\
\hline ASTMOD & ASTMOD & Aster modestus & Few-flowered Aster \\
\hline ASTOCC & ASTOCC & Aster occidentalis & Western Aster \\
\hline ASTPER & ASTPER & Aster perelegans & Elegant Aster \\
\hline ASTSCO & ASTSCO & Aster scopulorum & Crag Aster \\
\hline ASTSIB & ASTSIB & Aster sibiricus & Artic Aster \\
\hline
\end{tabular}




\section{Appendix A. Flora of the Beaverhead Mountains Section Forbs}

\begin{tabular}{|c|c|c|c|}
\hline $\begin{array}{l}\text { USFS Reg. } \\
1 \text { Code }\end{array}$ & Synonymy & Species Name & Common Name \\
\hline ASTSTE & ASTSTE & Aster stenomeres & Rocky Mountain Aster \\
\hline ASTSUB & ASTSUB & Aster subspicatus & Douglas' Aster \\
\hline ASTABO & ASTAUS & Astragalus aboriginum & Indian Milk-vetch \\
\hline ASTADS & ASTADS & Astragalus adsurgens & Standing Milk-vetch \\
\hline ASTAGR & ASTAGR & Astragalus agrestis & Field Milk-vetch \\
\hline ASTALP & ASTALP & Astragalus alpinus & Alpine Milk-vetch \\
\hline ASTAME & ASTAME & Astragalus americanus & American Milk-vetch \\
\hline ASTARG & ASTARG & Astragalus argophyllus & Silver-leaved Milk-vetch \\
\hline ASTATR & ASTATR & Astragalus atropubescens & Kelsey's Milk-vetch \\
\hline ASTBOU & ASTBOU & Astragalus bourgovii & Bourgeau's Milk-vetch \\
\hline ASTCAN & ASTCAN & Astragalus canadensis & Canada Milk-vetch \\
\hline ASTCER & ASTCER & Astragalus ceramicus & Painted Milk-vetch \\
\hline ASTCRA & ASTCRA & Astragalus crassicarpus & Ground Plum \\
\hline ASTDRU & ASTDRU & Astragalus drummondii & Drummond's Milk-vetch \\
\hline ASTFLE & ASTFLE & Astragalus flexuosus & Wiry Milk-vetch \\
\hline ASTGIL & ASTGIL & Astragalus gilviflorus & Plains Orophaca \\
\hline ASTKEN & ASTKEN & Astragalus kentrophyta & Thistle Milk-vetch \\
\hline ASTLEN & ASTLEN & Astragalus lentiginosus & Freckled Milk-vetch \\
\hline ASTLEP & ASTLEP & Astragalus leptaleus & Park Milk-vetch \\
\hline ASTMIS & ASTMIS & Astragalus miser & Weedy Milk-vetch \\
\hline ASTMIO & ASTMIO & Astragalus missouriensis & Missouri Milk-vetch \\
\hline ASTPLA & ASTPLA & Astragalus platytropis & Broad-keeled Milk-vetch \\
\hline ASTPUR & ASTPUR & Astragalus purshii & Pursh's Milk-vetch \\
\hline ASTROB & ASTROB & Astragalus robbinsii & Robbins' Milk-vetch \\
\hline ASTSCA & ASTSCA & Astragalus scaphoides & Bitterroot Milk-vetch \\
\hline ATRARG & ATRARG & Atriplex argentea & Silverscale \\
\hline ATRPAT & ATRPAT & Atriplex patula & Common Orache \\
\hline BALHOO & BALHOO & Balsamorhiza hookeri & Hooker's Balsamroot \\
\hline BALSAG & BALSAG & Balsamorhiza sagittata & Arrowleaf Balsamroot \\
\hline BARORT & BARORT & Barbarea orthoceras & American Wintercress \\
\hline BESRUB & BESRUB & Besseya rubra & Bed Besseya \\
\hline BESWYO & BESWYO & Besseya wyomingensis & Wyoming Kittentail \\
\hline BRIGRA & BRIGRA & Brickellia grandiflora & Large-flowered Brickellia \\
\hline BUPAME & BUPAME & Bupleurum americanum & American Thorough-wax \\
\hline CALHER & CALHER & Callitriche hermaphroditica & Autumnal Water-starwort \\
\hline CALAPI & CALAPI & Calochortus apiculatus & Pointed Mariposa \\
\hline CALNUT & CALNUT & Calochortus nuttallii & Sego-lilly \\
\hline CALLEP & CALLEP & Caltha leptosepala & Elkslip Marshmarigold \\
\hline CALBUL & CALBUL & Calypso bulbosa & Fairy-slipper \\
\hline CAMQUA & CAMQUA & Camassia quamash & Common Camas \\
\hline CAMMIC & CAMMIC & Camelina microcarpa & Littlepod Falseflax \\
\hline CAMPAR & CAMPAR & Campanula parryi & Parry's Bellflower \\
\hline CAMROT & CAMROT & Campanula rotundifolia & Harebell \\
\hline CAMSCA & CAMSCA & Campanula scabrella & Rough Bellflower \\
\hline CAMUNI & CAMUNI & Campanula uniflora & Arctic Bellflower \\
\hline CAPBUR & CAPBUR & Capsella bursa-pastoris & Shepherd's-purse \\
\hline CAROLI & CAROLI & Cardamine oligosperma & Few-seeded Bittercress \\
\hline CARDRA & CARDRA & Cardaria draba & Hoary Pepperwort \\
\hline CARNUT & CARNUT & Carduus nutans & Musk Thistle \\
\hline
\end{tabular}




\section{Appendix A. Flora of the Beaverhead Mountains Section Forbs}

\begin{tabular}{|c|c|c|c|}
\hline $\begin{array}{l}\text { USFS Reg. } \\
1 \text { Code }\end{array}$ & Synonymy & Species Name & Common Name \\
\hline CARCAR & CARCAR & Carum carvi & Caraway \\
\hline CASANG & CASANG & Castilleja angustifolia & Northwest Paintbrush \\
\hline CASCHR & CASCHR & Castilleja chromosa & Desert Paintbrush \\
\hline CASCOV & CASCOV & Castilleja covilleana & Rocky Mountain Paintbrush \\
\hline CASCRI & CASCRI & Castilleja crista-galli & Cocks-comb Paintbrush \\
\hline CASCUS & CASCUS & Castilleja cusickii & Cusick's Paintbrush \\
\hline CASFLA & CASFLA & Castilleja flava & Yellow Paintbrush \\
\hline CASGRA & CASGRA & Castilleja gracillima & Slender Paintbrush \\
\hline CASHIS & CASHIS & Castilleja hispida & Harsh Paintbrush \\
\hline CASLIN & CASLIN & Castilleja linariifolia & Narrow-leaved Paintbrush \\
\hline CASLON & CASLON & Castilleja longispica & White Paintbrush \\
\hline CASLUT & CASLUT & Castilleja lutescens & Yellowish Paintbrush \\
\hline CASMIN & CASMIN & Castilleja miniata & Scarlet Paintbrush \\
\hline CASNIV & CASNIV & Castilleja nivea & Snow Paintbrush \\
\hline CASPAL & CASPAL & Castilleja pallescens & Palish Indian-paintbrush \\
\hline CASPUL & CASPUL & Castilleja pulchella & Showy Indian-paintbrush \\
\hline CASSUL & CASSUL & Castilleja sulphurea & Sulphur Paintbrush \\
\hline CENMAC & CENMAC & Centaurea maculosa & Spotted Knapweed \\
\hline CERARV & CERARV & Cerastium arvense & Field Chickweed \\
\hline CERBEE & CERBEE & Cerastium beeringianum & Alpine Chickweed \\
\hline CERNUT & CERNUT & Cerastium nutans & Nodding Chickweed \\
\hline CERVUL & CERVUL & Cerastium vulgatum & Common Chickweed \\
\hline CHADOU & CHADOU & Chaenactis douglasii & Hoary Chaenactis \\
\hline CHEALB & CHEALB & Chenopodium album & Lambsquarter \\
\hline CHEFRE & CHEFRE & Chenopodium fremontii & Fremont's Goosefoot \\
\hline CHEGLA & CHEGLA & Chenopodium glaucum & Oakleaf Goosefoot \\
\hline CHERUB & CHERUB & Chenopodium rubrum & Red Goosefoot \\
\hline CHITWE & CHITWE & Chionophila tweedyi & Chionophila \\
\hline CICDOU & CICDOU & Cicuta douglasii & Douglas Water-hemlock \\
\hline CIRARV & CIRARV & Cirsium arvense & Canada Thistle \\
\hline CIRFLO & CIRFLO & Cirsium flodmanii & Flodman's Thistle \\
\hline CIRFOL & CIRFOL & Cirsium foliosum & Leafy Thistle \\
\hline CIRLON & CIRLON & Cirsium longistylum & Long-styled Thistle \\
\hline CIRSCA & CIRSCA & Cirsium scariosum & Elk Thistle \\
\hline CIRSUB & CIRSUB & Cirsium subniveum & Jackson's Hole Thistle \\
\hline CIRUND & CIRUND & Cirsium undulatum & Wavy-leaved Thistle \\
\hline CIRVUL & CIRVUL & Cirsium vulgare & Bull Thistle \\
\hline CLALAN & CLALAN & Claytonia lanceolata & Western Springbeauty \\
\hline CLECVC & CLEOVC & Clematis columbiana var. columbi & Columbia Clematis \\
\hline CLEHIR & CLEHIR & Clematis hirsutissima & Vaseflower Clematis \\
\hline COLPAR & COLPAR & Collinsia parviflora & Small-flowered Blue-eyed Mary \\
\hline COLLIN & COLLIN & Collomia linearis & Narrow-leaf Collomia \\
\hline COLTEN & COLTEN & Collomia tenella & Diffuse Collomia \\
\hline COMUMB & COMUMB & Comandra umbellata & Bastard Toad-flax \\
\hline CONMAC & CONMAC & Conium maculatum & Poison Hemlock \\
\hline COPOCC & COPOCC & Coptis occidentalis & Western Goldthread \\
\hline CORAUR & CORAUR & Corydalis aurea & Golden Corydalis \\
\hline CREACU & CREACU & Crepis acuminata & Tapertip Hawksbeard \\
\hline CREATR & CREATR & Crepis atribarba & Slender Hawksbeard \\
\hline
\end{tabular}




\section{Appendix A. Flora of the Beaverhead Mountains Section Forbs}

\begin{tabular}{|c|c|c|c|}
\hline $\begin{array}{l}\text { USFS Reg. } \\
1 \text { Code } \\
\end{array}$ & Synonymy & Species Name & Common Name \\
\hline CREINT & CREINT & Crepis intermedia & Gray Hawksbeard \\
\hline CREMOD & CREMOD & Crepis modocensis & Low Hawksbeard \\
\hline CREOCC & CREOCC & Crepis occidentalis & Western Hawksbeard \\
\hline CRERUN & CRERUN & Crepis runcinata & Meadow Hawksbeard \\
\hline CRYAFF & CRYAFF & Cryptantha affinis & Slender Cryptantha \\
\hline CRYAMB & CRYAMB & Cryptantha ambigua & Obscure Cryptantha \\
\hline CRYCEL & CRYCEL & Cryptantha celosioides & Northern Cryptantha \\
\hline CRYFEN & CRYFEN & Cryptantha fendleri & Fendler's Cryptantha \\
\hline CRYHUM & CRYHUM & Cryptantha humilis & Small Cryptantha \\
\hline CRYINT & CRYINT & Cryptantha interrupta & Bristly Cryptantha \\
\hline CRYSPI & CRYINT & Cryptantha spiculifera & Bristly Cryptantha \\
\hline CYMBIP & CYMBIP & Cymopterus bipinnatus & Hayden's Cymopterus \\
\hline CYMGLA & CYMGLA & Cymopterus glaucus & Grayish Cymopterus \\
\hline CYNOFF & CYNOFF & Cynoglossum officinale & Common Hound's-tongue \\
\hline DELAND & DELAND & Delphinium andersonii & Anderson's Larkspur \\
\hline DELBIC & DELBIC & Delphinium bicolor & Little Larkspur \\
\hline DELDEP & DELDEP & Delphinium depauperatum & Slim Larkspur \\
\hline DELGLU & DELGLA & Delphinium glaucescens & Pale Larkspur \\
\hline DELGLA & DELGLA & Delphinium glaucum & Pale Larkspur \\
\hline DELOCC & DELOCC & Delphinium occidentale & Western Larkspur \\
\hline DESPIN & DESPIN & Descurainia pinnata & Pinnate Tansymustard \\
\hline DESSOP & DESSOP & Descurainia sophia & Flixweed Tansymustard \\
\hline DIADEL & DIADEL & Dianthus deltoides & Maiden Pink \\
\hline DICUNI & DICUNI & Dicentra uniflora & Steer's Head \\
\hline DISTRA & DISTRA & Disporum trachycarpum & Wartberry Fairy-bell \\
\hline DODCON & DODCON & Dodecatheon conjugens & Slimpod Shooting Star \\
\hline DODJEF & DODJEF & Dodecatheon jeffreyi & Tall Mountain Shooting Star \\
\hline DODPUL & DODPUL & Dodecatheon pulchellum & Few-flowered Shooting Star \\
\hline DOUMON & DOUMON & Douglasia montana & Rocky Mountain Douglasia \\
\hline DRAAPI & DRAAPI & Draba apiculata & Pointed Draba \\
\hline DRAAUR & DRAAUR & Draba aurea & Golden Draba \\
\hline DRACAN & DRALAN & Draba cana & Lance Leaved Draba \\
\hline DRACRA & DRACRA & Draba crassifolia & Thickleaved Draba \\
\hline DRADEN & DRADEN & Draba densifolia & Nuttall's Draba \\
\hline DRAINC & DRAINC & Draba incerta & Yellowstone Draba \\
\hline DRALAN & DRALAN & Draba lanceolata & Lance Leaved Draba \\
\hline DRALON & DRALON & Draba lonchocarpa & Lancefruit Draba \\
\hline DRANEM & DRANEM & Draba nemorosa & Woods Draba \\
\hline DRAOLI & DRAOLI & Draba oligosperma & Few-seeded Draba \\
\hline DRASTE & DRASTE & Draba stenoloba & Slender Draba \\
\hline ECHANG & ECHANG & Echinacea angustifolia & Pale Purple Coneflower \\
\hline EPIALP & EPIALP & Epilobium alpinum & Alpine Willow-herb \\
\hline EPIANG & EPIANG & Epilobium angustifolium & Fireweed \\
\hline EPICIL & EPICIL & Epilobium ciliatum & Common Willow-herb \\
\hline EPIGLA & EPIGLA & Epilobium glaberrimum & Smooth Willow-herb \\
\hline EPIMIN & EPIMIN & Epilobium minutum & Small-flowered Willow-herb \\
\hline EPIPAL & EPIPAL & Epilobium palustre & Swamp Willow-herb \\
\hline ERIASP & ERIASP & Erigeron asperugineus & Rough Fleabane \\
\hline ERICAE & ERICAE & Erigeron caespitosus & Tufted Fleabane \\
\hline
\end{tabular}




\section{Appendix A. Flora of the Beaverhead Mountains Section Forbs}

\begin{tabular}{|c|c|c|c|}
\hline $\begin{array}{l}\text { USFS Reg. } \\
1 \text { Code }\end{array}$ & Synonymy & Species Name & Common Name \\
\hline ERICOM & ERICOM & Erigeron compositus & Cut-leaved Daisy \\
\hline ERICOR & ERICOR & Erigeron corymbosus & Long-leaved Fleabane \\
\hline ERIDIV & ERIDIV & Erigeron divergens & Spreading Fleabane \\
\hline ERIGLA & ERIGLA & Erigeron glabellus & Smooth Daisy \\
\hline ERIGRA & ERIGRA & Erigeron gracilis & Slender Fleabane \\
\hline ERIHUM & ERIHUM & Erigeron humilis & Arctic-alpine Daisy \\
\hline ERIOCH & ERIOCH & Erigeron ochroleucus & Buff Fleabane \\
\hline ERIPER & ERIPER & Erigeron peregrinus & Subalpine Daisy \\
\hline ERIPHI & ERIPHI & Erigeron philadelphicus & Philadelphia Fleabane \\
\hline ERIPUM & ERIPUM & Erigeron pumilus & Shaggy Fleabane \\
\hline ERIRAD & ERIRAD & Erigeron radicatus & Taprooted Fleabane \\
\hline ERIRYD & ERIRYD & Erigeron rydbergii & Rydberg's Daisy \\
\hline ERISIM & ERISIM & Erigeron simplex & Alpine Daisy \\
\hline ERISPE & ERISPE & Erigeron speciosus & Showy Fleabane \\
\hline ERISTR & ERISTR & Erigeron strigosus & Branching Daisy \\
\hline ERISUB & ERISUB & Erigeron subtrinervis & Three-veined Fleabane \\
\hline ERITWE & ERITWE & Erigeron tweedyi & Tweedy's Daisy \\
\hline ERIURS & ERIURS & Erigeron ursinus & Bear River Fleabane \\
\hline ERICAS & ERICAS & Eriogonum caespitosum & Mat Buckwheat \\
\hline ERICER & ERICER & Eriogonum cernuum & Nodding Wild Buckwheat \\
\hline ERIFLA & ERIFLA & Eriogonum flavum & Yellow Buckwheat \\
\hline ERIHER & ERIHER & Eriogonum heracleoides & Wyeth Buckwheat \\
\hline ERIMAN & ERIMAN & Eriogonum mancum & Imperfect Buckwheat \\
\hline ERIOVA & ERIOVA & Eriogonum ovalifolium & Cushion Buckwheat \\
\hline ERIOVE & ERIOVE & Eriogonum ovalifolium var. nevac & c Yellow Cushion Buckwheat \\
\hline ERIUMB & ERIUMB & Eriogonum umbellatum & Sulfur Buckwheat \\
\hline ERILAN & ERILAN & Eriophyllum lanatum & Common Eriophyllum \\
\hline ERINAN & ERINAN & Eritrichium nanum & Pale Alpine Forget-me-not \\
\hline EROCIC & EROCIC & Erodium cicutarium & Stork's-bill \\
\hline ERYASP & ERYASP & Erysimum asperum & Plains Wallflower \\
\hline ERYCAP & ERYASP & Erysimum capitatum & Western Wallflower \\
\hline ERYCHE & ERYCHE & Erysimum cheiranthoides & Wormseed Wallflower \\
\hline ERYINC & ERYINC & Erysimum inconspicuum & Smallflowered Wallflower \\
\hline ERYGRA & ERYGRA & Erythronium grandiflorum & Glacier-lily \\
\hline ESCVIV & CORVIV & Escobaria vivipara & Spinystar \\
\hline EUPESU & EUPESU & Euphorbia esula & Leafy Spurge \\
\hline FRAVES & FRAVES & Fragaria vesca & Woods Strawberry \\
\hline FRAVIR & FRAVIR & Fragaria virginiana & Virginia Strawberry \\
\hline FRAALB & FRAALB & Frasera albicaulis & White-stemmed Frasera \\
\hline FRASPE & FRASPE & Frasera speciosa & Giant Frasera \\
\hline FRIATR & FRIATR & Fritillaria atropurpurea & Checker Lily \\
\hline FRIPUD & FRIPUD & Fritillaria pudica & Yellow Bell \\
\hline GAIARI & GAIARI & Gaillardia aristata & Blanket-flower \\
\hline GALAPA & GALAPA & Galium aparine & Goose-grass \\
\hline GALBIF & GALBIF & Galium bifolium & Thinleaf Bedstraw \\
\hline GALBOR & GALBOR & Galium boreale & Northern Bedstraw \\
\hline GALTRF & GALTRF & Galium trifidum & Small Bedstraw \\
\hline GALTRI & GALTRI & Galium triflorum & Sweetscented Bedstraw \\
\hline GAUCOC & GAUCOC & Gaura coccinea & Scarlet Gaura \\
\hline
\end{tabular}




\section{Appendix A. Flora of the Beaverhead Mountains Section Forbs}

\begin{tabular}{|c|c|c|c|}
\hline $\begin{array}{l}\text { USFS Reg. } \\
1 \text { Code } \\
\end{array}$ & Synonymy & Species Name & Common Name \\
\hline GAYDEC & GAYDEC & Gayophytum decipiens & Deceptive Groundsmoke \\
\hline GAYDIF & GAYDIF & Gayophytum diffusum & Spreading Groundsmoke \\
\hline GAYRAC & GAYRAC & Gayophytum racemosum & Racemed Groundsmoke \\
\hline GENAFF & GENAFF & Gentiana affinis & Pleated Gentian \\
\hline GENALG & GENALG & Gentiana algida & Siberia Gentian \\
\hline GENAQU & GENAQU & Gentiana aquatica & Wet Meadow Gentian \\
\hline GENCAL & GENCAL & Gentiana calycosa & Explorer's Gentian \\
\hline GENAMA & GENAMA & Gentianella amarella & Northern Gentian \\
\hline GENPRO & GENPRO & Gentianella propinqua & Four-parted Gentian \\
\hline GERRIC & GERRIC & Geranium richardsonii & White Geranium \\
\hline GERVIS & GERVIS & Geranium viscosissimum & Sticky Geranium \\
\hline GEUALE & GEUALE & Geum aleppicum & Yellow Avens \\
\hline GEUMAC & GEUMAC & Geum macrophyllum & Large-leaved Avens \\
\hline GEUROS & GEUROS & Geum rossii & Ross's Avens \\
\hline GEUTRI & GEUTRI & Geum triflorum & Prairie Smoke \\
\hline GLAMAR & GLAMAR & Glaux maritima & Sea-milkwort \\
\hline GLEHED & GLEHED & Glecoma hederacea & Ground Ivy \\
\hline GLYLEP & GLYLEP & Glycyrrhiza lepidota & American Licorice \\
\hline GNAVIS & GNAVIS & Gnaphalium viscosum & Sticky Cudweed \\
\hline GOOOBL & GOOOBL & Goodyera oblongifolia & Western Rattlesnake-plantain \\
\hline GRISQU & GRISQU & Grindelia squarrosa & Curlycup Gumweed \\
\hline HACDEF & HACDEF & Hackelia deflexa & Nodding Stickseed \\
\hline HACFLO & HACFLO & Hackelia floribunda & Showy Stickseed \\
\hline HACMIC & HACMIC & Hackelia micrantha & Blue Stickseed \\
\hline HACPAT & HACPAT & Hackelia patens & Spreading Stickseed \\
\hline HAPACA & HAPACA & Haplopappus acaulis & Cushion Goldenweed \\
\hline HEDBOR & HEDBOR & Hedysarum boreale & Northern Hedysarum \\
\hline HEDSUL & HEDSUL & Hedysarum sulphurescens & Yellow Hedysarum \\
\hline HELQUI & HELQUI & Helianthella quinquenervis & Nodding Helianthella \\
\hline HELUNI & HELUNI & Helianthella uniflora & One-flowered Helianthella \\
\hline HELNUT & HELNUT & Helianthus nuttallii & Nuttall's Sunflower \\
\hline HERSPH & HERLAN & Heracleum sphondylium & Eltrot \\
\hline HETVIL & CHRVIL & Heterotheca villosa & Hairy Golden-aster \\
\hline HETVIL & CHRVIL & Heterotheca villosa & Hairy Golden-aster \\
\hline HEUCYL & HEUCYL & Heuchera cylindrica & Roundleaf Alumroot \\
\hline HEUGRO & HEUGRO & Heuchera grossulariifolia & Gooseberry-leaved Alumroot \\
\hline HEUPAR & HEUPAR & Heuchera parvifolia & Small-leaved Alumroot \\
\hline HIEALB & HIEALB & Hieracium albiflorum & White-flowered Hawkweed \\
\hline HIECYN & HIECYN & Hieracium cynoglossoides & Houndstongue Hawkweed \\
\hline HIEGRA & HIEGRA & Hieracium gracile & Alpine Hawkweed \\
\hline HIPVUL & HIPVUL & Hippuris vulgaris & Common Mare's-tail \\
\hline HYDCAP & HYDCAP & Hydrophyllum capitatum & Ballhead Waterleaf \\
\hline HYMFIL & HYMFIL & Hymenopappus filifolius & Columbia Cut-leaf \\
\hline IPOCON & IPOCON & Ipomopsis congesta & Ballhead Gilia \\
\hline IPOSPI & IPOSPI & Ipomopsis spicata & Spicate Gilia \\
\hline IRIMIS & IRIMIS & Iris missouriensis & Rocky Mountain Iris \\
\hline IVAAXI & IVAAXI & Iva axillaris & Poverty-weed \\
\hline IVEGOR & IVEGOR & Ivesia gordonii & Gordon's Ivesia \\
\hline LACSER & LACSER & Lactuca serriola & Prickly Lettuce \\
\hline
\end{tabular}




\section{Appendix A. Flora of the Beaverhead Mountains Section Forbs}

\begin{tabular}{|c|c|c|c|}
\hline $\begin{array}{l}\text { USFS Reg. } \\
1 \text { Code } \\
\end{array}$ & Synonymy & Species Name & Common Name \\
\hline LAPECH & LAPECH & Lappula echinata & Bristly Stickseed \\
\hline LAPRED & LAPRED & Lappula redowskii & Western Stickseed \\
\hline LEPCAM & LEPCAM & Lepidium campestre & Field Pepperweed \\
\hline LEPDEN & LEPDEN & Lepidium densiflorum & Prairie Pepperweed \\
\hline LEPPER & LEPPER & Lepidium perfoliatum & Clasping Pepperweed \\
\hline LEPRAM & LEPRAM & Lepidium ramosissimum & Branched Pepperweed \\
\hline LEPVIR & LEPVIR & Lepidium virginicum & Tall Pepperweed \\
\hline LEPPYR & LEPPYR & Leptarrhena pyrolifolia & False Saxifrage \\
\hline LESALP & LESALP & Lesquerella alpina & Alpine Bladderpod \\
\hline LESCAR & LESCAR & Lesquerella carinata & Keeled Bladderpod \\
\hline LESLUD & LESLUD & Lesquerella ludoviciana & Silvery Bladderpod \\
\hline LESPAY & LESPAY & Lesquerella paysonii & Payson Bladderpod \\
\hline LEWPYG & LEWPYG & Lewisia pygmaea & Dwarf Lewisia \\
\hline LEWRED & LEWRED & Lewisia rediviva & Bitterroot \\
\hline LIAPUN & LIAPUN & Liatris punctata & Dotted Blazing-star \\
\hline LIGCAN & LIGCAN & Ligusticum canbyi & Canby's Licorice-root \\
\hline LIGFIL & LIGFIL & Ligusticum filicinum & Fearn-leaf Lovage \\
\hline LIGTEN & LIGTEN & Ligusticum tenuifolium & Slender-leafed Licorice-root \\
\hline LINSEP & LINSEP & Linanthus septentrionalis & Northern Linanthus \\
\hline LINVUL & LINVUL & Linaria vulgaris & Butter-and-eggs \\
\hline LINLEW & LINPER & Linum lewisii & Wild Blue Flax \\
\hline LINPER & LINPER & Linum perenne & Blue Flax \\
\hline LINUSI & LINUSI & Linum usitatissimum & Cultivated Flax \\
\hline LISCOR & LISCOR & Listera cordata & Heart-leaf Twayblade \\
\hline LITGLA & LITGLA & Lithophragma glabrum & Smooth Woodlandstar \\
\hline LITPAR & LITPAR & Lithophragma parviflorum & Smallflower Woodlandstar \\
\hline LITTEN & LITTEN & Lithophragma tenellum & Slender Woodlandstar \\
\hline LITINC & LITINC & Lithospermum incisum & Yellow Gromwell \\
\hline LITRUD & LITRUD & Lithospermum ruderale & Western Gromwell \\
\hline LLOSER & LLOSER & Lloydia serotina & Alpine Lily \\
\hline LOMAMB & LOMAMB & Lomatium ambiguum & Swale Desert-parsley \\
\hline LOMATT & LOMATT & Lomatium attenuatum & Tapertip Desertparsley \\
\hline LOMCOU & LOMCOU & Lomatium cous & Cous Biscuit-root \\
\hline LOMCUS & LOMCUS & Lomatium cusickii & Cusick's Desert-parsley \\
\hline LOMDIS & LOMDIS & Lomatium dissectum & Fern-leaved Desert-parsley \\
\hline LOMFOE & LOMFOE & Lomatium foeniculaceum & Fennel-leaved Desert-parsley \\
\hline LOMMAC & LOMMAC & Lomatium macrocarpum & Bigseed Desert-parsley \\
\hline LOMTRI & LOMTRI & Lomatium triternatum & Nine-leaf Lomatium \\
\hline LOMROT & LOMROT & Lomatogonium rotatum & Felwort \\
\hline LUPARB & LUPLVL & Lupinus arbustus & Spurred Lupine \\
\hline LUPARG & LUPARG & Lupinus argenteus & Silvery Lupine \\
\hline LUPLEP & LUPLEP & Lupinus lepidus & Prairie Lupine \\
\hline LUPLEU & LUPLEU & Lupinus leucophyllus & Velvet Lupine \\
\hline LUPSER & LUPSER & Lupinus sericeus & Silky Lupine \\
\hline LUPWYE & LUPWYE & Lupinus wyethii & Wyeth's Lupine \\
\hline LYGJUN & LYGJUN & Lygodesmia juncea & Rush-like Skeletonweed \\
\hline MACCAN & MACCAN & Machaeranthera canescens & Hoary Aster \\
\hline MAIRAC & SMIRAC & Maianthemum racemosum & False Spikenard \\
\hline MAISTE & SMISTE & Maianthemum stellatum & Starry Solomon-plume \\
\hline
\end{tabular}




\section{Appendix A. Flora of the Beaverhead Mountains Section Forbs}

\begin{tabular}{|c|c|c|c|}
\hline $\begin{array}{l}\text { USFS Reg. } \\
1 \text { Code }\end{array}$ & Synonymy & Species Name & Common Name \\
\hline MEDLUP & MEDLUP & Medicago lupulina & Black Medic \\
\hline MEDSAT & MEDSAT & Medicago sativa & Alfalfa \\
\hline MELLIN & MELLIN & Melampyrum lineare & Narrow-leaved Cow-wheat \\
\hline MELALB & MELALB & Melilotus alba & White Sweet-clover \\
\hline MELOFF & MELOFF & Melilotus officinalis & Yellow Sweet-clover \\
\hline MENARV & MENARV & Mentha arvensis & Field Mint \\
\hline MENALB & MENALB & Mentzelia albicaulis & White-stemmed Mentzelia \\
\hline MENDEC & MENDEC & Mentzelia decapetala & Evening Star \\
\hline MENDIS & MENDIS & Mentzelia dispersa & Bushy Mentzelia \\
\hline MENLAE & MENLAE & Mentzelia laevicaulis & Blazing-star Mentzelia \\
\hline MERALP & MERALP & Mertensia alpina & Alpine Bluebells \\
\hline MERCIL & MERCIL & Mertensia ciliata & Mountain Bluebells \\
\hline MERLAN & MERLAN & Mertensia lanceolata & Lanceleaf Bluebells \\
\hline MERLON & MERLON & Mertensia longiflora & Small Bluebells \\
\hline MEROBL & MEROBL & Mertensia oblongifolia & Oblongleaf Bluebells \\
\hline MERPAN & MERPAN & Mertensia paniculata & Panicle Bluebells \\
\hline MERVIR & MERPER & Mertensia viridis & Obscure Bluebells \\
\hline MICNUT & MICNUT & Microseris nutans & Nodding Microseris \\
\hline MIMDEN & MIMDEN & Mimulus dentatus & Tooth-leaved Monkey-flower \\
\hline MIMGLA & MIMGLA & Mimulus glabratus & Glaberous Monkey-flower \\
\hline MIMGUT & MIMGUT & Mimulus guttatus & Common Monkey-flower \\
\hline MIMLEW & MIMLEW & Mimulus lewisii & Lewis' Monkey-flower \\
\hline MIMPRI & MIMPRI & Mimulus primuloides & Primrose Monkey-flower \\
\hline MITBRE & MITBRE & Mitella breweri & Brewer's Mitrewort \\
\hline MITCAU & MITCAU & Mitella caulescens & Leafy Mitrewort \\
\hline MITPEN & MITPEN & Mitella pentandra & Five-stamened Mitrewort \\
\hline MITSTA & MITSTA & Mitella stauropetala & Side-flowered Mitrewort \\
\hline MOELAT & MOELAT & Moehringia lateriflora & Bluntleaf Sandwort \\
\hline MONFIS & MONFIS & Monarda fistulosa & Horsemint \\
\hline MONCHA & MONCHA & Montia chamissoi & Water Montia \\
\hline MUSDIV & MUSDIV & Musineon divaricatum & Leafy Musineon \\
\hline MYOALP & MYOASI & Myosotis alpestris & Wood Forget-me-not \\
\hline MYOSYL & MYOSYL & Myosotis sylvatica & Wood Forget-me-not \\
\hline MYRSPI & MYRSPI & Myriophyllum spicatum & Spiked Water-milfoil \\
\hline NEMBRE & NEMBRE & Nemophila breviflora & Great Basin Nemophila \\
\hline OENCES & OENCAE & Oenothera cespitosa & Desert Evening-primrose \\
\hline OENFLA & OENFLA & Oenothera flava & Long-tubed Evening-primrose \\
\hline ONOMOL & ONOMOL & Onosmodium molle & False Gromwell \\
\hline OPUFRA & OPUFRA & Opuntia fragilis & Brittle Prickly-pear \\
\hline OPUPOL & OPUPOL & Opuntia polyacantha & Plains Prickly-pear \\
\hline OROCOR & OROCOR & Orobanche corymbosa & Flat-topped Broomrape \\
\hline OROFAS & OROFAS & Orobanche fasciculata & Clustered Broomrape \\
\hline OROLUD & OROLUD & Orobanche ludoviciana & Suksdorf's Broomrape \\
\hline OROUNI & OROUNI & Orobanche uniflora & Naked Broomrape \\
\hline ORTSEC & PYRSEC & Orthilia secunda & One-sided Wintergreen \\
\hline ORTSEC & PYRSEC & Orthilia secunda & One-sided Wintergreen \\
\hline ORTLUT & ORTLUT & Orthocarpus luteus & Yellow Owl-clover \\
\hline ORTTEN & ORTTEN & Orthocarpus tenuifolius & Thin-leaved Owl-clover \\
\hline OSMBER & OSMCHI & Osmorhiza berteroi & Sweetcicely \\
\hline
\end{tabular}




\section{Appendix A. Flora of the Beaverhead Mountains Section Forbs}

\begin{tabular}{|c|c|c|c|}
\hline $\begin{array}{l}\text { USFS Reg. } \\
1 \text { Code } \\
\end{array}$ & Synonymy & Species Name & Common Name \\
\hline OSMDEP & OSMDEP & Osmorhiza depauperata & Blunt-fruit Sweet-cicely \\
\hline OSMOCC & OSMOCC & Osmorhiza occidentalis & Western Sweet-cicely \\
\hline OXYDIG & OXYDIG & Oxyria digyna & Mountain Sorrel \\
\hline OXYCAM & OXYCAM & Oxytropis campestris & Slender Crazyweed \\
\hline OXYDEF & OXYDEF & Oxytropis deflexa & Pendent-pod Crazyweed \\
\hline OXYLAG & OXYLAG & Oxytropis lagopus & Rabbit-foot Crazyweed \\
\hline OXYRIP & OXYRIP & Oxytropis riparia & Riparian Crazyweed \\
\hline OXYSER & OXYSER & Oxytropis sericea & Silky Crazyweed; White Locoweed \\
\hline OXYVIS & OXYVIS & Oxytropis viscida & Sticky Crazyweed \\
\hline PARPEN & PARPEN & Parietaria pensylvanica & Pennsylvania Pellitory \\
\hline PARFIM & PARFIM & Parnassia fimbriata & Fringed Grass-of-parnassus \\
\hline PARPAL & PARPAL & Parnassia palustris & Northern Grass-of-parnassus \\
\hline PARPAR & PARPAR & Parnassia parviflora & Small-flowered Grass-of-parnassus \\
\hline PARSES & PARSES & Paronychia sessiliflora & Stemless Whitlow-wort \\
\hline PEDBRA & PEDBRA & Pedicularis bracteosa & Bracted Lousewort \\
\hline PEDCON & PEDCON & Pedicularis contorta & Coiled-beak Lousewort \\
\hline PEDCYS & PEDCYS & Pedicularis cystopteridifolia & Fern-leaved Lousewort \\
\hline PEDGRO & PEDGRO & Pedicularis groenlandica & Elephant's Head \\
\hline PEDPAR & PEDPAR & Pedicularis parryi & Parry's Lousewort \\
\hline PEDPUL & PEDPUL & Pedicularis pulchella & Pretty-dwarf Lousewort \\
\hline PEDRAC & PEDRAC & Pedicularis racemosa & Sickletop Lousewort \\
\hline PEDSIM & PEDSIM & Pediocactus simpsonii & Simpson's Hedgehog Cactus \\
\hline PENALE & PENALE & Penstemon albertinus & Alberta Penstemon \\
\hline PENARI & PENARI & Penstemon aridus & Stiff-leaf Penstemon \\
\hline PENATT & PENATT & Penstemon attenuatus & Sulphur Penstemon \\
\hline PENCON & PENCON & Penstemon confertus & Yellow Penstemon \\
\hline PENDIP & PENDIP & Penstemon diphyllus & Diphyllus Penstemon \\
\hline PENELL & PENELL & Penstemon ellipticus & Elliptic-leaved Beardtongue \\
\hline PENERI & PENERI & Penstemon eriantherus & Fuzzytongue Penstemon \\
\hline PENMON & PENMON & Penstemon montanus & Mountain Beardtongue \\
\hline PENPRO & PENPRO & Penstemon procerus & Small-flowered Penstemon \\
\hline PENRAD & PENRAD & Penstemon radicosus & Mat-root Penstemon \\
\hline PENRYD & PENRYD & Penstemon rydbergii & Rydberg's Penstemon \\
\hline PENWHI & PENWHI & Penstemon whippleanus & Whipple's Penstemon \\
\hline PENWIL & PENWIL & Penstemon wilcoxii & Wilcox's Penstemon \\
\hline PERGAI & PERGAI & Perideridia gairdneri & Gairdner's Yampah \\
\hline PHAFRA & PHAFRA & Phacelia franklinii & Franklin's Phacelia \\
\hline PHAGLA & PHAGLA & Phacelia glandulosa & Glandular Phacelia \\
\hline PHAHAS & PHAHAS & Phacelia hastata & Silverleaf Phacelia \\
\hline PHAHET & PHAHET & Phacelia heterophylla & Varileaf Phacelia \\
\hline PHAINC & PHAINC & Phacelia incana & Hoary Phacelia \\
\hline PHALIN & PHALIN & Phacelia linearis & Threadleaf Phacelia \\
\hline PHASER & PHASER & Phacelia sericea & Silky Phacelia \\
\hline PHLALB & PHLALB & Phlox albomarginata & White-margined Phlox \\
\hline PHLALY & PHLALY & Phlox alyssifolia & Alyssum-leaved Phlox \\
\hline PHLBRY & PHLBRY & Phlox bryoides & Moss Phlox \\
\hline PHLCAE & PHLCAE & Phlox caespitosa & Tufted Phlox \\
\hline PHLHOO & PHLHOO & Phlox hoodii & Hood's Phlox \\
\hline PHLKEL & PHLKEL & Phlox kelseyi & Kelsey's Phlox \\
\hline
\end{tabular}




\section{Appendix A. Flora of the Beaverhead Mountains Section Forbs}

\begin{tabular}{|c|c|c|c|}
\hline $\begin{array}{l}\text { USFS Reg. } \\
1 \text { Code } \\
\end{array}$ & Synonymy & Species Name & Common Name \\
\hline PHLLON & PHLLON & Phlox longifolia & Long-leaf Phlox \\
\hline PHLMUL & PHLMUL & Phlox multiflora & Many-flowered Phlox \\
\hline PHLPUL & PHLCAE & Phlox pulvinata & Cushion Phlox \\
\hline PHYDID & PHYDID & Physaria didymocarpa & Common Twinpod \\
\hline PHYGEY & PHYGEY & Physaria geyeri & Geyer's Twinpod \\
\hline PLAERI & PLAERI & Plantago eriopoda & Saline Plantain \\
\hline PLAMAJ & PLAMAJ & Plantago major & Common Plantain \\
\hline PLAPAT & PLAPAT & Plantago patagonica & Indian-wheat \\
\hline PLATWE & PLATWE & Plantago tweedyi & Tweedy's Plantain \\
\hline POLOCC & POLOCC & Polemonium occidentale & Western Polemonium \\
\hline POLPUL & POLPUL & Polemonium pulcherrimum & Skunk-leaved Polemonium \\
\hline POLVIS & POLVIS & Polemonium viscosum & Sticky Polemonium \\
\hline POLAMP & POLAMP & Polygonum amphibium & Water Smartweed \\
\hline POLAVI & POLAVI & Polygonum aviculare & Prostrate Knotweed \\
\hline POLBIS & POLBIS & Polygonum bistortoides & American Bistort \\
\hline POLDOU & POLDOU & Polygonum douglasii & Douglas' Knotweed \\
\hline POLRAM & POLRAM & Polygonum ramosissimum & Bushy Knotweed \\
\hline POLVIV & POLVIV & Polygonum viviparum & Alpine Bistort \\
\hline POTFRI & POTFRI & Potamogeton friesii & Flat-stalked Pondweed \\
\hline POTGRM & POTGRM & Potamogeton gramineus & Grass-leaved Pondweed \\
\hline РОТРЕС & POTPEC & Potamogeton pectinatus & Fennel-leafed Pondweed \\
\hline POTARG & POTARG & Potentilla arguta & Tall Cinquefoil \\
\hline POTCON & POTCON & Potentilla concinna & Early Cinquefoil \\
\hline POTDIV & POTDIV & Potentilla diversifolia & Diverse-leaved Cinquefoil \\
\hline POTFLA & POTFLA & Potentilla flabellifolia & Fan-leaf Cinquefoil \\
\hline POTGLA & POTGLA & Potentilla glandulosa & Sticky Cinquefoil \\
\hline POTGRA & POTGRA & Potentilla gracilis & Slender Cinquefoil \\
\hline POTHIP & POTHIP & Potentilla hippiana & Woolly Cinquefoil \\
\hline POTNIV & POTNIV & Potentilla nivea & Snow Cinquefoil \\
\hline POTOVI & POTOVI & Potentilla ovina & Sheep Cinquefoil \\
\hline POTPEN & POTPEN & Potentilla pensylvanica & Prairie Cinquefoil \\
\hline POTRIV & POTRIV & Potentilla rivalis & Brook Cinquefoil \\
\hline PRIINC & PRIINC & Primula incana & Mealy Primrose \\
\hline PRUVUL & PRUVUL & Prunella vulgaris & Self-heal \\
\hline PSOTEU & PSOTEN & Psoralidium tenuiflorum & Slender-flowered Scurf-pea \\
\hline PTEAND & PTEAND & Pterospora andromedea & Woodland Pinedrops \\
\hline PYRASA & PYRASA & Pyrola asarifolia & Pink Wintergreen \\
\hline PYRCHL & PYRCHL & Pyrola chlorantha & Green Wintergreen \\
\hline PYRMIN & PYRMIN & Pyrola minor & Snowline Pyrola \\
\hline PYRPIC & PYRPIC & Pyrola picta & White-vein Pyrola \\
\hline RANACR & RANACR & Ranunculus acris & Tall Buttercup \\
\hline RANALI & RANALI & Ranunculus alismifolius & Water-plantain Buttercup \\
\hline RANAQU & RANAQU & Ranunculus aquatilis & White Water-buttercup \\
\hline RANCYM & RANCYM & Ranunculus cymbalaria & Shore Buttercup \\
\hline RANESC & RANESC & Ranunculus eschscholtzii & Subalpine Buttercup \\
\hline RANFLA & RANFLA & Ranunculus flammula & Creeping Buttercup \\
\hline RANGLA & RANGLA & Ranunculus glaberrimus & Sagebrush Buttercup \\
\hline RANINA & RANINA & Ranunculus inamoenus & Unlovely Buttercup \\
\hline RANJOV & RANJOV & Ranunculus jovis & Jove's Buttercup \\
\hline
\end{tabular}




\section{Appendix A. Flora of the Beaverhead Mountains Section Forbs}

\begin{tabular}{|c|c|c|c|}
\hline $\begin{array}{l}\text { USFS Reg. } \\
1 \text { Code }\end{array}$ & Synonymy & Species Name & Common Name \\
\hline RANNAT & RANNAT & Ranunculus natans & Floating Water-buttercup \\
\hline RANPYG & RANPYG & Ranunculus pygmaeus & Dwarf Buttercup \\
\hline RANSCE & RANSCE & Ranunculus sceleratus & Blister Buttercup \\
\hline RANUNI & RANUNI & Ranunculus uncinatus & Little Buttercup \\
\hline RORISL & RORPAL & Rorippa islandica & Marsh Yellowcress \\
\hline RORNAS & NASOFF & Rorippa nasturtium-aquaticum & Water-cress \\
\hline RUDHIR & RUDHIR & Rudbeckia hirta & Black-eyed Susan \\
\hline RUDOCC & RUDOCC & Rudbeckia occidentalis & Black Head \\
\hline RUMACE & RUMACE & Rumex acetosella & Sheep Sorrel \\
\hline RUMCRI & RUMCRI & Rumex crispus & Curly Dock \\
\hline RUMMAR & RUMMAR & Rumex maritimus & Golden Dock \\
\hline RUMPAU & RUMPAU & Rumex paucifolius & Mountain Sorrel \\
\hline RUMSAL & RUMSAL & Rumex salicifolius & Willow Dock \\
\hline SALKAL & SALIBE & Salsola kali & Russian Thistle \\
\hline SAUWEB & SAUWEB & Saussurea weberi & Weber's Sawwort \\
\hline SAXARG & SAXARG & Saxifraga arguta & Brook Saxifrage \\
\hline SAXBRO & SAXBRO & Saxifraga bronchialis & Spotted Saxifrage \\
\hline SAXCES & SAXCES & Saxifraga cespitosa & Tufted Saxifrage \\
\hline SAXFLA & SAXFLA & Saxifraga flagellaris & Stoloniferous Saxifrage \\
\hline SAXLYA & SAXLYA & Saxifraga lyallii & Red-stemed Saxifrage \\
\hline SAXOCC & SAXOCC & Saxifraga occidentalis & Western Saxifrage \\
\hline SAXODO & SAXARG & Saxifraga odontoloma & Brook Saxifrage \\
\hline SAXOPP & SAXOPP & Saxifraga oppositifolia & Purple Saxifrage \\
\hline SAXORE & SAXORE & Saxifraga oregana & Oregon Saxifrage \\
\hline SAXRHO & SAXRHO & Saxifraga rhomboidea & Diamondleaf Saxifrage \\
\hline SAXTEM & SAXTEM & Saxifraga tempestiva & Storm Saxifrage \\
\hline SCHLIN & SCHLIN & Schoenocrambe linifolia & Flaxleaf Plainsmustard \\
\hline SCUGAL & SCUGAL & Scutellaria galericulata & Marsh Skullcap \\
\hline SEDBOR & SEDLEI & Sedum borschii & Leiberg's Stonecrop \\
\hline SEDLAN & SEDLAN & Sedum lanceolatum & Lance-leaved Stonecrop \\
\hline SEDSTE & SEDSTE & Sedum stenopetalum & Wormleaf Stonecrop \\
\hline SENCAN & SENCAN & Senecio canus & Woolly Groundsel \\
\hline SENCRA & SENCRA & Senecio crassulus & Thick-leaved Groundsel \\
\hline SENCYM & SENCYM & Senecio cymbalarioides & Few-leaved Groundsel \\
\hline SENDEB & SENDEB & Senecio debilis & Weak Butterweed \\
\hline SENDIM & SENDIM & Senecio dimorphophyllus & Payson's Groundsel \\
\hline SENFRE & SENFRE & Senecio fremontii & Dwarf Mountain Butterweed \\
\hline SENHYR & SENFVH & Senecio hydrophiloides & Sweet-marsh Butterweed \\
\hline SENHYD & SENHYD & Senecio hydrophilus & Alkali-marsh Butterweed \\
\hline SENINT & SENINT & Senecio integerrimus & Western Groundsel \\
\hline SENPAU & SENPAU & Senecio pauciflorus & Rayless Alpine Butterweed \\
\hline SENPSE & SENPSE & Senecio pseudaureus & Streambank Groundsel \\
\hline SENSER & SENSER & Senecio serra & Tall Butterweed \\
\hline SENSPH & SENSPH & Senecio sphaerocephalus & Mountain-marsh Butterweed \\
\hline SENSTR & SENSTR & Senecio streptanthifolius & Rocky Mountain Butterweed \\
\hline SENTRI & SENTRI & Senecio triangularis & Arrowleaf Groundsel \\
\hline SIBPRO & SIBPRO & Sibbaldia procumbens & Creeping Sibbaldia \\
\hline SILACA & SILACA & Silene acaulis & Moss Campion \\
\hline SILDOU & SILDOU & Silene douglasii & Douglas' Silene \\
\hline
\end{tabular}




\section{Appendix A. Flora of the Beaverhead Mountains Section Forbs}

\begin{tabular}{|c|c|c|c|}
\hline $\begin{array}{l}\text { USFS Reg. } \\
1 \text { Code } \\
\end{array}$ & Synonymy & Species Name & Common Name \\
\hline SILMEN & SILMEN & Silene menziesii & Menzies' Silene \\
\hline SILORE & SILORE & Silene oregana & Oregon Silene \\
\hline SILPAR & SILPAR & Silene parryi & Parry's Silene \\
\hline SILREP & SILREP & Silene repens & Creeping Silene \\
\hline SILSCO & SILSCO & Silene scouleri & Scouler's Silene \\
\hline SISLOE & SISLOE & Sisymbrium loeselii & Loeselii Tumblemustard \\
\hline SISOFF & SISOFF & Sisymbrium officinale & Hedge Mustard \\
\hline SISANG & SISIDA & Sisyrinchium angustifolium & Blue-eyed Grass \\
\hline SISMON & SISMON & Sisyrinchium montanum & Mountain Blue-eyed Grass \\
\hline SIUSUA & SIUSUA & Sium suave & Hemlock Water-parsnip \\
\hline SMECAL & SMECAL & Smelowskia calycina & Alpine Smelowskia \\
\hline SOLCAN & SOLCAN & Solidago canadensis & Canada Goldenrod \\
\hline SOLGIG & SOLGIG & Solidago gigantea & Late Goldenrod \\
\hline SOLMIS & SOLMIS & Solidago missouriensis & Missouri Goldenrod \\
\hline SOLMUL & SOLMUL & Solidago multiradiata & Northern Goldenrod \\
\hline SOLNAN & SOLNAN & Solidago nana & Low Goldenrod \\
\hline SOLNEM & SOLNEM & Solidago nemoralis & Gray Goldenrod \\
\hline SOLRIG & SOLRIG & Solidago rigida & Stiff Goldenrod \\
\hline SOLSPA & SOLSPA & Solidago spathulata & Dune Goldenrod \\
\hline SONASP & SONASP & Sonchus asper & Prickly Sow-thistle \\
\hline SONULI & SONASU & Sonchus uliginosus & Marsh Sow-thistle \\
\hline SPEARV & SPEARV & Spergula arvensis & Cornspurry \\
\hline SPHCOC & SPHCOC & Sphaeralcea coccinea & Red Globe-mallow \\
\hline SPIROM & SPIROM & Spiranthes romanzoffiana & Hooded Ladies-tresses \\
\hline STAVIR & STAVIR & Stanleya viridiflora & Perennial Stanleya \\
\hline STEAME & STEAME & Stellaria americana & American Starwort \\
\hline STECAL & STECAL & Stellaria calycantha & Northern Starwort \\
\hline STELON & STELON & Stellaria longipes & Longstalk Starwort \\
\hline STEUMB & STEUMB & Stellaria umbellata & Umbellate Starwort \\
\hline STERUN & STERUN & Stephanomeria runcinata & Runcinate-leaved Skeltonweed \\
\hline STESPI & LYGSPI & Stephanomeria spinosa & Spiny Skeletonweed \\
\hline STRAMP & STRAMP & Streptopus amplexifolius & Clasping-leaved Twisted-stalk \\
\hline SUADEP & SUADEP & Suaeda depressa & Pahute Weed \\
\hline SWEPER & SWEPER & Swertia perennis & Swertia \\
\hline SYMOFF & SYMOFF & Symphytum officinale & Common Comfrey \\
\hline SYNPIN & SYNPIN & Synthyris pinnatifida & Featherleaf Kittentails \\
\hline TARERI & TARERI & Taraxacum eriophorum & Rocky Mountain Dandelion \\
\hline TARLAE & TARLAE & Taraxacum laevigatum & Red-seeded Dandelion \\
\hline TARLYR & TARLYR & Taraxacum lyratum & Dwarf Alpine Dandelion \\
\hline TAROFF & TAROFF & Taraxacum officinale & Common Dandelion \\
\hline THAALP & THAALP & Thalictrum alpinum & Alpine Meadowrue \\
\hline THAFEN & THAFEN & Thalictrum fendleri & Fendler's Meadowrue \\
\hline THAOCC & THAOCC & Thalictrum occidentale & Western Meadowrue \\
\hline THAVEN & THAVEN & Thalictrum venulosum & Veiny Meadowrue \\
\hline THEINT & THEINT & Thelypodium integrifolium & Entire-leaved Thelypody \\
\hline THEPAN & THEPAN & Thelypodium paniculatum & Northwestern Thelypody \\
\hline THESAG & THESAG & Thelypodium sagittatum & Slender Thelypody \\
\hline THEMON & THEMON & Thermopsis montana & Mountain Thermopsis \\
\hline THLARV & THLARV & Thlaspi arvense & Field Pennycress \\
\hline
\end{tabular}




\section{Appendix A. Flora of the Beaverhead Mountains Section Forbs}

\begin{tabular}{|c|c|c|c|}
\hline $\begin{array}{l}\text { USFS Reg. } \\
1 \text { Code }\end{array}$ & Synonymy & Species Name & Common Name \\
\hline THLMON & THLFEN & Thlaspi montanum & Fendler's Pennycress \\
\hline THLPAR & THLPAR & Thlaspi parviflorum & Small-flowered Pennycress \\
\hline TOFGLU & TOFGLU & Tofieldia glutinosa & Sticky Tofieldia \\
\hline TOWCON & TOWCON & Townsendia condensata & Cushion Townsendia \\
\hline TOWHOO & TOWHOO & Townsendia hookeri & Hooker's Townsendia \\
\hline TOWPAR & TOWPAR & Townsendia parryi & Parry's Townsendia \\
\hline TRADUB & TRADUB & Tragopogon dubius & Goat's Beard \\
\hline TRAMIS & TRAMIS & Tragopogon miscellus & Hybrid Salsify \\
\hline TRAPRA & TRAPRA & Tragopogon pratensis & Jack-go-to-bed-at-noon \\
\hline TRITER & TRITER & Tribulus terrestris & Puncture-vine \\
\hline TRIHAY & TRIHAY & Trifolium haydenii & Hayden's Clover \\
\hline TRILON & TRILON & Trifolium longipes & Long-stalked Clover \\
\hline TRINAN & TRINAN & Trifolium nanum & Dwarf Clover \\
\hline TRIPAR & TRIPAR & Trifolium parryi & Parry's Clover \\
\hline TRIPRA & TRIPRA & Trifolium pratense & Red Clover \\
\hline TRIREP & TRIREP & Trifolium repens & White Clover \\
\hline TRIMAR & TRIMAR & Triglochin maritimum & Seaside Arrow-grass \\
\hline TRIPAL & TRIPAL & Triglochin palustre & Marsh Arrow-grass \\
\hline TRIOVA & TRIOVA & Trillium ovatum & White Trillium \\
\hline TROLAX & TROLAX & Trollius laxus & American Globeflower \\
\hline TYPLAT & TYPLAT & Typha latifolia & Common Cattail \\
\hline URTDIO & URTDIO & Urtica dioica & Stinging Nettle \\
\hline UTRVUL & UTRVUL & Utricularia vulgaris & Common Bladderwort \\
\hline VALACU & VALACU & Valeriana acutiloba & Downy-fruit Valerian \\
\hline VALDIO & VALDIO & Valeriana dioica & Northern Valerian \\
\hline VALEDU & VALEDU & Valeriana edulis & Edible Valerian \\
\hline VALOCC & VALOCC & Valeriana occidentalis & Western Valerian \\
\hline VALSIT & VALSIT & Valeriana sitchensis & Sitka Valerian \\
\hline VERVIR & VERVIR & Veratrum viride & Green False Hellebore \\
\hline VERTHA & VERTHA & Verbascum thapsus & Common Mullein \\
\hline VERAME & VERAME & Veronica americana & American Speedwell \\
\hline VERBIL & VERBIL & Veronica biloba & Bilobed Speedwell \\
\hline VERCUS & VERCUS & Veronica cusickii & Cusick's Speedwell \\
\hline VERSCU & VERSCU & Veronica scutellata & Marsh Speedwell \\
\hline VERSER & VERSER & Veronica serpyllifolia & Thyme-leaved Speedwell \\
\hline VERVER & VERVER & Veronica verna & Spring Speedwell \\
\hline VERWOR & VERWOR & Veronica wormskjoldii & Wormskjold Speedwell \\
\hline VICAME & VICAME & Vicia americana & American Vetch \\
\hline VIOADU & VIOADU & Viola adunca & Hook Violet \\
\hline VIOCAN & VIOCAN & Viola canadensis & Canada Violet \\
\hline VIOGLA & VIOGLA & Viola glabella & Pioneer Violet \\
\hline VIOMAC & VIOMAC & Viola macloskeyi & Small White Violet \\
\hline VIONEP & VIONEP & Viola nephrophylla & Northern Bog Violet \\
\hline VIONUT & VIONUT & Viola nuttallii & Yellow Prairie Violet \\
\hline VIOORB & VIOORB & Viola orbiculata & Round-leaved Violet \\
\hline VIOPAL & VIOPAL & Viola palustris & Marsh Violet \\
\hline VIOVAL & VIONVV & Viola vallicola & Valley Yellow Violet \\
\hline WYEAMP & WYEAMP & Wyethia amplexicaulis & Northern Mule's-ears \\
\hline WYEHEL & WYEHEL & Wyethia helianthoides & White-head Mule's-ears \\
\hline
\end{tabular}


Appendix A. Flora of the Beaverhead Mountains Section

Forbs

\begin{tabular}{llll}
$\begin{array}{l}\text { USFS Reg. } \\
\text { 1 Code }\end{array}$ & Synonymy & Species Name & Common Name \\
\hline XANSTR & XANSTR & Xanthium strumarium & Common Cockleburr \\
XERTEN & XERTEN & Xerophyllum tenax & Beargrass \\
ZIGELE & ZIGELE & Zigadenus elegans & Glaucous Zigadenus \\
ZIGPAN & ZIGPAN & Zigadenus paniculatus & Panicled Death-camas \\
ZIGVEN & ZIGVEN & Zigadenus venenosus & Meadow Death-camas \\
ZIZAPT & ZIZAPT & Zizia aptera & Heart-leaved Alexanders \\
ZYGFAB & ZYGFAB & Zygophyllum fabago & Syrian Bean-caper
\end{tabular}




\section{Appendix A. Flora of the Beaverhead Mountains Section Graminoids}

\begin{tabular}{|c|c|c|c|}
\hline $\begin{array}{l}\text { USFS Reg. } \\
1 \text { Code }\end{array}$ & Synonymy & Species Name & Common Name \\
\hline AGRINT & ELYINE & Agropyron intermedium & Intermediate Wheatgrass \\
\hline AGRREP & ELYREE & Agropyron repens & Quackgrass \\
\hline AGRALB & AGRSTO & Agrostis alba & Redtop \\
\hline AGRDIE & AGRDIE & Agrostis diegoensis & Thin Bentgrass \\
\hline AGREXA & AGREXA & Agrostis exarata & Spike Bentgrass \\
\hline AGRHUM & AGRHUM & Agrostis humilis & Alpine Bentgrass \\
\hline AGRIDA & AGRIDA & Agrostis idahoensis & Idaho Bentgrass \\
\hline AGRSCA & AGRSCA & Agrostis scabra & Tickle-grass \\
\hline AGRSTO & AGRSTO & Agrostis stolonifera & Redtop \\
\hline AGRTHU & AGRTHU & Agrostis thurberiana & Thurber Bentgrass \\
\hline AGRVAR & AGRVAR & Agrostis variabilis & Variant Bentgrass \\
\hline ALOAEQ & ALOAEQ & Alopecurus aequalis & Short-awn Foxtail \\
\hline ALOALP & ALOALP & Alopecurus alpinus & Alpine Foxtail \\
\hline ALOGEN & ALOGEN & Alopecurus geniculatus & Water Foxtail \\
\hline ALOPRA & ALOPRA & Alopecurus pratensis & Meadow Foxtail \\
\hline ARILON & ARILON & Aristida longiseta & Red Threeawn \\
\hline BOUGRA & BOUGRA & Bouteloua gracilis & Blue Grama \\
\hline BROANO & BROANO & Bromus anomalus & Nodding Brome \\
\hline BROCAR & BROCAR & Bromus carinatus & Mountain Brome \\
\hline BROCIL & BROCIL & Bromus ciliatus & Fringed Brome \\
\hline BROINE & BROINE & Bromus inermis & Smooth Brome \\
\hline BROISP & BROISP & Bromus inermis ssp. pumpellianus & Pumpelly Brome \\
\hline BROJAP & BROJAP & Bromus japonicus & Japanese Brome \\
\hline BROMAR & BROCVL & Bromus marginatus & Mountain Brome \\
\hline BROMOL & BROMOL & Bromus mollis & Soft Brome \\
\hline BROTEC & BROTEC & Bromus tectorum & Cheatgrass \\
\hline BROVUL & BROVUL & Bromus vulgaris & Columbia Brome \\
\hline CALCAN & CALCAN & Calamagrostis canadensis & Bluejoint Reedgrass \\
\hline CALMON & CALMON & Calamagrostis montanensis & Plains Reedgrass \\
\hline CALPUR & CALPUR & Calamagrostis purpurascens & Purple Reedgrass \\
\hline CALRUB & CALRUB & Calamagrostis rubescens & Pinegrass \\
\hline CALSTR & CALSTR & Calamagrostis stricta & Narrow-spiked Reedgrass \\
\hline CARALB & CARALB & Carex albonigra & Black-and-white-scaled Sedge \\
\hline CARAQU & CARAQU & Carex aquatilis & Water Sedge \\
\hline CARATH & CARATH & Carex atherodes & Awned Sedge \\
\hline CARATO & CARATO & Carex athrostachya & Slender-beaked Sedge \\
\hline CARATR & CARATR & Carex atrata & Blackened Sedge \\
\hline CARAUR & CARAUR & Carex aurea & Golden Sedge \\
\hline CARBRE & CARBRE & Carex brevior & Short-beaked Sedge \\
\hline CARBUX & CARBUX & Carex buxbaumii & Buxbaum's Sedge \\
\hline CARCAN & CARCAN & Carex canescens & Gray Sedge \\
\hline CARCON & CARCON & Carex concinnoides & Northwestern Sedge \\
\hline CARDIA & CARDIA & Carex diandra & Lesser-panicled Sedge \\
\hline CARDIS & CARDIS & Carex disperma & Soft-leaved Sedge \\
\hline CARDOU & CARDOU & Carex douglasii & Douglas's Sedge \\
\hline CARELY & CARELY & Carex elynoides & Kobresia-like Sedge \\
\hline CAREXS & CARVES & Carex exsiccata & Inflated Sedge \\
\hline CARFIL & CARFIL & Carex filifolia & Thread-leaved Sedge \\
\hline CARGEY & CARGEY & Carex geyeri & Elk Sedge \\
\hline CARHAY & CARHAY & Carex haydeniana & Hayden's Sedge \\
\hline
\end{tabular}




\section{Appendix A. Flora of the Beaverhead Mountains Section Graminoids}

\begin{tabular}{|c|c|c|c|}
\hline $\begin{array}{l}\text { USFS Reg. } \\
1 \text { Code } \\
\end{array}$ & Synonymy & Species Name & Common Name \\
\hline CARHOO & CARHOO & Carex hoodii & Hood's Sedge \\
\hline CARIDA & CARPSI & Carex idahoa & Idaho Sedge \\
\hline CARILL & CARILL & Carex illota & Sheep Sedge \\
\hline CARINT & CARINT & Carex interior & Inland Sedge \\
\hline CARLAN & CARLAN & Carex lanuginosa & Woolly Sedge \\
\hline CARLAS & CARLAS & Carex lasiocarpa & Slender Sedge \\
\hline CARLEN & CARLEN & Carex lenticularis & Lentil-fruited Sedge \\
\hline CARLIM & CARLIM & Carex limosa & Mud Sedge \\
\hline CARLUZ & CARLUZ & Carex luzulina & Woodrush Sedge \\
\hline CARMIC & CARMIC & Carex microptera & Small-winged Sedge \\
\hline CARNAR & CARNAR & Carex nardina & Spikenard Sedge \\
\hline CARNEB & CARNEB & Carex nebrascensis & Nebraska Sedge \\
\hline CARNEU & CARNEU & Carex neurophora & Alpine Nerved Sedge \\
\hline CARNIG & CARNIG & Carex nigricans & Black Alpine Sedge \\
\hline CARNOV & CARNOV & Carex nova & New Sedge \\
\hline CAROBT & CAROBT & Carex obtusata & Blunt Sedge \\
\hline CARPAC & CARPAC & Carex pachystachya & Thick-headed Sedge \\
\hline CARPAR & CARPAR & Carex parryana & Parry's Sedge \\
\hline CARPAY & CARPAY & Carex paysonis & Payson Sedge \\
\hline CARPED & CARPED & Carex pedunculata & Woodland Sedge \\
\hline CARPEN & CARPEN & Carex pensylvanica & Long-stolon Sedge \\
\hline CARPET & CARPET & Carex petasata & Liddon's Sedge \\
\hline CARPHA & CARPHA & Carex phaeocephala & Dunhead Sedge \\
\hline CARPRA & CARPRA & Carex praegracilis & Clustered Field Sedge \\
\hline CARPRT & CARPRT & Carex praticola & Meadow Sedge \\
\hline CARPRI & CARPRI & Carex prionophylla & Saw-leaved Sedge \\
\hline CARPYR & CARPYR & Carex pyrenaica & Pyrenaean Sedge \\
\hline CARRAY & CARRAY & Carex raynoldsii & Raynolds' Sedge \\
\hline CARROI & CARROI & Carex rossii & Ross Sedge \\
\hline CARRUP & CARRUP & Carex rupestris & Curly Sedge \\
\hline CARSCI & CARSCI & Carex scirpoidea & Canadian Single-spike Sedge \\
\hline CARSCO & CARSCO & Carex scopulorum & Holm's Rocky Mountain Sedge \\
\hline CARSIM & CARSIM & Carex simulata & Short-beaked Sedge \\
\hline CARSPE & CARSPE & Carex spectabilis & Showy Sedge \\
\hline CARUTR & CARUTR & Carex utriculata & Beaked Sedge \\
\hline CARVAL & CARVAL & Carex vallicola & Valley Sedge \\
\hline CARVES & CARVES & Carex vesicaria & Inflated Sedge \\
\hline CARXER & CARXER & Carex xerantica & Dryland Sedge \\
\hline CATAQU & CATAQU & Catabrosa aquatica & Brookgrass \\
\hline CINLAT & CINLAT & Cinna latifolia & Drooping Woodreed \\
\hline DACGLO & DACGLO & Dactylis glomerata & Orchard-grass \\
\hline DANCAL & DANCAL & Danthonia californica & California Oatgrass \\
\hline DANINT & DANINT & Danthonia intermedia & Timber Oatgrass \\
\hline DANSPI & DANSPI & Danthonia spicata & Poverty Oatgrass \\
\hline DANUNI & DANUNI & Danthonia unispicata & Onespike Oatgrass \\
\hline DESCES & DESCES & Deschampsia cespitosa & Tufted Hairgrass \\
\hline DISSPI & DISSPI & Distichlis spicata & Inland Saltgrass \\
\hline DISSTR & DISSPI & Distichlis stricta & Alkali Saltgrass \\
\hline ELEACI & ELEACI & Eleocharis acicularis & Needle Spike-rush \\
\hline ELEPAL & ELEPAL & Eleocharis palustris & Common Spikesedge \\
\hline
\end{tabular}




\section{Appendix A. Flora of the Beaverhead Mountains Section Graminoids}

\begin{tabular}{|c|c|c|c|}
\hline $\begin{array}{l}\text { USFS Reg. } \\
1 \text { Code }\end{array}$ & Synonymy & Species Name & Common Name \\
\hline ELEPAU & ELEPAU & Eleocharis pauciflora & Few-flowered Spike-rush \\
\hline ELYCAN & ELYCAN & Elymus canadensis & Canada Wildrye \\
\hline ELYELY & SITHYS & Elymus elymoides & Bottlebrush Squirreltail \\
\hline ELYGLA & ELYGLA & Elymus glaucus & Blue Wildrye \\
\hline ELYLAN & ELYLAN & Elymus lanceolatus & Thick-spike Wheatgrass \\
\hline ELYREP & ELYREE & Elymus repens & Quackgrass \\
\hline ELYSCR & ELYSCR & Elymus scribneri & Spreading Wheatgrass \\
\hline ELYTRA & ELYTRA & Elymus trachycaulus & Bearded Wheatgrass \\
\hline ELYVIR & ELYVIR & Elymus virginicus & Virginia Wildrye \\
\hline ERIANG & ERIPOL & Eriophorum angustifolium & Many-spiked Cotton-grass \\
\hline ERICHA & ERICHA & Eriophorum chamissonis & Chamisso's Cotton-grass \\
\hline ERIVIR & ERIVIR & Eriophorum viridicarinatum & Green-keeled Cotton-grass \\
\hline FESCAM & FESSCA & Festuca campestris & Rough Fescue \\
\hline FESIDA & FESIDA & Festuca idahoensis & Idaho Fescue \\
\hline FESKIN & LEUKIN & Festuca kingii & Spike-fescue \\
\hline FESOCC & FESOCC & Festuca occidentalis & Western Fescue \\
\hline FESOVI & FESOVI & Festuca ovina & Sheep Fescue \\
\hline FESPRA & FESPRA & Festuca pratensis & Meadow Fescue \\
\hline FESRUB & FESRUB & Festuca rubra & Red Fescue \\
\hline GLYBOR & GLYBOR & Glyceria borealis & Northern Mannagrass \\
\hline GLYELA & GLYELA & Glyceria elata & Tall Mannagrass \\
\hline GLYGRA & GLYGRA & Glyceria grandis & American Mannagrass \\
\hline GLYSTR & GLYSTR & Glyceria striata & Fowl Mannagrass \\
\hline HORBRA & HORBRA & Hordeum brachyantherum & Meadow Barley \\
\hline HORJUB & HORJUB & Hordeum jubatum & Foxtail Barley \\
\hline HORPUS & HORPUS & Hordeum pusillum & Little Barley \\
\hline JUNALP & JUNALP & Juncus alpinus & Northern Rush \\
\hline JUNBAL & JUNBAL & Juncus balticus & Baltic Rush \\
\hline JUNCAS & JUNCAS & Juncus castaneus & Chestnut Rush \\
\hline JUNCON & JUNCON & Juncus confusus & Colorado Rush \\
\hline JUNDRU & JUNDRU & Juncus drummondii & Drummond's Rush \\
\hline JUNEFF & JUNEFF & Juncus effusus & Soft Rush \\
\hline JUNENS & JUNENS & Juncus ensifolius & Dagger-leaf Rush \\
\hline JUNLON & JUNLON & Juncus longistylis & Long-styled Rush \\
\hline JUNMER & JUNMER & Juncus mertensianus & Merten's Rush \\
\hline JUNNEV & JUNNEV & Juncus nevadensis & Sierra Rush \\
\hline JUNPAR & JUNPAR & Juncus parryi & Parry's Rush \\
\hline JUNTEN & JUNTEN & Juncus tenuis & Slender Rush \\
\hline JUNTOR & JUNTOR & Juncus torreyi & Torrey's Rush \\
\hline JUNTRA & JUNTRA & Juncus tracyi & Tracy's Rush \\
\hline KOEMAC & KOECRI & Koeleria macrantha & Prairie Junegrass \\
\hline LEYCIN & LEYCIN & Leymus cinereus & Giant Wildrye \\
\hline LUZCAM & LUZCAM & Luzula campestris & Field Woodrush \\
\hline LUZGVH & LUZHIT & Luzula glabrata var. hitchcockii & Smooth Woodrush \\
\hline LUZPAR & LUZPAR & Luzula parviflora & Small-flowered Woodrush \\
\hline LUZSPI & LUZSPI & Luzula spicata & Spike Woodrush \\
\hline MELBUL & MELBUL & Melica bulbosa & Oniongrass \\
\hline MELSPE & MELSPE & Melica spectabilis & Showy Oniongrass \\
\hline MELSUB & MELSUB & Melica subulata & Alaska Oniongrass \\
\hline MUHASP & MUHASP & Muhlenbergia asperifolia & Alkali Muhly \\
\hline
\end{tabular}




\section{Appendix A. Flora of the Beaverhead Mountains Section Graminoids}

\begin{tabular}{|c|c|c|c|}
\hline $\begin{array}{l}\text { USFS Reg. } \\
1 \text { Code }\end{array}$ & Synonymy & Species Name & Common Name \\
\hline MUHCUS & MUHCUS & Muhlenbergia cuspidata & Plains Muhly \\
\hline MUHFIL & MUHFIL & Muhlenbergia filiformis & Slender Muhly \\
\hline MUHRIC & MUHRIC & Muhlenbergia richardsonis & Mat Muhly \\
\hline NASVIR & STIVIR & Nassella viridula & Green Needlegrass \\
\hline ORYEXI & ORYEXI & Oryzopsis exigua & Little Ricegrass \\
\hline ORYHYM & ORYHYM & Oryzopsis hymenoides & Indian Ricegrass \\
\hline PASSMI & PASSMI & Pascopyrum smithii & Western Wheatgrass \\
\hline PHAARU & PHAARU & Phalaris arundinacea & Reed Canarygrass \\
\hline PHLALP & PHLALP & Phleum alpinum & Alpine Timothy \\
\hline PHLPRA & PHLPRA & Phleum pratense & Common Timothy \\
\hline POAALP & POAALP & Poa alpina & Alpine Bluegrass \\
\hline POAAMP & POASEC & Poa ampla & Alkali Bluegrass \\
\hline POAARC & POAARC & Poa arctica & Artic Bluegrass \\
\hline POAARI & POAARI & Poa arida & Plains Bluegrass \\
\hline POABUL & POABUL & Poa bulbosa & Bulbous Bluegrass \\
\hline POACAN & POASEC & Poa canbyi & Canby's Bluegrass \\
\hline POACOM & POACOM & Poa compressa & Canada Bluegrass \\
\hline POACUS & POAFEN & Poa cusickii & Cusick's Bluegrass \\
\hline POAFEN & POAFEN & Poa fendleriana & Muttongrass \\
\hline POAGLA & POAGLA & Poa glaucifolia & Pale-leaf Bluegrass \\
\hline POAGRA & POAARC & Poa grayana & Gray's Bluegrass \\
\hline POAINT & POAGLU & Poa interior & Inland Bluegrass \\
\hline POAJUN & POASEC & Poa juncifolia & Alkali Bluegrass \\
\hline POALEP & POALEP & Poa leptocoma & Bog Bluegrass \\
\hline POALET & POALET & Poa lettermanii & Letterman's Bluegrass \\
\hline POANER & POANER & Poa nervosa & Wheeler's Bluegrass \\
\hline POANEV & POASEC & Poa nevadensis & Nevada Bluegrass \\
\hline POAPAL & POAPAL & Poa palustris & Fowl Bluegrass \\
\hline POAPRA & POAPRA & Poa pratensis & Kentucky Bluegrass \\
\hline POAREF & POAREF & Poa reflexa & Nodding Bluegrass \\
\hline POASAN & POASEC & Poa sandbergii & Sandberg's Bluegrass \\
\hline POASCA & POASEC & Poa scabrella & Pine Bluegrass \\
\hline POASEC & POASEC & Poa secunda & Sandberg's Bluegrass \\
\hline POASTE & POASTE & Poa stenantha & Trinius' Bluegrass \\
\hline POATRI & POATRI & Poa trivialis & Roughstalk Bluegrass \\
\hline PSESPI & PSESPI & Pseudoroegneria spicata & Bluebunch Wheatgrass \\
\hline PUCDIS & PUCDIS & Puccinellia distans & Weeping Alkaligrass \\
\hline PUCNUT & PUCNUT & Puccinellia nuttalliana & Nuttall's Alkaligrass \\
\hline SCIACU & SCIACU & Scirpus acutus & Hardstem Bulrush \\
\hline SCITAB & SCIVAL & Scirpus tabernaemontani & Softstem Bulrush \\
\hline SPAGRA & SPAGRA & Spartina gracilis & Alkali Cordgrass \\
\hline SPAPEC & SPAPEC & Spartina pectinata & Prairie Cordgrass \\
\hline SPHOBT & SPHOBT & Sphenopholis obtusata & Prairie Wedgegrass \\
\hline SPOAIR & SPOAIR & Sporobolus airoides & Alkali Sacaton \\
\hline SPOCRY & SPOCRY & Sporobolus cryptandrus & Sand Dropseed \\
\hline STICOM & STICOM & Stipa comata & Needle-and-thread \\
\hline STIOCC & STIOCC & Stipa occidentalis & Western Needlegrass \\
\hline STIRIC & STIRIC & Stipa richardsonii & Richardson's Needlegrass \\
\hline TRISPI & TRISPI & Trisetum spicatum & Spike Trisetum \\
\hline TRIWOL & TRIWOL & Trisetum wolfii & Wolf's Trisetum \\
\hline
\end{tabular}




\section{Appendix A. Flora of the Beaverhead Mountains Section Fern and Fern Allies}

\begin{tabular}{llll}
$\begin{array}{c}\text { USFS Reg. } \\
\text { 1 Code }\end{array}$ & Synonymy & Species Name & Common Name \\
\hline CHEFEE & CHEFEE & Cheilanthes feei & Fee's Lip-fern \\
CYSFRA & CYSFRA & Cystopteris fragilis & Brittle Bladder-fern \\
EQUARV & EQUARV & Equisetum arvense & Field Horsetail \\
EQUFLU & EQUFLU & Equisetum fluviatile & Water Horsetail \\
EQUHYE & EQUHYE & Equisetum hyemale & Common Scouring-rush \\
EQULAE & EQULAE & Equisetum laevigatum & Smooth Scouring-rush \\
SELDEN & SELDEN & Selaginella densa & Compact Selaginella \\
SELWAT & SELWAT & Selaginella watsonii & Watson's Selaginella \\
WOOORE & WOOORE & Woodsia oregana & Oregon Woodsia \\
WOOSCO & WOOSCO & Woodsia scopulina & Rocky Mountain Woodsia
\end{tabular}




\section{Appendix A. Flora of the Beaverhead Mountains Section Non-Vascular}

USFS Reg. Synonymy $\quad$ Species Name Common Name
1 Code

Non-vascular Algae

$\begin{array}{lll}\text { PEDBRA } & \text { PEDBRA } & \text { Pediastrum braunii } \\ \text { PEDSIM } & \text { PEDSIM } & \text { Pediastrum simplex }\end{array}$

Non-vascular Lichen

$\begin{array}{lll}\text { COLTEN } & \text { COLTEN } & \text { Collema tenax } \\ \text { MELALB } & \text { MELALB } & \text { Melanelia albertana } \\ \text { MELSUB } & \text { MELSUB } & \text { Melanelia substygia } \\ \text { VERVIR } & \text { VERVIR } & \text { Verrucaria viridula }\end{array}$

Non-vascular Moss

\begin{tabular}{|c|c|c|c|}
\hline CALSTR & CALSTR & Calliergon stramineum & Pale Spoon Moss \\
\hline LEPPYR & LEPPYR & Leptobryum pyriforme & Pear Moss \\
\hline
\end{tabular}


Appendix B.

\section{Natural Heritage Global Rank Definitions and Their Modifiers}

\section{Rank Global Rank Definitions}

GX Presumed Extinct - Believed to be extinct throughout its range. Not located despite intensive searches of historic sites and other appropriate habitat, and virtually no likelihood that it will be rediscovered.

GH Possibly Extinct - Known from only historical occurrences. Still some hope of rediscovery.

G1 Critically Imperiled - Critically imperiled globally because of extreme rarity or because of some factor(s) making it especially vulnerable to extinction. Typically 5 or fewer occurrences or very few remaining individuals $(<1,000)$ or acres $(<2,000)$ or stream miles $(<10)$.

G2 Imperiled - Imperiled globally because of rarity or because of some factor(s) making it very vulnerable to extinction. Typically 6 to 20 occurrences or few remaining individuals $(1,000$ to 3,000$)$ or acres $(2,000$ to 10,000$)$ or stream miles $(10$ to 50).

G3 Vulnerable - Vulnerable globally either because very rare and local throughout its range, found only in a restricted range (even if abundant at some locations), or because of other factors making it vulnerable to extinction. Typically 21 to 100 occurrences or between 3,000 and 10,000 individuals.

G4 Apparently Secure - Uncommon but not rare, and usually widespread. Possibly cause for long-term concern. Typically more than 100 occurrences and more than 10,000 individuals.

G5 Secure - Common, typically widespread and abundant. Typically with considerably more than 100 occurrences and more than 10,000 individuals.

GU Unrankable - Currently unrankable due to lack of information or due to substantially conflicting information about status or trends.

G? Unranked - Global rank not yet assessed

? Inexact numeric rank 


\section{Appendix C. Plant Associations of the Beaverhead Mountains Section Evergreen Forest}

\begin{tabular}{|c|c|c|}
\hline Association & State Rank & Global Rank \\
\hline Abies lasiocarpa / Actaea rubra Forest & $\mathrm{S} 2$ & G4? \\
\hline Abies lasiocarpa / Alnus viridis ssp. sinuata Forest & S4 & G4 \\
\hline Abies lasiocarpa / Arnica cordifolia Forest & S5 & G5 \\
\hline Abies lasiocarpa / Arnica latifolia Forest & S3 & G4 \\
\hline Abies lasiocarpa / Calamagrostis canadensis Forest & S5 & G5 \\
\hline Abies lasiocarpa / Calamagrostis rubescens Forest & S3 & G4G5 \\
\hline Abies lasiocarpa / Carex geyeri Forest & S3 & G5 \\
\hline Abies lasiocarpa / Clematis columbiana var. columbiana Forest & S3? & G3? \\
\hline Abies lasiocarpa / Galium triflorum Forest & S4 & G4 \\
\hline Abies lasiocarpa / Ledum glandulosum Forest & S4 & G4 \\
\hline Abies lasiocarpa / Linnaea borealis Forest & S5 & G5 \\
\hline Abies lasiocarpa / Luzula glabrata var. hitchcockii Forest & S5 & G5 \\
\hline Abies lasiocarpa / Menziesia ferruginea Forest & S5 & G5 \\
\hline Abies lasiocarpa / Ribes (montigenum, lacustre, inerme) Forest & S4 & G5 \\
\hline Abies lasiocarpa / Streptopus amplexifolius Forest & S3 & G4? \\
\hline Abies lasiocarpa / Symphoricarpos albus Forest & S3 & G3 \\
\hline Abies lasiocarpa / Thalictrum occidentale Forest & S3 & G4 \\
\hline Abies lasiocarpa / Vaccinium cespitosum Forest & S5 & G5 \\
\hline Abies lasiocarpa / Vaccinium membranaceum Rocky Mountain Forest & S5 & G5 \\
\hline Abies lasiocarpa / Vaccinium scoparium Forest & S5 & G5 \\
\hline Abies lasiocarpa / Xerophyllum tenax Forest & S5 & G5 \\
\hline Picea (engelmannii X glauca, engelmannii) / Carex disperma Forest & S2? & G2Q \\
\hline Picea (engelmannii X glauca, engelmannii) / Galium triflorum Forest & S4 & G4 \\
\hline Picea (engelmannii X glauca, engelmannii) / Juniperus communis Forest & S2 & G2Q \\
\hline Picea (engelmannii X glauca, engelmannii) / Linnaea borealis Forest & S4 & G4 \\
\hline Picea (engelmannii X glauca, engelmannii) / Maianthemum stellatum Forest & S3 & G3 \\
\hline Picea (engelmannii X glauca, engelmannii) / Physocarpus malvaceus Forest & S3 & G3 \\
\hline Picea (engelmannii X glauca, engelmannii) / Senecio streptanthifolius Forest & S4 & G4 \\
\hline Picea (engelmannii X glauca, engelmannii) / Vaccinium cespitosum Forest & S4 & G4 \\
\hline Picea engelmannii / Calamagrostis canadensis Forest & S3 & G4 \\
\hline Picea engelmannii / Equisetum arvense Forest & S2 & G4 \\
\hline Picea engelmannii / Hypnum revolutum Forest & SP & G2 \\
\hline Picea engelmannii / Vaccinium scoparium Forest & SP & G3G5 \\
\hline Pinus albicaulis / Carex rossii Forest & SP & G3? \\
\hline Pinus albicaulis / Vaccinium scoparium Forest & S3 & G4 \\
\hline Pinus contorta / Arnica cordifolia Forest & S3? & G4? \\
\hline Pinus contorta / Calamagrostis rubescens Forest & S5 & G5 \\
\hline Pinus contorta / Carex geyeri Forest & SP & G4? \\
\hline Pinus contorta / Carex rossii Forest & $\mathrm{SP}$ & G5 \\
\hline Pinus contorta / Linnaea borealis Forest & S5 & G5 \\
\hline Pinus contorta / Spiraea betulifolia Forest & SP & G3G4 \\
\hline Pinus contorta / Vaccinium cespitosum Forest & S5 & G5 \\
\hline Pinus contorta / Vaccinium scoparium Forest & S5 & G5 \\
\hline Pinus contorta / Xerophyllum tenax Forest & S4 & G5 \\
\hline Pseudotsuga menziesii / Arnica cordifolia Forest & S4 & G4 \\
\hline Pseudotsuga menziesii / Calamagrostis rubescens Forest & S5 & G5 \\
\hline
\end{tabular}




\section{Appendix C. Plant Associations of the Beaverhead Mountains Section Evergreen Forest}

\begin{tabular}{lcc} 
Association & State Rank Global Ran \\
\hline Pseudotsuga menziesii / Carex geyeri Forest & S4 & G4? \\
Pseudotsuga menziesii / Juniperus communis Forest & S4 & G4 \\
Pseudotsuga menziesii / Linnaea borealis Forest & S4 & G4 \\
Pseudotsuga menziesii / Mahonia repens Forest & S3 & G5 \\
Pseudotsuga menziesii / Physocarpus malvaceus Forest & S5 & G5 \\
Pseudotsuga menziesii / Spiraea betulifolia Forest & S4 & G5 \\
Pseudotsuga menziesii / Symphoricarpos albus Forest & S5 & G5 \\
Pseudotsuga menziesii / Symphoricarpos oreophilus Forest & S3 & G5 \\
Pseudotsuga menziesii / Vaccinium membranaceum Forest & S5 & G5?
\end{tabular}




\section{Appendix C. Plant Associations of the Beaverhead Mountains Section Evergreen Woodland}

\begin{tabular}{lcc} 
Association & State Rank & Global Ra \\
\hline Abies lasiocarpa - Pinus albicaulis / Vaccinium scoparium Woodland & S5 & G5? \\
Abies lasiocarpa / Juniperus communis Woodland & S3 & G4G5 \\
Abies lasiocarpa Scree Woodland & S5 & G5? \\
Cercocarpus ledifolius / Festuca idahoensis Woodland & S2 & G3 \\
Juniperus scopulorum / Artemisia nova Woodland & S2 & G2? \\
Juniperus scopulorum / Artemisia tridentata Woodland & S2? & G3Q \\
Juniperus scopulorum / Cercocarpus ledifolius Woodland & S3? & G3? \\
Juniperus scopulorum / Cornus sericea Woodland & S4 & G4 \\
Juniperus scopulorum / Pseudoroegneria spicata Woodland & S4 & G4 \\
Picea engelmannii / Cornus sericea Woodland & S3 & G3 \\
Pinus albicaulis - Abies lasiocarpa Woodland [Provisional] & S5 & G5? \\
Pinus albicaulis / Carex geyeri Woodland & S1 & G2G3 \\
Pinus albicaulis / Festuca idahoensis Woodland & S4 & G4 \\
Pinus albicaulis / Juniperus communis Woodland & SP & G4? \\
Pinus albicaulis Woodland [Provisional] & S5 & G5? \\
Pinus contorta / Juniperus communis Woodland & S3 & G5 \\
Pinus contorta Scree Woodland & S5Q & G5? \\
Pinus flexilis / Cercocarpus ledifolius Woodland & S3 & G3G4 \\
Pinus flexilis / Festuca idahoensis Woodland & S5 & G5 \\
Pinus flexilis / Juniperus communis Woodland & S4 & G5 \\
Pinus flexilis / Pseudoroegneria spicata Woodland & S4 & G4 \\
Pinus flexilis / Scree Woodland & S3 & G3Q \\
Pinus ponderosa / Pseudoroegneria spicata Woodland & S4 & G4 \\
Pseudotsuga menziesii / Cercocarpus ledifolius Woodland & S? & G4? \\
Pseudotsuga menziesii / Cornus sericea Woodland & S3 & G4 \\
Pseudotsuga menziesii / Festuca campestris Woodland & S4 & G4 \\
Pseudotsuga menziesii / Festuca idahoensis Woodland & S4 & G4 \\
Pseudotsuga menziesii / Juniperus scopulorum Woodland & S3 & G3 \\
Pseudotsuga menziesii / Pseudoroegneria spicata Woodland & S4 & G4 \\
Pseudotsuga menziesii Scree Woodland & S4 & G5
\end{tabular}




\section{Appendix C. Plant Associations of the Beaverhead Mountains Section Deciduous Forest and Woodland}

\begin{tabular}{lcc} 
Association & State Rank Global Rank \\
\hline Larix lyallii - Abies lasiocarpa Forest [Provisional] & S4 & G4 \\
Populus angustifolia / Cornus sericea Woodland & S3 & G4 \\
Populus balsamifera ssp. trichocarpa / Poa pratensis Forest & S? & G? \\
Populus tremuloides / Amelanchier alnifolia Forest & S3 & G4 \\
Populus tremuloides / Bromus carinatus Forest & S? & G5 \\
Populus tremuloides / Calamagrostis canadensis Forest & S2 & G3 \\
Populus tremuloides / Calamagrostis rubescens Forest & S4 & G5? \\
Populus tremuloides / Cornus sericea Forest & S3 & G4 \\
Populus tremuloides / Poa pratensis Forest & S? & G? \\
Populus tremuloides / Symphoricarpos oreophilus Forest & S3 & G5 \\
Populus tremuloides / Tall Forbs Forest & S3 & G5 \\
Populus tremuloides / Thalictrum fendleri Forest & S? & G5
\end{tabular}




\section{Appendix C. Plant Associations of the Beaverhead Mountains Section Shrubland}

\begin{tabular}{|c|c|c|}
\hline Association & State Rank & Global Rank \\
\hline Alnus incana Shrubland & S3 & G?Q \\
\hline Alnus viridis ssp. sinuata Shrubland [Provisional] & $\mathrm{S} 2$ & G2Q \\
\hline Artemisia cana / Festuca idahoensis Shrub Herbaceous Vegetation & S3? & G3? \\
\hline Artemisia cana / Leymus cinereus Shrubland & S? & G1 \\
\hline Artemisia cana / Pascopyrum smithii Shrubland & S4 & G4 \\
\hline Artemisia cana / Poa pratensis Shrub Herbaceous Vegetation & $\mathrm{S} ?$ & S? \\
\hline Artemisia cana / Stipa comata Shrub Herbaceous Vegetation & S3 & G3 \\
\hline Artemisia tridentata / Festuca campestris Shrub Herbaceous Vegetation & S3 & G3 \\
\hline Artemisia tridentata / Festuca idahoensis Shrub Herbaceous Vegetation & S4? & G4Q \\
\hline Artemisia tridentata / Stipa comata Shrubland & $\mathrm{S} 4$ & G4Q \\
\hline Artemisia tridentata ssp. tridentata / Festuca idahoensis Shrubland & SP & G4? \\
\hline Artemisia tridentata ssp. tridentata / Leymus cinereus Shrubland & S1 & G2 \\
\hline Artemisia tridentata ssp. tridentata / Pascopyrum smithii Shrubland & S3 & G3? \\
\hline $\begin{array}{l}\text { Artemisia tridentata ssp. tridentata / Pseudoroegneria spicata Shrub } \\
\text { Herbaceous Vegetation }\end{array}$ & SP & G2G4 \\
\hline $\begin{array}{l}\text { Artemisia tridentata ssp. vaseyana / Festuca campestris Shrub Herbaceous } \\
\text { Vegetation }\end{array}$ & S? & $\mathrm{G} ?$ \\
\hline $\begin{array}{l}\text { Artemisia tridentata ssp. vaseyana / Festuca idahoensis Shrub Herbaceous } \\
\text { Vegetation }\end{array}$ & S4 & G5 \\
\hline $\begin{array}{l}\text { Artemisia tridentata ssp. vaseyana - Symphoricarpos oreophilus / Festuca } \\
\text { idahoensis Shrubland }\end{array}$ & S? & G4 \\
\hline $\begin{array}{l}\text { Artemisia tridentata ssp. vaseyana / Festuca idahoensis - Elymus trachycaulus } \\
\text { Shrubland }\end{array}$ & S? & G? \\
\hline $\begin{array}{l}\text { Artemisia tridentata ssp. vaseyana / Festuca idahoensis - Geranium } \\
\text { viscossimum Shrubland }\end{array}$ & S? & G? \\
\hline $\begin{array}{l}\text { Artemisia tridentata ssp. vaseyana / Festuca idahoensis - Pascopyrum smithii } \\
\text { Shrubland }\end{array}$ & S? & G? \\
\hline $\begin{array}{l}\text { Artemisia tridentata ssp. vaseyana / Festuca idahoensis - Pseudoroegneria } \\
\text { spicata Shrubland }\end{array}$ & S? & G? \\
\hline Artemisia tridentata ssp. vaseyana / Leymus cinereus Shrubland & S? & G4? \\
\hline Artemisia tridentata ssp. vaseyana / Pascopyrum smithii Shrubland & S3? & G3? \\
\hline Artemisia tridentata ssp. vaseyana / Pseudoroegneria spicata Shrubland & S? & G5 \\
\hline $\begin{array}{l}\text { Artemisia tridentata ssp. vayesana / Festuca idahoensis - Stipa occidentalis } \\
\text { Shrubland }\end{array}$ & S? & $\mathrm{G} ?$ \\
\hline $\begin{array}{l}\text { Artemisia tridentata ssp. wyomingensis / Elymus lanceolatus ssp. albicans } \\
\text { Shrubland }\end{array}$ & S? & G4 \\
\hline Artemisia tridentata ssp. wyomingensis / Poa secunda Shrubland & SP & G4 \\
\hline $\begin{array}{l}\text { Artemisia tridentata ssp. wyomingensis / Pseudoroegneria spicata Shrub } \\
\text { Herbaceous Vegetation }\end{array}$ & S3 & G4 \\
\hline Artemisia tridentata ssp. wyomingensis / Pseudoroegneria spicata Shrubland & S5? & G5? \\
\hline Artemisia tripartita / Elymus lanceolatus Shrub Herbaceous Vegetation & S? & $\mathrm{G} ?$ \\
\hline Artemisia tripartita / Festuca idahoensis Shrub Herbaceous Vegetation & S3? & G3 \\
\hline Artemisia tripartita / Pseudoroegneria spicata Shrub Herbaceous Vegetation & S? & G2G3 \\
\hline Betula glandulosa / Carex utriculata Shrubland & S4 & G4? \\
\hline Betula occidentalis Shrubland & S3 & G3Q \\
\hline Cercocarpus ledifolius / Pseudoroegneria spicata Shrubland & S4 & G4Q \\
\hline Chrysothamnus viscidiflorus / Stipa comata Shrubland & S? & $\mathrm{S} ?$ \\
\hline Cornus sericea Shrubland [Provisional] & S3 & G4 \\
\hline Pentaphylloides floribunda / Carex utriculatra Shrubland & S? & G? \\
\hline Pentaphylloides floribunda / Deschampsia cespitosa Shrubland & S4 & G4 \\
\hline
\end{tabular}




\section{Appendix C. Plant Associations of the Beaverhead Mountains Section Shrubland}

\begin{tabular}{|c|c|c|}
\hline Association & State Rank & Global Rank \\
\hline Pentaphylloides floribunda / Festuca campestris Shrub Herbaceous Vegetation & S3 & G4 \\
\hline Pentaphylloides floribunda / Festuca idahoensis Shrub Herbaceous Vegetation & S2S3 & $\mathrm{G} 3$ \\
\hline Pentaphylloides floribunda / Potentilla ovina Shrubland & S? & G? \\
\hline Purshia tridentata / Festuca idahoensis Shrub Herbaceous Vegetation & S3 & G3G5 \\
\hline Purshia tridentata / Pseudoroegneria spicata Shrub Herbaceous Vegetation & SP & G3 \\
\hline Rhus trilobata / Pseudoroegneria spicata Shrub Herbaceous Vegetation & S4 & G4 \\
\hline Rosa woodsii Shrubland & S5 & G5 \\
\hline Salix bebbiana Shrubland & S3? & G3? \\
\hline Salix boothii / Calamagrostis canadensis Shrubland & SR & G3G4Q \\
\hline Salix boothii / Carex utriculata Shrubland & S? & G4 \\
\hline Salix boothii / Mesic Graminoids Shrubland & S? & G3? \\
\hline Salix brachycarpa / Carex aquatilis Shrubland & S? & G2G3 \\
\hline Salix candida / Carex lasiocarpa Shrubland & S? & G? \\
\hline Salix candida / Carex utriculata Shrubland & S3 & G3 \\
\hline Salix drummondiana / Calamagrostis canadensis Shrubland & SP & $\mathrm{G} 3$ \\
\hline Salix drummondiana / Carex utriculata Shrubland & S5 & $\mathrm{G} 3$ \\
\hline Salix drummondiana Shrubland [Provisional] & S3 & G3Q \\
\hline Salix exigua Temporarily Flooded Shrubland & S5 & G5 \\
\hline Salix geyeriana / Calamagrostis canadensis Shrubland & S4 & G5 \\
\hline Salix geyeriana / Carex rostrata Shrubland & S5 & G5 \\
\hline Salix geyeriana / Carex utriculata Shrubland & S4 & G4 \\
\hline Salix geyeriana / Deschampsia cespitosa Shrubland & S3 & G4 \\
\hline Salix glauca Shrubland & S2 & G3? \\
\hline Salix lutea / Calamagrostis canadensis Shrubland & S3? & G3? \\
\hline Salix planifolia / Carex aquatilis Shrubland & S3 & G5 \\
\hline Salix planifolia / Carex nebrascensis Shrubland & S? & G? \\
\hline Salix planifolia / Carex scopulorum Shrubland & S? & $\mathrm{G} 4$ \\
\hline Salix wolfii / Carex aquatilis Shrubland & S3 & G4 \\
\hline Salix wolfii / Deschampsia cespitosa Shrubland & S3 & G3 \\
\hline Sarcobatus vermiculatus / Distichlis spicata Shrubland & S2 & G4 \\
\hline Sarcobatus vermiculatus / Elymus lanceolatus Shrub Herbaceous Vegetation & S3 & G3 \\
\hline Sarcobatus vermiculatus / Leymus cinereus Shrubland & S2S3 & G3 \\
\hline Sarcobatus vermiculatus / Pascopyrum smithii Shrub Herbaceous Vegetation & S4 & G4 \\
\hline Shepherdia argentea Shrubland [Provisional] & S3? & G3G4 \\
\hline Symphoricarpos occidentalis Shrubland [Provisional] & S4S5 & G4G5 \\
\hline
\end{tabular}




\section{Appendix C. Plant Associations of the Beaverhead Mountains Section Dwarf Shrubland}

\begin{tabular}{lcc} 
Association & State Rank Global R \\
\hline $\begin{array}{l}\text { Artemisia arbuscula / Festuca idahoensis Dwarf-shrub Herbaceous } \\
\text { Vegetation }\end{array}$ & S3 & \\
$\begin{array}{l}\text { Artemisia arbuscula / Pseudoroegneria spicata Dwarf-shrub } \\
\text { Herbaceous Vegetation }\end{array}$ & S3 & G5 \\
$\begin{array}{l}\text { Artemisia arbuscula ssp. longiloba / Elymus lanceolatus Dwarf-shrub } \\
\text { Herbaceous Vegetation }\end{array}$ & S? & G? \\
$\begin{array}{l}\text { Artemisia arbuscula ssp. longiloba / Festuca idahoensis Dwarf-shrub } \\
\text { Herbaceous Vegetation }\end{array}$ & S2 & G3? \\
$\begin{array}{l}\text { Artemisia nova / Festuca idahoensis Dwarf-shrub Herbaceous } \\
\text { Vegetation }\end{array}$ & S1S2 \\
$\begin{array}{l}\text { Artemisia nova / Pseudoroegneria spicata Dwarf-shrubland } \\
\text { Artemisia pedatifida / Festuca idahoensis Dwarf-shrub Herbaceous }\end{array}$ & S3 \\
$\begin{array}{l}\text { Vegetation } \\
\text { Atriplex gardneri / Oryzopsis hymenoides Dwarf-shrubland }\end{array}$ & G4G5 \\
$\begin{array}{l}\text { Cassiope mertensiana / Carex paysonis Dwarf-shrubland } \\
\text { Dryas octopetala - Carex rupestris Dwarf-shrub Herbaceous Vegetation }\end{array}$ & G2 \\
$\begin{array}{l}\text { Dryas octopetala - Carex spp. Dwarf-shrub Herbaceous Vegetation } \\
\text { Dryas octopetala - Polygonum viviparum Dwarf-shrub Herbaceous }\end{array}$ & S3? & G3 \\
Vegetation & S2 & G2? \\
$\begin{array}{l}\text { Kalmia microphylla / Carex scopulorum Dwarf-shrubland } \\
\text { Phyllodoce empetriformis / Antennaria lanata Dwarf-shrubland }\end{array}$ & G3? \\
$\begin{array}{l}\text { Salix arctica / Polygonum bistortoides Dwarf-shrubland } \\
\text { Salix reticulata / Caltha leptosepala Dwarf-shrubland }\end{array}$ & S3 & G3G4 \\
\end{tabular}




\section{Appendix C. Plant Associations of the Beaverhead Mountains Section Herbaceous}

\begin{tabular}{|c|c|c|}
\hline Association & State Rank & Global Rank \\
\hline Agrostis stolonifera Herbaceous Vegetation & S5 & GM \\
\hline Alpine Fellfield / Dry & S? & $\mathrm{G} ?$ \\
\hline Alpine Fellfield / Moist & S? & $\mathrm{G} ?$ \\
\hline Bromus carinatus - Bromus anomalus Herbaceous Vegetation & S? & $\mathrm{G} ?$ \\
\hline Calamagrostis canadensis Western Herbaceous Vegetation & S4 & G4Q \\
\hline Calamagrostis montanensis Herbaceous Vegetation & S? & $\mathrm{G} ?$ \\
\hline Calamagrostis purpurascens - Carex rupestris Herbaceous Vegetation & S? & $\mathrm{G} ?$ \\
\hline Carex aquatilis Herbaceous Vegetation & S4 & G5 \\
\hline Carex atherodes Herbaceous Vegetation & S5 & $\mathrm{G} ?$ \\
\hline Carex buxbaumii Herbaceous Vegetation & S3 & G3 \\
\hline Carex elynoides - Geum rossii Herbaceous Vegetation & S4 & G4 \\
\hline Carex elynoides Herbaceous Vegetation & S4 & G4 \\
\hline Carex lanuginosa Herbaceous Vegetation & SP & G3? \\
\hline Carex lasiocarpa Herbaceous Vegetation & S4 & G4 \\
\hline Carex nebrascensis Herbaceous Vegetation & S4 & G4 \\
\hline Carex nigricans Herbaceous Vegetation & S3 & G4 \\
\hline Carex rupestris - Potentilla ovina Herbaceous Vegetation & S3 & G3 \\
\hline Carex scirpoidea - Geum rossii Herbaceous Vegetation & S3 & G3 \\
\hline Carex scirpoidea - Potentilla diversifolia Herbaceous Vegetation & S2 & G3? \\
\hline Carex scopulorum - Caltha leptosepala Herbaceous Vegetation & S3 & G4 \\
\hline Carex scopulorum Herbaceous Vegetation & S4 & G5 \\
\hline Carex simulata Herbaceous Vegetation & S3 & G4 \\
\hline Carex spp. - Geum rossii Herbaceous Vegetation & S4Q & G4Q \\
\hline Carex utriculata Herbaceous Vegetation & S5 & G5 \\
\hline Deschampsia cespitosa - Caltha leptosepala Herbaceous Vegetation & S3 & G4 \\
\hline Deschampsia cespitosa - Carex spp. Herbaceous Vegetation & S4Q & G4Q \\
\hline Deschampsia cespitosa - Potentilla diversifolia Herbaceous Vegetation & S2 & G5 \\
\hline Deschampsia cespitosa Herbaceous Vegetation & S4 & G4 \\
\hline Distichlis spicata Herbaceous Vegetation & S4 & G5 \\
\hline Eleocharis palustris Herbaceous Vegetation & S5 & G5 \\
\hline Eleocharis quinqueflora Herbaceous Vegetation & SP & G4 \\
\hline Elymus elymoides Herbaceous Vegetation & S? & $\mathrm{G} ?$ \\
\hline Elymus lanceolatus - Phacelia hastata Herbaceous Vegetation & S2 & G2 \\
\hline Equisetum fluviatile Herbaceous Vegetation & S4 & G4 \\
\hline Festuca campestris - Festuca idahoensis - Stipa richardsonii Herbaceous Vegetation & S? & $\mathrm{G} ?$ \\
\hline Festuca campestris - Festuca idahoensis Herbaceous Vegetation & S3 & G3 \\
\hline Festuca campestris - Pseudoroegneria spicata Herbaceous Vegetation & S4 & G4 \\
\hline Festuca idahoensis - Carex filifolia Herbaceous Vegetation & S3 & G3 \\
\hline Festuca idahoensis - Danthonia intermedia Herbaceous Vegetation & S? & G3? \\
\hline Festuca idahoensis - Deschampsia cespitosa Herbaceous Vegetation & S3 & G3 \\
\hline Festuca idahoensis - Elymus trachycaulus Herbaceous Vegetation & S3S4 & G4 \\
\hline Festuca idahoensis - Festuca kingii Herbaceous Vegetation & SP & G2? \\
\hline Festuca idahoensis - Pascopyrum smithii Herbaceous Vegetation & S4 & G4 \\
\hline Festuca idahoensis - Potentilla diversifolia Herbaceous Vegetation & S3 & G3 \\
\hline Festuca idahoensis - Pseudoroegneria spicata Herbaceous Vegetation & S4 & G4 \\
\hline Festuca idahoensis - Stipa richardsonii Herbaceous Vegetation & S3 & G3 \\
\hline Festuca idahoensis Herbaceous Vegetation & S? & G3Q \\
\hline Festuca kingii - Oxytropis campestris Herbaceous Vegetation & S2 & G3? \\
\hline Festuca kingii Herbaceous Vegetation & SP & G3Q \\
\hline Geum rossii - Minuartia obtusiloba Herbaceous Vegetation & S3 & G3 \\
\hline Glyceria borealis Herbaceous Vegetation & S3 & G4 \\
\hline Hordeum jubatum Herbaceous Vegetation & S4 & G4 \\
\hline Juncus balticus - Carex praegracilis Herbaceous Vegetation & S? & $\mathrm{G} ?$ \\
\hline
\end{tabular}




\section{Appendix C. Plant Associations of the Beaverhead Mountains Section Herbaceous}

\begin{tabular}{|c|c|c|}
\hline Association & State Rank & Global Rank \\
\hline Juncus balticus Herbaceous Vegetation & S5 & G5 \\
\hline Juncus drummondii - Antennaria lanata Herbaceous Vegetation & S2 & G3? \\
\hline Juncus parryi - Erigeron ursinus Herbaceous Vegetation & S2? & $\mathrm{G} 2 ?$ \\
\hline Leymus cinereus - Festuca idahoensis Herbaceous Vegetation & S2? & $\mathrm{G} 2 ?$ \\
\hline Leymus cinereus - Pascopyrum smithii Herbaceous Vegetation & S3? & G3Q \\
\hline Leymus cinereus - Puccinellia nuttalliana Herbaceous Vegetation & S? & $\mathrm{G} ?$ \\
\hline Leymus cinereus Herbaceous Vegetation [Provisional] & S2 & G2G3Q \\
\hline Pascopyrum smithii Herbaceous Vegetation [Provisional] & S4 & G3G5Q \\
\hline Phalaris arundinacea Western Herbaceous Vegetation & S4 & G5 \\
\hline Phragmites australis Temperate Herbaceous Vegetation & $\mathrm{S} 2$ & G4 \\
\hline Poa palustris Herbaceous Vegetation & S4 & GW \\
\hline Poa pratensis Herbaceous Vegetation [Provisional] & SW & GW \\
\hline Poa juncifolia Herbaceous Vegetation & S? & G4? \\
\hline Pseudoroegneria spicata - Bouteloua gracilis Herbaceous Vegetation & S3 & G4 \\
\hline Pseudoroegneria spicata - Cushion Plant Herbaceous Vegetation & S3 & G3 \\
\hline Pseudoroegneria spicata - Koeleria macrantha Herbaceous Vegetation & S4? & G4? \\
\hline Pseudoroegneria spicata - Oryzopsis hymenoides Herbaceous Vegetation & S? & G3 \\
\hline Pseudoroegneria spicata - Pascopyrum smithii Herbaceous Vegetation & S4 & G4 \\
\hline Pseudoroegneria spicata - Poa secunda - Stipa comata Herbaceous Vegetation & S? & $\mathrm{G} ?$ \\
\hline Pseudoroegneria spicata - Poa secunda Herbaceous Vegetation & S4 & G4? \\
\hline Pseudoroegneria spicata - Stipa comata Herbaceous Vegetation & S3 & G4 \\
\hline Puccinellia nuttalliana Herbaceous Vegetation & S? & G3? \\
\hline Scirpus acutus Herbaceous Vegetation & S5 & G5 \\
\hline Scirpus tabernaemontani Temperate Herbaceous Vegetation & S3 & G4 \\
\hline Senecio triangularis Herbaceous Vegetation & S3? & G3? \\
\hline Silene acaulis Herbaceous Vegetation & S5 & G5? \\
\hline Sporobolus airoides Herbaceous Vegetation & S2 & G3Q \\
\hline Stipa comata - Bouteloua gracilis - Pascopyrum smithii Herbaceous Vegetation & S? & $\mathrm{G} ?$ \\
\hline Stipa comata - Bouteloua gracilis Herbaceous Vegetation & S5 & G5 \\
\hline Stipa comata - Psoralidium tenuiflorum Herbaceous Vegetation & S? & $\mathrm{G} ?$ \\
\hline Typha latifolia Western Herbaceous Vegetation & S5 & G5 \\
\hline
\end{tabular}


Appendix D.

Plant Associations: Principal and Substantiating References

\begin{tabular}{|c|c|}
\hline Plant Association Name & Principle Reference Source \\
\hline $\begin{array}{l}\text { Abies lasiocarpa - Pinus albicaulis / } \\
\text { Vaccinium scoparium Woodland }\end{array}$ & Cole 1982, Pfister et al. 1977 \\
\hline Abies lasiocarpa / Actaea rubra Forest & $\begin{array}{l}\text { Hansen et al. 1991, Johnston 1987, Mauk and Henderson 1984, Padgett } \\
\text { et al. 1988, Steele et al. 1983, Youngblood and Mauk } 1985\end{array}$ \\
\hline $\begin{array}{l}\text { Abies lasiocarpa / Alnus viridis ssp. } \\
\text { sinuata Forest }\end{array}$ & Pfister et al. 1977, Steele et al. 1981 \\
\hline $\begin{array}{l}\text { Abies lasiocarpa / Arnica cordifolia } \\
\text { Forest }\end{array}$ & $\begin{array}{l}\text { Hoffman and Alexander 1976, Pfister et al. 1977, Reed 1969, Steele et al. } \\
\text { 1981, Steele et al. 1983, Youngblood and Mueggler } 1981\end{array}$ \\
\hline Abies lasiocarpa / Arnica latifolia Forest & Cooper and Pfister 1981, Johnston 1987, Steele et al. 1983 \\
\hline $\begin{array}{l}\text { Abies lasiocarpa / Calamagrostis } \\
\text { canadensis Forest }\end{array}$ & $\begin{array}{l}\text { Cooper et al. 1987, Hess and Alexander 1986, Johnston 1987, Mauk and } \\
\text { Henderson 1984, Pfister et al. 1977, Richard et al. 1996, Steele et al. 1981, } \\
\text { Steele et al. } 1983\end{array}$ \\
\hline $\begin{array}{l}\text { Abies lasiocarpa / Calamagrostis } \\
\text { rubescens Forest }\end{array}$ & $\begin{array}{l}\text { Cooper et al. 1987, Johnson and Simon 1987, Komarkova et al. 1988, } \\
\text { Mauk and Henderson 1984, Pfister et al. 1977, Steele et al. 1981, Steele } \\
\text { et al. 1983, Williams and Lillybridge 1985, }\end{array}$ \\
\hline Abies lasiocarpa / Carex geyeri Forest & $\begin{array}{l}\text { Alexander et al. 1986, Hess and Alexander 1986, Johnson and } \\
\text { Clausnitzer 1992, Johnston 1987, Komarkova 1982, Komarkova et al. } \\
\text { 1988, Pfister et al. 1977, Steele et al. 1981, Steele et al. 1983, Terwilliger et } \\
\text { al. 1979, Wasser and Hess 1982, Youngblood and }\end{array}$ \\
\hline $\begin{array}{l}\text { Abies lasiocarpa / Clematis columbiana } \\
\text { var. columbiana Forest }\end{array}$ & Pfister et al. 1977 \\
\hline $\begin{array}{l}\text { Abies lasiocarpa / Galium triflorum } \\
\text { Forest }\end{array}$ & et al. 1977 \\
\hline $\begin{array}{l}\text { Abies lasiocarpa / Juniperus communis } \\
\text { Woodland }\end{array}$ & $\begin{array}{l}\text { Henderson et al. 1989,Johnston 1987, Mauk and Henderson 1984, } \\
\text { Pfister et al. 1977, Roberts 1980, Steele et al. 1981, Steele et al. 1983, } \\
\text { Youngblood and Mauk } 1985\end{array}$ \\
\hline $\begin{array}{l}\text { Abies lasiocarpa / Ledum glandulosum } \\
\text { Forest }\end{array}$ & Hansen et al. 1991, Hansen et al. 1995 \\
\hline $\begin{array}{l}\text { Abies lasiocarpa / Linnaea borealis } \\
\text { Forest }\end{array}$ & $\begin{array}{l}\text { Henderson et al. 1985, Johnson and Simon 1987, Pfister et al. 1977, } \\
\text { Steele et al. 1981, Steele et al. 1983, Williams and Lillybridge 1985, } \\
\text { Williams and Smith 1990, Williams et al. } 1990\end{array}$ \\
\hline $\begin{array}{l}\text { Abies lasiocarpa / Luzula glabrata var. } \\
\text { hitchcockii Forest }\end{array}$ & $\begin{array}{l}\text { Cooper et al. 1987, Pfister et al. 1977, Steele et al. 1983, Williams and } \\
\text { Smith } 1990\end{array}$ \\
\hline $\begin{array}{l}\text { Abies lasiocarpa / Menziesia ferruginea } \\
\text { Forest }\end{array}$ & $\begin{array}{l}\text { Cooper et al. 1987, Daubenmire and Daubenmire 1968, Johnson and } \\
\text { Simon 1987, Pfister et al. 1977, Steele et al. 1981, Steele et al. } 1983\end{array}$ \\
\hline $\begin{array}{l}\text { Abies lasiocarpa / Ribes (montigenum, } \\
\text { lacustre, inerme) Forest }\end{array}$ & $\begin{array}{l}\text { Henderson et al. 1977, Johnston 1987, Mauk and Henderson 1984, } \\
\text { Osborn et al. 1998, Peet 1975, Pfister 1972, Pfister et al. 1977, Steele et al. } \\
\text { 1981, Steele et al. } 1983\end{array}$ \\
\hline $\begin{array}{l}\text { Abies lasiocarpa / Streptopus } \\
\text { amplexifolius Forest }\end{array}$ & $\begin{array}{l}\text { Cooper et al. 1987,Hansen et al. 1995, Johnson and Simon 1987, Mauk } \\
\text { and Henderson 1984, Padgett et al. 1988, Steele et al. 1981, Steele et al. } \\
\text { 1983, Youngblood and Mauk 1985 }\end{array}$ \\
\hline $\begin{array}{l}\text { Abies lasiocarpa / Symphoricarpos } \\
\text { albus Forest }\end{array}$ & Cooper and Pfister 1981, Steele et al. 1983 \\
\hline $\begin{array}{l}\text { Abies lasiocarpa / Thalictrum } \\
\text { occidentale Forest }\end{array}$ & Cole 1982, Cooper 1975, Johnston 1987, Steele et al. 1983 \\
\hline $\begin{array}{l}\text { Abies lasiocarpa / Vaccinium } \\
\text { cespitosum Forest }\end{array}$ & $\begin{array}{l}\text { Kerr and Henderson 1979, Mauk and Henderson 1984, Pfister et al. } \\
\text { 1977, Steele et al. 1981, Williams et al. 1990, Youngblood and Mauk } 1985\end{array}$ \\
\hline \begin{tabular}{|l|} 
Abies lasiocarpa / Vaccinium \\
membranaceum Rocky Mountain Forest
\end{tabular} & $\begin{array}{l}\text { Cooper 1975, Johnston 1987, Kerr and Henderson 1979, Mauk and } \\
\text { Henderson 1984, Pfister et al. 1977, Steele et al. 1981, Steele et al. 1983, } \\
\text { Terwilliger et al. } 1979\end{array}$ \\
\hline
\end{tabular}


Appendix D.

Plant Associations: Principal and Substantiating References

\begin{tabular}{|c|c|}
\hline Plant Association Name & Principle Reference Source \\
\hline $\begin{array}{l}\text { Abies lasiocarpa / Vaccinium scoparium } \\
\text { Forest }\end{array}$ & $\begin{array}{l}\text { Alexander et al. 1986, Daubenmire and Daubenmire 1968, DeVelice 1983, } \\
\text { Dix and Richards 1976, Hall 1973, Hess and Alexander 1986, Hoffman } \\
\text { and Alexander }\end{array}$ \\
\hline $\begin{array}{l}\text { Abies lasiocarpa / Xerophyllum tenax } \\
\text { Forest }\end{array}$ & $\begin{array}{l}\text { Cooper 1975, Cooper et al. 1987, Daubenmire and Daubenmire 1968, } \\
\text { Horton 1971, Pfister et al. 1977, Steele et al. 1983, Williams and } \\
\text { Lillybridge 1985, Williams et al. } 1990\end{array}$ \\
\hline Abies lasiocarpa Krummholz Shrubland & Habeck and Choate 1963 \\
\hline \begin{tabular}{|l|} 
Abies lasiocarpa Scree Woodland \\
\end{tabular} & Pfister et al. 1977 \\
\hline $\begin{array}{l}\text { Agrostis stolonifera Herbaceous } \\
\text { Vegetation }\end{array}$ & Hansen et al. 1995 \\
\hline \begin{tabular}{|l|} 
Alnus incana Shrubland \\
\end{tabular} & Evans 1989, Hansen et al. 1990, Hansen et al. 1991 \\
\hline $\begin{array}{l}\text { Alnus viridis ssp. sinuata Shrubland } \\
\text { [Provisional] }\end{array}$ & Hansen et al. 1991 \\
\hline Alpine Fellfield / Dry & Cooper et al. 1997 \\
\hline \begin{tabular}{|l} 
Alpine Fellfield / Moist \\
\end{tabular} & Cooper et al. 1997 \\
\hline $\begin{array}{l}\text { Artemisia arbuscula / Festuca } \\
\text { idahoensis Dwarf-shrub Herbaceous } \\
\text { Vegetation }\end{array}$ & $\begin{array}{l}\text { Blackburn et al. 1968, Blackburn et al. 1969, Hall 1973, Hess and Wasser } \\
\text { 1982, Lewis 1975, Mueggler and Stewart 1980, Tweit and Houston 1980, } \\
\text { Zamora and Tueller } 1973\end{array}$ \\
\hline $\begin{array}{l}\text { Artemisia arbuscula / Pseudoroegneria } \\
\text { spicata Dwarf-shrub Herbaceous } \\
\text { Vegetation }\end{array}$ & $\begin{array}{l}\text { Blackburn et al. 1971, Hall 1973, Jensen et al. 1988, Lewis 1975, } \\
\text { Mueggler and Stewart 1980, Schuller and Evans 1986, Tiedemann and } \\
\text { Klock 1977, Zamora and Tueller } 1973\end{array}$ \\
\hline $\begin{array}{l}\text { Artemisia arbuscula ssp. longiloba / } \\
\text { Elymus lanceolatus Dwarf-shrub } \\
\text { Herbaceous Vegetation }\end{array}$ & Mueggler and Stewart 1980 \\
\hline $\begin{array}{l}\text { Artemisia arbuscula ssp. longiloba / } \\
\text { Festuca idahoensis Dwarf-shrub } \\
\text { Herbaceous Vegetation }\end{array}$ & $\begin{array}{l}\text { Caicco and Wellner 1983, Hironaka et al. 1983, Jensen et al. 1988, } \\
\text { Mueggler and Stewart 1980, Schlatterer 1972, Tisdale et al. 1965, Zamora } \\
\text { and Tueller } 1973\end{array}$ \\
\hline $\begin{array}{l}\text { Artemisia cana / Festuca idahoensis } \\
\text { Shrub Herbaceous Vegetation }\end{array}$ & Mueggler and Stewart 1980 \\
\hline $\begin{array}{l}\text { Artemisia cana / Leymus cinereus } \\
\text { Shrubland }\end{array}$ & Oregon Natural Heritage Program n.d. \\
\hline $\begin{array}{l}\text { Artemisia cana / Pascopyrum smithii } \\
\text { Shrubland }\end{array}$ & Hansen et al. 1991, Hansen et al. 1995 \\
\hline \multicolumn{2}{|l|}{$\begin{array}{l}\text { Artemisia cana / Poa pratensis Shrub } \\
\text { Herbaceous Vegetation }\end{array}$} \\
\hline $\begin{array}{l}\text { Artemisia cana / Stipa comata Shrub } \\
\text { Herbaceous Vegetation }\end{array}$ & DeVelice et al. 1991 \\
\hline $\begin{array}{l}\text { Artemisia nova / Festuca idahoensis } \\
\text { Dwarf-shrub Herbaceous Vegetation }\end{array}$ & Mueggler and Stewart 1980 \\
\hline $\begin{array}{l}\text { Artemisia nova / Pseudoroegneria } \\
\text { spicata Dwarf-shrubland }\end{array}$ & $\begin{array}{l}\text { Baker 1983, Baker and Kennedy 1985, Blackburn et al. 1968, Blackburn } \\
\text { et al. 1971, Fisser 1970, Hughes 1977, Tweit and Houston 1980, Zamora } \\
\text { and Tueller } 1973\end{array}$ \\
\hline $\begin{array}{l}\text { Artemisia pedatifida / Festuca } \\
\text { idahoensis Dwarf-shrub Herbaceous } \\
\text { Vegetation }\end{array}$ & Mueggler and Stewart 1980 \\
\hline $\begin{array}{l}\text { Artemisia tridentata / Festuca } \\
\text { campestris Shrub Herbaceous } \\
\text { Vegetation }\end{array}$ & Mueggler and Stewart 1980 \\
\hline $\begin{array}{l}\text { Artemisia tridentata / Festuca } \\
\text { idahoensis Shrub Herbaceous } \\
\text { Vegetation }\end{array}$ & $\begin{array}{l}\text { Blackburn et al. 1968, Daubenmire 1970, Hall 1973, Hironaka et al. 1983, } \\
\text { Mclean 1970, Mueggler and Stewart 1980, Poulton 1955, Tisdale } 1947\end{array}$ \\
\hline
\end{tabular}


Appendix D.

Plant Associations: Principal and Substantiating References

\begin{tabular}{|c|c|}
\hline Plant Association Name & Principle Reference Source \\
\hline $\begin{array}{l}\text { Artemisia tridentata / Stipa comata } \\
\text { Shrubland }\end{array}$ & $\begin{array}{l}\text { Blackburn et al. 1968, Caicco and Wellner 1983, Daubenmire 1970, } \\
\text { Hironaka et al. 1983, Lesica and DeVelice 1992, Mclean 1970, Poulton } \\
\text { 1955, Tueller and Blackburn } 1974\end{array}$ \\
\hline $\begin{array}{l}\text { Artemisia tridentata ssp. tridentata / } \\
\text { Festuca idahoensis Shrubland }\end{array}$ & Hironaka et al. 1983, Jensen et al. 1988 \\
\hline $\begin{array}{l}\text { Artemisia tridentata ssp. tridentata / } \\
\text { Leymus cinereus Shrubland }\end{array}$ & Baker 1982, Baker 1983, Hess 1981, Kittel et al. 1994, Osborn et al. 1998 \\
\hline $\begin{array}{l}\text { Artemisia tridentata ssp. tridentata / } \\
\text { Pascopyrum smithii Shrubland }\end{array}$ & Francis 1983, Keammerer 1977, Strong 1980, Tiedemann et al. 1987 \\
\hline $\begin{array}{l}\text { Artemisia tridentata ssp. tridentata / } \\
\text { Pseudoroegneria spicata Shrub } \\
\text { Herbaceous Vegetation }\end{array}$ & $\begin{array}{l}\text { Caicco and Wellner 1983, Daubenmire 1970, Hironaka et al. 1983, Jensen } \\
\text { et al. 1988, Mooney 1985, Mueggler and Stewart 1980, Tweit and } \\
\text { Houston } 1980\end{array}$ \\
\hline $\begin{array}{l}\text { Artemisia tridentata ssp. vaseyana - } \\
\text { Symphoricarpos oreophilus / Festuca } \\
\text { idahoensis Shrubland }\end{array}$ & Caicco and Wellner 1983, Hironaka et al. 1983 \\
\hline $\begin{array}{l}\text { Artemisia tridentata ssp. vaseyana / } \\
\text { Festuca campestris Shrub Herbaceous } \\
\text { Vegetation }\end{array}$ & \\
\hline $\begin{array}{l}\text { Artemisia tridentata ssp. vaseyana / } \\
\text { Festuca idahoensis - Elymus } \\
\text { trachycaulus Shrubland }\end{array}$ & Mueggler and Stewart 1980 \\
\hline $\begin{array}{l}\text { Artemisia tridentata ssp. vaseyana / } \\
\text { Festuca idahoensis - Geranium } \\
\text { viscossimum Shrubland }\end{array}$ & Mueggler and Stewart 1980 \\
\hline $\begin{array}{l}\text { Artemisia tridentata ssp. vaseyana / } \\
\text { Festuca idahoensis - Pascopyrum } \\
\text { smithii Shrubland }\end{array}$ & Mueggler and Stewart 1980 \\
\hline $\begin{array}{l}\text { Artemisia tridentata ssp. vaseyana / } \\
\text { Festuca idahoensis - Pseudoroegneria } \\
\text { spicata Shrubland }\end{array}$ & Mueggler and Stewart 1980 \\
\hline $\begin{array}{l}\text { Artemisia tridentata ssp. vaseyana / } \\
\text { Festuca idahoensis Shrub Herbaceous } \\
\text { Vegetation }\end{array}$ & $\begin{array}{l}\text { Bramble-Brodahl 1978, Despain 1973, Hess and Wasser 1982, Hironaka } \\
\text { et al. 1983, Jensen et al. 1988, Johnston 1987, Lewis 1975, Mooney 1985, } \\
\text { Mueggler and Stewart } 1980\end{array}$ \\
\hline $\begin{array}{l}\text { Artemisia tridentata ssp. vaseyana / } \\
\text { Leymus cinereus Shrubland }\end{array}$ & Hironaka et al. 1983, Jensen et al. 1988, Mooney 1985 \\
\hline $\begin{array}{l}\text { Artemisia tridentata ssp. vaseyana / } \\
\text { Pascopyrum smithii Shrubland } \\
\end{array}$ & Rzedowski 1981, Smith 1966, Terwilliger et al. 1978 \\
\hline $\begin{array}{l}\text { Artemisia tridentata ssp. vaseyana / } \\
\text { Pseudoroegneria spicata Shrubland }\end{array}$ & $\begin{array}{l}\text { Baker 1983, Baker and Kennedy 1985, Bramble-Brodahl 1978, Current } \\
\text { 1984, Hironaka et al. 1983, Jensen et al. 1988, Lewis 1975, Mooney 1985, } \\
\text { Smith 1966, Terwilliger et al. } 1978\end{array}$ \\
\hline $\begin{array}{l}\text { Artemisia tridentata ssp. vayesana / } \\
\text { Festuca idahoensis - Stipa occidentalis } \\
\text { Shrubland }\end{array}$ & Mueggler and Stewart 1980 \\
\hline $\begin{array}{l}\text { Artemisia tridentata ssp. wyomingensis } \\
\text { / Elymus lanceolatus ssp. albicans } \\
\text { Shrubland }\end{array}$ & $\begin{array}{l}\text { Giese 1975, Gross 1955, Marr et al. 1979, Steger 1970, Terwilliger et al. } \\
1979\end{array}$ \\
\hline $\begin{array}{l}\text { Artemisia tridentata ssp. wyomingensis } \\
\text { / Poa secunda Shrubland }\end{array}$ & Caicco and Wellner 1983, Hironaka et al. 1983, Jensen et al. 1988 \\
\hline $\begin{array}{l}\text { Artemisia tridentata ssp. wyomingensis } \\
\text { / Pseudoroegneria spicata Shrub } \\
\text { Herbaceous Vegetation }\end{array}$ & $\begin{array}{l}\text { Baker 1983, Baker and Kennedy 1985, Brown 1971, Fisser 1964, Fisser } \\
\text { 1970, Hansen and Hoffman 1988, Hess 1981, Knight et al. 1987, Strong } \\
1980\end{array}$ \\
\hline
\end{tabular}


Appendix D.

\section{Plant Associations: Principal and Substantiating References}

\begin{tabular}{|c|c|}
\hline Plant Association Name & Principle Reference Source \\
\hline $\begin{array}{l}\text { Artemisia tridentata ssp. wyomingensis } \\
\text { / Pseudoroegneria spicata Shrubland }\end{array}$ & $\begin{array}{l}\text { Blackburn 1967, Blackburn et al. 1968, Blackburn et al. 1969, Blackburn } \\
\text { et al. 1971, Brotherson and Brotherson 1981, Daubenmire 1970, Hall } \\
\text { 1973, Heinze et al. 1962, Hironaka et al. 1983, Lewis 1975, Mclean 1970, } \\
\text { Moretti 1979, Moretti and Brotherson 1982, }\end{array}$ \\
\hline \multicolumn{2}{|l|}{$\begin{array}{l}\text { Artemisia tripartita / Elymus lanceolatus } \\
\text { Shrub Herbaceous Vegetation }\end{array}$} \\
\hline \begin{tabular}{|l|} 
Artemisia tripartita / Festuca idahoensis \\
Shrub Herbaceous Vegetation
\end{tabular} & $\begin{array}{l}\text { Caicco and Wellner 1983, Daubenmire 1970, Hess 1981, Hironaka et al. } \\
\text { 1983, Johnston 1987, Mueggler and Stewart } 1980\end{array}$ \\
\hline \begin{tabular}{|l|l} 
Artemisia tripartita / Pseudoroegneria \\
spicata Shrub Herbaceous Vegetation
\end{tabular} & $\begin{array}{l}\text { Caicco and Wellner 1983, Daubenmire 1970, Hironaka et al. 1983, } \\
\text { Mclean } 1970\end{array}$ \\
\hline $\begin{array}{l}\text { Atriplex gardneri / Oryzopsis } \\
\text { hymenoides Dwarf-shrubland }\end{array}$ & Baker 1983, Baker and Kennedy 1985, DeVelice 1992 \\
\hline $\begin{array}{l}\text { Betula glandulosa / Carex utriculata } \\
\text { Shrubland }\end{array}$ & Hansen et al. 1991 \\
\hline Betula occidentalis Shrubland & Hansen et al. 1991, Kittel and Lederer 1993 \\
\hline \multicolumn{2}{|l|}{$\begin{array}{l}\text { Bromus carinatus - Bromus anomalus } \\
\text { Herbaceous Vegetation }\end{array}$} \\
\hline $\begin{array}{l}\text { Calamagrostis canadensis Western } \\
\text { Herbaceous Vegetation }\end{array}$ & $\begin{array}{l}\text { Cooper 1986, Cooper and Cottrell 1990, Hansen et al. 1988, Hansen et al. } \\
\text { 1991, Kovalchik 1993, Mattson 1984, Mutz and Queiroz 1983, Padgett et } \\
\text { al. 1989, Wilson } 1969\end{array}$ \\
\hline \multicolumn{2}{|l|}{\begin{tabular}{l|l} 
Calamagrostis montanensis Herbaceous & \\
Vegetation &
\end{tabular}} \\
\hline \multicolumn{2}{|l|}{$\begin{array}{l}\text { Calamagrostis purpurascens - Carex } \\
\text { rupestris Herbaceous Vegetation }\end{array}$} \\
\hline Carex aquatilis Herbaceous Vegetation & $\begin{array}{l}\text { Baker 1984, Baker and Kennedy 1985,Briggs and MacMahon 1983, Hall } \\
\text { 1971, Hansen et al. 1987, Hansen et al. 1995, Hess and Wasser 1982, }\end{array}$ \\
\hline \multicolumn{2}{|c|}{\begin{tabular}{|l|l|} 
Carex atherodes Herbaceous Vegetation & \\
\end{tabular}} \\
\hline Carex buxbaumii Herbaceous Vegetation & $\begin{array}{l}\text { Hansen et al. 1988, Mattson 1984, Moseley et al. 1991, Moseley et al. } \\
\text { 1994, Padgett et al. 1989, Pierce 1986, Pierce and Johnson } 1986\end{array}$ \\
\hline $\begin{array}{l}\text { Carex elynoides - Geum rossii } \\
\text { Herbaceous Vegetation }\end{array}$ & Bamberg 1961 \\
\hline Carex elynoides Herbaceous Vegetation & Baker 1980, Baker 1983, Cooper and Lesica 1992, Moir and Smith 1970 \\
\hline $\begin{array}{l}\text { Carex lanuginosa Herbaceous } \\
\text { Vegetation }\end{array}$ & $\begin{array}{l}\text { Hansen et al. 1987, Kittel et al. 1995, Kovalchik 1987, Padgett et al. 1988, } \\
\text { Padgett et al. } 1989\end{array}$ \\
\hline Carex lasiocarpa Herbaceous Vegetation & $\begin{array}{l}\text { Hansen et al. 1988, Hansen et al. 1991, Kovalchik 1987, Kovalchik 1993, } \\
\text { Padgett et al. } 1989\end{array}$ \\
\hline $\begin{array}{l}\text { Carex nebrascensis Herbaceous } \\
\text { Vegetation }\end{array}$ & $\begin{array}{l}\text { Baker 1982, Cooper and Cottrell 1990, Hall 1973, Hansen et al. 1995, } \\
\text { Kittel et al. 1996, Kovalchik 1987, Mutz and Queiroz 1983, Padgett et al. } \\
\text { 1989, Youngblood et al. } 1985\end{array}$ \\
\hline Carex nigricans Herbaceous Vegetation & $\begin{array}{l}\text { Agee and Kertis 1987, Cooper and Lesica 1992, Douglas and Bliss 1977, } \\
\text { Hamann 1972, Henderson 1973, Henderson and Peter } 1982\end{array}$ \\
\hline $\begin{array}{l}\text { Carex rupestris - Potentilla ovina } \\
\text { Herbaceous Vegetation }\end{array}$ & Cooper and Lesica 1992 \\
\hline $\begin{array}{l}\text { Carex scirpoidea - Geum rossii } \\
\text { Herbaceous Vegetation }\end{array}$ & Cooper and Lesica 1992, Cooper et al. 1997 \\
\hline $\begin{array}{l}\text { Carex scirpoidea - Potentilla diversifolia } \\
\text { Herbaceous Vegetation }\end{array}$ & Cooper and Lesica 1992, Cooper et al. 1997 \\
\hline $\begin{array}{l}\text { Carex scopulorum - Caltha leptosepala } \\
\text { Herbaceous Vegetation }\end{array}$ & $\begin{array}{l}\text { Clements 1904, Cooper and Lesica 1992, Hess 1981, Hess and Wasser } \\
\text { 1982, Johnston 1987, Kettler and McMullen 1996, Kittel et al. 1994, } \\
\text { Willard } 1963\end{array}$ \\
\hline $\begin{array}{l}\text { Carex scopulorum Herbaceous } \\
\text { Vegetation }\end{array}$ & Hansen et al. 1991, Kovalchik 1993, Manning and Padgett 1991 \\
\hline
\end{tabular}


Appendix D.

Plant Associations: Principal and Substantiating References

\begin{tabular}{|c|c|}
\hline Plant Association Name & Principle Reference Source \\
\hline Carex simulata Herbaceous Vegetation & $\begin{array}{l}\text { Hansen et al. 1987, Hansen et al. 1991, Kovalchik 1987, Padgett et al. } \\
\text { 1988, Padgett et al. 1989, Sanderson and Kettler 1996, Tuhy and Jensen } \\
\text { 982, Youngblood et al. 1985 }\end{array}$ \\
\hline $\begin{array}{l}\text { Carex spp. - Geum rossii Herbaceous } \\
\text { Vegetation }\end{array}$ & Bamberg 1961 \\
\hline Carex utriculata Herbaceous Vegetation & $\begin{array}{l}\text { Andrews 1983, Baker 1983, Benedict 1983, Franklin and Dyrness 1973, } \\
\text { Hansen et al. 1987, Hansen et al. 1991, Hess and Wasser 1982, Kerr and } \\
\text { Henderson 1979, Kittel et al. } 1995\end{array}$ \\
\hline $\begin{array}{l}\text { Cassiope mertensiana / Carex paysonis } \\
\text { Dwarf-shrubland }\end{array}$ & Cooper and Lesica 1992, Cooper et al. 1997 \\
\hline $\begin{array}{l}\text { Cercocarpus ledifolius / Festuca } \\
\text { idahoensis Woodland }\end{array}$ & Cooper et al. 1995, DeVelice 1992, Dealy 1975, Hall 1973, Nesser 1997 \\
\hline $\begin{array}{l}\text { Cercocarpus ledifolius / } \\
\text { Pseudoroegneria spicata Shrubland }\end{array}$ & $\begin{array}{l}\text { Baker 1983, Baker and Kennedy 1985, Knight et al. 1987, Lewis } 1975 \text {, } \\
\text { Miller 1964, Mueggler and Stewart 1980, Tisdale } 1986\end{array}$ \\
\hline $\begin{array}{l}\text { Chrysothamnus viscidiflorus / Stipa } \\
\text { comata Shrubland }\end{array}$ & \\
\hline Cornus sericea Shrubland [Provisional] & Hansen et al. 1991, Kittel et al. 1994, Kovalchik 1993, Osborn et al. 1998 \\
\hline $\begin{array}{l}\text { Deschampsia cespitosa - Caltha } \\
\text { leptosepala Herbaceous Vegetation }\end{array}$ & $\begin{array}{l}\text { Bonham 1966, Bonham and Ward 1970, Cooper et al. 1997, Hess 1981, } \\
\text { Hess and Wasser 1982, Johnson 1970, Johnston 1987, Komarkova 1986, } \\
\text { May 1973, Osburn 1958, Wilson } 1969\end{array}$ \\
\hline $\begin{array}{l}\text { Deschampsia cespitosa - Carex spp. } \\
\text { Herbaceous Vegetation }\end{array}$ & Mueggler and Stewart 1980 \\
\hline $\begin{array}{l}\text { Deschampsia cespitosa - Potentilla } \\
\text { diversifolia Herbaceous Vegetation }\end{array}$ & Baker 1983, Bonham 1966, Bonham and Ward 1970, Cooper et al. 1997 \\
\hline $\begin{array}{l}\text { Deschampsia cespitosa Herbaceous } \\
\text { Vegetation }\end{array}$ & $\begin{array}{l}\text { Bonham and Ward 1970, Briggs and MacMahon 1983, Crowe and } \\
\text { Clausnitzer 1997, Daubenmire and Daubenmire 1968, Franklin and } \\
\text { Dyrness 1973, Hall 1971, Hall 1973, Hamann 1972, Hansen et al. 1987, } \\
\text { Hansen et al. 1995, Hess and Wasser 1982, Jo }\end{array}$ \\
\hline $\begin{array}{l}\text { Distichlis spicata Herbaceous } \\
\text { Vegetation }\end{array}$ & $\begin{array}{l}\text { Baker 1984, Beatley 1976, Brotherson 1987, Daubenmire 1970, Franklin } \\
\text { and Dyrness 1973, Hansen et al. 1991, Hyder et al. 1966, Jones and } \\
\text { Walford 1995, Kittel and Lederer 1993 }\end{array}$ \\
\hline $\begin{array}{l}\text { Dryas octopetala - Carex rupestris Dwarf- } \\
\text { shrub Herbaceous Vegetation }\end{array}$ & $\begin{array}{l}\text { Cooper et al. 1997, Cox 1933, Eddleman 1967, Fritz 1981,Hess and } \\
\text { Wasser 1982, Holway 1962, Holway and Ward 1963, Johnston 1987, } \\
\text { Komarkova 1976, Willard 1963, Willard } 1979\end{array}$ \\
\hline $\begin{array}{l}\text { Dryas octopetala - Carex spp. Dwarf- } \\
\text { shrub Herbaceous Vegetation }\end{array}$ & Bamberg and Major 1968 \\
\hline $\begin{array}{l}\text { Dryas octopetala - Polygonum } \\
\text { viviparum Dwarf-shrub Herbaceous } \\
\text { Vegetation }\end{array}$ & Cooper and Lesica 1992, Cooper et al. 1997 \\
\hline $\begin{array}{l}\text { Eleocharis palustris Herbaceous } \\
\text { Vegetation }\end{array}$ & $\begin{array}{l}\text { Baker 1983, Baker and Kennedy 1985, Brotherson and Barnes 1984, } \\
\text { Hansen et al. 1987, Hansen et al. 1995, Kettler and McMullen 1996, } \\
\text { Kittel et al. 1994, Kovalchik 1993, M }\end{array}$ \\
\hline $\begin{array}{l}\text { Eleocharis quinqueflora Herbaceous } \\
\text { Vegetation }\end{array}$ & $\begin{array}{l}\text { Briggs and MacMahon 1983, Cooper 1990, Hansen et al. 1988, Jensen } \\
\text { and Tuhy 1981, Johnston 1987, Kittel et al. 1994, Kovalchik 1987, } \\
\text { Mattson 1984, Padgett et al. 1989 }\end{array}$ \\
\hline \multicolumn{2}{|l|}{$\begin{array}{l}\text { Elymus elymoides Herbaceous } \\
\text { Vegetation }\end{array}$} \\
\hline $\begin{array}{l}\text { Elymus lanceolatus - Phacelia hastata } \\
\text { Herbaceous Vegetation }\end{array}$ & DeVelice 1992 \\
\hline $\begin{array}{l}\text { Equisetum fluviatile Herbaceous } \\
\text { Vegetation }\end{array}$ & $\begin{array}{l}\text { Bursik and Moseley 1995, Hansen et al. 1991, Hansen et al. 1995, } \\
\text { Kovalchik } 1993\end{array}$ \\
\hline
\end{tabular}


Appendix D.

\section{Plant Associations: Principal and Substantiating References}

\begin{tabular}{|c|c|}
\hline Plant Association Name & Principle Reference Source \\
\hline \begin{tabular}{|l|} 
Festuca campestris - Festuca idahoensis \\
- Stipa richardsonii Herbaceous \\
Vegetation
\end{tabular} & \\
\hline \begin{tabular}{|l|} 
Festuca campestris - Festuca idahoensis \\
Herbaceous Vegetation
\end{tabular} & $\begin{array}{l}\text { Daubenmire 1970, Hodgkinson and Young 1973, Mclean 1970, } \\
\text { Mueggler and Stewart 1980, Tisdale 1947, Tisdale and Bramble-Brodahl } \\
\text { 1983, Tisdale and McLean 1957 }\end{array}$ \\
\hline $\begin{array}{l}\text { Festuca campestris - Pseudoroegneria } \\
\text { spicata Herbaceous Vegetation }\end{array}$ & Mueggler and Stewart 1980 \\
\hline $\begin{array}{l}\text { Festuca idahoensis - Carex filifolia } \\
\text { Herbaceous Vegetation }\end{array}$ & Mueggler and Stewart 1980 \\
\hline $\begin{array}{l}\text { Festuca idahoensis - Danthonia } \\
\text { intermedia Herbaceous Vegetation }\end{array}$ & Mattson and National n.d. \\
\hline $\begin{array}{l}\text { Festuca idahoensis - Deschampsia } \\
\text { cespitosa Herbaceous Vegetation }\end{array}$ & Mueggler and Stewart 1980 \\
\hline $\begin{array}{l}\text { Festuca idahoensis - Elymus } \\
\text { trachycaulus Herbaceous Vegetation }\end{array}$ & Hess and Wasser 1982, Johnston 1987, Mueggler and Stewart 1980 \\
\hline $\begin{array}{l}\text { Festuca idahoensis - Festuca kingii } \\
\text { Herbaceous Vegetation }\end{array}$ & $\begin{array}{l}\text { Idaho Conservation Data Center n.d., Montana Natural Heritage } \\
\text { Program n.d. }\end{array}$ \\
\hline $\begin{array}{l}\text { Festuca idahoensis - Pascopyrum } \\
\text { smithii Herbaceous Vegetation } \\
\end{array}$ & Mueggler and Stewart 1980 \\
\hline $\begin{array}{l}\text { Festuca idahoensis - Potentilla } \\
\text { diversifolia Herbaceous Vegetation }\end{array}$ & Caicco 1983, Cooper and Lesica 1992, Cooper et al. 1997, Moseley 1985 \\
\hline $\begin{array}{l}\text { Festuca idahoensis - Pseudoroegneria } \\
\text { spicata Herbaceous Vegetation }\end{array}$ & Mueggler and Stewart 1980, Tisdale 1986 \\
\hline $\begin{array}{l}\text { Festuca idahoensis - Stipa richardsonii } \\
\text { Herbaceous Vegetation }\end{array}$ & Mueggler and Stewart 1980 \\
\hline $\begin{array}{l}\text { Festuca idahoensis Herbaceous } \\
\text { Vegetation }\end{array}$ & Johnson 1970 \\
\hline $\begin{array}{l}\text { Festuca kingii - Oxytropis campestris } \\
\text { Herbaceous Vegetation }\end{array}$ & Cooper and Lesica 1992 \\
\hline Festuca kingii Herbaceous Vegetation & Montana Natural Heritage Program n.d. \\
\hline $\begin{array}{l}\text { Geum rossii - Minuartia obtusiloba } \\
\text { Herbaceous Vegetation }\end{array}$ & Cooper and Lesica 1992 \\
\hline $\begin{array}{l}\text { Glyceria borealis Herbaceous } \\
\text { Vegetation }\end{array}$ & Hansen et al. 1995 \\
\hline $\begin{array}{l}\text { Hordeum jubatum Herbaceous } \\
\text { Vegetation }\end{array}$ & Bunin 1985, Hansen et al. 1991, Redmann 1972, Reid 1974, Vestal 1914 \\
\hline \multicolumn{2}{|l|}{$\begin{array}{l}\text { Juncus balticus - Carex praegracilis } \\
\text { Herbaceous Vegetation }\end{array}$} \\
\hline Juncus balticus Herbaceous Vegetation & $\begin{array}{l}\text { Brotherson and Barnes 1984, Bunin 1985, Flowers 1962, Hansen et al. } \\
\text { 1988, Hansen et al. 1991, Hess 1981, Kittel et al. 1994, Komarkova 1986, } \\
\text { Manning 1988, }\end{array}$ \\
\hline $\begin{array}{l}\text { Juncus drummondii - Antennaria lanata } \\
\text { Herbaceous Vegetation }\end{array}$ & Cooper and Lesica 1992 \\
\hline $\begin{array}{l}\text { Juncus parryi - Erigeron ursinus } \\
\text { Herbaceous Vegetation }\end{array}$ & Cooper and Lesica 1992 \\
\hline $\begin{array}{l}\text { Juniperus scopulorum / Artemisia nova } \\
\text { Woodland }\end{array}$ & DeVelice and Lesica 1993, Lesica and DeVelice 1992 \\
\hline $\begin{array}{l}\text { Juniperus scopulorum / Artemisia } \\
\text { tridentata Woodland }\end{array}$ & $\begin{array}{l}\text { Cooper et al. 1995, DeVelice 1992, DeVelice et al. 1995, Hess 1981, Hess } \\
\text { and Alexander 1986, Jennings 1978, Jennings 1979, Johnston 1987, } \\
\text { Wasser and Hess } 1982\end{array}$ \\
\hline
\end{tabular}


Appendix D.

Plant Associations: Principal and Substantiating References

\begin{tabular}{|c|c|}
\hline Plant Association Name & Principle Reference Source \\
\hline $\begin{array}{l}\text { Juniperus scopulorum / Cercocarpus } \\
\text { ledifolius Woodland }\end{array}$ & DeVelice 1992 \\
\hline $\begin{array}{l}\text { Juniperus scopulorum / Cornus sericea } \\
\text { Woodland }\end{array}$ & $\begin{array}{l}\text { Hansen et al. 1991, Kittel et al. 1994, Kittel et al. 1996, Kittel et al. 1998, } \\
\text { Osborn et al. } 1998\end{array}$ \\
\hline $\begin{array}{l}\text { Juniperus scopulorum / } \\
\text { Pseudoroegneria spicata Woodland }\end{array}$ & $\begin{array}{l}\text { Brown 1971, Francis 1983, Hansen 1985, Hansen and Hoffman 1988, } \\
\text { Johnston 1987, Strong 1980, Tiedemann et al. } 1987\end{array}$ \\
\hline $\begin{array}{l}\text { Kalmia microphylla / Carex scopulorum } \\
\text { Dwarf-shrubland }\end{array}$ & Boggs et al. 1990, Hansen et al. 1988, Hansen et al. 1991 \\
\hline $\begin{array}{l}\text { Larix lyallii - Abies lasiocarpa Forest } \\
\text { [Provisional] }\end{array}$ & Pfister et al. 1977 \\
\hline $\begin{array}{l}\text { Leymus cinereus - Festuca idahoensis } \\
\text { Herbaceous Vegetation }\end{array}$ & Ross et al. 1973 \\
\hline $\begin{array}{l}\text { Leymus cinereus - Pascopyrum smithii } \\
\text { Herbaceous Vegetation }\end{array}$ & Mueggler and Stewart 1980 \\
\hline $\begin{array}{l}\text { Leymus cinereus - Puccinellia } \\
\text { nuttalliana Herbaceous Vegetation }\end{array}$ & \\
\hline $\begin{array}{l}\text { Leymus cinereus Herbaceous } \\
\text { Vegetation [Provisional] }\end{array}$ & $\begin{array}{l}\text { Aldous and Shantz 1924, DeVelice 1992, Johnson and Simon 1987, } \\
\text { Knight et al. 1976, Mueggler and Stewart 1980, Thilenius et al. 1995, } \\
\text { Walker and Brotherson } 1982\end{array}$ \\
\hline $\begin{array}{l}\text { Pascopyrum smithii Herbaceous } \\
\text { Vegetation [Provisional] }\end{array}$ & $\begin{array}{l}\text { Baker 1983, Baker and Kennedy 1985, Bunin 1985, Christensen and } \\
\text { Welsh 1963, Hansen et al. 1991, Marr and Buckner 1974, Ramaley 1942, } \\
\text { Shanks 1977, Thilenius et al. 1995 }\end{array}$ \\
\hline \multicolumn{2}{|l|}{$\begin{array}{l}\text { Pentaphylloides floribunda / Carex } \\
\text { utriculatra Shrubland }\end{array}$} \\
\hline $\begin{array}{l}\text { Pentaphylloides floribunda / } \\
\text { Deschampsia cespitosa Shrubland }\end{array}$ & $\begin{array}{l}\text { Hansen et al. 1991, Kettler and McMullen 1996, Mutz and Graham 1982, } \\
\text { Padgett et al. 1988, Padgett et al. 1989, Sanderson and March 1996, } \\
\text { Youngblood et al. 1985 }\end{array}$ \\
\hline $\begin{array}{l}\text { Pentaphylloides floribunda / Festuca } \\
\text { campestris Shrub Herbaceous } \\
\text { Vegetation }\end{array}$ & Mueggler and Stewart 1980 \\
\hline $\begin{array}{l}\text { Pentaphylloides floribunda / Festuca } \\
\text { idahoensis Shrub Herbaceous } \\
\text { Vegetation }\end{array}$ & $\begin{array}{l}\text { Mattson and National n.d., Mueggler and Stewart 1980, Tweit and } \\
\text { Houston 1980, Youngblood et al. } 1985\end{array}$ \\
\hline \multicolumn{2}{|l|}{$\begin{array}{l}\text { Pentaphylloides floribunda / Potentilla } \\
\text { ovina Shrubland }\end{array}$} \\
\hline $\begin{array}{l}\text { Phalaris arundinacea Western } \\
\text { Herbaceous Vegetation }\end{array}$ & Boggs et al. 1990, Hansen et al. 1991, Hansen et al. 1995 \\
\hline $\begin{array}{l}\text { Phragmites australis Temperate } \\
\text { Herbaceous Vegetation }\end{array}$ & Baker 1982, Hansen et al. 1991, Hansen et al. 1995, Kittel et al. 1995 \\
\hline $\begin{array}{l}\text { Phyllodoce empetriformis / Antennaria } \\
\text { lanata Dwarf-shrubland }\end{array}$ & Cooper and Lesica 1992, Cooper et al. 1997 \\
\hline $\begin{array}{l}\text { Picea (engelmannii X glauca, } \\
\text { engelmannii) / Carex disperma Forest }\end{array}$ & Steele et al. 1981, Steele et al. 1983 \\
\hline $\begin{array}{l}\text { Picea (engelmannii X glauca, } \\
\text { engelmannii) / Cornus sericea Forest }\end{array}$ & Hansen et al. 1991, Hansen et al. 1995 \\
\hline $\begin{array}{l}\text { Picea (engelmannii X glauca, } \\
\text { engelmannii) / Galium triflorum Forest }\end{array}$ & Pfister et al. 1977 \\
\hline $\begin{array}{l}\text { Picea (engelmannii X glauca, } \\
\text { engelmannii) / Juniperus communis } \\
\text { Forest }\end{array}$ & Roberts 1980 \\
\hline $\begin{array}{l}\text { Picea (engelmannii X glauca, } \\
\text { engelmannii) / Linnaea borealis Forest }\end{array}$ & Pfister et al. 1977 \\
\hline
\end{tabular}


Appendix D.

\section{Plant Associations: Principal and Substantiating References}

\begin{tabular}{|c|c|}
\hline Plant Association Name & Principle Reference Source \\
\hline $\begin{array}{l}\text { Picea (engelmannii X glauca, } \\
\text { engelmannii) / Maianthemum stellatum } \\
\text { Forest }\end{array}$ & Fisher and Clayton 1983, Pfister et al. 1977 \\
\hline $\begin{array}{l}\text { Picea (engelmannii X glauca, } \\
\text { engelmannii) / Physocarpus malvaceus } \\
\text { Forest }\end{array}$ & Pfister et al. 1977 \\
\hline $\begin{array}{l}\text { Picea (engelmannii X glauca, } \\
\text { engelmannii) / Senecio streptanthifolius } \\
\text { Forest }\end{array}$ & Pfister et al. 1977 \\
\hline $\begin{array}{l}\text { Picea (engelmannii X glauca, } \\
\text { engelmannii) / Vaccinium cespitosum } \\
\text { Forest }\end{array}$ & Pfister et al. 1977 \\
\hline $\begin{array}{l}\text { Picea engelmannii / Calamagrostis } \\
\text { canadensis Forest }\end{array}$ & Boggs et al. 1990, Youngblood et al. 1985, Zuck 1974 \\
\hline $\begin{array}{l}\text { Picea engelmannii / Caltha leptosepala } \\
\text { Forest }\end{array}$ & Johnston 1987, Mauk and Henderson 1984, Steele et al. 1983 \\
\hline $\begin{array}{l}\text { Picea engelmannii / Equisetum arvense } \\
\text { Forest }\end{array}$ & $\begin{array}{l}\text { Crowe and Clausnitzer 1997, Johnston 1987, Kettler and McMullen } \\
\text { 1996, Kovalchik 1987, Mauk and Henderson 1984, Osborn et al. 1998, } \\
\text { Padgett et al. 1989, Pfister et al. 1977, Steele et al. 1981 }\end{array}$ \\
\hline $\begin{array}{l}\text { Picea engelmannii / Hypnum revolutum } \\
\text { Forest }\end{array}$ & Johnston 1987, Steele et al. 1981, Steele et al. 1983 \\
\hline $\begin{array}{l}\text { Pinus albicaulis - Abies lasiocarpa } \\
\text { Woodland [Provisional] }\end{array}$ & Cooper et al. 1987, Pfister et al. 1977, Steele et al. 1981 \\
\hline $\begin{array}{l}\text { Pinus albicaulis / Carex geyeri } \\
\text { Woodland }\end{array}$ & $\begin{array}{l}\text { Arno and Weaver 1990, Bowerman et al. 1997, Cooper 1975, Hall 1973, } \\
\text { Johnston 1987, Murray 1996, Nesser 1997, Rust 1998, Schlatterer 1972, } \\
\text { Steele et al. 1983 }\end{array}$ \\
\hline Pinus albicaulis / Carex rossii Forest & Johnston 1987, Reed 1969, Steele et al. 1983 \\
\hline $\begin{array}{l}\text { Pinus albicaulis / Festuca idahoensis } \\
\text { Woodland }\end{array}$ & $\begin{array}{l}\text { Johnston 1987, Montana Natural Heritage Program n.d., Steele et al. } \\
1983\end{array}$ \\
\hline $\begin{array}{l}\text { Pinus albicaulis / Juniperus communis } \\
\text { Woodland }\end{array}$ & Johnston 1987, Reed 1969, Steele et al. 1983 \\
\hline $\begin{array}{l}\text { Pinus albicaulis / Vaccinium scoparium } \\
\text { Forest }\end{array}$ & Cooper 1975, Johnston 1987, Reed 1969, Steele et al. 1983, \\
\hline Pinus albicaulis Woodland [Provisional] & Pfister et al. 1977 \\
\hline Pinus contorta / Arnica cordifolia Forest & Johnston 1987, Steele et al. 1983 \\
\hline $\begin{array}{l}\text { Pinus contorta / Calamagrostis } \\
\text { rubescens Forest }\end{array}$ & $\begin{array}{l}\text { Horton 1971, Johnson and Clausnitzer 1992, Pfister et al. 1977, Steele et } \\
\text { al. 1983, Tisdale and McLean } 1957\end{array}$ \\
\hline Pinus contorta / Carex geyeri Forest & $\begin{array}{l}\text { Alexander et al. 1986, Hess 1981, Hess and Alexander 1986, Hess and } \\
\text { Wasser 1982, Johnston 1987, Moir 1969, Steele et al. 1981, Steele et al. } \\
\text { 1983, Wasser and Hess 1982 }\end{array}$ \\
\hline Pinus contorta / Carex rossii Forest & Alexander et al. 1986, Mauk and Henderson 1984, Steele et al. 1983 \\
\hline $\begin{array}{l}\text { Pinus contorta / Juniperus communis } \\
\text { Woodland }\end{array}$ & $\begin{array}{l}\text { Alexander 1981, Alexander et al. 1986,Hess and Alexander 1986, } \\
\text { Johnston 1987, Mauk and Henderson 1984, Moir 1969, Roberts 1980, } \\
\text { Steele et al. 1981, Steele et al. } 1983\end{array}$ \\
\hline Pinus contorta / Linnaea borealis Forest & Johnson and Simon 1987, Pfister et al. 1977, Steele et al. 1983 \\
\hline $\begin{array}{l}\text { Pinus contorta / Spiraea betulifolia } \\
\text { Forest }\end{array}$ & Steele et al. 1983 \\
\hline $\begin{array}{l}\text { Pinus contorta / Vaccinium cespitosum } \\
\text { Forest }\end{array}$ & $\begin{array}{l}\text { Cooper et al. 1987, Mauk and Henderson 1984, Pfister et al. 1977, Steele } \\
\text { et al. } 1981\end{array}$ \\
\hline
\end{tabular}


Appendix D.

Plant Associations: Principal and Substantiating References

\begin{tabular}{|c|c|}
\hline Plant Association Name & Principle Reference Source \\
\hline $\begin{array}{l}\text { Pinus contorta / Vaccinium scoparium } \\
\text { Forest }\end{array}$ & $\begin{array}{l}\text { Alexander et al. 1986, Cooper et al. 1987, Hall 1973, Harrington 1978, } \\
\text { Hess 1981, Hess and Alexander 1986, Hoffman and Alexander 1976, } \\
\text { Johnston 1987, Komarkova et al. 1988, Marr et al. 1973, Mauk and } \\
\text { Henderson 1984, Oswald } 1966\end{array}$ \\
\hline $\begin{array}{l}\text { Pinus contorta / Xerophyllum tenax } \\
\text { Forest }\end{array}$ & Arno et al. 1985, Cooper et al. 1987, Volland 1976 \\
\hline Pinus contorta Scree Woodland & Montana Natural Heritage Program n.d. \\
\hline $\begin{array}{l}\text { Pinus flexilis / Cercocarpus ledifolius } \\
\text { Woodland }\end{array}$ & $\begin{array}{l}\text { DeVelice 1992, Mauk and Henderson 1984, Steele et al. 1981, Steele et } \\
\text { al. } 1983\end{array}$ \\
\hline $\begin{array}{l}\text { Pinus flexilis / Festuca idahoensis } \\
\text { Woodland }\end{array}$ & Johnston 1987, Pfister et al. 1977, Steele et al. 1981, Steele et al. 1983 \\
\hline $\begin{array}{l}\text { Pinus flexilis / Juniperus communis } \\
\text { Woodland }\end{array}$ & $\begin{array}{l}\text { Alexander et al. 1986, Cole 1982, Hoffman and Alexander 1980, Johnston } \\
\text { 1987, Pfister et al. 1977, Steele et al. 1981, Steele et al. 1983, Wasser and } \\
\text { Hess } 1982\end{array}$ \\
\hline $\begin{array}{l}\text { Pinus flexilis / Pseudoroegneria spicata } \\
\text { Woodland }\end{array}$ & Pfister et al. 1977 \\
\hline Pinus flexilis / Scree Woodland & Pfister et al. 1977 \\
\hline $\begin{array}{l}\text { Pinus ponderosa / Pseudoroegneria } \\
\text { spicata Woodland }\end{array}$ & $\begin{array}{l}\text { Cooper et al. 1987, Daubenmire and Daubenmire 1968, Hall 1973, } \\
\text { Hansen and Hoffman 1988, Hoffman and Alexander 1976, Johnson and } \\
\text { Clausnitzer 1992, Johnson and Simon } 1985\end{array}$ \\
\hline Poa juncifolia Herbaceous Vegetation & Blackburn et al. 1969, Manning 1988 \\
\hline Poa palustris Herbaceous Vegetation & Hansen et al. 1991, Hansen et al. 1995 \\
\hline $\begin{array}{l}\text { Poa pratensis Herbaceous Vegetation } \\
\text { [Provisional] }\end{array}$ & Sawyer and Keeler-Wolf 1995 \\
\hline $\begin{array}{l}\text { Populus angustifolia / Cornus sericea } \\
\text { Woodland }\end{array}$ & $\begin{array}{l}\text { Hansen et al. 1995, Hess and Wasser 1982, Johnston 1987, Kittel and } \\
\text { Lederer 1993, Kittel et al. 1995, Muldavin and Johnson 1993,Padgett et } \\
\text { al. 1988, Padgett et al. 1989, Richard et a }\end{array}$ \\
\hline $\begin{array}{l}\text { Populus balsamifera ssp. trichocarpa / } \\
\text { Poa pratensis Forest }\end{array}$ & Hansen et al. 1995 \\
\hline $\begin{array}{l}\text { Populus tremuloides / Amelanchier } \\
\text { alnifolia Forest }\end{array}$ & Montana Natural Heritage Program n.d. \\
\hline $\begin{array}{l}\text { Populus tremuloides / Bromus carinatus } \\
\text { Forest }\end{array}$ & Mueggler 1988, Mueggler and Campbell 1986 \\
\hline $\begin{array}{l}\text { Populus tremuloides / Calamagrostis } \\
\text { canadensis Forest }\end{array}$ & Boggs et al. 1990, Hansen et al. 1991, Mutel 1973, Powell 1988 \\
\hline $\begin{array}{l}\text { Populus tremuloides / Calamagrostis } \\
\text { rubescens Forest }\end{array}$ & $\begin{array}{l}\text { Alexander et al. 1986, Cooper and Pfister 1981, Mueggler 1988, } \\
\text { Mueggler and Campbell 1982, Mueggler and Campbell 1986, Williams } \\
\text { and Lillybridge 1983, Youngblood and Mueggler } 1981\end{array}$ \\
\hline $\begin{array}{l}\text { Populus tremuloides / Cornus sericea } \\
\text { Forest }\end{array}$ & $\begin{array}{l}\text { Evans 1989, Hansen et al. 1990, Hansen et al. 1991, Kittel et al. 1994, } \\
\text { Richard et al. } 1996\end{array}$ \\
\hline \multicolumn{2}{|l|}{$\begin{array}{l}\text { Populus tremuloides / Poa pratensis } \\
\text { Forest }\end{array}$} \\
\hline $\begin{array}{l}\text { Populus tremuloides / Symphoricarpos } \\
\text { oreophilus Forest }\end{array}$ & $\begin{array}{l}\text { Hess and Wasser 1982, Hoffman and Alexander 1980, Hoffman and } \\
\text { Alexander 1983, Johnston 1987, Johnston and Hendzel 1985, Keammerer } \\
\text { and Peterson 1981, Keammerer and Stoecker 1975, Keammerer and } \\
\text { Stoecker } 1980\end{array}$ \\
\hline Populus tremuloides / Tall Forbs Forest & $\begin{array}{l}\text { Hess and Wasser 1982, Hoffman and Alexander 1980, Hoffman and } \\
\text { Alexander 1983, Johnston and Hendzel 1985,Kittel et al. 1994, Lewis } \\
\text { 1975, Marr et al. 1973, Mueggler } 1988\end{array}$ \\
\hline
\end{tabular}


Appendix D.

Plant Associations: Principal and Substantiating References

\begin{tabular}{|c|c|}
\hline Plant Association Name & Principle Reference Source \\
\hline $\begin{array}{l}\text { Populus tremuloides / Thalictrum } \\
\text { fendleri Forest }\end{array}$ & $\begin{array}{l}\text { Boyce 1977, Bunin 1975, Crouch 1983, Hess 1981, Hess and Alexander } \\
\text { 1986, Hoffman and Alexander 1980, Hoffman and Alexander 1983, } \\
\text { Johnston 1987, Johnston and Hendzel 1985, Komarkova et al. 1988, } \\
\text { Langenheim 1962, Mueggler } 1988\end{array}$ \\
\hline $\begin{array}{l}\text { Pseudoroegneria spicata - Bouteloua } \\
\text { gracilis Herbaceous Vegetation }\end{array}$ & $\begin{array}{l}\text { Bear Creek Uranium Mine Application n.d., Boutton et al. 1980, Francis } \\
\text { 1983, Mueggler and Stewart 1980, Terwilliger and Tiedemann 1978, } \\
\text { Thilenius et al. 1995, Tiedemann et al. } 1987\end{array}$ \\
\hline $\begin{array}{l}\text { Pseudoroegneria spicata - Cushion } \\
\text { Plant Herbaceous Vegetation }\end{array}$ & Lesica and DeVelice 1992 \\
\hline \begin{tabular}{|l|} 
Pseudoroegneria spicata - Koeleria \\
macrantha Herbaceous Vegetation
\end{tabular} & Ross et al. 1973 \\
\hline $\begin{array}{l}\text { Pseudoroegneria spicata - Oryzopsis } \\
\text { hymenoides Herbaceous Vegetation }\end{array}$ & Baker 1983, Baker 1984, DeVelice 1992 \\
\hline $\begin{array}{l}\text { Pseudoroegneria spicata - Pascopyrum } \\
\text { smithii Herbaceous Vegetation }\end{array}$ & Hansen and Hoffman 1988, Mueggler and Stewart 1980 \\
\hline $\begin{array}{l}\text { Pseudoroegneria spicata - Poa secunda - } \\
\text { Stipa comata Herbaceous Vegetation }\end{array}$ & \\
\hline $\begin{array}{l}\text { Pseudoroegneria spicata - Poa secunda } \\
\text { Herbaceous Vegetation }\end{array}$ & $\begin{array}{l}\text { Daubenmire 1970, Fisser et al. 1965, Hall 1973, Johnson and Simon } \\
\text { 1985, Johnson and Simon 1987,Mueggler and Stewart 1980, Poulton } \\
\text { 1955, Price and Brotherson 1987 }\end{array}$ \\
\hline $\begin{array}{l}\text { Pseudoroegneria spicata - Stipa comata } \\
\text { Herbaceous Vegetation }\end{array}$ & $\begin{array}{l}\text { Anderson 1956,Daubenmire 1970, Hyde 1964, Montana Natural } \\
\text { Heritage Program n.d., Mueggler and Stewart 1980, Poulton 1955, Tweit } \\
\text { and Houston } 1980\end{array}$ \\
\hline $\begin{array}{l}\text { Pseudotsuga menziesii / Arnica } \\
\text { cordifolia Forest }\end{array}$ & Johnston 1987, Pfister et al. 1977, Steele et al. 1981, Steele et al. 1983 \\
\hline $\begin{array}{l}\text { Pseudotsuga menziesii / Calamagrostis } \\
\text { rubescens Forest }\end{array}$ & $\begin{array}{l}\text { Cooper et al. 1987, Daubenmire 1952, Daubenmire and Daubenmire } 1968 \\
\text { Hall 1973, Horton 1971, Johnson and Clausnitzer 1992, Johnson and } \\
\text { Simon 1987, Johnston } 1987\end{array}$ \\
\hline $\begin{array}{l}\text { Pseudotsuga menziesii / Carex geyeri } \\
\text { Forest }\end{array}$ & $\begin{array}{l}\text { Clausnitzer and Zamora 1987, Cooper et al. 1987,Hess 1981, Hess and } \\
\text { Alexander 1986, Johnston 1987,Pfister et al. 1977, Steele et al. 1981, } \\
\text { Williams and Smith } 1990\end{array}$ \\
\hline $\begin{array}{l}\text { Pseudotsuga menziesii / Cercocarpus } \\
\text { ledifolius Woodland }\end{array}$ & $\begin{array}{l}\text { DeVelice 1992, Johnson and Clausnitzer 1992, Mauk and Henderson } \\
\text { 1984, Steele et al. 1981, Steele et al. 1983, Youngblood and Mauk } 1985\end{array}$ \\
\hline $\begin{array}{l}\text { Pseudotsuga menziesii / Cornus sericea } \\
\text { Woodland }\end{array}$ & $\begin{array}{l}\text { Hansen et al. 1990, Hansen et al. 1991, Hansen et al. 1995, Kittel and } \\
\text { Lederer 1993, Kittel et al. 1994, Kittel et al. 1995, Kittel et al. 1997, } \\
\text { Osborn et al. 1998, Richard et al. } 1996\end{array}$ \\
\hline $\begin{array}{l}\text { Pseudotsuga menziesii / Festuca } \\
\text { campestris Woodland }\end{array}$ & Pfister et al. 1977 \\
\hline $\begin{array}{l}\text { Pseudotsuga menziesii / Festuca } \\
\text { idahoensis Woodland }\end{array}$ & Johnston 1987, Pfister et al. 1977, Steele et al. 1983 \\
\hline $\begin{array}{l}\text { Pseudotsuga menziesii / Juniperus } \\
\text { communis Forest }\end{array}$ & $\begin{array}{l}\text { Giese 1975, Johnston 1987, Murphy 1982, Pfister et al. 1977, Steele et al. } \\
\text { 1981, Steele et al. 1983, Vories } 1974\end{array}$ \\
\hline $\begin{array}{l}\text { Pseudotsuga menziesii / Juniperus } \\
\text { scopulorum Woodland }\end{array}$ & Roberts et al. 1979 \\
\hline $\begin{array}{l}\text { Pseudotsuga menziesii / Linnaea } \\
\text { borealis Forest }\end{array}$ & Pfister et al. 1977, Steele et al. 1981 \\
\hline $\begin{array}{l}\text { Pseudotsuga menziesii / Mahonia } \\
\text { repens Forest }\end{array}$ & $\begin{array}{l}\text { Alexander et al. 1984, Hoffman and Alexander 1976, Larson and Moir } \\
\text { 1987, Mauk and Henderson 1984, Roberts 1980, Roberts et al. 1992, } \\
\text { Steele et al. 1981, Steele et al. 1983, Youngblood and Mauk } 1985\end{array}$ \\
\hline
\end{tabular}


Appendix D.

Plant Associations: Principal and Substantiating References

\begin{tabular}{|c|c|}
\hline Plant Association Name & Principle Reference Source \\
\hline $\begin{array}{l}\text { Pseudotsuga menziesii / Physocarpus } \\
\text { malvaceus Forest }\end{array}$ & $\begin{array}{l}\text { Clausnitzer and Zamora 1987, Cooper et al. 1987, Daubenmire 1952, } \\
\text { Daubenmire and Daubenmire 1968, Hall 1973, Johnson and Simon 1987, } \\
\text { Johnston 1987, Mauk and Henderson 1984, Pfister et al. 1977, Steele et } \\
\text { al. 1981, Steele et al. } 1983\end{array}$ \\
\hline $\begin{array}{l}\text { Pseudotsuga menziesii / } \\
\text { Pseudoroegneria spicata Woodland }\end{array}$ & Cole 1982, Pfister et al. 1977 \\
\hline $\begin{array}{l}\text { Pseudotsuga menziesii / Spiraea } \\
\text { betulifolia Forest }\end{array}$ & $\begin{array}{l}\text { Cooper et al. 1987, Horton 1971, Johnson and Simon 1987, Johnston } \\
\text { 1987, Oswald 1966, Pfister et al. 1977, Steele et al. 1983, Youngblood and } \\
\text { Mueggler } 1981\end{array}$ \\
\hline $\begin{array}{l}\text { Pseudotsuga menziesii / } \\
\text { Symphoricarpos albus Forest }\end{array}$ & $\begin{array}{l}\text { Cooper et al. 1987, Daubenmire and Daubenmire 1968, Johnson and } \\
\text { Simon 1987, Pfister et al. 1977, Steele et al. 1981, Steele et al. 1983, } \\
\text { Williams and Lillybridge } 1983\end{array}$ \\
\hline $\begin{array}{l}\text { Pseudotsuga menziesii / } \\
\text { Symphoricarpos oreophilus Forest }\end{array}$ & $\begin{array}{l}\text { Hess and Wasser 1982, Johnson and Simon 1987, Johnston 1987, Mauk } \\
\text { and Henderson 1984, Muldavin 1994, Pfister et al. 1977, Reed 1976, } \\
\text { Steele et al. 1981, Steele et al. } 1983\end{array}$ \\
\hline $\begin{array}{l}\text { Pseudotsuga menziesii / Vaccinium } \\
\text { membranaceum Forest }\end{array}$ & Cooper et al. 1987,Johnston 1987, Pfister et al. 1977, Steele et al. 1983 \\
\hline \begin{tabular}{|l|} 
Pseudotsuga menziesii Scree Woodland \\
\end{tabular} & DeVelice et al. 1986, Fitzhugh et al. 1987, Pfister et al. 1977 \\
\hline \begin{tabular}{|l|l} 
Puccinellia nuttalliana Herbaceous \\
Vegetation
\end{tabular} & Ungar 1972, Ungar 1974, Heidel and Cooper 1997 \\
\hline $\begin{array}{l}\text { Purshia tridentata / Festuca idahoensis } \\
\text { Shrub Herbaceous Vegetation }\end{array}$ & $\begin{array}{l}\text { Daubenmire 1970, Hall 1973, Johnson and Simon 1987, Lewis 1975, } \\
\text { Mueggler and Stewart 1980, Tweit and Houston } 1980\end{array}$ \\
\hline $\begin{array}{l}\text { Purshia tridentata / Pseudoroegneria } \\
\text { spicata Shrub Herbaceous Vegetation }\end{array}$ & $\begin{array}{l}\text { Daubenmire 1970, Hall 1973, Hironaka et al. 1983, Johnson and Simon } \\
\text { 1987, Mueggler and Stewart } 1980\end{array}$ \\
\hline $\begin{array}{l}\text { Rhus trilobata / Pseudoroegneria } \\
\text { spicata Shrub Herbaceous Vegetation }\end{array}$ & $\begin{array}{l}\text { Bighorn Coal Mine n.d., Brown 1971, Hansen and Hoffman 1988, } \\
\text { Mueggler and Stewart 1980, Tweit and Houston } 1980\end{array}$ \\
\hline Rosa woodsii Shrubland & Hansen et al. 1990, Hansen et al. 1991 \\
\hline $\begin{array}{l}\text { Salix arctica / Polygonum bistortoides } \\
\text { Dwarf-shrubland }\end{array}$ & Cooper and Lesica 1992, Cooper et al. 1997 \\
\hline Salix bebbiana Shrubland & $\begin{array}{l}\text { Baker 1982, Dick-Peddie 1993, Hansen et al. 1991, Osborn et al. 1998, } \\
\text { Szaro } 1989\end{array}$ \\
\hline $\begin{array}{l}\text { Salix boothii / Calamagrostis canadensis } \\
\text { Shrubland }\end{array}$ & $\begin{array}{l}\text { Mutz and Graham 1982, Mutz and Queiroz 1983, Norton et al. 1981, } \\
\text { Padgett et al. 1988, Tuhy and Jensen 1982, Youngblood et al. } 1985\end{array}$ \\
\hline Salix boothii / Carex utriculata Shrubland & $\begin{array}{l}\text { Kittel and Lederer 1993, Kittel et al. 1994, Kovalchik 1987, Mutz and } \\
\text { Graham 1982, Mutz and Queiroz 1983, Padgett et al. 1988, Padgett et al. } \\
\text { 1989, Tuhy and Jensen } 1982\end{array}$ \\
\hline $\begin{array}{l}\text { Salix boothii / Mesic Graminoids } \\
\text { Shrubland }\end{array}$ & Padgett et al. 1988, Padgett et al. 1989, Reid 1990 \\
\hline $\begin{array}{l}\text { Salix brachycarpa / Carex aquatilis } \\
\text { Shrubland }\end{array}$ & Colorado Natural Heritage Program n.d., Osborn et al. 1998 \\
\hline \multicolumn{2}{|l|}{$\begin{array}{l}\text { Salix candida / Carex lasiocarpa } \\
\text { Shrubland }\end{array}$} \\
\hline $\begin{array}{l}\text { Salix candida / Carex utriculata } \\
\text { Shrubland }\end{array}$ & Hansen et al. 1991, Steele et al. 1981 \\
\hline $\begin{array}{l}\text { Salix drummondiana / Calamagrostis } \\
\text { canadensis Shrubland }\end{array}$ & $\begin{array}{l}\text { Baker 1986, Baker 1989, Johnston 1987, Kettler and McMullen 1996, } \\
\text { Kittel and Lederer 1993, Komarkova 1986, Sanderson and Kettler 1996, } \\
\text { Tuhy and Jensen } 1982\end{array}$ \\
\hline $\begin{array}{l}\text { Salix drummondiana / Carex utriculata } \\
\text { Shrubland }\end{array}$ & Kovalchik 1993, Moseley et al. 1994 \\
\hline $\begin{array}{l}\text { Salix drummondiana Shrubland } \\
\text { [Provisional] }\end{array}$ & $\begin{array}{l}\text { Baker 1989, Boggs et al. 1990, Hansen et al. 1989, Hansen et al. 1991, } \\
\text { Kittel and Lederer 1993, Phillips } 1977\end{array}$ \\
\hline
\end{tabular}


Appendix D.

\section{Plant Associations: Principal and Substantiating References}

\begin{tabular}{|c|c|}
\hline Plant Association Name & Principle Reference Source \\
\hline $\begin{array}{l}\text { Salix exigua Temporarily Flooded } \\
\text { Shrubland }\end{array}$ & $\begin{array}{l}\text { Evenden 1990, Foti et al. 1994, Hansen et al. 1989, Hansen et al. 1991, } \\
\text { Hansen et al. 1995, Hoagland 1997, Kittel and Lederer 1993, Kovalchik } \\
\text { 1987, Phillips 1977 }\end{array}$ \\
\hline $\begin{array}{l}\text { Salix geyeriana / Calamagrostis } \\
\text { canadensis Shrubland }\end{array}$ & $\begin{array}{l}\text { Cooper and Cottrell 1990, Hansen et al. 1991, Johnston 1987, Kettler and } \\
\text { McMullen 1996, Kittel et al. 1994, Osborn et al. 1998, Padgett et al. 1989, } \\
\text { Tuhy and Jensen 1982, Youngblood et al. } 1985\end{array}$ \\
\hline $\begin{array}{l}\text { Salix geyeriana / Carex utriculata } \\
\text { Shrubland }\end{array}$ & $\begin{array}{l}\text { Hansen et al. 1991, Kettler and McMullen 1996, Kittel and Lederer 1993, } \\
\text { Mutz and Queiroz 1983, Padgett et al. 1989, Tuhy and Jensen 1982, } \\
\text { Youngblood et al. 1985 }\end{array}$ \\
\hline $\begin{array}{l}\text { Salix geyeriana / Deschampsia cespitosa } \\
\text { Shrubland }\end{array}$ & $\begin{array}{l}\text { Jensen and Tuhy 1981, Montana Natural Heritage Program n.d., Padgett } \\
\text { et al. 1988, Padgett et al. } 1989\end{array}$ \\
\hline Salix glauca Shrubland & Cooper and Lesica 1992 \\
\hline $\begin{array}{l}\text { Salix lutea / Calamagrostis canadensis } \\
\text { Shrubland }\end{array}$ & Hansen et al. 1991 \\
\hline $\begin{array}{l}\text { Salix planifolia / Carex aquatilis } \\
\text { Shrubland }\end{array}$ & $\begin{array}{l}\text { Baker 1989, Cooper and Cottrell 1990, Hansen et al. 1988, Hansen et al. } \\
\text { 1991, Hess 1981, Hess and Wasser 1982, Jensen and Tuhy 1981, } \\
\text { Johnston 1987, Kittel and Lederer 1993, Kittel et al. 1994, Kittel et al. } \\
\text { 1995, Kittel et al. 1996, Komarkova 1986, Lewis }\end{array}$ \\
\hline \multicolumn{2}{|l|}{\begin{tabular}{|l|} 
Salix planifolia / Carex nebrascensis \\
Shrubland \\
\end{tabular}} \\
\hline $\begin{array}{l}\text { Salix planifolia / Carex scopulorum } \\
\text { Shrubland }\end{array}$ & Hess 1981, Hess and Wasser 1982, Komarkova 1976, Komarkova 1986 \\
\hline $\begin{array}{l}\text { Salix reticulata / Caltha leptosepala } \\
\text { Dwarf-shrubland }\end{array}$ & Cooper and Lesica 1992, Cooper et al. 1997 \\
\hline Salix wolfii / Carex aquatilis Shrubland & $\begin{array}{l}\text { Baker 1986, Baker 1989, Hansen et al. 1991, Johnston 1987, Kettler and } \\
\text { McMullen 1996, Kittel and Lederer 1993, Kittel et al. 1996, Mattson } 1984\end{array}$ \\
\hline $\begin{array}{l}\text { Salix wolfii / Deschampsia cespitosa } \\
\text { Shrubland }\end{array}$ & $\begin{array}{l}\text { Hansen et al. 1991, Padgett et al. 1988, Padgett et al. 1989, Youngblood } \\
\text { et al. } 1985\end{array}$ \\
\hline $\begin{array}{l}\text { Sarcobatus vermiculatus / Distichlis } \\
\text { spicata Shrubland }\end{array}$ & $\begin{array}{l}\text { Daubenmire 1970, Franklin and Dyrness 1973, Mueggler and Stewart } \\
1980\end{array}$ \\
\hline $\begin{array}{l}\text { Sarcobatus vermiculatus / Elymus } \\
\text { lanceolatus Shrub Herbaceous } \\
\text { Vegetation }\end{array}$ & Jorgensen 1979 \\
\hline $\begin{array}{l}\text { Sarcobatus vermiculatus / Leymus } \\
\text { cinereus Shrubland }\end{array}$ & Mueggler and Stewart 1980 \\
\hline $\begin{array}{l}\text { Sarcobatus vermiculatus / Pascopyrum } \\
\text { smithii Shrub Herbaceous Vegetation }\end{array}$ & $\begin{array}{l}\text { Hansen and Hoffman 1988, Mueggler and Stewart 1980, Olson and } \\
\text { Gerhart } 1982\end{array}$ \\
\hline Scirpus acutus Herbaceous Vegetation & Hansen et al. 1991, Hansen et al. 1995 \\
\hline $\begin{array}{l}\text { Scirpus tabernaemontani Temperate } \\
\text { Herbaceous Vegetation }\end{array}$ & Hansen et al. 1988, Kovalchik 1993, Sanderson and Kettler 1996 \\
\hline $\begin{array}{l}\text { Senecio triangularis Herbaceous } \\
\text { Vegetation }\end{array}$ & Hansen et al. 1991, Hansen et al. 1995, Komarkova 1976 \\
\hline $\begin{array}{l}\text { Shepherdia argentea Shrubland } \\
\text { [Provisional] }\end{array}$ & $\begin{array}{l}\text { Hansen et al. 1991, Kittel and Lederer 1993, Kittel et al. 1994, Osborn et } \\
\text { al. } 1998\end{array}$ \\
\hline Silene acaulis Herbaceous Vegetation & Bamberg 1961 \\
\hline $\begin{array}{l}\text { Sporobolus airoides Herbaceous } \\
\text { Vegetation }\end{array}$ & $\begin{array}{l}\text { Aldous and Shantz 1924, Cooper 1984, Francis 1986, Johnston 1987, } \\
\text { Kittel and Lederer 1993, Lesica and DeVelice 1992, Steward 1982, Ungar } \\
1974\end{array}$ \\
\hline $\begin{array}{l}\text { Stipa comata - Bouteloua gracilis - } \\
\text { Pascopyrum smithii Herbaceous } \\
\text { Vegetation }\end{array}$ & \\
\hline
\end{tabular}


Appendix D.

Plant Associations: Principal and Substantiating References

\begin{tabular}{|l|l|}
\hline Plant Association Name & Principle Reference Source \\
\hline $\begin{array}{l}\text { Stipa comata - Bouteloua gracilis } \\
\text { Herbaceous Vegetation }\end{array}$ & $\begin{array}{l}\text { Hess 1981, Johnston 1987, Mueggler and Stewart 1980, Ramaley 1916, } \\
\text { Soil Conservation Service 1978, Stoecker-Keammerer Consultants n.d. }\end{array}$ \\
\hline $\begin{array}{l}\text { Stipa comata - Psoralea tenuiflora } \\
\text { Herbaceous Vegetation }\end{array}$ & \\
\hline $\begin{array}{l}\text { Symphoricarpos occidentalis Shrubland } \\
\text { [Provisional] }\end{array}$ & $\begin{array}{l}\text { Christy 1973, Clark 1977, Clark et al. 1980, Hansen et al. 1991, Kittel et al. } \\
\text { 1994, Osborn et al. 1998 }\end{array}$ \\
\hline $\begin{array}{l}\text { Typha latifolia Western Herbaceous } \\
\text { Vegetation }\end{array}$ & $\begin{array}{l}\text { Bunin 1985, Christy 1973, Hansen et al. 1991, Kittel et al. 1996, } \\
\text { Kovalchik 1993, Lindauer and Christy 1972, Muldavin and Johnson } \\
1993, \text { Padgett et al. 1989, Youngblood et al. 1985 }\end{array}$ \\
\hline
\end{tabular}




\section{Index to D escriptions of Types}

Needle-leaved Evergreen Forests ........................................................................................................................... 76

Abies lasiocarpa / Arnica cordifolia Forest ....................................................................................... 76

Abies lasiocarpa / Calamagrostis canadensis Forest ………………………………………………..... 76

Abies lasiocarpa / Calamagrostis rubescens Forest .............................................................................. 77

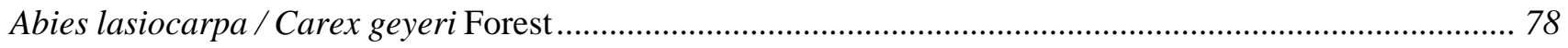

Abies lasiocarpa / Ribes montigenum Forest .................................................................................... 78

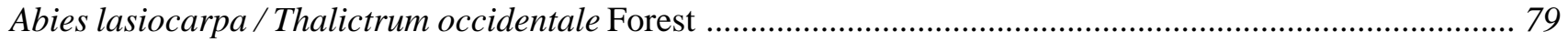

Picea (engelmannii x glauca, engelmannii) / Equisetum arvense Forest ..................................................... 80

Picea engelmannii / Calamagrostis canadensis Forest .......................................................................... 81

Picea (engelmannii x glauca, engelmannii) / Galium triflorum Forest.........................................................82

Picea (engelmannii x glauca, engelmannii) / Senecio streptanthifolius Forest ............................................... 83

Pinus contorta / Carex rossii Forest .................................................................................................. 84

(Pseudotsuga menziesii / Arnica cordifolia Forest. ................................................................................ 84

Pseudotsuga menziesii / Calamagrostis rubescens Forest...…………………………………………….... 85

(Pseudotsuga menziesii / Juniperus communis Forest ....................................................................... 86

Pseudotsuga menziesii / Physocarpus malvaceus Forest ............................................................... 86

Pseudotsuga menziesii / Spiraea betulifolia Forest …………....................................................... 87

Pseudotsuga menziesii / Symphoricarpos oreophilus Forest ........................................................... 88

Needle-leaved Evergreen Woodlands.................................................................................................................. 89

Abies lasiocarpa / Juniperus communis Woodland …………………………………………………....... 89

Cercocarpus ledifolius / Festuca idahoensis Woodland ........................................................................... 89

Juniperus scopulorum / Artemisia tridentata Woodland .......................................................................... 90

Juniperus scopulorum / Cercocarpus ledifolius Woodland .......................................................................... 90

Juniperus scopulorum / Pseudoroegneria spicata Woodland .................................................................... 91

Pinus albicaulis - Abies lasiocarpa Woodland [Provisional] ........................................................................... 92

Pinus albicaulis / Festuca idahoensis Woodland .................................................................................. 92

Pinus flexilis / Cercocarpus ledifolius Woodland ……………………………………………………...... 93

Pinus flexilis / Festuca idahoensis Woodland ........................................................................................ 94

Pinus flexilis / Juniperus communis Woodland ................................................................................. 95

Pinus flexilis / Pseudoroegneria spicata Woodland ...………………………………………………….... 95

Pseudotsuga menziesii / Cercocarpus ledifolius Woodland .................................................................... 96

Pseudotsuga menziesii / Festuca idahoensis Woodland ........................................................................... 97

Pseudotsuga menziesii / Scree Woodland ............................................................................................ 97

Montane or Boreal Cold-deciduous Forest ……………………………................................................................ 98

Populus balsamifera ssp. trichocarpa / Poa pratensis Forest ................................................................. 98

Populus tremuloides / Bromus carinatus Forest …………………………………………………….... 99

Populus tremuloides / Calamagrostis canadensis Forest …………………………………......................... 99

Populus tremuloides / Calamagrostis rubescens Forest …………………………………………….... 100

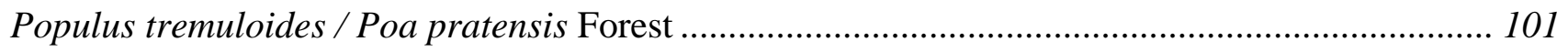

Populus tremuloides / Tall Forbs Forest ............................................................................... 102

Populus tremuloides / Thalictrum fendleri Forest ...................................................................... 102 


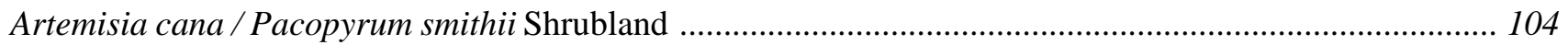

Artemisia tridentata ssp. tridentata / Festuca idahoensis Shrubland ...................................................... 104

Artemisia tridentata ssp. tridentata / Pascopyrum smithii Shrubland ...................................................... 105

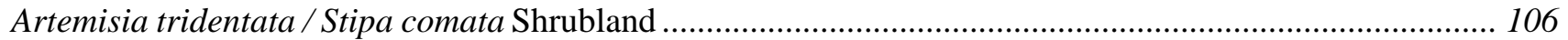

Artemisia tridentata ssp. vaseyana / Leymus cinereus Shrubland ............................................................. 106

Artemisia tridentata ssp. vaseyana / Pseudoroegneria spicata Shrubland .............................................. 107

Artemisia tridentata ssp. wyomingensis / Elymus lanceolatus Shrubland .................................................... 108

Betula glandulosa / Carex utriculata Herbaceous Vegetation ................................................................ 108

Cercocarpus ledifolius / Pseudoroegneria spicata Shrubland ................................................................. 109

Chrysothamnus viscidiflorus / Stipa comata Shrubland …..................................................................... 110

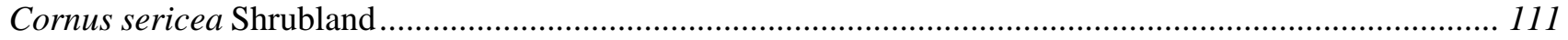

Pentaphylloides floribunda / Deschampsia cespitosa Shrubland .............................................................. 112

Pentaphylloides floribunda / Potentilla ovina Shrubland ........................................................................ 113

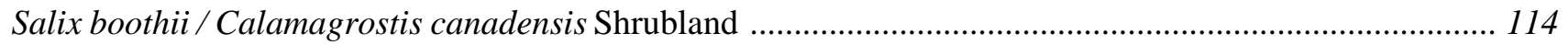

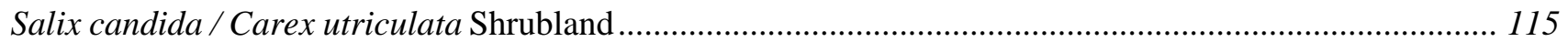

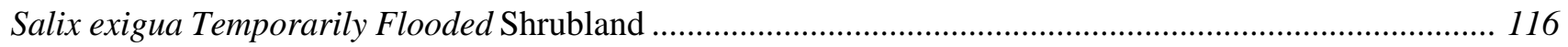

Salix drummondiana / Calamagrostis canadensis Shrubland ................................................................... 116

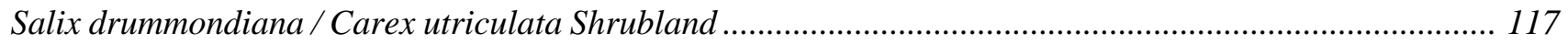

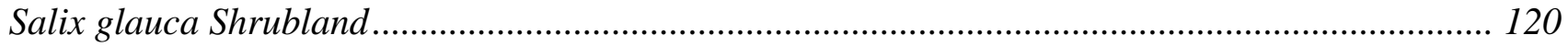

Salix geyeriana / Calamagrostis canadensis Shrubland ....................................................... 121

Salix geyeriana / Carex utriculata Shrubland ........................................................................ 122

Salix planifolia / Carex nebrascensis Shrubland ................................................................. 123

Sarcobatus vermiculatus / Distichlis spicata Shrubland..........................................................124

Sarcobatus vermiculatus / Leymus cinereus Shrubland ....................................................................... 124

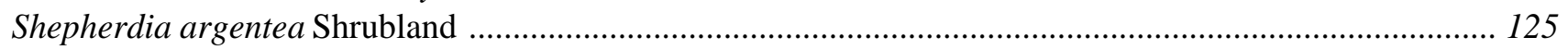

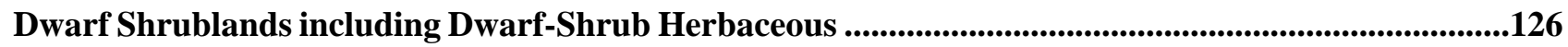

Artemisia arbuscula ssp. longiloba / Elymus lanceolatus Dwarf-shrub Herbaceous Vegetation .................. 126

Artemisia arbuscula ssp. longiloba / Festuca idahoensis Dwarf-shrub Herbaceous Vegetation .................. 127

Artemisia nova / Pseudoroegneria spicata Dwarf-shrubland ................................................................ 127

Atriplex gardneri / Oryzopsis hymenoides Dwarf-shrubland .............................................................. 128

Cassiope mertensiana / Carex paysonis Dwarf-shrubland ...................................................................... 129

Dryas octopetala / Carex rupestris Dwarf-shrub Herbaceous Vegetation ................................................. 129

Dryas octopetala / Polygonum viviparum Dwarf-shrub Herbaceous Vegetation ....................................... 131

Phyllodoce empetriformis / Antennaria lanata Dwarf-shrubland ............................................................. 132

Salix reticulata / Caltha leptosepala Dwarf-shrubland .......................................................................... 133

Herbaceous Vegetation with a Sparse Needle-leaved or Microphyllus Evergreen Shrub Layer .......... 134

Artemisia cana / Elymus trachycaulus Shrub Herbaceous Vegetation ....................................................... 134

Artemisia cana / Festuca idahoensis Shrub Herbaceous Vegetation ..................................................... 134

Artemisia cana / Poa pratensis Shrub Herbaceous Vegetation ................................................................ 135

Artemisia tridentata ssp. vaseyana / Festuca idahoensis Shrub Herbaceous Vegetation.............................. 135

Artemisia tridentata ssp. wyomingensis / Pseudoroegneria spicata Shrub Herbaceous Vegetation...............136

Artemisia tripartita / Elymus lanceolatus Shrub Herbaceous Vegetation.....................................................136

Artemisia tripartita / Festuca idahoensis Shrub Herbaceous Vegetation.....................................................137

Artemisia tripartita / Pseudoroegneria spicata Shrub Herbaceous Vegetation .......................................... 138

Pentaphylloides floribunda / Festuca campestris Shrub Herbaceous Vegetation ....................................... 139

Pentaphylloides floribunda / Festuca idahoensis Shrub Herbaceous Vegetation ....................................... 140

Salix arctica / Polygonum bistortoides Dwarf Shrubland ...................................................................... 141

Sarcobatus vermiculatus / Pascopyrum smithii Shrub Herbaceous Vegetation .......................................... 142 


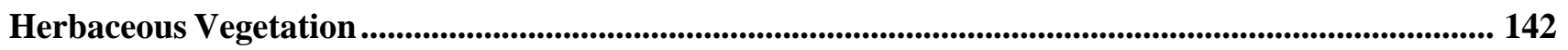

Calamagrostis canadensis Herbaceous Vegetation .......................................................................... 144

Calamagrostis purpurascens - Carex rupestris Herbaceous Vegetation ................................................. 145

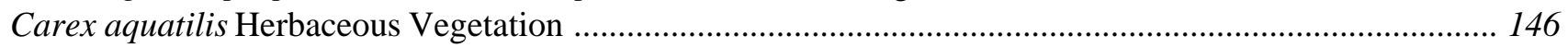

Carex buxbaumii Herbaceous Vegetation ...................................................................................... 146

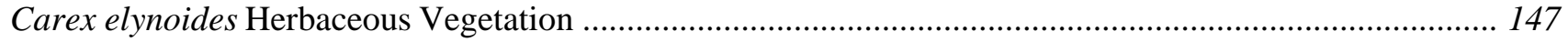

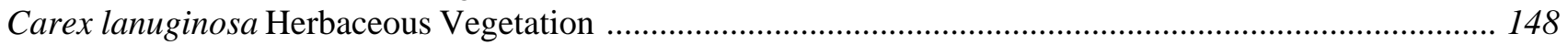

Carex lasiocarpa Herbaceous Vegetation ............................................................................................... 149

Carex nebrascensis Herbaceous Vegetation ......................................................................................... 150

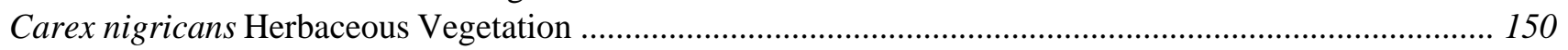

Carex rupestris / Potentilla ovina Herbaceous Vegetation ..................................................................... 151

Carex scirpoidea / Geum rossii Herbaceous Vegetation.............................................................................153

Carex scirpoidea / Potentilla diversifolia Herbaceous Vegetation ............................................................. 154

Carex scopulorum / Caltha leptosepala Herbaceous Vegetation ........................................................... 155

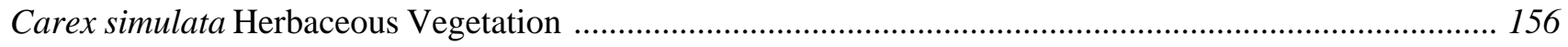

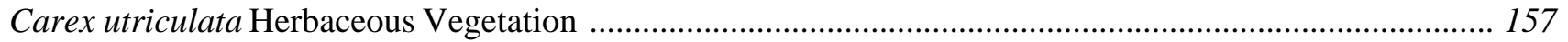

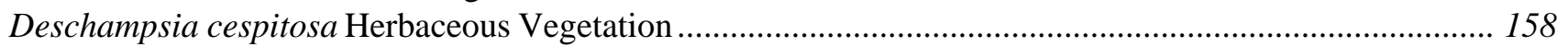

Deschampsia cespitosa - Caltha leptosepala Herbaceous Vegetation ..................................................... 158

Deschampsia cespitosa - Potentilla diversifolia Herbaceous Vegetation .................................................... 160

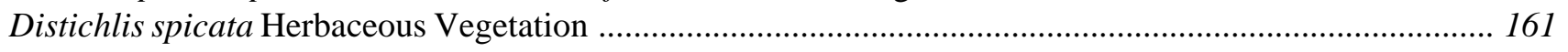

Eleocharis palustris Herbaceous Vegetation ............................................................................... 161

Elymus lanceolatus - Phacelia hastata Herbaceous Vegetation ............................................................... 162

Equisetum fluviatile Herbaceous Vegetation ...................................................................................... 163

Festuca idahoensis - Deschampsia cespitosa Herbaceous Vegetation ...................................................... 164

Festuca idahoensis - Elymus trachycaulus Herbaceous Vegetation ........................................................ 164

Festuca idahoensis - Potentilla diversifolia Herbaceous Vegetation ....................................................... 165

Festuca idahoensis - Pseudoroegneria spicata Herbaceous Vegetation ................................................... 166

Festuca kingii - Oxytropis campestris Herbaceous Vegetation ............................................................ 167

Geum rossii - Minuartia obtusiloba Herbaceous Vegetation ................................................................. 168

Hordeum jubatum Herbaceous Vegetation .......................................................................................... 169

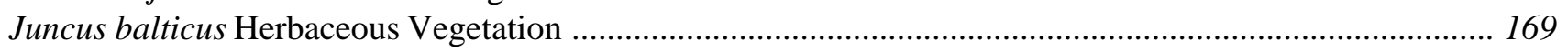

Phalaris arundinacea Western Herbaceous Vegetation ....................................................................... 174

Poa juncifolia Herbaceous Vegetation .......................................................................................... 174

Poa pratensis Herbaceous Vegetation [Provisional] ........................................................................... 175

Pseudoroegneria spicata - Cushion plant Herbaceous Vegetation .......................................................... 175

Pseudoroegneria spicata - Oryzopsis hymenoides Herbaceous Vegetation ........................................... 176

Pseudoroegneria spicata - Pascopyrum smithii Herbaceous Vegetation .................................................. 176

Pseudoroegneria spicata - Poa secunda Herbaceous Vegetation .............................................................. 177

Puccinellia nuttalliana Herbaceous Vegetation ............................................................................... 178

Scirpus acutus Herbaceous Vegetation ......................................................................................... 178

Scirpus tabernaemontani Herbaceous Vegetation ............................................................................ 179

Stipa comata - Bouteloua gracilis Herbaceous Vegetation ..................................................................... 180

Stipa comata - Psoralidium tenuiflorum Herbaceous Vegetation .......................................................... 181

Stipa richardsonii - Festuca idahoensis Herbaceous Vegetation .......................................................... 181

Typha latifolia Western Herbaceous Vegetation ............................................................................... 182 